

$$
\text { ST } 1085
$$







T'HE volumes of the University of Michigan Studies are published by authority of the Executive Board of the Graduate School of the University of Michigan. A list of the volumes thus far published or arranged for is given at the end of this volume. 
OHnibrrsity of Mirbigan Studirs

HUMANISTIC SERIES

VOLUME $\mathrm{X}$

THE COPTIC MANUSCRIPTS IN THE

FREER COLLECTION 


\section{The $>$ X}

THE MACMILLAN COMPANY NEW YORK - BOSTON CHICAgO DALLAS ATLANTA - SAN FRANCISCO

MACMILLAN \& CO., LIMITED LONDON - BOMBAY - CALCUTTA

THE MACMILLAN CO. OF CANADA, LTD. TORONTO 



\section{PLATE I.}

\section{A}
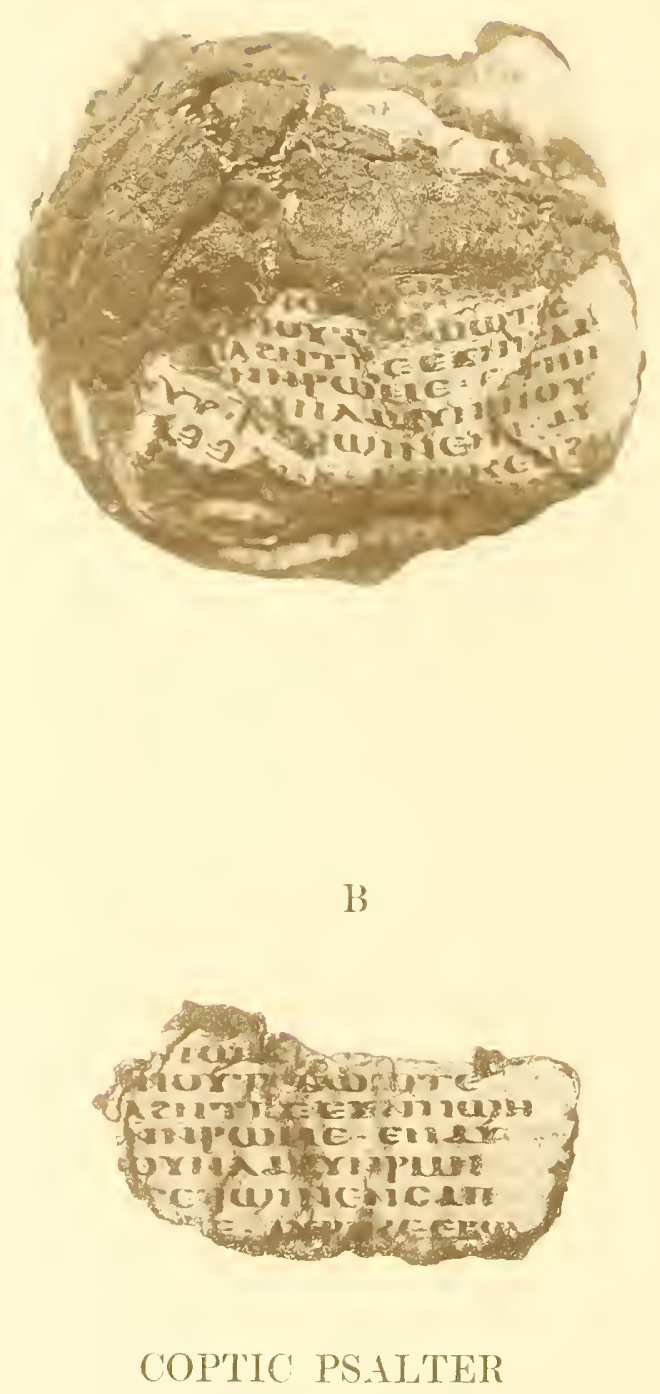

A. Ottside, BEFore separation OF LEAVES

B. Sirall regular hand [p. 256] 


\section{THE \\ COPTIC MANUSCRIPTS \\ IN THE \\ FREER COLLECTION}

EDITED BY

WILLIAM H. WORRELL

HARTFORD SEMINARY FOUNDATION

New Mark

THE MACMILLAN COMPANY

LONDON: MACMILLAN AND COMPANY

I923

All rights reserved
Freer Gallery of Art
Washington, D. C. 
PRINTED IN THE UNITED STATES OH AMERICA

Copyright, I923,

BY WILLIAM H. WORRELL.

Set up and electrotyped. Published July, 1923.

Nortoond 租rsgs

J. S. Cushing Co. - Berwick \& Smith Co.

Norwood, Mass., U.S.A. 


\section{PREFACE}

Part I of this volume was published in a small edition in r916. The publication of Part II was delayed by conditions resulting from the War. In reprinting Part I in this volume no changes have been made.

The editor desires to express his gratitude for the unfailing interest and support with which Mr. Charles L. Freer followed the preparation of this volume until his lamented death, in I9I9.

Professor Henry A. Sanders separated the leaves of the Psalter manuscript, read the proof-sheets throughout, and gave manifold advice in matters of palaeography and publication.

Professor Henri Hyvernat very kindly went over the entire material of the second part of this volume in advance of publication, and also read the proof-sheets. To him is due the credit of discovering that British Museum Manuscripts Or. 6780 and 7028 belonged with each other and with the leaves in the Freer collection.

Mr. Walter E. Crum loaned to the editor his personal collation of The Earliest Known Coptic Psalter with the original manuscript, obtained photographs of British Museum Manuscript Or. 6780, and gave much helpful advice.

Dr. L. D. Barnett obtained photographs of British Museum Manuscript Or. 7028, and kindly granted permission to publish both this and Or. 6780; and Mr. E. O. Winstedt generously waived a prior claim to this material.

Sir Frederic G. Kenyon kindly gave his opinion on the age of the handwriting of the Psalter manuscript.

Dr. A. A. Vaschalde, Dr. W. F. Albright, and Professor A.E. R. Boak gave assistance which has been acknowledged in its place. Dr. A. M. Perry kindly prepared the indices.

The libraries of the University of Michigan and of the Hartford Seminary Foundation gave much assistance through the purchase and loan of necessary books and photographs.

To all of these the eritor wishes to express his gratitude and indebtedness. 
The second homily is published as a new text, based upon a single manuscript; only the title is given by Budge, Miscellaneous Coptic Texts in the Dialect of Upper Egypt (I9I5), p. I I92. Since work was commenced upon it another, and apparently older, manuscript of the same homily has been brought to light which, however, has not been accessible to me; reference thereto is made in the article Coptic Literature, by Professor Henri Hyvernat, in the Catholic Encyclopedia, Vol. XVI, pp. 27 ff., and in A Check List of Coptic Manuscripts in the Pierpont Morgan Library, I9I9, under No. XII (M 600) of A.D. 906. It is to be hoped that when the other manuscript has been made available by publication, many of the emendations which I have felt obliged to suggest will no longer be necessary.

WILLIAM H. WORRELL.

Hartford, Connecticut,

May, I923. 


\section{CONTENTS}

\section{PART I}

INTRODUCTION :

PAGE

The Coptic Manuscripts and Fragments in the Freer Collection . . ix

Appearance and Make-up of the Collection . . . . . . xi

Vellum, Writing, Date . . . . . . . . . . $\mathrm{xv}$

Punctuation and Spelling $\quad . \quad$. $\quad . \quad$. $\quad . \quad$. . . . . . xviii

Affinities of the Text . . . . . . . . . . . . xxiii

Plan of this Edition . . . . . . . . . . . xxiii

The Reproduction of the Text . . . . . . . . $\mathrm{xxv}$

Text :

Coptic Psalter . . . . . . . . . . . . . . I

Psalter Fragment .

Job Fragment $\cdot$.

\section{PART II}

INTRODUCTION :

Manuscript No. 2 and Fragmeńt No. Io of the Freer Collection . . I I5

Appearance and Make-up of Ms. No. 2 and its Complements from the

British Museum . . . . . . . . . . . II6

Vellum, Writing, Date . . . . . . . . . . . 1 I 6

Punctuation and Spelling . . . . . . . . . . . .

Peculiarities of Inflection, Construction, and Vocabulary . . . $\quad$ I22

The Homilies . . . . . . . . . . . . 124

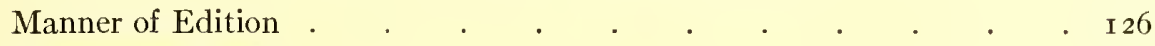

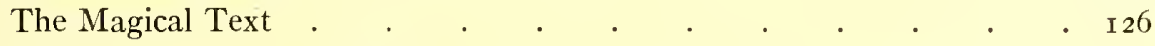

TEXT:

A Homily on the Archangel Gabriel by Celestinus, Archbishop of Rome I 29

A Homily on the Virgin by Theophilus, Archbishop of Alexandria . $\quad 249$

First Colophon . . . . . . . . . . . 32 I

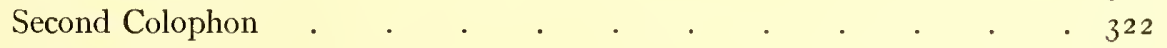

Magical Text . . . . . . . . . . . . . . 323

Translation :

A Homily on the Archangel Gabriel by Celestinus, Archbishop of Rome 327

A Homily on the Virgin by Theophilus, Archbishop of Alexandria . $\quad 359$

First Colophon . . . . . . . . . . . . . 379

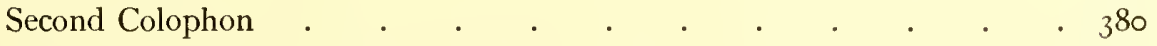

Magical Text • • $\cdot \cdot \cdot_{\text {vii }} \cdot \cdot \cdot \cdot \cdot \cdot 38$ r 
INDEXES :

Index of Coptic Words and Forms . • • • • . 385

Index of Greek Words . . . . . . . . . . . 386

Index of Biblical Passages . . . . . . . . . 392

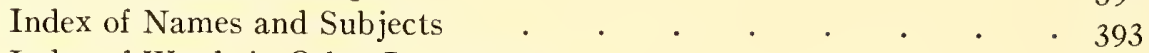

Index of Words in Other Languages . . . . . . . 396

\section{PLATES}

Plate I. Coptic Psalter . . . . . . Frontispiece

A. Outside, before separation of leaves.

B. Small regular hand.

Plate II. Coptic Psalter . . . . . . facing page xiv

A. Round or "Coptic" hand.

B. Change to square or "Greek" hand.

Plate III. Coptic Psalter . . . . . . . . xvi

A. Round hand: Coronis and number in primary hand.

B. Square hand: Chapter sign and number in secondary hand.

Plate IV. Coptic Psalter . . . . . . . . xviii

A. Round hand and square hand on the same leaf.

B. Square hand, with example of round form.

Plate V. Coptic Fragments . . . . . . . . 106

A. Fragment No. 9 [Job xxiv, $24 \mathrm{ff}$.$] .$

B. Fragment No. I [Ps. xliii, 24 ff.].

Plate Vi. Coptic Fragments . . . . . . . . . II 2 Fragment No. 2 [Mat. i, 2 I ff.].

Plate VII. Coptic Homily on the Archangel Gabriel . . . II4 British Museum Manuscript Oriental No. 7028 [p. I]

Plate VIII. Coptic Homily on the Virgin. . . . . . il 8 British Museum Manuscript Oriental No. 6780 [p. 6o]

Plate IX. Coptic Homily on the Virgin . . . . . . I 22

British Museum Manuscript Oriental No. 6780 [p. 6I]

Plate X. Coptic Homily on the Virgin. . . . . 248 Washington Coptic Manuscript No. 2 [p. 64]

Plate XI. Coptic Homily on the Virgin. : . . . 268

Washington Coptic Manuscript No. 2 [p. 7o]

Plate XiI. Coptic Fragments . . . . . . . . 322

Fragment No. Io, Magical Text 


\section{PART I}

THE PSALTER AND TWO FRAGMENTS 



\section{INTRODUCTION}

i. The Coptic Manuscripts and Fragments in the Freer Collection

The Freer collection contains the following Coptic manuscripts and fragments:

$\checkmark$ 08.32 Manuscript No. I, a large portion of the Psalter. (Vautt ; ms. boc 4)

08.33 Manuscript No. 2, part of a homily on the Virgin. (Vault; ms. bou 5)

09,148 Fragment No. I, a small portion of the Psalter on three pieces of vellum (Plate $\mathrm{V}, \mathrm{B})$.

08.34 Fragment No. 2, a small portion of Matthew (Plate VI; i, $22 \mathrm{ff}$.). ${ }^{1}$ voutt; ms. $60 x$ 5)

02.35 Fragment No. 3, three large leaves and four small pieces, having originally two columns of writing in a large, neat, Fayyûmic

(?) hand. In its present state the parchment is too badly crumpled to permit decipherment.

Q8.36 Fragment No.4, one fly-leaf having pen-trials: rixo $\overline{\mathbf{C C}} \overline{\mathbf{X C}}$ etc.

$08.3 \uparrow$ Fragment No. 5, a fragmentary single leaf, having upon one side a half cursive, very black hand, and upon the other a pale slanting hand. Unintelligible.

08.38 Fragment No. 6, two small pieces with a very defective text, (Vault; ms.bux 5) possibly magical. Unintelligible.

08.39 Fragment No. 7, three pieces of papyrus binding bearing letters (vault; ms. box 5 ) in a large hand. Illegible.

o8.40 Fragment No. 8, a parchment leaf from the inside of a book cover. It has a rude portrait of Christ (?), and an inscription of which one may still trace the letters:

\section{- - TIAxOeIC \\ EK(B)INNI $2 \epsilon--$ \\ EKMNTEPY}

r. 29 Fragment No. 9, a small portion of Job (Plate V, A). (Vault: ms. box 4)

08.41 Fragment No. IO, a magical text.

\$8,42 Fragment No. I I, piece of vellum once part of a book cover. Illegible.

09.199 Fragment No. I 2 , two pieces of vellum. Illegible. (Ms. box 5, vaut

ob. 43 Fragment No. I3, piece of vellum bearing an early hand. Illegible.

09. 150 a Fragment No. I4, two pieces of vellum. Illegible. (Vault: ans. bo 5)

1 The text, as far as legible, agrees with Horner's except for the reading ENTגq®ure pro птдqшшпє. 
? Fragment No. I 5, piece of vellum. Illegible. (See 14)

c. Fragment No. 16 , piece of vellum. Illegible. ${ }^{1}(" u)$

All of these except fragment No. Io are in the Sahidic dialect.

In the present publication we are concerned with all of them except manuscript No. 2 and fragment No. IO, which have been reserved for the second part of the volume.

Manuscript No. I and fragments 2 to 8 were bought by Mr. Charles L. Freer in May, I9o8, a year and a half after the purchase of the well-known Greek manuscripts of Deuteronomy and Joshua, the Psalms, the Four Gospels, and the fragments of the Pauline Epistles. Fragments I, I6 were bought in I909, and 9 was brought from Egypt in the spring of IgI 2.

In Volume IX of this series, The Washington Manuscript of the Four Gospels (1912), p. 3 f., ${ }^{2}$ Professor Sanders has stated about all that can as yet be said of the provenance of the collection. The dealer who sold the manuscripts, ${ }^{3}$ Ali al Arabi of the village of Gizeh, near Cairo, at first asserted that they had been acquired in Akhmîm, Upper Egypt. Later he owned that the statement was false, and produced the actual finders, who maintained that the manuscripts had been discovered at a spot which, though it cannot at present be named, is quite consistent with all the other evidence; it would have been a likely refuge for fugitives from the ruined Monastery of the Vinedresser, which lay near the third Pyramid, and was near enough to modern Gizeh so that the manuscripts would naturally be offered there for sale. It is therefore impossible to believe that the manuscripts were found near the White Monastery at Akhmîm, or at Eshmunên.

Manuscripts Nos. I and 2 and fragment No. Io appear to have been damaged by insects or worms working around the edges and making superficial holes, but not boring deeply. One of the Greek manuscripts was found to contain a living bookworm, so identified by biologists at the University of Michigan. This circumstance also connects the manuscripts with the Fayyûm. It must, however, be admitted that manuscript No. 2 was written in Esneh, and that other parts of the same manuscript, now in the British Museum, are said to have been found at Edfu.

The report that new finds had been made at Akhmîm-Sohâg, at the famous White Monastery, and that the Freer Greek manuscripts had come from that place, is easily explained by the fact that

1 The last three were reconstructed out of eleven small pieces.

${ }^{2}$ See also Vol. VIII, p. I, and references to earlier literature. $\quad{ }^{3}$ Except No. 9. 
Mr. Robert de Rustafjaell in February, 1907, heard of the discovery of manuscripts in Upper Egypt, and found upon investigation that part of them had been taken from an old Coptic monastery, about five miles south of Edfu, within whose ruins now stands a modern structure popularly known as the New White Monastery. Another story given him at the same time was that they had come from Thebes (Akhmîm-Sohâg). ${ }^{1}$

\section{ii. Appearance and Make-up of the Collection}

The theory of the Fayyumic origin of the collection - excepting the two large pieces, manuscript No. 2 and fragment No. IOis supported by an examination of the format (see plates) in connection with other manuscripts of known Fayyûmic provenance.

Manuscript No. I must have been, when complete, a volume of seven hundred pages of about $2 \frac{3}{4} \times 3 \frac{1}{8}$ inches, which would have been a size very difficult to bind or to use, as is recognized by the editors of the Oxyrhynchus Papyri, Vol. VII, No. Ioro. It is possible that the leaves were intended to be bound into small volumes, or not at all. The latter case would explain the extensive displacements hereafter to be discussed. As in the case of all the small manuscripts enumerated below (excepting the papyrus manuscript No. 28 of the John Rylands collection, Manchester), there is no trace of binding. Perhaps such sma!l-sized copies of the Psalms and Gospels may have been in vogue at one time and in one community, in which a use had thus been discovered for the trimmings of larger books. To reduce a large number of skins to a given size, strips of some width must often be cut off. These ordinarily include the rough and buckled parts around the extremities of the animal. Noteworthy in this connection is the rough hair-grain of parts of our manuscript. Such trimmings would furnish folio quires of small size; and the format, once established, may have been found convenient for certain purposes. The manuscripts mentioned by Horner, Coptic Version of the New Testament in the Southern Dialect (Oxford, I9 I I), Vol. III, Appendix, p. 377 ff., though small, appear to be somewhat larger than one would expect to have been made from scraps of parchment.

For purposes of comparison I append a list of the small manuscripts which have come to my notice:

${ }^{1}$ See The Light of Egypt, by Mr. de Rustafjaell, London, I909; Coptic Apocrypha in the Dialect of Upper Egypt, by E. A. W. Budge, London, I903; a review of Budge's Coptic Apocrypha in Zeitschrift der deutschen morgenländischen Gesellschaft, Vol. 68, p. I 76 f., by Mr. W. E. Crum; also an article in the Theologische Literaturzeitung, 1908 , No. 12, by Dr. Carl Schmidt. 
I. Size, $5.6 \times 8.4 \mathrm{~cm}$. Vellum. $4^{\text {th }}$ century. Greek. Fayyûm. I 2 lines of Io letters each. Large hand. Oxyrhynchus Papyri, Vol. VII, igro, No. iо Iо.

2. Size, $5.8 \times 9 \mathrm{~cm}$. Vellum. 5th century (?). Coptic. Fayyûm. I 2 lines of 8 letters each. Large hand. Freer collection, fragment No. 2.

3. Size, $6.4 \times 7 \mathrm{~cm}$. Vellum. 5th century (?). Coptic. Fayyûm. I 5 lines of I I letters each. Illegible. Freer collection, fragment No. 3. Possibly a part of No. Io below.

4. Size, $6.6 \times 7.5 \mathrm{~cm}$. Papyrus. 3d or 4th century. Greek. Provenance unknown. I 5 lines of 18 letters each. Large hand. John Rylands Library, Manchester, No. 28. See Hunt, Catalogue of the Greek Papyri in the John Rylands Library, Manchester, I9I I, plate 5, I 8 . Traces of binding.

5. Size, $7 \times 8 \mathrm{~cm}$. Vellum. 5th century (?). Coptic. Fayyûm. I 8 lines of 12 letters each. Large hand. Freer collection, No. I. This is the Psalter manuscript here published.

6. Size, $7 \times 8.5 \mathrm{~cm}$. Vellum. Two fragments, now separated, but once a double leaf of a quire - perhaps the second and seventh of an eight-leaved quire. 5th century (?). Coptic. Fayyûm. 20 lines of $\mathrm{I} 3$ letters each. Small hand. Freer collection, fragment No. 9. This is the Job fragment here published.

7. Size, $7 \times 9 \mathrm{~cm}$. Vellum. $5^{\text {th }}$ century (?). Coptic. Provenance unknown. I I lines of I l letters each. Very large hand. British Museum, manuscript Or. 35I8. See Crum, Catalogue of the Coptic Manuscripts in the British Museum, I90I, No. I42. Kenyon, Handbook to the Criticism of the New Testament, I90 I, p. I60. The Berlin Museum manuscript Or. oct. 408 is said to be a part of the same.

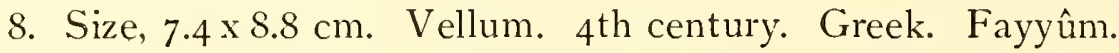
22 lines of 27 letters each. Very small hand. Oxyrhynchus Papyri, Vol. V, i 908, No. 840.

9. Size, $8 \times$ Iо. I cm. Vellum. Early 4 th century. Akhmimic Coptic. I 2 lines of about I I letters each. Thought by Crum to be written in a special Middle Egyptian hand. Crum, Coptic Manuscripts Brought from the Fayyum, London, I893, p. 2 f., plate I.

IO. Size, $9 \times 9.8 \mathrm{~cm}$. Vellum. 4th century. Greek. Fayyûm. I 4 lines of 18 letters each. Medium large, thin hand. Oxyrhynchus Papyri, Vol. VI, No. 849.

I I. Size, 9 x Io $\mathrm{cm}$. Vellum. 5th century. Greek. Fayyûm. I 2 letters to a line. Large hand. Oxyrhynchus Papyri, Vol. VI, No. 848 , cf. No. 847 . 
1 2. Size, 9x ro cm. Vellum. 5th century (?). Coptic. Fayyûm. 24 lines preserved, of I 8-20 letters each. Small, beautiful, ancient hand. Freer collection, fragment No. I.

The remaining Freer fragments are not in a condition to be utilized in the discussion.

When brought to Detroit the Psalter manuscript (No. I) appeared as in Plate I. It had been attacked by insects or worms. Decay, and perhaps fire, had reduced the edges to carbon, under which, especially at one corner, the parchment had melted into a gelatinous mass containing particles of sand. Shrinkage at the edges was apparent. Within the mass were three fresh breaks, undoubtedly modern, and without displacement of the leaves.

The separation of the leaves, which were very thin and brittle, was successfully accomplished by Professor Sanders through the use of a damp sponge and penknife. Often two leaves would adhere so firmly as to seem in every respect to be one. They were largest at the middle of the mass, and decreased toward the ends until they became small fragments. Not all of the smallest pieces, containing sometimes only a few letters, could be positively identified.

A remarkable and difficult feature was the displacement of leaves, which is shown in the following comparative tables, the first of which indicates the order of the leaves when the manuscript was discovered, the second the true order in which they must have been arranged in the beginning.

Fig. I. Table showing the Arrangement of Leaves and Pages in the Coptic Psalter when Discovered

\begin{tabular}{|c|c|c|c|c|c|}
\hline $\begin{array}{c}\text { ORDER OF } \\
\text { LEAVES }\end{array}$ & PAGING & BLOCKS & $\begin{array}{l}\text { ORDER OF } \\
\text { LEAVES }\end{array}$ & PAGING & BLOCKS \\
\hline I $28-13^{\circ}$ & fragments & \multirow{7}{*}{ I 5} & 54 & \multirow{8}{*}{$\begin{array}{c}\text { I } 38-137 \\
\text { 1 } 79-180 \\
200-199 \mathrm{frg} . \\
\text { 1 } 79-180 \mathrm{frg} . \\
200-18 \text { I } \\
\text { 1 } 78-165 \\
201-202 \mathrm{frg} . \\
\text { fragment }\end{array}$} & 8 \\
\hline $125-127$ & $24^{-19}$ & & 53 & & 7 \\
\hline $123^{-124}$ & $25^{-26}$ & & $5^{2}$ & & \\
\hline I $14-122$ & $44^{-27}$ & & $5^{1}$ & & \\
\hline lost & $45^{-46}$ & & $4^{I-5^{\circ}}$ & & 6 \\
\hline I OO-I I 3 & $74-47$ & & $34-40$ & & 5 \\
\hline 99 & fragment & & 33 & & \\
\hline $9^{2-9^{8}}$ & I $5 I-I 64$ & I 4 & $3^{2}$ & & \\
\hline $89-91$ & I $43^{- \text {I }} 4^{8}$ & I 3 & $6-3 i$ & $25 \mathrm{I}-20 \mathrm{I}$ & 4 \\
\hline 88 & I $40-139$ & & $4-5$ & $25^{2-2} 5^{6}$ & 3 \\
\hline 87 & $\mathrm{I} 42-\mathrm{I} 4 \mathrm{I}$ & 12 & 3 & $257^{-2} 5^{8}$ & 2 \\
\hline $8 I-86$ & $75^{-86}$ & I I & $I-2$ & I 7-I 8 & I \\
\hline $5^{6}-80$ & I $36-87$ & 10 & $\circ$ & fragments & \\
\hline 55 & I $50-149$ & 9 & & & \\
\hline
\end{tabular}


Fig. 2. Table showing the Proper or Original Arrangement of Leaves and Pages in the Psalter

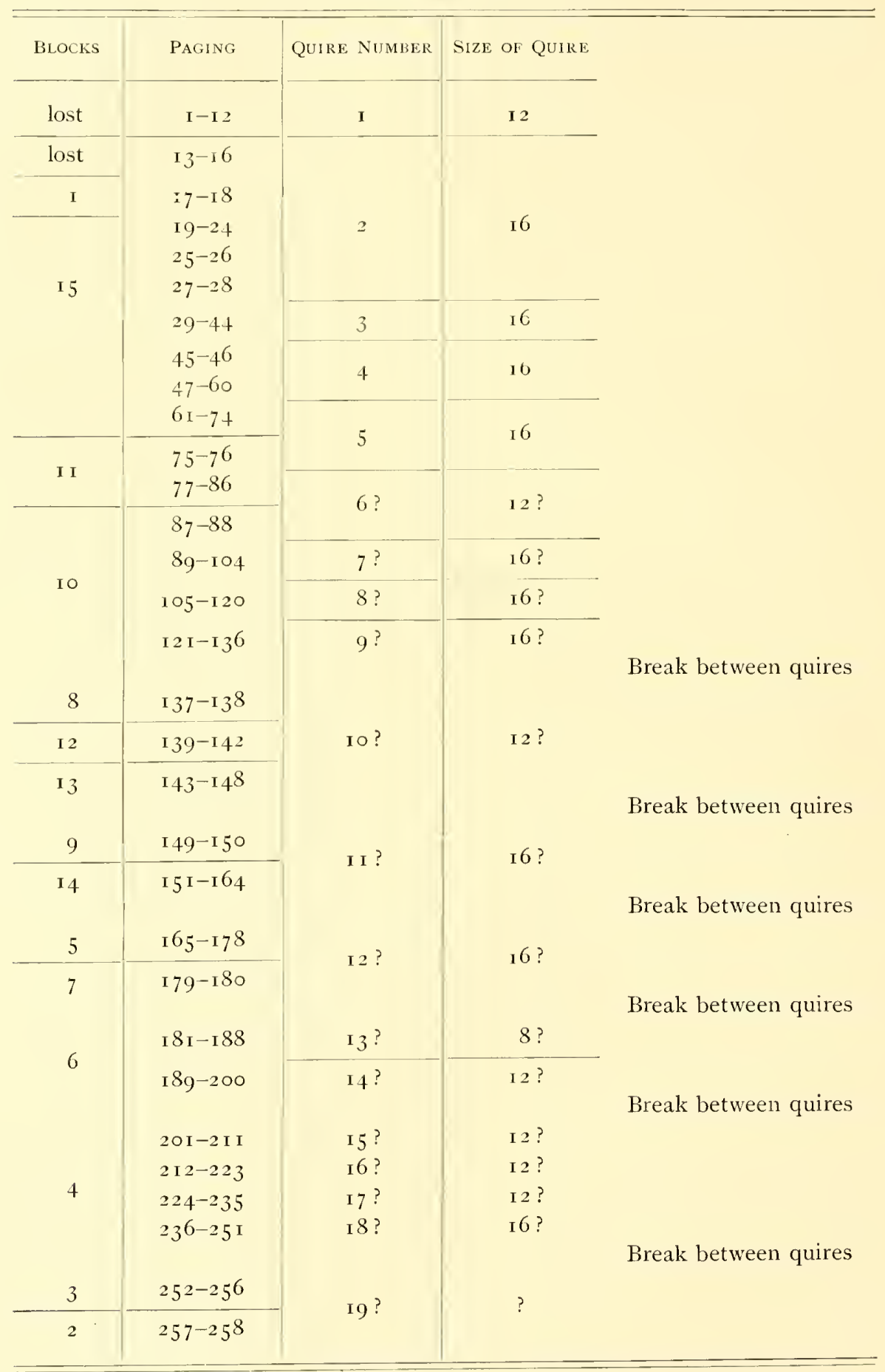




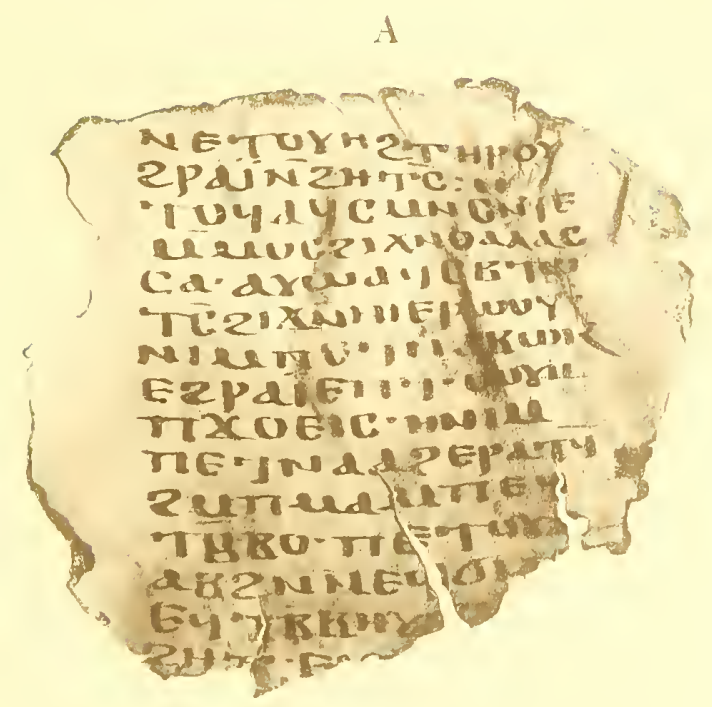

B

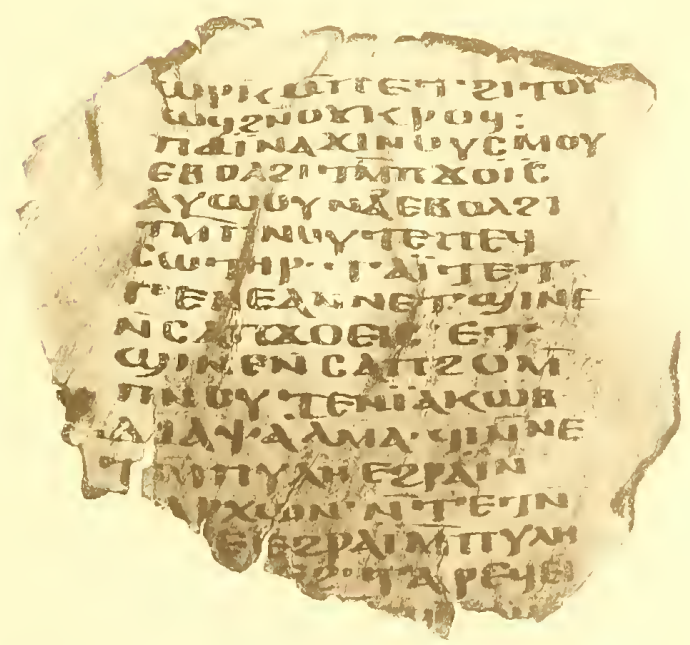

\section{('OPTIC' PSAITER}

A. RoCNe or "Coptec" hand [p. 10:3]

B. ("HAN(iE TO SQUARE OR "GREEK" HAND, I. :3 [p. 104] 

The quire signature $\overline{\boldsymbol{\epsilon}}$ in the upper right hand corner of page 76 shows that the latter closed the fifth quire of the volume or of the first of several small volumes, and that probably the first five quires, allowing four blank pages at the beginning, were arranged as $12+\mathrm{I} 6+\mathrm{I} 6+\mathrm{I} 6+\mathrm{I} 6$. The scheme of the remaining quires is purely hypothetical, but supplies an arrangement by which the breaks occur for the most part between quires. Yet even so there is a break within the fifth quire (pages $74-75$ ). There are no traces of binding; and if there ever were quires of any size they must have been reduced to loose leaves before the ancient displacements took place.

\section{iii. Vellum, Writing, Date}

Of the illegible fragments $3^{-8}$ nothing need be said.

Fragments I, 2, and 9, and manuscript No. I are written upon similar material, in handwriting which is similar yet not identical, and they are all doubtless of about the same period. They have been described above (pages xii f.), and the dating is discussed below. Cf. plates 5 and 6 .

The Psalter manuscript No. I is mostly or entirely of goat skin, varying in thickness from eight to seventeen hundredths of a millimeter. The leaves were originally about $7 \times 8 \mathrm{~cm}$., and contained from 13 to 18 lines of from 8 to i 8 letters each: a quite extraordinary fluctuation. The upper lines are generally shortest, and the last line or two longest, but not invariably so (cf. page I40). Note the short line 15 on page 153 . Ruling is visible in a few places only; and is provided sometimes for every line, - especially for the first two lines, - sometimes for alternate lines. The characters are placed usually just above or just below, rarely upon the lines. The marginal limits are maintained by perpendiculars.

The writing is clear but rude. One is inclined at first to recognize three distinct hands (as shown in Plates I-IV), which for convenience we shall call $A, B$, and $C$.

$A$ is found pages i 8 -ro4, line 3 a ; I 6 -I I 7 ; I 26-2 37; 248-25 2 .

$B$ is found pages 104, line $3 \mathrm{~b}-\mathrm{II} 5 ;$ I $18-\mathrm{I} 25 ; 238-247$.

$C$ is found pages $253^{-258}$.

On page 240 a single upsilon occurs, and on pages 239 and $244 \mathrm{a}$ mu, which, though found in the midst of a $B$ section, would have to be assigned to $A$.

$A$ is characterized by "round" forms of the three letters alpha, mu, ${ }^{1}$ and upsilon: $\boldsymbol{A} \boldsymbol{\mu} \boldsymbol{\gamma}$.

1 Another form $\boldsymbol{u}$ is occasionally used for condensation; cf. pages $198,229$. 
$B$ is characterized by "square" forms of the same: $\boldsymbol{A} \mathbf{M} \boldsymbol{Y}$.

$C$ is a very small, regular hand, having exceptionally long and upright stems to alpha and mu, and a shima which leans backward.

$A$ more careful examination reveals the fact that $A$ and $B$ are, except for the three letters mentioned, identical ; cf. especially the ugly form of hori. With less certainty one must also admit that shrinkage of the parchment might explain the condensed, upright, rigid character of $C$. The hori of $C$ is not always as bad as in $A$ and $B$, however. Although not entirely certain, it is probable that the whole manuscript is from one hand.

The few corrections in the manuscript are in dark ink, and were probably made by the original scribe before leaving the page, and without blotting off. There was probably no diorthotes, since other errors remain uncorrected (cf. page r 40). The coronis and chapter number (or other sign) are written in dark ink on pages 87,90 , IOO, I 24, I 3 I, 169, I 88,2 I 0,224 , probably simultaneously with the text; but in light or blotted off ink on pages I06, I I 2, I I 5, I 27, very likely at a later time, by the same or another scribe. Similarly the page numbers 72 , 88 , I I 7 , seem to be in darker ink than pages $73,76,8_{3}, 86$, and the quire signature 5 .

It remains to explain why a scribe should employ for pages at a time, alternately, and with occasional slips, first the "round" and then the "square" forms of alpha, mu, and upsilon; and to determine whether such use has any bearing upon the date of the manuscript.

It must be accepted as evident from our manuscript, if it were not otherwise established, that both the "round" and "square" forms were in use at the same period. The fluctuation of the copyist may have been fanciful, or due to the use of different exemplars on different occasions. The "round" forms greatly predominate. None of the theories suggested to me, as e.g. the influence of the hair-side and flesh-side of the parchment, seems applicable.

The dating of Coptic manuscripts is notoriously difficult. Professor Hyvernat promises a revision of accepted opinion on the basis of the Morgan collection; but his publications are not yet available, and our data are meager.

The London Sahidic Psalter manuscript (Brit. Mus. Ms. Or. 5000 ) has been dated by its editor, E. A. W. Budge, at about 700 A.D.; the Berlin Sahidic manuscript by its editor, Alfred 


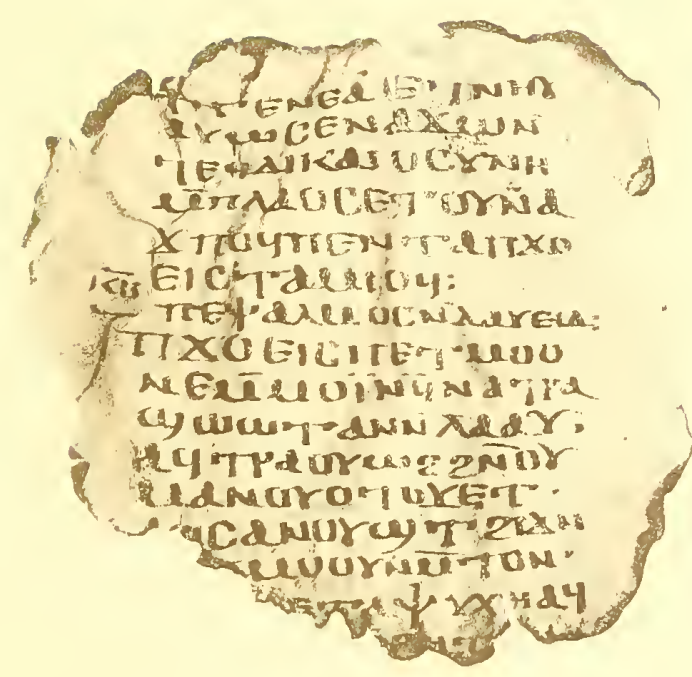

B)

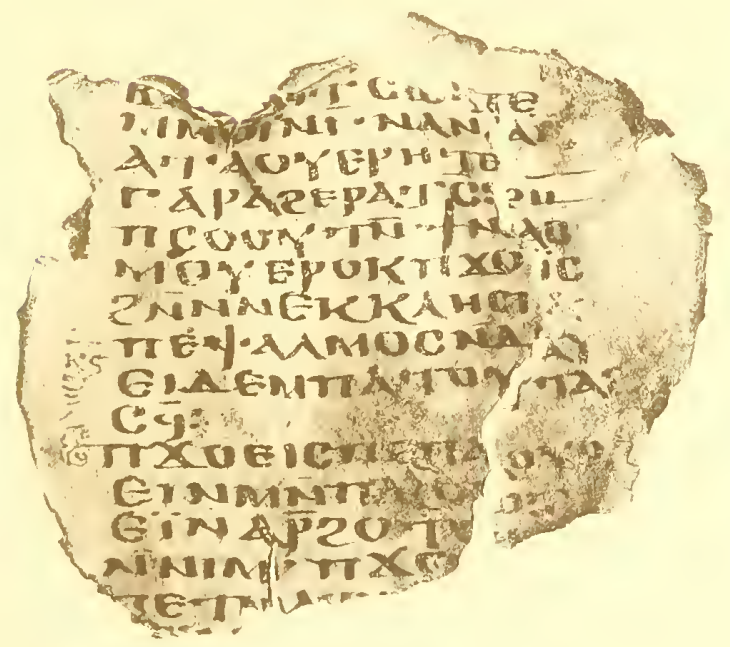

COPTIC' PSAITER

A. RoCND HaND: CORONis AND NEMBER IN PRIMART HAND [p. 100]

B. S(QUARE HAND: ('HAPTER SI(XN AND NUMBR IN SE(ONDART HAND [p. 115] 

Rahlfs, at about 400 A.D. The Freer manuscript may be presumed to lie between these two. Dr. Kenyon is inclined to put it at the extreme end of this period or later. On the other hand, the small sized Fayyûmic manuscripts mentioned, which resemble it in appearance, are dated by their editors from the third to the fifth centuries, and no later.

Rahlfs, in the introduction to his edition of the Berlin manuscript, Die Berliner Handschrift des sahidischen Psalters (Berlin, I90 I), page I 2, says: "Auch die Schrift beweist, dass der Berliner Psalter zu den ältesten koptischen Pergamenthss. gehört. Die griechischen Buchstaben zeigen keine Spur von dem specifisch koptischen Ductus, sondern sind den Buchstaben der ältesten griechischen Pergamenthss. sehr ähnlich ...." It is difficult to understand how the presence or absence of the "round," so-called "Coptic" forms of alpha, mu, and upsilon (if that is the editor's meaning) could be of much service in dating a manuscript which in any case is later than the third century.

At the beginning of Coptic Christianity and of Coptic literature, the Egyptians simply used the contemporary Greek hands, of which a great many were current in Egypt. ${ }^{1}$ The so-called Coptic forms were not used exclusively by Copts, nor exclusively for writing Coptic. In all the centuries of the uncial one may find varying degrees of admixture of these forms; and not alone in Egypt. They are really cursive forms, which came to be used more and more by writers of Coptic in proportion to their disuse by writers of Greek uncial. There seems to be some doubt as to the order of their introduction into Greek manuscripts. Thompson, in his Introduction to Greck and Latin Palacography (I 9 I 2), pages I $90 \mathrm{ff.}$, gives upsilon from the third century B.C. onward; alpha from the first century B.C. onward; and mu from the first century A.D. onward. Gardthausen traces alpha back to the Ptolemaic period, and mu and upsilon to the first century A.D., after which time they are said to have been in continued but not exclusive use. For instance, one manuscript of $8 S$ A.D. has alpha only; another, of 99-IOO A.D., has all three letters; two others, of the first or second centuries A.D., have mu and upsilon only; another, of 200-295, has none of the three; the Sinaiticus, of 400 A.D., has mu only; the Vaticanus, of the fourth century, and the Alexandrinus, of the fifth century, use mu in numerals, in titles, and at the ends of lines. Thirteen others, from the early sixth to the tenth century, lack all

${ }^{1}$ Cf. Gardthausen, National- und Provincialschriften, in the Byzantinische Zitschrift, Vol. XV, I906; and Griechische Paläographie, I9I3, Vol. II, page $248 \mathrm{f}$. 
three of the forms, except upsilon in one manuscript of the tenth century.

As for the Coptic manuscripts, the Berlin Psalter, of about 400 A.D., has none of the forms; but a manuscript, dated by Kenyon 350 A.D. and published by Budge in his Coptic Biblical Texts in the Dialect of Upper Egypt (London, I912), employs mu at the end of a line (Plate 2), mu and upsilon at the end of a line (Plate I), all three forms (Plate 4), and none of the forms (Plates 3, 5, 6, $7,8,9)$. Plate 4 exhibits both "round" and "square" forms on the same page. The London Coptic Psalter, published by Budge as The Earliest Known Coptic Psalter (London, I898), and dated, as we have seen, about 700 A.D., shows in the plates all three of the letters, with no exceptions. Yet the sixth-century manuscript in Fïhrer durch die Ausstellung der Sammlung Erzherzog Rainer, Vienna, I 894 (Plate 7, Nos. I 33-140), mixes the forms.

\section{iv. Punctuation and Spelling}

The manuscript employs no capitals and no coloring. At the end of Psalm 49 a simple ornament is used, as also on page II 5 (Plate III). A coronis is generally used to mark the actual beginning of a psalm, and the first word of the psalm or of the title may be indicated by slight extension into the margin; but there is no invariable rule. There is no spacing between words. The usual abbreviations $\overline{\boldsymbol{\Pi} N \boldsymbol{\lambda}}$ and $\overline{\boldsymbol{\Pi} \boldsymbol{T H \boldsymbol { \lambda }}}$ are employed. As in Greek manuscripts, final nun at the end of a line is sometimes indicated by a short dash above the vowel (see pages 52, I4I, 190, 207). A similar dash is placed over a single syllabic vowel at the end of a line and belonging to the first word in the following line (see pages 54, I93, line I 3, 196, 207, 21 7), but not invariably so (page I93, lines 2,3). A short dash sometimes occurs in place of the familiar double dot over the iota (pages I IO, 245). On page 207 is found по $\mathbf{Y} \boldsymbol{X} \boldsymbol{x} \mathbf{}$. The five signs - - , are used indiscriminately above or to the right of the last letter of the word, as hereafter described. The last three are rare (pages $16_{3}, 224$ ), and the - is by far the commonest. They are employed in the following positions:

I. With a final consonant before an initial consonant.

2. With a final vowel before an initial vowel.

3. With a final consonant before an initial vowel.

4. With a final vowel before an initial consonant.

The first of these occurs about twice as often as any of the others, which are about equally represented; and it is the first of 
PLATE IV.

A

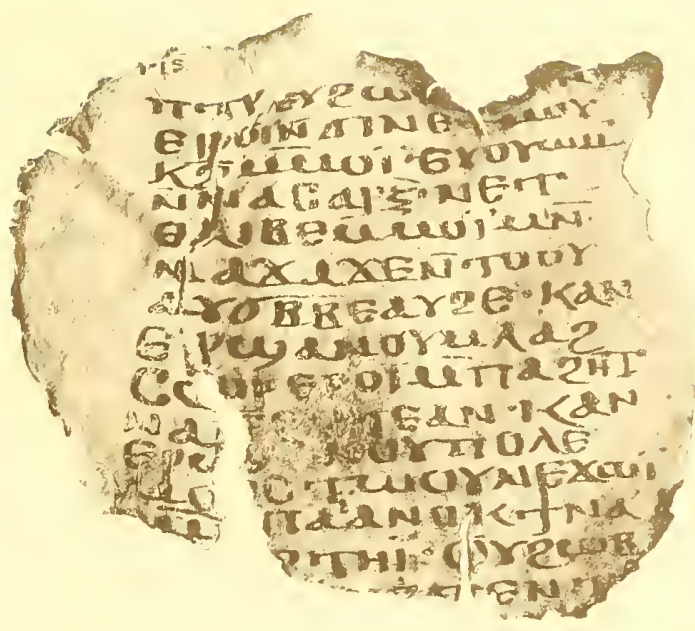

B

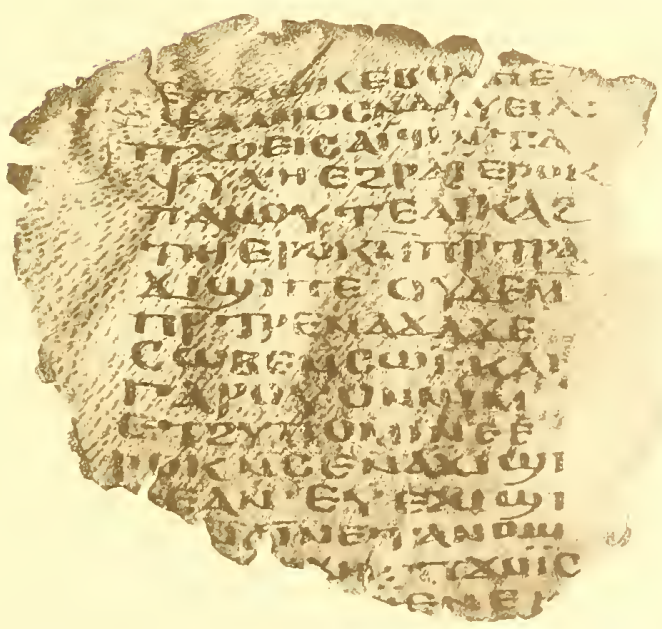

COPTIC: PSALTER

A. Rolid hand and square hand on the same leaf [p. 116, cf. p. $11 j]$

B. S(QUARE HAND: ROTND FORM IN $1.1 \pm[\mathrm{p} .106]$ 

these which most nearly resembles the Greek usage with foreign words, particularly proper names having un-Hellenic endings. Correct division of the syllables in Coptic being vital to the sense, the functions of the sign were extended.

A single point, in one of three positions $(\cdot)$ without distinction, is used for primary divisions of punctuation. The double point (:) has greater force. Instead of the usual lines placed over

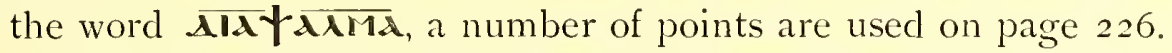

The superlinear dashes are usually placed or extended too far to the right, as is common in manuscripts. Since there is a general tendency to make swinging off-strokes (pages I $85,232,142$ ) and long dashes (pages $38,196,236$ ) at the margin, there can hardly be any significance in such forms as MNOY-for MN OYpage 49 , or 2NNET - for $2 \bar{N}$ NET- page 77 , or NINET- for NNNETpage 249. But a helping vowel, before or after the first member of a double consonance, may be indicated in $\overline{2}$ THC for 2 THC

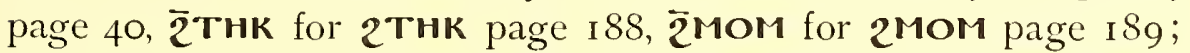
and between a liquid and a following consonant in Mīleq- page

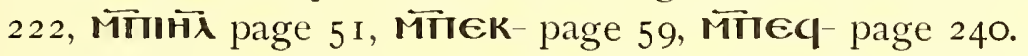

Like the London Psalter ${ }^{1}$ our manuscript writes XolC instead of $\mathbf{x o e i c}$ when it is necessary to save space at the end of a line. In other positions it has the latter form. This alone would show that no phonetic difference is involved in the two ways of writing the vowel.

The present manuscript, $\mathbf{F}$, greatly favors the use of $\mathbf{I}$ for $\mathbf{E}$. The following is a list of the instances in which $\mathrm{F}$ differs from $\mathrm{L}$, with the readings of all available manuscripts at these points.

A. I for $\mathbf{E}$ in Greek loan words:

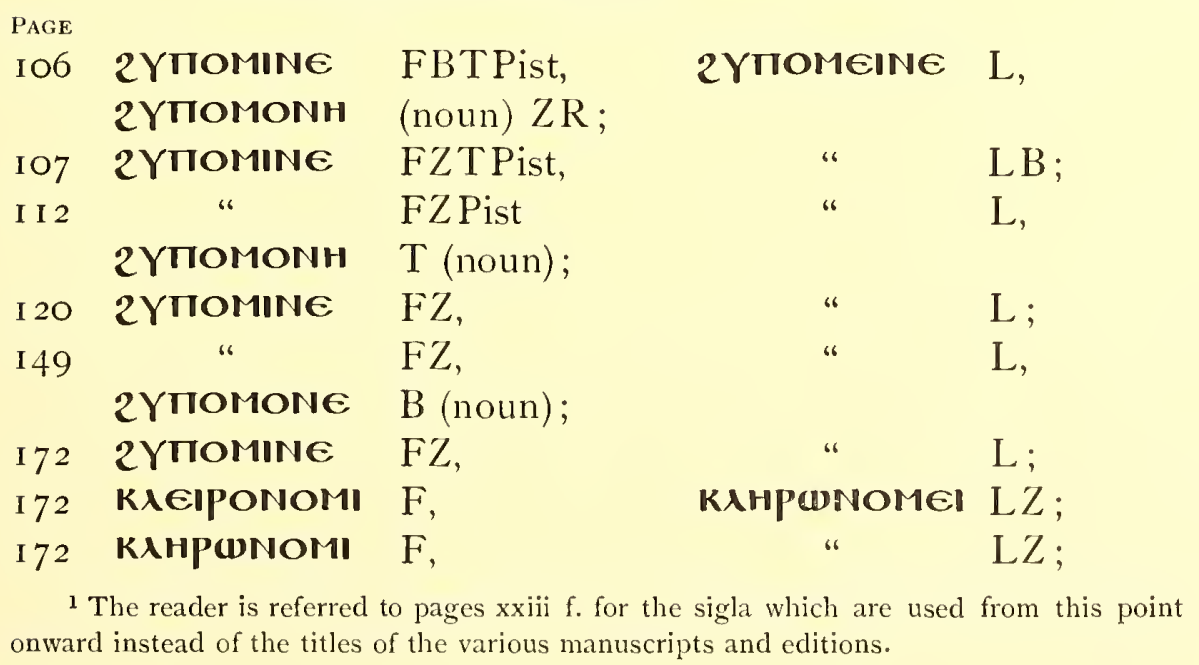




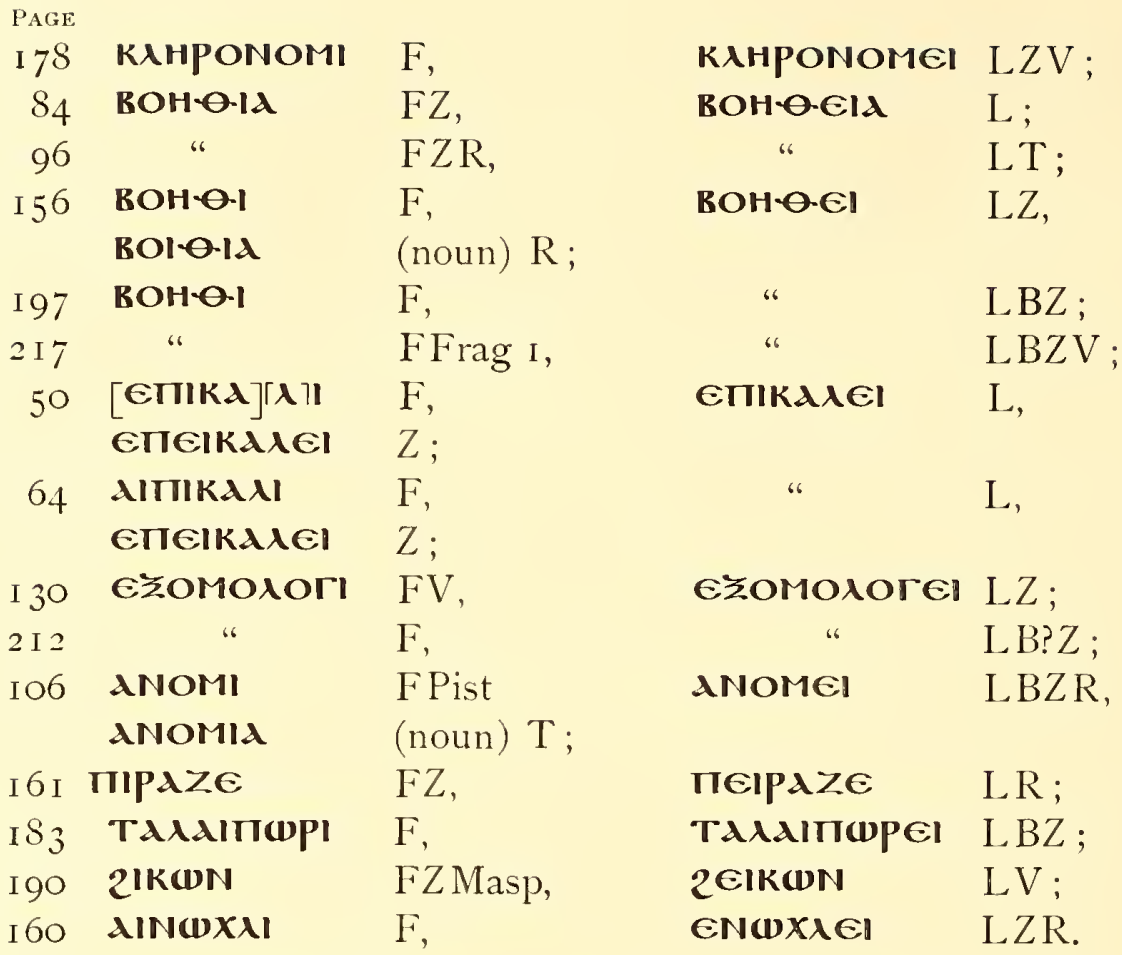

B. Al for $\mathbf{\lambda} \mathbf{\epsilon}$ in Coptic words:

$\begin{array}{ccccc}\text { I67 } & \text { OגIBEC } & \text { FBZV, } & \text { OגeıвEC } & \text { L; } \\ \text { 2I5 } & \text { " } & \text { FBZV, } & \text { " } & \text { L. }\end{array}$

C. Ol for OEl in Coptic words :

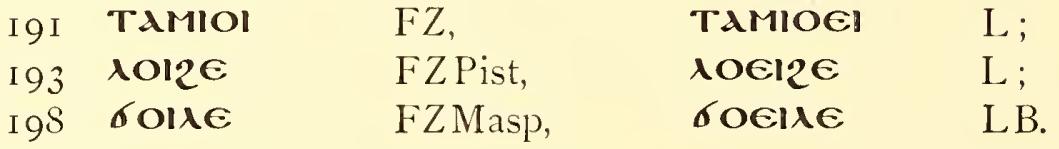

D. OYI for OYGl in Coptic words:
6 I NNIMOYI $\mathrm{FZ}$, NNEIMOYI R.
NNIMOYEI L,

E. I for $\mathbf{\epsilon}$ as initial consonant of a syllable in Coptic words:

\begin{tabular}{|c|c|c|c|c|}
\hline \multirow{3}{*}{$\begin{array}{r}73 \\
\text { I } 26\end{array}$} & NI-E-IOYA & $\mathrm{F}$, & NI_e_eloYt & $\mathrm{L} Z$; \\
\hline & $\mathrm{N}-\mathrm{G}-\operatorname{IOY} \lambda$ & $\mathrm{F}$, & N-E-EloY $\lambda$ & $\mathrm{L}$ \\
\hline & N-le-OYג & (metathesis) Z R & & \\
\hline 20 & OY-E-IOYג & $\mathrm{F}$, & OY-E-EIOYA & $\mathrm{LZ}$; \\
\hline 21 & Td-10 & $\mathrm{F}$ & Td-ClO & LZ; \\
\hline 22 & MП-IE-PO & $\mathrm{F}$, & Mா-€IE-PO & LZR Masp. \\
\hline
\end{tabular}

It will be seen at once that $F$ has $\mathbf{I}$ and $L$ has $\boldsymbol{\epsilon}$ without exception, the remainder being divided. 
The manuscripts exhibit the usual, and some unusual, cases of confusion between vowels, diphthongs, and consonants. In Greek words :

$\mathbf{H}$ and $\mathbf{\epsilon}, \boldsymbol{H}$ and $\mathbf{Y}, \boldsymbol{H}$ and $\mathbf{I}, \boldsymbol{\epsilon}$ and $\boldsymbol{\lambda} \mathbf{I}, \boldsymbol{\epsilon}$ and $\boldsymbol{A}, \boldsymbol{H}$ and $\boldsymbol{A}$ (Doric), $\boldsymbol{\omega}$ and $\boldsymbol{O}, \boldsymbol{T}$ and $\boldsymbol{A}, \boldsymbol{\Gamma}$ and $\boldsymbol{K}, \boldsymbol{\Gamma}$ 尘 and

\begin{tabular}{|c|c|c|c|c|}
\hline $\begin{array}{l}\text { PAGE } \\
207\end{array}$ & TEAYTTH & $\mathrm{F}$, & TEAYTE & $\mathrm{LZ}$; \\
\hline 209 & " & $\mathrm{F}$, & " & $\mathrm{L} Z$; \\
\hline 67 & ПНГН & $\mathrm{LB}$ & ПҮГн & FZ; \\
\hline I 68 & “ & $\mathrm{LB}$ & “" & $\mathrm{FZ}$; \\
\hline I 27 & КАТАКАНСМОС & FR, & КАТАКАҮСMOC & $\mathrm{LZ}$; \\
\hline I 4 I & " & $\mathrm{F}$, & “ & $\mathrm{LZ}$; \\
\hline I 56 & ВОН-O-(E) & FLZ, & BOl-0.1d & $\mathrm{R}$; \\
\hline 64 & $\epsilon \Pi(\Theta) \| \mathbf{K \lambda \lambda \epsilon ।}$ & $\mathrm{L}, Z$ & АITIKA入I & $\mathrm{F}$ \\
\hline 66 & XEPOYR(E) IN & $\mathrm{LB}$ & XAIPOYBIN & Z ; \\
\hline I 60 & ENAXA(G)I & LZR, & AINOXXI & $\mathrm{F}$ \\
\hline I 70 & етнMА & $\mathrm{F}$ & АITHMA & $\mathrm{LZ}$; \\
\hline I 26 & MONOK $[\Gamma] \in P W\left[\mathrm{O}^{-} \mathrm{C}\right.$ & LZ, & MONOKAPOC & $\mathrm{F}$ \\
\hline $\begin{array}{r}83 \\
\text { I } 26\end{array}$ & $\begin{array}{l}\text { MEᄉЕTH } \\
\text { (supra); }\end{array}$ & $\mathrm{L} Z$, & MeגeTג & FB ; \\
\hline I 72,6 & КАHPWNOMEI & $\mathrm{L} Z$ & KגєIPONOMI & $\mathrm{F}$; \\
\hline $\begin{array}{l}253 \\
\text { I } 26\end{array}$ & $\begin{array}{l}\text { TYNATOC } \\
\text { (supra); }\end{array}$ & $\mathrm{F}$ & AYNATOC & $\mathrm{LZ}$; \\
\hline I 42 & MACTIГ亡 & FBZ, & MACTI乏 & $\mathrm{L} ;$ \\
\hline I 6 I & “ & FZR, & " & $\mathrm{L}$ \\
\hline I 86 & " & FBR V, & “ & $\mathrm{LZ}$ \\
\hline I 9 I & “ & $\mathrm{FB}$, & “ & LZ \\
\hline
\end{tabular}

I note also the spelling $\mathbf{X} \boldsymbol{A} \mathbf{A} \boldsymbol{A C Z \boldsymbol { A }}$ (page 67 ) which may be an error merely.

$I V$ preceded by a vowel is written oY or $\mathrm{Y}$ in $\mathrm{F}$.

\begin{tabular}{|c|c|c|c|c|}
\hline 73 & $\begin{array}{l}\text { XIcieoY } \\
\text { XIce€Y€ (erro }\end{array}$ & $\begin{array}{l}\mathrm{F} \\
\mathrm{r} \text { ?) } \mathrm{Z}\end{array}$ & XICIE€Y & $\mathrm{L}$, \\
\hline 94 & MOOY & FBZT, & MOY & $\mathrm{L}$; \\
\hline $8 \mathbf{I}$ & meOYoel(1) & $\mathrm{LZ}$ & пеүоєা(1) & $\mathrm{F}$; \\
\hline I68, IC & eYOYOEIN & LBZ, & EYOEIN & F (doubled). \\
\hline
\end{tabular}

F shows a preference for the doubling or "breaking" of vowels:

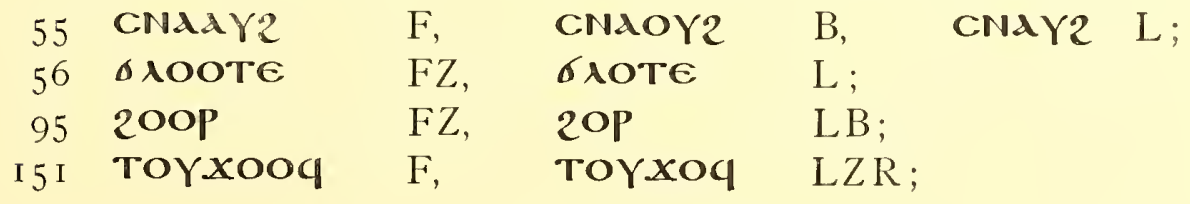


PAGE

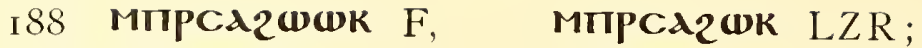

2 I 5 CA2waq F,

$\mathrm{Cd} \mathbf{\omega q}$

LZV.

On the other hand, $F$ writes $\hat{\mathrm{u}}-\mathrm{o}$ and $\hat{\mathrm{u}}$-e for $\hat{\mathrm{u}}$-wo and $\hat{\mathrm{u}}-\mathrm{w}^{\mathrm{e}}$ :

$\begin{array}{lllll}\text { I33 OYOCTN } & \text { FZ, } & \text { OYOYOCTN } & \text { L; } \\ \text { I60 EKOYNT } & \text { FR, } & \text { EKOYOYNT } & \text { LZ; }\end{array}$

Old forms of the article appear in two instances:

$$
8 \text { I (bis) NNıKOYI F, NNKOYI LZ; }
$$

as also the uncontracted forms:

$\begin{array}{lllll}39 & \text { пеET- } & \text { F, } & \text { пеT- } & \text { T; } \\ 50 & \text { nEET (?) } & \text { F, } & \text { NET- } & \text { LZ. }\end{array}$

A striking feature of our manuscript is the doubling of intervocalic $n$, noted by Stern (Koptische Grammatik, § 72) as a peculiarity of Middle-Egyptian and, rarely, of Sahidic manuscripts. It occurs not only with $\overline{\mathbf{N}}$ and $\overline{2 N}$ (Stern), but also with $\mathbf{M N}$, $2 \| \overline{T N}, X I N, 2 \in N$, NNA2 $\overline{P N}$, and $A \overline{X N}$ :

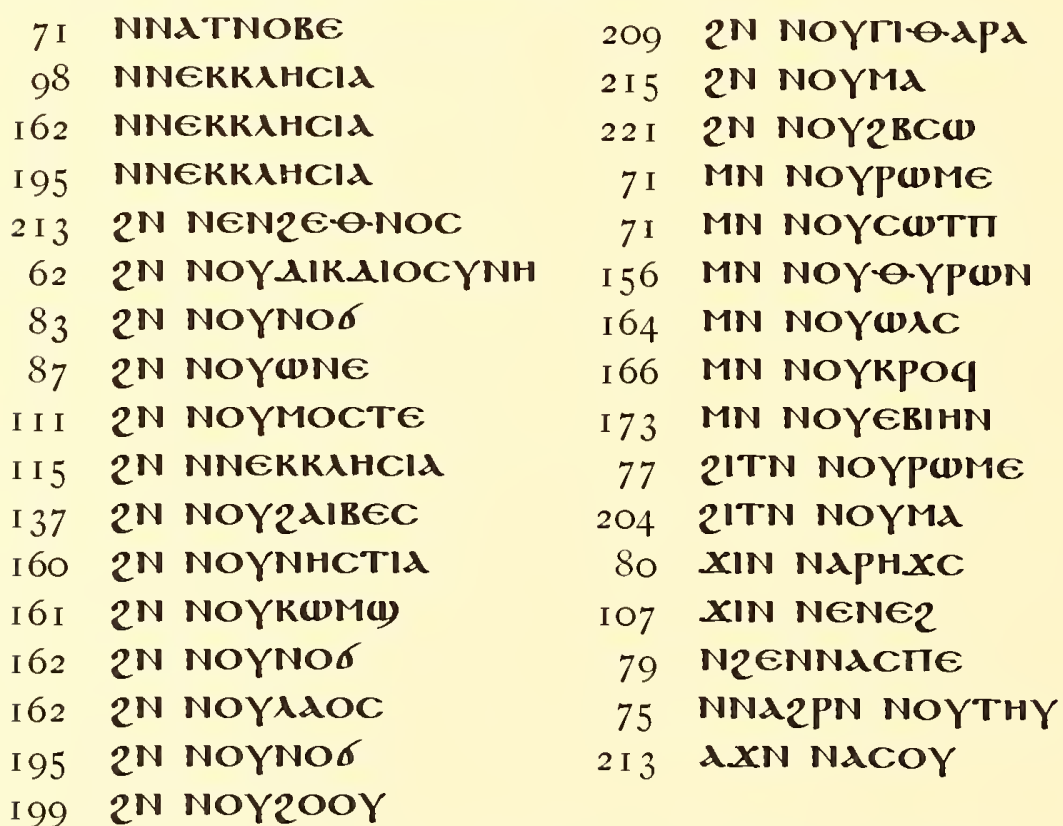

In an effort to avoid the mannerism the scribe has written one $n$ where there should be two, and two where there should be three.

$\begin{array}{ll}208 \text { NAKEEC } & 223 \text { NOYEIOTE } \\ \text { I } 45 \text { NNOYN } & \end{array}$

The other manuscripts show no trace of this tendency. 


\section{v. Affinities of the Text}

The present manuscript possesses no remarkable textual features. It is closely related to the other Sahidic Psalter manuscripts, one of which, L, being complete and old, furnishes for us, as for previous editors, a means of comparison.

If we take the cases in which $\mathrm{F}$ differs from $\mathrm{L}$, and compare $F$ in all such instances with all available manuscripts, we discover that most of the cases involve mere orthographic, or perhaps in part, dialectic peculiarities. These have been discussed in the preceding section. Only a few cases seem to lead back to a difference in text in the Greek; and the significance of these cannot, of course, be interpreted at present. Even the interrelation of the Sahidic manuscripts is not clear. F appears, when differing from $\mathrm{L}$, to favor B in matters other than orthography.

\begin{tabular}{|c|c|c|c|c|c|}
\hline PAGE & $(5)^{\mathrm{B}}$ & (5) $\mathrm{U}$ & (5) $₹ c, a R$ & $9 \mathfrak{i}$ & UNIDENTIFIED \\
\hline 39 & $\mathrm{FZT}$ & \multirow{14}{*}{ LZR } & \multirow[t]{14}{*}{$\mathrm{L}$} & & \\
\hline 72 & $\mathrm{~L}$ & & & & F? Z \\
\hline 78 & $\mathrm{LV}$ & & & $\mathrm{LV}$ & FZ \\
\hline $8_{5}$ & $\mathrm{~L}$ & & & L. & $\mathrm{FZ}$ \\
\hline 93 & $\mathrm{~F}$ & & & & \\
\hline 93 & LZT & & & & $\mathrm{F}$ \\
\hline 94 & FBZT & & & & $\mathrm{L}$ \\
\hline 103 & LZT & & & LZT & $\mathrm{F}$ \\
\hline I I 7 & $\mathrm{~F}$ & & & & LZT \\
\hline I 65 & FBZR & & & FBZR & $\mathrm{L}$ \\
\hline 168 & $\mathrm{~L}$ & & & FBZ & \\
\hline 216 & $\mathrm{LZV}$ & & & & $\mathrm{F}$ \\
\hline 240 & & & & $\mathrm{~L}$ & FBZR \\
\hline 246 & FBZGrol & & & & $\mathrm{L}$ \\
\hline
\end{tabular}

\section{vi. Plan of This Edition}

The list of available texts of the Sahidic Psalter has been published by both Rahlfs and Wessely in their editions; and from these I have taken the following list, adapting it to the purposes of the present edition, which does not extend beyond Psalm 53 . At the same time I have indicated the sigla employed respecting them in this introduction and in the following text.

F The Freer Coptic Psalter manuscript, No. I, here published.

B The Berlin manuscript, dated about 400 A.D., edited by Rahlfs in Abhandlungen der königlichen Gesellschaft der Wissenschaften zu Göttingen, I901. 
L The London manuscript, British Museum, Or. 5000, dated in the late sixth century, edited by Budge as The Earliest Known Coptic Psalter, London, I 898.

$Z$ The de la Zouche (later Parham) British Museum manuscript, dated in the ninth or tenth century, published by de Lagarde as an appendix to the Bohairic Psalter, Psalterii Versio Memphitica, accedunt Psalterii Thebani Fragmenta Parhamiana, Proverbiorum Memphiticorum Fragmenta Berolinensa, Berlin, Is 75 .

$\mathrm{T}$ The Turin manuscript, dated about the fourteenth century, edited by Peyron in Memoria della Regia Accademia di Torino, Ser. II, 28.

R Fragments now partly in Rome and partly in Naples, formerly of the Borgian Museum, dated ninth to twelfth century, edited by Ciasca in Sacrorum Bibliorum Fragmenta CoptoSahidica Musei Borgiani, Rome, I889.

V Vienna fragments, dated early sixth century, edited by Wessely in Sitzungsberichte der kaiserlichen Akademie der Wissenschaften in Wien, Vienna, 1907.

Masp Fragments copied by Maspero in Egypt, dated fifth to sixth centuries, edited by him in Etudes Égyptiennes, I, Paris, I 88 I fol. See remarks below, page xxvi.

Gol Manuscript of Golenischtschew, dated in the ninth century, edited by von Lemm in Bulletin de l'Académie de St. Pétersbourg, new series, I, I890.

Bour Fragments edited by Bouriant in Mémoires publiés par les membres de la mission archéologique française au Caire, I, 3, Paris, I 887.

Pist The Psalm texts embedded in the Pistis Sophia, published by Schwartze-Petermann, Berlin, 1851 .

Winst Fragments published by Winstedt in Proceedings of the Society of Biblical Archeology, xxv, 7 .

Frag I Part of the Freer collection, described above, page xiii.

Schm Schmidt's review of the Budge edition of L, Göttinger gelehrte Anzeigen, igor.

$\mathrm{L}^{\mathrm{b}}$ and $\mathrm{L}^{\mathrm{c}}$ The printed text of the Budge edition of $\mathrm{L}$, and the collation of the same with the original manuscript by Mr. W. E. Crum.

(4) indicates the Greek text of Swete's Old Testament in Greek, Cambridge, I896, which is, for our passages, the Vaticanus. Other symbols added to $\$$ h have been taken from Swete.

$\mathfrak{M}$ indicates the Massoretic Hebrew text. 


\section{vii. The Reproduction of the Text}

The manuscript has been reproduced as far as possible line for line. The original relative position of the words on the page has been observed as nearly as the word division would permit. The actual length of incomplete lines and pages could only be conjectured, since they are subject to wide variation in the manuscript. There is evidence for supposing it to be safe to employ as many as eighteen lines to a page, and an equal number of letters to a line, when restoring defective pages.

Missing portions of the text have been supplied from L, and indicated by [] . Letters which can be recognized from the context and a comparison with $\mathrm{L}$, but which are not independently recognizable, are indicated by \ulcorner\urcorner . It is impossible in this to avoid the exercise of a degree of personal judgment, with results which may not be approved by others in every case. When $\mathrm{L}$ is corrupt, the bracketed reading is taken from another source, indicated in the notes. No superlineation is employed within the brackets unless actually visible in the manuscript. It is not intended to exaggerate the importance of the manuscript by printing the text in full; but it seems necessary to do this if the reader is to distinguish easily between textual omissions and lacunæ in the parchment. The word-separation is not as extreme as that of Steindorff on the one hand nor of Budge on the other. In this matter there is always room for criticism, but it can be seriously offered only when the method is uneven, or when sense or grammar is violated. The chapter and verse numbers follow Swete's edition of the Greek text. The strokes over the letters are discussed above, pages xviii $\mathrm{f}$.

The collation of the manuscript with $\mathrm{L}$ has been described on page xxiii. Mere typographical slips or scribal errors, in L or F, did not require the mention of other manuscript readings. For the sake of completeness, however, the regular and unimportant variation of $\mathbf{X O \Theta I C}$ and $\boldsymbol{X O I C}$ was noted. No comparison of punctuation was attempted.

In the portion of the Psalter covered by our manuscript the Budge edition contains some sixty-five errors; and it was found necessary to indicate these, together with the corrected readings of the collation kindly loaned me by Mr. Crum, in order not to misrepresent the manuscript. Some of these were noted by Schmidt in his review. A few are paralleled in the earlier editions of $Z$ and $R$, being incorrect division of letters into words. 
The apparatus on pp. I07-I 12 of this book requires the explanation that the words secunda manu refer to the retracing of certain letters in a very black ink, and not to any change in the reading nor added letters. The Job fragment has been collated with the edition of Ciasca (cf. p. 329, 1l. 7 ff.).

The British Museum manuscript Or. 756I, known as the Kennard Papyri, and consisting of some hundred and fifty biblical and patristic fragments, does not appear in the catalogue of Crum (closed in 1905), and must have been acquired since that date.

Manuscript Or. 756r b is a volume containing an edition of these fragments, and bears the title גp-O-YP NNIEP(woY Xe

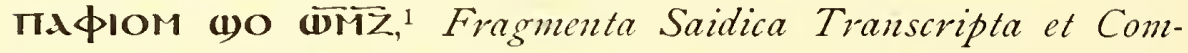
mentata ab Arthurio des Revières, I848. On the following page is the note: "This book contains the transcript and notes by Arthurio des Revières, from the original papyri, in my possession, from which the Gospels of our Bible were translated. Henry Martyn Kennard, 27th May, I906. Another transcript is said to be in the Munich Museum. My papyri, the originals, were supposed to have been destroyed during a bombardment of Alexandria."

If we compare the Psalm fragments in the Kennard collection with those published by Maspero (page xxiv), we find the passages to be the same. The latter alludes to "un homme dont l'œevre entière, restée manuscrite, a été dispersée " . . . after having been prepared for publication thirty years before. "Ce curieux personnage" is apparently de Revières, who wrote in 1848 . The bombardment of Alexandria occurred in 1882, Maspero copied them in $188_{3}$, and the British Museum acquired them in 1906.

1 "Arthur of the Rivers, that is, of the Fayyum, Thousand 847." 


\section{COPTIC PSALTER}

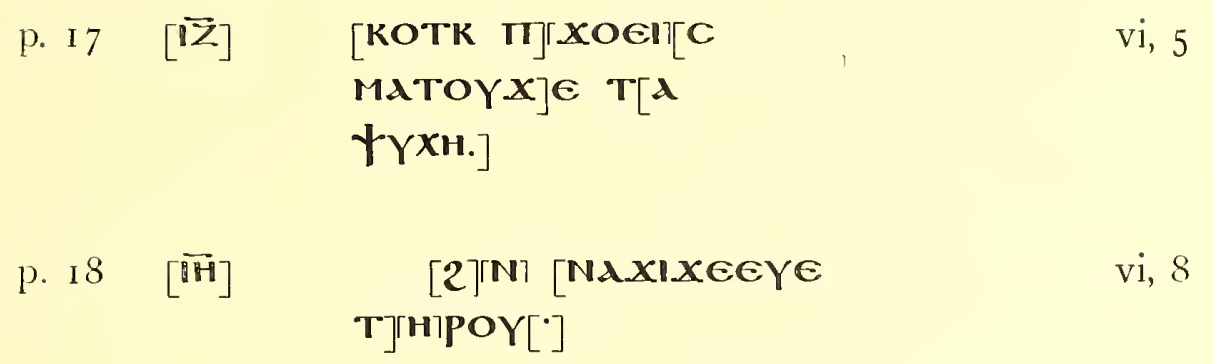

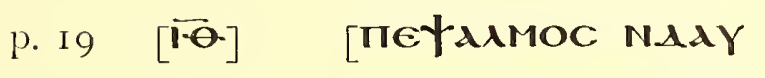

vii, I-2

eIA mentaqXoOq

eIIXOEIC $2 A$ Nadxe

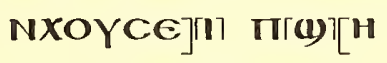

PE NIEME]NEIn $[-$

TIXO]GIC [TANOY

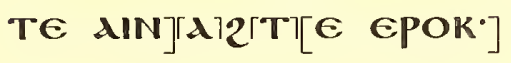

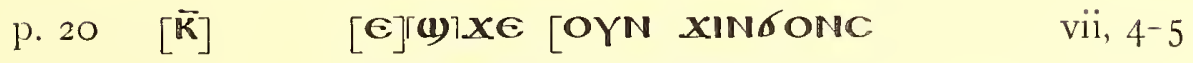

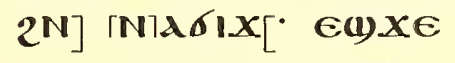

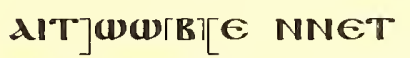

TWW] [BE] [NAI N2ENIIE

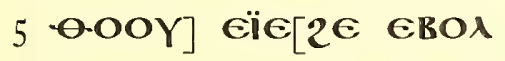

$2 ! T][N]$ NA[XAXE El(i)

oYel] $\left.\mathrm{T}^{\circ}\right]$

p. $2 \mathrm{I} \quad[\mathrm{K} \overline{\boldsymbol{A}}]$

[2M] ITIOYC[2CA

vii, 7-8

2NE] GNTAK2ГCi? [N

MM] [O'Q. AYra? [OYN

OYCYN]ATE[TH NAA

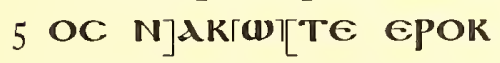

AY(D) 2 A T[Al KOTK

eYXI] rCle: 
p. $22 \quad[\overleftarrow{\mathrm{K} \dot{\mathbf{K}}}]$

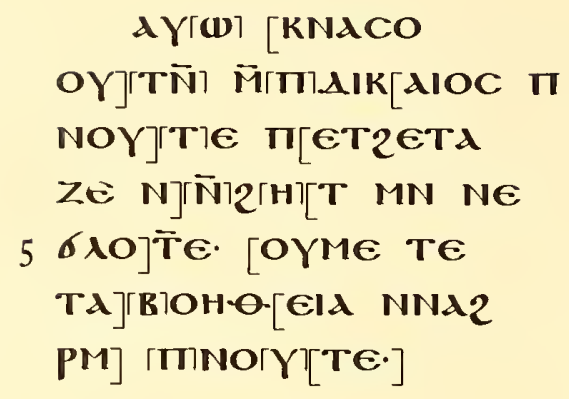

p. 23 [Kॉ $]$

ГАपC][WMT

vii, I 3-14

$$
\text { NT] Сपп[Іте Аप }
$$

p. $24[\widehat{\mathbf{K A}}] \quad[\mathbf{E I C} 2 \mathbf{H H}][\mathbf{T E}][\boldsymbol{A}$ vii, I 5

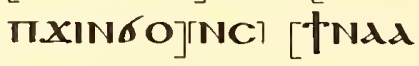
ке· גqw] ГMाா[2ІCE AqXIE] TA[NOMIA]

p. 27 [ $\overline{\mathrm{KZ}}]$

[ETRE

viii, 3

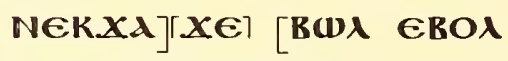

NOYXd]XE][- MN OY

peqxIKBd.]
p. $28 \quad[\overline{\mathrm{KH}}] \quad[\mathrm{OY} \mathbf{E}][\mathrm{OOY}][\mathbf{M N}$
OY]TГA [IO AKAגY $N$

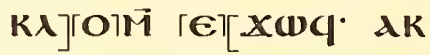
Kd]-O-ICT[A MMOQ

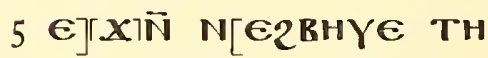
POY] [ÑN][EKGIX]


p. $29 \quad[\overline{\mathrm{K} \theta}]$

[IIXO

viii, Io-ix, I

EIC TENXOEIC NeE

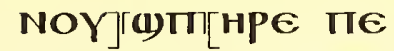

IIEK]PAN [2M IIKA2 TH

5 P] $[4]$ :

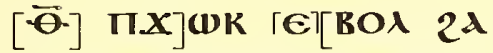

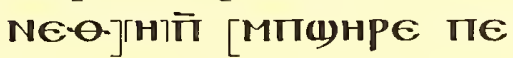

†ANMOC NAגYeIA:]

p. $30 \quad[\overline{\mathbf{\lambda}}]$

[CENA

ix, $4-5$

\author{
NCE2E] ГMT[TEKM

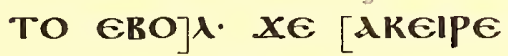

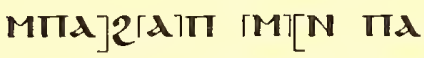 \\ $5 \mathrm{KBd}] \cdot[\mathrm{AK2}][\mathrm{MOOC} 21$

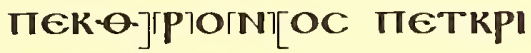 \\ NE NT] AI] [KAlOCY \\ NH: - ]
}

p. $3 \mathrm{I}[\overline{\mathbf{A d}}]$

пाГX][OEIC (1)

ix, 8

OाI (1) $]$ ג $E\left[\mathrm{NE}_{2}\right.$ ג

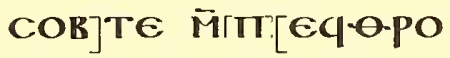

NOC $2 \mathrm{~N}$ OY2AI·]

p. $32 \quad[\overrightarrow{\mathbf{A B}}]$

[XE MாєK

ix, I I I 2

K(1) NCWK NNET

(1)][N]E [NCWK ПTXO

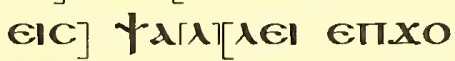

5 El] пе[ТоYн2 $2 \mathrm{~N}$

$\mathrm{C}]\left[1 \boldsymbol{\omega} \boldsymbol{\omega}\left[\mathbf{N}^{\circ}\right]\right.$

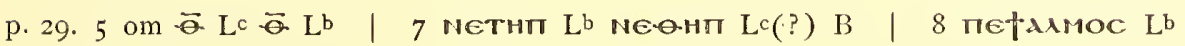
пеభגAMOC Lc 
p. $33[\overline{\mathbf{A r}}]$

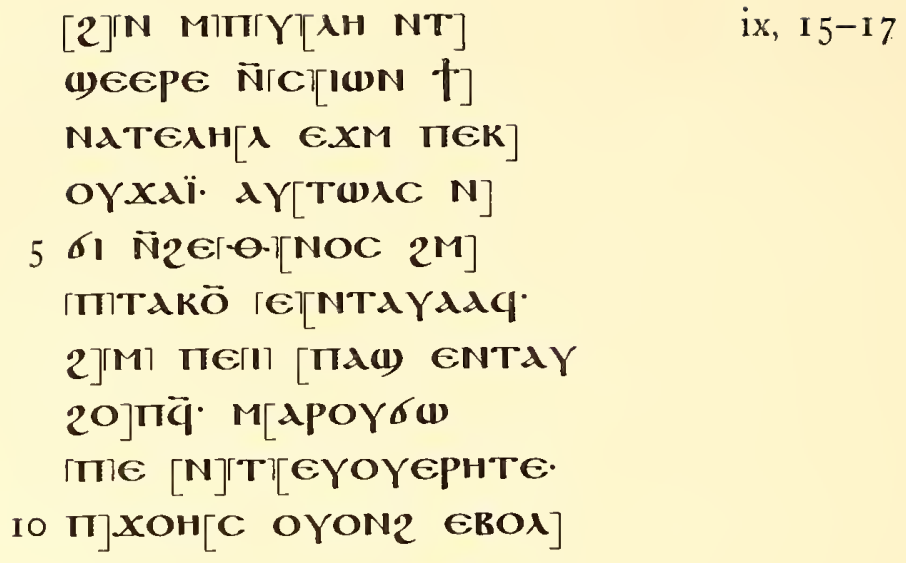

p. 34 [A]

[XE] $[\mathbf{N}] \mathrm{C}[\mathrm{E} / \mathrm{N}\lceil\boldsymbol{A P}]$

ix, $19^{-20}$

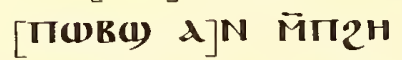

[KE (1)גR] OA. O.YTO

[MONH M]ITlERIHN

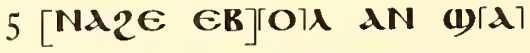

[ПTHPG T][M]OY[N]

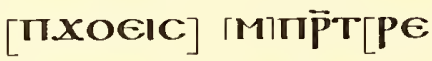

пршме $\sigma] \bar{M} 160 \mathrm{OM:}$

[MАPOYKPI]NE $\mathbf{N}[\mathbf{N}$

IO 2E-NNOC MПEКMTO

EBOA]

p. $35[\overline{\mathrm{AE}}] \quad[\mathrm{GTBE}$ OY] $[\Pi] \mathbf{X}[\mathbf{O G I C}$

ix, 22-23

ג]

MON: A[K][OB(1)K]

$2 \ddot{M}$ TEYrol[eId) NNE]

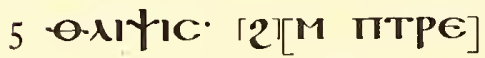

пגСєR[HC XICE]

[M̈]MOQ Г(1)][APE ПтH

к]е xepo [Cendáo

T]OY $2 \bar{N}$ [NEYUOXNE

ro e]ToY[MEeYe epooY'] 


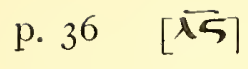

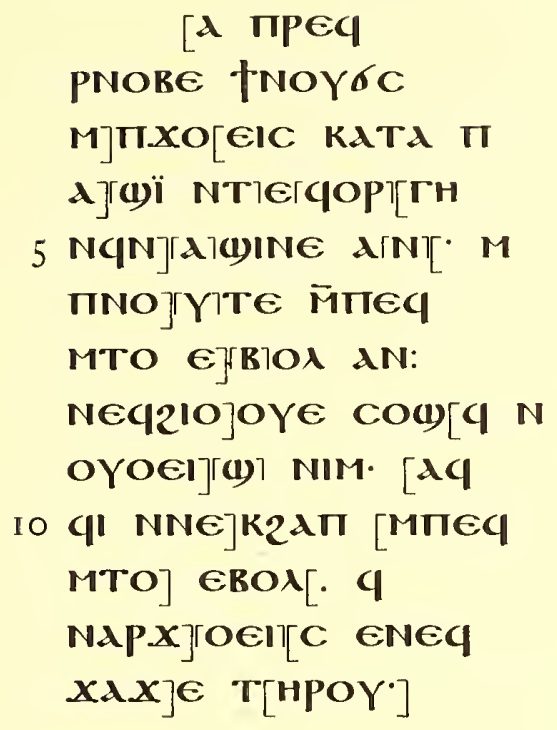

PNORE PNOÝ́C

M]T.XO[GIC KATd $\Pi$

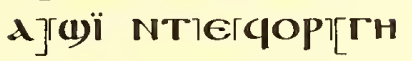

5 NGN][A]WINE AIN][י M

INO][YTE MTIEA

MTO E]RाO入 AN:

NEGIIO]OYE COW[ $[4$

OYOEI](1)] NIM. [AC

IO qI NNE]K2גI [MTEQ

MTO] EBON[. 4

N.APX][OEI[C ENGq

$\boldsymbol{X \lambda X}] \Theta$ T[HPOY']

p. $37 \quad[\overline{\mathbf{A Z}}]$

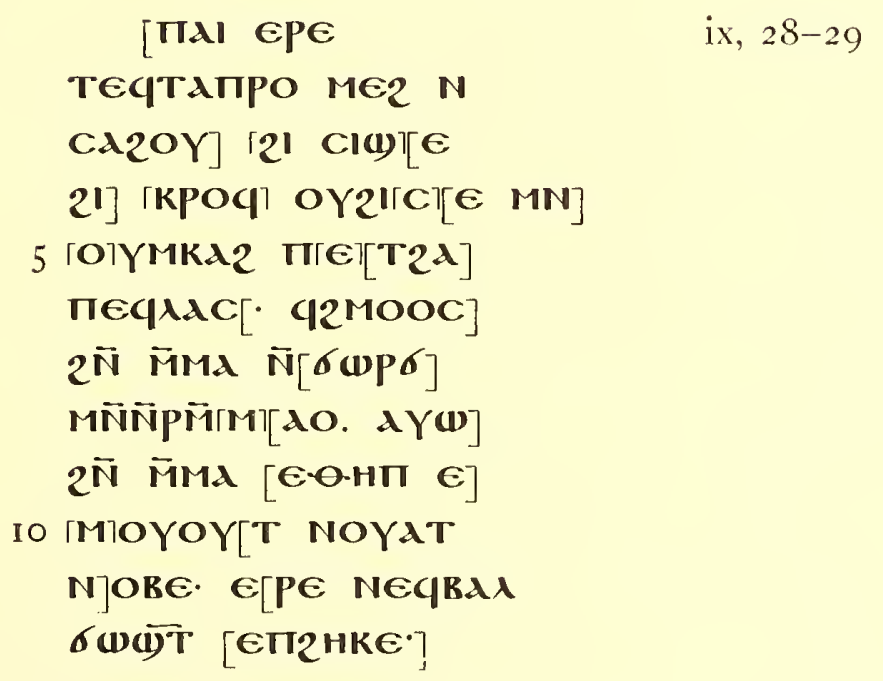


p. $38 \quad[\overline{\mathbf{A}} \overline{\mathbf{H}}]$

$$
\begin{aligned}
& \text { [CNATI2TC } \\
& \text { N42E } 2 M \text { пTPEप } \\
& \text { PXO]ढIC EाN2HKE. } \\
& \text { Aq]XOOG [एAP } 2 \mathrm{M}\rceil \\
& 5 \text { [Пеप]2нт } \boldsymbol{x \in ~ А ा ~}
\end{aligned}
$$

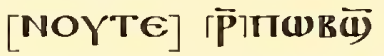

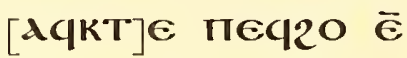

$$
\begin{aligned}
& \text { [BOA ET] }
\end{aligned}
$$

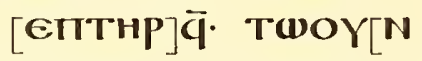

$$
\begin{aligned}
& \text { IO IXOEIC] [ITNOYГT'[E } \\
& \text { MAPEC } x \mathrm{l}] \mathrm{Ce} \overline{\mathrm{N}} \sigma[1]
\end{aligned}
$$

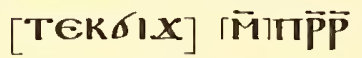

$$
\begin{aligned}
& \text { [IUB(I) NN2HKE'] }
\end{aligned}
$$$$
\text { ix, } 3 \mp-33
$$

p. $39 \quad[\overline{\boldsymbol{A} \cdot} \cdot]$

$$
\begin{aligned}
& \text { [GTPEY]TAdY } \Theta[2] \\
& \text { pdï ENeKбוx [ } \mathrm{E}] \\
& \text { PE пгнке бге] [Nd] } \\
& \text { NOXવ̄ EPO[K NTOK] } \\
& 5 \text { пеєтвГO[[HO-I EIIOP] } \\
& \text { PANO[C OYWWq] }
\end{aligned}
$$

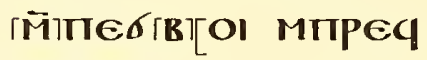

$$
\begin{aligned}
& \text { P]NOBE [MN ITITO] } \\
& \text { NHPO }[C][\cdot \text { CENAGI] } \\
& \text { Io Niel [NCA nEqNOBE } \\
& \text { NCETM2E EPOC } \\
& \text { єтвннтч'] }
\end{aligned}
$$

$\mathrm{ix}, 35-36$ 
p. $40 \quad[\ddot{\mathbf{M}}]$

\author{
[A TIXOEIC

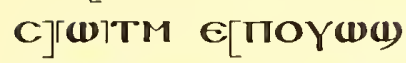 \\ N]ர̄i]2HKE: АाЕप[MA \\ גxe] †йтня еता \\ 5 [COQT] \\ [ERPINE] МाTIOPфA

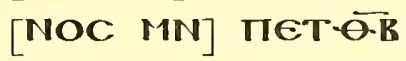 \\ [BIHY.XE] ГNTNEITPГW][ME

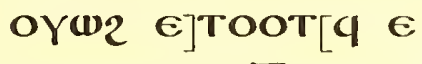

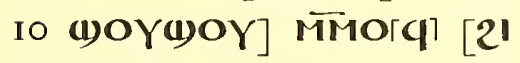 \\ XM חाKA2']
}

ix, $3^{8-39}$

p. $4 \mathrm{I}[\overline{\mathrm{MA}}]$

$$
\begin{aligned}
& \text {-XE EIC }[N \\
& \text { PEq][Р̈NOlBe AYC[(w) } \\
& \text { MNT NTEYT[ITE:] } \\
& \text { AYCOBTIE] [NNEY] } \\
& 5 \text { COTE ET[EYRNAAY] } \\
& \text { NंtCoT[E ENEX } \\
& \text { с]оте 2[M Пгшा } \\
& \text { E]NETC[OYTWN 2M] } \\
& \text { пEY2H[T' } x \in \text { NEN] } \\
& \text { IO TГA][KCBTWTOY AY } \\
& \text { TAYOOY E2PAI] }
\end{aligned}
$$


p. 42 [MR

[NECRAd бW(T)

$x, 4-6$

E] XN̄ TOII ROYME

$\mathbf{N}]^{[H]} \cdot$ NEqROYГ2][E XNO

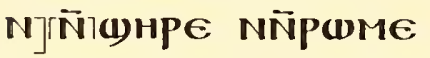

$5[\Pi X]$ roE $[1] C$ NAXNE

[ПגIKג] IIOC MÑாவ

[CевнC] тетме $\Lambda €$

[MIXIN] 'ONC̄ qMIOI[C

TE NTEq] †YXH IM][AYA

IO Aq' $^{\circ}$ qNA $] 2$ WOY $E[X N$

NPECP]NOBE $\bar{N}$

[2ENIIAW OY] $]^{[K \omega} 12 \bar{T}$

[MN OYOHN MN OY

Пत̄̃ N2dTHY ---]

p. $43[\overrightarrow{M T}]$

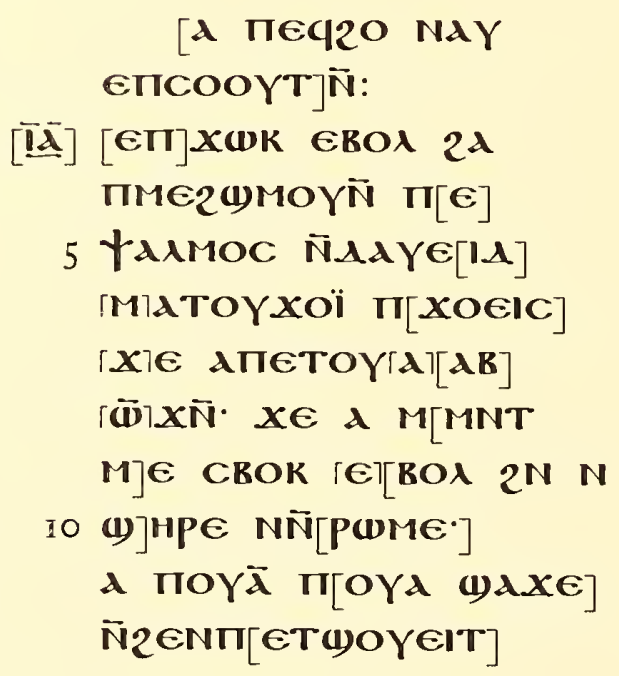


p. $44 \quad[M \Lambda]$

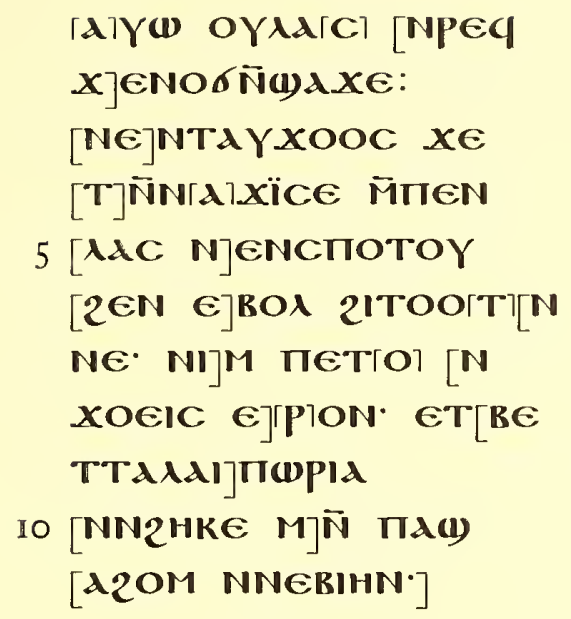

p. 47 [MZ]

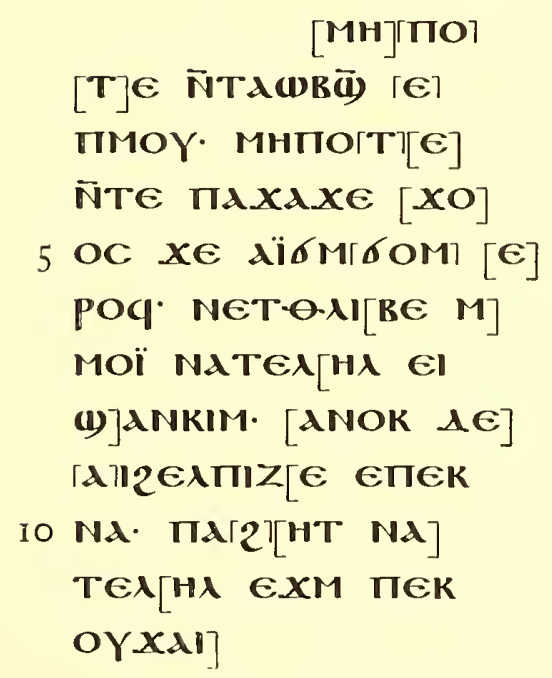


p. 48

[ัMH]

[iī]

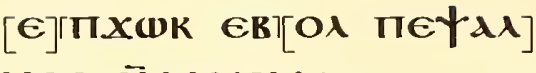

xiii, I-2

MOC ÑAdYelA:

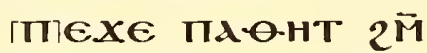

[I]єЧ2HT $x \in$ MÑ NOY

5 [T]€ Гબ]

[KO AY] CWUG $2 \bar{N}$

[NEY]MIEEYE. MÑ $\Pi[\epsilon T$

EIPE] ГNIOYMNTXP[HC

TOC MMN $]$ OYON 1$)[\lambda\rceil[2$

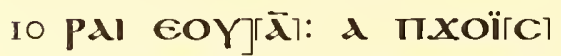

[бШ(1) $]$ EROA $2 \bar{N} T$

[ПЄ EXN N(1)]Ple NÑ

[P(DME']

p. 49 [M-O $]$

[MMN пாТ

xiii, 3

EIPE NOY][M][NTXPH

CT][OC1 MÑ OYON (1)d2

PAï COYর̃: OYTA

5 фOC EqOYHN TГE]

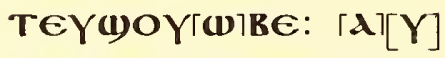

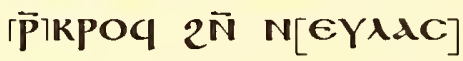

IOIYMATOY [N2OG]

ГTרET2ג N[EYCIO]

Io TOY EPE [TEYTd]

про̄ Me2 [NCd2OY]

$21 \mathrm{CI}\left[(1) \epsilon^{\circ}\right]$ 
p. 50 [N]

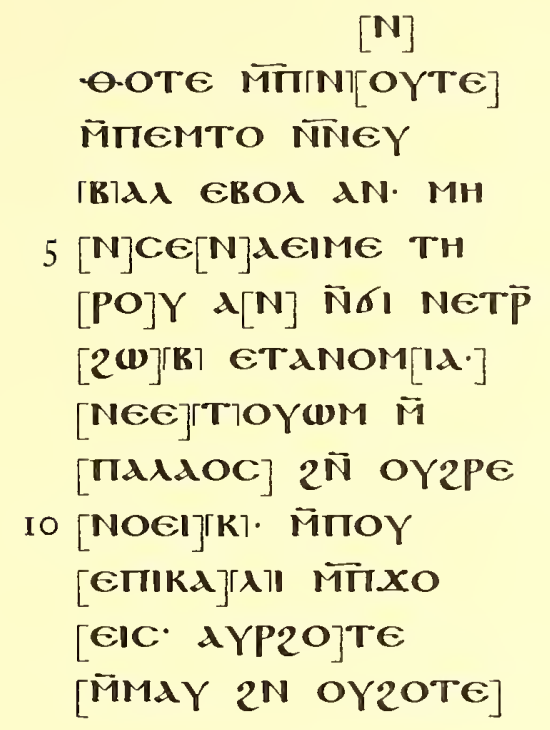

xiii, $3-5$

p. $5 \mathrm{I} \quad[\overline{\mathbf{N}} \mathbf{A}]$

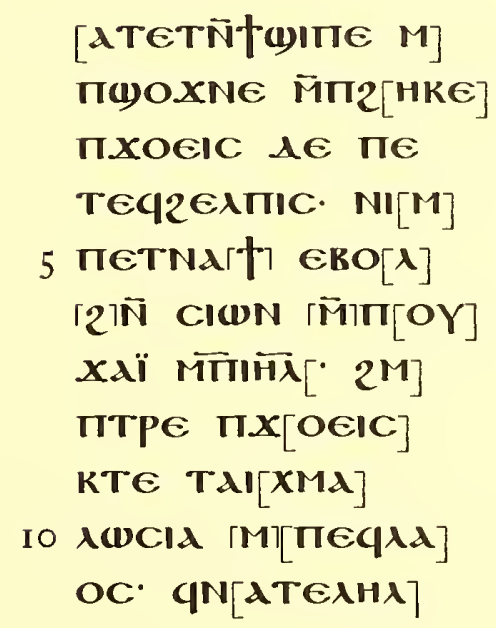


p. 52 [Tें [IIXOEIC NIM TET xiv, $I-3$ NAOYW2 $2 \bar{M}$ ПЕК

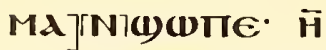
NIM ПாЕTNАMTŌ

5 MMOG $2 \bar{M}$ ПЕК TOOY [E]TOYAגB. $[\pi][\epsilon] T M O O \emptyset) ~ п[\epsilon$ ETo]YAdB ET $\overline{\mathrm{P}}$ [20B] ETAIKAIO

I0 [CYNH] єqФגXe [NTM] $2 \bar{M}$ ПЕС2HT

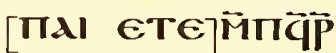

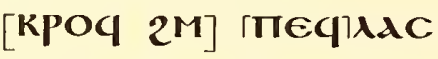
[OYАє MாવPा]є I 5 [बOOY MTET? TOYw4']

p. 53 [Nं]

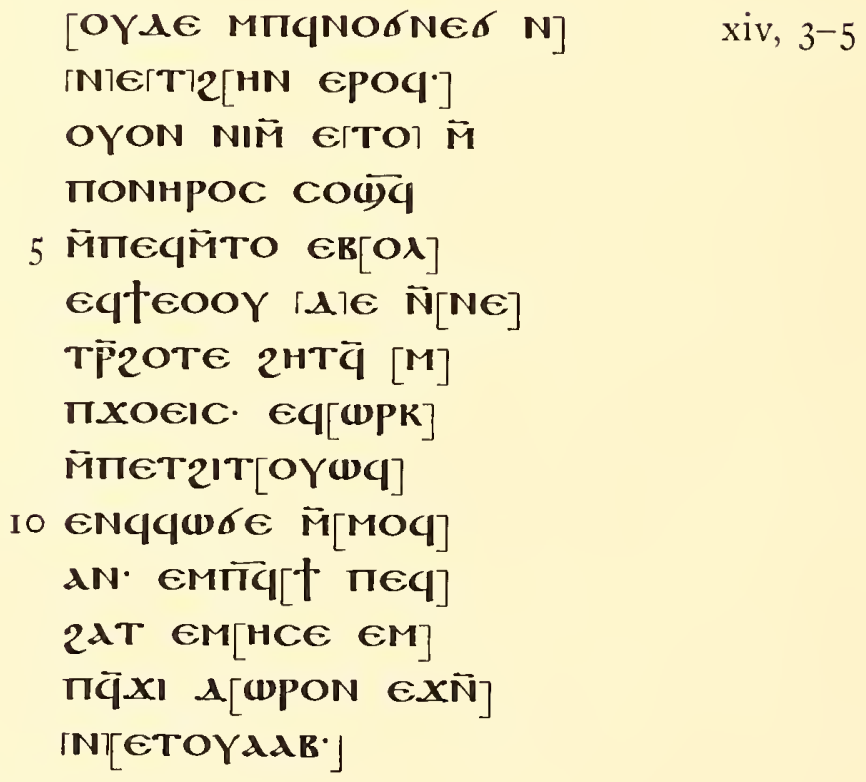


p. 54 [त्रA]

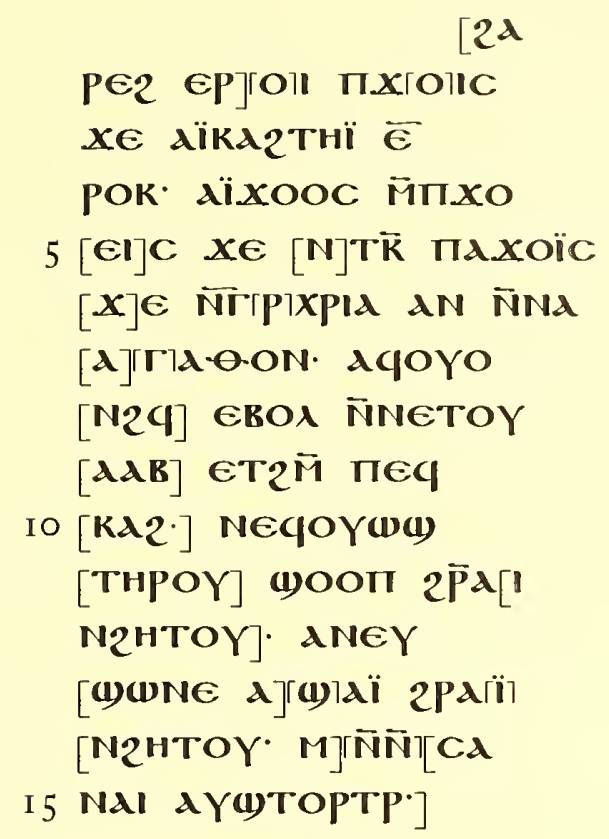

XV, I- -4

p. 55 [NG]

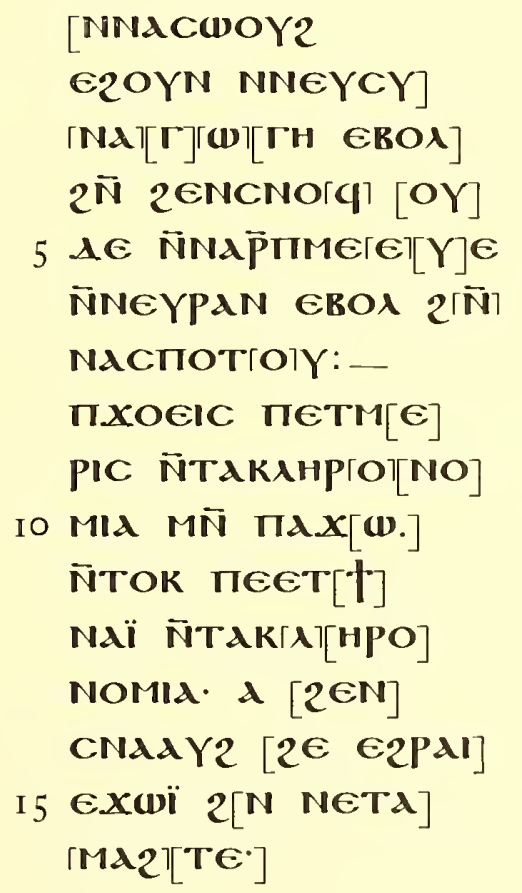


p. 56 [N5]

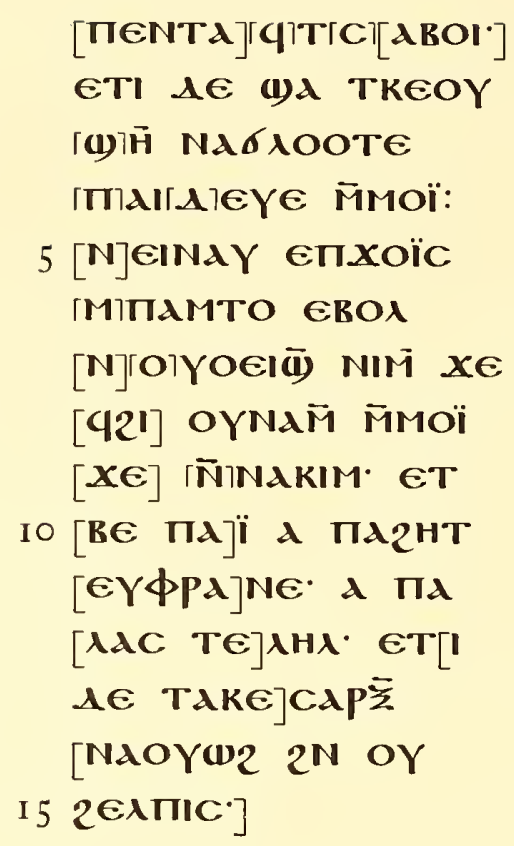

p. $57 \quad[N Z]$

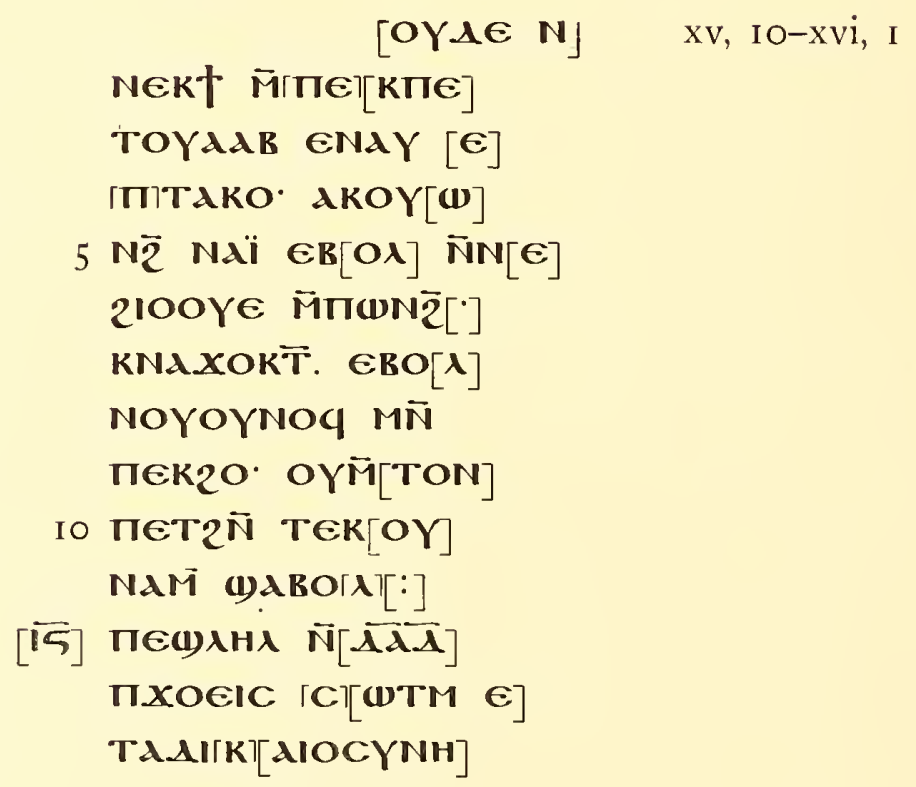


p. $5^{8}[\overline{\mathbf{N H}}]$

[ePE חId

xvi, $2-4$

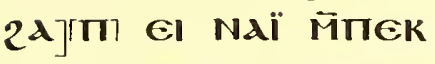

[M]TO EROA. EPE

$[\mathrm{N}] \mathrm{ARA \lambda}$ NAY Eח

5 [C]OOYГTाN̄: AKАO

[KI]M[A]Z€ M̈ாג2HT

[AK]

[T]

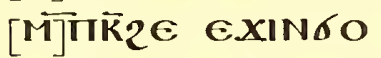

Io [NC] $\overline{\mathrm{N}} 2 \mathrm{HT}^{*} x \in \overline{\mathrm{N}}$

[NE] TATAாPŌ $X(1)$

[NNE2]RHYE ÑNPW

[ME E] TTRE Ñ(1)גXe

[NNERCMO]TOY גï

I 5 [2APE2 ENE2IOOYE

ETNA(1)T:]

p. 59 [N- $]$

[AICOQTe nNATAG]

xvi, 5-7

CE $2^{N}$ [NeK2IOOYE']

XEKAC ENNEY[KIM]

[N] I NATגбCE:

5 АNOK АÏXI Ф)КАГK]

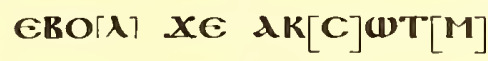

ePO[l] пNOYTE[']

РІКе М̈̈ாЄКМА[А]

$x \in$ epoï NĩCWT[M]

IO ENACAXE: MaP[OY]

Р̈)пне ÑNE[KNa']

ПЕTNOYZM̈ [NNET]

zeAIIIZE E[POK·]

$[\mathrm{N}] \mathrm{A2ME[T}][2 \mathrm{~N}$ TE

I $5 \mathrm{~K}] \mathrm{OYN}\left[\mathrm{AM}-\mathrm{N}^{-}\right]$ 
p. 60 [层

[2APE2 EPOI N-OE

xvi, 8-I I

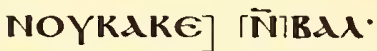

[KINAPै2dïrec EPOï

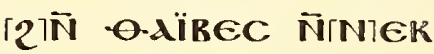

5 ГTाNू

ERO[A] NÑACERHC

[N]Aï [EINTAYMOK

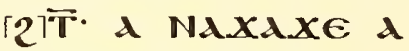

MA2TE त̄TAభY

I0 [X]H' АY२®Xī

[I]]

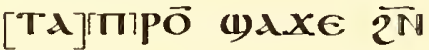

[OYM] $[\mathrm{N}] \mathrm{TX} X \mathrm{Cl}$ HT [

EYNOY

I 5 [EROA TE]INIOY AYY]

[КШTE EPOI А КШ

NNEYRAd EPI]

p. 6 I

KE $2^{\lceil\bar{M}\rceil} \pi K A 2 \cdot A Y$

XVi, I I-I 3

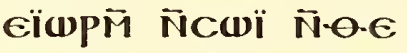

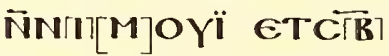

TWT EYГII $7 \overline{2 C}$ [AYW]

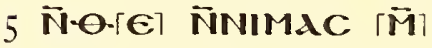

MOYГï? ET(1)OOIT?

$2 \bar{N}$ NIMAE-H.HII[]

TWOYN ПIXO[EIC]

APIळOPत̈ EP[OOY]

ro N"̈TAYOO[Y E2PAl]

NETM TA[YYXH]

[E]TOOГTদद [Mाय

C] Євн[C. 'Тснсє

NNXAXE EROA

I5 $2 \mathrm{~N}$ текбוx пхо

EIC qUTE MMO]

p. 60. 2 кєкє Lb кдкє prima manu кєкє emendatoris manu Lc кекє RZ

p. 6I. 3 NNIMOYGI L NNIMOYIZ NNGIMOYIR / 4 om AYa? 
p. 62 玄

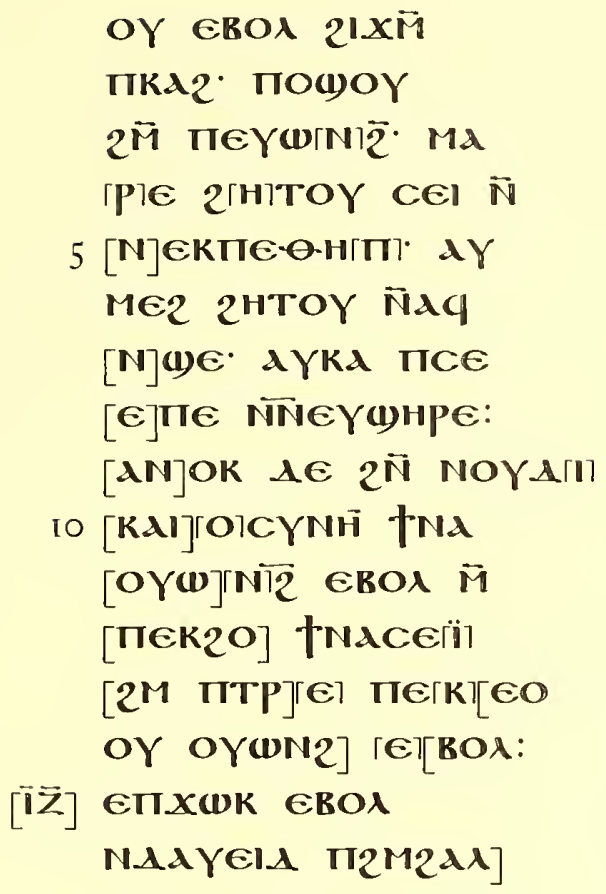


p. 64 [立]

TE EPOY' TANAC)

xvii, $3-7$

те пе АYल ПтАП

ज̈пגоYXגї: пा

реqшоTт EPOC.

5 Ë̈сIM)YY TNAd

пIKAג M̈ாxої

AYM TnAOYXAi

EROA $2 \bar{N}$ NAXAXE.

[d] NNAdke M̈ImOY

IO [A]MA2TE M̈MÖ̈.

[M]MOY NCAPM Nं

[TAN]OMIA AYU)

[TPT][M]PT. A NNA

[AKE N]ГגרMÑTTE]

I5 [AMA2T] $]$ ' 1 MMO $[1$.

А Mாג(y) M̈IIM[OY

РщОРा EPOї 2M пा

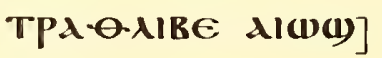

p. 65 [宝 $]$

erpaï emxoeic.

xvii, $7-9$

дїхімкגK E2Pגіi]

EIANOYTE Aq

СШTM̆ EII2PO

5 OY EROA $2 \bar{M}$ пеप $\bar{P}$

TIE GTOYAגR:

AYW ПIAGKA[K]

M̈пеqMัто $\epsilon[\mathrm{BO \lambda}]$

NARUK E2O/Y][N]

Io eNEqMadXIE][']

А ПாКА2 KIM $Г A][Y(\omega)]$

ACCTET [ A NCN]

TE NÑT[OOY

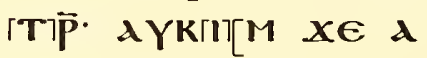

I5 INOYTE NOÝC $E$

POOY AYKATINOC]

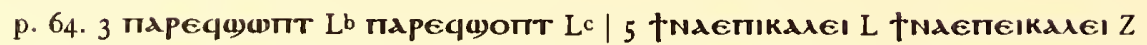


p. 66 [广5]

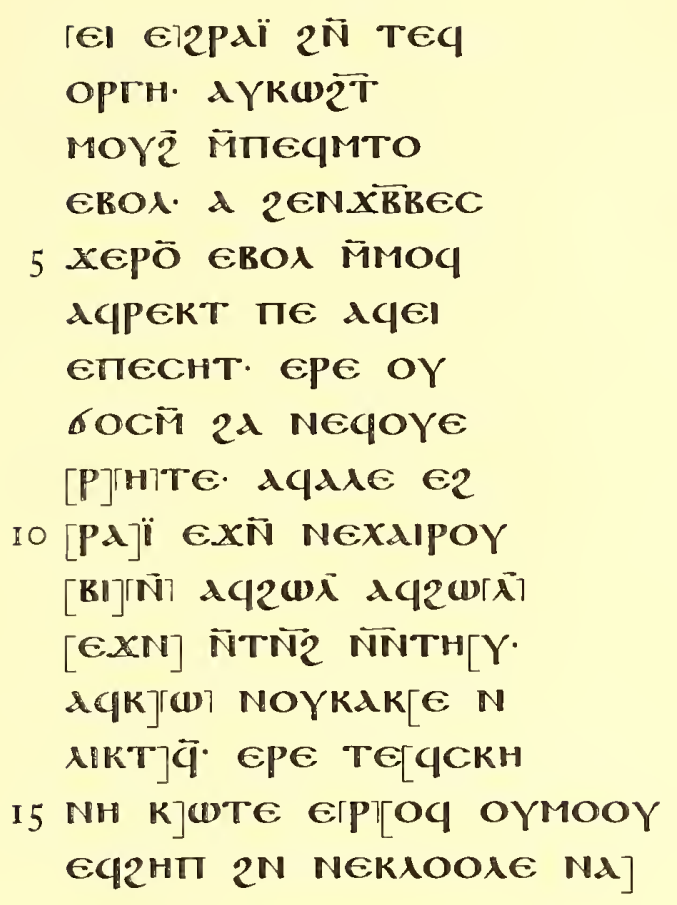

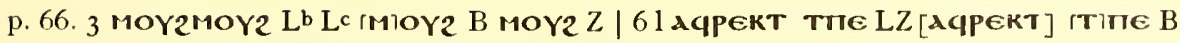

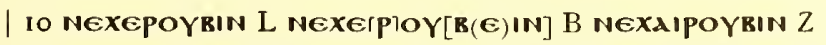

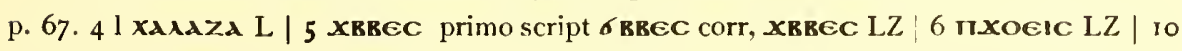

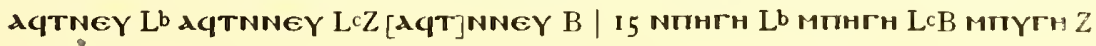


p. 68 [寅]

ГKOYIMENH. EROA

xvii, I6-I9

जTEкEாITIMId

[ா]XOEIC. EROA $\bar{M}$

пाIqE MīT̃ त̄

5 'Тєк[O]РГн' АपТT̄

NOOY ERON $2 \bar{M} \Pi \times X I$

ce АqXIт Ачш)

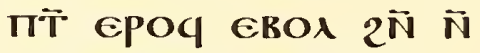

[2] ENMOOY ENAฒW

IO [O]Y' qNATOYXOÏ

$[\Theta R] O \lambda 2 \bar{N}$ Naxdxe

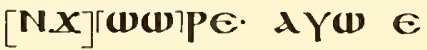

[ROA 2N] NETMOCTE

[MMOI X] АYбMбГOা[M

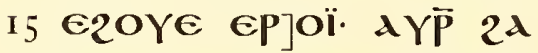

[PUI MாE]

MKג2. А пXOEIC]

p. 69 [iं日]

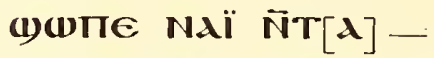

xvii, $19-22$

XPO АपÑT EROA $E Y$

OYOCTÑ. GNATOY xої xE גqOYגबT

5 GNANA2MET ERIOIX

$2 \bar{N}$ Naxdxe NXx

WPE AYW EROA $2 \bar{N}$

NETMOCTE MTM[OI']

ITXOEIC NATUW

IO RE NAï KATd T[AגI]

КАIOCYNH. AY[w]

qNגTOYEIO [NAI KA]

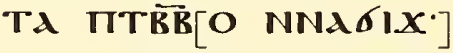

$x \in \lambda \ddot{2} \lambda I P]\left[\epsilon_{2}\right.$ ENE2l]

I 5 ГOOYe M̈ாI XOElC.

Аү(1) MाIP(1)גपTE

EITANOYTE $x \in$ NEq]

p. $68.812 \mathrm{~N} 2 \in \mathrm{N} \mathrm{LZ} 2[\mathrm{~N}][2 \in][\mathrm{N}) \mathrm{B}$ 
p. $70 \quad[\overline{\mathbf{O}}]$

p. $7 \mathrm{I}[\overline{\mathrm{Od}}]$

$$
\begin{aligned}
& \text { ATNOBE MÑ NOY } \\
& \text { PEME NINATNO } \\
& \text { вE- KNaP̈CCTIT̃ } \\
& \text { MÑ NOYCWTIT. } \\
& 5 \text { АYल KNÁ́⿴囗十 } \\
& \text { ME MÑ петбடOO। } \\
& \text { ME. XE ÑTOK K[NA] } \\
& \text { xice їIIAdoc E[T] } \\
& \text { - } \overline{\mathrm{B} B I H Y} \cdot \mathbf{A} \text { Y }
\end{aligned}
$$

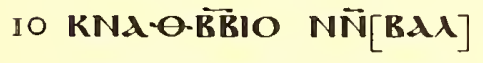

$$
\begin{aligned}
& \text { NN̄XACI2HT' }\left[{ }^{\circ} X \in\right] \\
& \text { N̄TOK ETP्OI[YOEIN] }
\end{aligned}
$$

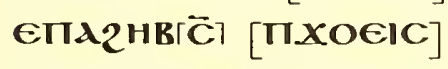

$$
\begin{aligned}
& \text { IINOY[TE KPOYO } \\
& \text { I5 EIN ETגरגKe } \\
& \text { xe tNanoyzm } \\
& \text { N2HTK EYMA N] }
\end{aligned}
$$

xvii, $26-29$ xvii, $23-26$ 
p. $72 \overline{\mathrm{OB}}$

\author{
COONE AYW $2^{\bar{M}}$ \\ IINOYTE TNAOY \\ WT $\overline{\mathbf{B}}$ NOYCOB $\overline{\mathrm{T}}$. \\ ПINOYTE TEq2IH

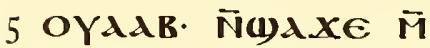 \\ пxоeIC посе $\widetilde{T}$ \\ NAGTE ПIE NOYON \\ NIM ET2EATIZC \\ EPOQ $x \in$ NIM пє \\ IO \\ [I]NOYTE ÑRAt M̄ \\ [T][EINXOEIC ï NIM

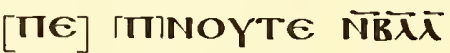 \\ [MITENN]OYTE: IIN[OY \\ TE ET]IMOYP MTMO[I \\ I5 NOYбO]ГM): АКк[А ТА2Ін

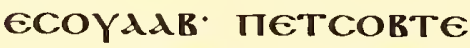 \\ NNAOYEPHTE NOE NNA]
}

xvii, $30-33$

p. 73 OT

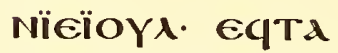

xvii, $34-37$

20 M̄MOÏ epd $\bar{T}$ E

$x \bar{N}$ NAXICIEOY eq

TCABO NINAGIX

5 еाтाтолемOC $\lambda k$

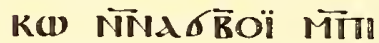

TE Ñ2OMNTT AK†

NAї NOYNAGTE

NOYXגï TEKOY

IO NAM̄ TENTACrM][O]

ताT EPOC TEK[CRw]

TENTACCA2「W][1 (1)ג]

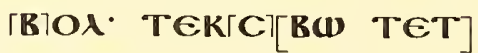

[NA] †CRE $[N]\left[A !^{\circ}\right]$ 
p. $74 \widehat{O A}$

†NגாயT त̄cd

xvii, $3^{8-39}$

NAXAXE N̄Td

TA2OOY AYW N

NAKTOї ємпоY

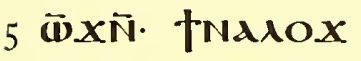

2OY ÑCETMিE(1)

б бембом еdzерג

[T]OY cendze $2 A$

[N]גо ҮЕРнте. АK

Io $[M][O 1 P T$ NOYбOM

[єП]ПОגеMOC:

[АKT] АYO ЄПєCH[T

2 Pd]

OYN THPOY $\epsilon$

I 5 2Pג єXฒ1 גK†

NNAXAXe $2 d$ NA]

p. $75 \overrightarrow{\mathrm{OE}}$

$$
\begin{aligned}
& \text { оүернте АК } \\
& \text { qUTE EBOA } \vec{N} \\
& \text { NETMOCTE M } \\
& \text { MOї· АYXI(1)КАK } \\
& 5 \text { EROX AYW NE MÑ } \\
& \text { пеTNOY2M̈ } \mathrm{C}_{2} \\
& \text { pdï enXoelc AY }
\end{aligned}
$$

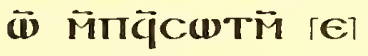

$$
\begin{aligned}
& \text { POOY TNATP }[E Y] \\
& \text { IO ПגKe N̈.eE NIO][Y] } \\
& \text { (1) OEI(1) } \mathrm{NIN}[A][2] \\
& \text { PÑ NOYTHTY [t } \\
& \text { NAO]CGIOI[Y NaE } \\
& \text { NOYOME N2IP. } \\
& \text { I } 5 \text { KNdTOYXOї } \mathrm{e} \\
& \text { BOA } 2 \mathrm{~N} \text { NOYW2M] }
\end{aligned}
$$


p. $76 \quad \overline{05}$

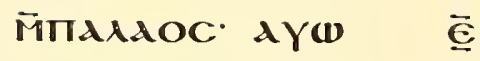

$$
\begin{aligned}
& \text { KNAKA-OHCTA } \bar{M}
\end{aligned}
$$

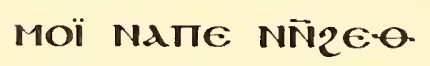

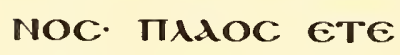

$$
\begin{aligned}
& 5 \text { MTाсO YwNä Aq }
\end{aligned}
$$

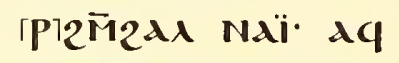

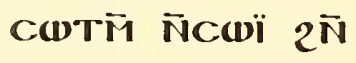

$$
\begin{aligned}
& \text { OYCWTM M̄MAd }
\end{aligned}
$$

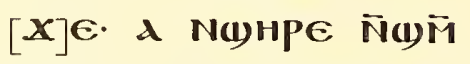

$$
\begin{aligned}
& \text { го }[\mathrm{M}] \overline{\mathrm{O}} \text { Xiбо人 epö̈ a } \mathrm{N} \\
& \text { [(1)H]PE ज்̃ }
\end{aligned}
$$

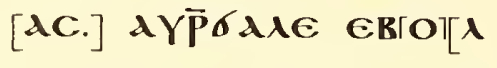

$$
\begin{aligned}
& \text { 2N NE]Yi2IrOOIYE. } \\
& \text { [IXOEl]C ION][2] IAY }[\mathbf{W} \\
& \text { I } 5 \text { GCMAMAd } \vec{T} \text { NGI } \\
& \text { пגNOYTE MAPEq } \\
& \text { XIce NбI TNOY] }
\end{aligned}
$$

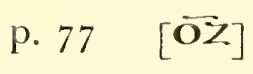

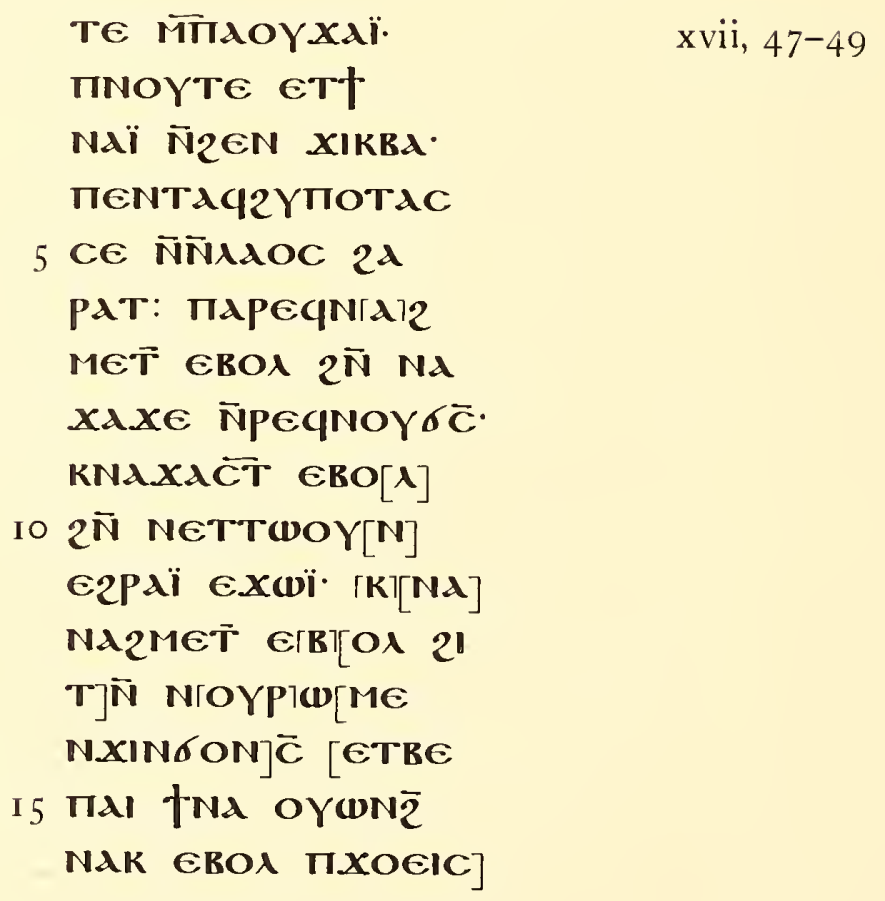


p. $78 \quad \overrightarrow{O H}$

$$
\begin{aligned}
& 2^{\bar{N}} \bar{N} 26 \theta N O C \cdot d Y \\
& \text { (v) TNatanael } \\
& \text { धпекPAN пеT } \\
& \text { XICE ÑNEYXAI M }
\end{aligned}
$$

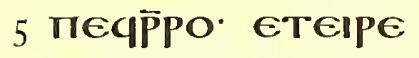

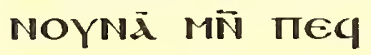

$$
\begin{aligned}
& \text { XPHCTOC AAYEIA } \\
& \text { Mі̄ пєqсперMд } \\
& \text { (a)d GNE2: }
\end{aligned}
$$

p. $79[\overline{\mathbf{O}} \boldsymbol{\theta}]$

$$
\begin{aligned}
& \text { TE2OOY XW NOY } \\
& \text { (1) XX MாE2OOY. } \\
& \text { TEY山H XW NOY } \\
& \text { COOYN NTEYMH } \\
& 5 \text { N̄2ENNACTE AN } \\
& \text { NE. OY } 1 \in \overrightarrow{N 2} \in N \\
& \text { (1) } \lambda X E \text { AN } N E \text { : } \\
& \text { CENACWTT AN } \\
& \text { єாєY2POOY. A } \\
& \text { IO пEY2POOY El EB[O][A] }
\end{aligned}
$$

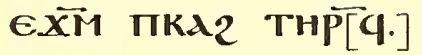

$$
\begin{aligned}
& \text { AY(D A NEY(1)d [XE] } \\
& \text { ח112 (1)d NEK[PW } \\
& \text { OY] [N]TOIKO [Y }
\end{aligned}
$$

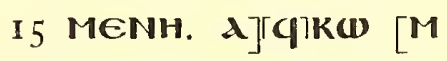

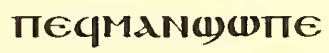

$$
\begin{aligned}
& 2 M \text { прн. Аүш NTOq] }
\end{aligned}
$$

xvii, 50-xviii, 2 
p. 80 โī

eqO N̈OE NOYNYM

xviii, 6-8

\$IOC. EqNHY EROA

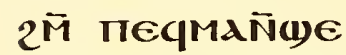

лEET पNATENH

5 N̈eE NOYTIrac E

ПIWT $2 \bar{N}$ TEq21H.

XIN NAPH $\overline{X C}$ NTTIE

IE IIEqMANEI EBOA

Аүज (1)ג АрнX $\bar{C}$

Io ГNרTाЕ пाе пеqMA

[N]BWK G2OYN. MÑ

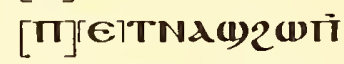

[ET] ]EIG 2MME:

[INO]MOC IM̄][IXOEIC OYA

I 5 АB ЄqKTO NNEҰYXH. TMNT]

p. 8 I $\overline{\text { IId }}$

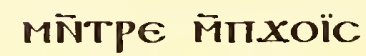

xviii, 8-IO

Ñ2OT ECTCARO

ÑNIKOYï: NAIKAI

wMA M̈TxOEIC.

5 COYTWÑ EYEYфPA

NE M̈ா2нT. TEN

ToגH M̈пxoeIC

oYoein Te. ec $\vec{p}$

OYOEIN ENRAג N̄

Io NIKOYï: O.OTE $\vec{M}$

IIXOEIC OYAA[B]

EC(1)OOI W[A] [ENE2]

N̈ENE2. जर[AT Mा

XOEl]C 2E[NME

I 5 NE EYTMAEIHY

21 OYCOII. NEqOY]

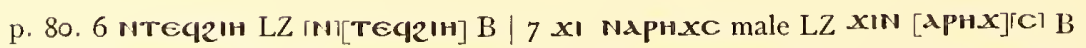

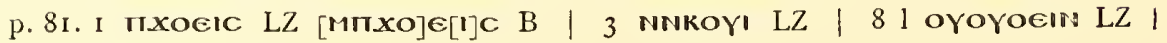
Io NNKOYI LZ 
p. 82 TतB

$$
\begin{aligned}
& \text { (1) COTIT EINOYB } \\
& \text { MÑ TWNE M̄ME }
\end{aligned}
$$

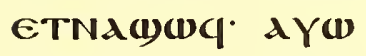

$$
\begin{aligned}
& \text { се2олб́ єாєвіш }
\end{aligned}
$$

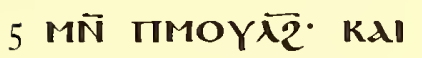

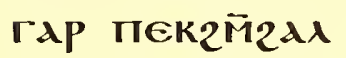

$$
\begin{aligned}
& \text { 2APE2 EPOOY } 2 \bar{M} \\
& \text { птреq2גре2 } \Lambda \in \epsilon \\
& \text { POOY OYTOYEIO IIE } \\
& \text { IO ENAWWC NIM IIET } \\
& \text { [N1גEIME EMTIAPd } \\
& \text { [Пт] }
\end{aligned}
$$

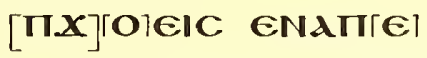

$$
\begin{aligned}
& \text { ['-HIT.] Г†'ClO] [ETEK } \\
& \text { I } 5 \text { M2גA EBOA } 2 \mathrm{~N} \\
& \text { N(1)MMO - - ] }
\end{aligned}
$$

Xviii, I I-I 4

p. 83 ता

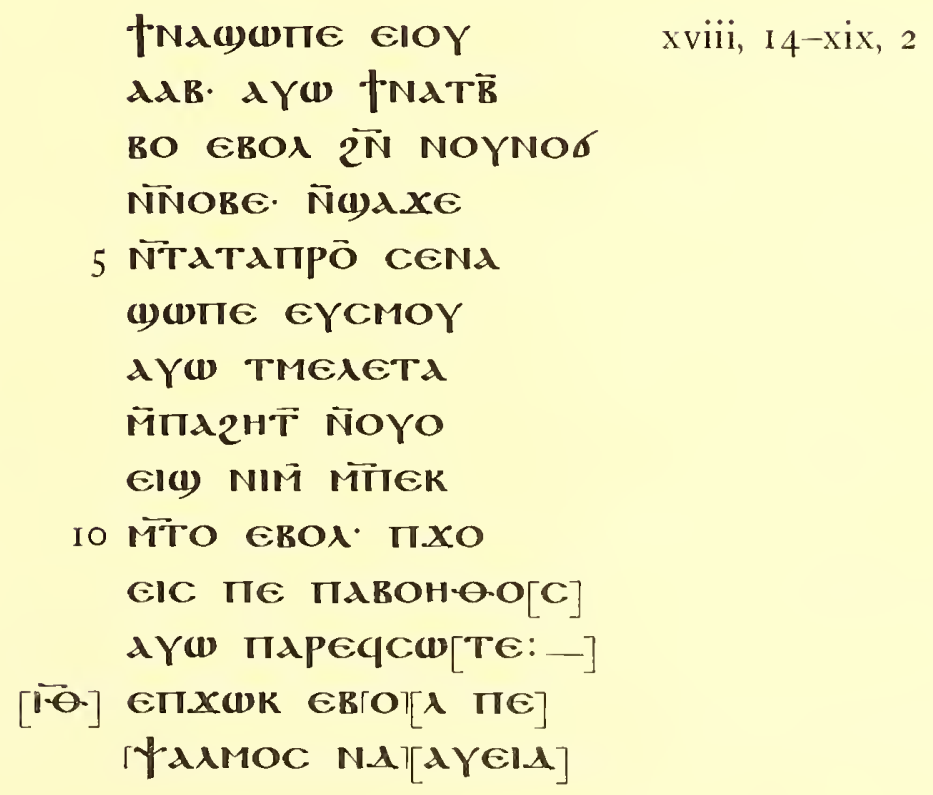


p. 84 近

єqер̈NA(1)TE ерок

xix, 2-6

N̄бI IPAN Mİ̃NOY

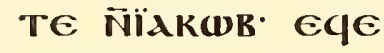

TĨNOOY NAK NOY

5 ROH-OIA EROA $2 \bar{M}$

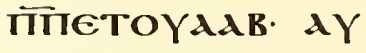

(i) Єqє(1)Oпाँ $\epsilon$

POC EBOA 2ÑN CION

еqерттмєеүє ì

Iо TEK-YYIA THPC -

ePE NEKG

(1)

Г(1)

[Eq] $\mathrm{CP}$ NAK Kd

I 5 [Tג ПЕК2нT --

TINNATEAHA E]

p. 85 गंध

XM̈ пекоYXגї.

xix, $6-7$

AYW TNT̃ dïג

2M̈ ITPAN MTEN

NOYTE EPE пхO

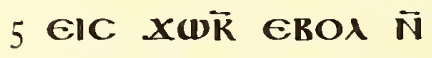

NERAITHMA TH

POY. TENOY dï

eIMe $x \in$ a пxö́c

тоүХе печхрнс

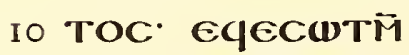

ЕРок $2 \bar{M}$ пєчп[є]

TOYגAв EPE[ITOY]

Xגï N̈TEKOYY[NAM $2 \mathrm{~N}$

zENGOM. NAI 2N 2EN

I 5 2APMA AYW NEIKO

OYE 2N 2EN2TWMP.]

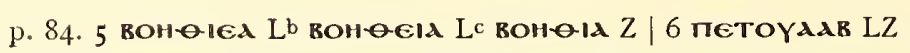

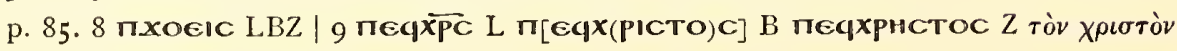

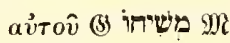


p. $86 \sqrt{15}$

$$
\begin{aligned}
& \text { ANON le ENAdïaï }
\end{aligned}
$$

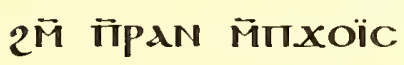

$$
\begin{aligned}
& \text { TENNOYTE: NTO } \\
& \text { Oү АүбломлM Аүш } \\
& 5 \text { AY2E ANON AE AN } \\
& \text { TWOYÑ AYW ANCO } \\
& \text { OYTT̃ ITXOEIC NE } \\
& 2 \ddot{M} \text { пекррӧ Аүш } \\
& \text { NÏCWTM EPON }
\end{aligned}
$$

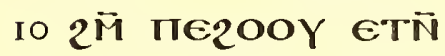

$$
\begin{aligned}
& \text { [N]ג山() Є2Pגї ЄРок. }
\end{aligned}
$$

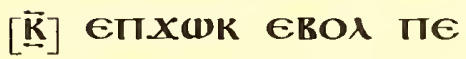

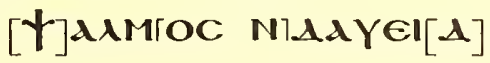

p. $87 \overline{\Pi Z}$

$$
\begin{aligned}
& \text { גК† NAq М̈пOY } \\
& \text { (1) М̈пєС2НТ } \\
& \text { Аүल М̈ாட̈2ОҮР } \\
& \text { wP M̈ICOITC ÑNEq } \\
& 5 \text { сIIOTOY } \boldsymbol{X} \in \boldsymbol{\lambda K} \\
& \text { тресї)ор̈त N } \\
& \text { NECMOY NTEK } \\
& \text { MNंТ Х्वस००. } \\
& \text { Ald talMa. }
\end{aligned}
$$

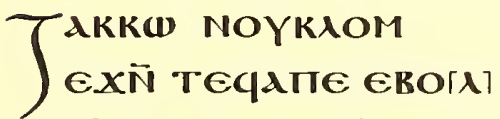

$$
\begin{aligned}
& 2 \bar{N} \text { NOYWNE M̄M[E]. } \\
& \text { А'C][-AITEI MMOK - -]: }
\end{aligned}
$$$$
x x, 2-4
$$
oYwNe LZR 
p. 88 Тัषิ

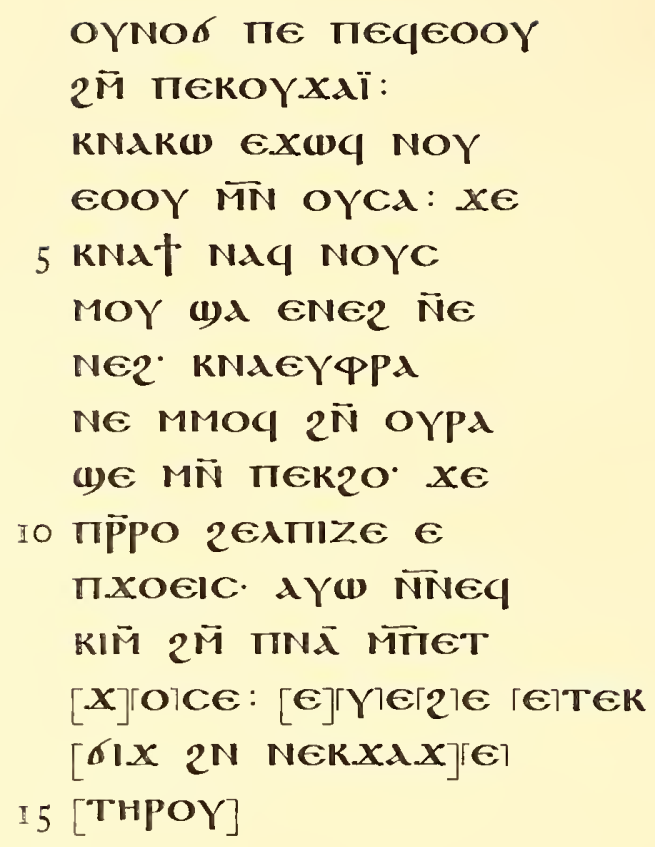

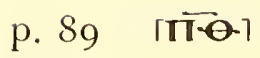

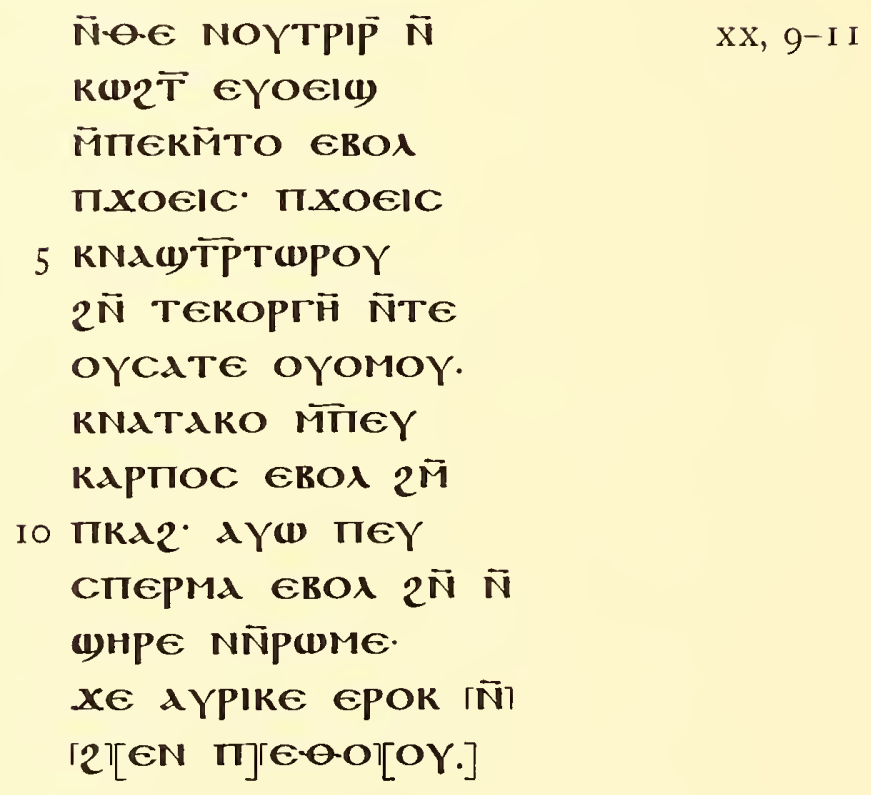


p. 90 [ั]

$$
\begin{aligned}
& \text { ХE КNАKळ M[MOO] } \\
& \text { етреҮгоMOY. } \\
& \text { кNACOBTE ज̈пеY } \\
& 20 \text { 2 } 2 \bar{N} \text { ÑCGEITE. } \\
& 5 \text { XICE ITXOeIC } 2 \bar{N} \\
& \text { текбоM TÑNA XW }
\end{aligned}
$$

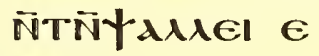

$$
\begin{aligned}
& \text { NeкбоM: } \\
& \text { 育 } \\
& \text { Iо п†тоота ї̈тNAY }
\end{aligned}
$$

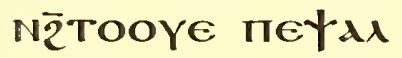

$$
\begin{aligned}
& \text { MOC Ñ } \lambda A Y \in I \lambda \text { : } \\
& \text { [MNOY]TTE MANOY } \\
& \text { [Tе MA†] } \overline{\mathcal{2}}[\text { THк] [е] } \\
& \text { I } 5 \text { [POI eTRE OY dK } \\
& \text { KAגT ÑCWK. CE } \\
& \text { OYHY EROА MITAOY] }
\end{aligned}
$$$$
\text { XX, I 2-XXI, I }
$$

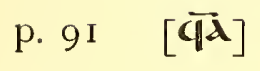

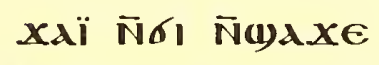

$$
\text { NÏITAPAITTWM[A][.] }
$$

manoYTE TNAC(i)

Є2Рגї ЄРОК $2 \bar{M}$ пє

5 2OOY NITTM̈COTM̃

EPOї. $2 \bar{N}$ TEY(1) NC̈

TM̄⿴囗шாє NaÏ NOY

MNTA $\theta \cdot$ T $^{\circ} \bar{N}$

ток $\lambda \epsilon$ єКОүн2 $2 \bar{N}$

Io NeTOYAגв חTA

धाO Мाँामें NTג

NENEIOTE NIAT[2TE]

ePГOTK. AYNAI2][TE]

[AY][M d][K][NA2MOY.

15 Aү(W) E2PAї EP

OK AYOYXAl. AY]

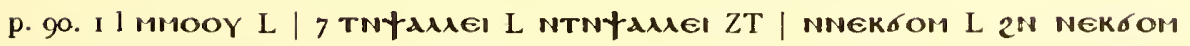

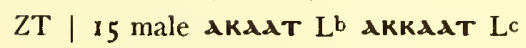

p. 9 I. I 5 eqoK male $\mathrm{L}^{\mathrm{b}}$ tacet $\mathrm{L}^{\mathrm{c}}$ 


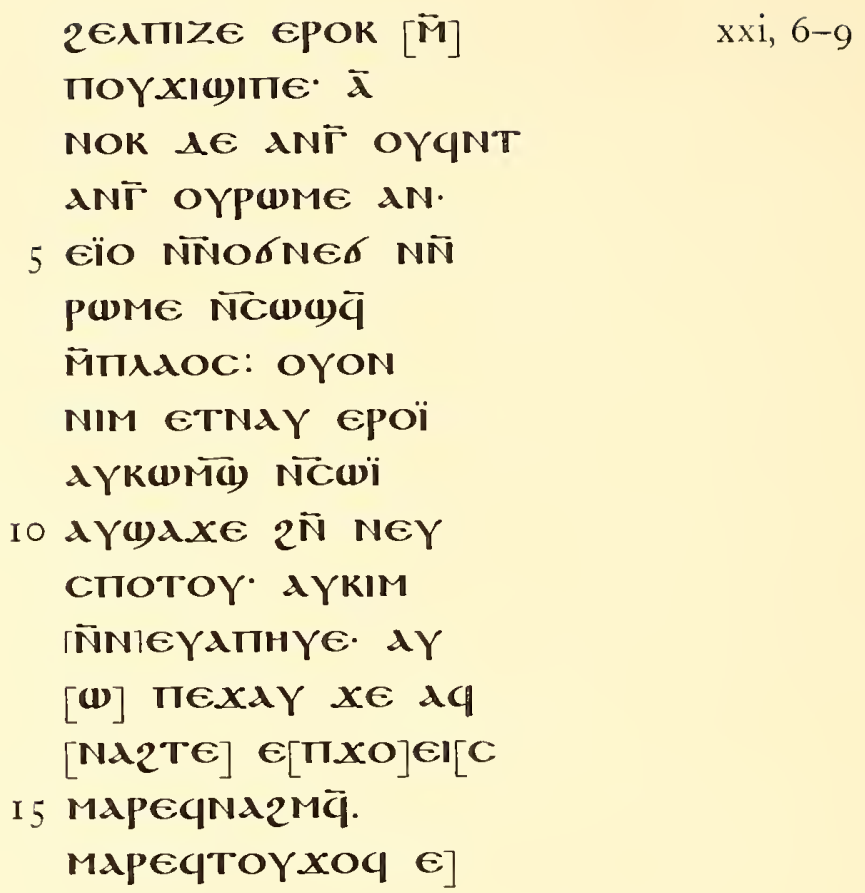

I 5 Oel. Аүмннфе M̈Mace kwTE EPOI. A zeNMACE] 


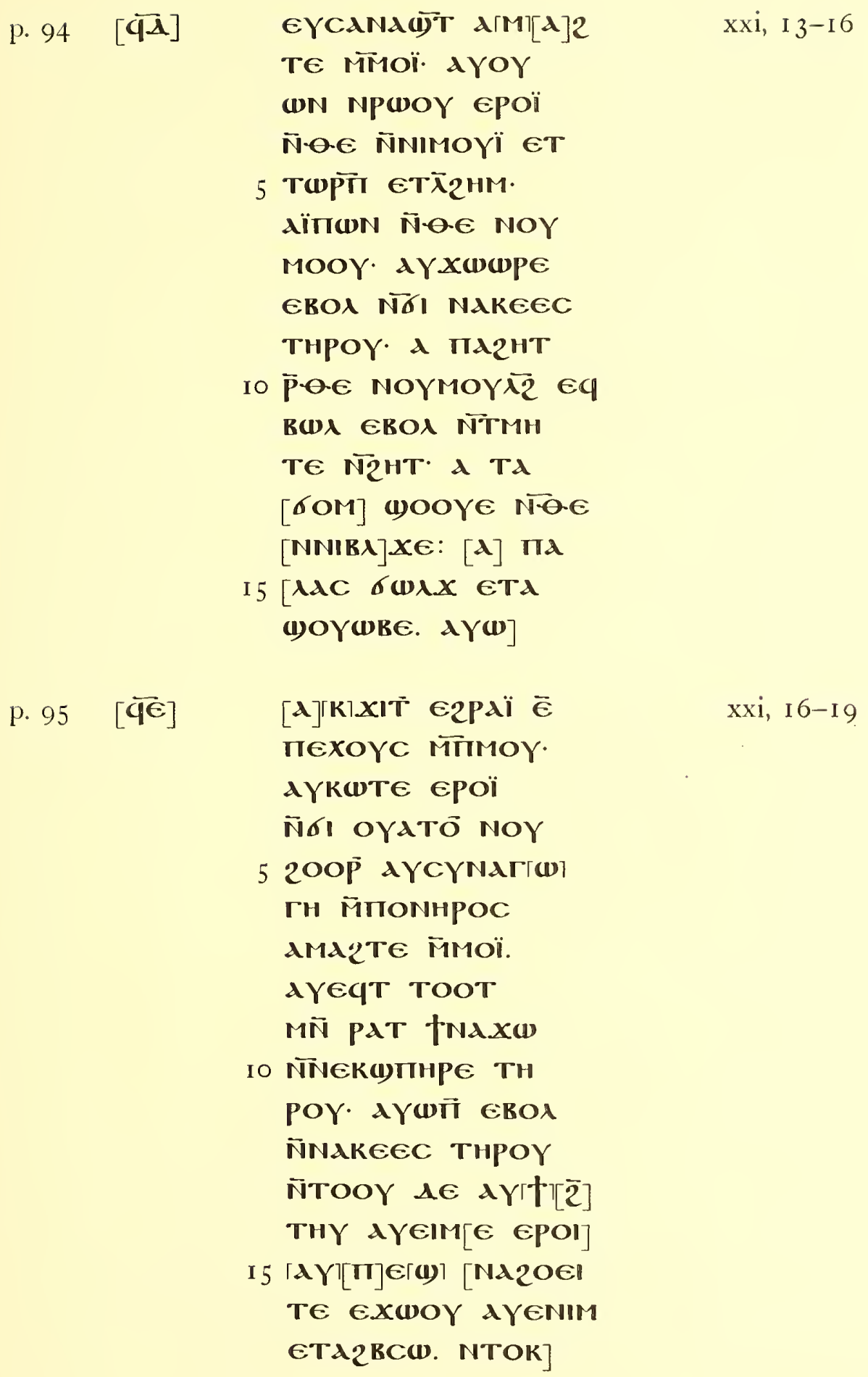


p. $96 \quad[\overline{4}] 5$

LE пXOEIC MTI $[\mathrm{P}]$

$\mathrm{xxi}, 2 \mathrm{O}-24$

тре теквон-өІd

oYe MMOÏ: †'

тнк єщотТ єрок.

5 MAтоYXE TA†Y

ХH Eтснде АYw

TAMNT(1) NE NOY

wT ET

2OOP MATOYXOÏ

го еттגпро जाтмоүї.

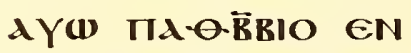

TAII ÑNA NITAT̃

NOYWT. TNAXw

[MIE]KPAN ÑNACNHY

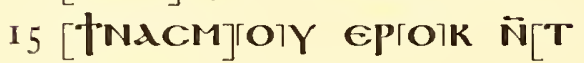

МнTE NTEKКגH

CId. NETP2OTE]

p. 97 [वz]

[2][нान्व जा XOEIC

$x x i, 24-26$

CMOY EPOY ПIEC

перMА тнр̈ її

KaR teOOY NAq.

5 MAPOY Р̈2OTE 2HTA

N̄б। песперMA

тнр्व Мाтामर $x \in \bar{M}$

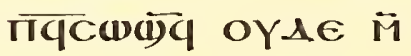

пїмєсте псопс̈

о ज̈ா2нке: оүАє М̈

пाव் кште їтеq

$20 \overline{N C}$ ṄOA M̄MÖ̈.

AY(1) $2 \vec{M} \Pi \pi[P \lambda(W)]$

E2एрาגï EP[Oq Aq

I5 CWTM EPOI. EPE IId

TMAEIO 2A 2THK 2N]

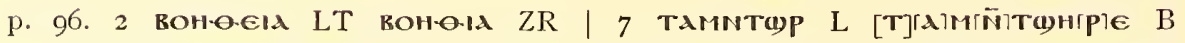

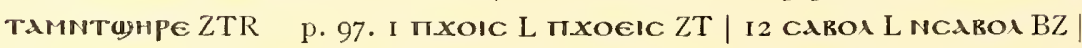

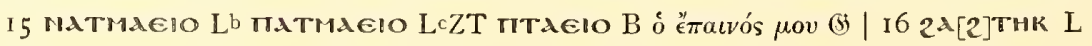




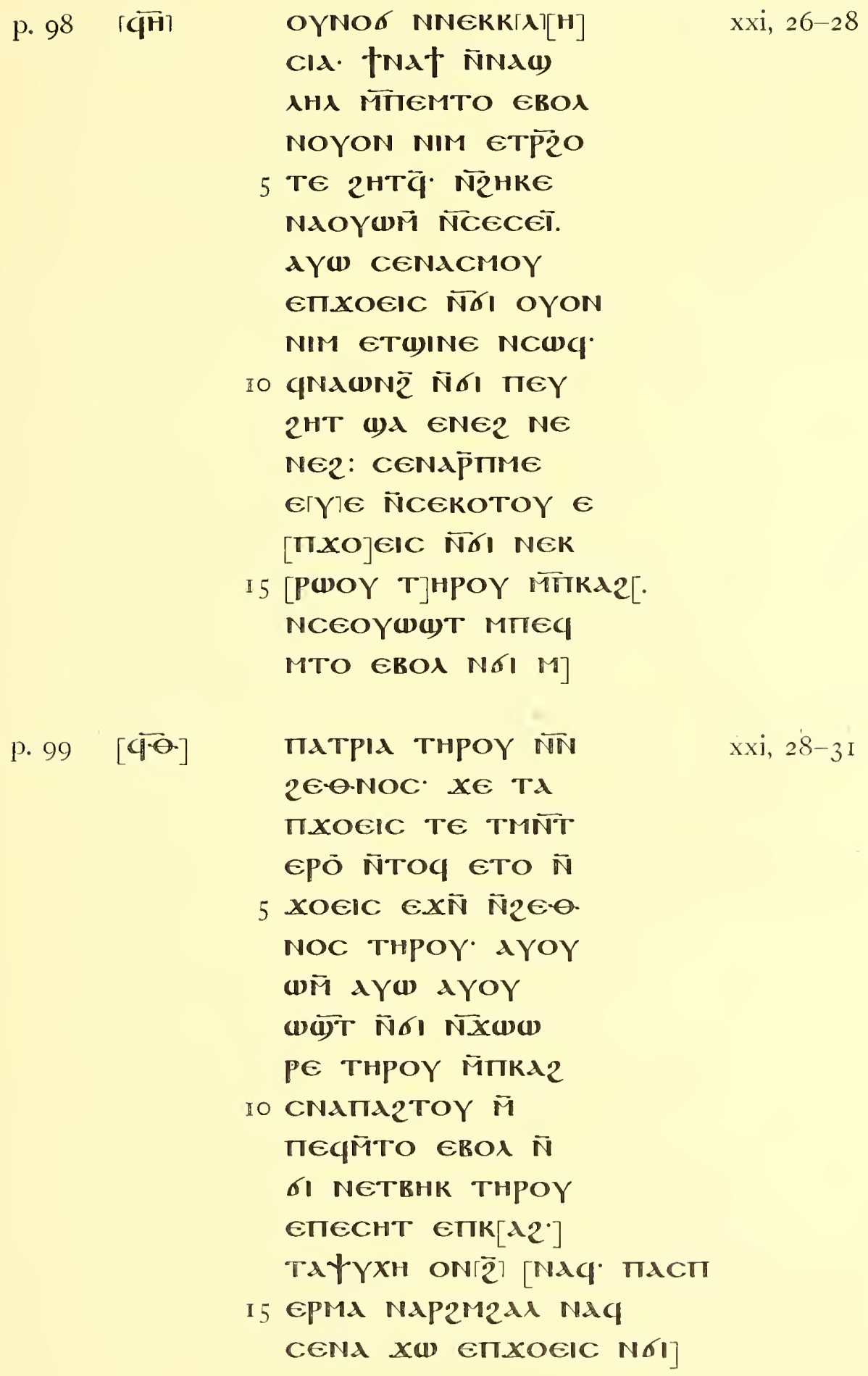




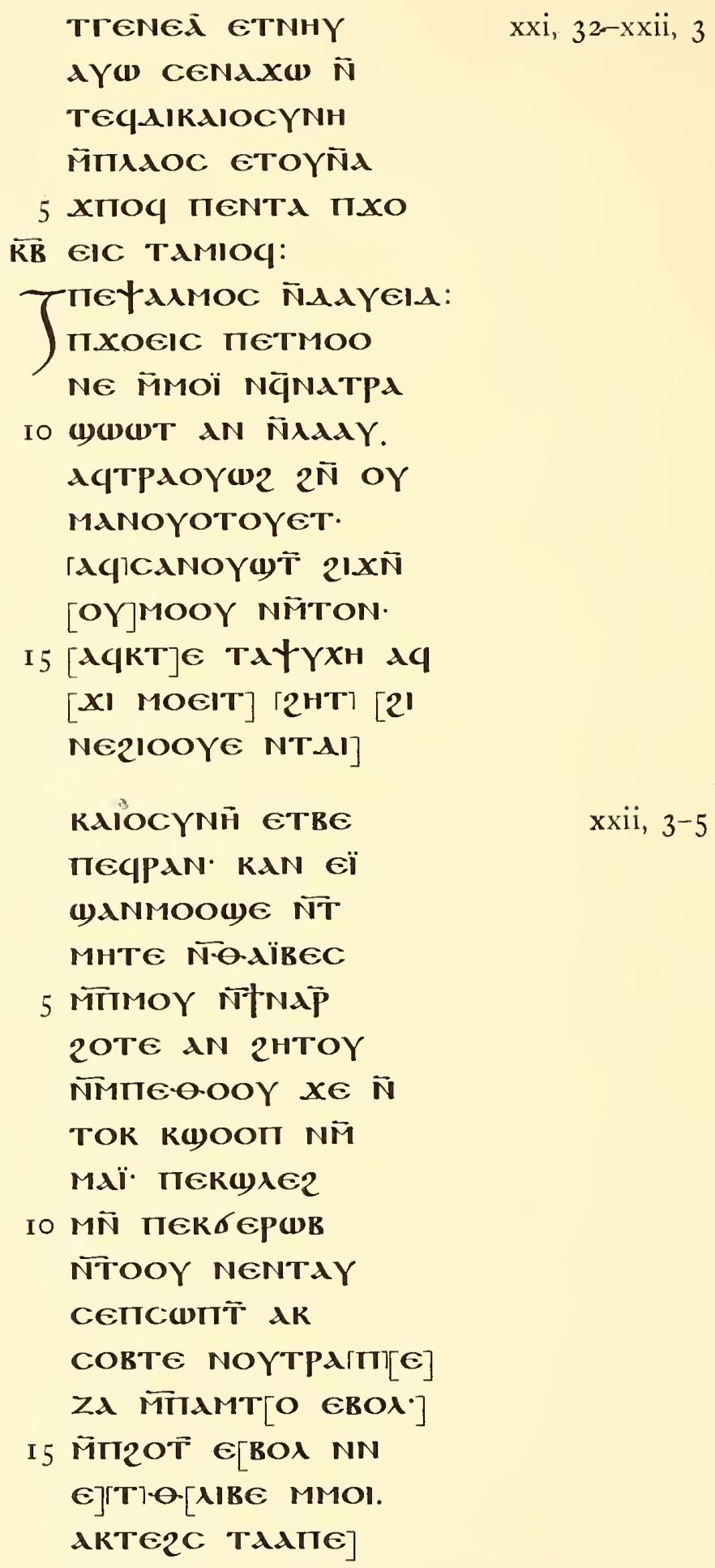


p. IO2 $\overrightarrow{P B}$

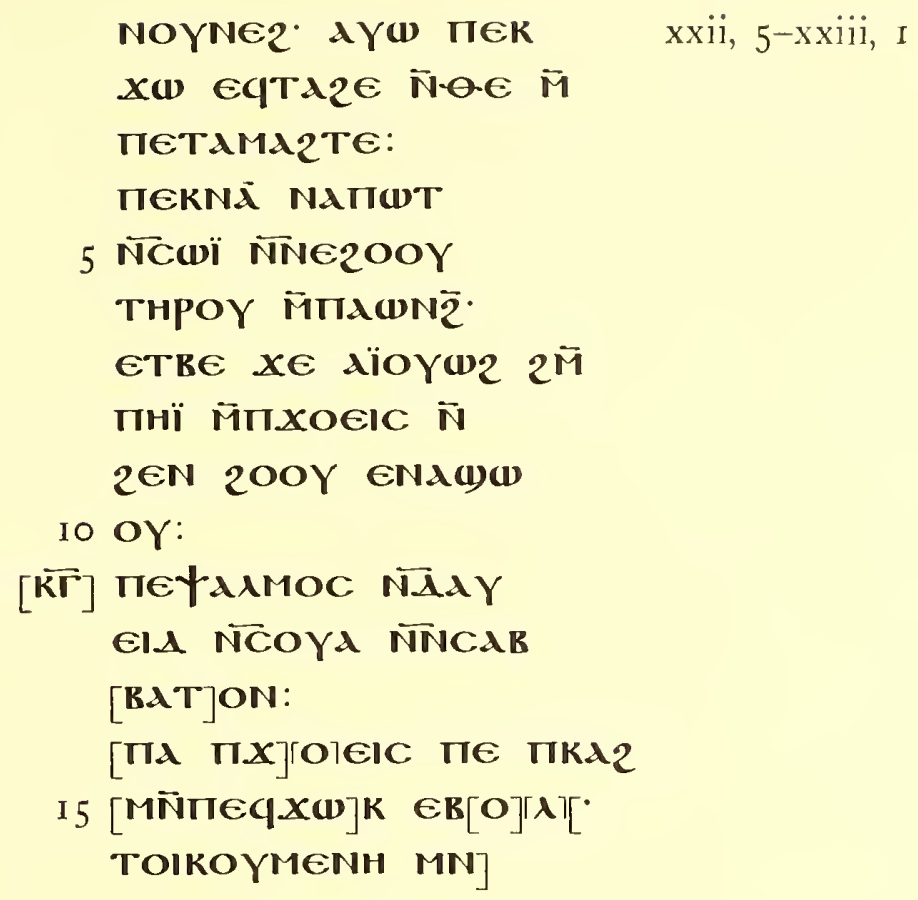

p. 103 PT

NETOYH2 THPOY

xxiii, I-4 2Pגї जूнTढ̈: [N]

TOQ AQCMÑCNTE MMOC 2IXÑ OAdAC

5 Cd. AYU AqC̈̈TU

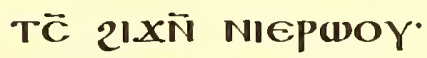

NIM пाETNAROK

E2Pגї E[ITTOOY M

пXOEIC H NIM

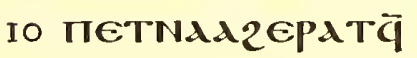

2'̈ TाM MாEq

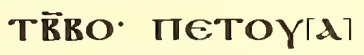

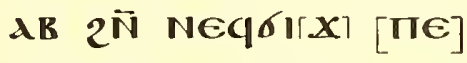

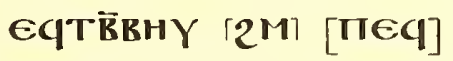

I 5 2HT' E[MTqXI NTEq

†YXH $\in X N$ OY

MNTETTPA EMTI] 
p. IO4 $[\overline{\mathrm{PA}}]$

Фрё їпет2ІтоY

xxiii, $4-8$

ल4 $2 \bar{N}$ OYKPOप:

IIÏ NAXI NOYCMOY

EBOA ITTM IIXOÏC

5 AYU OYNÄ GBOA $2 !$

TM̆ пNOYTE пеq

сштнр таї те т

reNEA N̈NETMINE

N̈Cd TXXEIC eT

IO MINE NCA $\Pi 20$ M

INOYTE NIIAKWB

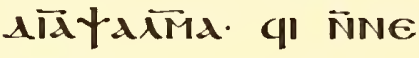

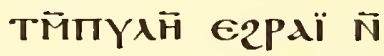

$[\mathrm{N}][\lambda] P X \mathscr{N} \cdot \overline{\mathrm{NTETN}}$

I 5 [ХIC]€ є2Pגї м̈пүגн

[N(1) EN] $\epsilon_{2}$ TAPEqGi

[E2OYN NGI] [ITP][P][O M]

[IEOOY. NIM пе пе।]

p. IO5 $\overrightarrow{\text { P「E } 1 ~}$

Р̈Po м̈пєоOY пхо

xxiii, 8-IO

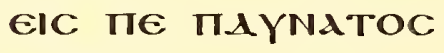

етТАхрнү пхо

EIC ETE OYN 6 OM

5 M̈MOQ 2ज̈ пाTOAE

MOC. $\lambda I \bar{\lambda} \psi \overline{\lambda i M}$.

qI ÑNETM̈TYAн $\epsilon_{2}$

Paï $\bar{N} \bar{N} \lambda$ PXuN.

N̄TETÑXICE $\epsilon_{2}$

Io paï M̈tтYAн waene?

Tapeqel E2OYN N̄

бI IPPO MİEOOY.

NIM пє пाиPPO [Mாє]

OOY $\Pi X[\mathrm{OEIC} N \mathrm{NN}]$

I 5 бOM̈ Гत̄][TOQ пе

ПРPО MIIEOOY]

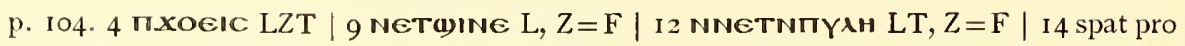

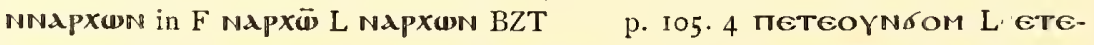

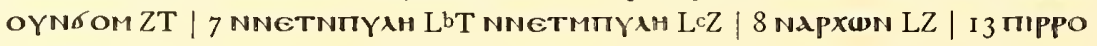
Z пеIPPO LT 


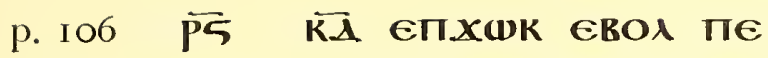
Xxiv, $I-4$

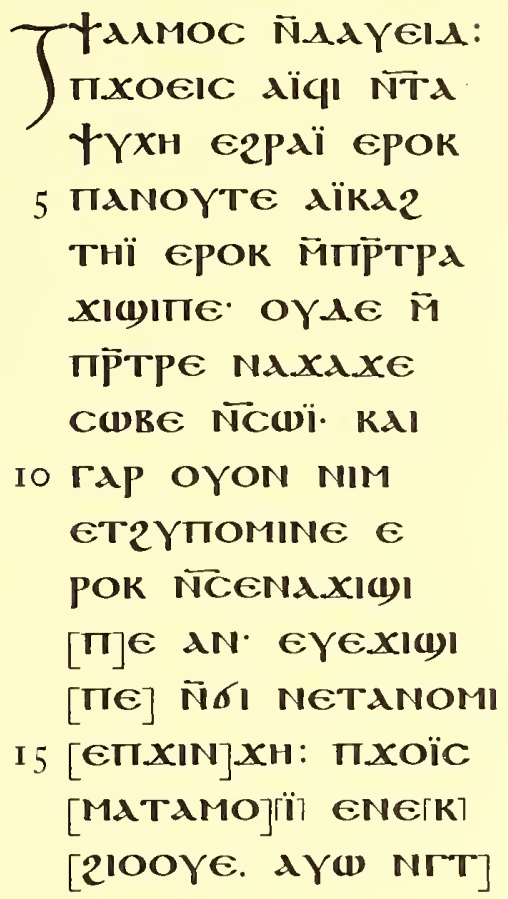

p. $107 \overline{\mathrm{PZ}}$

CגBö̈ ENEKMגM

MOO(1) XIMOEIT

2HT 21 TEKME:

АY山 NTT'TABOÏ

5 XE N̄TOK пе пाNOY

те плсштнр.

AYw TNA2YIIO

MINE EPOK MTIE

2OOY тнрव̈ं ApI

Io IIMEeYE N̄NEK

MNT(1)גN2THq

пХOEIC AYW NEK

Nì $x \in$ ce(1)OOT

XIN NENE2: NINIOT

I 5 BE N̈TגMN̄T[KOY]

MÑ NAMÑ]TAT

C]OOYYN] [MПPPाEY

MEEYE API ПME]

p. Iо6. II 2YחOMEINE L, BTPist $=\mathrm{F}$ 2YாOMONH $Z \mathrm{R} \mid$ I4 NeTANOMEI LBZR NCTANomid $T$, Pist $=\mathrm{F} \mid$ I 5 пx xoelc LZTPist 


\section{p. I08 $\widehat{\mathrm{PH}}$}

$$
\begin{aligned}
& \text { EYE N̄TOK KATd } \\
& \text { пдщגї MாడкNג } \\
& \text { ETВе TEКMÑT } \\
& \text { хрнстос пхоїс } \\
& 5 \text { оүХрнстос Аүш } \\
& \text { EqCOYTWN TE } \\
& \text { П.XOCIC. ETRE }
\end{aligned}
$$

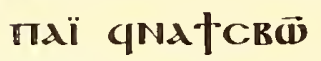

$$
\begin{aligned}
& \text { NNETPNNORE } 21 \\
& \text { IO TE2IH. qNAXIMO } \\
& \text { EIT 2HTOY Nĩ } \\
& \text { [P]M̈Pג(1) } 2^{\bar{N}} \text { OY2Аा厂 } \\
& \text { [QN]ATCARE N̄PM } \\
& \text { [Pd()] reINEIq12IOOYE. } \\
& \text { I } 5 \text { [Ne2IOO] } \\
& \text { [OY MITXOIC 2EN } \\
& \text { NA NE } 21 \text { ME NNET] }
\end{aligned}
$$

p. $\log \overrightarrow{\mathbf{P A}}$

$$
\begin{aligned}
& \text { wINE NCEA TEY } \\
& \text { AIA-OHKH АYW } \\
& \text { NEपMNTTMNTPE } \\
& \text { Стве пाКрАN } \\
& 5 \text { TIXOGIC KA TIANO } \\
& \text { re Naï erod Xe } \\
& \text { पO() ГAP NIM пе }
\end{aligned}
$$

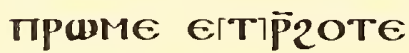

$$
\begin{aligned}
& \text { гнтव̈ М̈ХХिधाC. } \\
& \text { IO GNACMÑ NOMOC } \\
& \text { NAq } 21 \text { TE2IḦ EN }
\end{aligned}
$$

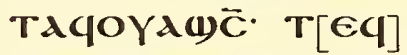

$$
\begin{aligned}
& \text { †Yxh ndew }[\pi \epsilon] \\
& \text { 2Ñ 2ENINIA[TAOON. } \\
& \text { I } 5 \text { п] } \\
& \text { кАHPONOMEI MTKA2] }
\end{aligned}
$$

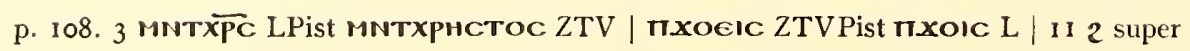
$\epsilon$ in ras

p. 109. 14 zenardeon LBZTPist 
p. i io קิ

p. II I Pid
пIXOEIC пाE ГTाT) хPÖ ĪNETP̄२OTE 2HTq̆ АYल IIPAN ज̈IXXOE IIA NETP 5 रОте २HTव் пाЕ. AYW ETAMOOY $\bar{\epsilon}$

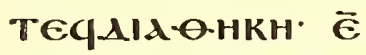
PE NARAl EİOTM NOYOEII) NIM $E$ Iо ПХXOGIC XE N̄TOY ПетNגTEKM̄ NA [O]ГYาернте EBOA $\overline{2 M}$

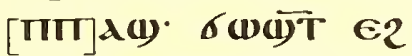

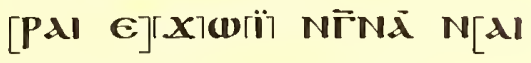
I5 XE ANT OYMH]PE [NOYWT AYW ANT]

OY2ГH]KE ANOK: $\bar{\lambda}$

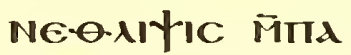
2HT OYWMC EROA ANIT GROX $2 \bar{N}$ NA

5 ANATKH. ANAY E

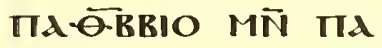
2ICE NT̈KW EBOA NINANORE THPOY. ANAY ENAXAXE I0 $x \in$ AYOYwa) $\bar{\epsilon}$ BOA- AYW AYME[C] Twi 2Ñ NOYMO[CT]E N̄XINGOT̄' 2[APE2] ETA†XH N[TTOY] I 5 Гxาoї Mाா][PTPAXI

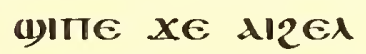
пIZE ЕРОК' NBAג] xxiv, $14-16$

xxiv, I6-2 I 
P. I I 2 PIB

2HT MÑ NE[TC]OY

xxiv, $2 \mathrm{I}-\mathrm{xxv}, 2$ TWÑ AYTOKOY

epoï xe dïzYпा

MINE EPOK пxOÏC

5 INOYTE CET ПIHस

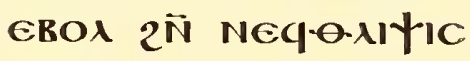

тнроY:

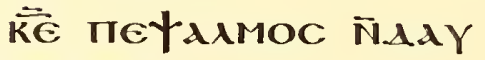

$\int$

eld:

KPINE M̄MOÏ пxXḮ

$\lceil X\rceil €$ ANOK AïmoO

[(1)]Є 2ர̄ TAMÑT

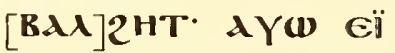

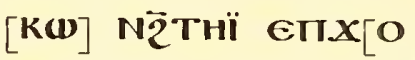

I 5 GIC N†NA $]\lceil K] I M\lceil\lambda\rceil[N$.

AOKIMAZE MMOI

TXOGIC NTIEIPA]

p. I I 3 PII

ze MMÖ̈ IICE

$\mathrm{xxv}, 2-5$

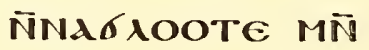

ГІАгHT XE ГIEK

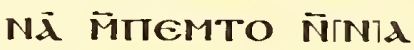

5 BAג EBOA AYW dï

PिANAK 2ї TEKГM]E.

MTIIIMOOC MÑ OY

CYN2EAPIOÑ Eq

ФOYEIT AYW

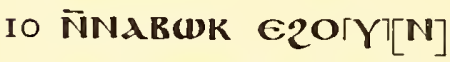

MÑ NPEGİN [ORE.]

AïECTE T[COOY]

2C ÑNETO [MIIO]

ГN]HPOC. AГY][W NNA2]

I 5 ГMרOO[C MN NACERHC.

TNAGIG NNAGIX $2 \mathrm{~N}$

NETOYАAв TAkwTE E?

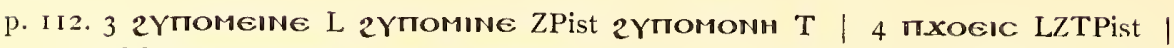

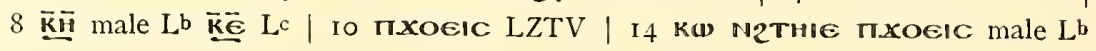

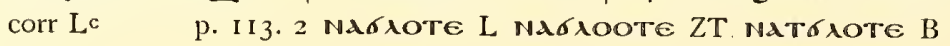


p. I I 4 PIA

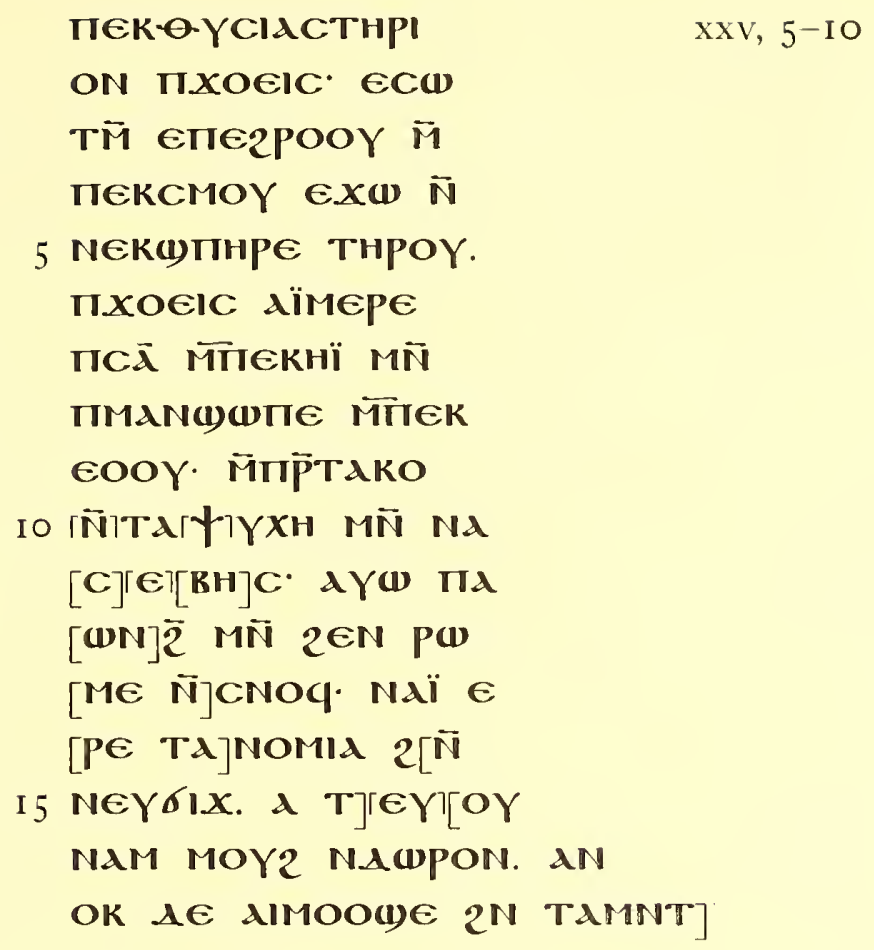




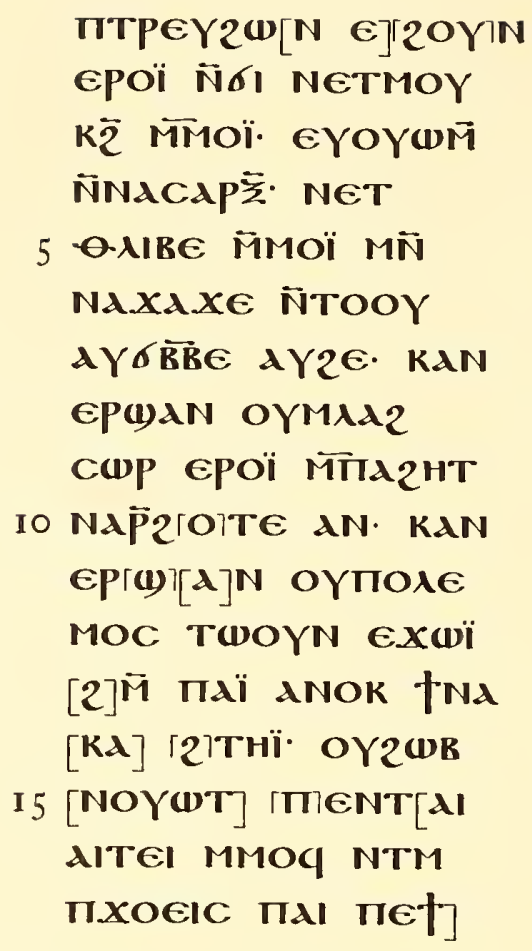


p. I I 8 PIH

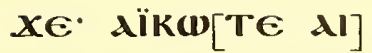

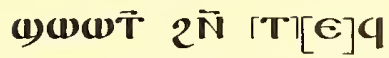

$$
\begin{aligned}
& \text { CKHNḦ NOYOYCIA } \\
& \text { NC̈MOY †NAXw }
\end{aligned}
$$

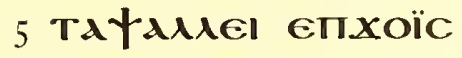

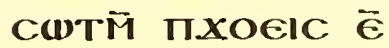

$$
\begin{aligned}
& \text { пе2POOY ENTAї } \\
& \text { w(1) EROA M̈MOq' } \\
& \text { NA Naï mXocic Nĩ } \\
& \text { Io CUTM̃ EPOї. NTA } \\
& \text { Пג2Hт (1)גXE } \bar{\epsilon} \\
& {[P][O 1 K \text { AqฒINE ÑCd }} \\
& \text { [пєк]20. пєк2о } \\
& \text { [IXOEIC †][N]A(1)! } \\
& \text { I } 5 \text { [NE NCWq. МПркळTE } \\
& \text { MПєК2O CABOA MMOI] }
\end{aligned}
$$$$
\text { xxvi, 6-9 }
$$

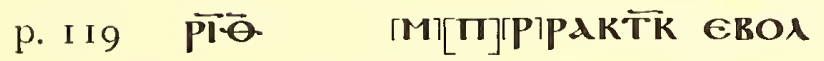

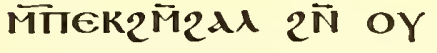

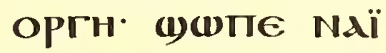

ज̄вон-ООС М̈пі

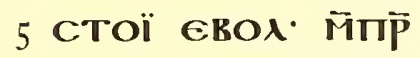

KAdT $\overline{\mathrm{NC}}(\mathrm{NK}$ TINOY

те пАсштнр $x \in A$

ПגEIWT MÑ TAMA

dY KAd $\bar{T}$ NCWOY.

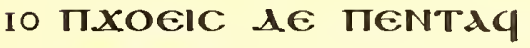

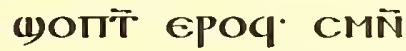

$$
\begin{aligned}
& \text { NOMOC Naï пxö̈c }
\end{aligned}
$$

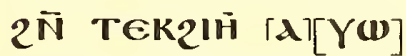

$$
\begin{aligned}
& \text { Nए̃̄XI] [MOEIT 2HT } \\
& \text { I5 } 2 \mathrm{~N} \text { OY2IH ECCOYTWN } \\
& \text { eTRe Naxaxe } \\
& \text { MாртАлT ETOO] }
\end{aligned}
$$

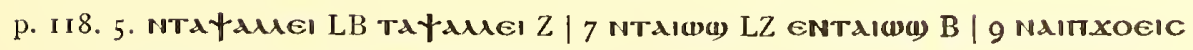
male $\mathrm{L}^{\mathrm{b}}$ cor $\mathrm{Lc} \mid$ to ep(i) $\mathrm{L}^{\mathrm{b}}$ epor $\mathrm{Lc}$

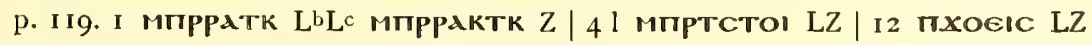


p. I $20 \quad \widehat{P K}$

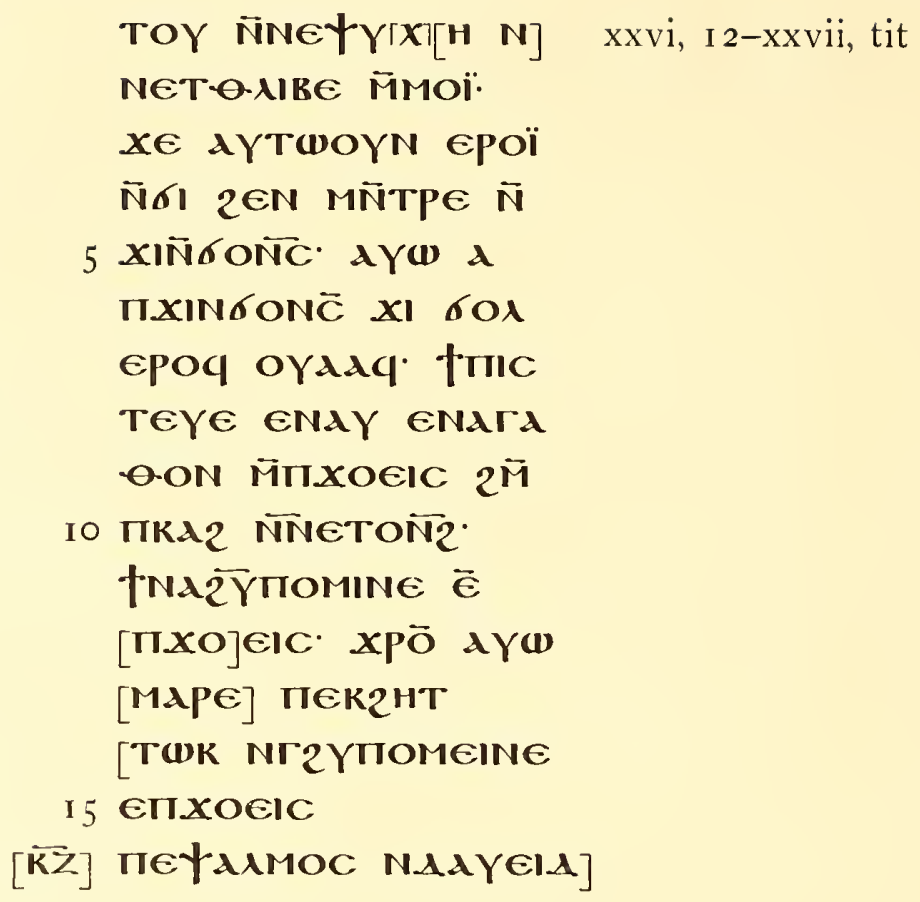

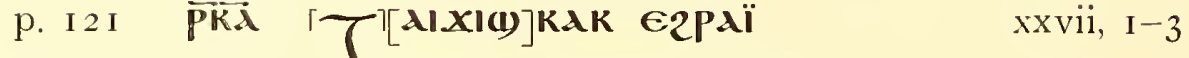 ePOK пХOEIC пג

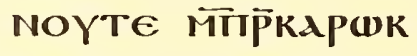 epoï- MHTालC $\mathrm{Ni}$
5 кגP®K epoï Tג $\bar{p}$ өє N̄NETBHK $\bar{\epsilon}$ теснт етाшні. СШTM̆ EாERPOOY MाTACOா̈ $2 \bar{M}$


p. 122 PKk

$$
\begin{aligned}
& \text { NETUגX[E } 2 \text { N OYEI] } \\
& \text { PHNH MÑ NE[T]2ITOY } \\
& \text { WOY EPE MTIE-OOOY } \\
& 2 \text { N NEY2HT T NAY } \\
& 5 \text { RATA NEY2RHYE. } \\
& \text { AYW KATA TIIONH }
\end{aligned}
$$

PIA ÑNEYMEEYE.

TWWBE NAY KATA

NE2RHYE ÑNEYGIX.

IO † NAY MTIEYTOY

EIO XE MITOYEI

ME ENE2RHYE M̈

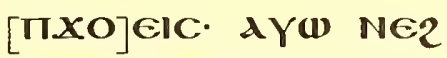

[RHYE] [NNEqGIX]

I 5 [KNגबP(1)

TMKOTOY IXXOEIC CMA]

p. I $23 \overrightarrow{\mathrm{Pk} \overrightarrow{\boldsymbol{T}}}$

[MAdT XE] АपCWTM

xxvii, 6-9

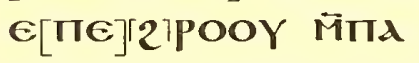

$\mathrm{CO}[\Pi] \overrightarrow{\mathrm{c}} \cdot \pi X O \mathrm{XeIC} \Pi \mathrm{TE}$

ГТАВОН-ОС Аүш

5 TANAWTE NTA

Пл2нT КА2Тнप

epoq' dïр20те

A TACAP玄 † OYĀ

TNAE乏OMOAOTI

Io NAq $2 \mathrm{M}$ TIAOYU(1).

пХOEIC пе птגX

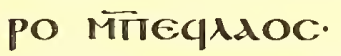

АYW TNACTE пе

NN̈EYXAÏ Mi[TEq

I 5 XPC MATOYXE

TIEKAAOC NTC

MOY ETEKRגH]

xxvii, $3^{-6}$ 
p. 124 PKג

(1)

PONOMIA [NTMO]

xxvii, 9-xxviii, 3

ONE MMOO[Y $\mathrm{Nr}]$

XACTOY W[A [E]NE2.

пеҰגАMOIC $[\mathrm{N}] \boldsymbol{\Lambda A Y}$

5 EIA EROA $2 \bar{N}$ TE2IḦ

KH NTECKHN[H]:

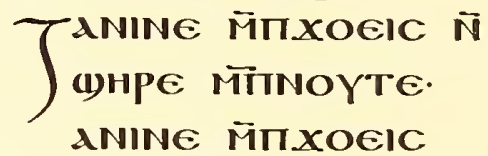

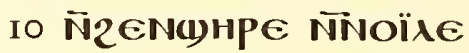

ANI OYTAÏO MÑ

OүTIMH MाTx

EIC. ANI OYEOOY

[MIIE] पPAN' OYN

I 5 [山T ПXXEIC $2 \mathrm{~N}$

теqגүAн ETOYAגR

тсмн мпхоєIC]

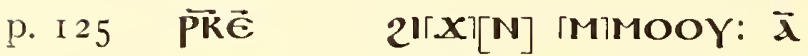

xxviii, 3-6 пN[O]YTE M̈ாСOOY

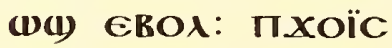

2IXÑ 2EN MOOY

5 ENAT(1) TOY: TEC

MH MTXXEIC $2 \bar{N}$

OүбОМ ТЕСМн M

ПXOEIC $2 \bar{N}$ OYMNT

No6. TесMH $\bar{M}$

IO ПXOEIC EqOYW

(1)व̄ NNKEAPOC.

TIXOEIC NA OY(D)

NN̄KEAPOC M̄[ாג]

BAN][OC1. N[G̈TPEY

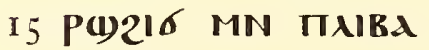

NOC NaE MTMACE

Аүळ пाЕРIT Eq]

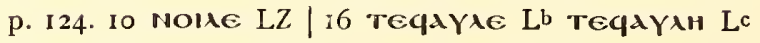

p. $125 \cdot 3$ IтxOGic LZ 
p. $126 \quad \overrightarrow{\mathbf{P K S}}$

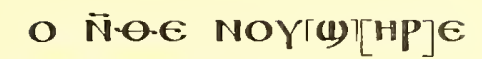

xxviii; 6-9

MMONOKAPOC

тесмн ज̈пxої

EqOYळ(a) N̄oY

5 山) 2 ÑCdTE. TEC

MH MTx

KIM ETEPHMOC.

ПIXOEIC NA KIM Eे

TEPHMOC NKA

Iо АнC. ТЕСМн ї

IIXOEIC EqCORT [E

N̈][N]Ë̈OYA A YU qNA

[бOAT] GBOA NM̈MA [N]

[山HN OYON NIM

I5 ET $2 M$ IЕ

MाIEqGOOY (1)גPE]

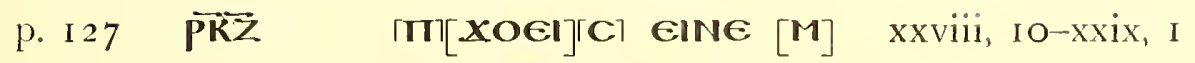
IIKגTגКАHCГM][OC]

AYW Nव̈TPEq2[MO]

OC. ПIXOEIC NATPP1

5 PO עd ENE2 TIXO

EIC Nat 6OM

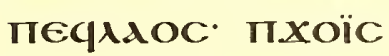

NACMOY ETIEqAג

OC $2 \bar{N}$ OY EIPHNH:

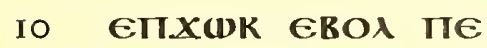

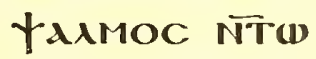

[र]-

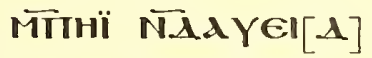

TNגXגстй П[XOGIC -- ]

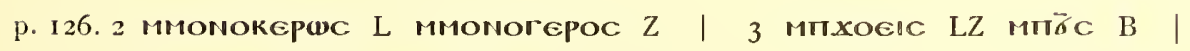

12 nNeEloYe L nNIEOYA ZR

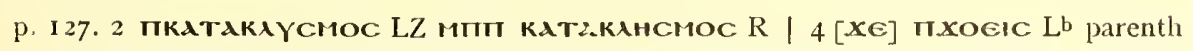
del Lc | Nappo LbLc Napppo ZR | 7 пхоєзc LZV пб̆c R 
p. I 28 РК[Н̈]

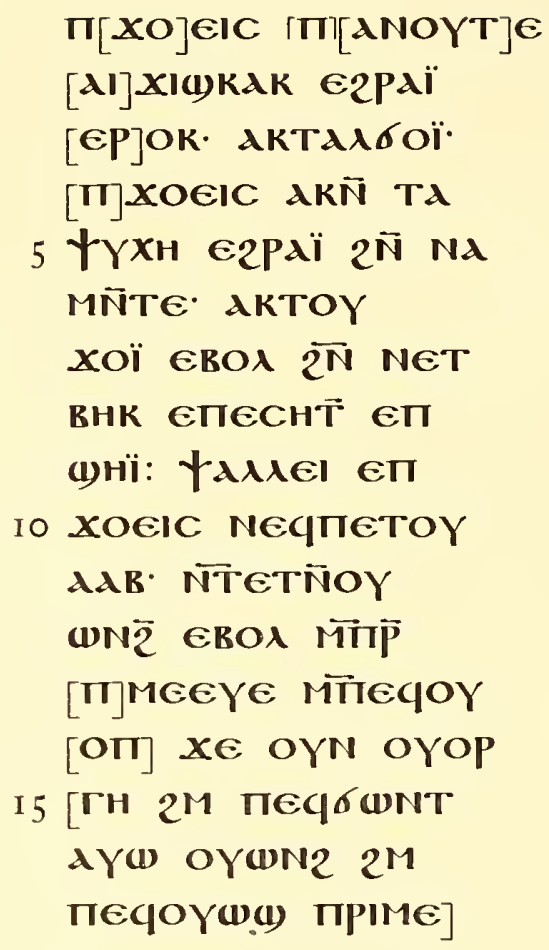

p. 129 PKO.

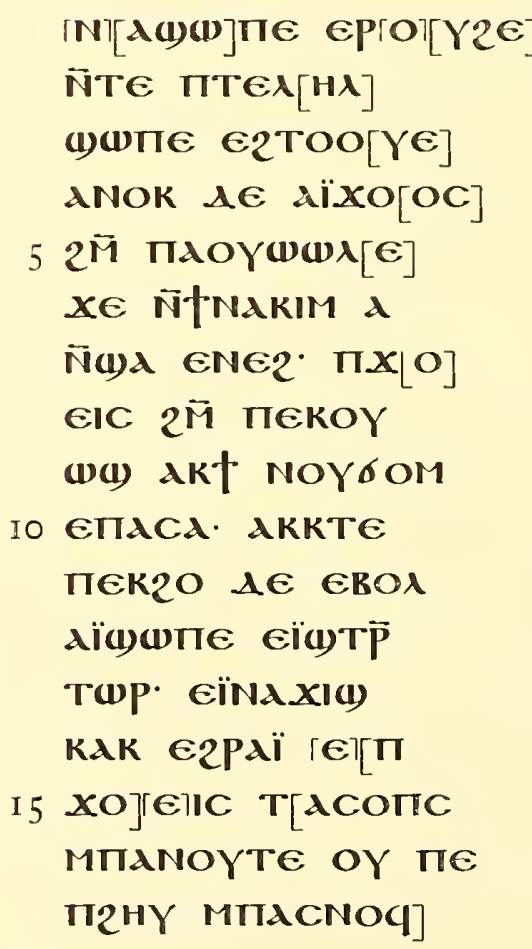




\section{p. $130 \quad[\overline{\mathbf{P \lambda}}]$}

$\left[\begin{array}{ll}2 M & \text { II }\end{array}\right]$ TP $А[$ ВСК

Xxix, $10-13$

єпєс]нт Єп[Т]Ако.

[MH] ере пехоүс

[Nd] E乏OMOAOrI

$5[\mathrm{~N}][\lambda] K . ~ M H ~ E q N \lambda X w$

[N]TEKME. АाXO

ГEIIC CUTM A INA

NAї d пXOGIC (1)

пе Nגї ̄̄воноOC.

Iо גqKTе пגNE2ПЕ

Naï eYpגae. AK

IIE2 TA

AKMOPT NOYOY

[NO]q хе ере тגOY

15 [NA] $\vec{M}$ cMOY EPOK

[NTA T $] M \bar{M} / M][K d 2 N$

2HT пXOGL пाג]

p. I 3 I $[\overline{\mathrm{PAX}}]$

[NOYTE fNגezo

xxix, $13^{-x x x}, 3$

MON]Or[el] $N[A K]$

(1)d ENE2:

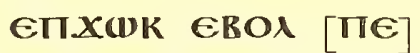

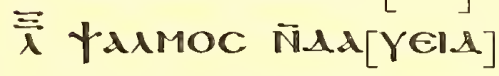

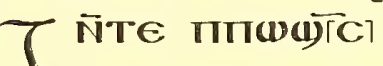

)גї кג2тні ерок

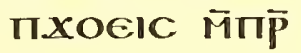

трдхा(1) шd $€$

Io Ne2. NazMET Ni

TOYXOÏ $2 \bar{N}$ TEKAI

КАIOCYNH. PIKE M

пекMdגхe ерої

Nїбетн eTOYX|Oї

I 5 ш)ाTE NAï EY[NOY]

TE NINA(i)TE] [AY

(1) Eүнї $\bar{M}[M A M$

п(1)] $\epsilon[T][O Y X O i]$

p. I30. 4 धzomoxore1 LZ ezomoxori V

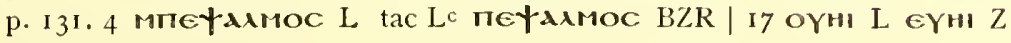




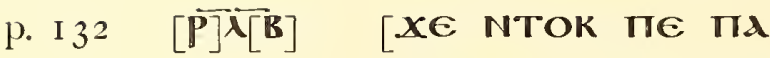
$\mathrm{xxx}, 4-7$

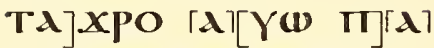

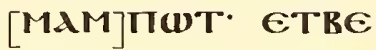

[T]€KPAN KNA.XI

$5[\mathrm{M}]$ OEI $\ddot{\mathrm{T}} 2 \mathrm{H} \overrightarrow{\mathrm{T}}$ Aү山

[N]TCANOYŪT.

「KTNANT EROA $2 \bar{N}$

ГTาєїорбС ENTAY

2оттї єрої $x \in \bar{N}$

IO TOK ПЕ TANAL)

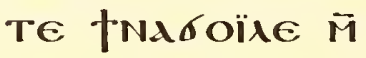

ПАПINA ENEKGIX.

Аксште जัмої

IXXOEIC IINOYTE

I $5[\mathbf{N}]^{\top T} \mathrm{~T} / \mathrm{C} \cdot \mathbf{A K M E C}$

[TE N]ET 2AP $\left[\epsilon_{2}\right.$

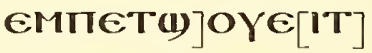

p. I $33[\overline{\mathrm{P}} \overline{\mathrm{r}}]$

[ETXINXH ANOK $\lambda \epsilon$

$\mathrm{xxx}, 7-\mathrm{IO}$

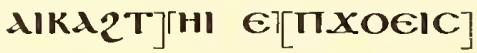

†Nגтелнर т[ג]єY

фPANE E.XM пाEKINA

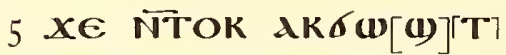

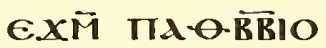

AYW AKTOYXE TA

†YXH EBOA $2 \bar{N}$ NA

АNАГKH. АYW M̈

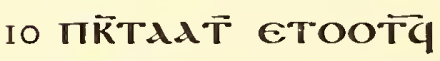

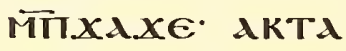

2є NAOYePHTE $\ddot{E}$

PגTOY 2त̄ OYOГCT][N].

Nd Ndï חXXelrch [XE]

I 5 H.

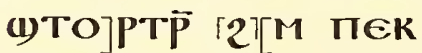

б 


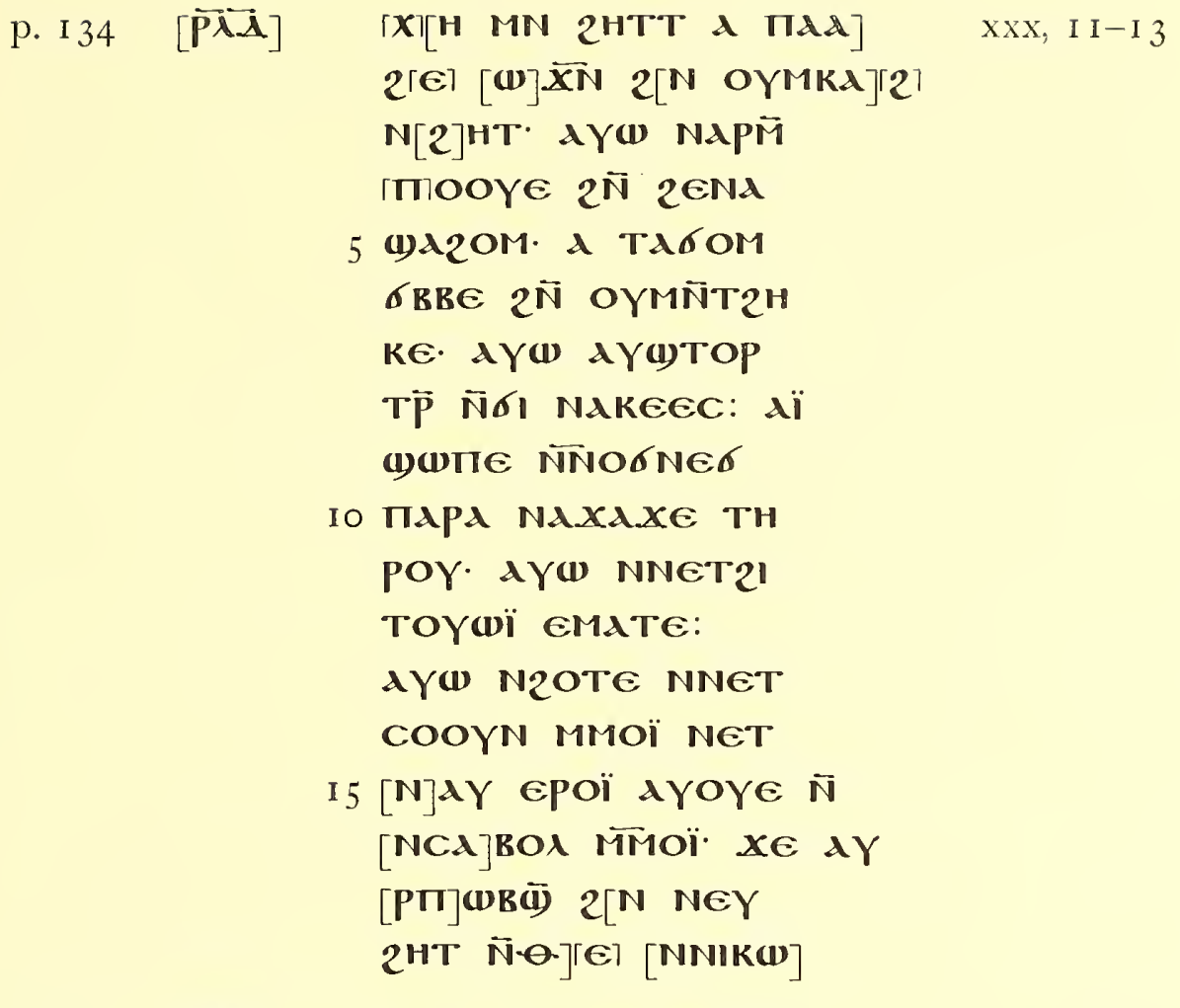

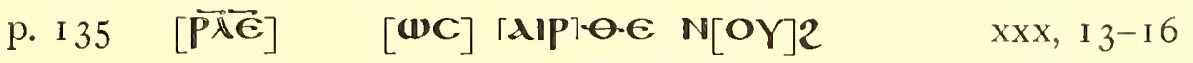

NAגY EAqCWPM̈

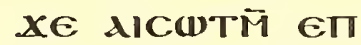

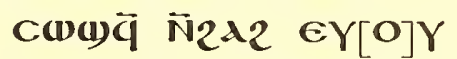

5 H2 2ї пАКШТЕ:

2М̈ птреүсшоY2

21 oүсоர̈ epoï aY

(1)OXNE E.XI N̄TA

†YXH. גNOK גï

Iо кА2тнї ЕРОк

пхOEIC. dïxoOC

xe N̄TOK пе пג

NOYTE EPE NARГגH]

POICl $2 \overline{\mathrm{N}}$ NEKбוx

I 5 [MAT]OYXÖ̈ $[$ ET

GIX NN]AX[AxE

MN NETTHT NC(1)

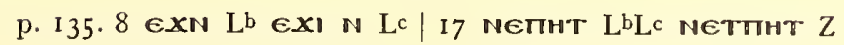




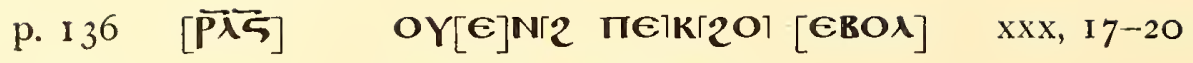

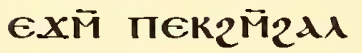
MАTOYXOї $2 \bar{M}$

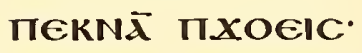

5 мпїтрдхимıпе $x \in$ dï(1) E2Pdi $\epsilon$ рок EYeXIGITE N̈бI NАCERHC АYळ N̈CEXITOY EAMÑTE

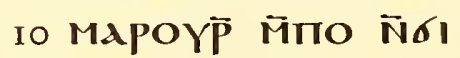
NECITOTOY N̄KPOГq1 NETXW NOYANO MId E2OYN EாגI KAIOC $2 \bar{N}$ OYMÑT

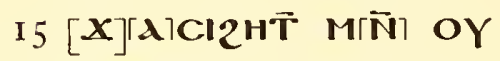

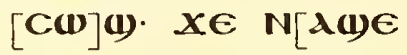
II(1)גI] NัT[EKMNT]

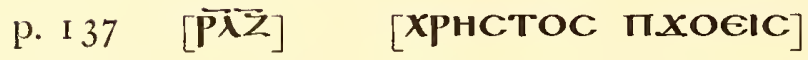
$\mathrm{xxx}, 20-22$ ГT][AI E][N]TA「K12O「ITC $]$ N̈NETPि2OTE 2HTK АKTOWC INNET

5 2єАПIZЄ ЄРОК М̆ ПЕМTO EROA NTN घнре ÑNP(B). KNA2OTOY $2 \bar{M}$ пாєОні̄ М̈пек

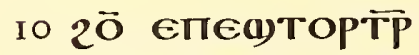
NN̈PGME. KNAP 2AївеC EPOOY $\overline{2 N}$ NOY2AÏBEC EITOY $\omega_{2} \bar{M} \bar{N}_{2} \in N$ AAC.

I5 ПXOEIC CMAMГA A $[T]\ulcorner X] \Theta$ Aqt $\ulcorner E]$ [OOY MITEqNA E2PAI] 


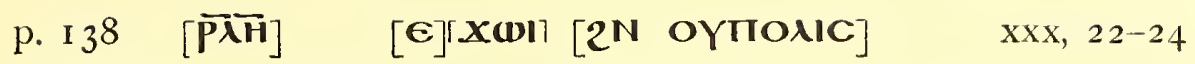
ECTAXIPHY ANOK

Le $\lambda \ddot{X X O C} 2^{\bar{N}}$ TA

ERCTACIC xE ME

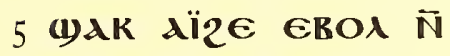

NA2PN̄ NERBdA.

ЄTRE пגї АKCW

Tї єात्2POOY $\bar{M}$

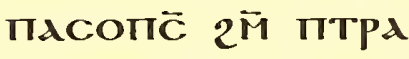

Iо (1) E2Pגï Oүвнк:

Mepe nXOEIC NE[4]

ПетоYАAв TH

poY $x \in$ epe пxoï

WINE N̄CA T̄MÑT

I $5[M][E]^{\circ}$ AYW CINATOFY]

[EIO NNE][T][XOCE]

p. I 39 Pᄉ्.

[N2HT EM]

XPO ГNITE пETN

2НT бМбОМ. OY

ON NIM ET2EᄉTI

5 Z€ eाIXo€IC:

ता TMNTTPMÑ

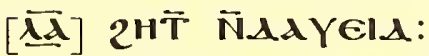

NAÏATOY ÑNENTAY

K@ EBOA ÑNEYA

IO NOMIA MÑ NENTAY

$2 \mathrm{WBC}$ GROA EXÑ

NEYNORE. NAIATA

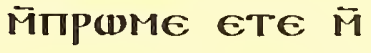

IIXOEIC NAET NO

I 5 BE EPOQ $\lambda \bar{N} \cdot$ OY $[\lambda \epsilon$

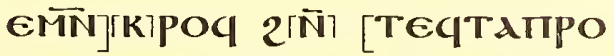

AlKAPWI XE a NAKEeG pAC]

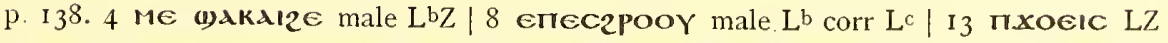

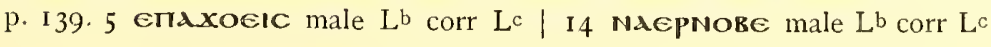


СвOX М̈пє2OOY

тнР̈ $x \in 2 \bar{M}$ пе

2OOY M̄N TEY山H

5 а текбוx 2POW $e_{2}$

pdi exwi. лїтої

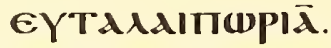

$2 \bar{M}$ Птрдтшї

Q.E ÑNILONTE.

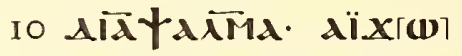

Mitanore Mitt

M̈ாI2ET TAANO

MId dïxooc $x \in \dagger$

NA][X]€ ITANOBE E[T

I5 ЮYвн]ї єп[XOEIC

АYW NTOK ПЕT]

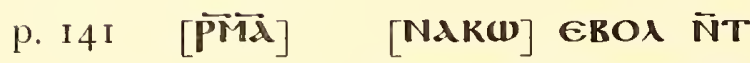

$[\mathrm{M}] \mathrm{N}[\mathrm{T}](1) \lambda q T \mathrm{~T}$ M

пג2HT: AIA†Aג

MA EPE OYON NIM

5 ETOYAגB NAG

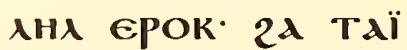

M̈теOYOEI() $\mathrm{ET}$

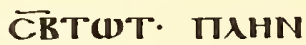

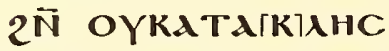

IO MOC M̈MOOIYI ENA

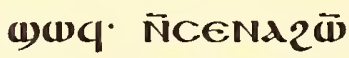

ePOY AN' NTTOK ITE

пАMАM̈ாШT $\epsilon$

ROA $2 \bar{N}$ TEentic

I 5 ЄTкште E[Pा[OI]

[ாАТТЕ人нर ГC][ОT'Т

GROA 2N NET2M]

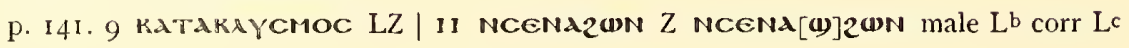


p. 142 [TMB]

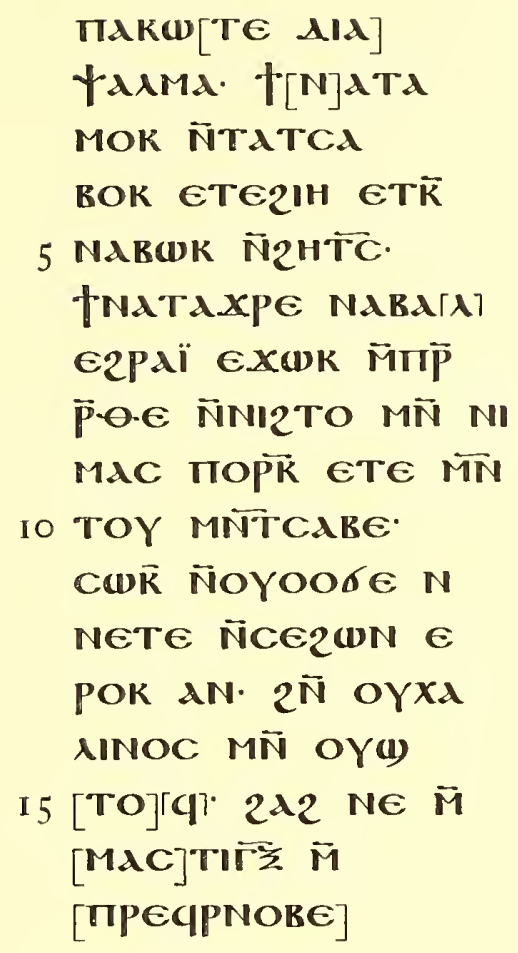

p. $143 \quad \overrightarrow{\mathbf{P}} \overrightarrow{\boldsymbol{N}}[\overrightarrow{\boldsymbol{T}}]$

[IINA $\Lambda \in$ Nd]KC

xxxi, 10-xxxii, 2

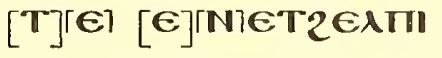

Ze EIXXOEIC ī

AIKAIOC EYфPA

5 NE EXM TाXOEIC.

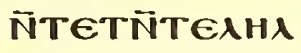

N̄TETÑฒOY

aOY M̄MCTÑ

THPTÑ NETCOY

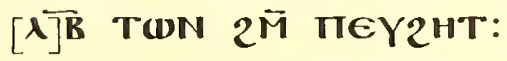

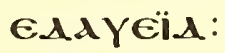

NAIKAIOC TEגHA

м̈пxoEIC E[P][E]

песMOY $\Pi[\mathrm{PE} \Pi \mathrm{CI}]$

I 5 Гก̄ंาNETCO[YTWN

OYWN2 EBOA M]

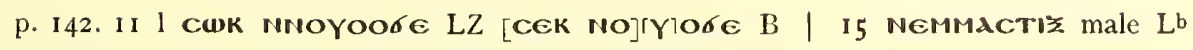

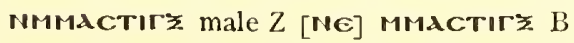


p. 44 PM̄A

ПХXOEIIC 2$][\mathrm{N}$ OYKI]

xxxii, 2-6

odpd †direl el

POq $2 \bar{N}$ OY†AגTH

PION M̄MHT NKAT.

$5 x \omega$ ePOq ÑOYXw

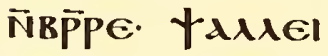

epoq kגАwc $2 \overline{\mathrm{N}}$ OY

loyddï $x \in$ mildxe

M̄TIXOEIC coYTwN

IO AYU NEG2BHYE

тнроY ФоŌं $2 \mathrm{~N}$ OY

ПIGTIC. पME NT

[M]NTNĀ MÑ Tा2גா.

[I2ג2] тнРव ME2

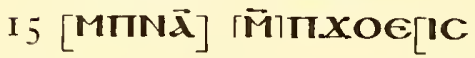

Aү@ $2^{M}$ пеqu)d.xe]

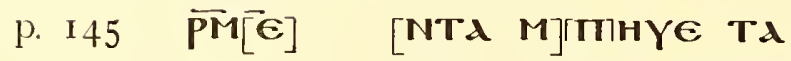

xxxii, 6-9

$[X]$ TPO ATYW NEYGOM

THPOY $2 \bar{M}$ ПЕாज̃

N̈ршq. петсшоY2

5 NMMOOY $\bar{N}$ OdגdC

Cd N̈.e ÑNIACKOC.

пеNTגqKU N̄N̄OYN

२N̄ NEYAzWWP. MA

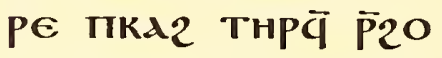

IO TE 2HTव̈ MTIXOEIC.

MAPE MA NIM CTAT

2HTव̈ MÑ OYON NIM

ЄTOYн2 ${ }^{2} 2$ TоY.

XE N̄TOQ TENTTI[Aq]

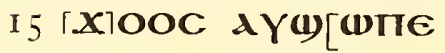

NTO] IIE][NTAq2WN

AYW AYCWNT]

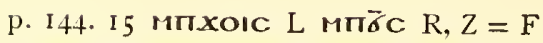

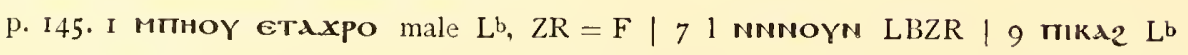
пKג2 Lc 


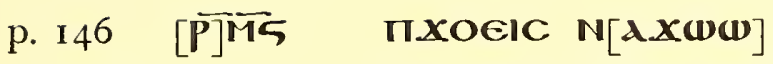
Xxxii, $10-12$

PE EROA MTIM[O] $[X][N E]$

N̄Ñze-ONOC. qNalA 1

-.eTEI NM̄MEeYE

5 ÑÑAdOC AYU qNA

AөETE ÑÑaOX

NE ÑÑAPXUN.

IMOXNE ÑTOY $\bar{M}$

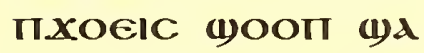

IO ENE2 MMEEYE

М̈песІ2н $\bar{T}$ XIN OY

XWM Ud OYXWM

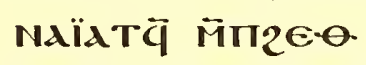

[NO]C EாXOEIC пе

I 5 [IEqN]OYTE. IIN/A]

[OC ENTA] пாX][OEIC]

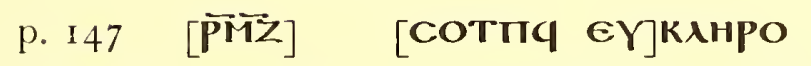

Xxxii, I 2-I 6

[NOMI][A] NAq. A TIXO

IEIC бWMT EROA

2त̃ TIIE ENAY EN

5 Фнре THPOY ÑN̄

PФME EBOA 2 M̃

IIEqMANMWTE

ЄTСК̈ТШT' Аप

бฒ(1) EXÑ NE

Iо TоYн2 тнроY $2 \bar{M}$

IIKA2 IIENTAC

IIACCE M̈MAY

ААq N̈NEY2HT.

ITETEIME ENTEY

15 2внүе тнрго [Y MN

oYPिO ÑAoYXA] 


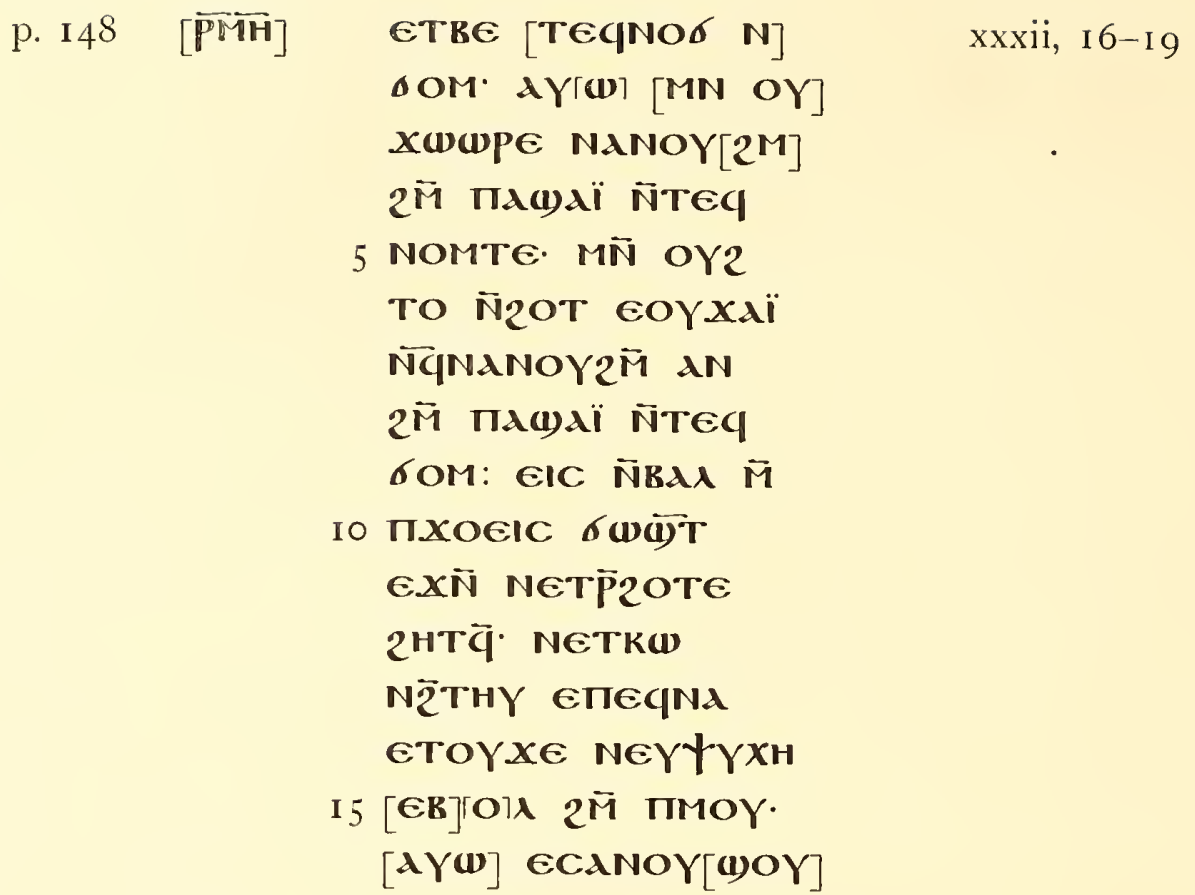

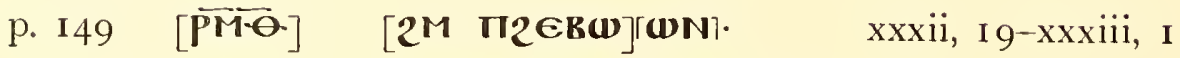
[TENY]ГYX]H NA2Y [IIO]MINE EIIXOÏ XE TENROH-OOC

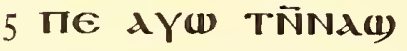

тє пе $x \in$ EPE

IEN2HT NAEY

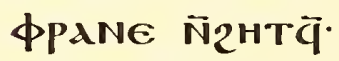
AYU N̄TANNA2 io TE 2M̈ пEqPAN E TOYAdв єPE ПЕК

NA חIXOEIC w) TE EXWT̄ KATA oE eNTAN2EA I 5 пाZ[Є Є]РОк:

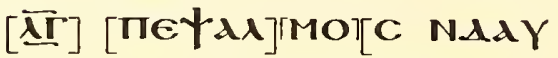
EIA NTEPEquIRE] 


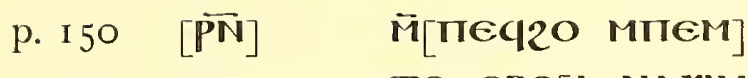

Xxxiii, $\mathbf{I}-5$

TO ERO $[\lambda$ NAXIME]

АєX АqкגГגq?

$\int$

ACBRWK:

tNACMOY ETIXOIC

NOYOEI(I) NITI NOY

OEI(1) NIM пеqCMOY

2Ñ Pшї. TAభYXH

NAXITAÏO $2^{\bar{M}}$ пXO

Io धIC MAPE N̄PM

PA(1) C(1TM̆ ÑCE

OYNOY XICE NM̆

Maï їாхоEIC.

AYW N̄TETÑ $X I$

I 5 CE M̈TIEqPAN 21

$[\mathrm{OYC}] \mathrm{OI} \cdot[\mathrm{A}][\mathrm{NO}][\mathrm{K}][\mathrm{d}] \mathrm{ii}]$

[aINE] $\bar{N}[\mathrm{CA}$ IIXOEIC]

p. I 5 I [PNমA] [AqCWTM E]POI?

xxxiii, $5^{-8}$

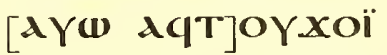

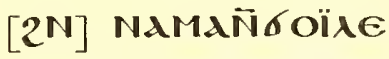

тнроY † M̈ாЕTÑ

5 OYOÏ EPOC ÑTETĨ

POYOEIÑ AYW NE

TÑ2Ö ÑNEYXI(1)।

пе. паї пе П2н

ke N̄TAq(w) EROA

IO A п.XOEIC CWTM

EPOC AYW АqTOY

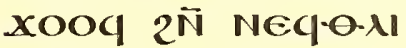

†ic тнроү as

pe marteaoc M

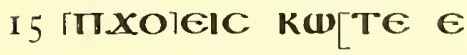

NET] P̄]2[OTE 2H

TQ NEQNA2MOY]

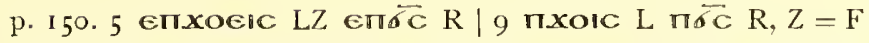

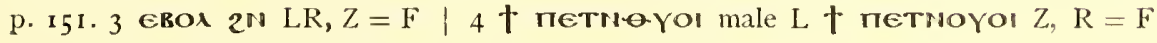
II 1 АqтоYXoq LZR 
p. 15

$[\overline{\mathrm{PN}}] \mathrm{B}$

xl Trie nTetnel]

xxxiii, 9-1 2

Me $x \in$ пा $x$ [OEIC]

2016. NAÏAT4 [M]

IтिME ETNA2E[A]

5 IIZE EPOq API

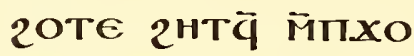

EIC NEqITETOYA

גB. $x \in \overline{M N}$ бP(2

ฒOOİ ÑNETP̄2O

Io TE 2 HTिं A NPM

MAO Р̈2HKE AYW

АY२KO NET(1)

NE $\lambda \in$ N̄Cd пxOÏC

N̄CENAGMUT

I 5 AN N̈NATAO-ON

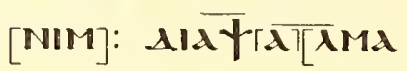

АMHI]TN̄] [NAตHPE NTETN]

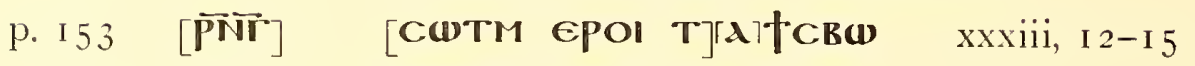

[NHTN] NO.OTE $\bar{M}$

$[\Pi]$ XOEIC NIM TIE

пршме ЄтоYє()

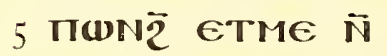

NAY ENEZOOY ET

NANOYOY MATAג

бE TERAגC EROA

$2 \bar{M}$ пеө.ooY dүa

IO NERCTIOTOY $\overline{\mathrm{e}}$

тйхш N̄oүкроч.

PARTK̈ EROX $\bar{M}$

TITIE-O.OY ÑreI

pe M̄IITIETNA

I5 โNרOYG' (1)I[NE

NCA] TP[HNH

NזTIT NCWC $x \in E]$

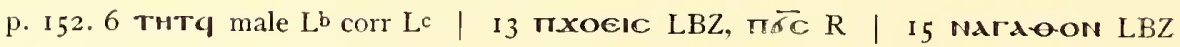
edrdeon R

p. I 33.9 мாєе-ooY Lb пாєе-ooY LcZRBour 


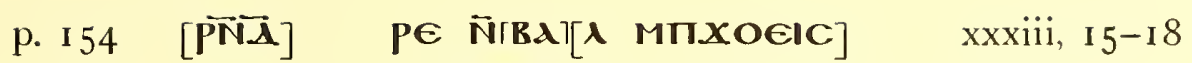

б(1) $\overparen{T} \in X[N$ Ñ.AIKAl]

Oc. AYw EPE $N[E q]$

MAdXE POKE $\epsilon$

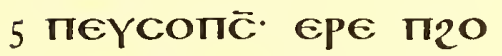

$\lambda \epsilon$ M̈ாXOEIC $\sigma \omega$

(1) $\bar{T}$ EXT̄ NETEIPE

NM̈ாE-OOY. Єqu

те м̈ாЕҮР̈ாте

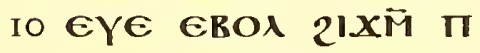

KA2: A NAIKAIOC

ХІ(кКАK ЄBOג А

IIXOEIC CUT"̈ $\epsilon$

POOY AYU AqTOY

I5 [XO]OY $2 \bar{N}$ NEY

$\left[\cdot \cdot \lambda \| Y_{1}\right] \mathrm{C}$ Тн $[$ POY

TIXOE]IIC] [2HN $\left.E_{2} O Y N\right]$

p. I 55 Pस्E ENETOYO][M]व $2 \mathrm{M}$

xxxiii, $19-23$

TEY2]HT' AYW qNA

TAN2E NET⿱一⿻上丨BI

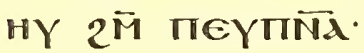

5 NAduOY NEANITIC

NN̈AIKAIOC'AYW

TIXOGIC NANA2

MOY N̈2HTOY TH

POY TXOEIC NA

IO 2APE2 ENEYREEO

THPOY ĨNE OYA

OYलवप EROA N̈2H

TOY IIMOY NंTOOY

NÑPECIPNORE 2OIOY

I 5 AYU NETMrO][CTE

MT] AlIKג[IOG NAP̈NOBE

пXOEIC NACWTE NNEYYXH]

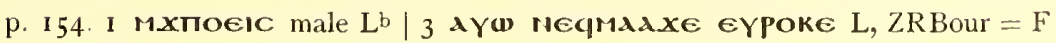

p. 155.14 NNPEqNore $\mathrm{LbLc}, \mathrm{BZR}=\mathrm{F}$ 


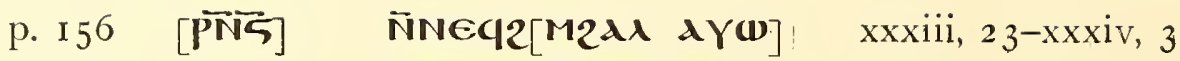
ÑNEYT̄NORE [NǴl OY]

ON NIM ET2EN[II]

$\overline{\lambda i}$ ZG epoq:

ITA AגYeIA:- :

† 2Аा пXOEIC ENET

xı M̄MÖ̈ $\bar{N} \sigma O \overline{N C}$.

M(1)E MÑ NETMI

(1) NM̈MÄ̈ AMA2

Io TE NOYZOTINON

MÑ NOYAYPWN $\overline{N T}$

TWOYN̄ EROH-I $€$

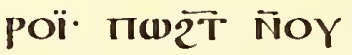

снqе Nï2OXITOY

I 5 M̈тtеMTо EROA

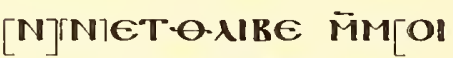

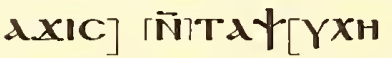

$x \in$ АNOK пе поY]

p. $\left.157 \mathrm{P}[\overline{\mathrm{NZ}}] \quad[\mathrm{CU}]]^{[\mathbf{H P}}\right]$ MAPOГY

xxxiv, 3-6

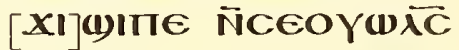

N̄́l OYON NIM ET

wINE ÑCA TAYY

5 XH. MAPOYKOTOY

Eாג2OY N̈CEXIฒI]

пте ÑбI NeTME

eYe epoï ezentTe

oOOY. MAPOYWW

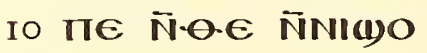

धı) ज̈пємто $\bar{\epsilon}$

вол М̈ттнү єрє

пдггелос Mाt $[X O]$

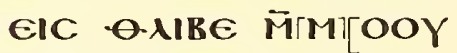

I 5 MAPE] ГT/EY[2IH P

KAKE NCCAAdTE] 
p. I 58 PNTH

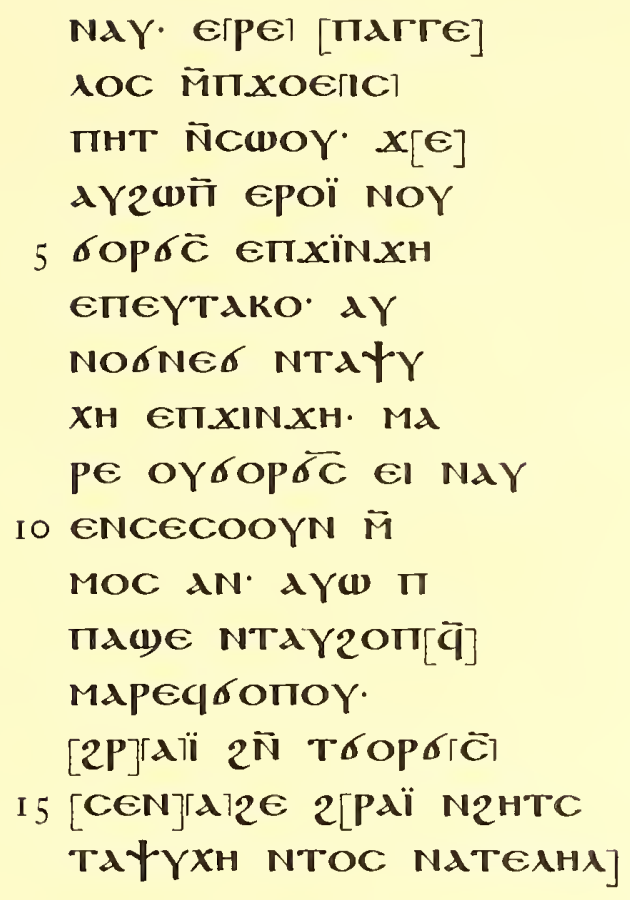

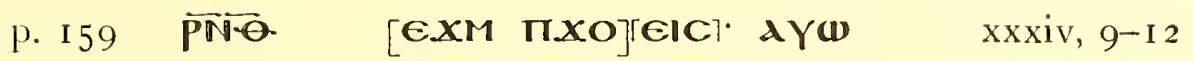
[CNAO]YNOY EXM̈ [IT] $е$ YOYXAï Nake ec THPOY NAxOOC

5 XE пXOEIC NIM петеINE M̈MOK. ITETNOY2M̄ ÑOY 2HKE EROA $2 \bar{N}$ T бIX ÑNETXOOP $\overline{\mathrm{E}}$

Iо POY OY२HкE MÑ OYERIHN EROA 2 TÑ NET'TMP M̈MOQ' AYTWOYN N̄бI 2ENMÑTPE

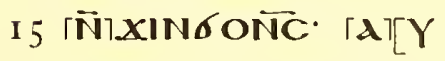
XNO]IYī ENET[E NTCOOYN MMOOY AN AYTWWBE NAl]

p. 158.13 scriptum erat $\ddot{i}$ corr $\pi$

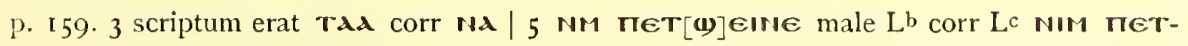
radeerre $\mathrm{ZR}$ 
P. I60 $[\bar{P}]^{2}$

ÑzeN[ா6-0OOY TIMA İZEN[ாGT]

Xxxiv, I 2-I 4

NANOYOY AY[W]

MNTAтшнре N̄

5 TAXYXH' ANOK

גє $2 \bar{M}$ птреүג

Noxג Nגï גïT2।

लDT NOYGOOY

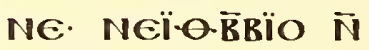

IO TAభYXH $2 \bar{N}$ NOY

NHCTIA AYW $\Pi$ I

(1) Анर NАKOTव̄ E2

Pdï EKOYNT NE[1]

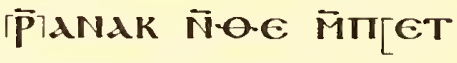

I 5 2IT]OYฒї MN [OY

CON NEIOBRIHY

N-E Мाетр2нвE]

p. I6 I $\overline{P(x d}$

[AYल пाET]

xxxiv, I 4-I 7

[AYC(1)]OY2 EPoï

[ATYC AYEY४PANE.

A 2ENMACTIГ亡

5 сшO $\overline{2}$ Epoï aYw

ज̈пIEIME AYX(1)

ape EBOA AYO $\bar{M}$

ПIOYЙKA2 N̄2HT.

AYTIPAZE MMOÏ

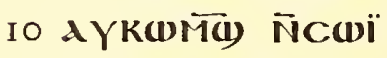

$2 \bar{N}$ NOYK(N) $A Y$

2POXPEX ÑNEY

or2e ezpdï exwï

ПXOEIC ERNA
I $5[$ б(w) $)$ T TN TNAY
TA2E] ГTाג[ҰҮXH EPd
TC EROA $2 M$ TIEY]

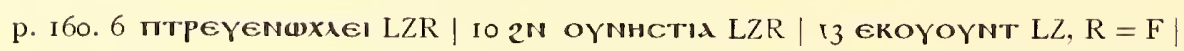

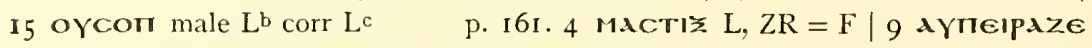

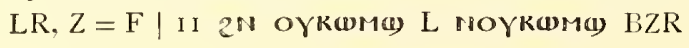


p. 162 PخR

$$
\begin{aligned}
& \text { MOXNIE] [EOOOY] } \\
& \text { TAM̈NTT(1) [HPE NOY } \\
& \text { WT EROA 2ITN } \\
& \text { NMMOYï. TNגOY } \\
& 5 \text { WN } \overline{\mathcal{L}} \text { NAK EROA } \\
& \text { TXOEIC } 2 \bar{N} \text { NOY } \\
& \text { NOF NTNERRAHCIA } \\
& \text { PNACMOY EPOK } \\
& \text { 2त̄ NOYAdOC EqOW } \\
& \text { Iо М̈ттртреүрдше } \\
& \text { MTMö̈ Ñ́́ Neto }
\end{aligned}
$$

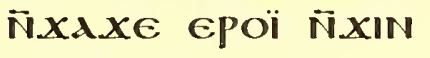

$$
\begin{aligned}
& \text { бONC̈ NETMOC } \\
& \text { те İMOї ї.XINX[H }
\end{aligned}
$$

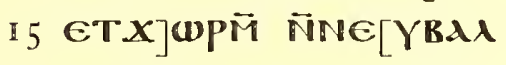

$$
\begin{aligned}
& \text { XE NEYX(1) MEN } \\
& \text { NAI N2ENCAXE] }
\end{aligned}
$$

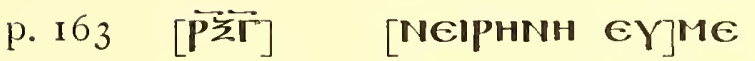

[EYE 16 E]2ENKPOC

xxxiv, $17-20$ [2N] оYOPГH. АYOY

wN N̈PwOY $\epsilon_{2}$

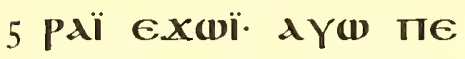

$x d Y x \in \epsilon_{2 \epsilon}, \epsilon_{2 \epsilon}$

ANME2 EIATN̈ M̈MOq.

AKNAY TIXOEIC $\bar{M}$

пРКАРФК' пХO

Io EIC MITTिOYE MMMÖ

TMOYN TIXOEIC T2

THK ETI2АT' TIA

NOYTE AYW TId

XOEIC ETARBA.

I5 [K] [PINE MMOÏ ГK][ATA

TEKAIKAI] $\mathrm{O}[\mathrm{CYNH}$

IIXOGIC IIANOYTE]

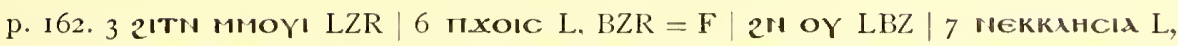

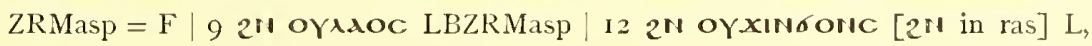
$\mathrm{ZR}=\mathrm{F}$ 


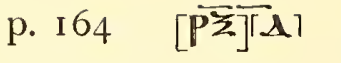

MாГї][TPEYPג心e M]

xxxiv, $24-27$

MOї. М̈п[РтРЕYXO]

OC $2 \bar{M}$ пеY२ГнТา [XE]

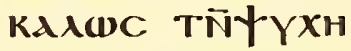

5 Аүल M̈ாрТреҮXO

OC $x \in$ ANOMKव் $E Y$

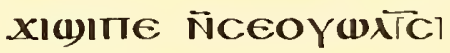

210 YCOT N̄́I NET

pגae exÑ Natte

IO GOOY MAPOYT

2IWOY NOYMITIE

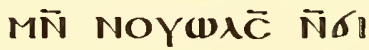

NeTXE Nor Nald

$x \in \epsilon_{2 p \lambda i} \in x \omega \ddot{~}$

I 5 MApoYTEגнג $\bar{N}$

[TOO]Y ÑCEOY[NOG

NǴ NETOYE(I) TAAI]

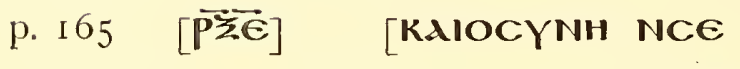

xxxiv, $27-x x x v, 2$

XOOC NOYOEI]

$[$ NIM XE EP]E пX[O]

[EIC] AII AI] NETOY

5 E() $\operatorname{TP}[\mathrm{H}] \mathrm{NH}$ M̈ாеप

रM2Aג AYW TाA

AגC NATEAHA $2 \bar{N}$

TEKAIKAIOCYNH.

MÑ пЕКTגїO

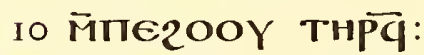

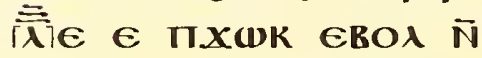

Adyeld $\pi \overline{2 M} 2 \lambda \lambda$

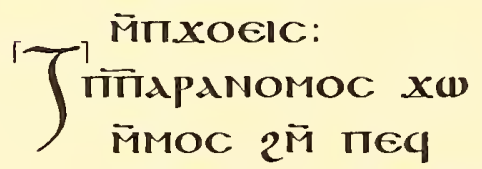

$[2 H]^{\Gamma \vec{T} 7} x \in$ TNA $\bar{P}[N O R][\epsilon$

NAOTE MINOYTE]

p. I64. 61 eyexianine LZR | I2 mN oyaxe LZR NM oywac B | i3 Noc male Lb corr $L^{c}$

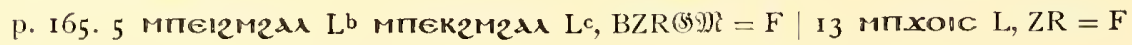




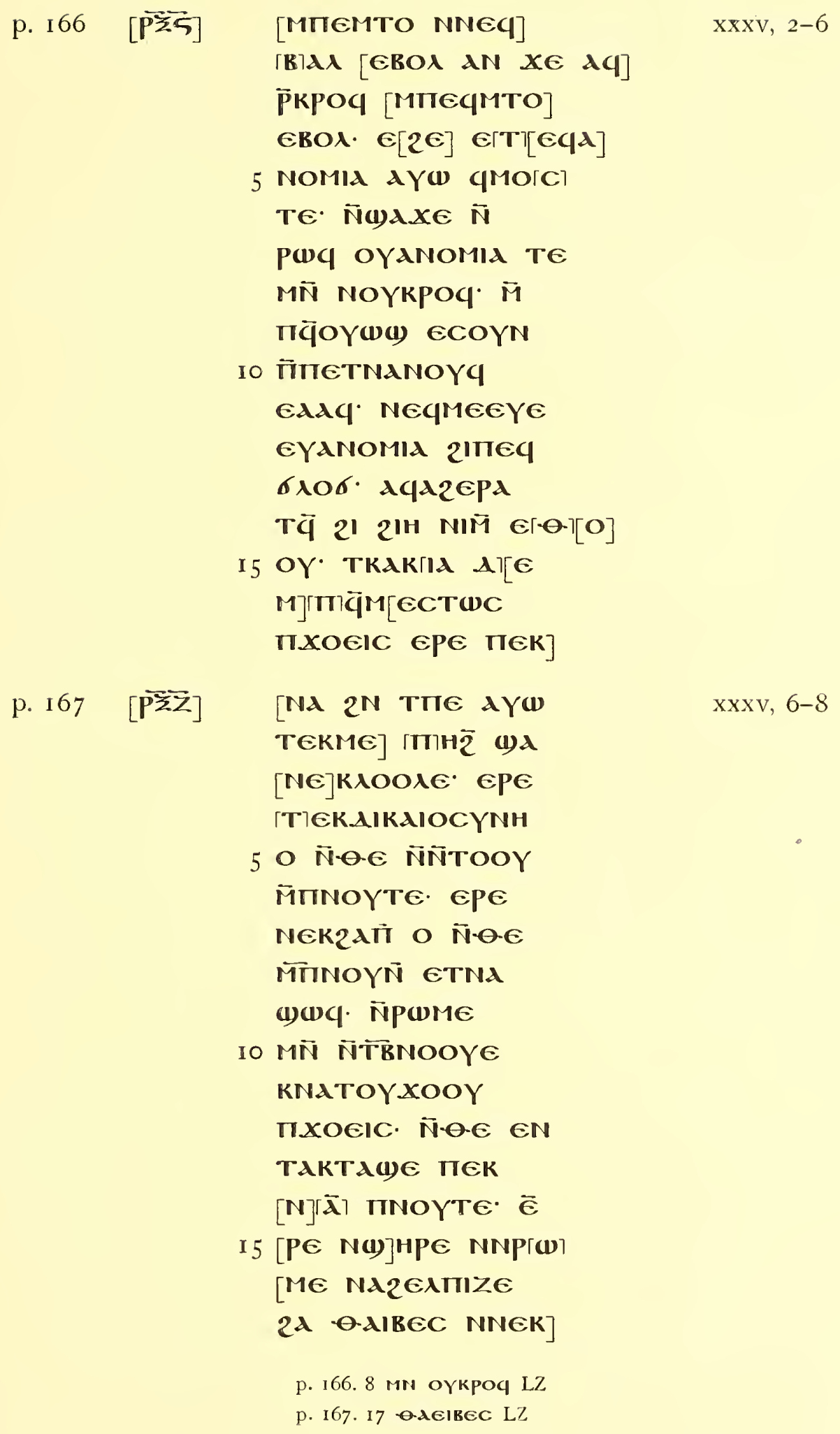


p. 168

[P $\overline{\mathrm{P}} \overrightarrow{\mathrm{H}}]$

TN2 [CendCel E]

$\operatorname{xxxv}, 8-\mathrm{I} 2$

BOA $2 M$ [ITCEI M]

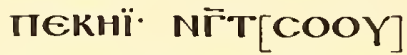

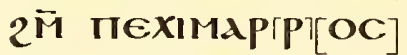

5 ÑNEKTPҮфн.

Xe epe тाтүгн

M̈IIUN2 2АTHк

2Pגї 2"̈ пекоY

OEIN TÑNANAY

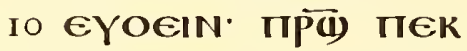

NȦ ERON ÑNET

COOYN MIMOK.

AYW TEKAIKAI

OCYNḦ ÑNETC[OY]

I5 TWN 2M̈ ПIE][Y2HT

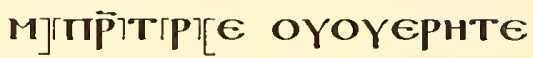

N.XACI 2HT El NAl AYW]

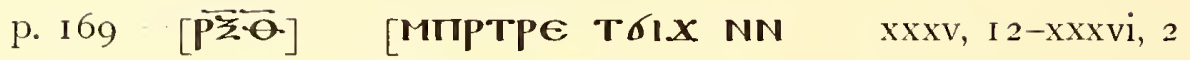
PECPNORE] KIM E

[POI XE] NTAY2E M

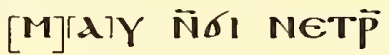

5 2UR ETANOMId.

АүтАKO NCE

NА(1)

Eג2ердтоY:-

$\overline{15}$

NंAdYeld.

Mัтр̄кш2 ENETO

M̈IIONHPOC. OY

גє M̈ாр̈кш2 ENE

TEIPE N̈TANOMIA

[X]E ÑaE NOYXOP

I 5 [T]

[2N OY]бEIH. AYT[W

N-E.E NNIO]Y[OOTE ETOYET]

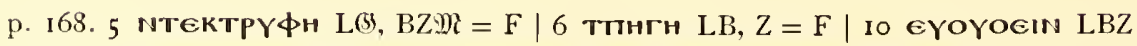

p. I69. I npeqpNoke L nNpeqpNoRe Z 12 mipko2 male $\mathrm{L}^{\mathrm{b}}$ corr $\mathrm{L}^{\mathrm{c}}$ 


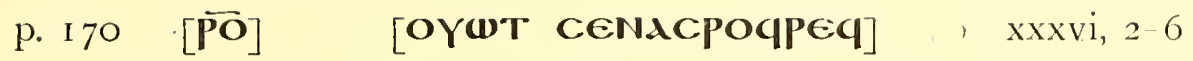

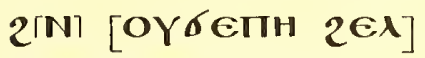

IIZE ETIX[OEIC]

N̄KeIPE NOY[MNT]

5 XPнстос N̄ГOY

W2 2IXÏ пKג2

N̈CEMOONE MMMO

2IXÑ TEqMNT

PM̄MAO. CANOY

IO

Peqt NaK ÑÑe

THMA ज̈ПСК2HT

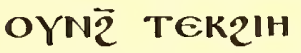

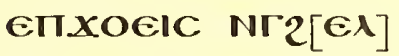

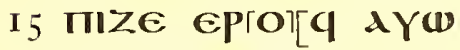

qNA] E[IPE NAK

qNAGINE EROX]

p. I 7 I $[\overrightarrow{\mathrm{POA}}] \quad[$ NTEKAIKAI $] \mathrm{O}$

xxxvi, 6-9

[CYNH N]-OE MTIOY

[OE]IIÑ AYW NEK

[2] גÜ

5 Mмеере 2 Үाо

TAcCe M̈ாশXo

धाC AYW NTिCा

сшпवं мпґ̈кш2

EாETCOYTWN

Io $2 \bar{M}$ пEqWÑ $2 \bar{N}$

oYpUME eqeipe

NMTIPANOMIA.

CA2WWK EROA

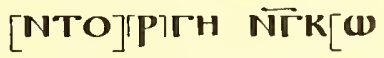

I 5 NCWK] $[\bar{M}] \pi[\sigma][\omega N T$

Мாркш2 2шСТе

EPTONHPOC]

p. 170. 4 NTEIPE LZ I I NNAITHMA LZ

p. I7I, 13 сагшк LZ 
p. 17

[ $\overrightarrow{\mathrm{POB}}]$

$x \in\lceil N][E T O$ MIIONH]

xxxvi, 9-1 2

POC CIENA][GOTOY]

EROA NET2[YTO]

MINE $\lambda \in$ ETXIO][IC]

5 N̄TOOY NETNA

KגeIPONOMI $\bar{M}$

חKג2 ETI KE

коүї пє ज̄TETM

пPEqT̄PORE ФW

IO TIE NT̈mINE $\bar{N}$

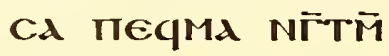

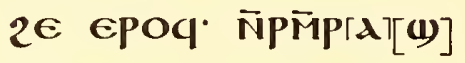

N̄TOOY NAKגHPW

NOMI MTIKג21

I $5[$ AY][W] CE[N][ACANG

2N O][YА][山H NEI

РнNн прєСР]

p. $173[\overrightarrow{\mathrm{PO}}$

[NORE NAT2TH]

xxxvi, I 2-I 5

[EI.AIKAIOC N] $92 P O X$

[PE] $] x]$ ÑNEqOR2E $E$

$[\mathrm{P}] \omega \mathrm{M}$ пxOEIC $\lambda \epsilon$

5 NACWBE NCWq.

$x \in q \sigma \omega(\bar{T}) x \in$

пеव2OOY NHY A $N$

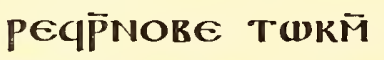

NंTEYCHqE AYCW

I0 MÑT N̄TEYIITE

[E]TAYO E2PAi NOY

2HKE MÑ NOYERI

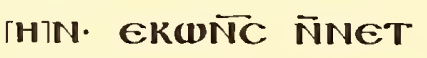

[COYT]WN 2M̈ ताE][Y

I5 2HT EPE] T[EYCHqE

RWK E2OYN $E$

пEY२HT AYW NTE]

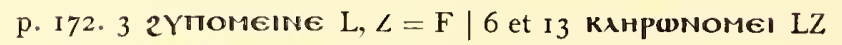

p. I73. I2 MN OYєBıнN LZ 
p. I74 [ $[\widehat{\mathrm{POA}}]$ [NEYCOTE OYW]

xxxvi, I 5-I9

(1).' N[ANOY OYROYi

MIIAIKAIOC IE][2OYE]

OYMNTPTIMAГO1

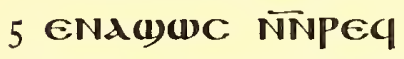

PNNORE $x \in$ NÉRO

N̄NPECIT̄NORE NAOY

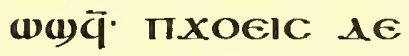

TAXPO NÑ̄AIRAIOC.

IO TIXOEIC COOYN ÑNE

2IOOYE ÑNEqTETOY

AAB· AYW TEYKIA][H]

ГPาONOMIA 「NרA[WWTE

(b) $][\lambda]$ ENE[2] [NCENA

I 5 XIEIIIE AN $2 \mathrm{~N}$ OY

OYOEI(I) EपIOOY

AYU CENACEI $2 N]$

p. I75 [P्POE] [NE2OOY MT2][E]BW

xxxvi, I9-22 [WN] $x \in$ NPECPNO [BE] NAT'AKO. NXX

xe MाIXOEIC $2 \bar{M}$

5 птРЕYXIEOOY

ÑCEXICE 2N OY

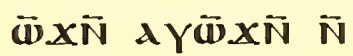

QE NOYKAIINOC.

(1)גPE IтРСप्̈NO

IO RE $X I$ EXuq Nq̄TM

'TAגY' (IAPE חגАI

ГKTAIOC $\lambda \in \omega \overline{N 2}$

[THq] גY山 Nāt $x \in$

[NETCMOY] EP[OG NA

I 5 KАHPONOMEI

MTKA2 NETCA]

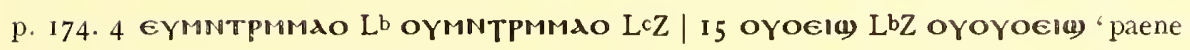
evanuerunt litterae 3 . et 4 .' Lc

p. I75. I5 RגнPONOMגє1 male Lb corr Lc 
p. 176

[

2OY [A][E MMOG CE

$x x x v i, 22-25$

NAqOTOY E[R][OA]

ePE NE2IOOYE [M]

TIPळME COOYTN

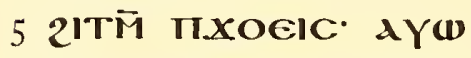

qNAOYW(1) TEQRI[H]

EP(I)AN IIAIKAIOC

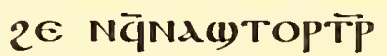

AN XE IXXOEIC

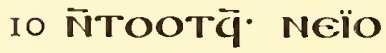

ज̄koYï пе גYळ גї

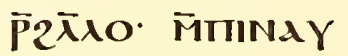

[ETYAIKAIOIC] [EAq

KAגG NCWG OYAE]

p. I77 [

xxxvi, $25-28$

[ФINE N]/CA OTEIK.

[MIT] E2OOY THPG

Г(1) $\lambda$ पNर्ब AYW NGT.

5 Aүल пеqспермд

NAฒ(1) EYC

MOY. CAzWWK

EROX M̈TIE-OOOY:

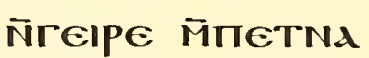

IO NOYq N̄roYw2 $\bar{N}$

(1) ENE2 N̄ENE2

XE пXOEIC ME $\bar{M}$

II2Aர̄ AYW Nव̄NA

$[\mathrm{K}] \boldsymbol{\omega}$ AN $\overline{\mathbf{N} C \omega q} \overline{\mathbf{N}}$

I 5 [NEqIET] OYdג $[\mathrm{B}$

CENA2APE2 EPOOY] 
p. $178[\widetilde{\mathrm{POH}}] \quad \boldsymbol{\omega}[\boldsymbol{d}$ ENE2 CENAP]

xxxvi, $28-3$ I

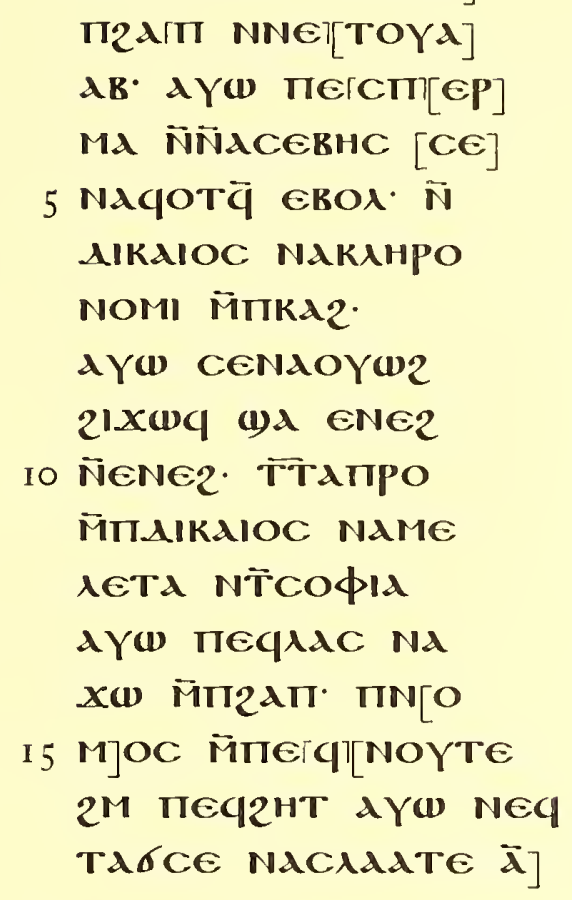

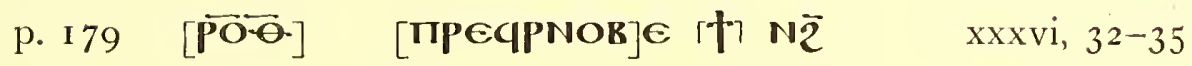
[THQ E]I.AIRAIOC

[AY]W qUINE N̄CA MOOYTव̈ं ÑNE

5 m.xoeIc $\lambda \in$ TגAq

ezpaï eNeq́lx.

AYW ÑNeq́r dïOq

equaN XI2Aİ NT

MAq. 2YTIOMINE

Io emXoeIC Nï2A

PE2 ETEपा?

AYW GNAXACTK

еTPEКкАнРОNO

[MEI M][T]KA2* KN[A]

I 5 [NAY ENPECIPNOBE

EYNACOTOY EROA] 
p. 180

[T़]

Гג][INAY EாגАERHC Eq] xxxvi, 35-38 XIC][E MMOQ AYW Eq]

TWOYN E2TP?[Al N-O.E]

NN̈KEגPOC M[TIXl]

5 BANOC dÏCAג[Tq]

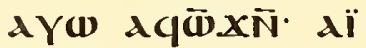

(1)INE N̄Cd IIEqMA

M̈пा2є EPOQ $2 \lambda$

PE2 ETMÑTRAג

IO $2 H \bar{T}$ NTNAY EIICO

OYTN' XE OYN OY

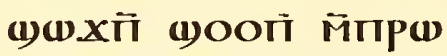

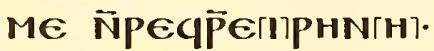

M̈IAPANOMOC $\lambda \epsilon$

15 N̄TOOY CENAqO

TOY EROA 2[1 OYCOT

N(1) $\boldsymbol{X}$ NI NACERHC]

p. I8I [PTाA] [CENATגKoOY EPE xxxvi, 38-xxxvii, 2 TIOYXAl $\Lambda \in$ NNAl

KAIOC NT][MIIXO

[EIC] AYW TEYNAG

5 [T]

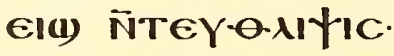

IIXOGIC NAROH-I

EPOOY AYW NGNA2

MOY: AYW qNATOY

IO XOOY EROA 2IT'̄

N̄N̄PEq̄̄NOBE' AYW

NḠNA2MOY $x \in A Y$

2EAIIZE EPOQ:

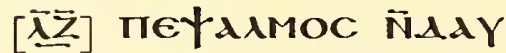

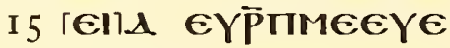

[GTB][E] IICABRA[TON]

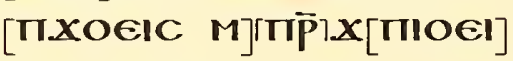

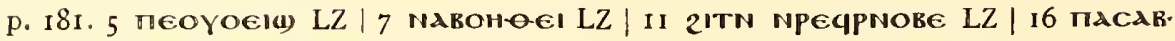
BdTON male Lb corr Lc 
p. I 82 [ $\widehat{\mathrm{PITB}}$

[2M TEКб WNT OY]

xxxvii, 2-6

Г.А [E MITP†CBW]

NAï $2 \mathrm{~N}$ TEK[OPГH]

$x \in$ А NEKCOT [E]

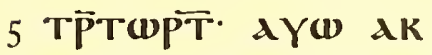

тахро స̈текбıх є2

pגï Exwï MÑ TAג

$602 \bar{N}$ TACגP啇

TEMTO EROA N̄

г текоргн. М̃N eI

PHNH שOOT ÑNA

KEEC N̈NA2PN̄ NA

NORE. $x \in$ a NAANO

MIA XICE ETAdITI[E]

I 5 AY2PO(1) E2एрג][ï

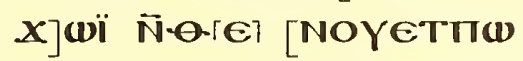

EC2OP() AYKNOC AYW]

p. I $8_{3}$ [ [TाT]

[АYлослеq N6I NA

xxxvii, 6-10 Cג(1) MTEM]TO EBOג N̄

[Tג]MNTA-OHT

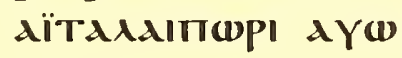

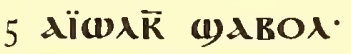

NeiMOOGE Eïo

кM̈ MTE

Pप̈ XE ATAYYXH

MOY2 ÑNOKNEG.

Io AYW MÑ Tal6o

कOOT $2 \bar{N}$ TACAP立.

Аї̈кג2 Aүज dï

O.RBIO EMATE.

dïxI(1)кAK EROA

I [2M] пג(1)ג2OM $\bar{M}$

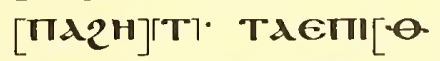

YMIA THP]C M̈[пек]

p. 183. 4 AITAlaImopeI LBZ 
p. $8_{4}[\widehat{\mathrm{PTA}}] \quad$ [MTO EBOA ПXOEIC] XXXvii, IO-I 3

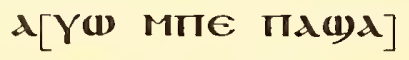

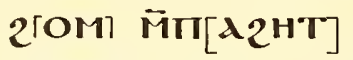

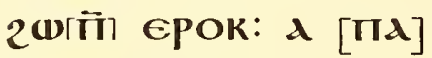

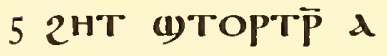

тАбOM KАגT

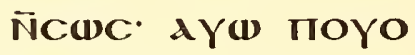

EIN̄ N̄NARגA M̄

пEїKe NMMAï AN.

Io NAüEep MÑ NET

2ІTOYWÏ AYZWN

E2OYN EPOÏ AYA26

PATOY AYW NET

2HN EPOÏ AYA2E

I 5 рАтоY м̈поYе.

AYXINGONC Ñ $[1]$

NET(1) [E NCATA

YY]XH. [AYW NET(1) $]$

p. $8_{5}[\overrightarrow{\mathrm{PTIE}}] \quad[\mathrm{NE}$ NCd 2ENTEO-O

xxxvii, $13^{-1} 6$

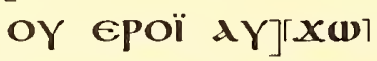

[N2ENT] $\mathrm{ET(1)OY}$

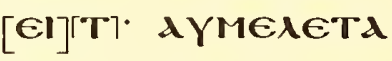

$52 \bar{N}$ NoYkPOG M̈пE

2OOY тнрवं גNOK

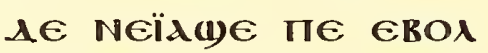

ITTOOTOY Aүa גï

$\bar{p}$ O-E NOYג $\overline{\mathbf{\lambda}}$ eMEq

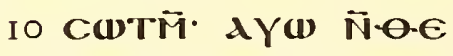

NOYMTIO EMEqOY

aN N̄Pag גīp

o-e NOYPMME EN

Гq1CWTM AN.

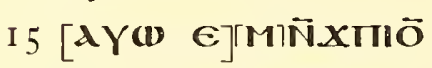

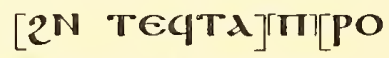

AINA2TE EPOK] 
p. 18

$$
\begin{aligned}
& \text { [TTIS] [NTOK ETNACW] } \\
& \text { ГT७M EPO[l } 17 . x O I C]
\end{aligned}
$$

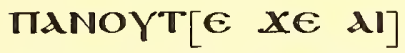

$$
\begin{aligned}
& \text { XOOC XE MHITO][TE] } \\
& 5 \text { ÑTE NAXAXE PA } \\
& \text { ()e M̈MOї } 2 \bar{M} \Pi \\
& \text { TPE NAOYEPHTE } \\
& \text { KIM AYXe Nor Ñ } \\
& \text { (1) } \lambda \in \epsilon_{2 p \lambda i ̈ ~}^{\epsilon x \omega i . ~} \\
& \text { IO } X E \text { ANOK TCË } \\
& \text { TWT E2ENMAC } \\
& \text { TITें AYW IIAM } \\
& \text { кА2 М̈текM̈то } \\
& \text { EBOA NOYOEI(1)] N[[M] } \\
& \text { I } 5 \text { xe fivaxim] a[NOL: } \\
& \text { NTAANOMIA AYW TNA } \\
& \text { GIPOOYU } 2 A \text { TINOKE] }
\end{aligned}
$$$$
\text { xxxvii, I6-I } 9
$$

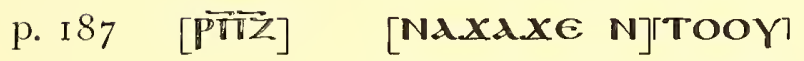

[ON2 AY][W] CEбM̆

[бO][M] E2OYe epoï.

ГATYOYW(1)C̈ EROA

5 N̄চI NETMOCTE

MMOÏ $2 \bar{N}$ OYXIN

бONC: NETTWA

re Naï ÑzentTe

-OOOY ETIMAN

IO 2ENTETNANOY

OY. АY†(1)TOYнT

epoï erod $x \in$ גï

ПIڤT N̄CA TAIKAI

[OC] YרNH. AYNOIX][T

I 5 ERON] Гா]MГEIPIT NOE.

NOYPEqMOOYT

ЄqRHT MITPKגАT] 


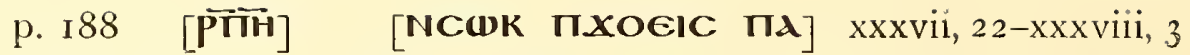
[NOYTH[C MTPCA] 2WWK E[BOX MMOI] †т̆тнк етגгвог[н]

5 - Qïд пxOGIC חNOY те їாдоYXגї:

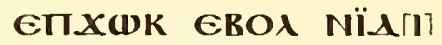

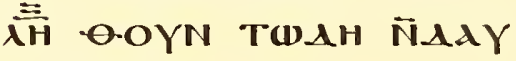
EIA:

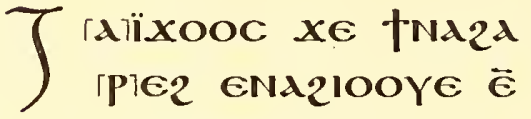
TMिलMRE $2 M$ Tा גAc. dïka NOY2d

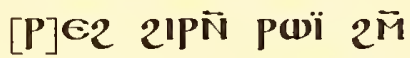

I 5 [ாт]

ве А2EP]А[ТА Мाג MTO GROA גITM по АाएTOPTP]

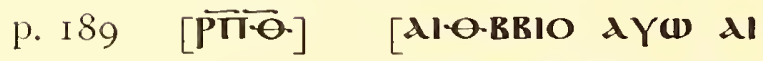
xxxviii, 3-6 KAPWI E]BOA $2 \mathrm{~N}$

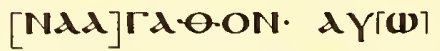

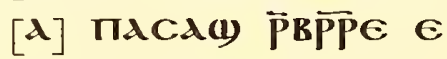

5 POї А пाА2HT $\overline{2}$ MOM̈ M̈ாגCd $N$ 2OYN AYW OYN OY

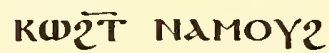

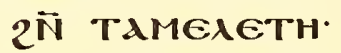

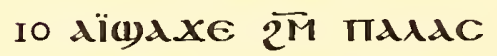
xe MAtגMoï пхO GIC ETג2גH. АYW тнпе N̈NA2OOY [XE] оYнP TE. I 5 [XE eleeIME $X \in$ EId)AT NOY EIC 2нHTE АKTPE]

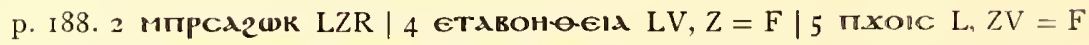
p. $189.62 M$ пrdCd LZ, BMasp $=F$ 
p. $190 \quad[\overline{\mathrm{PQ}}]$

[NA2OOY PAC AYU

xxxviii, $6-8$

ере пגтגхро о]

$\bar{N} \boldsymbol{A} \Theta \mathrm{N}[\mathrm{\lambda d \lambda} \mathrm{Y} M]$

IІЕКМ̆T[O ЄROA]

5 IIHN ПтТнРФ (1) [OY]

धाT्र PAME NIT̃

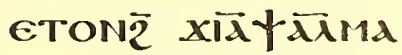

MENTOITE EPE

прФме MOOWE

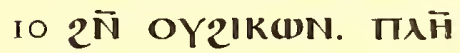

धq(1)T

XH. qCWOY2 E2OYN

NĞCOOYN AN $x \in \in q$

C@OY2 M̈MOOY $N[\mathrm{NIM}]$

I 5 TENOY NIM IIE[T] [d

2] TYITOMONH [...- ]

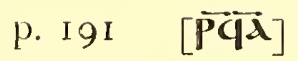

[MATOYXOI EROX

xxxviii, 9-I 2

2N NAdN][OTMIA TH

[POY AK†] MMOÏ $\bar{N}$

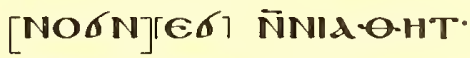

5 גї̄̄ாO M̈ாIOY

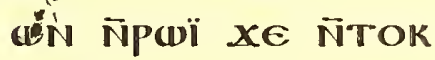

ПЕNTAKTAMI

oï: COO2E ÑNEK

MACTIT्र ÑCd

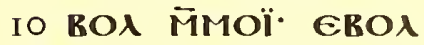

гар $2 \overline{\mathrm{N}}$ тбом

N̄TEKбוX ANOK $\lambda і$

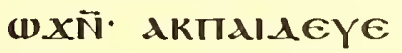

M̈TршME $2 \bar{N}$ 2EN

I5 [XT]InO 2A TeqdNO

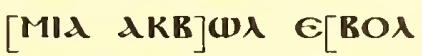

NTEqTYXH N-OE]

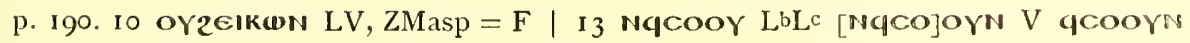
Masp, $Z=F \mid$ i6 2үпомолнмн male $L^{b}$

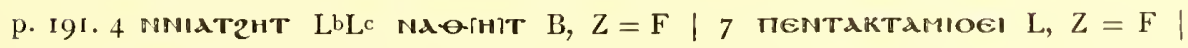

9 MActiz $\mathrm{LZ}, \mathrm{B}=\mathrm{F}$ 


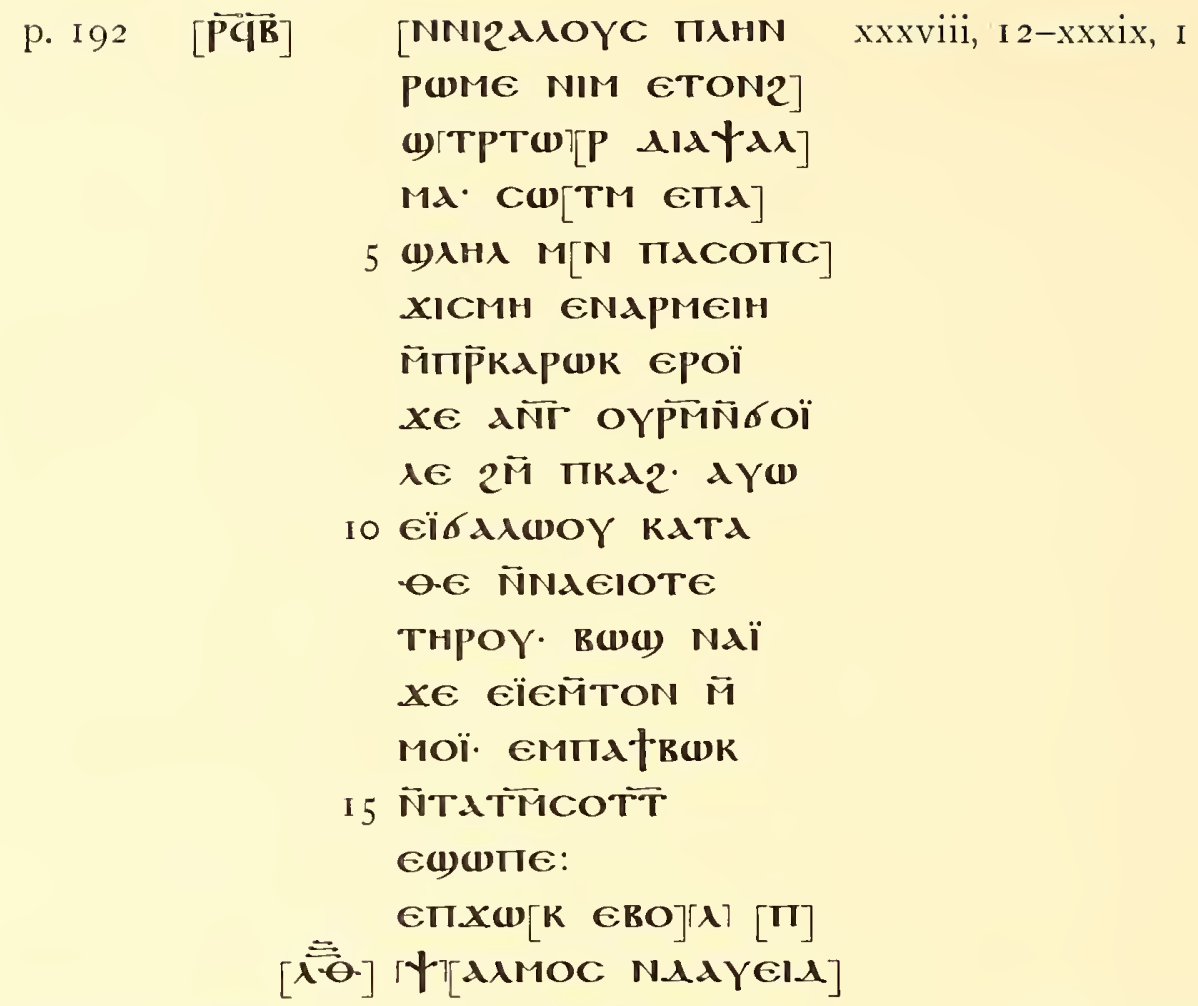

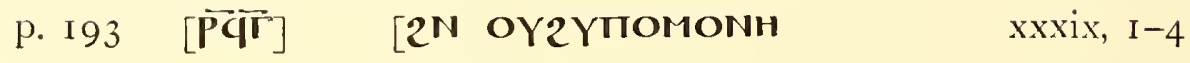

AI2YTIOMEINE $E$

пxOEIC Aqt2T][н]q $\mathrm{E}$

[POI AYG] ГАТवC्)TM̈

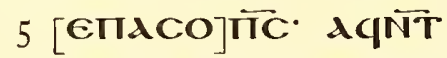

e2pגï $\overline{2^{M}}$ пिнї

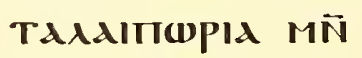

IIOME NTAOÏ2E.

АqTג2е NAOYEPH

Iо TE EPATOY $21 X \bar{N}$

петрג. AYW גQ

COOYTN̈ ÑNATA6

ce. גqNOYXE $\bar{\epsilon}$

PUï NOYX(1) $\overline{\mathrm{N}} \overrightarrow{\mathrm{PP}}$

I 5 PE OYCMOY M̈ITEN

[NOYTE OYN 242 NA

NAY NCEP2OTE AYW NCE]

p. I92. 8 оүрмпбоєıлє LB, ZMasp $=F$

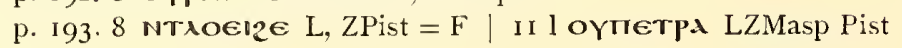




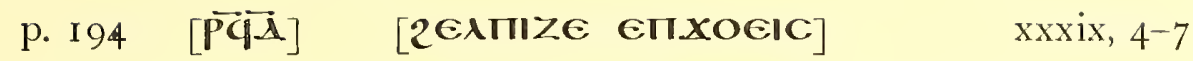
$\lceil N][\lambda I A T 4$ MIPWME] EIPAN [MIIXOEIC]

пе теq[2ENIIC]

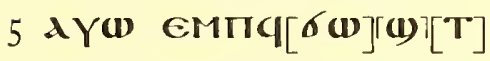
EXN̈ 2ENாET(1)OY

EIT MĪN 2ENMA

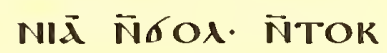

IXOEIC INOYTE

Iо АКTА(1) NEК(1)

PE AYW MEN IIE

TEINE M̈MOK $2 \bar{N}$

NERMEEYE dixOOY

AITAYOOY AYA(1)גï

I 5 e2OY coYнतе. OY

OYCIA IM[N

[ФOPA MIIROYAEOY]

p. 195 [Pवल]

[OYCWMA $\lambda \in$ AKCB

xxxix, 7-IO

TUTA NAI NEGAIA MN

NE(I) XTAY 2 A NORE] [M]

[IROYA(1)]OY. TOTE

$5[$ AlXOO $] \mathrm{C} \mathrm{XE} \mathrm{EIC2H}$

HTE †NHY 4СH2

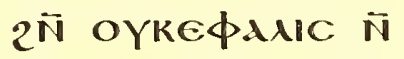

XWUME ЄTRHHT

етPдеIPE пINOYTE

Iо ज̈ாढкоүш(1):

AYW IIEKNOMOC

जTMHTE МाIА2HT.

גїєүАГГеגZе

NOYAIKAIOCYNH

I $52^{\bar{N}}$ NOYNOK NNEK

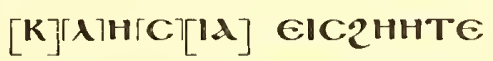

[NNAKWגY NN]A[CIO]

p. I94. I I 1 11N L mon BZ | i6 גүupocфopd male Lb corr Lc

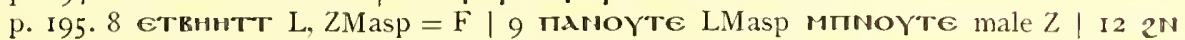

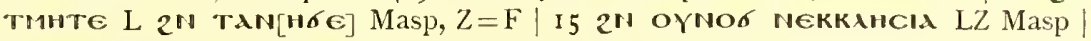
I7 NMakgare Z, Masp $=\mathrm{L}$ 
eNTAREIME $E$

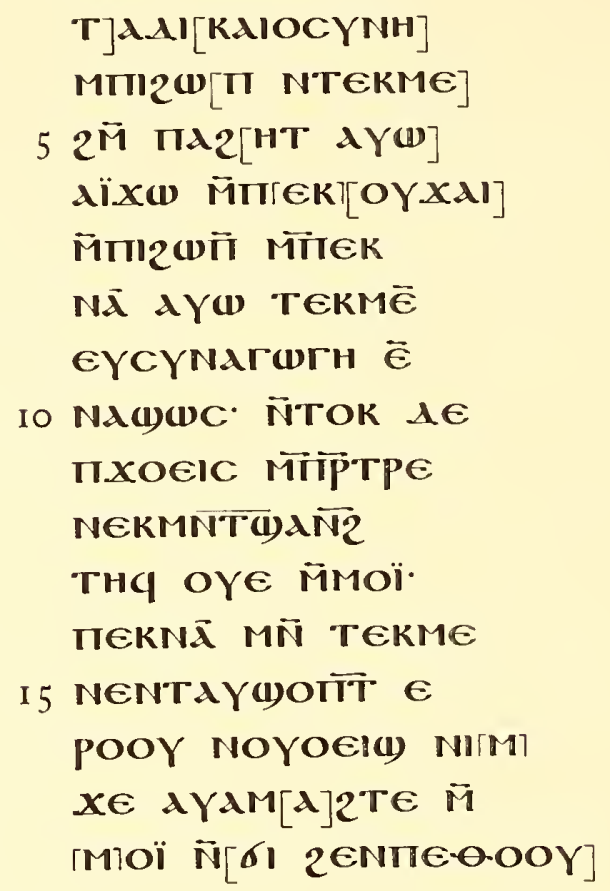


p. 198 [Pपण्]

[epd MapoyXI

xxxix, I 5-1 8

(1IIE NTEYNOY]

N[GI NETXW MMOC]

Nai xe E[YГE EYГE]

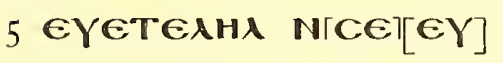

фpane ezpai exuK

N̄бI OYON NIM ET

(1)INE ÑCWR ITXO

EIC. AYW NGEXO

IO OC NOYOCIN NIM

xe MAPE пxOE\|C

Aïï NINETME M

IIEKOYXAï NOY[O]

EIU) NIM. ANOK $\Lambda[\Theta]$

I 5 ANT OYR[H]KE AINT[T]

ГOYาER[IHN IT.XOEIC

ITE TגPOOY(1) NTOK

ПЕ ПТВОН-O.OC]

p. 199 [

[AYW TANAETE TI

Xxxix, I 8-x], 4 NOYTE MIPUCK

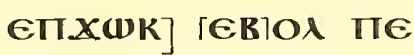

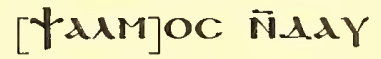

[ï] Elג:

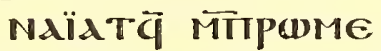

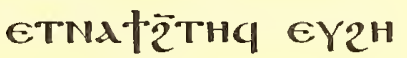

RE MÑ NOYEBIHN

ITXOEIC NATOY

IO XOG $2 \bar{N}$ NOY2OOY II

TIONHPON' GNA2A

PE2 EPOY ÑTAN

2Oq NGMAKגPI

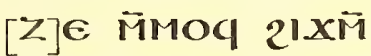

$15[$ IIKA2 AYW] NNIG][C

TAdq ETOOTq M

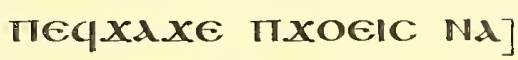

p. I98. I2 netme LZ NeEt[Me] Masp

p. 199. 8 MN OYeBнHN LZRMasp | 10 2N OYzOOY BZRMasp 
p. 200

$[\overline{\mathbf{C}}]$

[КОН-О.ध EPOप 21]

$\mathrm{xl}, 4-7$ ГX][M пеб М̈ாкА2 [АККTO] M̈ாЄQMANINK][OTK]

5 Тнस् $2 \bar{M}$ пЕपФ) NE ANOK AÏXOOC xe mXoelc Na Naï MATגАбE TA广Y ХH $x \in$ ä̈рNoRE $\bar{\epsilon}$ Io POK. a NAXAxe xw epoï ÑzeNIE -oOY $x \in$ EqNamoY TNAY N̈TE пEqPA[N]

TAKO. АY(1) NE[C]

I5 B/H]K E[2OYN ENAY EपXW N2ENாET wOYEIT - - - ]

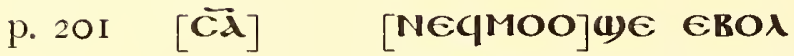
$\mathrm{xl}, 7-\mathrm{IO}$

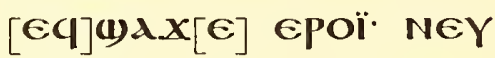
KAC $\overparen{K C}$ EPOї T[HPO]'Y] N̄́l Naxdxe 21 OY

5 COI' NEYMEEYE

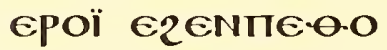
OY. AYCMINE NOY (1)גXe M̈IIAPANO MON [EP]Ö̈ MH пє

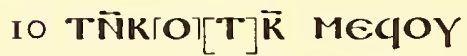
12 $\epsilon[$ TOO]Tä $\in T \omega$ [OYN KAI] IT/AP ITPW [ME NTAEIPHNH NEIKU N2THI EPOQ -- ] 
p. $202 \quad[\overline{\mathbf{C B}}]$

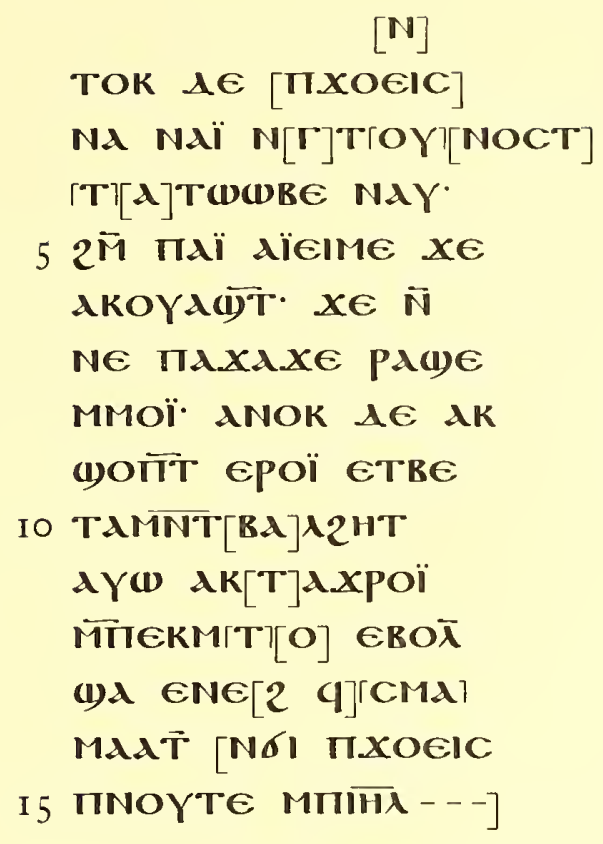$$
\mathrm{xl}, \mathrm{I} \mathrm{I}-\mathrm{I} 4
$$

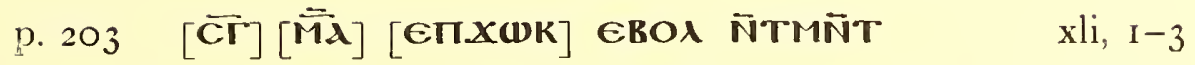
[PMN]2Hत̄ ÑN̄aHPE N̄KOPE:

NOE ETEPE OYEI

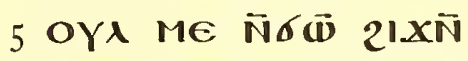
2EN2ONBE M̄MOOY

тגï те өe eтере

TAYYXH Me Nel é

PATK IIANOYTE.

Io a TA TYXH eIre M

INOYTE ETON2.

$[X] \Theta$ Eï[NHY] TNAY TA

[OYWN2 EBOA MIRO

MTANOYTE -...- ] 
[A NAPMEIOOYE wa

xli, 4-6

TE NAI NOEIK M

ПЕ2OOY MN TEY]

шн. [2M пTPEYXO]

5 OC NAÏ M̈M[HNE $x \in]$

Eq TWN IIEK[NOYTE]

Ndï dїрпеYMєєY[є]

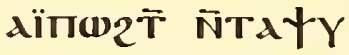

XH erpaï exü̈ $x \in \dagger$

IO NHY EROA 2ITT̃ NOY

MA సัNКнNH N(ब)

пнре (1) пнї ї

IINOYTE. $2 \bar{N}$ OY

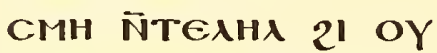

I 5 WNरू GROX N2POOГY

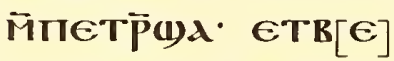

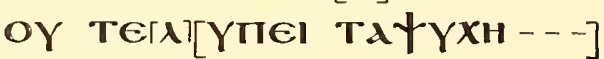

p. $205[\overline{\mathbf{C E}}]$

$[$ IIOYXAl MTI] $][20$

xli, 6-8

пе IIN]OYTE.

[A Tג

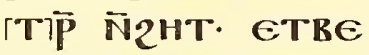

5 паї ГNap̈пекме

eYe пXOEIC $\overline{2 M}$ пкג2

M̈TIOPAANHC MÑ

2EPMUN. EROA $\overline{2 M}$

ПTOOY ФHM. ПINOYN

IO NACW OYBE IINOYN.

пERPOOY ÑNEKKA

TA2PAKTHĆ NEK

[P] [OlOYY(1) THPOY MÑ

[NERzOEIM A] J'Y] [I

I 5 E2PAl EX(1) ----] 
p. 206

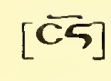

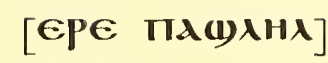

xli, 9- I I

N̄TOOT [EINOY]

те Мाтג(1)[N][2] [†]

NAXOOC MIIAN[OY]

5 TE $x \in$ NTTे пגреq

()OाT EPOK.

GTRE OY АKग̄IIA

(wB(i) ETBE OY AK

KAd $\bar{T}$ N̄CQK.

Iо AYW ETRE OY TMO

oye ध̈̈окM $2 \bar{M}$

птреq-Aाве M

Moï N̄́l TIAxגxe

$2 \bar{M} \pi T[P E Y \lambda O X 2$

I 5 NNAKEEC AYNEб

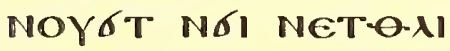

RE MMOI 2M TITPEYXOOC]

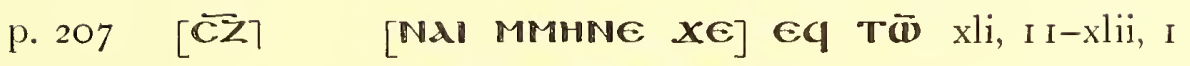
[TER]NГOTYTE' ET

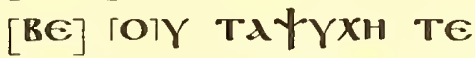

Аүтн. АүЩ ЕTве

5 OY TEWTOPTि $\bar{M}$

Mö̈ zєATIZ€ $\bar{\epsilon}$

IINOYTE XE †NA

OYWN2 NAq EBOA

noȲXaï M̈Td20

IO TE TINOYTE:

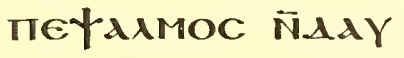

[M] B Elג:

[KPINE MMO]II $\mathrm{TI}[\mathrm{N}]$ OY

[TE - - - ] 
p. 208

$[\overline{\mathrm{CH}}]$

[MATOYXOI ERON]

xlii, $1-3$

2[N] [OYPWME NXIN]

бГO१NC [AYM NKPOC]

$x \in$ N̈TOK IIE] [INOYTE]

5 плтАХ्РО єтвГе] [OY]

AKKAd $T$ NCWK.

AYU ETRE OY TMO

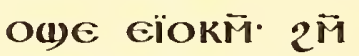

птре пахגхE

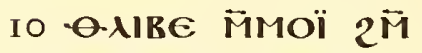

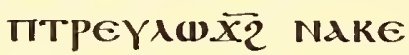

EC. AYNEбNOYбT

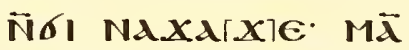

T[NNOOY MTEKOYO

I 5 EIN MN TEKME NTO

OY NENTAYXIMO

EIT 2HT AYNT EXM]

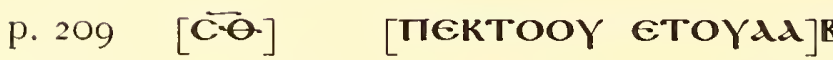

xlii, $3-5$

[AYW ENEKMAN][(I)

[†NAB(1)] e2OYN $_{2}$

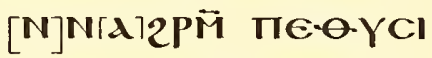

5 ACTHPION MIINOY

TE. NNA2TM TINOY

TE EтEYфPANE.

ก̄TAMÑT)н

ФнM 'ТNAOYलNГ2?

IO NAK EBOA TIANO[Y]

TE $2 \bar{N}$ NOYбl-APd.

етве оY телүтї

[TA †YXH] [AY] [W] ET

[BE OY TEMTOPTP

I 5 MMOI - - - ]

p. 208. II NNגKeec LBZ | i 3 MdTnooy male L

p. 209. II 2N OYKIOAPA LZ| 12 теAYחEI LZ 
p. 210

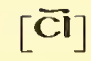

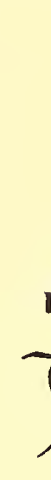

[IIOYXAI MIIA2O]

IIE III[ANOYTE]

ЄПХШК [ЄROA NN]

Фнре ர̄когр [Є пє]

5 †גАMOC ETMNT

Мाँ рM̄̄̃2HT:

$\int \begin{aligned} & \text { INOYTE ANCWTM } \\ & 2 \bar{N} \text { NENMAAXE. } \\ & \text { NENEIOTE AYTA }\end{aligned}$

Io MON EIIL $\mathrm{EN}$

TAKגAq $2^{\bar{N}}$ NEY2O

OY $2 \bar{N}$ Ne2OOY $\bar{N}$

[(1)][OPII - - - ]

p. 2 I I $[\overrightarrow{\mathbf{C i d}}]$

[NTA YKAHPONO

xliii, 4-6

xlii, 5-xliii, 2

MeI IAP AN MIIK]A2

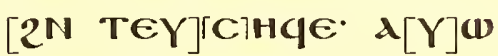

[Mாє]Yб́вої АN ПIEIN

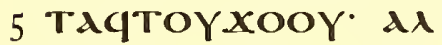

ld TEROYNAM TE

MÑ пாєќROї AYW

пOYOEIN ज̈ாЕк2O

xe גкоүАшоY $\bar{N}$

IO TOK IIE IIAP̄PO AYw

IINOYTE. пIET

2WN N̄NEYXAï N̄ïA

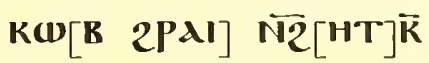

[TNNAKWNC NNEN

I 5 XגXe AYW $2 M$ ПIEK

PAN TNNACG(i)q

NNET"TWOYN E] 


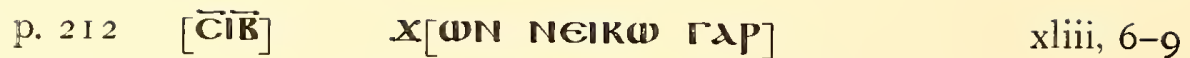
$\lambda[\mathrm{N}$ N2THI ETATITE]

AYW $N[$ TACHqE AN] TETNATO[YXOI Ak]

5 TOYXON TAP EBIOA IITÑ NETA-AIRE

MMON. AYW AK† घITE ÑNeTMOCTE MMON. 2PAÏ $2 \bar{M}$ IO TIENNOYTE TÑNA хाтגїо जाँ тнрवे AYW TNNA

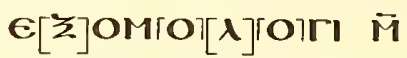
[TERPAN ฒג E]IN]E2: I 5 [AId †גАMd - - - ]

p. 2 I 3 [त्तiT]

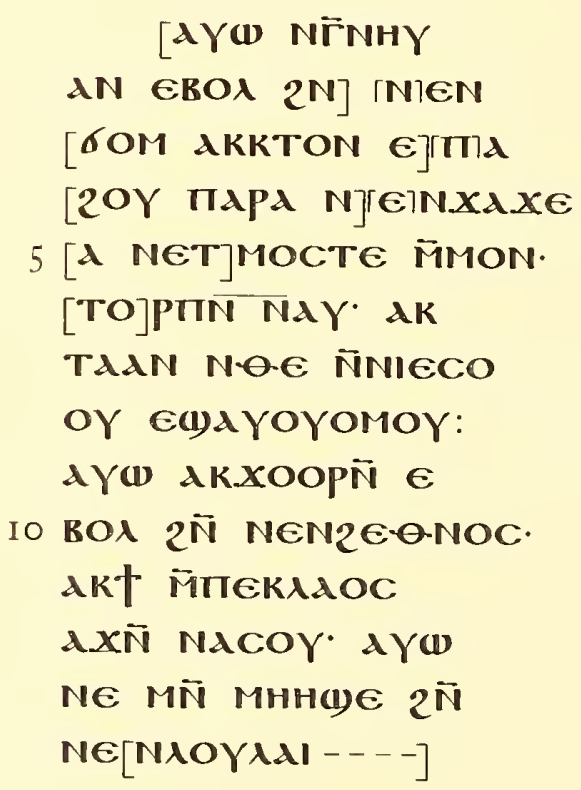


p. 2 I $[\overrightarrow{\mathrm{CI}}]$

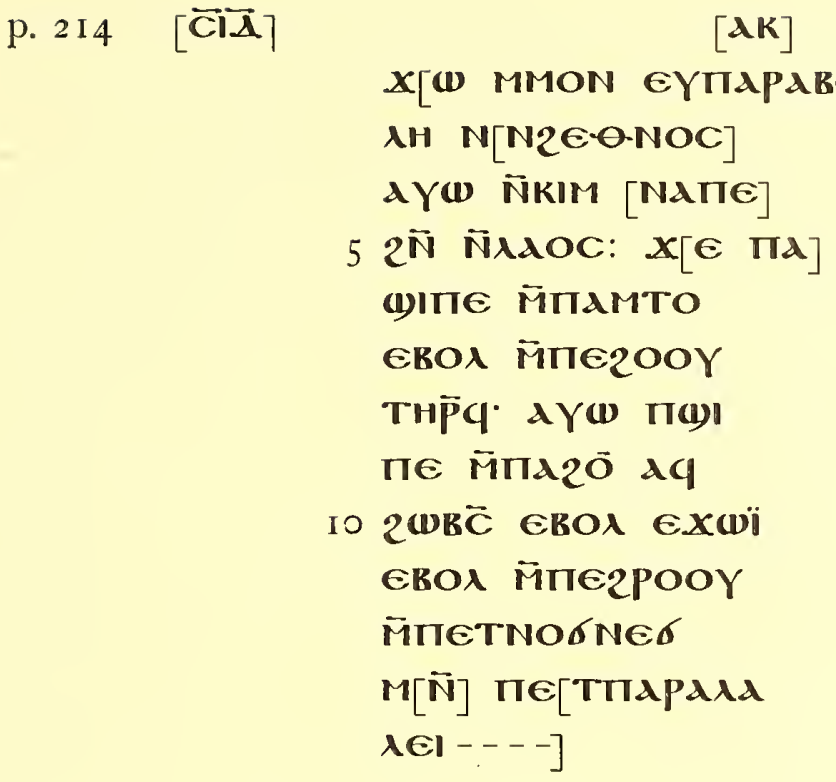

xliii, I 5-I 7

p. 2 I 5 [त्बiE]

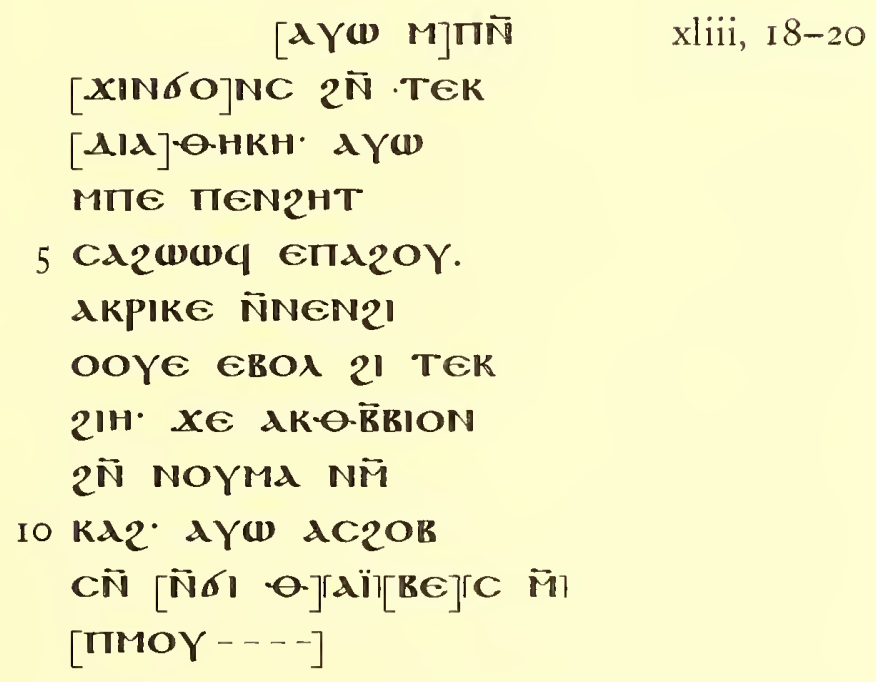

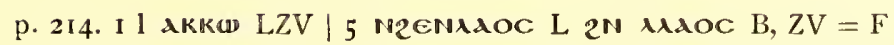

p. 215.5 cגzag LZV | 7 eror $2 \mathrm{~N}$ LZV | $92 \mathrm{~N}$ orma LZV | II aderbec L. $\mathrm{BZV}=\mathrm{F}$ 


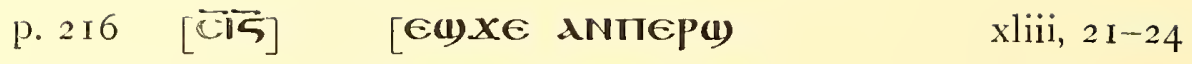
NENGIX EROA EY]
N[OYTE NGMMO MH]
M̈INIOYTE AN]
5 IIETUINE N[CA NAI]
N̄TOG гAP ET[CO]
OYÑ N̄NE-QHTT M̀

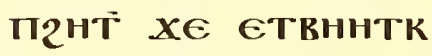
CEMOYOYT M̄MON

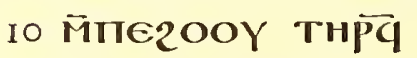
AYOTIN N̈.QE N̈NIE
COOY EKONCOY.
TWOYT TIXOEIC
[ЄTBE OY KNKO]TГK?:
I $5[---]$

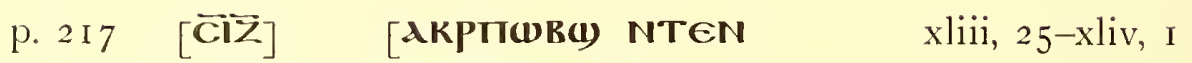

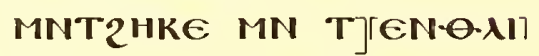

[HIC xe] ra]TerNt'Y

[XH] FO-TRRIO (1)ג ITKA2.

5 [d] 2нті̄ тшбе $\bar{\epsilon}$

пЕСнт' ТшоY

ROHAI EPON TIXO

EIC AYW Nïco

TÑ ETRE IIERAN:

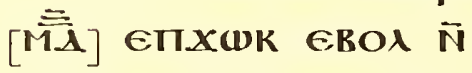

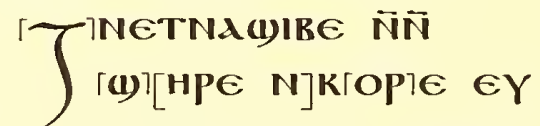

[MNTPMN2HT TWАH

2ג TIMEPIT - - - ]

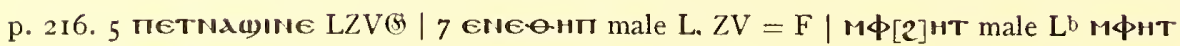
Schm

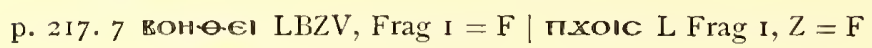



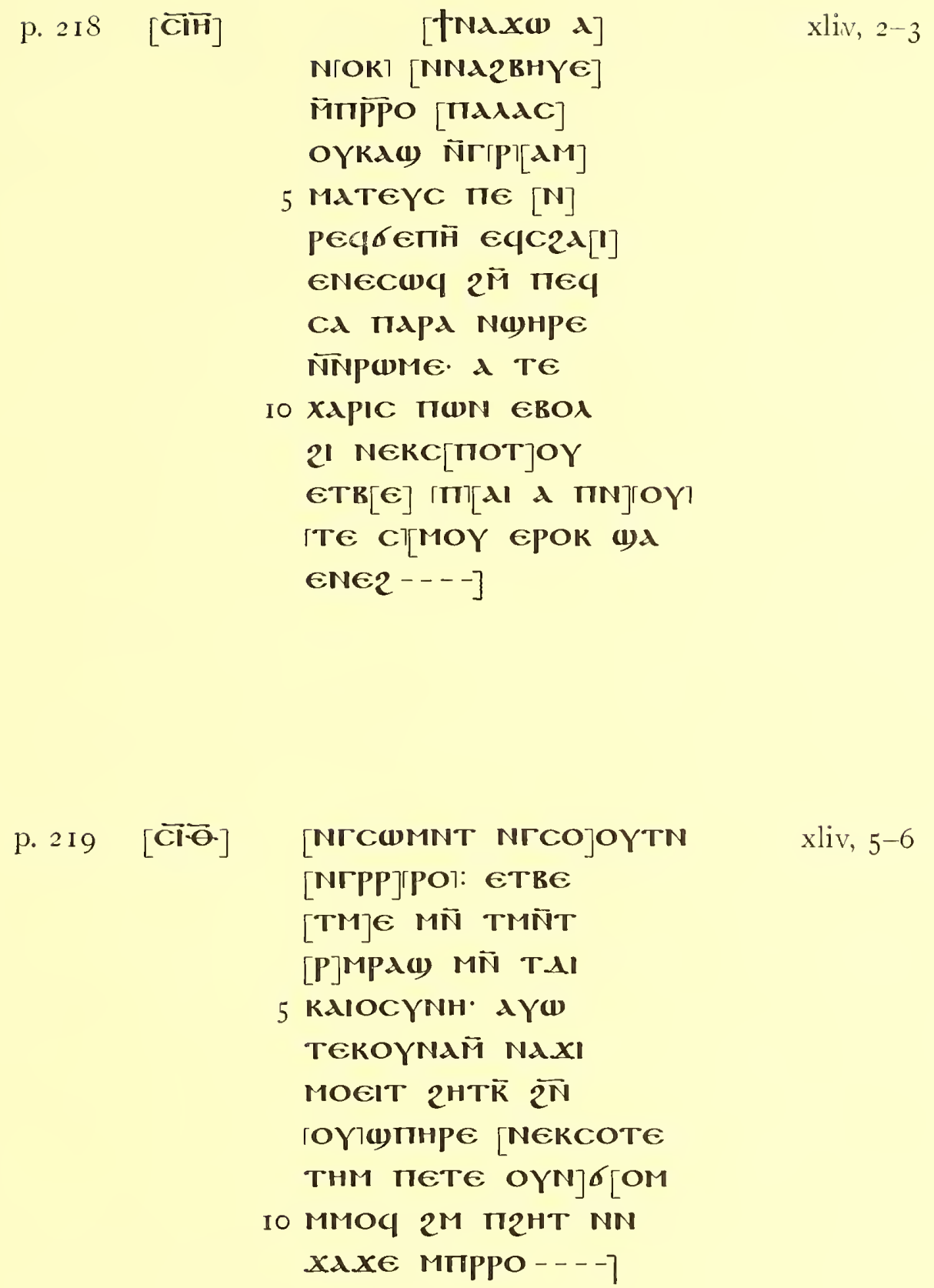
p. 220

$[\overline{\mathbf{C K}}]$

[OYбEPER NCOOY

xliv, $7-8$

TN пє пбершв N]

TEK[MNTEPO]

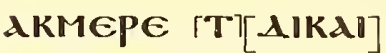

5 OCYNH АKM[EC]

TE ПIXINGONC

етве пдї Аqтג[2]

cK N̄6। пNOYTE

ПЕKNOYTE' NOY

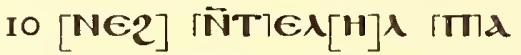

[PA NET2ITOYWK -- - ]

p. 22 I $[\overrightarrow{\mathrm{CKX}}]$ [ENTAYEYQPANE MMOK xliv, IO-I N2HTOY Nбו N山EeP]e $\bar{N}$

[NEPP(1)]

[TגE]

5 [2]

M̈MOK $2 \overline{\mathrm{N}}$ NOY $\overline{2 B}$

Cii) ECO NEIEITNOY

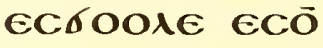

ÑNAYEI NAYAN.

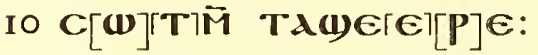

N[TENAY] IÑ]][TEPIKE

MाToYMddXe - - - ]

p. $222 \quad[\overline{\mathbf{G K B}}$

[CENA]

xliv, I 3-I 4

[OT[Y][(1)][UT NAG NGI N]

山EEPE [NTYPOC]

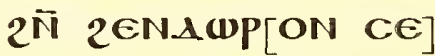

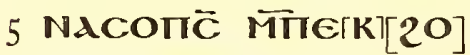

స̄б। N̄PM̄MAO $\bar{M}$

пкА2· ПడОOY Тн

Pवे NT(1)epe

ПPPO NECERWN.

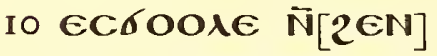

[AOOTY NIIN][OYB EYO

NAYEI NAYAN - - - ]

p. 220.7 АqTג2K male Lb corr Lc

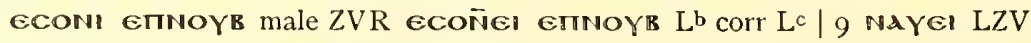




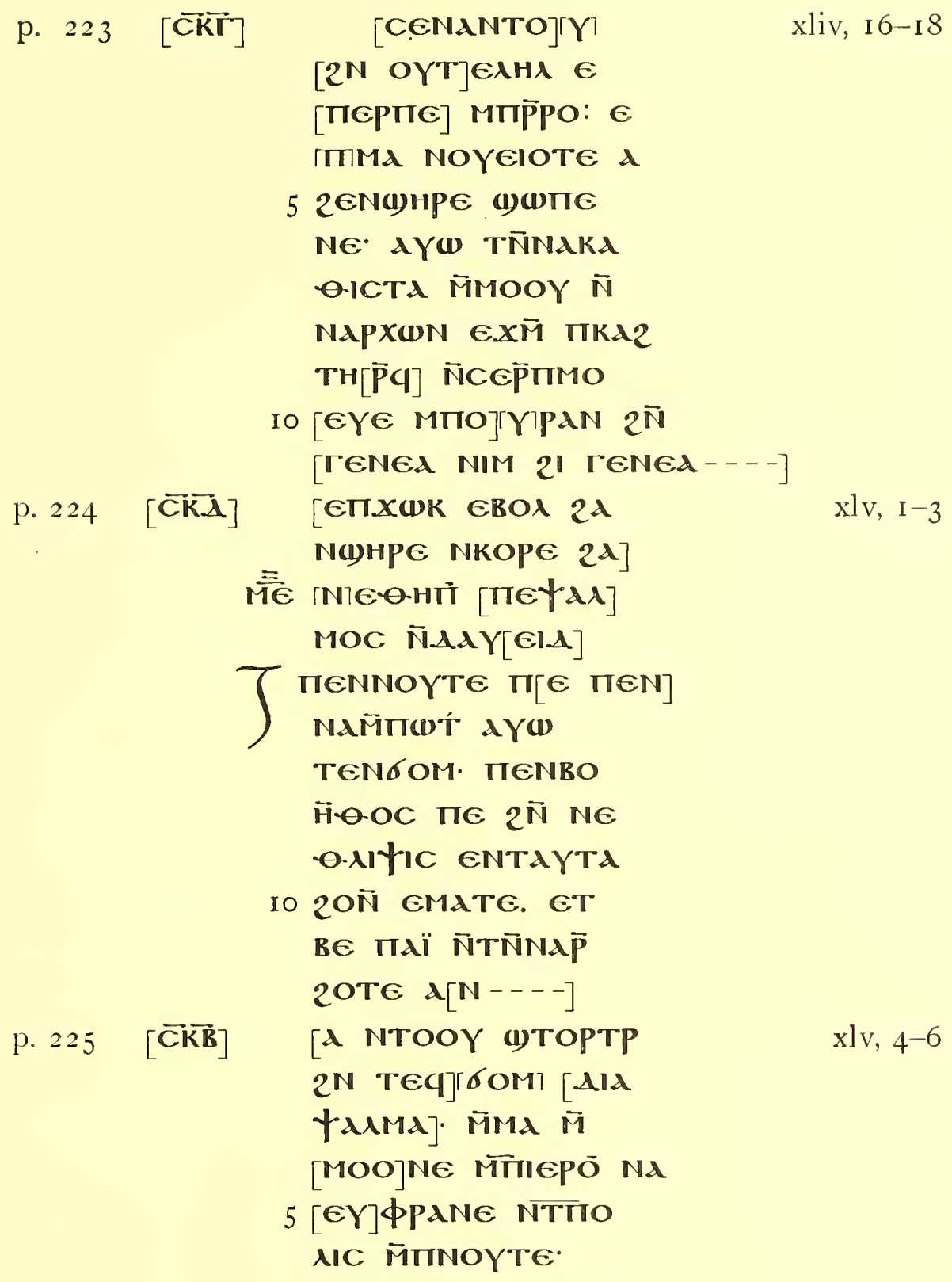




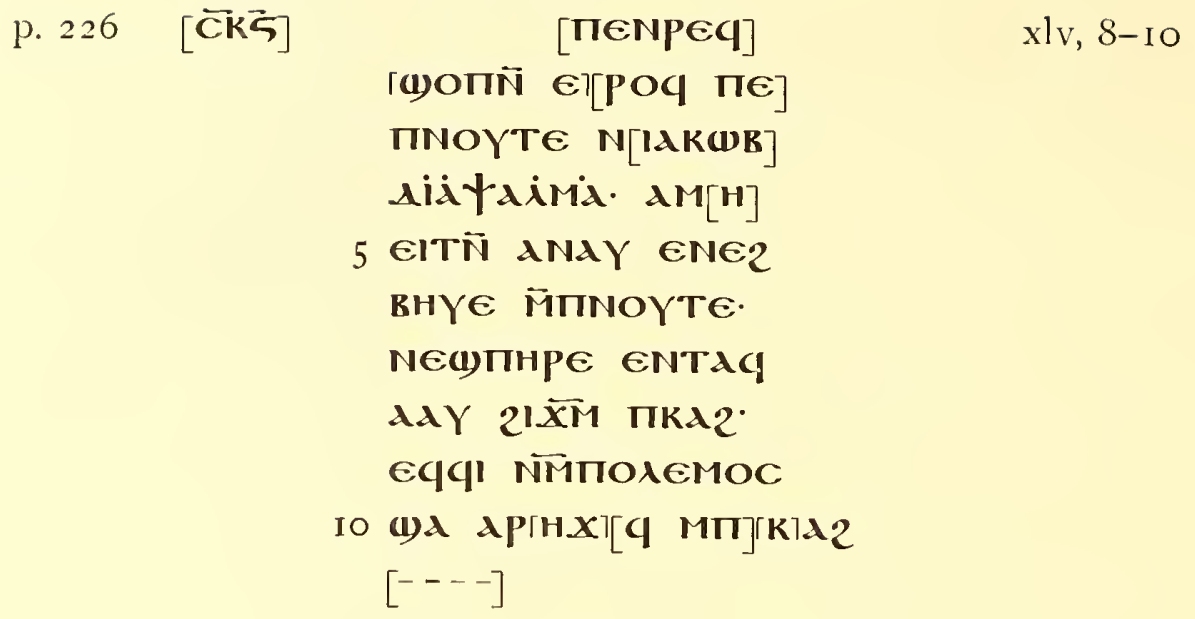

p. 227 [ $[\Theta-\mathrm{NO}] \mathrm{C} \cdot$ TNAXICE $[2]^{\bar{M}}$ пкג2: пхоїс пNOYTE NN̄́OOM

5 NM̈MAN: TIENPEC (1) TNOYTE NIAKWB:

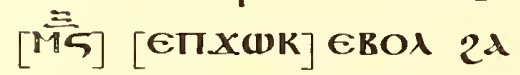
[N(1)нРе NK]OPE $[----]$

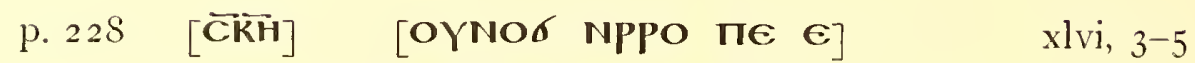

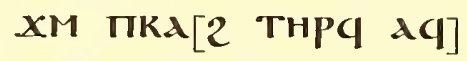

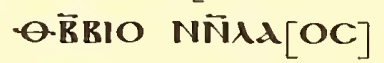
NAN' AYU Nं2[GO] 5 NOC 2 A NENOYEPH TE АपС山Tர் NAN N̄TеqкגнрONOMI

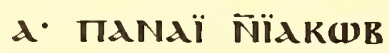
пЕNTגqMEP[ит̄̄ Io $\operatorname{lid} \nmid[\lambda \lambda M \lambda-.-]$ 


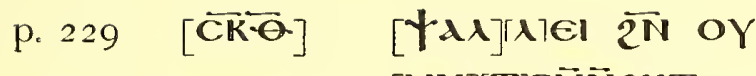

xlvi, 8- Io

[MN] TTPM̈N̄2H.

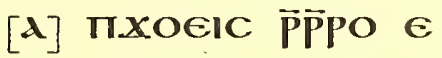

$x \bar{N}$ NÑ̄e-NOC TH

5 POY IINOYTE 2 MO

OC 21 пеq-O-PONOC

ETOYdAk' NAP

$X[\boldsymbol{W}][\mathrm{N}]$ NÑNAdOC $\mathrm{dY}$

[CWOY2 E2]OYN MIN

IO [IINOYTE NABPAAM - - - ]

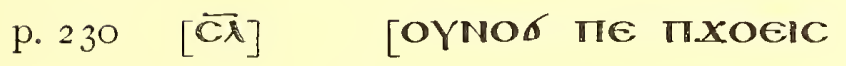

xlvii, $2-3$

AYW GCMAMANT]

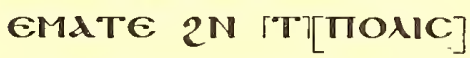

MாENNOYT[E 2M]

5 TIEqTOOY ETOY[A]

גв. EqOYECTWN

2М̈ ПтЕАнА М̈ாКג2

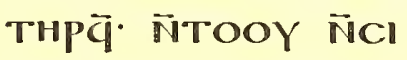

wÑ ETXOCE ÑTE

IO ПЕМ2ІT TाOAIC

MT̃NÓ [NPPO ----]

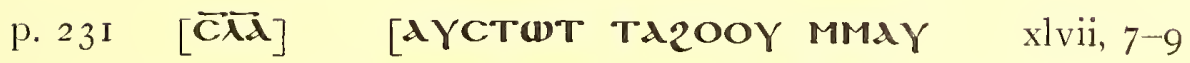
N-Ee] NNNAdKE N̄TET [N]TATMICE. 2N̄ OYTHY

N̄

5 (i)प N̄NEXHY N̈.QAP

CIC. KATd Q.C EN

TANCWTIM Tגi

ON TE O-E ENTIN

N[AY $2 N$ T]TIOÄIC $\bar{M}$

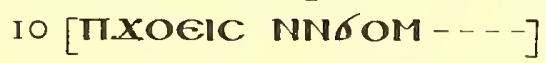

p. 229. 3 EXN N2E-ONOC LBZR

p. 230.9 NeT:Xoce L, $Z R=F$ 

p. $232[\overline{\mathbf{C A B}}] \quad[$ KATA пеKPAN $] \quad$ xlvii, I I-I 2 п[NO] TYTE TrA] [I ON TE] ө. М̈ாєкКєс[MOY] ExIN NapHxă ज̈ா
5 кג2. ере текоY NAM ME2 Ñ.LIKAI OCYNH. MAPEqEY фPANE $\bar{N} \sigma\left[\begin{array}{ll}1 & \text { IT }\end{array}\right]$ TOOY [NCION - - - ]

p. $233[\overrightarrow{\mathbf{C \lambda}}]$

[ xlvii, I 4-I 5 NNE]TาTגїнY N̄TAC

xекגс ететNE xOOY ERETENEA

5 xe паї пе пNOYTE

ITENOYTE wd E NE2 [AY][W] a)d reinie2] [NENE2 NTO]4 ПTET NAMOONE MMON IO wd NIENE2 ----]

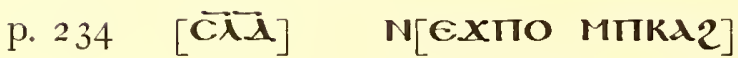
xlviii, 3-4

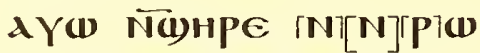
ME त̈PM̄MAÖ 21 OY COT M̈N

5 TATAтрӧ NAxल $[\mathrm{N}] \mathrm{OYCO} \phi][\mathbf{I d}]$ AYW [TMEגеTH MT] ג [2HT NOYMNTPMN]2[HT - - - ]

p. 235 [

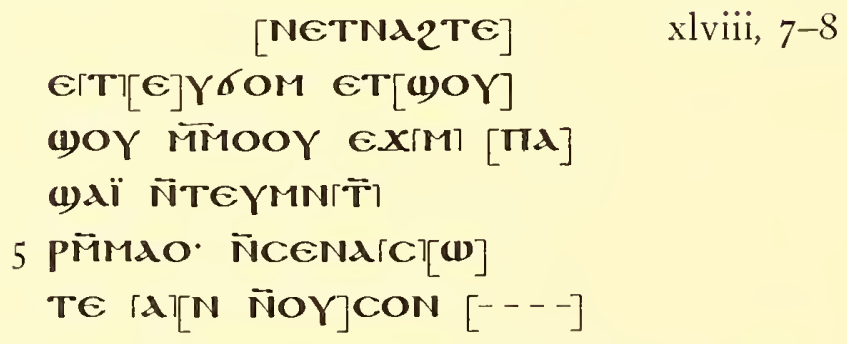
[NeTNA2TE] xlviii, 7-8 E[T][ย]YбOM ET[山OY] wOY MMOOY EXIM] [ח1] ()גï กัTEYMNГT̄]

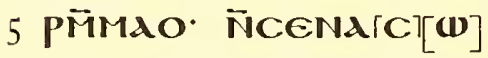
TE $[A][N$ ÑOY $]$ CON $[---]$ 


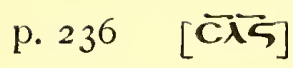

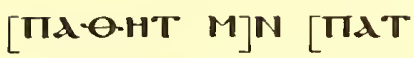

xlviii, I I-I 2

CK]W NATAK[O] [21]

[O]IYרCOT• CENAKW

Гก̄าTEYMÑTPM̄

5 IMIAO N̄2ENKOOYE.

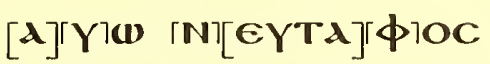

[NE NEYHI Wג ENE2---]

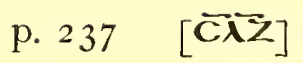

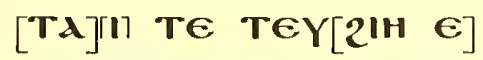

xlviii, I 4-I 5

cep̃xpō̃ NaY [MN]

ÑCd Naï Cendac

MOY $\overline{2 N}$ PEO/Y ${ }^{\circ}[\boldsymbol{A l A}]$

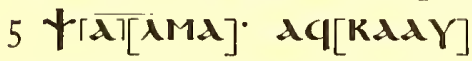

$\mathrm{N}[\cdot-\mathrm{E}$ N2ENCOOY

2N AMNTE ----]

p. $23^{8}[\overline{\mathbf{C \lambda H}}]$

[IIAHN INOYTE NA

xlviii, I6-I 7

CoTE NTAYYXH]

$e[\mathrm{BO}][\lambda] 2^{\bar{N}}$ T $[[I X][\mathrm{NA}]$

MN̄TE Eq(I)ANXIT

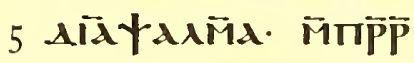

Г27OTE EP(I)AN OY

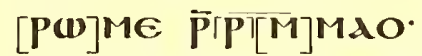

$[A Y(1)] \Theta[P(1) \lambda N \quad \pi] \in Q$

[GOOY ACI)AI MN TIA

Iо Пєфнा -..- ]

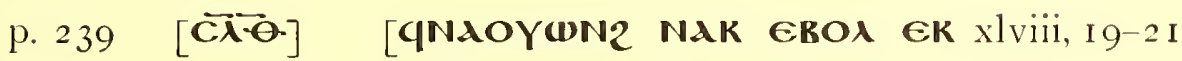
()ג] [NPIח[E]TT][NANOYG

N]גC. GNARIOK $\Theta$

2OYN U1 חIXWM

5 İNEqGOTE' NG

NANAY AN ETOY

oEIN [u]dA ENE[2]

"IPUME Eq 2N OYTA

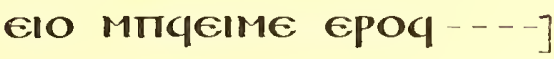

p. 236. 4 NTEYMnTpmio male $\mathrm{L}^{\mathrm{b}}$ corr $\mathrm{Lc}$

p. 239.9 емпфепме male $\mathrm{L}$ 
p. $240 \quad[\overline{\mathrm{CM}}]$

[AYW AqMOYTE

xlix, $1-3$

ПIKA2 EROА $2 N$ M

MAN][(1)][A M̈]T[PH]

(1)d NEqMAN2[C]][TII]

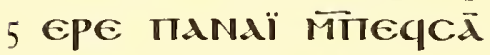

()OOT $2 \bar{N}$ CIWN.

IINOYTE NHY $2 \bar{N}$ OY

[OYO][N] $\overline{2}$ EROA. IIENNOY

[TE] $\Gamma \mathrm{NTC}[\mathrm{NAK \lambda P}] \mathrm{\omega q} \mathrm{AN}$.

p. $24 \mathrm{I} \quad[\overline{\mathrm{MM}}] \quad$ [NE]TCMINE $\overline{\mathrm{N}}[\mathbf{T E q}]$

$x \operatorname{lix}, 5-6$

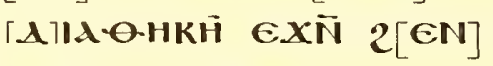

өYCId ज̈тHYE

Naxu ÑTeqdal

5 KAIOCYNH. AYW

$2 \mathrm{~N} \cdot \mathrm{O} \cdot[\mathrm{AH}]\lceil\overline{\mathrm{N}}] \mathrm{NE} 2[\mathrm{OO}\rceil[\mathrm{Y}]$

П[NOYTE NAMEWT

ӨІІХHM MN OY2HBC ----]

p. 242 [त्वB$]$

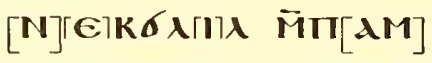

xlix, 9- Io

ГT1O EBOA NOY[W][EI()]

NIM. ÑNAXI MA

CE EBOX $\overline{2 M}$ Пек

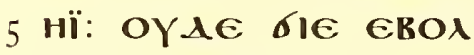

[2N̄] NEKГO [2E] ГX]E

[NOYI THPOY] NE

[NGOHPION MIIEAPYMOC - - - ]

p. $243[\overrightarrow{\mathrm{CM}}]$

[TU1 гAP

$\mathrm{xlix}$, I 2-I 4
TE] ГT'OIKOГYTM[ENH
M]N пєСХХK $\Theta[B][O \lambda]$
MH EINAOYM̃ $[A][G]$
5 M̄Mace H Eïn[A]
Ce CNOY N̄GIE

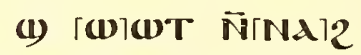
PM̈ T[NO]YT[E NOY]
O[YCId N̈CMOY ----]

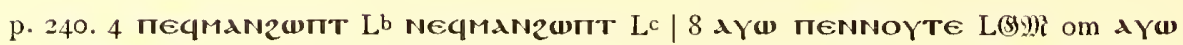
BZR

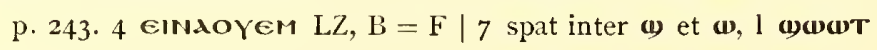




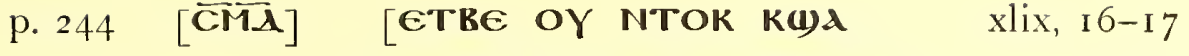 $x \in]\ulcorner 2\rceil[N]$ NA「A]IK[AIOMA
AY](1) KXI N̄TAA[IA
Ө]ГНाКн 2ІTÑ PWГK1

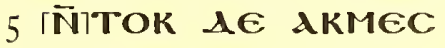

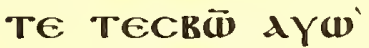
AГKINOYXE 「ĪNTIA)d

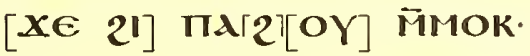
$[---$ ]
p. 245 [ד̈E] [NEK2MOOC EKKA
xlix, 2O-2 I
TAגANEI NCA TTEK
CO $][N] \cdot A \Gamma Y][\boldsymbol{W}]$ NE[KKA
C]KANAגAON $\bar{N}[\mathrm{CA}]$
5 П(1)не ज̈текMГג][AY]
AT्रP NAī AīkAPWII]
ARMEEYE EYANO

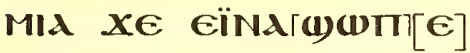

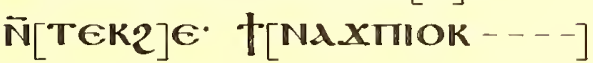

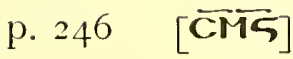

[AYw] ГE] [PE

xlix, 23-1, I

TE2I][H] M̈MAY ETOГYAT

[A]R E†NATCd

[k]ГO14 єрос поY

5 Xגї MாINOYTE:

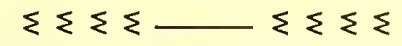

$[\overline{\overline{\hat{N}}}][\epsilon \pi x] \omega k$ єгво

[tdג ${ }^{-}$MO][C NAdYeIA - - - ]

p. 247 [ $\overline{\mathrm{CMZ}}]$

EIM] AdT EMA

$1,4-5$

T']€ EBOג $2 \overline{\mathrm{N}}[\mathrm{TAd}]$

NOMIA AYrW] [NT]

Tёвої ЄROA $2[\mathrm{M}]$

5 паNOBE. xe ††

COOYN ANOГK] [N]

T[AANO][MIIA $[----]$

p. 246. 3 e†NAтсגвок L, BZGol(5) = F 
p. 24

$$
\text { [CMH] }
$$

[EIC 2HHTE ГAP NTAY

$1,7-8$

wW MMOl 2][N] 2E[NANO

MIA] AYW N̄Td [TA

M][dA7Y XlOYä IM][MOI

$\left.\begin{array}{ll}5 & 2\end{array}\right][\bar{N}]$ 2ENNORE [E][IC

2H][H]Tе ГГАาP АКME

[PE T]ME [NEQH]

[MN NETE NCEOYO

N2 EROX AN NTEK

IO COQIA AKOYON2OY

NAI EBOA ----]

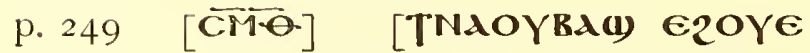

OYX][IW][N KNATPA

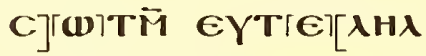

M] Ñ NOYOYNO[G

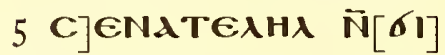

NKEEC NNET[- $-\mathrm{R}]$

BITH][Y] КTE п[СК2O

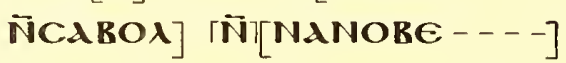

p. $250 \quad[\overline{\mathrm{CN}}]$

[AYW IERாIN]d ETTOYר

1, I $3^{-1} 4$

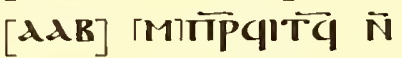

[CגB]]O]入 MMOÏ: MA

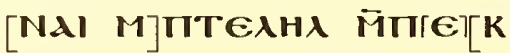

5 OYX]dï AYW $[\overline{N T}] T d$

[XPOI] [2]Nं lOY][INA

N2HTEMONIKON - - - ]

p. $25 \mathrm{I}$ [CNA]

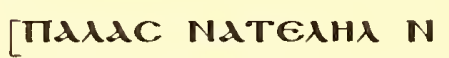

1, I 7-I 8

TERAIKA]IOCYNH ГT][XO

GIC KN]AOYWN ÑNA

[CII][O]TOY ÑTE T[ATd

5 IIP][O] XW M̈ாек[CMOY

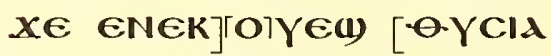

NeINAt $\Pi \in---$ ]

p. 248.6 eкMePe L, ZVGol $=\mathrm{F} \mid 8$ netenceoYon LbV NeTeNceoYon2 LcZ

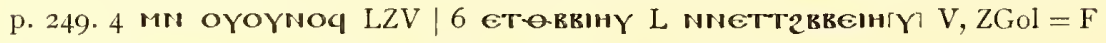

p. $25 \mathrm{I} .3$ NNNגctrotor male Lb corr Lc 
p. 252 [ $\overrightarrow{\mathrm{CNK}}]$

[AYU) MA

$1,20-2 \mathrm{I}$

POYKW] TT NNCO [RT

N-O.IA][H]M TO[TE

K][N]dOY[U] 2E[N][-OY

5 Cld] N̄AIrKAIO1G[YNH

2EN]TAN/OT [MN 2EN

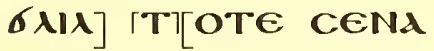

TAגe zenMACE ----]

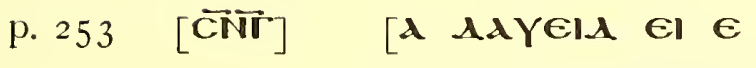

li, I-4

пн]Ги NAXIMEГA][GX

ग2PO]C प(1)OY(1)O $[Y$

MM]'OTA NGI NTYNATT" [OC

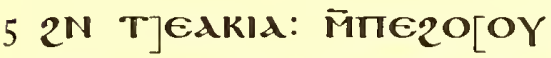

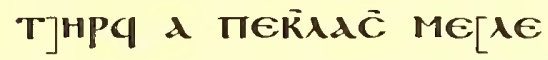

T]A N[OY]XINGONC $\Gamma \mathrm{N}][-\theta \mathrm{E}$

NOYTOK Eq] TT/HM [AK

eIPE NOYKPOC -.-- ]

p. $254 \quad[\overline{\mathbf{C N \lambda}}]$

[d]YW TEKIN][OYNE

li, 6-9

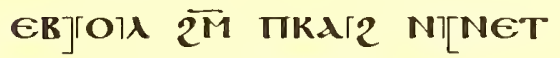

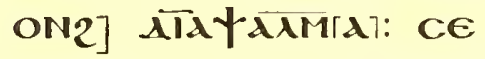

[NANA]Y NGI NAIKAIOC [N]

5 [CE]P2OTE. N̄CECU

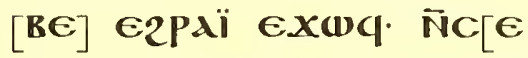

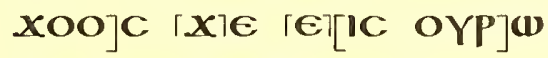

[ME EMTIRA IINOY

TE NAC NBOH-OOC ----$]$

p. 255 [ $\overline{\mathrm{CNE}}]$ [AI2EATIZE ETNA li, IO-I I

MINOYTE (1)d EN] $\epsilon_{2}$ A[Y

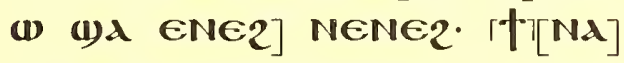

OYWN2 NAK ERO[A]

5 ud ENE2 $x \in$ גKeI $[\mathrm{PE}]$

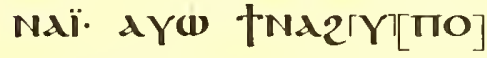

MINE ETEKNA $x[\epsilon$ OY]

ХPнстос пе Мा[GM]

TO E[ROA N][Ne][RMETOYAגR - - - ] 
tadmoc nadyela

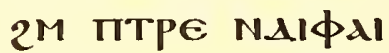

OC el NCEXOOJC

5 [NCAOYA $X E \mathrm{El}] \mathrm{C}$ AdY

[धा. 2HIT 2ג]THN

[MATOYXE TIA]NOY

[T€ 2M пекPג][N]. AY

[(1) KPINE MMO]i $2 \mathrm{~N}$

Iо [TекбоM - - - - 
$A$

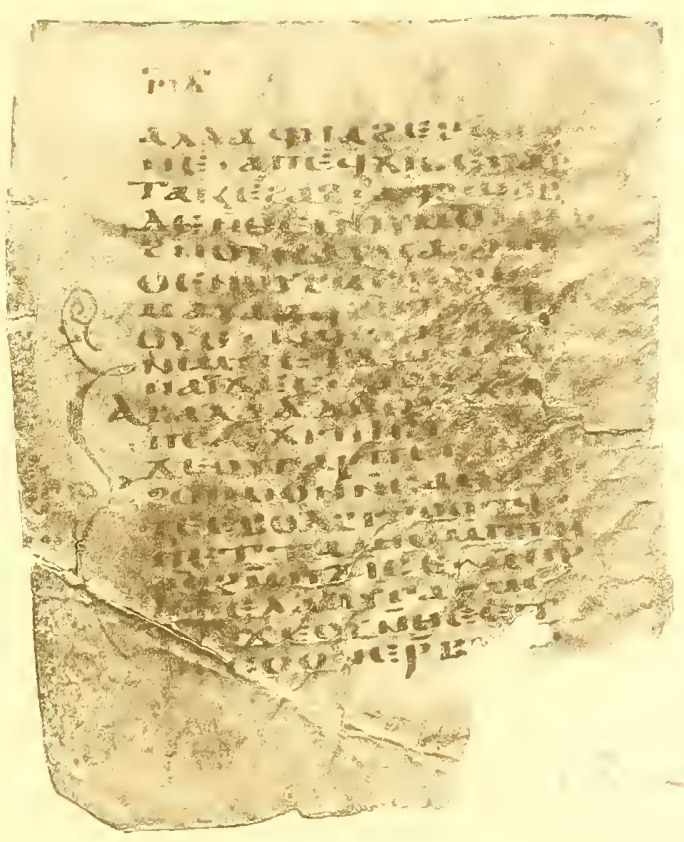

B

$$
\begin{aligned}
& \text { ars-sade }
\end{aligned}
$$

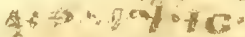

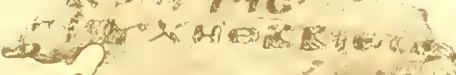

a.

a

4. 5 . तो

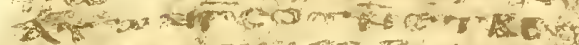

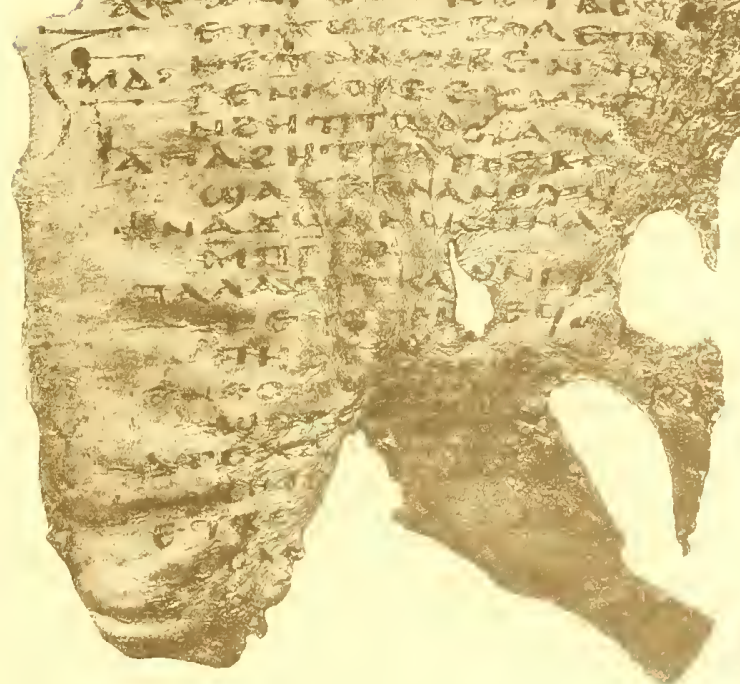

\section{COPTIC FRAGMEN'LS}

A. Fraguent No. 9 [Job ximt, $24 \mathrm{ff}$. $]$

B. Fraguent No. 1 [Ps. ximi, $24 \mathrm{ff}$.] 

PSALTER FRAGMENT

[Акрпова) N]TїмгN][T2нке xliii, 25-xliv, 3 MN TEN]-A.NTIC.

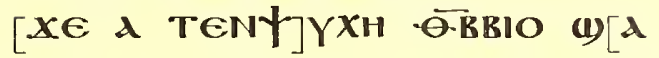

пाкג]2:

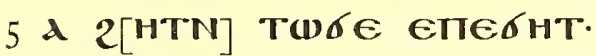

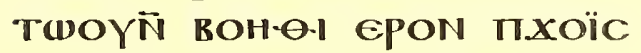

АY(1) NITCOTIN ETRE ITKPAN.

T єпхшк євол етве

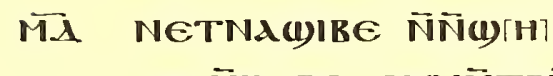

Iо PE ÑKope EYMÑTPM̄

Ñ2HT T(D/AH] $2 \lambda$ TTIME][PIT]

A ITA2HT [TA?YIO] EBOA [NOY]

(1) AXE eNANOYq.

†NAXW ANOK ÑNA2[BHYE]

I 5 їпाтро

TTAגAC OYKAC) N̈rPA[MMA]

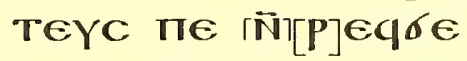

пи ec2Aï

ENECOC [2]M̄ TTEqCA [TIA]PA [N]

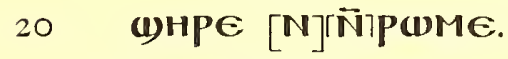

a Texa[PI]C ITWNe E[ROA 21

NE]кс[потто'Y]

ETBE [TAI] A ITNOY[TE]

cM[OY] герO1K (1)ג E[NG2]

I NTENMNT2нке L | 6 вон-еє L пхоеіс L | 7 пекрдN L | KAN secunda

manu | 9 NNetNdajıe L | I7 6 e secunda manu | I8 eqc2dl L | I9 pd secunda manu | 2 I пам L 
[MOрк N]текГс][нqе ЄXM пек xliv, 3-8

MHP]OC петеГO][YN 6 OM]

MMOQ

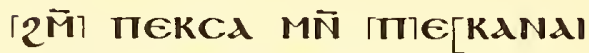

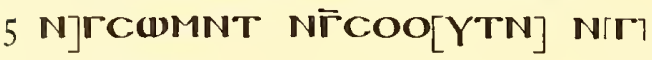

Pि̄PO

ETRE TME MN TMNTPM

PA(I) MT̃ TAIKAIOCYNH.

AYW TEKOYNAM NAXIMO

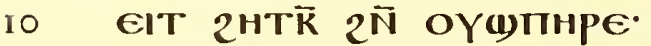

[N]

бОМ М̈MOप $2 \bar{M}$ п2нТ

ГN̄]NXXXE MITPPO.

[NAA]OC NA2E 2APATK.

I 5 [ПЕ][K]-PONOC IINOYTE WO

[O]TT (1) reTNE2 ÑENE2

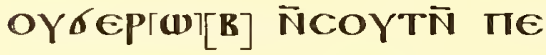

пб ершв NTEкMÑ

TEPO

$20[\boldsymbol{\lambda}]$ KMЕРе T[ $\boldsymbol{\lambda}]$ IKגIOCY

$[\mathrm{N}][\mathrm{H}]$ АKMГC]CTE TIXIN

Гด ОNC̈.

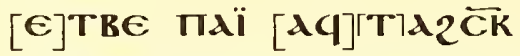

[NGI IINOYTE] пTEK[NOYTE]

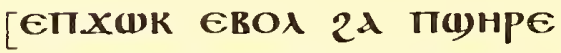

xlvi, I -7

NкоPE пाе]ГтגА [MOC

N2E-NOG TH]

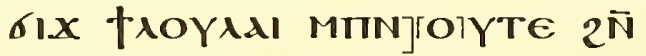

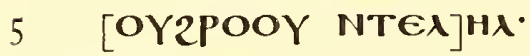

[XE пाXOEIC] XOCE OYZOTE

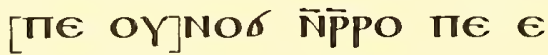

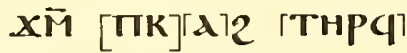

AC[- 


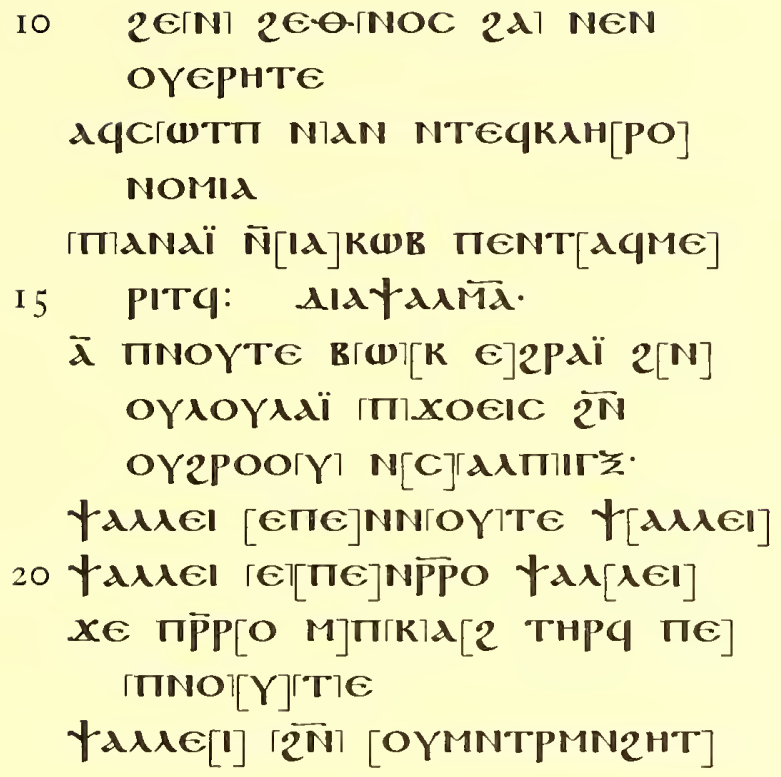

Io N2e NoC L / I2 qKגH secunda manu

[The remainder is illegible.] 


\title{
JOB FRAGMENT
}

P. II 3 PIT

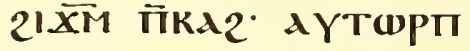

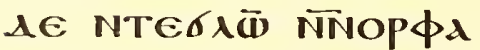 \\ NOC EITA AYPחME \\ EYe M̈ாе $[4]$ NOB M̈ாOY

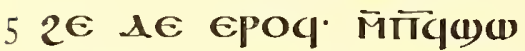

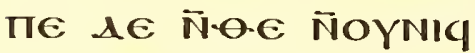 \\ NEIWTE EYETA \\ WBE $\lambda E$ NAq ÑNEÑTAq \\ гАาגY EPE PEqXIÑ́ OÑ \\ IO NIM TגKo ÑoYGE Eq \\ 2OOY. М̈ாपРாЕTNA \\ NOYG $\lambda \in$ ÑOYÁPHN.

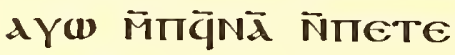 \\ M̄NTCेC2IME. $2 \bar{M}$ \\ I5 пеqбயNT ГАP Аq \\ TAKE NбWR. EqE \\ TWOYN N̈TOOYN NG \\ TM̈TAN2ET пЕqWN2.

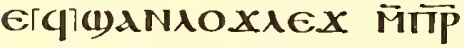 \\ 20 [TP]
}

xxiv, $19-23$

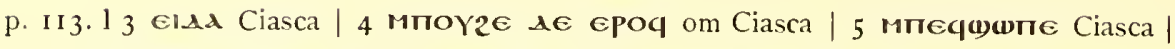

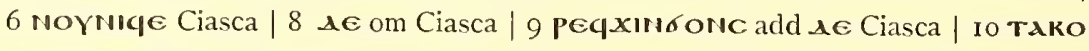

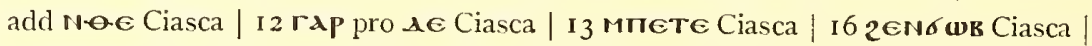
I9 equarroxix Ciasca 


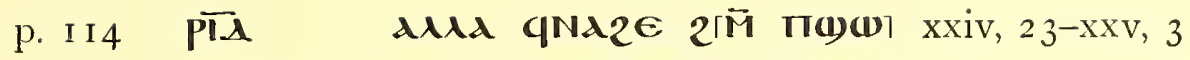
Ne. a пеqXICE таке 2А2. Аाप12@6в

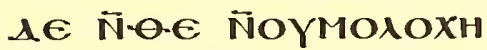

$5 \overline{2 N}$ OYKגYMA. Г̈̈ $N 1$ oe NOYRT̈C redq2El MAYdAq $\overline{2 N}$ ГTEq $\bar{C} B P O$ oYe. E(I)rWTTE MM][ON]

( ) NIM петхш MMOC

¿ nai xe erïxíol.

$\triangle$ Baldal $\lambda \in$ o/YW(1)B

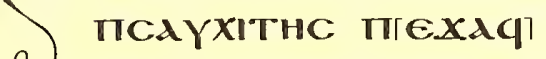

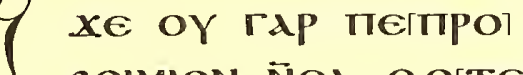
2OIMION N̄CA O-OTTE]

I5 TE ERON 2ITOOTq. пाетTАMIO MTTTH

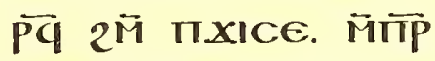
гтре MdגY ГגP ME EYE XE OYÑ $\theta \epsilon \in T$ 20 PE coONe $\overline{\mathrm{P}} \mathrm{BO}[\mathrm{\lambda}]^{\circ}$

p. I xxvii, IO-I 3

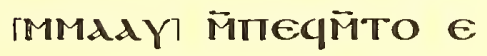

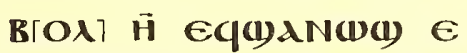
2ГPגіi OYBГHาq qNACW 5 [T]

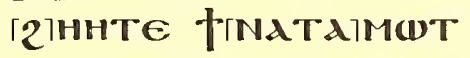
$[N$ X]E OY TIET $2 N$ TAIX ГMTIIXOEIC ArYh⿻ t

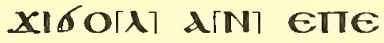
IO [TN̄T] IOIOTC MTITIAN [TWK] 
[HTE] ГTרH ГTN ГT]ETIN]CO OYГN X1E Г2lEN пETUOГY

[EIT N]E[T]NH[Yา EX「N̄]

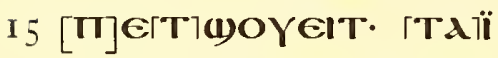

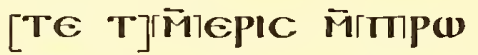

[M]

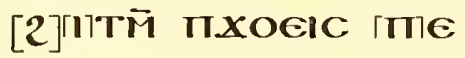

[TXп]

20 [NX][N1N2 NHГY E2Pגï

EXwOY

p. I $20 \quad \overline{P K}$

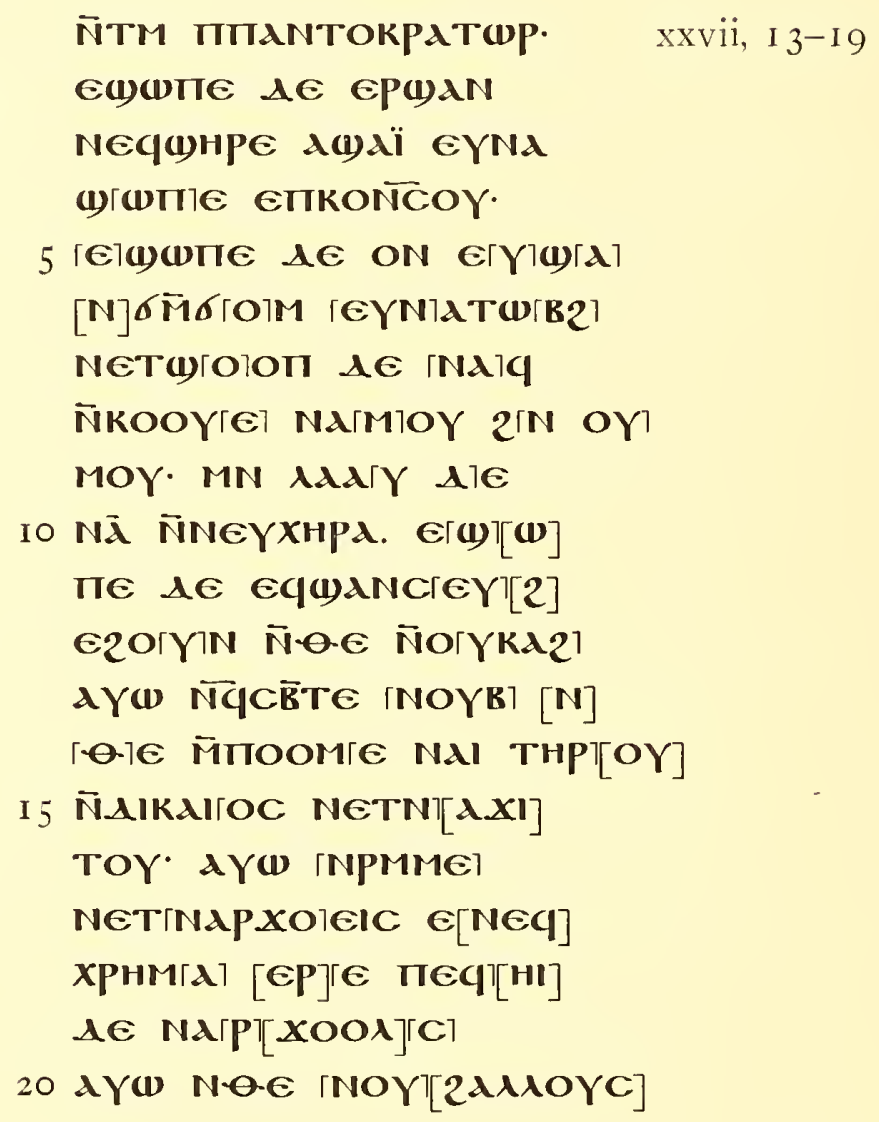




\section{J'LATE VI.}

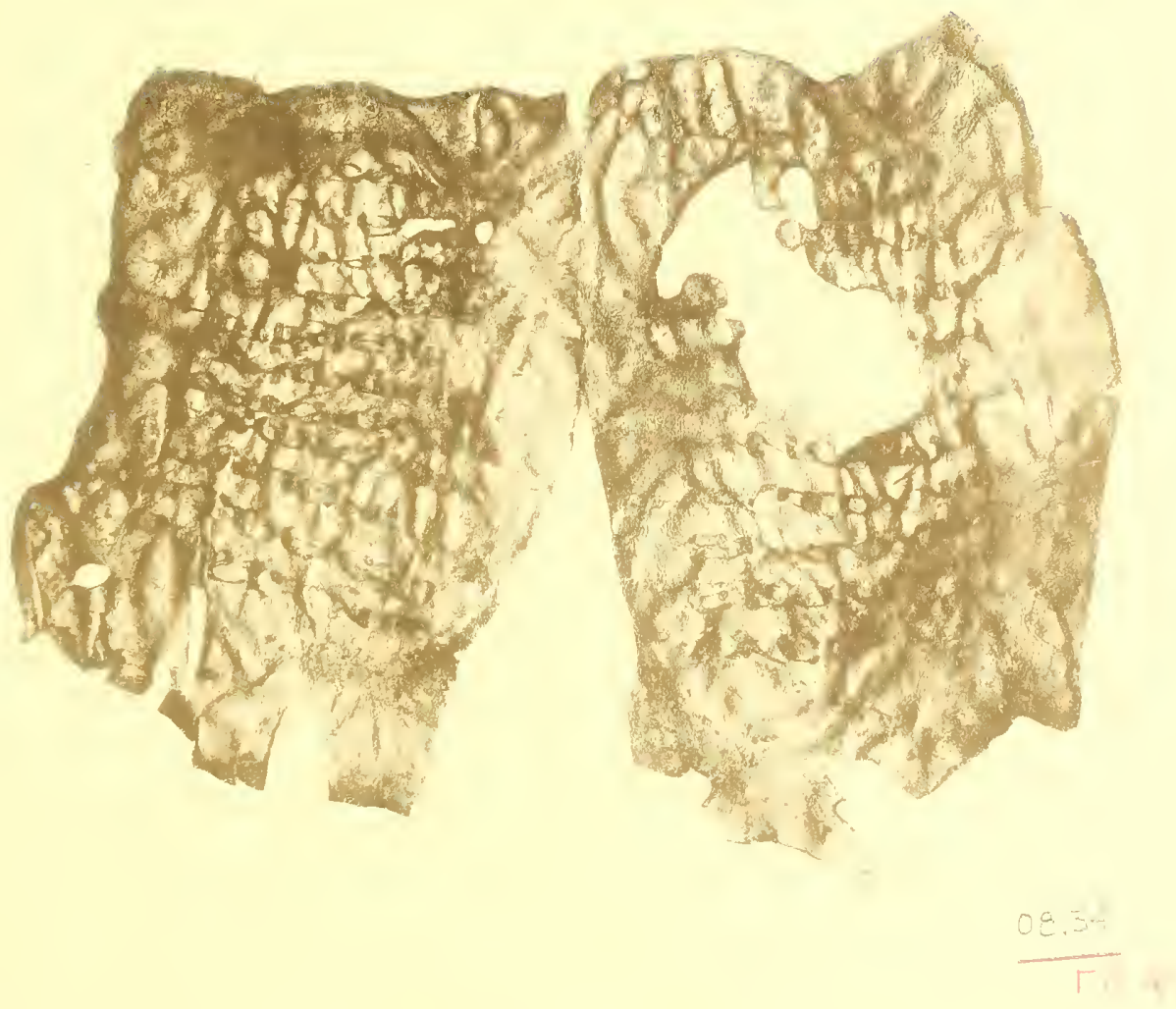

COPTIC FRAGMENTS

Fragment No. '2 [Mat. I, 21 ff.] 



\section{PART II}

TWO HOMILIES AND A MAGICAL

TEXT 
$\therefore \quad 1$ 


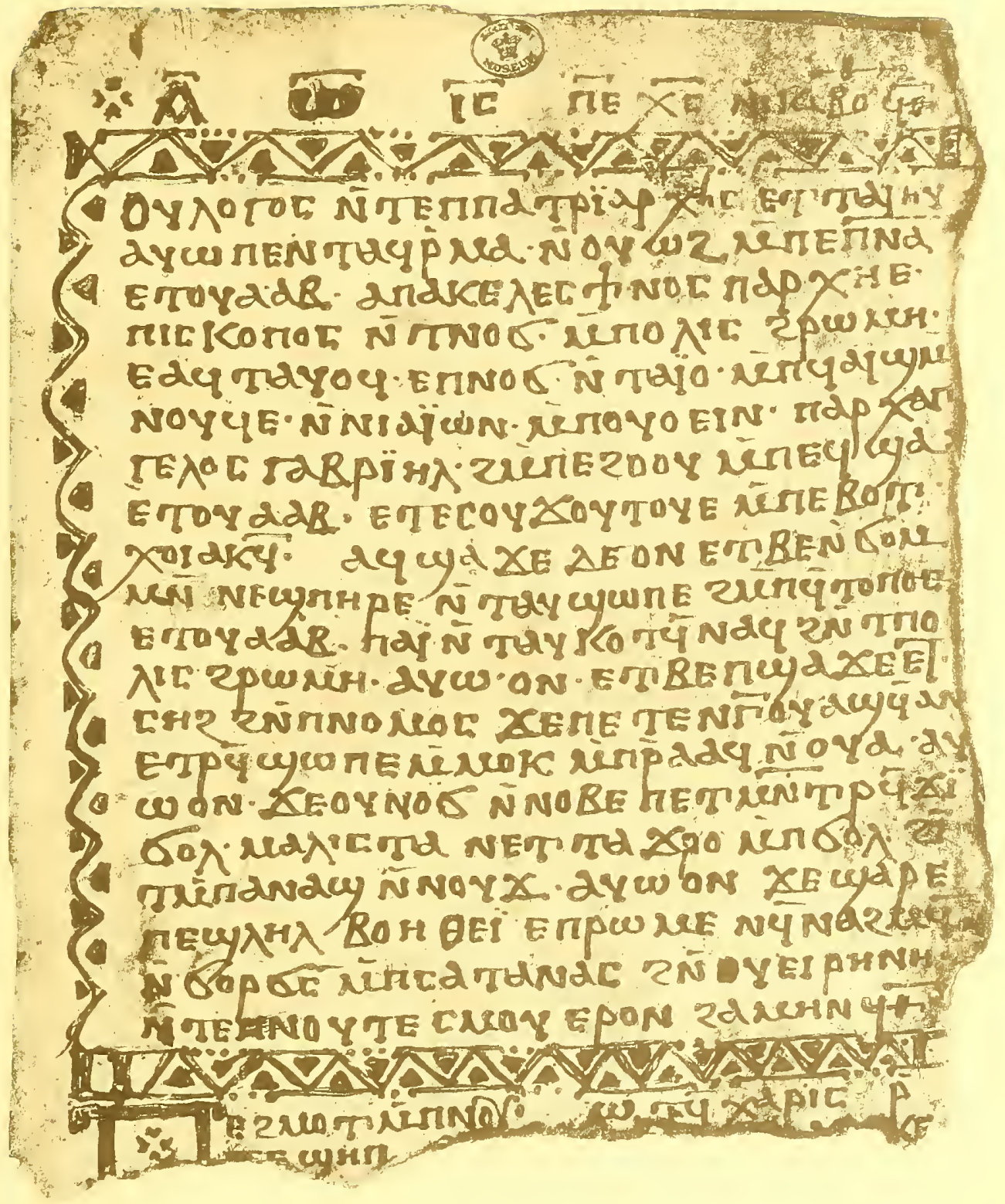

COPTIC HOMIIY ON THE ARCHANGEL, GABRIEL,

British Museum Manuscript Oriental No. 70:28 [p. 1] 



\section{INTRODUCTION}

\section{i. Manuscript No. 2 and Fragment No. 10 of the Freer Collection}

As it may be assumed that readers of this second part of the volume on the Coptic Manuscripts in the Freer Collection have access to the first part, published in April, igi6, it seems unnecessary to repeat here what has there been said on the constitution and provenance of the collection as a whole. The view there expressed ${ }^{1}$ of the provenance of the collection, with the possible exception of manuscript No. 2 and fragment No. Io, which are to be treated of in the present work, is that it was brought from a place near the Fayûm where, it is said, all its members were dug up. The reservation in the case of the two manuscripts must now be justified.

Manuscript No. 2, as conjectured by Mr. W. E. Crum in a personal letter in 1909, proves to be a part of the British Museum Manuscript Oriental No.6780. These two were seen by Professor Hyvernat in I9I I to belong to B. M. Ms. Or. No. 7028. Combining the three gives the following result:

\begin{tabular}{|c|}
\hline $\begin{array}{l}\text { B. M. Ms. Or. No. } 7028=\text { quire } \\
\text { B. M. Ms. Or. No. } 6780=\text { quire }\end{array}$ \\
\hline Freer Ms. No. \\
\hline B. M. Ms. Or. No. $6780=$ quire \\
\hline
\end{tabular}

each quire consisting of sixteen pages. ${ }^{2}$

The colophons, discussed below, assert that the volume was written at Esne. The British Museum authorities believe their portions to have come from Esne and Edfu. ${ }^{3}$ Against such evidence as to ultimate provenance the evidence of immediate

1 University of Michigan Studies, Humanistic Series, Vol. X, Part I, pp. ix-xi.

${ }^{2}$ Cf. Budge, Miscellaneons Coptic Texts in the Dialect of Upper Egypt, I9I5, p. li. With No. 7028 had been bound two irrelevant leaves at the end; and at the beginning is another leaf, which is not a colophon, but seems to refer somewhat remotely to the history of the volume or a similar one. It refers to events some fifteen years later than our manuscript date (A.D. $989=$ A.M. $705=$ A.H. 378), mentioning the rebuilding of a church of the Archangel Gabriel in Edfu (?) and of a monastery of St. Mercurius there.

${ }^{8}$ Cf. Budge, Miscellaneous Coptic Texts, pp. xxiii ff., and Budge, Coptic Apocrypha in the Dialect of Upper Egypt, I9I3, p. xvi, in addition to what has been said in Part I of the present volume. The complete list of these manuscripts, according to Hyvernat in the Catholic Encyclopedia, Vol. XVI, pp. 27 ff., is B. M. Ms. Or. Nos. 6780-6784, 6799-6804, $6806,702 \mathrm{I}-7030$. 
Fayûmic provenance and the Fayûmic tendencies of the scribe must count for little, especially as Fayûmic scribes were employed all over Egypt.

Fragment No. Io, of larger format than any of the other fragments and written in an uncouth hand without analogies (Plate XII), might seem to have no relation to them; but its dialect is Middle-Egyptian, and it may therefore be Fayûmic, although its uniqueness should be emphasized.

\section{ii. Appearance and Make-up of Ms. No. 2, and its Comple- ments from the Britisi Museum}

Manuscript No. 2 can be conveniently considered only in conjunction with its complements, B. M. Ms. Or. Nos. 6780 and 7028 , with which, as has been said, it constituted a single volume. ${ }^{1}$ This volume consisted of six quires of sixteen pages each, plus a single leaf on which the second colophon was written. ${ }^{2}$.

The paging from pp. $\overline{\boldsymbol{M}}$ to $\overline{\boldsymbol{M}}$, both inclusive, runs ahead one number.

The leaves are of unequal size and average $25 \times 30 \mathrm{~cm}$.

The breaks seem to have occurred in ancient times, for all adjacent surfaces show wear. The leaf which is pp. $\overline{\mathbf{2} \boldsymbol{\Gamma}}$ - $\mathbf{\Sigma} \boldsymbol{\lambda}$ was detached and reversed, so that the inner side became more worn than the outer.

The presence of worm-holes, alluded to in Part $\mathrm{i}$, is doubted by Professor Hyvernat on the ground that the holes do not penetrate the mass of leaves, but only single ones.

\section{iii. Vellum, Writing, and Date}

The eight parchment leaves of the Freer manuscript ${ }^{3}$ are of coarse, heavy sheepskin, cut so as to obtain the large pages necessary for the large, sprawling script of the period. It is yellow on one side, and the hair follicles are plainly visible. Both decay and water have done their destructive work. All but the first and last leaves remain intact at the fold, and a bit of binding thread remains. In other parts of the volume ${ }^{4}$ there are large holes in the skin which however do not interfere with the text, as they are older than the latter.

1 B. M. Ms. Or. Nos. 6780 and 7028 are described by Budge, Miscellaneons Coptic Texts, pp. xlix ff.; p. II II ; Plates xix, xx. Plates xxi, xxii are the irrelevant leaves mentioned.

${ }^{2}$ Some calculation must have been necessary to ensure the exact completion of the text.

${ }^{3}$ The editor has had only the photographs of the British Museum manuscripts to work from.

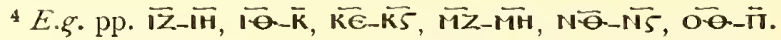


The writing is in two columns ${ }^{1}$ of approximately $2 i-26$ lines each, and 8-I 2 letters to the line. ${ }^{2}$ There is no ruling; but perpendiculars, made with some kind of crayon or other dry point, irregularly appear.

Specimens of the writing are to be seen in the plates. It resembles most of the other hands of the Esne-Edfu collection, ${ }^{3}$ but it is not identical with any of them nor as refined as most of them.

The letters are about $.7 \mathrm{~cm}$. high and not carefully formed.

There is a tendency to elongate $\boldsymbol{\lambda}, \boldsymbol{\phi}, \boldsymbol{t}$.

To economize space at the end of a line the usual ligature for

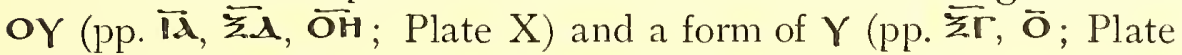
$\mathrm{XI}$ ) embracing the preceding letter with its left arm, and a large $\mathbf{T}$

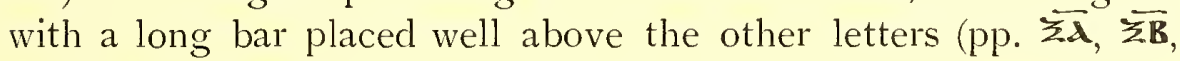
落, 兹; Plates IX, X) are frequently employed.

To fill out space at the end of a line the arms of $\mathbf{C}$ are extended

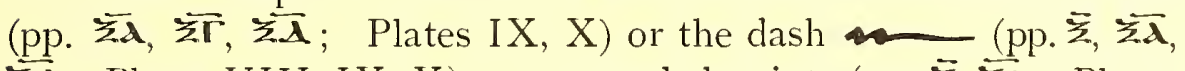

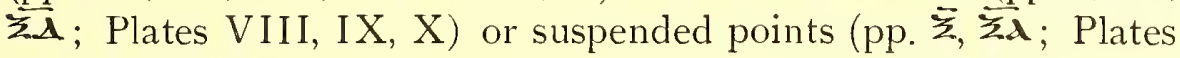
VIII, IX) employed.

- Paragraph divisions are often shown by enlarged letters set in the margin, even though for reasons of economy of space the paragraph may really begin in the middle of the same line and this enlarged letter may belong to some previous word. Throughout the manuscript large red-and-black marginal letters are employed for such first letters. P occurs enlarged but not extended into the margin (p. $\overline{\mathbf{O B}}$ ). For this purpose $\mathbf{A}, \mathbf{M}, \mathbf{N}$ and $\mathbb{\dagger}$ seem to be preferred.

The vocative and interjectional $\overrightarrow{\boldsymbol{\omega}}$ is regularly enlarged and colored.

Zigzag ornaments are placed above the second homily and its title and above and below the title of the first homily (pp. $\overline{\mathbf{\lambda}}$, $\bar{\Sigma}$, 莣; Plates VII, VIII, IX). A grapevine border appears at the left of the title of the first homily (p. $\overline{\boldsymbol{x}}$ ). A sort of croix pattee formed of three triangular dots stands before the title. Something like an interrogation point is placed above most of the page numerals.

That the scribe was a Fayûmite may be inferred from the tendency to substitute $\boldsymbol{\lambda}$ for $\mathbf{P}$ (p. i5. MEגOC for MEPOC; p.

The text and both colophons (pp. 32 I, 322) are in one hand.

\footnotetext{
1 Excepting the title on p. $\overline{\boldsymbol{\lambda}}$.

${ }^{2}$ The archetype must have had ten letters to the line and therefore two narrow columns to the page (cf. p. $\bar{\nabla} \cdot \bar{\theta}$ ).

${ }^{3}$ Cf. the plates in Budge, Coptic Martyrdoms and Miscellaneons Coptic Texts.
} 
The cursive in which the second colophon is written intrudes itself in the text (p. $\overline{\boldsymbol{X}}, 1.2$; p. $\overline{\mathbf{B}}$, col. ii, 1. I 8; p. $\overline{\mathbf{E}}$, col. i, 1. 22 ; p. $\overline{\mathbf{\epsilon}}$, col. ii, l. 3 ; p. $\overline{\mathbf{H}}$, col. i, 1. 3 ; p. $\overline{\mathbf{H}}$, col. ii, 1. 25 ; cf. Plate VII); and is shown to be the same hand as the uncial. The cursive additions (p. $\overline{\boldsymbol{\theta}}, 11.2 \mathrm{I}, 23$ ) are by the same hand as the uncial additions (p. $\overline{\boldsymbol{\Gamma}}$, col. ii, 11. 8, I6), including such as are placed in the margin with the obelus $\therefore$ in margin and text (pp. 帘-, $\overline{\mathbf{O}}$; Plate XI).

The only possible signs of a second hand are the marginal corrections without the obelus (pp. $\overline{\mathbf{\Sigma} \boldsymbol{\epsilon}}$, col. ii, 1. 2I; $\overline{\mathbf{O}}$, col. i, 1. I 7, col. ii. 1. 22; cf. Plate XI) and the Copto-Arabic numerals in the margin (pp. $\overline{\mathbf{Z}}, \overline{\mathbf{K}}, \overline{\mathbf{K B}}, \overline{\mathbf{K}}, \overline{\mathbf{K} Z}$ ).

The manuscript is fortunate in possessing two colophons: one in the corrupt Copto-Greek of such documents, giving the exact date according to three eras, and the other in Coptic, which supplies some interesting information. ${ }^{1} \quad$ These are given on p. 32 I.

According to this colophon the correct date of the manuscript is Saturday, February 28th, A.D. 974, which is Saturday, Gumâdâ 1-âhira 2d, A.H. 363 , or Paremhot 4th, in the $2 \mathrm{~d}$ year of the $45^{\text {th }}$ Indiction, or the year 690 of the Era of Martyrs.

\section{iv. Punctuation and Spelling}

The superlinear dash is seldom omitted (p. $\overline{\mathbf{z}} \mathbf{B})$. It is regularly placed far to the right of its proper place. From being used

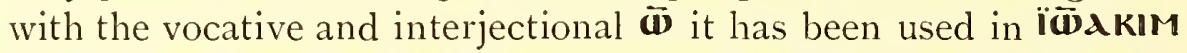

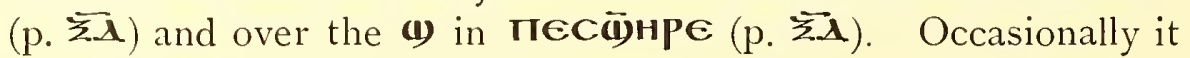
is used for " over the $\boldsymbol{i}$ (p. $\overline{\boldsymbol{i}} \boldsymbol{\lambda})$.

The dotted and undotted $\mathbf{I}$ are completely confused, with a preference for the former (pp. $\overline{\mathrm{ZA}}$ bis, $\overline{\mathrm{z}} \mathrm{B}$ bis). The dots are twice placed over $\mathbf{H}$ (pp. $\overline{\boldsymbol{\lambda}}, \overline{\mathbf{q}} \boldsymbol{\lambda})$.

The single dot seems to be used to prevent a false division

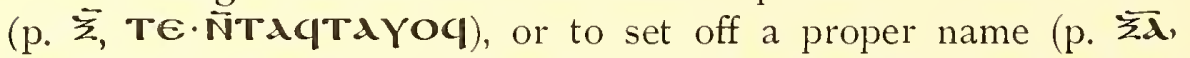

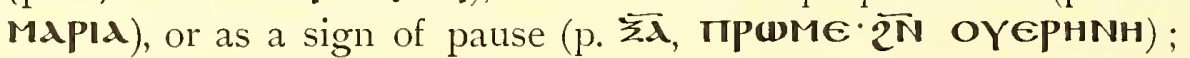
but its excessive use, even within a word (p. $\overline{\text { G }, ~ N E N ~ E I O O T E, ~}$ p. $\bar{\Sigma} \bar{\Gamma}, \overline{\Pi \pi} \mathbf{N} \cdot \boldsymbol{\lambda}$ ), shows that the scribe had no clear idea of its function.

A heart-shaped sign upon its side indicates great divisions (p. $\overline{\boldsymbol{N T}})$; but it is also carelessly used (pp. $\overline{\mathbf{K}}$, $\overline{\mathbf{K H}}$ ).

The marginal signs $\approx$ or (less frequently) $\therefore$, whose influ-

${ }^{1}$ Professor Hyvernat kindly assisted me with the paleography of these in the summer of I9II. 


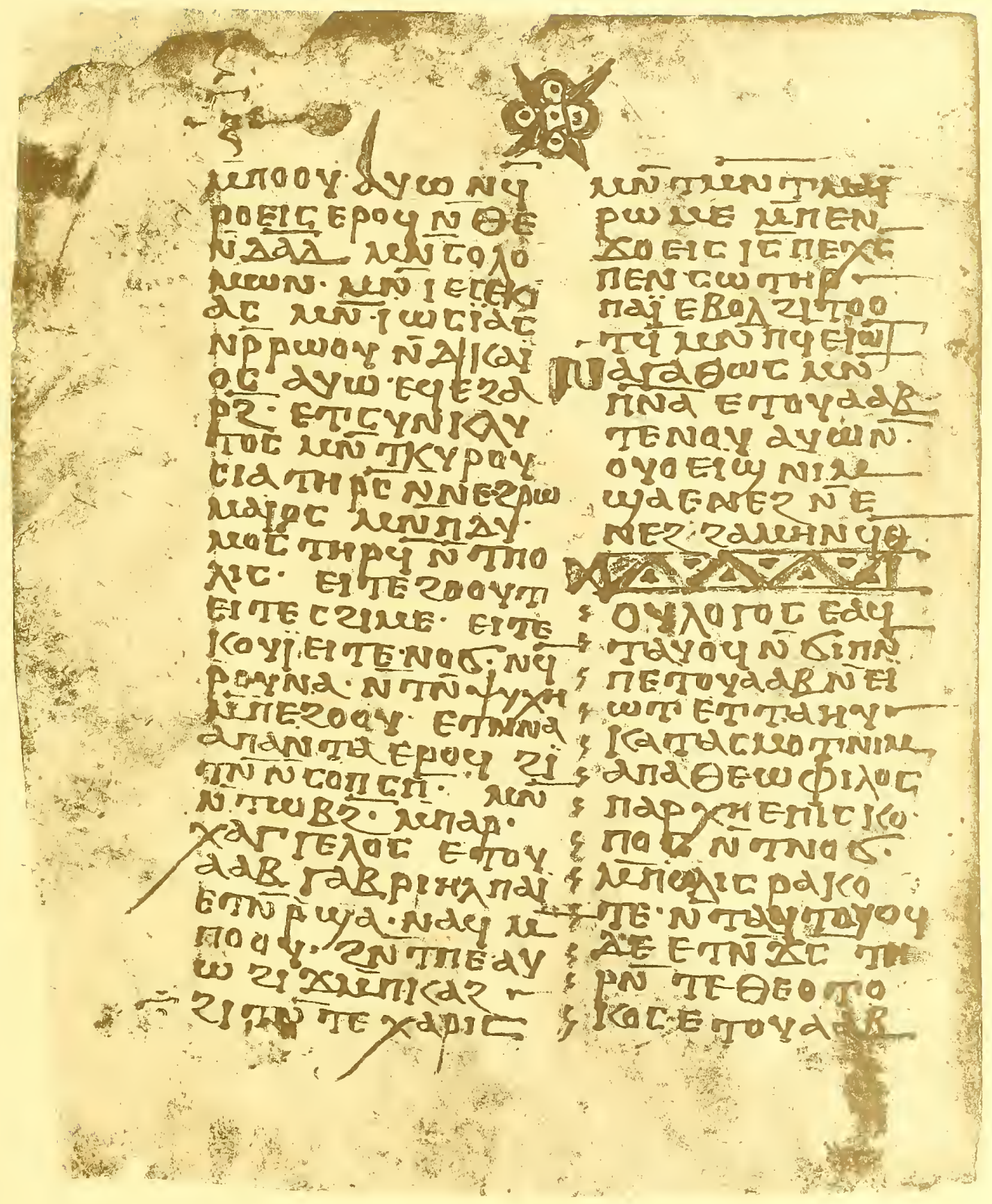

COPTIC HOMILY ON THE VIRGIN

British Museum Manuscript Oriental No. 6780 [p. 60] 

ence is often extended down the columns by the sign $\mathbf{S}$ opposite each line, indicates emphasis or special appeal (p. $\overline{\boldsymbol{\pi}}$ ), or short exclamatory sentences (p. $\overline{\boldsymbol{\Sigma}_{\boldsymbol{T}}}$ ), or invocations (p. $\overline{\mathrm{\Sigma}}$ ), or transi-

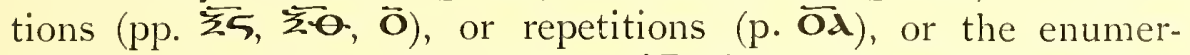
ation of important items (pp. $\overline{\mathbf{O A}}-\overline{\mathbf{O E}}$ ), or (and most frequently) a quotation. It is not used on p. $\bar{\Sigma}$, i, io ff., where expected.

Omissions within the text are occasionally indicated by the obelus before the correction in the margin and at the place of omission; but it is not always used.

The dash usually employed to fill out a line, occurs at the end of a quotation on p. $\overline{\boldsymbol{\Sigma}}$, end of col. ii, probably by mere chance. It enables (p. $\overline{\mathbf{O} \boldsymbol{d}}$ ) the scribe to bring his initials in the margin at the beginning of a phrase.

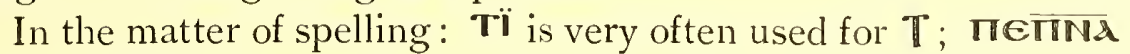
is written $\overline{\mathbf{\Pi N \boldsymbol { \lambda }}}$ everywhere but p. $\mathbf{\boldsymbol { \pi } \cdot \boldsymbol { O }}$, ii, I 7 . The other peculiarities are best treated as phonetic and grammatical phenomena.

The syllable junction, as indicated by the word division at the end of the line, is loose. ${ }^{1}$ Thus one finds $\boldsymbol{A}$-(1) $\mathbf{\lambda} 20 \mathrm{M}$ for

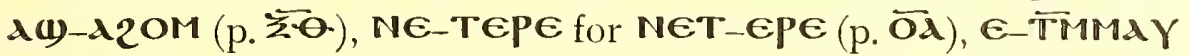
for $\mathbf{E T - M M A Y}$ (p. $\overline{\boldsymbol{\lambda} \boldsymbol{A}}$ ). This being the case, it is evident that

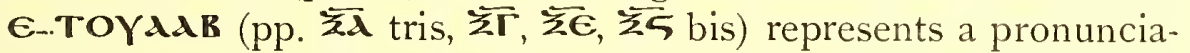
tion $e+t \hat{u} a+a b$ or $e+t w a+a b$, and not the conventional $e t+$ $w a+a b$. That the first of these, $e+t \hat{u} a+a b$, is the true form is

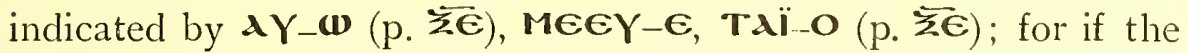
$Y$ and $\mathbf{I}$ were consonantal they would go with the following vowel. The combinations which are commonly pronounced as diphthongs are therefore probably, like the Italian, not true diphthongs, but a succession of syllabic vowels.

Haplological syllable ellipsis is fairly common, as in $\mathbf{\Gamma \in T N A}$ [NA]2MNT (p. $\overline{\mathbf{O B}}$ ), and may occasion the omission of the copula

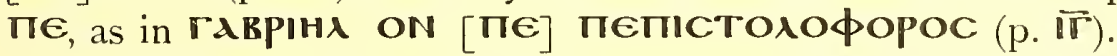

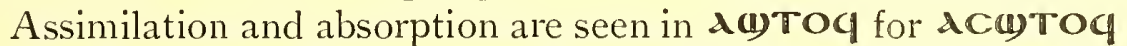

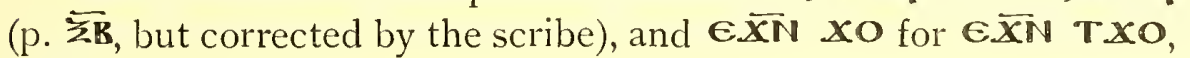
p. $\overline{\Pi \lambda}$.

${ }^{1}$ The difference between tight and loose syllable junction (Sweet's "close and open stress," Sievers' "stark- und schwachgeschnittener Akzent," Jespersen's " fester und loser Anschluss") is illustrated by English isolate as contrasted with French isolé. In the former the syllabic boundary lies within the $s$ and uncertainty in division results, so that one hears both $i+$ solate and $i s+$ olate. In the latter the boundary lies between $i$ and $s$, and every one writes and speaks confidently $i+$ solé. The former is found in Germanic languages, the latter in Romance and Slavic, Arabic, Turkish, and quite generally. Cf. Jespersen, Lehrbuch der Phonetik, 1913, pp. 204 ff. 
The latter makes it likely that $\boldsymbol{X}$ was still pronounced as a true palatal, as is Arabic $\boldsymbol{Z}$ in Upper Egypt to the present day, and not as English $j$.

The usual assimilation of $\overline{\mathbf{N}}-, \overline{2 \mathbb{N}}$, and similar words to a

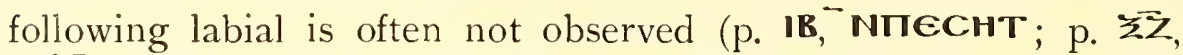

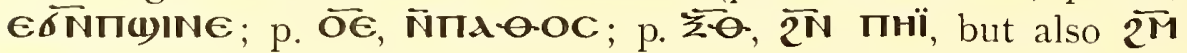
пніі).

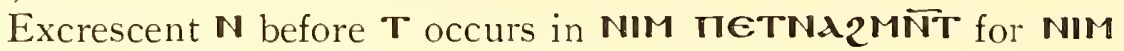
TETNANA2TTT (p. $\overline{\mathrm{OB}}$ ) WOMNTE (pp. $\overline{\mathrm{NB}}, \widehat{N S}$ ) is a conflation of the masculine and feminine, under the influence of the dental $\mathbf{T}$.

Shortening of long sounds is not common. It occurs e.g. in the

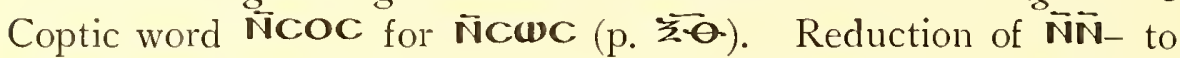
$\overline{\mathbf{N}}-$ and of MMd- to Md- occurs (p. TT, $\overline{\mathbf{N} 2 O T}$ for $\overline{\mathbf{N}} \mathbf{N} 20 \mathrm{~T}$;

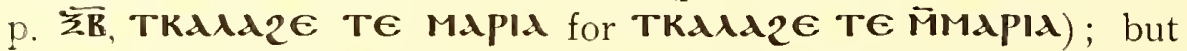
whether this is shortening of a single syllabic $\overline{\mathbf{N}}, \overrightarrow{\mathbf{M}}$ or haplological syllable ellipsis cannot be ascertained. Even $\overline{\mathbf{N} N O \overline{N 2}}$ occurs for NNNENON2 (p. $\overline{\mathbf{R}}$ ). TA-for TAd- on p. $\overline{\mathbf{I}} \mathbf{R}$ may be shortening or haplological ellipsis.

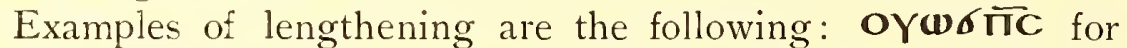
oүoб

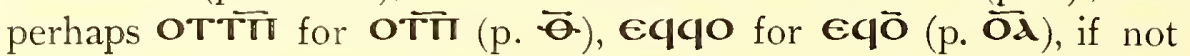

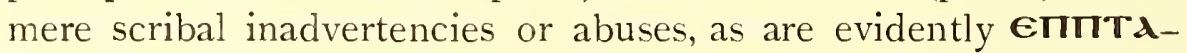

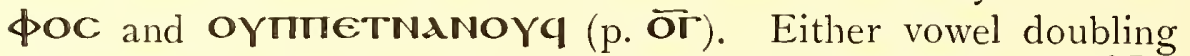

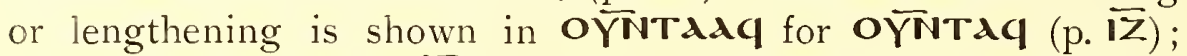

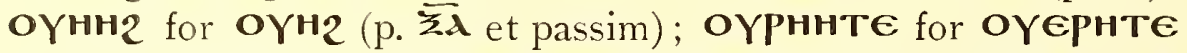
(p. ī et passim); 2loome for 2lOME (p. $\overline{\mathbf{O A}}$ ); $\overline{\mathbf{\omega}} \overline{\mathbf{\omega}}$ for $\overline{\mathbf{\omega}}$

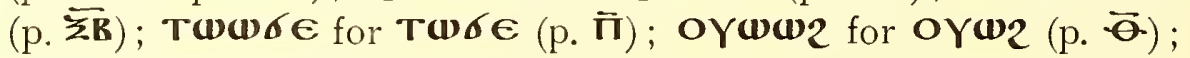
EBRIHN for EBIHN (pp. 玄, Ö).

The following are examples of confusion between voiced and

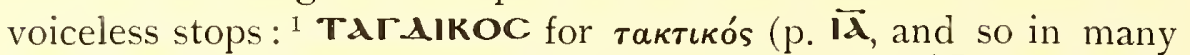

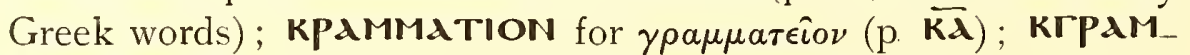

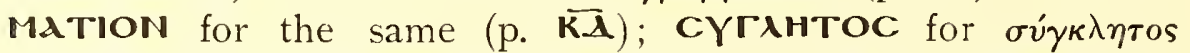
(p. $\overline{M H}$, cf. CYNГENHC for $\sigma v \gamma \gamma \epsilon \nu \eta^{\prime} s$, p. MBß $){ }^{2}$ АNAKГH for

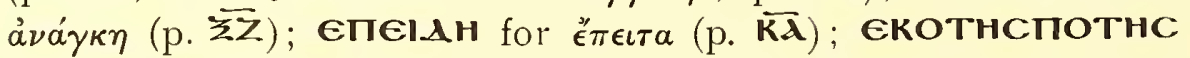

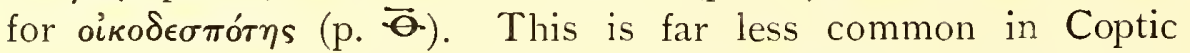

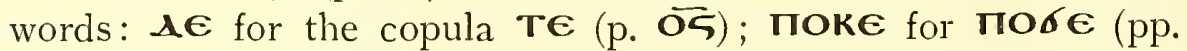

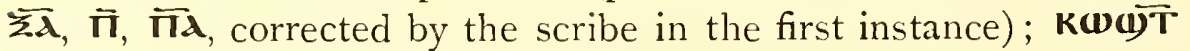

\footnotetext{
${ }^{1}$ Common in both Coptic and the Greek papyri ; for the phonetics of both languages must have been the same, as is the case in Alsace. and other border countries. There seems to have been no $k, t$, or $p$, aspirated as in English. $\mathbf{~}, \mathbf{T}$, and $\boldsymbol{\pi}$ were probably unaspirated (mediae). A was similar to $\mathbf{T}$, $\mathbf{B}$ to $\mathbf{O Y}, \boldsymbol{\sigma}$ to $\mathrm{K}$.

${ }^{2}$ The usual spelling in later Greek.
} 


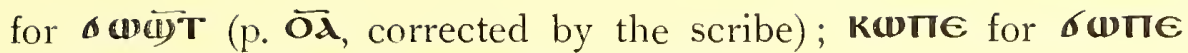

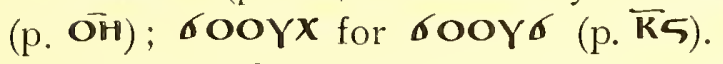

The use of $\mathbf{Y} Y$ and $\mathbf{R}$ is such as to suggest that they had but one value, and that this was not a bilabial fricative but a very open $u$ or even a close $o$. The following are examples.

B for [O]Y, OYe:

\begin{tabular}{|c|c|c|}
\hline p. $\overline{O Z}$ & ERE- & for EYE- \\
\hline p. $\overline{\mathrm{KS}} \overline{\pi \epsilon}$ & TARE & for $\mathbf{T} \mathbf{A} \gamma \mathbf{E}$ \\
\hline p. $\overline{\mathbf{B}}$ & COBTN & for COOYTN \\
\hline p. $\overline{\mathbf{B}}$ & COB2EN & for coOY $\overline{2 N}$ \\
\hline p. $\overline{\mathbf{K}}$ & OY $\overline{\mathrm{BB}}$ & for oyoroyed \\
\hline
\end{tabular}

OYB for OY and $\mathbf{R}$ :
p. $\overrightarrow{\mathbf{B}}$
p. $\overline{O Z}$

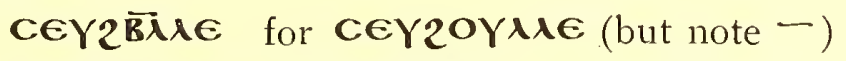
ПIOYВHMA for ПR⿴囗М

$[\mathrm{O}] \mathrm{Y}$ for $\mu \beta$ :

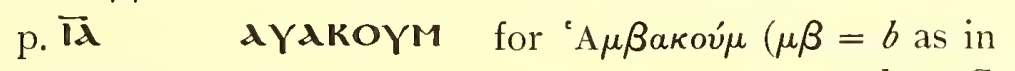

oY omitted before $\mathbf{\omega}$ :

modern Greek)

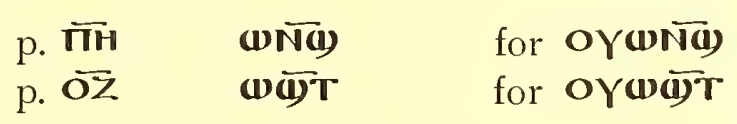

OY for OYO :
p. $\overline{\mathrm{KS}}$
OY $\overline{\mathrm{B}} \overline{6}$
for oyoroye6

oY wrongly placed before $\boldsymbol{\omega}$ :
p. ī
OYलम्ब(1)
for (1)
p. $\overline{\mathrm{OA}}$
OYMÑ?
for $\omega \overline{N 2}$
p. $\overline{\mathrm{O \theta}}$.
OY(1)
for (1)

The superlinear dash is widely used in place of the vowel $\mathbf{E}$ :

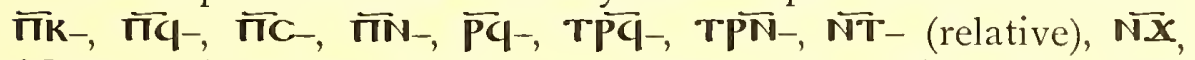

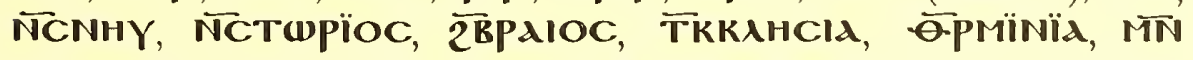
for MEN $\left(\mu \epsilon{ }^{\prime}\right)$, p. $\overline{\mathbf{T}}$. Rarely $\boldsymbol{\epsilon}$ is placed where it does not

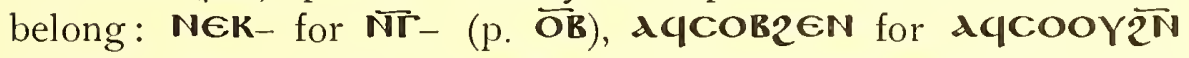
(p. $\overline{\mathbf{B}})$. It is very often omitted initially in the participial clause

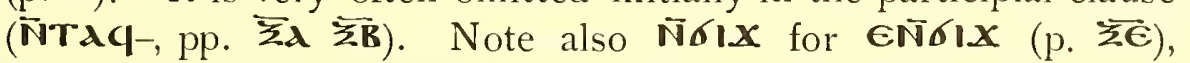

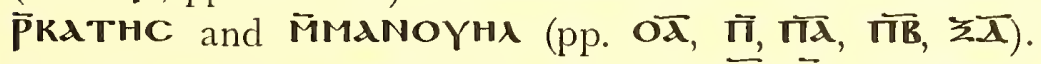

$\boldsymbol{\epsilon}$ is sometimes entirely omitted (p. $\overline{\boldsymbol{\Sigma}} \boldsymbol{\Gamma}, \overline{\mathbf{P}}-$ for $\mathbf{E P E}-$ and $\boldsymbol{\lambda} \mathbf{P}-$ for $\boldsymbol{A P E}-$ ). In CWTE for CWT it is added.

The following are examples of $\mathbf{O}$ for $\mathbf{O Y}$, $\boldsymbol{w}$ for $\mathbf{O Y}$, and $\mathrm{OY}$ for $\boldsymbol{\omega}$ : KONG for KOYTI (p. TाH), NO2M for NOY $\overline{2^{M}}$ (pp. $\overline{\mathrm{NZ}}$, $\overline{\mathbf{N H}}$ ), ENANOOY for ENANOYOY (p. $\overline{\mathrm{OA}}$ ). $\overline{\mathbf{W}}$ for OY (interroga- 


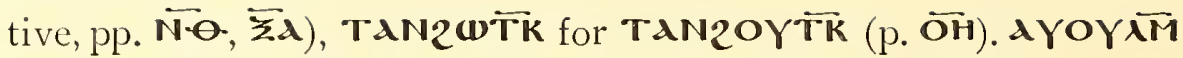

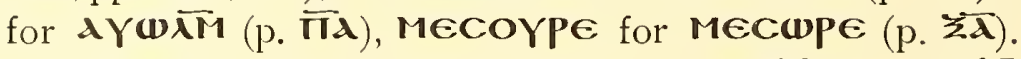

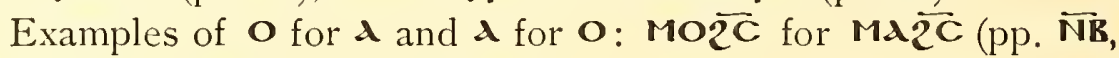
NE), גN for ON (several times).

There are the usual confusions between the vowels and diphthongs $\boldsymbol{d}, \boldsymbol{A l}, \mathbf{\epsilon}, \mathbf{E I}, \mathbf{H}, \mathbf{I}, \mathbf{O l}, \mathrm{Y}$.

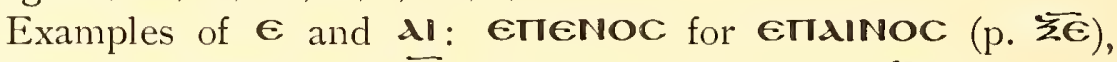

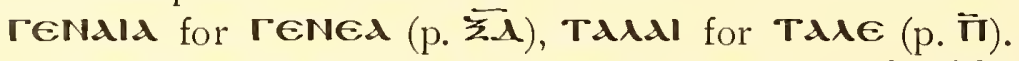

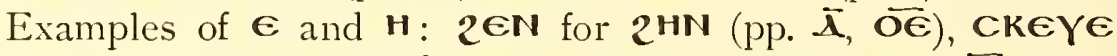

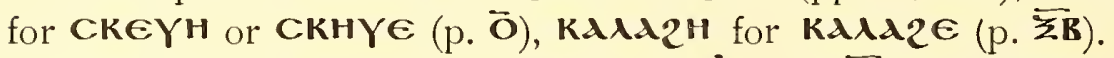

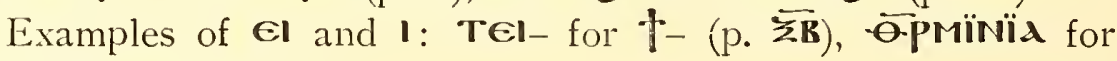
e.epmhneld (p. $\overline{\boldsymbol{\lambda}}$ ).

Example of $\mathbf{E l}$ and $\boldsymbol{H}$ : проскүNH for проскүNel (p. $\overline{\boldsymbol{\lambda}}$ ).

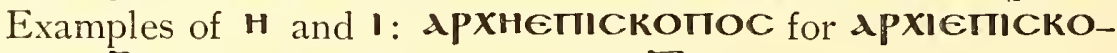

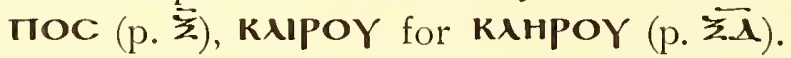

The interchange of $\mathbf{H}$ and $Y$ is constantly occurring.

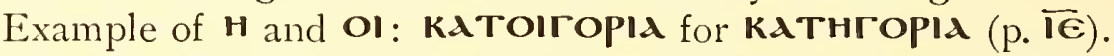

There is a single instance of $\boldsymbol{A}$ for $\boldsymbol{A l}$ and of $\ddot{\mathbf{i}}$ for $\boldsymbol{\epsilon}$ : גIIOQANE for ATIOQAINEI (from the aorist? p. $\overline{\mathrm{OB}}$ ), $\sigma i \mathrm{i}$ for $\sigma \in($ p. $\vec{\Gamma})$.

The combination $\mathbf{T} 2$ is usually rendered by $\mathbf{T} \cdot \boldsymbol{O} \cdot \mathbf{T}$ : $\mathbf{O} \cdot \mathbf{A} \mathbf{I} \mathbf{A}$

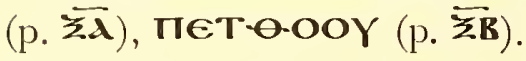

The aspirate 2 is lost in coyo $\overline{\text { T }}$ for czoyopT (p. $\overline{\mathbf{H}}$ ), and superfluous in 2גPHY for גPHY (р. זіZ).

\section{v. Peculiarities of Inflection, Construction, and Vocabulary}

On p. $\bar{\Sigma} \boldsymbol{\Gamma}$ three times and on p. $\overline{\mathrm{OE}}$ once $\overline{\mathbf{T P}}-$ occurs for either $\mathbf{T} \boldsymbol{\epsilon}$ - of the first present or EP€- of the second present, second person feminine singular; and its persistent use excludes the explanation of error.

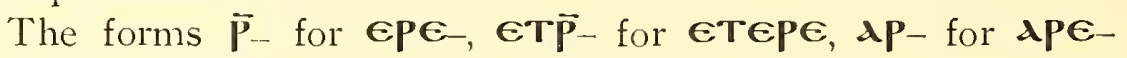
occur all on p. $\bar{\Sigma}$ with the peculiarity alluded to in the preceding paragraph; and they occur nowhere else in the manuscript.

The form TdxpoeiT for TdxpHy (cf. Boheiric TdxPHOYT) is found on p. बढร.

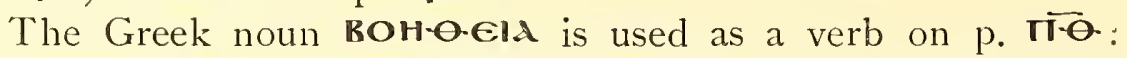

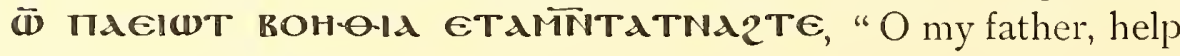
thou my unbelief." ${ }^{1}$

${ }^{1}$ Cf. Crum, Catalogue of the Coptic Manuscripts in the Rylands Library at Manchester, 1909, p. 16r. 
PLA'I'E IX.
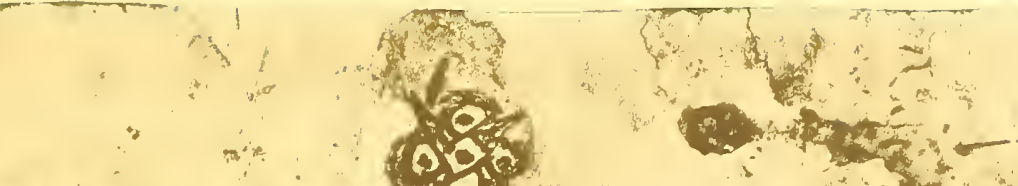

$-180$ hir IAY Nethen

and

$6 \mathrm{CH}, \mathrm{CO}$

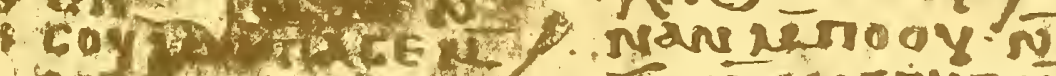

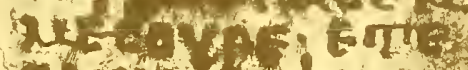

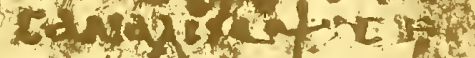
THUdB $N$, a

Xirte zuă

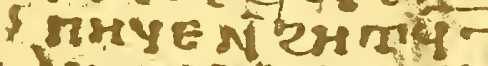
reterydx 26 on.

- Egrboypwre

SBAdpor Enayn

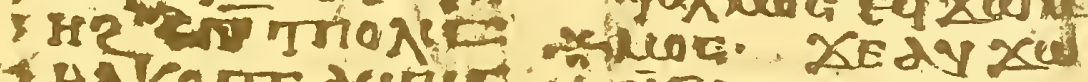
OIIPINREEYEN IEQEOTOIOCE

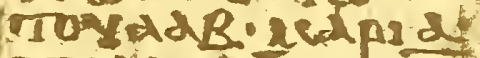
Trudayierre: $x p$

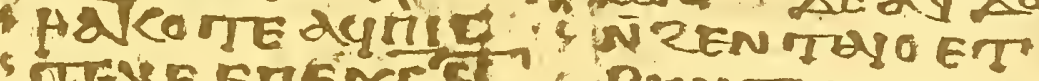
TIGY EREXEE

- BEOEI JCUNO NT

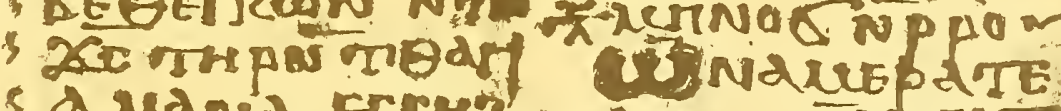

a Mapia Ecem?

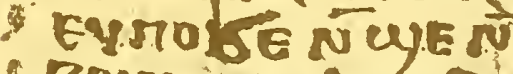

- ZOYN- EVPAT QY

164 depaleze 21 रer IN

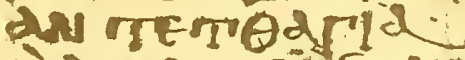

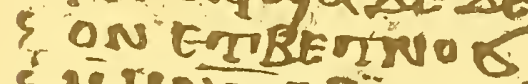

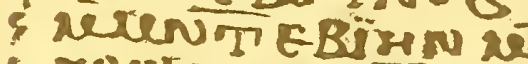

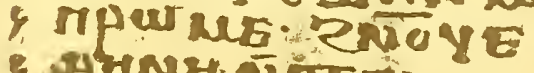

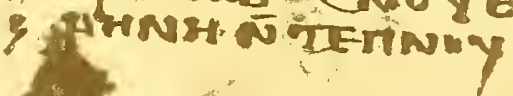

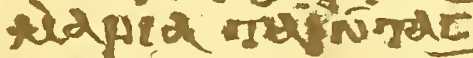
IPo कH TIE E IaYd $2 R$ 2NA. Eी wey ERAX ZNंN है

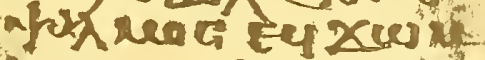
7
-4
-18

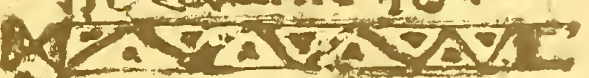



EாICкOாION (p. $\overline{\mathbf{O Q}}$ ) is treated as feminine.

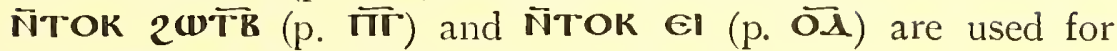

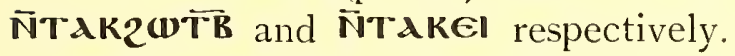

The second future tense is used for the third future in

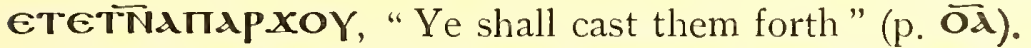

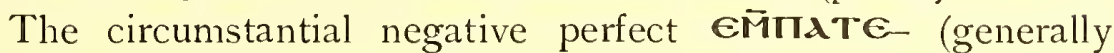

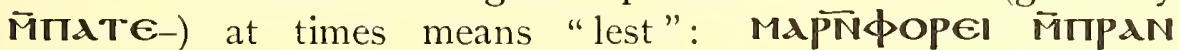

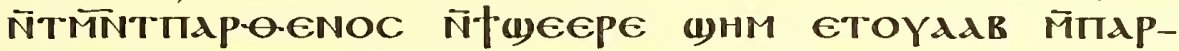

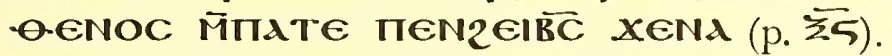

In relative clauses the preposition and its object when tem-

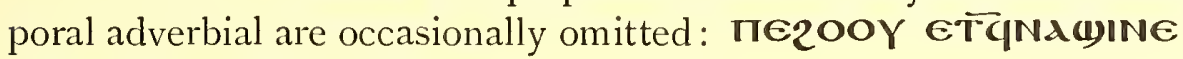

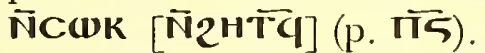

GITINAPION is evidently to be read GINTINAPION = $\kappa \epsilon \nu \tau \eta \nu a ́ \rho \iota \nu^{1}$ or $\kappa \epsilon \nu \tau \eta \nu a ́ \rho ı s=$ centenarius, cf. Crum, Catalogue of the Coptic manuscripts in the British Museum, pp. 259, 43 I, 462, 463. 477, 500; Crum, Catalogue of the Coptic Manuscripts in the Collection of the John Rylands Library, Manchester, pp. I06, 2 I9; Van Herwerden, Lexicon Graecum Suppletorium et Dialecticum.

The following words or expressions are new or strange in form or meaning:

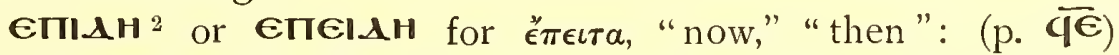

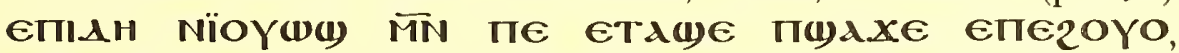
"Now I wished to prolong the discourse yet more." (p. $\overline{\mathbf{k} \lambda}$ )

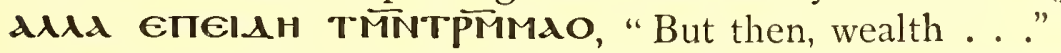

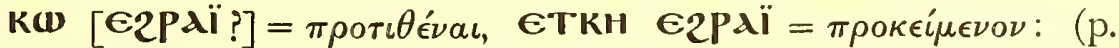

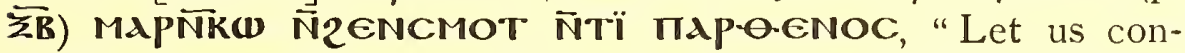
sider certain aspects of this Virgin"; (p. $\overline{\mathrm{OS}}$ ) 入OITION MAPNKTON E.XXN ITEITPKYMENON ETKH NAN TENOY, "But let us return to the subject which now lies before us for consideration."

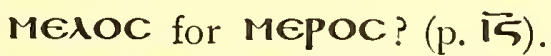

MONH, stage of a journey (p. $\overline{\mathbf{I}})$.

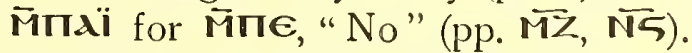

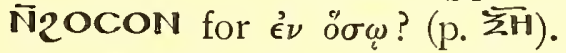

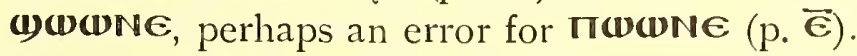

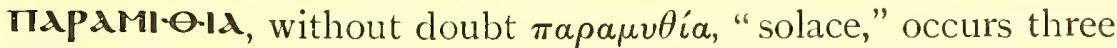

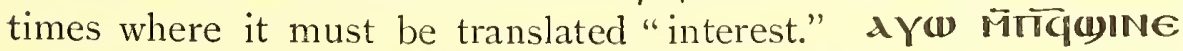

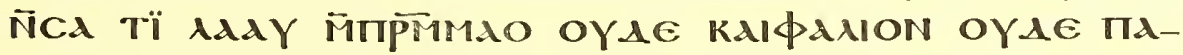

${ }^{1}$ I am indebted for this emendation to Mr. W. E. Crum, who has written me also that "this is a well known Byzantine weight (generally of coin), a "hundredweight" in short."

${ }^{2}$ Cf. Crum, Coptic Manuscripts in the Rylands Library at Manchester, p. 126 EחITd = Ula 
PAMï-öd (p. $\overrightarrow{\mathbf{k}}$ ), "and he sought not to give the rich man anything either in the way of principal or interest." ג пртммдо

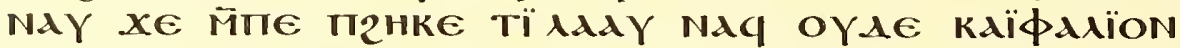

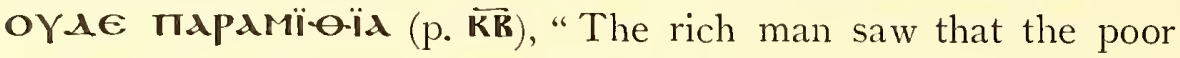
man would not give him anything either in the way of principal or

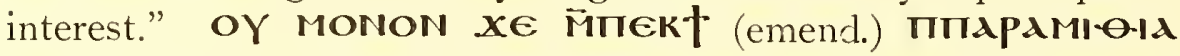

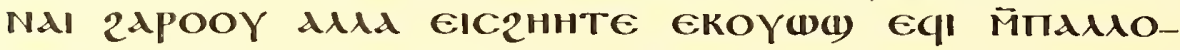
TPION (p. $\widetilde{\mathbf{K T}}$ ), "Not only wouldst thou not give me the interest on them (i.e., the denarii), but thou wouldst actually take what is the property of another (i.e., the principal)." 1

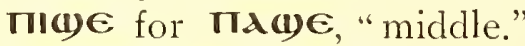

CWMNT for CWNT, "creation" (p. $\overline{\mathbf{O}}$ ).

TI (1)MNOYce, "to bear good tidings" (p. $\overline{\mathbf{\theta}}$ ).

TON for GTWN, "whence" or "whither" (p. MH).

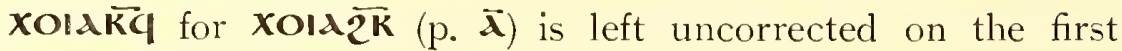
page of the manuscript, in the title.

(1)MON perhaps $=2$ MON $(\mathrm{p} . \widehat{O K})$.

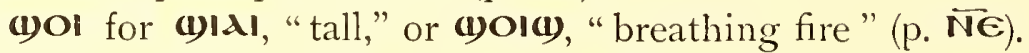

2А2THN for 2A2TN (pp. $\overline{\mathrm{TE}}, \lambda \mathrm{H})$.

2їве for 2lalBE (p. İ̈).

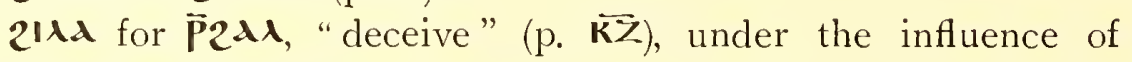
Arabic حيلة, "a trick."

xo€ for $\boldsymbol{x O}$ (p. īi), cf. Boheiric $\boldsymbol{x O I}$.

\section{vi. The Homilies}

If the two homilies contained in the manuscript are indeed of Theophilus of Alexandria and Celestine of Rome, they are, with their allusions to Cyril and Nestorius, of great interest to the student of the history of dogma and homiletics, and even to a wider circle of readers.

The precise dating and final evaluation of the homilies must await fuller evidence. The reader who is accustomed only to the language of the New Testament and to the modern types of homiletic address in the various Christian denominations, may find himself at a loss to understand the purpose and content of these ancient Coptic homilies. Unfortunately the internal evidence is not sufficient to warrant a conclusion regarding the century in which they were produced.

We may be warranted in observing, however, that the free use

${ }^{1}$ He feels that the principal belongs to him in a truer sense than the interest. It is not uncommon for Copts at the present day to lend money to members of their own families for educational purposes at ten per cent, payable yearly. 
of allegorical interpretation at the beginning of the homily on the Virgin seems to imply the continuation of the Alexandrine tradition. Origen himself held that Scripture has three senses; a grammatical, a moral and a spiritual or allegorical; and it seems to be clear that he considered the allegorical of great if not of relatively the greatest importance. Consistent with such a view was the practice of applying texts of Scripture to a kind of mosaic composition around personal or institutional conceits with which they could not be connected by what seems to us today to be a sound exegesis.

The unquestioning acceptance of the Theotokos doctrine in the homily on the Virgin might well be expected of Theophilus and his contemporaries. On the other hand the allusions to the beliefs and practices associated with the intercession of saints and angels and the use of ikons, are not sufficiently definite to furnish evidence of the period of composition.

In writings of this nature we expect to find traces of idioms or transfer of meanings which would point to an original composition in another language. Thus far I have not been able to collect such evidence as would warrant the conclusion that either homily was not composed originally in the Coptic language.

It is not improbable that further study will dissociate these homilies from Theophilus, who died in 4I 3 A.D., and Celestinus, whose death occurred in 432 A.D. The only certain date is fixed by the colophon of 975 A.D., which shows that both homilies were composed before that date.

The content of the homilies, while full of human interest and of great value for the interpretation of the intellectual life of the period in which they were composed, is not free from the faults of composition which one expects to find in similar works of antiquity. We find a somewhat limited range of ideas, a restricted vocabulary, the heaping up of anecdotes, and a free use of apostrophic exclamation, together with a certain degree of repetition and exaggeration. On the other hand both preachers are fearless in attacking the vices and the petty faults of the people, such as coming late to church and leaving before services are over, and both discourses are pervaded by a moral idealism and earnestness which is worthy of all praise.

I am indebted to Professor A. E. R. Boak of the University of Michigan for the opinion that "The title King, i.e. Basileus, was used throughout the Eastern Empire from the fourth century, although it was not employed in strictly official documents until 629 A.D. Since Egypt was not relinquished to the Saracens until 
64I, it would be natural for an Alexandrine writing before that date to speak of the image or the statue of the Basileus as still existing there. This will leave you a period of 300 years during which such a statement could have been made." The inference is that while the homily on the Archangel may or may not be authentic, it was probably composed before 64I A.D.

\section{vii. Manner of Edition}

Following the practice adopted in the first part of this volume, the manuscript has been reproduced line for line. The original relative position of the words has been preserved as far as possible. Missing portions of the text have been supplied conjecturally and indicated by [] . Letters which can be recognized from the context, but which are not independently recognizable, are indicated by $\ulcorner$. In the nature of the case critics may not always agree with the editor's use of the latter in every case. No superlineation is employed in the text unless actually visible in the manuscript. The superlinears are placed in a standardized position, and not far to the right, as in the manuscript. The word separation, as in the first part of the volume, aims at a medium between the usage of Steindorff on the one hand and that of Budge on the other; but consistency is difficult to maintain. The scriptural references follow the Septuagint version in the Old Testament. Ornaments have been described in the introduction, but omitted in the printing. Instead of reproducing the single dot between words, in its various positions, as was done in the first part, the evident fact of complete confusion in the usage of the scribe has led the editor to omit it entirely.

In the interests of those who, while not being acquainted with Coptic in any degree, may wish to employ the translation, or even follow the same in the original text, the annotations have been made somewhat fuller than would otherwise have been necessary. The notes on the Coptic text are concerned with the readings only. All other matters are treated in the notes to the translation.

The Coptic page numbers run ahead one unit from p. $\mathbf{\lambda} \overline{\boldsymbol{r}}-\mathbf{M Z}$ (actual p. $3^{2-p .46)}$, as there is no p. $\overline{\mathbf{A B}}$ and there are two pp. $\overline{\mathbf{M Z}}$. The references to pages by their Coptic numbers give the number on the page and not the number which should be there.

\section{viii. The Magical Text}

Nothing is known of the provenance of fragment No. Io beyond the fact that it was acquired along with the homily manuscript. 
It consists of a single leaf, c. $20 \times 3 \mathrm{I} \mathrm{cm}$., folded in the middle, as if for binding, so as to form two opposite pages, the reverse side remaining blank. Apparently it is not part of a volume; and yet it has never been folded or rolled compactly as are amulets. The material is thick vellum, very stiff and hard; and it has been eaten away considerably in many places (Plate XII) by some worm or insect, as is seen from the small specks surrounding the larger lacunae.

The writing, which almost entirely covers both pages, and which is from a single hand, with the exception of the letters $\mathbf{X I}$, p. i, l. 3, is rather smaller than that of the homily manuscript, stiff, inelegant, and unpractised. The forms of some of the letters are extremely peculiar, the added tail of the (I) and $\sigma$ being noteworthy. Considerable space has been left between letters. The lower third of the second page is occupied with letters, signs, ${ }^{1}$ and obscurely written ligatures, enclosed in pen-strokes, which are probably the result of innumerable copyings of an unintelligible text, if not the meaningless additions of a conscious impostor.

There is no way of dating the fragment beyond recording the opinion that no manuscript in the Fayumic dialect is probably older than the ninth century.

There is no punctuation of any kind, except the occasional dotting of the $\mathbf{I}$ and the superlineation of $\overline{\mathbf{\Pi O C}}$ and the magical names of God.

The language of the text is a Middle Egyptian dialect. The bad condition of the parchment, the corruption of the text, the hasty slovenliness and inconsistency of the scribe, and the obscure character of the subject matter, make it difficult to set the text to rights, or to study the spelling and grammatical peculiarities. The meaning, however, is as clear as is usually the case with such documents; perhaps more so.

An examination of any small collection ${ }^{2}$ of Abyssinian amulets reveals at once the fact that these depend upon a Coptic-Arabic tradition which, strange to say, seems to have exhausted itself in Egypt. Abyssinian amulets, ancient and modern, are abundant. The modern Copts do not, I believe, write many amulets, in either

1 Three of these are of the "ringed" type found in Hebrew, Greek, Coptic, and Abyssinian amulets. The age and history of these are unknown. Cf. Fournal of the American Oriental Society, Vol. XXXI, p. 272; Zeitschrift fiir Assyriologie, Vol. XX, pp. 405 ff.; XXIII, p. 153.

${ }^{2}$ There are excellent specimens in Princeton, N. J., in the British Museum, and in Berlin. Cf. the present writer's article, Charms, Abyssinian, in Hastings' Encyclopedia of Religion and Ethics. 
Coptic or Arabic; and the number of Coptic amulets preserved out of the past is not great. ${ }^{1}$

The text falls roughly into two halves: the first bearing upon perils of the deep, and the second upon sickness. The scribe could hardly have omitted so many words, here and there, if he had not been copying from another manuscript.

1 See especially W. E. Crum, La Magie Copte, in the Champollion centenary Recueil d'Études Égyptologiques, Paris, 1922. 
A HOMILY ON THE ARCHANGEL GABRIEL BY CELESTINUS ARCHBISHOP OF ROME

(BM MsOr 7028)

p. I

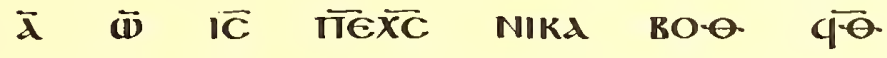

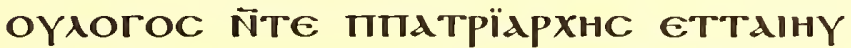

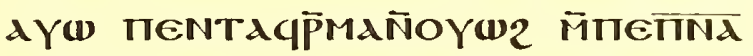

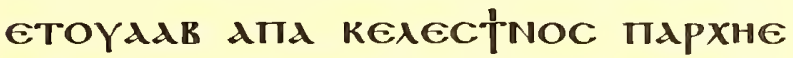

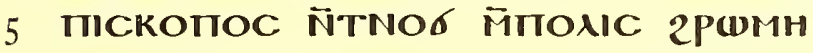

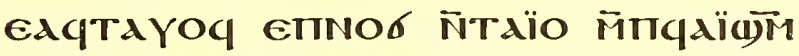

NOYGE ÑNIAİN M̄HOYOEIN MAPXar

гелос ГАврїнд $\overline{2 M}$ пе2ОоY їпєфшд

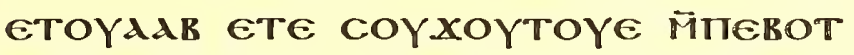

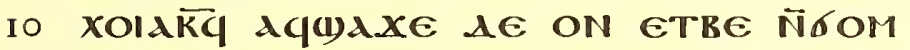

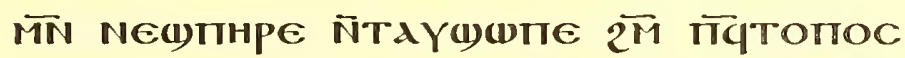

ETOYAAR TIÏ ÑTAYKOTG NAq $2 \bar{N}$ TIIO

NIC 2P(DMH AYW ON ETRE тा1)גхе ET

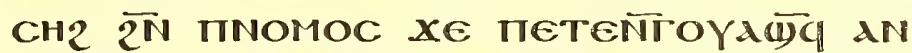

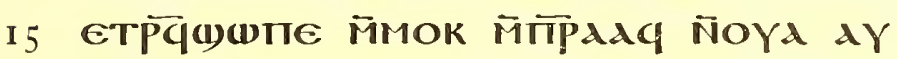

(1) ON Xe OYNOK ÑNOBE

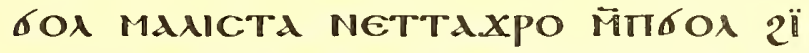

TM TINA(1) ÑNOYX AY(1) ON $x \in$ (1)ape

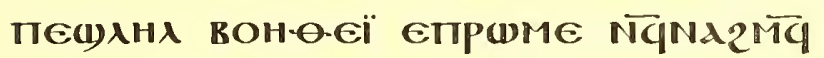

20 N̈бOPĞ

N̄TE INOYTE CMOY EPON 2AMHN $\overline{q . \theta}$.

Пе2MOT जिтाNOY (1) TqXapIC $\bar{p}$

ге (унтा [גY]

гдqсіке

9 post coyxoytoye suppl

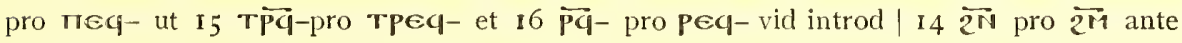

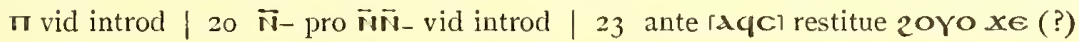


$\overrightarrow{\mathbf{B}}$

p. 2, col. i

TIZZE M̈MON AY

(1) АपР्PCORTT

EPON $\overline{2^{N}} \overline{T q} \operatorname{lox}$

NA2OPATON AC

5 N̄TN E2Pגï $\overline{2 M}$

TIKYKरOC N̈TE

POMTE $\bar{N} N O \overline{N 2}$

ACGOR2EN E2OYN

धПтопос M̈ாqגї

IO Ф'MNOYGE N̄

NAÏWN M̈TOY

OEIN חIPXAF

гENOC ETOYA

АB ГАврїн

I5 єтве тлї тї

Naxï Naï 2w

ज̄TEСМн $\bar{M}$

П2YMNOTOC

eTOYAdв $\overline{\Lambda A} \bar{\Lambda}$

20 Аүш пт̈पсеY2

OY $\overline{\mathrm{B \lambda} \lambda \epsilon}$ ETNO

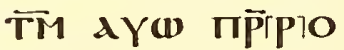

2 Аq $\overrightarrow{P C O B T N}$ sic pro Aq

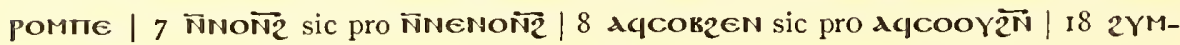

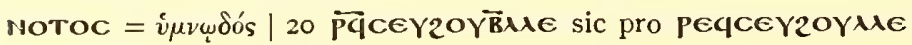


p. 2, col. ii

$$
\begin{aligned}
& \text { N̄AÏKAÏOC ĩ } \\
& \text { TAXOOC } \overline{2 M} \text { חय } \\
& \text { IAC Ñ2HKE XE } \\
& \text { пגї пе пє2оOY } \\
& 5 \text { N̄Td п } \overline{X C} \text { TגMï } \\
& \text { Oq MAPNCWOY2 } \\
& \text { N̈TNTEAнג } \bar{N} \\
& \text { TNOYNOQ } \bar{M} \\
& \text { MON N̄2HTQ } \\
& \text { IO ש)גqXOOC ON XE } \\
& \text { ÏOYAd APÏ NEK } \\
& \text { (a) A AY(1) NEK† } \\
& \text { ÑNEKEPHT } \\
& \text { ETRE Пגї еqCMA } \\
& \text { I } 5 \text { MdA N̄GI TNOY } \\
& \text { TE XE AqAaN T̃ } \\
& \text { A } \mathrm{N} I O C \text { ACCOB } \\
& \text { ZEN E2OYN E } \\
& \text { птопос जिता } \\
& 20 \text { оїкONOMOC } € \\
& \text { TOYAAR AYW } \\
& \text { Tावдї̈MNOYGE }
\end{aligned}
$$

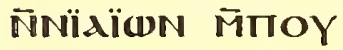

$$
\begin{aligned}
& \text { OEIN TIPXATГE }
\end{aligned}
$$

4-9 Ps cxvii, 24 (Budge: om T̈moN) | II-I3 Nahum i, 15. post Api videtur supplend wa $\overline{2 \pi}$ of Homil Liberii a Lefort editam (Muséon N.S. XII, I9II, p 12) | 17 Ассов2еN sic pro AqсOOY $\overline{\mathrm{TI}}$ 
I 32

p. 3, col. i
COPTIC HOMILIES

AOC ETOYAAR

ГАврїнд MA

PNCWOY2 $6 \ddot{\mathrm{i}}$

ТN OYT'BBO

5 Nं2HT MN OY

TिBO ĨCW

MA $\overline{\mathrm{N} T T} \overline{\mathrm{P}}(1)$

Mัпाєтере

INOYTE MNN

Io NंqагеАОC

P(1)d Naq

MAPNNOYXE

ÑCABOA $\bar{M}$

MON N̄TOAM

15 NIM MTN $2 Y$

покPICIC NIM

जัTत्रि(1) M

тाАрхагте

入OC ГАвPIHA

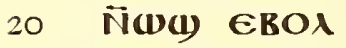

Nंxw M̈MOC

MN п2Yеро

†лАтнс $\overline{\text { AdA }}$

$36 \mathrm{i}^{\circ}$ sic pro $6 \mathrm{E} / 4 \overline{\mathrm{TB}} \mathrm{BO}$ prius scriptum $\overline{\mathrm{TB}} \mathrm{TO}$ 
p. 3, col. ii

$$
\begin{aligned}
& \vec{\Gamma} \\
& \text { XE CMOY Eா } \overline{X C} \\
& \text { NeqAгTEAOC TH } \\
& \text { PoY } \bar{N} x \omega a p e ~ \overline{2 N} \\
& \text { TeYбOM eтeIpe } \\
& 5 \text { Мтеүज्यА }
\end{aligned}
$$

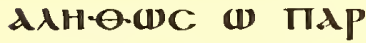

$$
\begin{aligned}
& \text { xArTEAOC ETOY } \\
& \text { AdR ГARPIHA OY } \\
& \text { No6 пе пाєOOY } \bar{N} \\
& \text { IO TA IINOYTE TA } \\
& \text { Aq NAK IIPA } \\
& \text { тАгГелIкH Тн } \\
& \text { PC } \bar{N} A C \omega M A T O C
\end{aligned}
$$

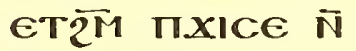

$$
\begin{aligned}
& \text { I5 ज̈пнүе } \omega \text { пגP } \\
& \text { XATREAOG N̄TAY } \\
& \text { MOYTE EPOK XIN } \\
& \text { NGOPTI EாEIPAN } \\
& \text { 2ITM INOYTE } \\
& 20 \text { ХE гАврїна тג }
\end{aligned}
$$

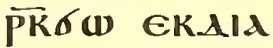

$$
\begin{aligned}
& \text { KONË̈ єாEXTO } \\
& \text { M̈ாINOYTE пגO }
\end{aligned}
$$

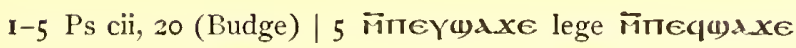


p. 4 , col. i

$\vec{\lambda}$

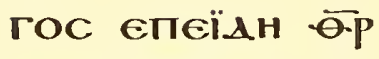

Mïnïd N̄гАk

PIHA пе NOYTE

2ï pWME KdTd

5 птҮாоС M̈ாеN

$\overline{X C}$ NTTAqE $\overline{2 N}$

oYe-RBIO Aq

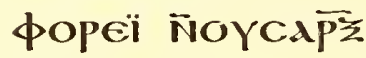

ЄTRHнTN $E Y$

IO NOYTE TE 2 P

ME M̈ாЕ TqMNT

NOYTE пथP्X

ETपMNTPसME

NOYPIKE N̈RAd

I 5 ME reNOÏTO dג

dA EqO ÑNOY

TE 21 PWME $\overline{2 N}$

OY XWK 21 OY

COाT $\overline{2 N}$ TKגAd

$202 \mathrm{H} \overline{\mathrm{N}} \widehat{\mathrm{Tq} M A \lambda Y}$

АCXTIOY EपO

ÑNOYTE 21 PW

ME АY $\overline{\mathrm{CPOY}} \overline{\mathrm{M}}$

MOQ EqO ÑNOY

25 TE 2 I PUME

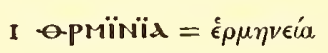


p. 4, col. ii

$$
\begin{aligned}
& \text { ACIP TCAP立 ÑYA } \\
& \text { NOYWT } \overline{2 N} \text { TMNT } \\
& \text { NOYTE } \overline{2 N} \text { OY } \phi Y \\
& \text { CIC N̄OYWT } \bar{N} \\
& 5 \text { АTाTलPX ÑAT } \\
& \text { 山וRE ÑdTOYE } \\
& \text { EBOA N̄NEYEPHY } \\
& \text { AMOY TENOY } \overline{\mathbf{w}} \\
& \text { N̄TEPIOOC } 1 \mathrm{~d} \\
& \text { io TNOYTE NAME } \\
& \text { АYळ ПАТТАПтО } \\
& \text { N̈(OYTOMC } \overline{N T} \\
& \text { cii ñyItTE AY } \\
& \text { (b) NTACXYMONH } \\
& \text { I } 5 \text { AMOY TENOY } \overline{N T} \\
& \text { NAY ETाPPO пе } \\
& \text { XC mIOYA miOY } \\
& \text { A ITE EqO ÑNOY } \\
& \text { te } 21 \text { PAME } \\
& 20 \text { PE NPिPEOY MN } \\
& \text { NCTPATEYMA } \\
& \text { NHY EYTिOCKY } \\
& \text { NH Naq AYW E } \\
& \text { PE TOÏKOYMENH }
\end{aligned}
$$

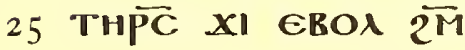

2 NOYaT prius scriptum ENYWT $\mid \overline{2 N}$ lege $\overline{m i n}$ cf boheir NEM in St. Bas. Anaph. (ed Labib p 407) AqAic NoYal NeM Teqmeanoyt (Vaschalde) 
p. 5 , col. i

\author{
TICGCOMA MTN \\ TTCNOY EYW(1) \\ EROX $\overline{2 N}$ TeфW \\ NH ज̈пMдкגрї \\ 5 OC ЄTM̈MAY \\ xe apї пIMEeY \\ $\epsilon \pi \overrightarrow{X C}$ EK(1)גN \\ Eï $\overline{2 N}$ TKMNT \\ ЄРО N̄TOK $\Lambda \epsilon$ \\ IO (1) IIAN2OCIOC \\ AKฒWWNE $\bar{M}$ \\ TEREOOY AYW \\ ERNAMOY $\overline{2^{N}}$ \\ TK乡OPIC|A \\ I 5 ETBE TKMNT \\ PQXIOYA $\overline{M N}$ \\ ПIEKAגC ET \\ ME2 M̈MATOY \\ AlAd тAPA Ke \\ 20 KOYї АNBWK

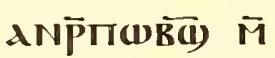 \\ пектגїо MN \\ пекєOOY $\boldsymbol{w}$ \\ ITNOK N̈APXAT
}

6-8 Luc xxiii, 42 ubi tamen Horner pro $\pi \overline{x C}$ habet $\overline{\mathbf{C}}$ sed cf Vulg memento mei

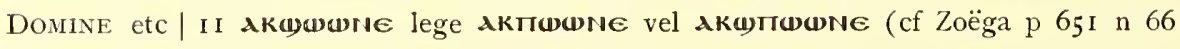
et Stern $\$ 97$ ) 
p. 5 , col. ii

\author{
$\overline{\mathrm{E}}$ \\ Гелос ГАврїн \\ ANAג MA NAN ÑOY \\ CYNTNGMH $x \in$ \\ тїбо $\overline{X B}$ EMATE \\ 5 शN NATPAそाC AY \\ (1) $\overline{M N}$ (1) $60 \mathrm{M}[\mathrm{N}]$

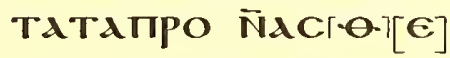 \\ NHC ЄX(1) M̈ாєK[є] \\ OOY TIÏ N̄TA TNIOY \\ IO TE TAAq NAK [W] \\ гАврїна пגрхаГГ

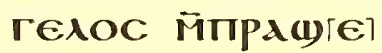 \\ d(1) ÑגdC ÑCdP方

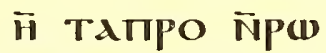 \\ I 5 N̈PWME петNА() \\ x(1) M̈пектגї \\ (i) тतITоYргос $\mathrm{e}$ \\ TOYAAB N̈TE TINO \\ ÑTe пाNoб ÑPPO $\omega$ \\ 20 пा2O ETUOYE \\ PAGE EROX (1) \\ ГАвPIHА пाАIE[MNOY] \\ qє N̈TAгтEAIK[H]
}

I3 2 prius scriptum $\boldsymbol{x} \mid$ I4 4 ĩpw a scriba (?) deletum | 18 îTe nro a scriba (?)

deletum | 20 плг2о lege папго 
p. 6, col. i

\section{$\overline{5}$}

(1) П.Ӓ̈коNїTHс

NAME ETAIAKO

Neï EтEXTIO M

ITq. $\overline{X C}$ ш пе2Pн

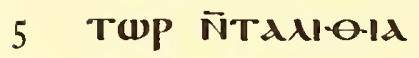

ITKPİ N̄TMNT

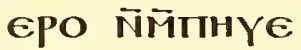

[T]OYW(I) ENAY e

POK \& пIAPXA

IO ГEגOC ETOYAגB

ГАврІн入 єкщ)

$x \in \overline{M N}$ TTIP-OE

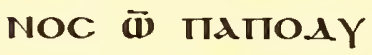

MOC N̄TATIOAY

I5 Mïд M̈ாNOYTE

ПாАNTшКРАTшP

ГNITMNTPAME

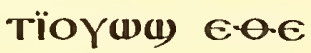

шреї ज̈MOK

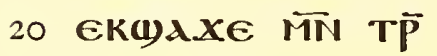

PW ÑNE2IOOME

"ToYwW ENAY $E$ 
p. 6, col. ii

$$
\begin{aligned}
& \text { пек2о ЕТ(IOYе } \\
& \text { PAdie EROA EK } \\
& \text { (1) } \lambda x \in \overline{M N} T E \\
& \text { TCOTT TIPA TITE } \\
& 5 \text { MT IIKA2 EK(1) } \\
& \text { erol ePOC xe xגï } \\
& \text { pe TNTacón } \\
& \text { 2MOT ПT्XC NM } \\
& \text { ME (1) пестPA } \\
& 10 \text { TOTIETAPXOC } \\
& \text { MIIPिPO N̄N̄P̄ } \\
& \text { OY } \\
& \text { M̈тtaldC N̄2H } \\
& \text { кE Ex山 MாER } \\
& \text { I5 GOOY ANA } \\
& \text { fNaxooc ema } \\
& \text { TE } x \in \text { qCMA } \\
& \text { MAAT N̄́I IINOY } \\
& \text { TE IINAHT ITN } \\
& 20 \text { TAqXOOC } \overline{2 N} \\
& \text { TqTampo } \bar{N} \\
& \text { NOYTE } X E \text { TIM } \\
& \text { eTEPE CNAY }
\end{aligned}
$$

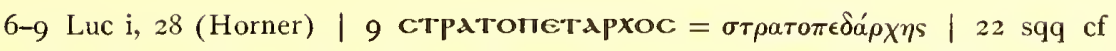
Mat xviii, 20 (Honner) ma rap eтере cNay н шойNт cooyz epoq emapan etc 
p. 7 , col. i

21 GOMNT $\bar{N}$

2HTС २T TAPAN

Т๘OOT NMMAAY

2N TEYMннте

5 Є()XE пNOYTE

()OOT MIN CNAY

H ตOMNT

NIM ПетNA()

хाнतе ज̈пеव

Io Pג®е M̃ாоOY

MN ПеяEIWT

N̈AГA-OOC MN

пEாNA ETOYA

AB EYNAY E

I5 ПЕINOб М̈MH

H(1) ÑARPOA

THC EYCWOY2

E2OYN EYteo

oY M̈т́тNó

20 N̄ApגАгтелоC

GTOYAdB rAB

Рінд пАрхАГ

гелос М̈трдае

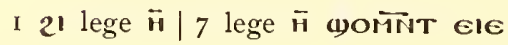


p. 7, col. ii $\bar{z}$

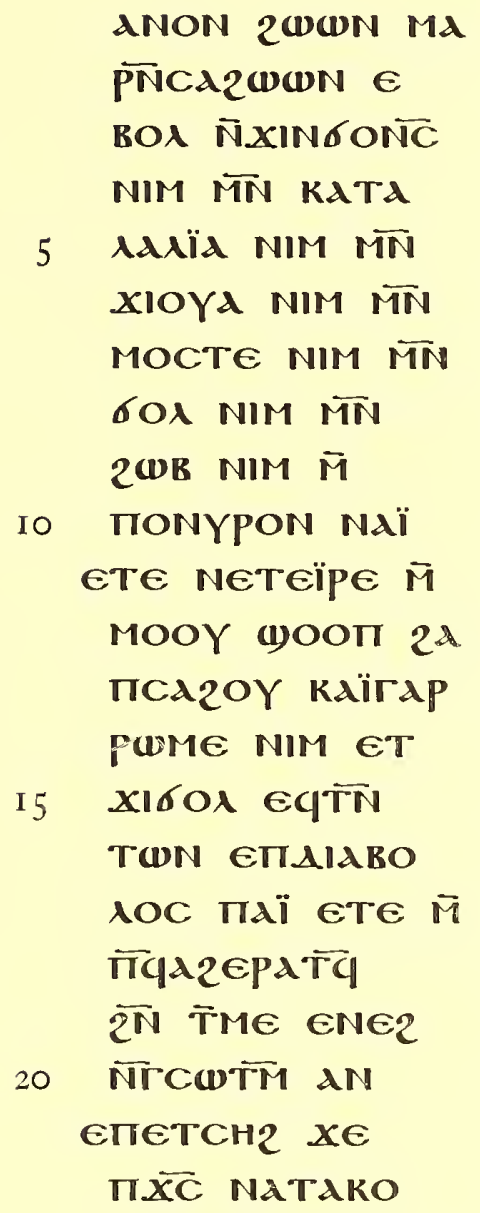




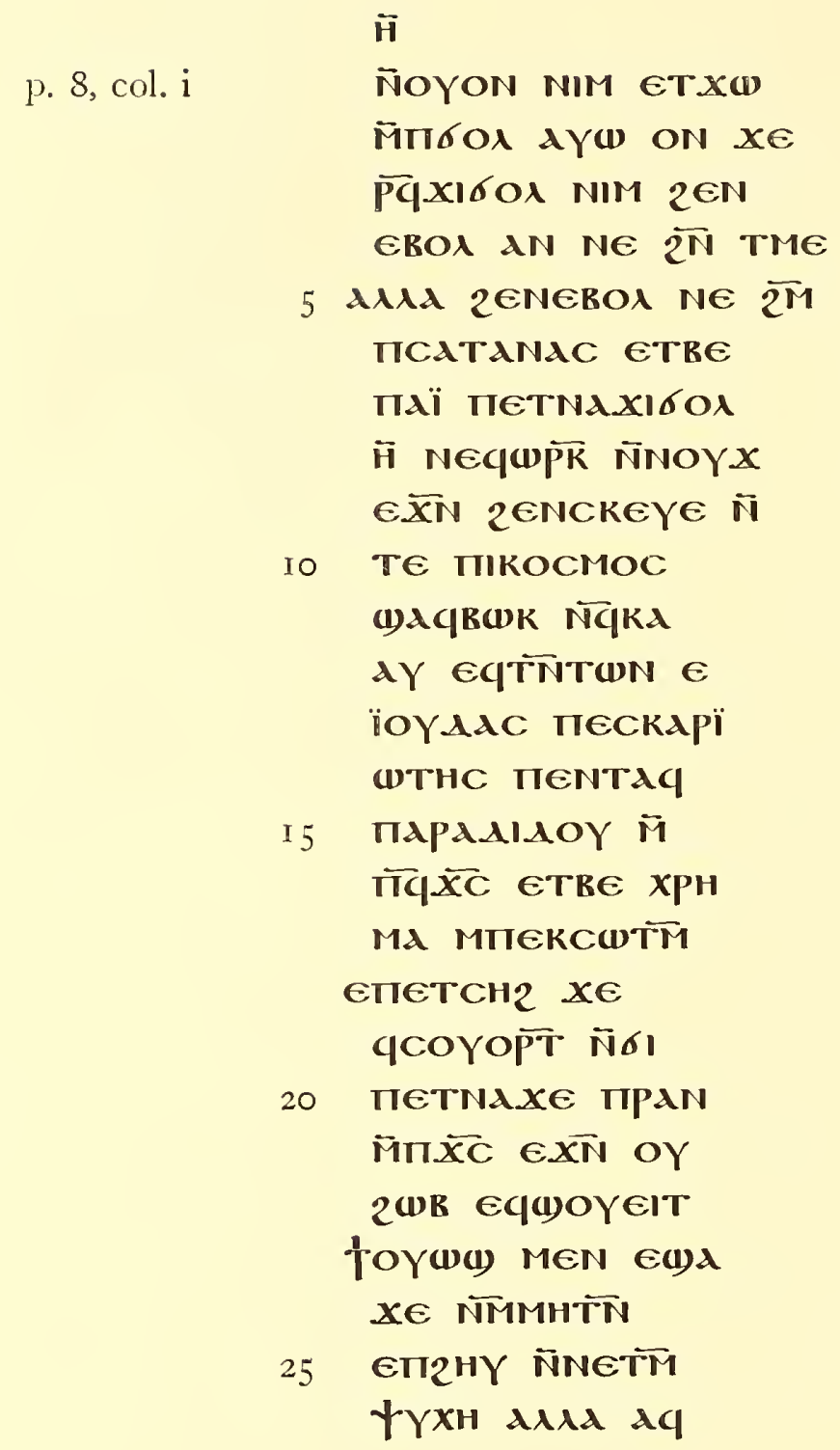

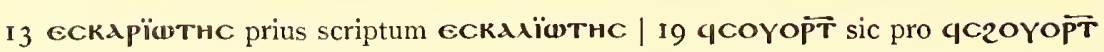

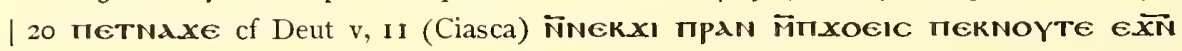
NoYzwr equoYeIT et lege петNaxı 
p. 8 , col. ii

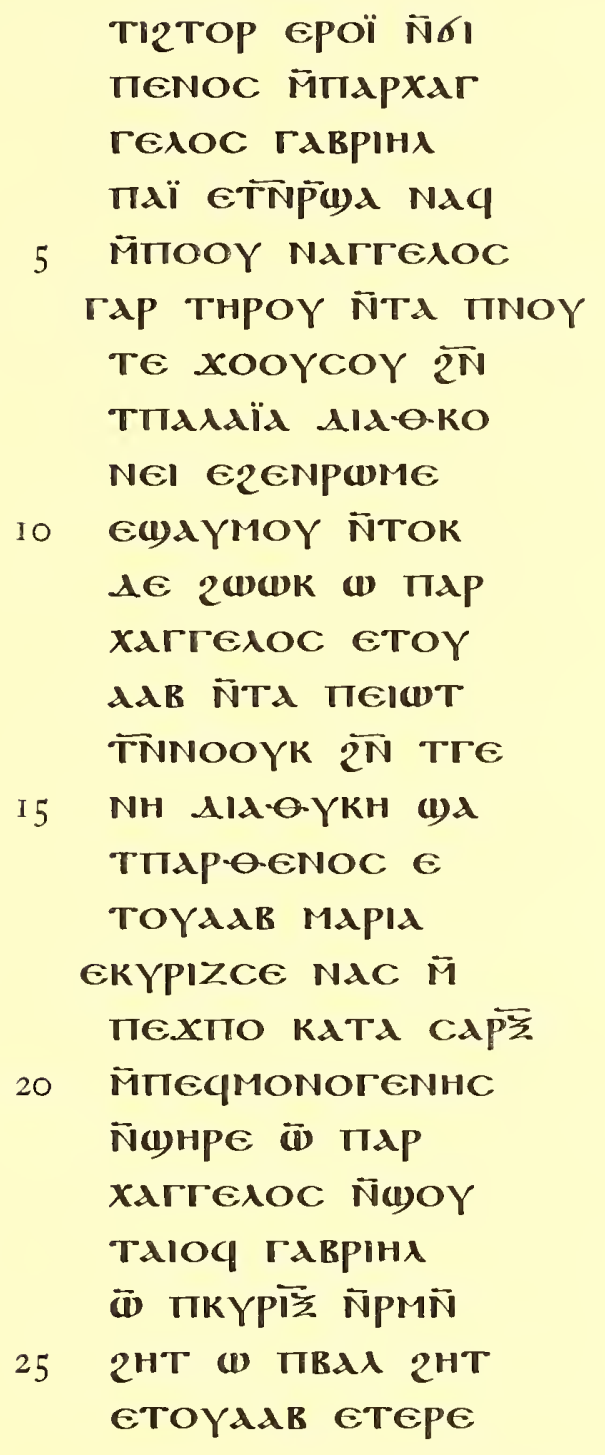


p. 9, col. i

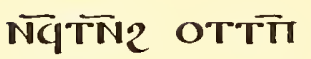 \\ ÑCTÏNOYGE \\ (i) ПЕкотнс \\ Потнс Єтсов \\ 5 TE AYW ET2Y \\ AYMAZE M̄ாнї \\ MாாENTAqCMTN \\ CENTE ÑTOIKOY \\ MENH THPC \\ IO A INOYTE $6 \mathrm{w}$ \\ (1)T EROX रM

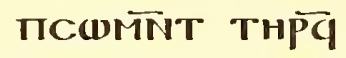 \\ जัтारह єпет \\ TTNTWN EMA \\ I5 PIA TM ПIEENOC \\ THPC ÑNE2IO \\ OME AपPि2NAG \\ EOYल2 \\ 2N OYOIKONOMï \\ 20 d (1)ANTqCu \\ TE MாாЕNIENOC \\ AqбW(i)T EROA

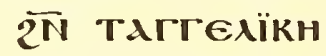 \\ тнре N̄Ñarге

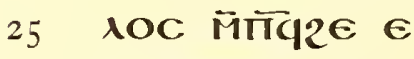 \\ ПETTNTWN \\ єрок $\overline{2 N}$ тоїко
}

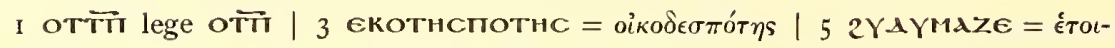

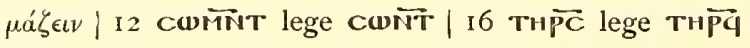


$\vec{\theta}$

p. 9, col. ii

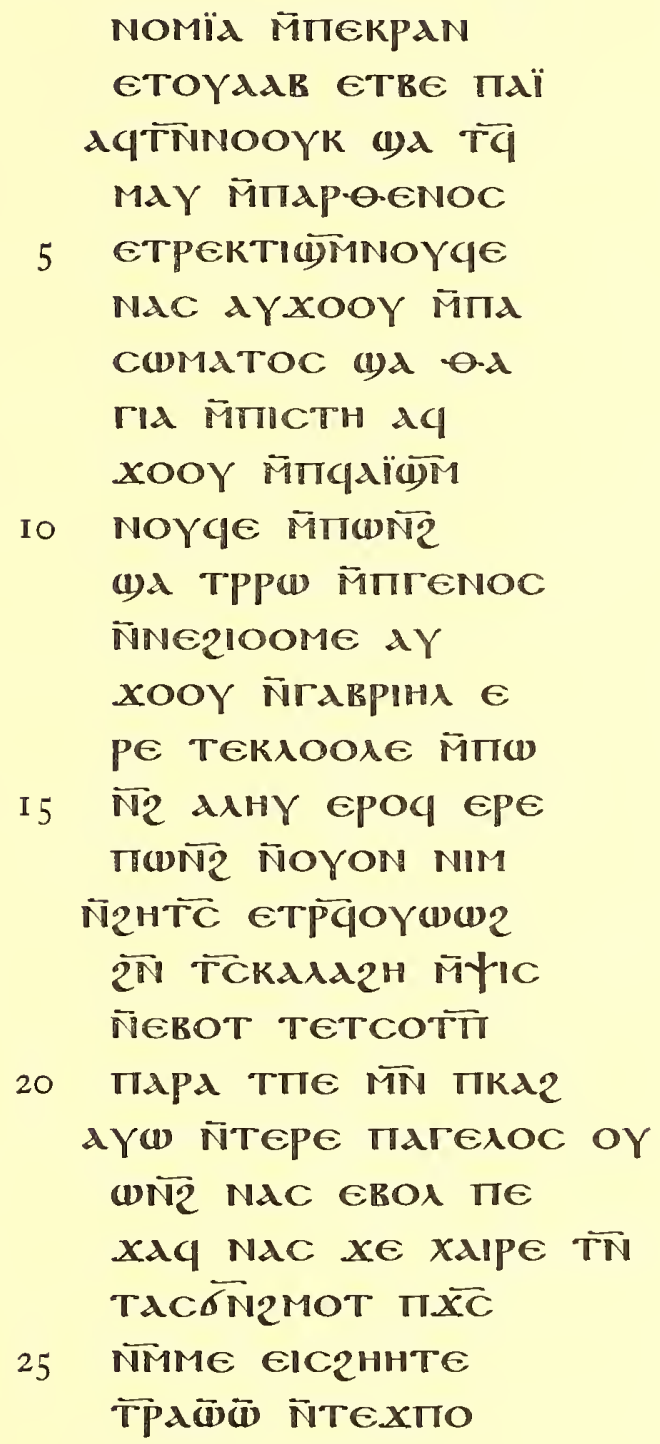

6 fortasse legendum aqxooy | I2 fortasse legendum aqxooy | I9 TETCoT⿱艹⿴囗十

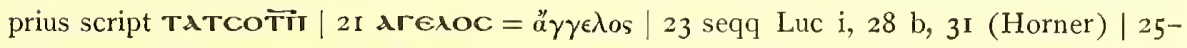

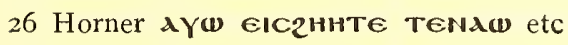


p. IO, col. i

$$
\begin{aligned}
& \text { i } \\
& \text { ÑOYฒHPE N̄TE } \\
& \text { MOYTE ETIAPAN } \\
& x \in \overparen{I C} \text { AY(1) N̈TEY } \\
& \text { NoY N̈TAqXe Nä̈ } \\
& 5 \text { NAC } \mathbf{A C \overline { \boldsymbol { i } } \overline { \mathbf { \omega } }} 2 \ddot{\mathbf{i}} \\
& \text { TTM TCWTM } \bar{N} \\
& \text { NCMAdxe MT TIC } \\
& \text { IACMOC M̈TIAPXA } \\
& \text { Гелос ГАврIHА } \\
& \text { Iо тाщн M̈ாNOY } \\
& \text { TE вШК EIIECHT }
\end{aligned}
$$

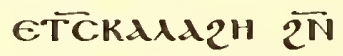

$$
\begin{aligned}
& \text { OүMNTATIME } \\
& \text { EPOC тїாגPAгג }
\end{aligned}
$$

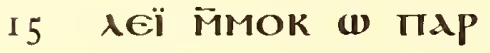

$$
\begin{aligned}
& \text { XATREAOC ETOY } \\
& \text { Аגв ГАвPIHA } \\
& \text { пqגї̈MNOYGe }
\end{aligned}
$$

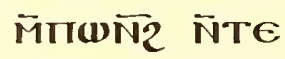

$$
\begin{aligned}
& 20 \text { РК Ё̈ ЕTMМнH } \\
& \text { TE MாTOOY } \overline{2 M} \\
& \text { TEINOK ज̄(1)d } \\
& \text { Єттор(1) Євоג } \\
& \text { ज̈ாOOY जٓTCMOY }
\end{aligned}
$$

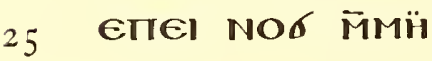


p. Io, col. ii

$$
\begin{aligned}
& \text { H(1) ETCWOY2 } \\
& \text { E2OYN TM חIEK } \\
& \text { PAN ETOYAdK } \\
& \text { Kגї̈גP EIC пеK } \\
& 5 \text { X्रC 2ג2THN } \bar{M} \\
& \text { TOOY AYW TIEN } \\
& \text { XOEIC THPN MT } \\
& \text { NEqAгтелOC TH } \\
& \text { POY ETOYAגK MIN }
\end{aligned}
$$

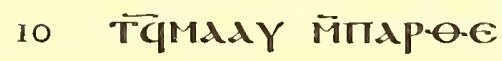

$$
\begin{aligned}
& \text { NOC EYP̈Wd NAK }
\end{aligned}
$$

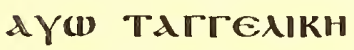

$$
\begin{aligned}
& \text { THP्P NंdCoMd } \\
& \text { TOC EYPAaE NM } \\
& \text { I5 MAK TM пाE2OOY } \\
& \text { MIIOYMN2 EBOA } \\
& \text { M̈ாекPAN ETOY } \\
& \text { AdB (1) ГABPï }
\end{aligned}
$$

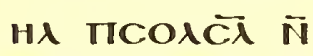

$$
\begin{aligned}
& 20 \text { TARгEAIKH AY } \\
& \text { (1) пा)OY(1)OY } \bar{N}
\end{aligned}
$$

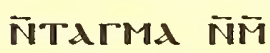

$$
\begin{aligned}
& \text { пाнYе OYNÓ } \\
& \text { TE TimH ÑTג } \\
& 25 \text { mNOYTE TAגC NגK }
\end{aligned}
$$

23 ornor te timm lege oynor te TfmH 
p. I I, col. i

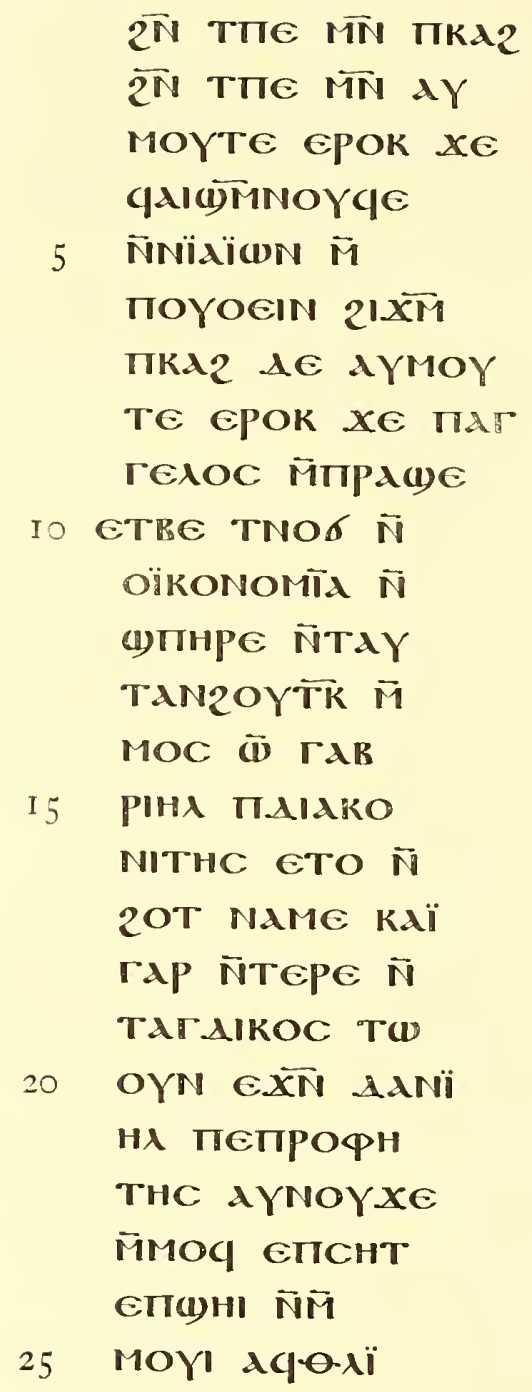


id

p. I I, col. ii

$$
\begin{aligned}
& \text { ве еMATE ETвE } \\
& \text { пегко МाN пाве } \\
& \text { TIAPXartedoc } \lambda \epsilon \\
& \text { GTOYAAB TABPI }
\end{aligned}
$$

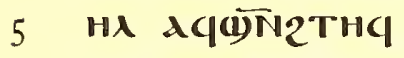

$$
\begin{aligned}
& \text { 2APOA 2ITN TKE } \\
& \text { АEYCIC MाTि } \overline{X C} \\
& \text { АqдMд2те } \bar{M} \\
& \text { піqW ÑTATIE N̄ } \\
& \text { IO AYAKOYM MTN TाA } \\
& \text { PICTON ETÑ } \\
& \text { TOOTq AqACT) }
\end{aligned}
$$

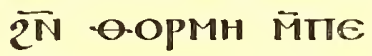

$$
\begin{aligned}
& \text { TINA XIN AIOY } \\
& 15 \text { AdA (I)d TRABY } \\
& \text { ION Ñ2METO } \\
& \text { ce MiMONH Aq } \\
& \text { XITY ÑTEYNOY } \\
& \text { Єп(1)нї ÑNIMOYI } \\
& 20 \text { aqt ت̈ாAPICTON }
\end{aligned}
$$

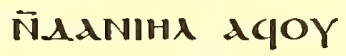

$$
\begin{aligned}
& \text { (DM AqCMOY } E \\
& \text { пNOYTE EqXu } \\
& \text { TिMOC } X E \text { АK户̈ }
\end{aligned}
$$

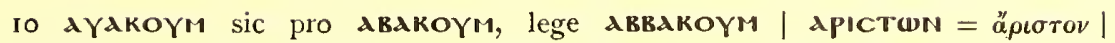

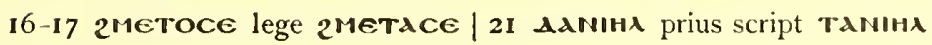


p. I 2, col. i

$\overline{\mathrm{IB}}$

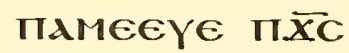

$$
\begin{aligned}
& \text { м̈теккW N̄CWK } \\
& \text { N̄NETME M̄MOK } \\
& \text { N̄TOG ON IIEN } \\
& 5 \text { Tдq(1)тגM N̄ } \\
& \text { TАПPO N̄M̄MOŸ } \\
& \text { xE ÑNEYENUX }
\end{aligned}
$$

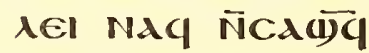

$$
\begin{aligned}
& \text { N̄2OOY EqN̄TE }
\end{aligned}
$$

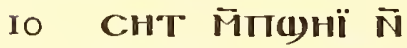

$$
\begin{aligned}
& \text { M̈MOYï ГАвpI } \\
& \text { нג пархагте } \\
& \text { AOC IIENTAGOY } \\
& \text { WN2 EBOA EAd } \\
& 15 \text { NIHX АqTCג } \\
& \text { BO4 Geopdcic } \\
& \text { KATA O.E ET } \\
& \text { Сн2 ХE ГАврї } \\
& \text { ha MATCABE } \\
& 20 \text { IIAI Goopdcic } \\
& \text { АY(u) } x \in \text { EIC прW } \\
& \text { ME ГАврIHА } \\
& \text { ACGE (1)גPoï גC } \\
& \text { тібоM NAï } \overline{\mathrm{N}} \\
& 25 \text { ब. N̄ฒOPா גq } \\
& \text { ГАвРІнА ПАР }
\end{aligned}
$$


p. I $2, \mathrm{col}$. ii

$$
\begin{aligned}
& \text { XATГEגOC IENTAG } \\
& \text { WÑ2 GBOA NZA } \\
& \text { xapidC } \overline{2 M} \text { пр̈пе } \\
& \text { גqKIPYZE NAq M } \\
& 5 \text { пехпо Nï̈口2АN } \\
& \text { NHC AYW ÑTET̈ } \\
& \text { PिAाICTOC АсдाтфА } \\
& \text { NE EXUQ ÑOYMT̃T } \\
& \text { M̈TIO ГАвPIHA } \\
& \text { IO ON TIENTAqOYW } \\
& \overline{N 2} \text { EBOl ÑNGOOC } \\
& \text { EqTגџ) }
\end{aligned}
$$

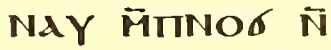

$$
\begin{aligned}
& \text { (ए)MNOYqE ETE } \\
& 15 \text { пе.Хாо пе M̈пNOY } \\
& \text { те плогос EBOA } \\
& \overline{2 N} \text { Te2їве M̈ME }
\end{aligned}
$$

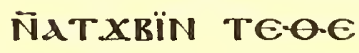

$$
\begin{aligned}
& \text { OTOKOC MAPIA } \\
& 20 \text { ГАвРІнА пАРХАГ } \\
& \text { гEגOC TENTAqEï }
\end{aligned}
$$

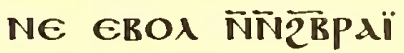

$$
\begin{aligned}
& \text { OC } \bar{N} T \overline{T K X M A \lambda O C I}
\end{aligned}
$$

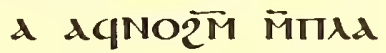

$$
\begin{aligned}
& 25 \text { OC } 2 M \text { TाKA2 } \bar{N} \\
& \text { TEYMT̃T2M2Aג }
\end{aligned}
$$

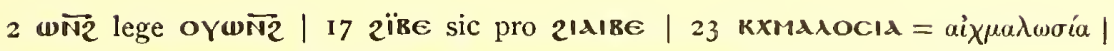

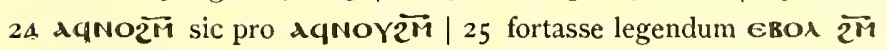


p. I 3, col. i

$$
\begin{aligned}
& \text { ATTOYXOOY 2N } \\
& \text { TEPIMOC AqEi } \\
& \text { pe ÑNeIMAEIN }
\end{aligned}
$$

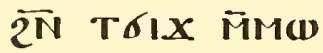

$$
\begin{aligned}
& 5 \text { ҮСнС ПАРХН } \\
& \text { профҮТнс } \\
& \text { ГАврннд ON те } \\
& \text { ПICTOגOфОРОС } \\
& \text { ETO Ñ2OT } \overline{2 N} \\
& \text { IO TMHHTE ÑTAT } \\
& \text { ГENIKH N̄TOG } \\
& \text { ON пе пqАा(i)M } \\
& \text { NoYqE } \overline{2 N} \text { TMH } \\
& \text { HTE N̄ÑATгEגOO } \\
& \text { I5 ГАвPIHA ON TाE } \\
& \text { ПMTАТшр } \bar{M} \\
& \text { TITANTलKPA } \\
& \text { тер Aүш пої } \\
& \text { KONOMOC } \overline{\mathrm{N}} \\
& 20 \text { TM̃NTEPO } \bar{M} \\
& \text { TIHYE } \overline{\mathbf{W}} \text { ГAB } \\
& \text { Рінд пслї̈м } \\
& \text { NOYGE ETO Ñ } \\
& \text { 2OT EPE NIM } \\
& 25 \text { Nd(1) } x(1) \text { M̈тек } \\
& \text { No6 ÑEOOY }
\end{aligned}
$$

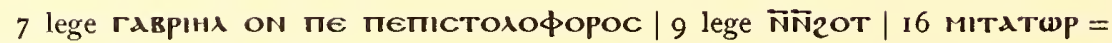

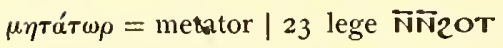


p. I 3 , col. ii

\section{p. I3. col. ii}

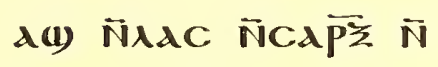

$$
\begin{aligned}
& \text { PWME N̄TAYWWTE }
\end{aligned}
$$

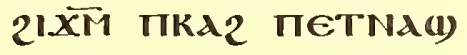

$$
\begin{aligned}
& \text { x(1) M̈TekNor N̄e } \\
& 5 \text { OOY Ф̈ं пIAPXAITE } \\
& \text { лос ГАврінд петА2 }
\end{aligned}
$$

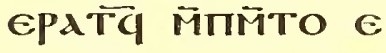

$$
\begin{aligned}
& \text { ROA MIINOYTE ÑOY } \\
& \text { OEI(1) NIM KATA } \\
& \text { Io OE NTTAK } \bar{P} \overline{M N T} \\
& \text { pe ÑZAXAPIAC }
\end{aligned}
$$

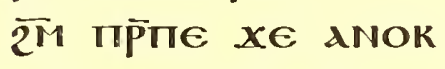

$$
\begin{aligned}
& \text { пе гАврIнд пета2 }
\end{aligned}
$$

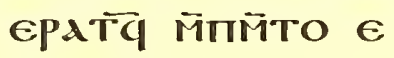

$$
\begin{aligned}
& \text { I } 5 \text { ROג MTINOYTE } \\
& \text { (a) } \bar{N} 2 H T \text { N̈PWME } \\
& \text { KAN OYCOфOC } \Pi \text { TE } \\
& \text { TIPA OYON NIM N̄ }
\end{aligned}
$$

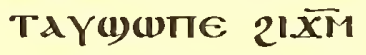

$$
\begin{aligned}
& 20 \text { пKג2 петNג(1) } \\
& \text { M̈фANHC-OAl NAN }
\end{aligned}
$$

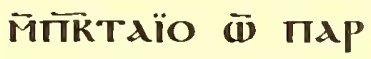

$$
\begin{aligned}
& \text { xATГEAOC ГАврї } \\
& \text { н入 ПАПгО GT(1) } \\
& 25 \text { EPд(1) EBOג } 21 \text { OY }
\end{aligned}
$$

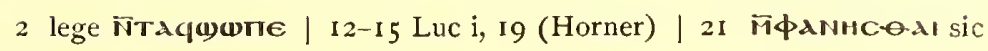


p. I4, col. i

ix

$$
\begin{aligned}
& \text { NOG KAI TAP MTN } \\
& \text { TגIO еqघнш) MN } \\
& \text { пектаїо евOג } \\
& \text { xе Екд2еРАTК }
\end{aligned}
$$

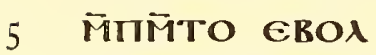

$$
\begin{aligned}
& \text { M̈ாNOYTE N̄OY } \\
& \text { OEI(1) NIM EK } \\
& \text { СОПत्ता М̈MOप } \\
& \text { E. } \overline{X M} \text { ITENOC TH } \\
& \text { Io } \widehat{\text { PQ }} \overline{\mathrm{N}} \lambda \Delta \lambda \mathrm{M} \\
& \text { AYa MIINAY } E \\
& \text { (1) N̄TAГMA } \\
& \text { THPOY N̄ÑAT } \\
& \text { reNOC MN NEï } \\
& \text { I5 XEIPOYREIN MTN } \\
& \text { N̈CEPAфEIN TIA2 } \\
& \text { TOY M̈ாTिTO } E \\
& \text { BOA M̈INOYTE } \\
& \text { eY† ÑTEニOMO }
\end{aligned}
$$

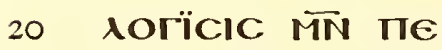

$$
\begin{aligned}
& \text { OOY MTN ITTAÏO } \\
& \text { MÑ TбOM M̀ } \\
& \text { пINOYTE пिРPO }
\end{aligned}
$$


p. I4, col. ii

$$
\begin{aligned}
& \text { NTTE MTN TIKA2 } \\
& \text { EY(W(I) EROA EY } \\
& \text { Xw M̄MOC ÑOY } \\
& \text { EI(1) NIM } x \in \text { kOY } \\
& 5 \text { AAR KOYAAR KOY } \\
& \text { AdR } \Pi \overline{X C} \text { CARA } \\
& \text { w-O. M̈THYE MTN } \\
& \text { пкג2 Me2 EROA } \\
& \text { 2M TEкOOY } \\
& \text { Io ตAPE IINOK } \bar{N} \\
& \text { АРХнСтрАті } \\
& \text { ГOC пाАїळM } \\
& \text { NOYqE M̈TIWÑ2 } \\
& \text { гАвPIHА MN пNOб } \\
& \text { I } 5 \text { ÑAPХHсTPАT } \\
& \text { ГOC MIXAHA } \\
& \text { IIAPXCWN } \bar{N} \bar{M} \\
& \text { пнYе घגYтा2 } \\
& \text { TоY їாїто } € \\
& 20 \text { ROג M̈INOYTE } \\
& \text { ET2MOOC } 2^{i} \\
& \text { IICA-PONOC EY } \\
& \text { Xw MMOC }
\end{aligned}
$$


p. I 5, col. i

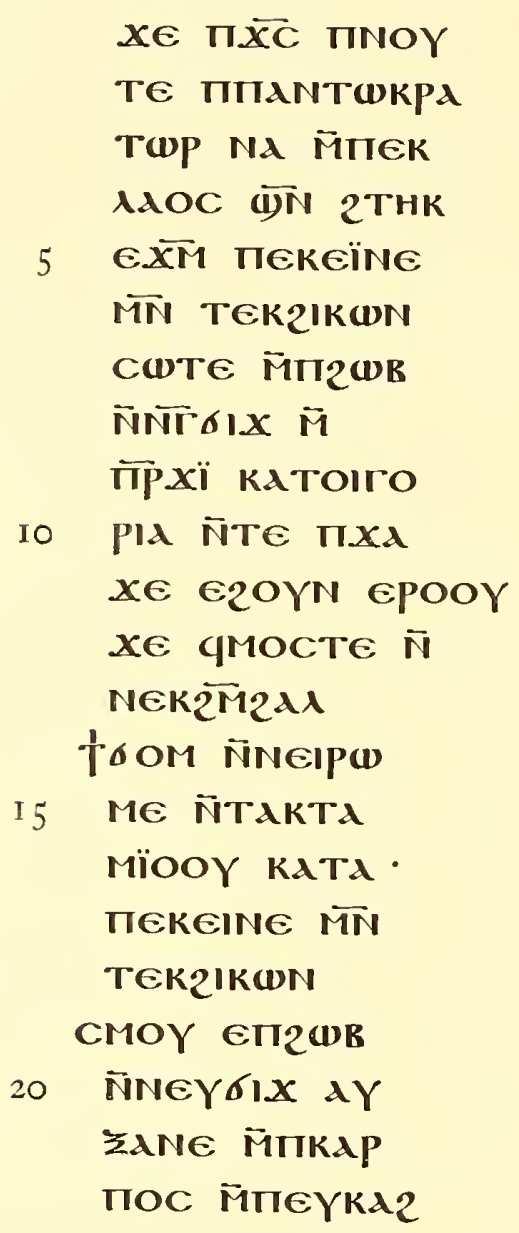

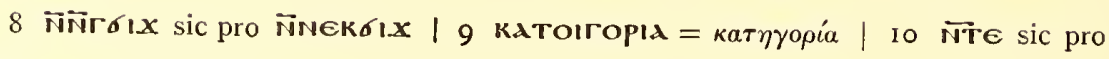
กิTกั 
p. I 5, col. ii

$$
\begin{aligned}
& \text { XOOY NAY N̄T } \\
& \text { WTE MN TMOYN } \\
& \text { 2WOY M̈TEYO } \\
& \text { EII) THप्PG ANï } \\
& 5 \text { Ne NaY E2PAI M } \\
& \text { пMOOY ज̈THEPO } \\
& \text { KATA TEKOY(1) } \\
& \text { K(1) NAY ÑNEY } \\
& \text { MNT(1)גGTE }
\end{aligned}
$$

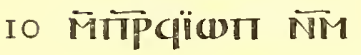

$$
\begin{aligned}
& \text { MAY ETRE NEY } \\
& \text { ANOMild } x \in \\
& \text { RCOOYN } x \in \pi i \\
& \text { BA2HT N̄AIABO } \\
& \text { I5 AOC ETCUPM } \\
& \text { MITEY2HT } \bar{N} \\
& \text { CABOA MTMOK }
\end{aligned}
$$

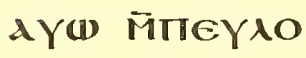

$$
\begin{aligned}
& \text { EYाTOF } \\
& 20 \text { пеY2O EYTाA }
\end{aligned}
$$

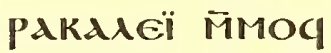

$$
\begin{aligned}
& \text { M̈Tढ2OOY MN } \\
& \text { TEY(1)H (1)AN }
\end{aligned}
$$$$
\text { IE }
$$

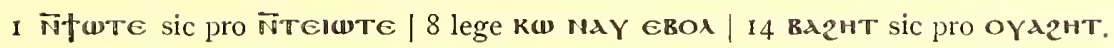

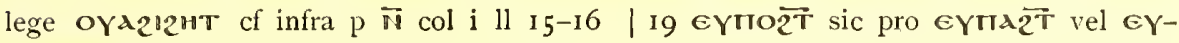
пाдтоY 


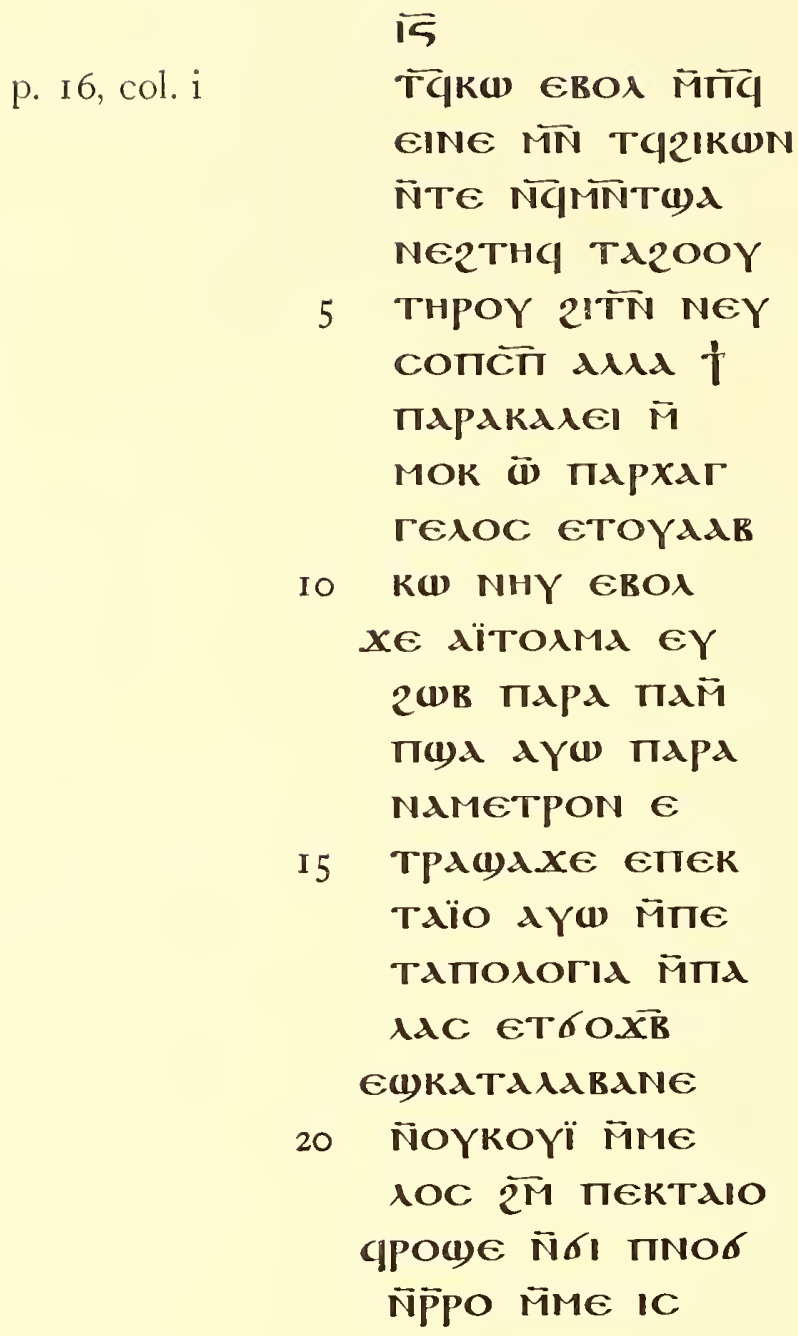

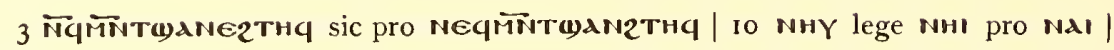

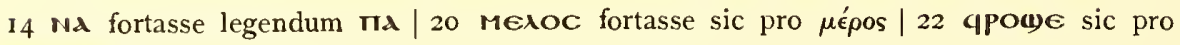
apowe 
p. I6, col. ii

$$
\begin{aligned}
& \text { пє } \overline{X C} \text { ЄТגїок } \\
& \text { кגTג пект̈п(ג }
\end{aligned}
$$

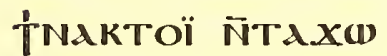

$$
\begin{aligned}
& \text { EPUTN Ñ2ENROYi } \\
& 5 \text { रू N̄ } \\
& \text { घтाне їтाNo6 } \\
& \text { Nipxarredoc rak } \\
& \text { PIHA NAï N̄TAY } \\
& \text { घ)ता 2M TाMAP } \\
& \text { Io TIPION ETOYA }
\end{aligned}
$$

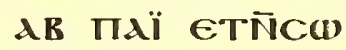

$$
\begin{aligned}
& \text { OY2 EPOY EYEO } \\
& \text { OY MIINOYTE }
\end{aligned}
$$

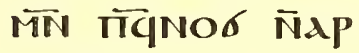

$$
\begin{aligned}
& \text { I5 XArTeAOC ETOY } \\
& \text { АגB ГАврIнА }
\end{aligned}
$$

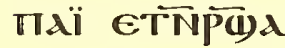

$$
\begin{aligned}
& \text { NAq M̈ாIOOY } \\
& \bar{N} \in \overline{Y N} \text { OYPWME } \\
& 20 \text { Aє N̄PMMAO } \\
& \text { 2N Troxic } \varepsilon \text { Tाप } \\
& \text { PAN TE фїIT } \\
& \text { moc maï } \Lambda \in \text { Ne }
\end{aligned}
$$


p. I 7 , col. i

$$
\begin{aligned}
& \text { OYPMMAO EMA } \\
& \text { TE TE } 2 M \text { TNOYB } \\
& \text { MN П2АT MN } \\
& \text { TBNOOYE AYW } \\
& 5 \text { KATA OE ETOY } \\
& \text { xw M̈MOC } \in T \\
& \text { RHнTी OYN } \\
& \text { TAdq zOYO E } \\
& \text { Tä̈OY N̄6ITï } \\
& \text { Io NAPION ÑNOYB } \\
& \text { ENE OYNOW TAP } \\
& \text { пе м̈трдгмд } \\
& \text { теYтнс EqXI } \\
& \text { Tï } \overline{2 N} 2+2 \bar{N} \\
& \text { I } 5 \text { Xupd AY(1) EPE } \\
& 212 \text { N̄PAME } \overline{\mathrm{P}} \\
& \text { 2WB 2APOG } \overline{2 N} \\
& \text { NEXWPA N̄BOA } \\
& \text { EYeINE NAC } \bar{N} \\
& 20 \text { OYNOб M̄TPAГ } \\
& \text { Matïa } 2 \bar{N} \text { NE } \\
& \text { X®PA THPOY } \\
& \text { AYW NEYCMOY }
\end{aligned}
$$

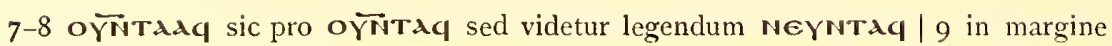

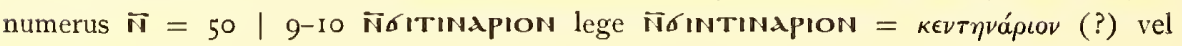

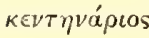




\section{$\overline{\mathbf{Z}}$}

p. I 7, col. ii

$$
\begin{aligned}
& \text { ePOq eMATE ET }
\end{aligned}
$$

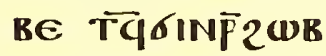

$$
\begin{aligned}
& \text { eTNANOYC mä̈ } \\
& \text { LE NE OYATATH } \\
& 5 \text { TOC пе M̈MAï } 2 \mathrm{H} \\
& \text { ke N̄Arג-0.OC }
\end{aligned}
$$

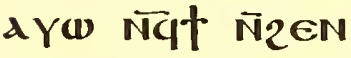

$$
\begin{aligned}
& \text { No6 } \bar{N} \lambda \text { ГАाн } \bar{N} \\
& \text { גUOOY İN̄2HKE } \\
& \text { IO } \overline{M N} \text { ÑOPфANOC } \\
& \text { AYW ON NG' } \\
& \text { N̄2ENNO6 } \bar{N} \lambda \\
& \text { ГАाTE E2OYN E } \\
& \text { ITTIIOC M̈IIAP } \\
& \text { I5 XAгTEAOC ETOY } \\
& \text { ААв ГАврIн入 } \\
& \text { EITEIAH NGOY } \\
& \text { Hн2 2ITOYलप } \\
& \text { ज̈птопос м̈пар } \\
& 20 \text { XATГEAOC ETOY } \\
& \text { ААв ГАврїн } \\
& \text { NETTN OYPWME } \\
& \text { AE Ñ2HKE OY } \\
& \text { нн2 } 2 \text { ITOYलQ }
\end{aligned}
$$

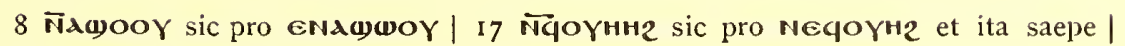
23-24 оүнн2 lege єсоүнг 


\section{iิi \\ p. 18, col. i \\ eITAPAN TE cTe \\ \$ANOC maï $\lambda \in$ \\ Nequdat $\bar{N}$ \\ те2PE їITE2O \\ 5 OY ITE2OOY

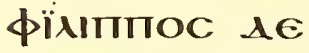 \\ пршме ज̈PM \\ MAO NEqX(1) \\ рнгеї ज̈тршме

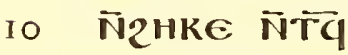

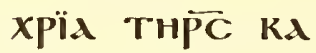 \\ TA OYArATE \\ N̄TE ITNOYTE \\ 2 HTN TQMNT \\ I5 Arג-OOC EாEї \\ Ан NGОYнH2 \\ 2їTOYलQ Kd

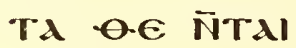 \\ (i)PITXOOC \\ 20 cTeфANOC \\ дє пршмє $\bar{N}$ \\ 2нke NeqNoï

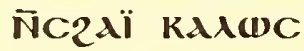

$22 \mathrm{NOÏ}=$ vo€îv 
p. I 8, col. ii

пртімдо $\lambda \epsilon$

Ne OYAQEANIC

He NeqNol AN

N̄C2dï dY(W $2 \lambda 2$

5 ज̄COI NE(1)ג

TPE CTEфANOC

прММе ज̃2нке

CMIN NECANOROC

NAG MTN NEqE

Iо пाтропос MÑ

ÑPकME EтTрдг

MATEYE zApOG

aYW NE ACTAN

2OYTA ENEqIO

15 ГOC THPOY Eq

C2Aї N̄Ca zoB

NIM EПTC ПाЕ

AYW 2 ג2 N̄COT

a NPWME ET

20 XITÏ ज़ ПएМ

MdO TÏ ÑzeN

No6 N̈XPHMA

ज̈праме ज̄2H

$2 \lambda \phi \epsilon \lambda \lambda I C=a \dot{\phi} \epsilon \lambda \eta^{\prime} / 3 \mathrm{NOI}=\nu 0 \epsilon \hat{\imath}$ 
p. I 9, col. i

\author{
KE $\overline{2 N} \quad O Y$ \\ रका Td $\overline{\mathrm{Pq}}$ \\ AdNOANE M̈ாPM \\ MAO $2 \bar{N}$ NEqAO \\ 5 ГOC TגPOYсї \\ ज̈пєТ2А2тнY \\ XE ÑNeqEIME \\ OYzOOY $\lambda \in$ EBOA \\ $\overline{2 N}$ OY2OOY \\ Iо А прФME $\overline{2}$ Hке \\ РPMMAO коYї \\ KOYï AlNA TMNT \\ PMMAO ETOY \\ NAcwOY2 $\bar{M}$ \\ I5 MOC E2OYN $\overline{2 N}$ \\ OYXIN口ONC \\ CNAXUMPE \\ EBO人 $\overline{2 N}$ OYбE \\ TIH KגTA O.E \\ 20 ETN̄NAOYEÑ2 \\ П2बB NHTÑ \\ EBOA KATA \\ тा(1)גXe MாME
}


p. I 9, col. ii

$$
\begin{aligned}
& \overline{\mathrm{I} \theta} \\
& \text { 入їгрдфос } \lambda \bar{\lambda} \\
& \text { xE M̄TिNA2TE } \\
& \text { EXIÑ } \\
& \text { ज̈тірк2тнк } 6 \\
& 5 \text { Tॅलित OYMNT } \\
& \text { PMMAo ec(1)ANEi } \\
& \text { м̈тркג2тнк } \\
& \text { POC ÑTEPE пथн } \\
& \text { KE } \lambda \in \text { Tï } 6-\mathbf{O H} \\
& \text { ı koүï koүï Аq2e } \\
& \text { EாOEIK AqOYO } \\
& \text { जव АqT пеqоY } \\
& \text { oï єாршмє N̄PM } \\
& \text { MAO ÑOYZOOY }
\end{aligned}
$$

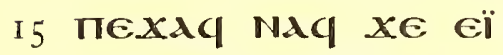

$$
\begin{aligned}
& \text { Oүw(1) ET } \overline{P K} \vec{P} \\
& \text { TINA NMMAi NT } \\
& \text { Tï Naï 2@ Nं2EN } \\
& \text { ROYï ÑNOYB Ī } \\
& 20 \text { TAחPAГMATEY } \\
& \text { E N̄2HTOY } \bar{N} \\
& \text { TAOYWN2 EPOOY } \\
& \text { MÑ Nג(н)е AY }
\end{aligned}
$$

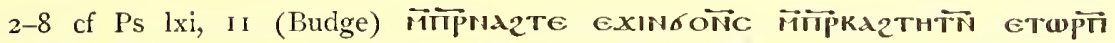

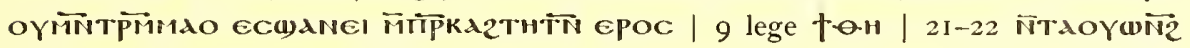

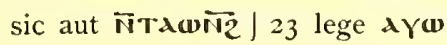


p. 20 , col. i

$$
\begin{aligned}
& \overline{\mathbf{K}} \\
& \text { TNAY ETKNA } \\
& \text { gINE ÑCA NeK } \\
& \text { NOYK TNATA } \\
& \text { AY NAK MTN TTK } \\
& 5 \text { MePOC } \overline{2 M} \text { Пе } \\
& \text { TiNAXIOG ī } \\
& \text { 2HTOY EIEY } \\
& \text { XAPICTOY NAK } \\
& \text { пехе пршME } \\
& \text { Io N̄PMMAO NAq } \\
& x \in \text { вшк ज̃TCMN } \\
& \text { пाекрАMMA† } \\
& \text { ON Naï EUETK } \\
& \text { рxxpia M̈MOq } \\
& \text { I5 AYW TindTA } \\
& \text { Aq NAK NAK } \\
& \text { ПभНке } \lambda \in \text { Аq } \\
& 2 \text { MOOC AपCMN } \\
& \text { пегрАMМА Ti } \\
& 20 \text { ON M̈TPAME } \\
& \text { N̄PMMAO } \\
& \text { cג(i) Ñ(1)E } \\
& \text { 2OЛOKOT"TINOC }
\end{aligned}
$$

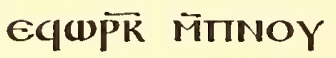

$$
\begin{aligned}
& 25 \text { TE TाTANTW }
\end{aligned}
$$

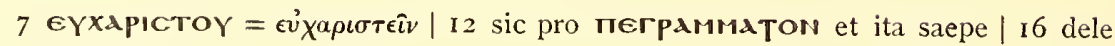
NAK posterius $\mid 22$ in margine numerus copto-arabicus 700 
p. 20, col. ii

$$
\begin{aligned}
& \text { кPd TwP } \\
& \text { TAPG TAdY } \\
& \text { NAq M̈ாxWK ī } \\
& \text { OYPOMTE MN } \\
& 5 \text { тпдуе ज̈пет̆ } \\
& \text { NAXпOQ N̈2HTOY } \\
& \text { тPMMAO } \\
& \text { xï мпाегрдмMд } \\
& \text { TON גqтї ज̈ாCג }
\end{aligned}
$$

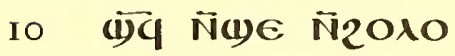

$$
\begin{aligned}
& \text { кOT'NOC ज̈ாPW } \\
& \text { ME N̄2HKE Аप } \\
& \text { ВШK АСПРАГMА } \\
& \text { TEYe Ñ2HTOY } \\
& \text { I } 5 \text { N̄ฒOMTE ÑगिМाE } \\
& \text { АपT12HY N̈2EN } \\
& \text { АФ) N̈XPIMA } \\
& \text { AYW M̈TாવINE } \\
& \text { ÑCd Tï AddY } \bar{M} \\
& 20 \text { тртMма OYAE } \\
& \text { KAIфANION OY } \\
& \text { LE пגPAMI-OId } \\
& \text { EïTd MNNN̄Cd } \\
& \text { 2EN2OOY ENd }
\end{aligned}
$$


p. 2 I, col. i

$$
\begin{aligned}
& \text { w)OY \& ПדР } \\
& \text { MAo xi м̄п2нке }
\end{aligned}
$$

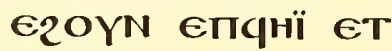

$$
\begin{aligned}
& \text { PGCMN NGAOTOC } \\
& 5 \text { NAq AYW ÑTE } \\
& \text { PEOYUN ÑTKA } \\
& \text { TCג ETEPE NEK } \\
& \text { PAMMATION } \overline{M N} \\
& \text { NंXW⿴囗十 } \bar{N}_{2 H} \\
& \text { Io TC eqoYwW } \mathrm{T} \\
& \text { 2E ENEXAPTHC } \\
& \text { ETqkwTE N̄CW } \\
& \text { oY N̄Tepe cTe } \\
& \text { фANOC } \lambda \in \text { прш } \\
& \text { I } 5 \text { Me ETÑadxe } \\
& \text { epoq } 2 \epsilon \text { eтісгрдm } \\
& \text { TiON AqTAdC } E \\
& \text { 2OYN } 2 A \text { TGKA } \\
& \text { MICION M̈ாE пिM } \\
& 20 \text { MAO eIME ETBE } \\
& \text { T्MNNTRAג2HT } \\
& \text { MN TिपूलT2AT } \\
& \text { AOYC AqEI EBOA } \\
& \text { ज̈пе пршме єї } \\
& 25 \text { ME OYzOOY AE }
\end{aligned}
$$

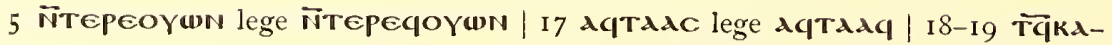
mciom lege пеqкаmсіо 
p. 2 I, col.ii

$$
\begin{aligned}
& \text { EBOA } \overline{2 N} \text { OY2OOY } \\
& \text { д приме N̄2HкE } \\
& \text { PिтMмдo koyï }
\end{aligned}
$$

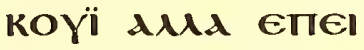

$$
\begin{aligned}
& 5 \text { АH TM̈NTPMTM][A] } \\
& \text { o eтOYCWOY2 } \\
& \text { MMMOC E2OYN } \overline{2 N} \\
& \text { OYXINGONC CNA } \\
& \text { TAkO NecXunpe } \\
& \text { IO EBOA } \overline{2 N} \text { OYбEITH } \\
& \text { KגTג O-E ÑTגї }
\end{aligned}
$$

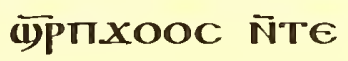

$$
\begin{aligned}
& \text { pe cteфanoc ae } \\
& \text { вшк Єாтинї Аq } \\
& \text { } 15 \text { пє() пекрАМмА } \\
& \text { TON AqAq ज̄AגKM } \\
& \text { АAKM AYW } \Pi \text { KE } \\
& \text { XAQ } \overline{2 M} \text { Пप12HT } \\
& \text { 2ITN NEqMEEY } \\
& 20 \text { C ETEOOY N̄Td } \\
& \text { IICATANAC NO } \\
& \text { XOY Eாपिरण } \\
& x \in \text { АAн-O.ШC }
\end{aligned}
$$

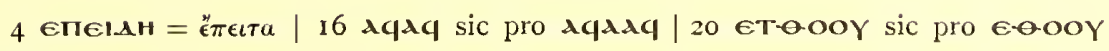
et ita saepe 
p. 22, col. i

$$
\begin{aligned}
& \overline{K B} \\
& \text { MїT ^גdY ज̈ாגї } \\
& \text { (1) ENE2 } \overline{2 M} \text { пї }
\end{aligned}
$$

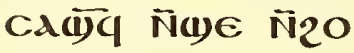

$$
\begin{aligned}
& \text { 入ОКОТTIINOC } \\
& 5 \text { єтеІхрешс } \\
& \text { M̈MOG NAG } \\
& \text { AYU EqudNXo } \\
& \text { oc Ndï } x \in \text { TAdY } \\
& \text { Ndï TïNdxoOc } \\
& \text { IO NAq } x \in \text { MNTTAK } \\
& \text { AddY EPOï dY } \\
& \text { EIC пекрגMMA } \\
& \text { TiON N̄TAMA2K } \\
& \text { जिМOप ЕшWाЕ } \\
& \text { I } 5 \text { 2О人ос тїхе } \\
& \text { wCTï NAK OY } \\
& \text { Ає } \\
& \text { pxpïd aN } \bar{N} \\
& \text { NOYB AlAd } \\
& 20 \text { Tïndiт̈ } \overline{\mathrm{N}} \\
& \text { таР̈РM }
\end{aligned}
$$

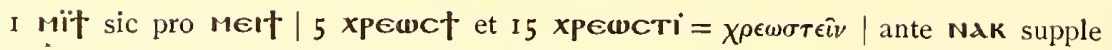
ninday (?) / I8 ñnoyb lege T̃neinoyb 
p. 22, col. ii

$$
\begin{aligned}
& \text { 2HTOY AYW } \\
& \text { Naï Napwae } \\
& \text { epoï N̄OYNOK } \\
& \text { N̄oYOEI(1) EïWM } \\
& 5 \text { EICW Ñ2HTOY } \\
& \text { еї-ердпеүе } \\
& \text { ज̄Tג†YXH }
\end{aligned}
$$

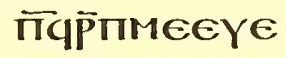

$$
\begin{aligned}
& \text { р๘ М̈петсн2 } \\
& \text { IO XE ПITOHT } \\
& \text { ceNdai } \bar{N} \overline{T K K} \\
& \text { ҰYXH N̈TOOTK } \\
& \text { NENTAK } \overline{C B} \\
& \text { TUTOY EYNA }
\end{aligned}
$$

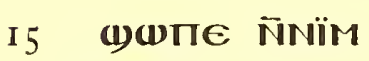

$$
\begin{aligned}
& \text { eITA } x \in \text { NENa } \\
& \overline{C K} 2^{M} \text { пथ) } x E \\
& \text { MNNÑCA qTOE } \\
& \text { de N̄POMாE d } \\
& 20 \text { прМмגO NAY } \\
& \text { xe М̈ாе Пभнке } \\
& \text { Tï AddY Ndq }
\end{aligned}
$$

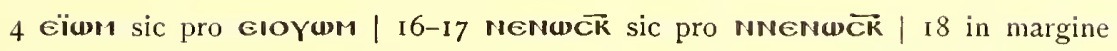
numerus copto-arabicus 4 
p. 23 , col. i

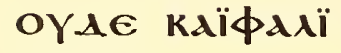 \\ ON OYAE TIPA \\ Mï日ïд aqбe \\ пн АqTNNO \\ 5 OY N̄CWG N̄Ñ \\ 2Mida AYw \\ пEXAq NAq

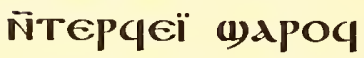 \\ xE MA Naï $\bar{M}$

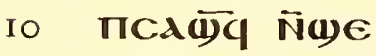 \\ N̄2O^ОКОT' \\ NOC ЄT2А2THK \\ $x \in$ TPxpld \\ MMOOY ПATNOY \\ I5 TE $1 \in$ ETM̃MAY \\ АपराTOOTG Nं \\ APNA M̈ாPM \\ MAO ÑEYCEBHC \\ ETM̆MAY XE \\ 20 MTTTK MAdY \\ 2ג2тнї Оүне \\ I7 Aprid $=\dot{\alpha} \rho \nu \epsilon \hat{\epsilon} \sigma \theta a t$
}


p. 23 , col. ii

$$
\begin{aligned}
& \text { M̈пек† גАdY } \\
& \text { Naï ene2 пExe } \\
& \text { TPMMAO NAq } \\
& \overline{2 N} \text { OYбWNT } x \in \\
& 5 \text { (i) TINOMOC } \\
& \text { NAME MH EIC } \\
& \text { петрдмMАTї } \\
& \text { ON ÑTOOT AN } \\
& \text { N̄TAKCMNTTA } \\
& \text { IO NMMAï ЄCA(T) } \\
& \text { N̄(1) N̄2O人OKOT } \\
& \text { TINOC EIC Te } \\
& \text { ÑPOMTE XIN ī } \\
& \text { TdïTdAY Nak } \\
& \text { I } 5 \text { OY MONON } x \in \\
& \text { M̈пекT пגPd } \\
& \text { paMlo-ld Naï } \\
& \text { 2APOOY dAlA } \\
& \text { धIC 2HHTE EK } \\
& 20 \text { OүW(1) Eqiі } \bar{M} \\
& \text { IIAXOTPION }
\end{aligned}
$$

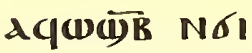

II in margine numerus copto-arabicus 700 I 2 in margine numerus copto-arabicus

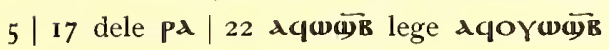




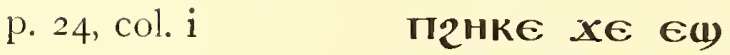

$$
\begin{aligned}
& \text { хе пекГрАМ } \\
& \text { MATON 2А2THK } \\
& \text { AYEIC̈ NÄ̈ } \\
& 5 \text { TANAY EPOQ } \\
& \text { АүМ N̈TАВШK } \\
& \text { กัтАтї пднї } \\
& \text { MTN NА(унре } \\
& \text { N̈TAMĀK } \bar{N}
\end{aligned}
$$

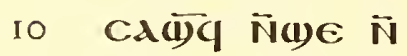

$$
\begin{aligned}
& \text { 2OגOKOTTIN } \\
& \text { OC прМмдо } \lambda \epsilon \\
& \text { AपME(T) NEK } \\
& \text { ГРАMMATION } \\
& \text { I5 TнроY } \overline{M N} \bar{N} \\
& \text { хартнс ज̈тाq } \\
& 26 \text { epoq } \lambda \\
& \text { EIME ÑTEYNOY } \\
& \text { XE N̄TAqROप̄ } \\
& 20 \text { Nं.XIOYE AY } \\
& \text { (1) пеXAq NAq } \\
& x \in \bar{N} C O O Y N \\
& \text { deïme } x \in \bar{N}
\end{aligned}
$$


p. 24, col. ii

$$
\begin{aligned}
& \text { TOK IIENTAK }
\end{aligned}
$$

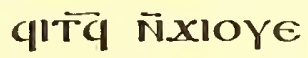

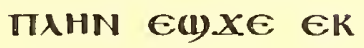

$$
\begin{aligned}
& \text { O-Apeï } \overline{2 M} \text { пек } \\
& 5 \text { 2нт } x \in \text { м̈пеї } \\
& \text { Tï NAK M̈trca(̄) }
\end{aligned}
$$

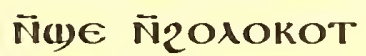

$$
\begin{aligned}
& \text { TINNOC AMOY } \\
& \text { MAPON EITTOIIOC }
\end{aligned}
$$

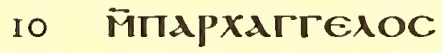

$$
\begin{aligned}
& \text { eтоYגАв гАвpї }
\end{aligned}
$$

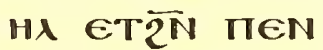

$$
\begin{aligned}
& \text { 2IP NTWT NAÏ } \\
& \text { जॉтाлнрофореї }
\end{aligned}
$$

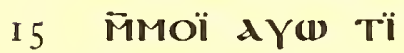

$$
\begin{aligned}
& \text { NABUA NMMAK } \\
& \text { ПIАТ2HT ETM̈MАY } \\
& \text { AqNEX OOTE M }
\end{aligned}
$$

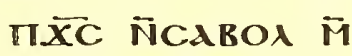

$$
\begin{aligned}
& 20 \text { MOG MNN TАщн } \\
& \text { N̄M̈ாETNANOYG } \\
& \text { N̈тגqпג-E M̈MO } \\
& \text { OY EBOX 2ITOOTA } \\
& 4 \text { - axp€ï }=\theta \alpha \rho \rho \epsilon \hat{\imath} v
\end{aligned}
$$


p. 25 , col. $\mathrm{i}$

$$
\begin{aligned}
& \text { М̈ாРМMAO } \bar{N} \\
& \text { EYCERHC ETM̆ } \\
& \text { MAY Eגवा̈ாक } \\
& \text { (सі) ज̈петсн2 } \\
& 5 \quad \mathrm{X} \in \Pi \overline{\mathrm{XC}} \text { NATA } \\
& \text { KO N̄OYON NIM } \\
& \text { етхш м̈пбол } \\
& \text { Аүш пеXАप M } \\
& \text { пртмдо } x \in \\
& \text { IO MAPON N̄TA } \\
& \text { WT NAK AY } \\
& \text { w N̄TEYNOY } \\
& \text { AYMOOwE } \overline{M N} \\
& \text { NEYEPHY ФAN } \\
& { }_{5} \text { TOYBWK E2OYN } \\
& \text { EпTопос } \bar{M} \\
& \text { парХАГГеАОС } \\
& \text { ГАврIHA Аүल } \\
& \text { AYNOK M̈MH } \\
& 20 \text { HШ) СWOY2 } \\
& \text { EPOOY АYР̈шпн } \\
& \text { pe M̈ாENTAq }
\end{aligned}
$$


p. 25 , col. ii

\author{
$\overline{\mathrm{KE}}$ \\ шщпе прММАо \\ de NeqXu M̃MOC \\ ज̈гтнке Xe MA \\ Nai M̈ாCגब̄ Ñ

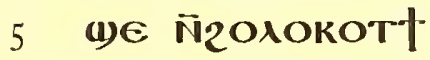 \\ NOC ÑTAITAAY \\ NAK ज̈TTPW $\overline{P K}$ \\ NOYX ÑTE OYTET \\ QOOY Tג2OK пג \\ I0 T-बHT $\lambda \in$ ETTि \\ MAY пеXגq XE \\ MTNTAK IAAY $2 d$ \\ 2тні пехе пाт̈ \\ MaO Naq xe EW

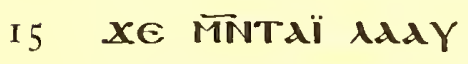 \\ 2А2THK WTK NAï \\ ÑTARQK NAÏ Tג \\ TNOYTE גE ETĨ \\ MAY АqKATA \\ 20 фPONH N̄N̄бOM

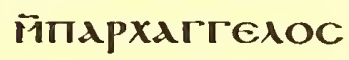 \\ eTOYAdB ГAвPI
}

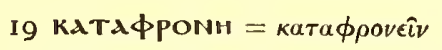


p. 26, col. $\mathrm{i}$

\section{रद}

H入 ETRE XPIMA

E()ג ТАКо АY

(1) AqTONMA $2^{N}$

TपMNTגT.HT

5 dqoye2 Tqбіx

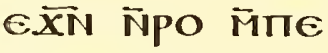

o.їїдctipion

AqWP̈ NAq $x \in$

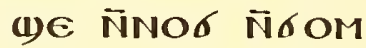

Iо M̈ாגрхגггеגос

гАврIнд М̈ТT

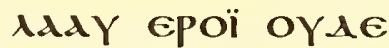

М̈пєкті АגАY

Naï ENE2 $\overline{2 M}$

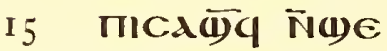

ज̄201ОктїNOC

eTERENETEi

MMMÖ̈ 2APOOY

AYU N̄TEYNOY

20 ЄTEї ЕРЕ пा)

$x \in \overline{2 N} \overline{T q T A}$

про АүАїл

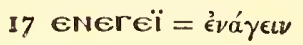


p. 26 , col. ii

$$
\begin{aligned}
& \text { MIC PA2T4 Aq } \\
& \text { 2є ERPai G. } \overline{X M}
\end{aligned}
$$

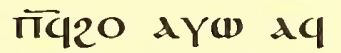

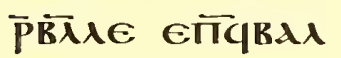

$$
\begin{aligned}
& 5 \text { CNAY A пाEप2O } \\
& \text { бOOYX N̄CA TIA } \\
& \text { 2OY M̄MOQ A }
\end{aligned}
$$

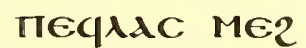

$$
\begin{aligned}
& \text { Poप ג42E E.XN }
\end{aligned}
$$

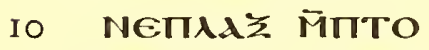

$$
\begin{aligned}
& \text { TOC AYw NG }
\end{aligned}
$$

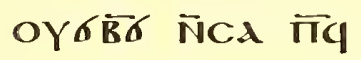

$$
\begin{aligned}
& \text { IAC M̄N NEqCTIO } \\
& \text { TOY MIMIN M̄MOG } \\
& \text { I5 } \overline{2 N} \text { OYNOK } \bar{N} \text { A } \\
& \text { ruNIA AYW Ní } \\
& \text { TARE 2RETE GROA } \\
& \text { 2N paq } \bar{N} \theta \in \bar{N} \\
& \text { ÑAXIMUNION }
\end{aligned}
$$

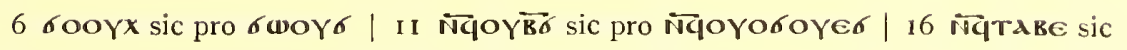
pro ñq̀ 
p. 27 , col. i

$$
\begin{aligned}
& \text { АүР̈Фтнре } A Y \\
& \text { (1) AYP̄ZRA AY } \\
& \text { (w) EROA } \overline{2 N} \text { OY } \\
& \text { Noб ज̄CMH } E Y \\
& 5 \text { X(1) जिMOC } X E \\
& \text { oYd пе пNOYTE } \\
& \text { M̈тАрХАГГЕАОС } \\
& \text { ГАвPIHA MTN } \\
& \text { N̄CA OYNor } A E \\
& \text { Iо N̄AItpHTE EqBA } \\
& \text { CANIZE EqAW } \\
& \text { RAK EBOA } \overline{2 N} \\
& \text { Noб N̄CMH } X \in \\
& \text { Ka NAI EBOA TI } \\
& \text { I5 } \overline{X C} \text { пАРХАГГе } \\
& \text { 入OC ГАвРIн入 } \\
& \text { xe Аїтолмд }
\end{aligned}
$$

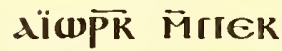

$$
\begin{aligned}
& \text { PAN ÑNOYX } \\
& 20 \text { А пеІршME } \bar{N} \\
& \text { PMMao † Ca }
\end{aligned}
$$

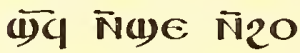

$$
\begin{aligned}
& \text { \OKOT'NOC }
\end{aligned}
$$


p. 27 , col. $\ddot{i i}$

$$
\begin{aligned}
& \overline{K Z} \\
& \text { Naï eIC Te INPOM } \\
& \text { пе тї̈qur } \bar{N} \\
& \text { 2HTOY AYW M } \\
& \text { TEITi IAdY NAG } \\
& 5 \text { 2APOOY NंTepï } \\
& \text { 2є मE GTEYKY } \\
& \text { pıd dïqi M̄Tek } \\
& \text { ГPAMMATION } \\
& \text { N̄xIOYE Edïzï } \\
& \text { IO } \lambda \text { ePOप EÏOYW( } \\
& \text { EqITOY N̈TA } \overline{P P M} \\
& \text { MAO N̄2HTOY }
\end{aligned}
$$

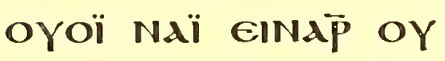

$$
\begin{aligned}
& x \in \text { dï山PK ÑNOYX } \\
& 15 \text { AYW ÑTEYNOY Aq } \\
& \text { TTNNOOY EITqHï } \\
& \overline{2 N} \text { OYбETH A } \\
& \text { TqC2IME EINE } \\
& \text { ज̈ாCג(1) N̄| } \\
& 20 \text { N̄2O人OKOT'TNOC } \\
& \text { АCTAdY ज̈ாTि } \\
& \text { MAO TिएМ } \\
& \text { LE ETMIMAY }
\end{aligned}
$$

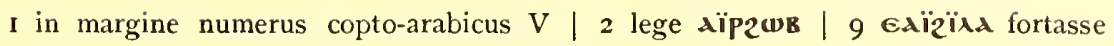

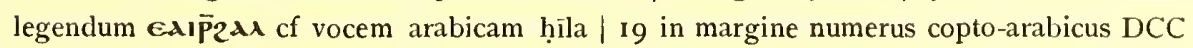




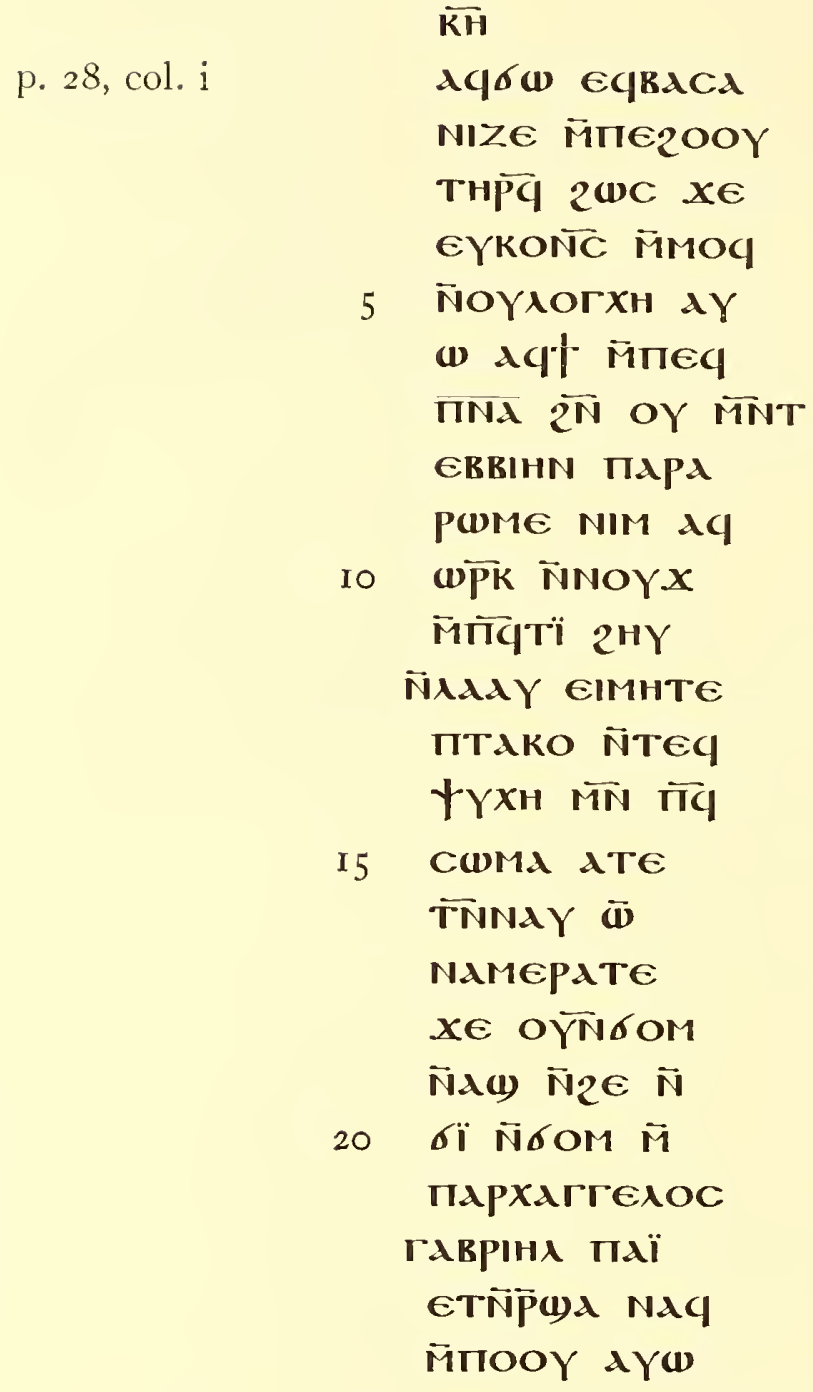

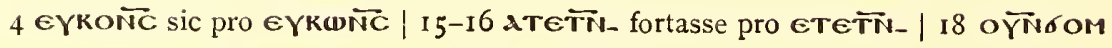

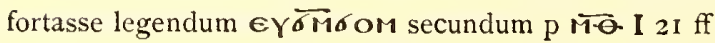


p. 28, col. ii

$$
\begin{aligned}
& x \in \pi \in T N \lambda \omega \overline{P K} \\
& \text { ÑTTPPAN NNOYX } \\
& \text { QN OYMOY GNA } \\
& \text { MOY } \overline{2 N} \text { OYMTIT } \\
& 5 \text { ERRIHN MAPN } \\
& \text { PNCA2WN EROA } \\
& \text { мாтбол пеXвIN } \\
& \text { ET2OOY MANIC } \\
& \text { TA NETTAXPO }
\end{aligned}
$$

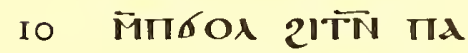

$$
\begin{aligned}
& \text { NA(1) ÑNOYX Nī } \\
& \text { CWTM dN EIT } \overline{X C} \\
& \text { Eq(D) ERON GOY } \\
& \text { ON NIM XE MA } \\
& \text { I } 5 \text { PE NETÑadxe }
\end{aligned}
$$

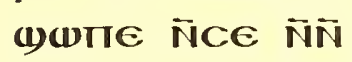

$$
\begin{aligned}
& \text { CE АүW петM } \\
& \text { MON N̄M̄MON } \\
& \text { xe ÑNeYKPINE } \\
& 20 \text { M̈MWTN ANON } \\
& \text { גE 2WWN MAPN } \\
& \text { 2APE2 ETNTA } \\
& \text { ITPO EROA } \overline{2 N} \text { Nei }
\end{aligned}
$$

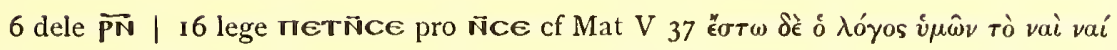

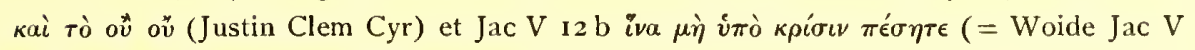
I2 b XeKגC ÑNeYKPINE F̈M(DTN) 
p. 29, col. i

$$
\begin{aligned}
& \text { NA(1) N̄2OTE }
\end{aligned}
$$

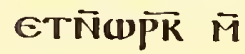

$$
\begin{aligned}
& \text { MOOY MANIC } \\
& \text { TA EXTN OYZWB } \\
& 5 \text { EXXN OYZWB Eq } \\
& \text { ФOYEiTT } X E \bar{N} \\
& \text { NeNXi N̄oYNor } \\
& \text { ÑKPIMA MTN } O Y \\
& \text { KOAגCIC N̈AT } \\
& \text { IO } \overline{X N} 2 \overline{2 M} \text { Tाय } \\
& \text { ETE } \bar{M} \text { TLגEN2 } \\
& \text { бINE AN ज̈TTq } \\
& \text { WINE TEIEII } \\
& \text { PE ГגP М̈тTMEеY } \\
& \text { I5 } \Theta x \in \text { лїєрнт } \\
& \text { EXW EPUT'T } \\
& \text { Ñ2ENKOYÏ EBOX } \\
& \text { टN ज̄ } \\
& \text { Neळтнре } \bar{M} \\
& 20 \text { пNOб } \bar{N} A p x A \Gamma \\
& \text { ГEגOC ГАвPї }
\end{aligned}
$$

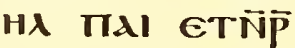

$$
\begin{aligned}
& \text { ()ג NAq M̈тlOOY }
\end{aligned}
$$

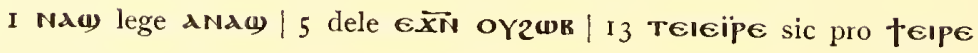


p. 29 , col. ii

K.̈.

EYEOOY M̈TNOYTE जTN TIqNOG ÑAPXAT гелос гаврїна пqаї

(1)MNOYqE NEYN

5 OYPWME $\lambda \in$ ON $\overline{2 N}$ тіполїс еqо de eTteqRal cNaY XIN TqMNTкоYї N̄TEPqCWTM $\lambda \epsilon$

Iо ЄтвЕ त̄боM МाN Ne(1)пне їпар хагтелос ГАврї

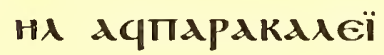
ÑNEqP(DME ET

I5 PEYXITA E2OYN EПтопос М̈пגP хаГГЄАOC ГАврї

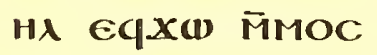
xe ПANTOC N̄TE Tாप 20 NA TA2OÏ 2w $\overline{N q}$ xapize NaÏ M̈ாOY OEIN ÑNARAA N̄ TOOY le AYXï Тq єптопос $\bar{M}$ 
p. 30, col. i 
p. 30, col. ii

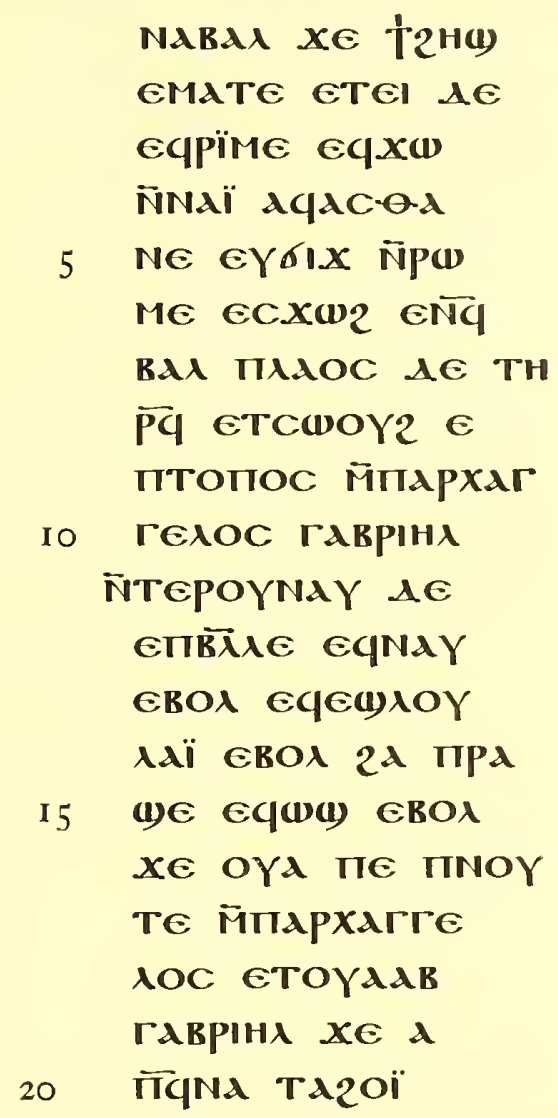

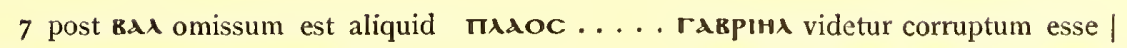
8 eтcworz supple ezorn 
p. 3I, col. i

\author{
AqXAPIZE \\ NAï M̈TOYOEIN \\ N̄NARAA AYW \\ АүтालT ExWप \\ 5 त̄б। Птмнне \\ тнРЯ AYXNOYG \\ EYXw ज̈MOc \\ $x \in X w$ ePON \\ M̈TENTAquW \\ IO TE M̈MOK $x \in$ \\ N̄TdKNAY E

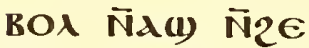 \\ Ачпдрдгтеле[ї \\ epooY ज̄zWB \\ I5 NIM N̄TAW( \\ TIE M̈MOG $x \in$ \\ Асฒште $\bar{M}$ \\ MOÏ MTN N̄CA \\ TPגEi C2OYN $^{-}$ \\ 20 ЕாЕІтопос 6 \\ TOYAגB גï

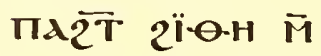 \\ пеөүсІдстї
}

3 post T̄irdrat omissum est aliquid 
p. 3 I, col. ii

\author{
$\bar{\lambda}$ \\ PION ETOYAגB \\ АїлРАГАлеI \\ пIPXAГTEAOC \\ GTOYAAB TAR \\ 5 PIHA $\overline{2 N}$ TEГYNTOY

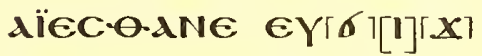 \\ ÑPAME ACEl [E] \\ пєснт $є \overline{X M} \Pi \mathbf{~}$ \\ 20 АсфрАгїє \\ IO İNARAA AYU Ñ \\ TEYNOY dïNAY \\ EROA AYU dï \\ СळTM EYCMH \\ ECXw M̈MOC \\ I5 Nai $x \in$ elc2H \\ HTE dïxגPIZe \\ NAK MTIOYOEIN \\ ÑNTRAג ITPOC O.E \\ ज̄TAKCักัICOTTT \\ 20 ANOK $\lambda \in \Pi \in X \lambda i$ \\ $x \in \overline{\mathrm{N} T} \overline{T K}$ NIM \\ II $\overrightarrow{X C}$ ÑTOQ \\ גe IEXגq Naï
}

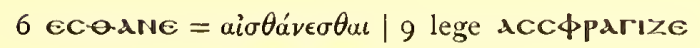


p. 32, col. i

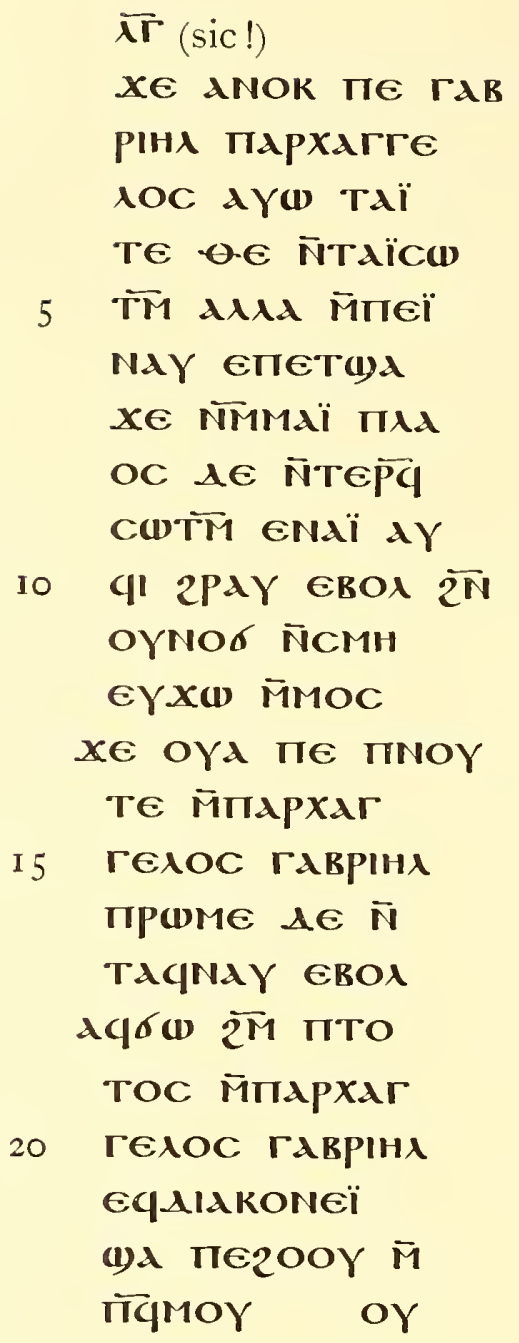


p. 32 , col. ii

$$
\begin{aligned}
& \text { pome } \lambda \in \\
& \text { OÑ N̈PĀMAO } \\
& \text { QN TNOYB MNN } \\
& \text { Пथट EQTO ÑM } \\
& 5 \text { TाO MIN ÑCOC Aq } \\
& \text { 2€ EYMUNe maï } \\
& \text { eTOYMOYTE } E \\
& \text { POQ } x \in[T] \text { Tגाחо } \\
& \text { тגкPïd epe oY } \\
& \text { IO NOG ÑTKגC OY } \\
& \text { WM N̄CA N̄qOYPH } \\
& \text { нPе जि̈Iе2OOY } \\
& \text { MN TеҮ(1) } е \\
& \text { גqXe пр(1) } \\
& \text { I5 AN2OXOMA EROA }
\end{aligned}
$$

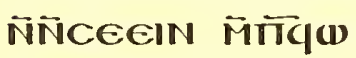

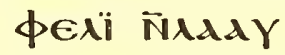

$$
\begin{aligned}
& \text { AlAd NंTAqP } \\
& \text { zOYO E.O.O } \\
& 20 \text { NंTE } \\
& \text { גe Ñ } \\
& \text { NE()тнPE ET } \\
& \text { (1)Oก } \overline{2 M} \text { ПтТ } \\
& \text { Пос м̈пגРXАГ }
\end{aligned}
$$

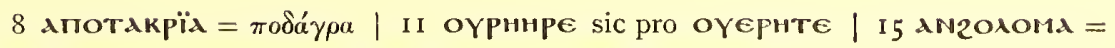

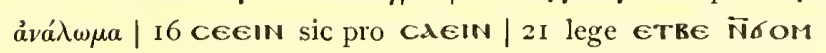


I 92

COPTIC HOMILIES

p. 33, col. i
ГEגOC ETOYAAB

ГАврІнА Аq

TPE NEYTTM2dA

TAlOq ÑceXITq

5 EIITTOIIOC ETOY

AגR Аq्NKKOTK

2M птопос $\mathrm{E}$

TM̈MdY ElquO

OाI $\overline{2 N}$ OrYNor ĩ

Io zïce ArYla Neq

(w) $[$ ERO $] \lambda$ EqX(1)

ज̈MOC $x \in \Pi \pi$

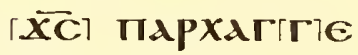

入O[C] ETOYAdB

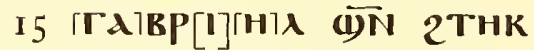

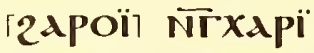

Z[G] Naï MĭtTdג

[6O] EROA $x \in d i$ 2їсе लMATE

20 OYPWME $\Lambda \in$ ON

2OMAIOC Eq

СHб ENEqOY

PHHTE EqCY

Pd $2 \bar{M}$ IIKd2 N

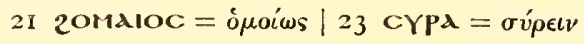


$\overline{\lambda . \lambda}$

p. 33, col. ii

NAY NIM NOE

ज̄N̄ฒнре шнм

NEq $\overline{2 M}$ ITTOIIOC

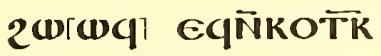

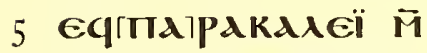

Гா]АPXAГГEגOC

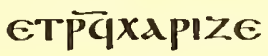

NAq їாTAлбо

пршме $\lambda \in$ eT

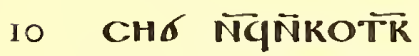

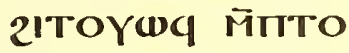

пOC EqTїткגс

ЕN̄̆ОҮРнте

AYW N̄TOOY $\bar{M}$

I 5 TECNAY NEYUï

NE M̈TTCNAY T̄

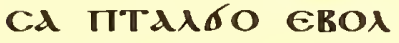

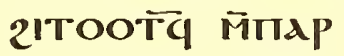

XAгTEAOC ETOY

20 ААВ ГАвРІнА

¿N TEYNOY E

TM̈MAY A TIP

XATTEAOC ज़N

2THq 2АPOOY

25 AqP2NAq $\overline{2^{N}}$ T 


\title{
p. 34, col. $\mathrm{i}$
}

\author{
$\overline{\mathbf{A \epsilon}}$ \\ MNTATA-OOC \\ exAPIZE NAY $\bar{M}$ \\ пталбо ज̈пес \\ NAY N̄OYCOIT $\bar{N}$ \\ 5 OүलT АYW $\bar{N}$ \\ TEI2E גपWN2

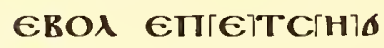 \\ ¿N OY2OPOMA M \\ ПIECMOT N̄OYPW \\ Io ME ÑoYOEIN $E$ \\ PE пеप2O NHX \\ AKTIN N̄OYOEIN \\ EвOА пеХАq $\bar{M}$ \\ пршме єтснб

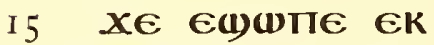 \\ OYW(I) EMATE \\ ज̈пталбо АY \\ (1) స̄TE поYXגï \\ घ)TIE NAK $\overline{N T}$ \\ 20 MOOळE ÑNEKOY

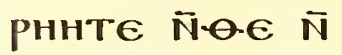 \\ PWME NIM IIPLC \\ EXEI EPOK (I)N \\ TE N̄PaME TH
}

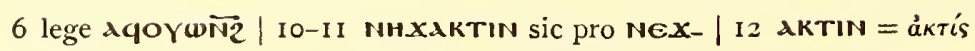


p. 34 , col. ii

$$
\begin{aligned}
& \text { POY ET 2M ПTO } \\
& \text { ПОС ज̄кOTK Ñ } \\
& \text { CE(WT) EK(I)AN } \\
& \text { eIME XE AY(D } \\
& 5 \text { (तi) TнроY Т(1 } \\
& \text { OYN 2WOK NTा } \\
& \text { ClNA ÑNEKGIX } \\
& \text { MN N[G]KOYPH } \\
& \text { нTE NTRळK } \\
& \text { IO 2A2TH[N] ITIMA } \\
& \text { ज̄N̄KOTK MПРМ } \\
& \text { MAO ETTITKגC } \\
& \text { ENGOYPHHTE } \\
& \text { NT̃ APXeï Ñqii } \\
& \text { I5 їпепрн(1) Ет } \\
& 21 \times(1) \mathrm{A} \text { A } y \\
& \text { ERNAMAdTE } \\
& \text { м̈ாтАлбO NTा } \\
& \text { MOOWE ĨNEK } \\
& 20 \text { OYPHнTE } \overline{\mathrm{N}} \\
& \text { TEYNOY NTTOY }
\end{aligned}
$$

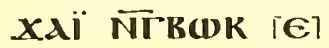

$$
\begin{aligned}
& \text { пєкнї і̄-өє } \\
& \text { N̄OYA ETE пєQ }
\end{aligned}
$$

$7 \mathrm{Cl \lambda d}=\sigma$ vó 24 пеq lege îneq 
p. 35, col. $i$

$$
\begin{aligned}
& \text { WWNE ENE? } \\
& \text { пршме } \lambda \in \bar{N} \\
& \text { TEPपNe2CE } \\
& \text { e2paï } \overline{2 M} \text { п20 } \\
& 5 \text { POMA Aqप्P } \\
& \text { (1) } \\
& \text { пехגq 2Pגi } \\
& \text { २सTद } x \in A \\
& \text { AHOCC NTAY } \\
& \text { io Pि2al M̄Moï } \overline{2 N} \\
& \text { TïpdcoY } \in i \\
& \text { (1) } \lambda N \bar{P} \text { Tגї } \bar{N} \\
& \text { тАвшк Xe eï } \\
& \text { Nacii MT2ai } \\
& \text { I5 TE ज̈пPिMA } \\
& \text { - CNAwnte } \\
& \text { NGMEZCE } E \\
& \text { 2pגï N̄TE Neq } \\
& \text { रM2AN боTाT } \\
& 20 \text { धпеїम MN } \\
& \text { (1) бом М̈мої }
\end{aligned}
$$

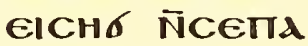

$$
\begin{aligned}
& \text { PAגIAOY } \bar{M} \\
& \text { MOÏ ETOOT̄C }
\end{aligned}
$$


p. 35 , col. ii

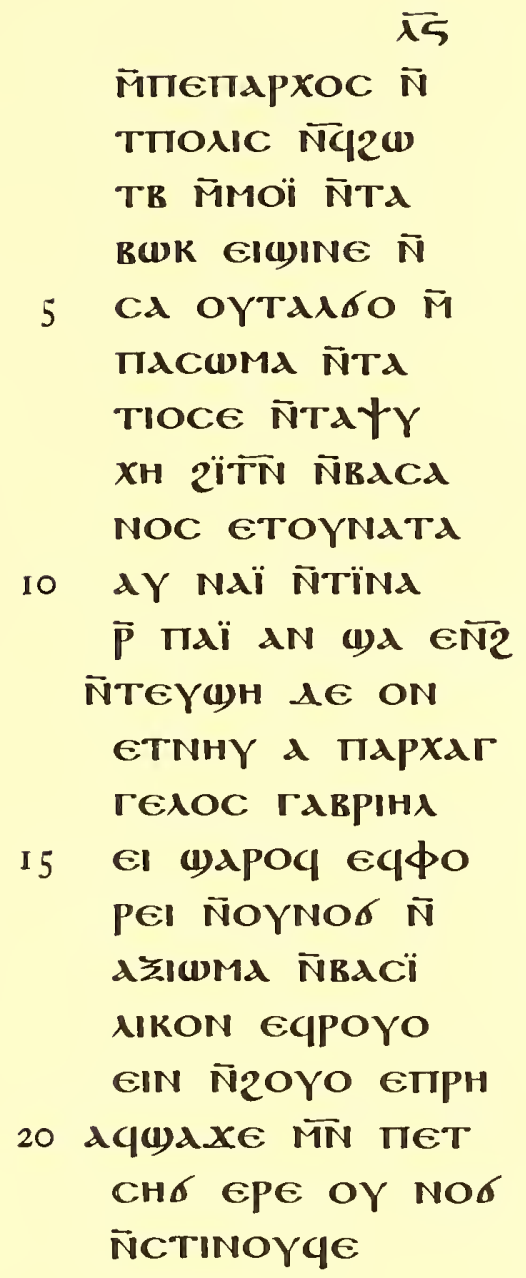


p. 36, col. i

\author{
$\overline{\lambda z}$ \\ NHY EROA $\overline{2 N}$ \\ Т̈тапро АY \\ (1) пехגq Naq \\ xe eтвE OY גK \\ 5 АМєлєї М̈тек \\ eIpe M̈tTeNTdï \\ oYeezCd2Ne \\ MMOG NAK \\ EIME NAK E \\ Io тиï $x \in$ ewwाe \\ जाTNACWTM \\ Ñcoil aN NTTNa

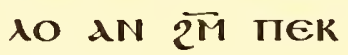 \\ WWNE WAN \\ I 5 TEKMOY EK \\ (1) גN" IIENT"גI \\ oYezcazNe $\bar{M}$ \\ MOG NAK KNA \\ OYXגï $\overline{2 N}$ OY \\ 20 бепн Аүल \\ Tере тірхаг \\ rexoc xe Naï \\ NAG ACBWK
}

7 lege oyezcazne 
p. $3^{6, \text { col. ii }}$

\author{
EBOA 2їTOOTA \\ N̄TEYNOY Aq \\ NE2CE $\overline{2 N}$ OY(WTOP \\ TP MTN OYzOTE \\ 5 Аүल Аqтגхре пाq \\ 2HT KAגलC XE \\ AגH-O.(WC TIPXAT \\ TEAOC ГARPIHA

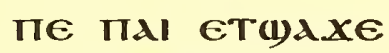 \\ Io NMMAI TENOY \\ бE EC(1)גN(1)(1) \\ N̄CEбOTTT ÑCEMOY

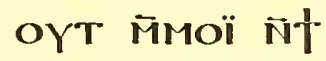 \\ NaP̃TCWTM \\ I5 AN Mitrel cont

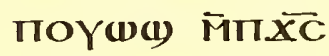

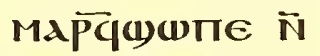 \\ Т2є а ттршме ка \\ п(1)dxe $\overline{2 M}$ пеq \\ $20 \quad 2 H T$

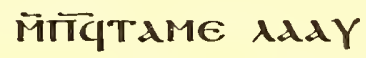 \\ ÑPUME EIIENTAq \\ ш)ाE POYze $\lambda \epsilon$ \\ ज̄TE
}

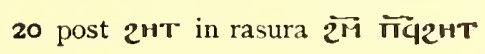


p. 37, col. i

$$
\begin{aligned}
& \text { петснб про } \\
& \text { CYXEI WAN } \\
& \text { TE N̄PwME } \\
& \text { THPOY ETत̃ } \\
& 5 \text { КОТК } 2 M \text { Пто } \\
& \text { TOC 2ÏNHE } \\
& \text { AqTWOYN Aq } \\
& \text { CYPA ÑNEq } \\
& \text { бIX MTN NEq } \\
& \text { IO OYРHнTE } \\
& \text { А पвळK } 2 \text { ג2 } \\
& \text { THN ITPEME } \\
& \text { धто N̄M̄ாо } \\
& \text { AYw Eq†TкגC } \\
& \text { I } 5 \text { Еत̄पОҮРннТЕ } \\
& \text { АqMA2TE } \bar{M} \\
& \text { п20їTе ET } \\
& \text { 2O } \overrightarrow{\mathrm{BC}} \text { EXWप } \\
& \text { AqגPXeI } \bar{N} \\
& 20 \text { Сшк M̄MOQ } \\
& \text { пршмє } \lambda \epsilon \\
& \text { eTT'TKגC } \\
& \text { AYw ETO } \bar{N} \\
& \text { MTIO AqNe? } \\
& 25 \text { CE E2PAI } \overline{2 M}
\end{aligned}
$$

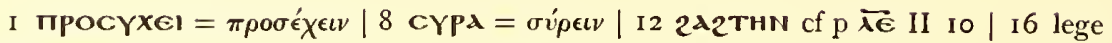
АqAMA2Tе 
$\overrightarrow{\mathrm{AH}}$

p. $37, \mathrm{col}$. ii

ПथINHB $\overline{2 N ~ O Y ~}$

(1)TOPТP AYA

A TINOYTE MN TIAP XARTEAOC ГAR

5 РІн入 जलि2THq E2PAl EXMG dC BWג EROA N̄TEY NOY N̄GI TMPPE

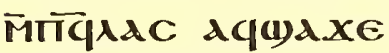

10 кגїпер ज̈пеquд $x \in$ ENE2 גqגa)

KAK EROA $\overline{2 N}$ OYNOб $\overline{\mathrm{N}} \mathrm{CMH} \mathrm{E}$

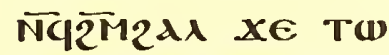

I5 OYN $\overline{2 N}$ OYбETH ज̄TET̄̄́ ПIXICTHC N̄TAq eï Аqपï ज̈ாג20ї TE ET2O $\overline{\mathrm{BC}} \mathrm{C}$

20 хшї петснб

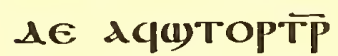
$x \in$ ÑNE N̄2M̃2A MाтिМмגO 60 ITव AYW N̈TEY I7 АICTHC $=\lambda \eta \sigma \tau \eta \dot{s}$ 
p. $3^{8, \text { col. } \mathrm{i}}$

\author{
$\overline{\lambda \theta}$ \\ NOY A ITCOOY \\ TN WWTTE $\bar{N}$ \\ NEqGIX MN \\ OYPHнTE Аप \\ 5 воб̆ е2рдї дq \\ IIWT $\overline{2 N} \mathrm{NEq}$ \\ оҮрннте N̄-QE \\ ÑOYTPOMEYC \\ (1) АNTСRWK \\ Iо ЄாЕपMAN̄N̄ \\ KOTK 2OMAï \\ OC IIENTA NG \\ OYPHнTE $\boldsymbol{W}$

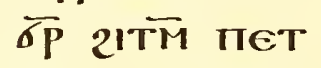 \\ I5 KAC A ITCOOY \\ TN WMTE NAC \\ मपप्ठबप EITECHT

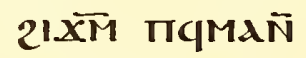 \\ ज̄KOT̄K $\bar{N}-\mathrm{O} \cdot \mathrm{C}$

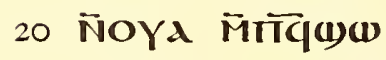 \\ NE ENE2 Aq \\ пUT N̄CA пET
}

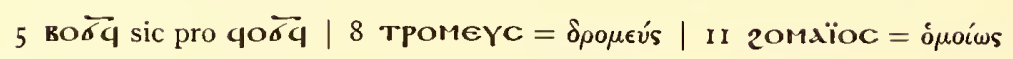




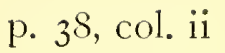

$$
\begin{aligned}
& \text { CHO MTN NEQ }
\end{aligned}
$$

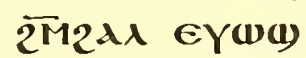

$$
\begin{aligned}
& \text { ध6отіप } \overline{M N} \overline{\mathrm{N}} \\
& \text { Cג OYATPHTE } \\
& 5 \text { АE त̄TEPOYגC } \\
& \text { OANE } \lambda \epsilon \text { EாE } \\
& \text { 2MOT ĨTAC } \\
& \text { TAzOOY ज̈ாE } \\
& \text { CNAY ETE TIPM } \\
& \text { Io MגO пе N̄Tdq } \\
& \text { 入O EqTITKגC } \\
& \text { AYu Aqio Eqo N̄ } \\
& \text { M̈ாо Аүल пет } \\
& \text { CHब ர̄TA пिО } \\
& \text { I } 5 \text { OYTT कWTE } \\
& \text { АYEI ETMMHн } \\
& \text { TE M̈ாECNAY dY } \\
& \text { 2OMONOrei M } \\
& \text { птАлбо ก̄тАq } \\
& 20 \text { घ)ाE M̈MOOY } \\
& \text { MாECNAY AY } \\
& \text { хІшкдK EBOA } \\
& \text { 2ï OYCOा } x \in
\end{aligned}
$$

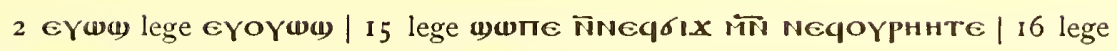
єтмннте 
p. 39, col. i

\author{
OYA пе пNOY \\ те М̈ாגрXar \\ ГEגOC ETOYAAB \\ ГАврінд $\overline{\mathrm{N}}$ \\ 5 тере птннше \\ मE TH $\overline{P q} \in T$ \\ GwOY2 E2OYN \\ EпTопос M̄IIAP \\ xגгеEגOC $\epsilon$ \\ IO TOYAAB ГAB \\ PIHA AYNAY \\ ETEi Nor N̄ฒாH \\ pe AYMOY2 $\bar{N}$ \\ 2OTE EMATE \\ I5 AYW AYCTWT \\ TdzOOY EI \\ TA AYXNE \\ ITPшME $\in T$ \\ снб $x \in$ OY ரTN \\ 20 TAquwte $\bar{M}$ \\ MOK (DANTKK \\ $\overline{\mathrm{P}}$ TId $\overline{\mathrm{N} T O Q} \boldsymbol{\lambda} \in$ \\ дqтардггелї $\mathrm{e}$
}

I A AYNAY lege NAY 
p. 39, col. ii

$$
\begin{aligned}
& \text { POOY ज̄₹Wr } \\
& \text { NIM ÑTAC) } \\
& \text { пе म̈MOq } \mathrm{Eq} \\
& x \omega \text { जिmoc } x \in \\
& 5 \text { GIC O.C MT O.C } \\
& \text { ज̃TAC(1) } \\
& \text { Moï AYW XE } \\
& \text { пархагтелос } \\
& \text { ГАвPIHA ПIEN } \\
& \text { IO TAqOYWÑ } E \\
& \text { POÏ NCTICNAY } \\
& \text { eqфopeï ÑoYC } \\
& \text { XHMA N̈OYOEIN } \\
& \text { c)ANTAए̈ חגї }
\end{aligned}
$$

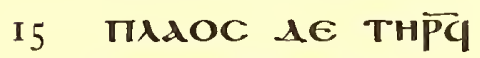

$$
\begin{aligned}
& \text { eTCWOY2 } \epsilon \\
& \text { ZOYN EITOIIOC } \\
& \text { ज̈пAPXAгтеגOC } \\
& \text { eTOYAdR rAR } \\
& 20 \text { PIHA AYQI 2PAY } \\
& \text { EROA } \overline{2 N} \text { OYNOG } \\
& \text { ÑCMH EYXW } \\
& \text { MOC XE OYA пIE } \\
& \text { IINOYTE MIIAP }
\end{aligned}
$$


p. 40, col. 1
$\overline{M d}$

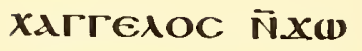

aPE 2ENNOK NE

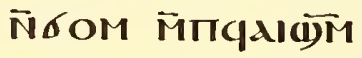

NOYGE MாMN2

5 ГАврІнд пл IтPAN

ÑฒOYTAIOG Пр⿴囗十

ME $\lambda \in$ ÑTAYMA

Ате м̈птגАбо

Аүб́⿴囗 $\overline{2 M}$ пто

Iо пос М̈пдрххГ

Гबגос ГАврIHА

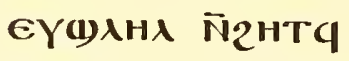

AYW EYNHCTEY

E (1)d пе2OOY $\bar{M}$

I 5 пеYMOY EINA

XE OY N̄TAKd OY

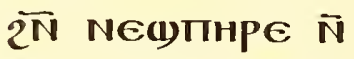

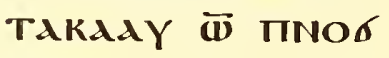

Ñ2PHTळP ÑTE

20 пNOYTE пиОГОС

пגрхагтелос

ETOYAdB Гגв

РІн入 АतHO.लC

EICANP TAOY

25 OEI(1) THप्रव Eï

6 lege mpome $\lambda \in$ cNAY 
p. 40 , col. ii

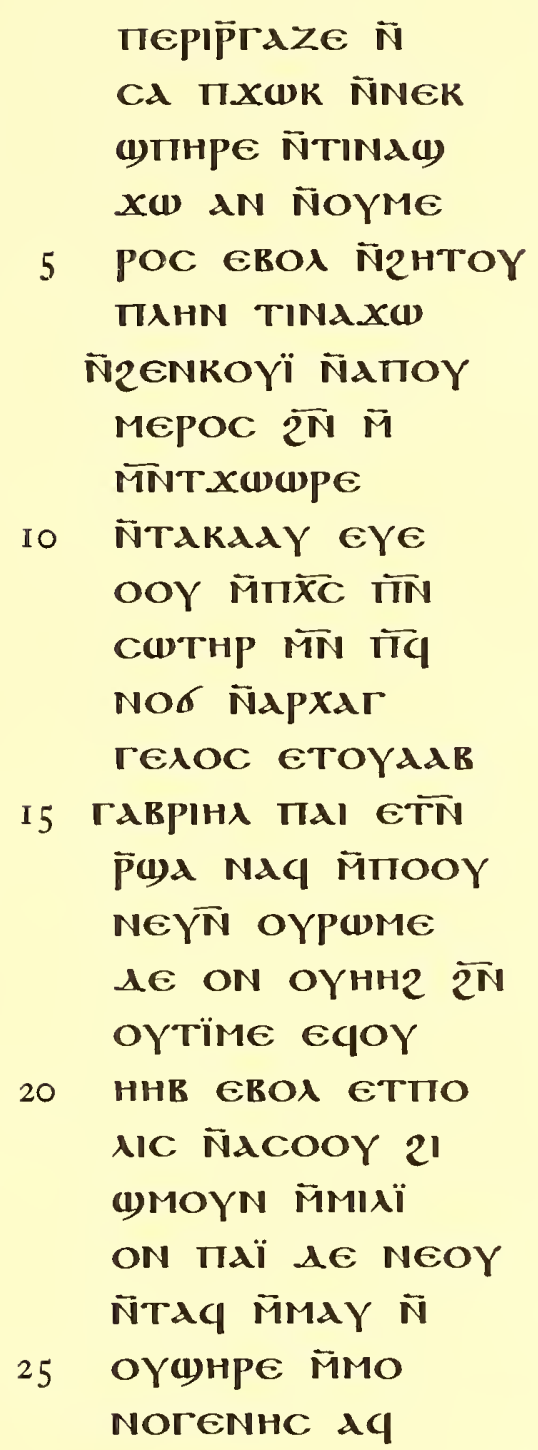

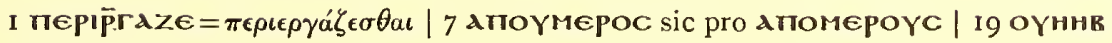
sic pro оүну 
p. 4 I, col. i

\author{
el EITTATMA

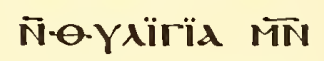 \\ NंCOC ACLE $\mathrm{E}$ \\ 2Paï eYwane \\ 5 eqTiткגс $\epsilon$ \\ NeqбIX MT \\ ज̄वYрннте \\ MITE2OOY MT \\ ТЕҮ(1) MTN(1) \\ I0 бом ज̄MOQ 6 \\ qï xuq E2pai \\ єптн $\overline{P G ~ E N E ~}$ \\ גqP्P COE ÑPOM \\ TE EqNHX $E$ \\ I5 $\overline{X N}$ OYMA N̄N \\ KOTTK EMEq \\ EщTWOYN $E$ \\ 2PAl 2M TIMA

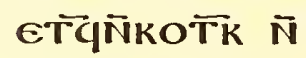 \\ 20 2HTि AYW \\ 242 N̄COI $A$ \\ NEqEIOOTE MTN \\ NEqCYNTENHC \\ EIATOOTOY $\bar{N}$ \\ 25 CWG EYXW $\bar{M}$ \\ MOC $X E$ AlH

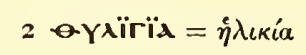


P. 4I, col. ii \\ O-WC OYMTTON \\ NAq TIE TMOY $\bar{N}$ \\ 2OYO EIIICE ET \\ 2IXU⿴ NeqEIO \\ 5 OTE $\lambda \in A Y X \in \overline{2^{N}}$ \\ Noб N̄XPHMA \\ EROA ĨNCEEIN

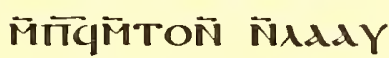 \\ ANAג N̈TAqPZOYO

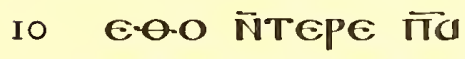 \\ EIWT $\lambda \in$ CWTM \\ eTBE $\overline{\text { N}}$ боM $\overline{\mathrm{MN}}$ \\ NЕ(тнРе ЄTळо \\ оп $\overline{2 M}$ птопос

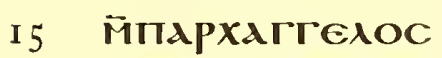 \\ ETOYAAB ГABPI

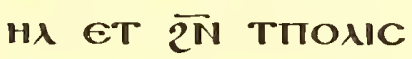 \\ 2РФМн АсеРнт \\ eqxu Mimoc $x \in$ \\ 20 P̈WAN TINOYTE \\ ज̈пגрхаггелос \\ ГАВРІнА СШTT

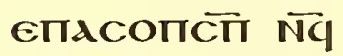 \\ XAPIZE M̈ாTAג \\ 25 бо М̈пдшнре \\ TNATI COOY Ñ
}

7 Ceein sic pro cdein 
p. 42, col. i

Mi

2OЛOK'NOC

2OYN EПKTOПOC

КАTA POMTE (1)

ПЕ2OOY ज̈ாАMOY

5 TCOOYN rap ī

пג $\overline{X C}$ пגрXАГ

гEגOC XE OYT

бОМ М̈Мок $\mathrm{C}$

2WB NIM AY

10 a $x \in$ MNNฒ6́⿴囗十

M̄MOÏ ЄXI M̄ாג

()нре ердт̈

М̈ாЕктопоC

xe Мัмабом

15 M̈MOQ EMOO

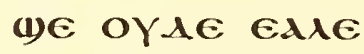

ЄT̈NH єTвE

пNO6 N̈2ICE

ET $21 . x \omega 9 d \lambda$

20 入А тIпICTEY

e $x \in$ текбом

eTOYגAR MOY2

M̈MA NIM MA

PE пеKNA TA

I lege 201око†Noc | $2 \mathrm{~K}$ supra lineam scripta 
p. 42, col. ii

$$
\begin{aligned}
& 20 і ̈ \text { NTxגPlZe } \\
& \text { м̈ாтАһбо ज̈ } \\
& \text { плкоүї їฒнре }
\end{aligned}
$$

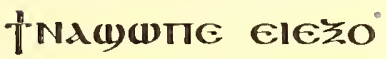

$$
\begin{aligned}
& 5 \text { MONOTEI ÑNEK } \\
& \text { 6OM yd nezOOY } \\
& \text { MITAMOY IN } \\
& \text { TEYNOY } \lambda \in A \\
& \text { тाАрхагтелос } \\
& \text { Iо ГАврIнд CWTM } \\
& \text { धाсопй М̈трш } \\
& \text { Me גqOYW(1) } е \\
& \text { xגpize M̈TTAג } \\
& \text { бо м̈тифшне } \\
& \text { I5 XE OYMANE? } \\
& \text { THप TIE AYW } \\
& \text { AqEINE ÑOYKOYi } \\
& \text { N̄2INHB } \in \overline{X M} \\
& \text { тाшнРе ФнМ }
\end{aligned}
$$

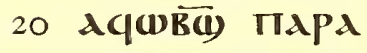

$$
\begin{aligned}
& \text { TिCYO-ï ENE }
\end{aligned}
$$

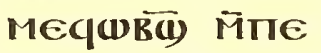

$$
\begin{aligned}
& \text { 2OOY MN TEY } \\
& \text { (1)H EqXI (1) }
\end{aligned}
$$

I 5 sic pro oy(mגnzthq of 
p. 43 , col. i

$$
\begin{aligned}
& \text { KגK EROג ET } \\
& \text { ве пNOб ज̄t } \\
& \text { TKגC ETOYWM } \\
& \text { - Mimog aya }
\end{aligned}
$$

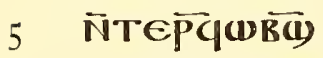

a mapxarteaoc eTOYAAB ГAk рінर eï (1)ג пФНमе ФнМ

IO $\overline{2 N}$ OY2OPOMA eqфopeï ÑoY Nó N̈eOOY NंגT(1)גХe $\epsilon$ poq epe oYc

I5 Tолн Nнх AKTIN N̄OY OEIN EROA боле ероч пе xaq Naq Xe

20 EIC 2нHTE dïcï $\overline{\mathrm{N}} 2 \mathrm{HTK}$ м̈петкגC AYW MN ldAY

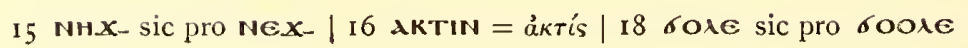


p. 43, col. ii

$$
\begin{aligned}
& \text { ÑZї̈E NATA2OK } \\
& \text { XIN MinïNAY } \Pi \overline{2 P} \\
& \text { wepe nexגq } x \in \\
& \text { TIX } \overline{X C} \text { NTTK NIM } \\
& 5 \text { N̄Tenze epe пеї } \\
& \text { NOr ÑEOOY KW } \\
& \text { тє єрок пєхגq } \\
& \text { NAq XE ANOK } \\
& \text { пе ГАврIHА ттар } \\
& \text { Io XATTEAOC AY } \\
& \text { (w) NTTEYNOY Aq } \\
& \text { रOाTि जिTाEपजि } \\
& \text { TO EROA ÑTE } \\
& \text { NE2CE AE E2TO } \\
& \text { I5 OYe Аप2є बताव } \\
& \text { COMM EqME2 } \\
& \text { N̄RWTE AYW Ñ } \\
& \text { TE } \overparen{P q} A I C \text { O.ANE } \\
& \text { eாTAגбo ÑTAq } \\
& 20 \text { ш)(ाЕ जिMOQ } \\
& \text { AYW XE MTN IAdY } \\
& \text { ÑTKגC 2ï ITqCW } \\
& \text { Md Аүए Аदq० } \\
& \overline{\sigma q} 21 \overline{X M} \text { TापMd }
\end{aligned}
$$

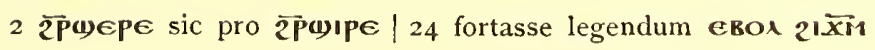


p. $44, \mathrm{col} . \mathrm{i}$

$$
\begin{aligned}
& \overline{M E} \\
& \text { N̄N̄KOTK גq } \\
& \text { म2EPATप Аq } \\
& \text { Moowe eqxï } \\
& \text { BOбC EqCMOY } \\
& 5 \text { EINOYTE AYW } \\
& \text { AqT̄e€ N̄oYA } \bar{M} \\
& \text { TIMUWNE } E \\
& \text { NE2 N̈TEYNOY } \\
& \text { Аप्YOW EBON } \\
& \text { Io } x \in \text { TITEOOY } \\
& \text { NAK TINOK } \overline{\mathrm{N}} \\
& \text { apxarTexoc } \\
& \text { ETOYAAв ГАR } \\
& \text { PIHA } x \in \lambda k \bar{p} \\
& \text { I } 5 \text { памеeүe } \\
& \text { akXapize Naï } \\
& \text { ज̈птдлбо Птव } \\
& \text { EIDT } \lambda \in \mathrm{MN} \\
& \text { T̈MMAXY N̈TE } \\
& 20 \text { PपNAY EITAג6o } \\
& \text { ज̄TOपшшाє } \\
& \text { ज̈теҮФнре } \\
& \text { टN OYФ்TाN } \\
& \text { Фलா АYPï } \\
& 25 \text { (1)E EMATE Ei } \\
& \text { TA AYXNE }
\end{aligned}
$$

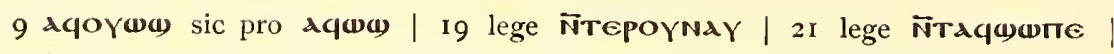
24 AYpiage fortasse legendum AYpime vel AYPAa) 25 a) prius scriptum $\mathbf{P}$ 
p. 44 , col. ii

$$
\begin{aligned}
& \text { пеҮшнре } x \in \text { оY } \\
& \text { пеNФшпе М̈MOK } \\
& \text { пеNMEPIT N̄(1) } \\
& \text { pe ÑTOq } \lambda \in \lambda q \times(1) \\
& 5 \text { epooY ज̈тторомд } \\
& \text { ÑTAqNAY epOq } \\
& \text { ÑTEYNOY AE AY } \\
& \text { (1) EROA } X \in \text { OY } \\
& \text { a ПIE TINOYTE } \\
& \text { Iо їпархגгтелос } \\
& \text { etoyadr rakpï } \\
& \text { HA OYNOE TE } \\
& \text { Auped ÑTdK } \\
& \text { AdC NIMMAN } \tilde{\omega}
\end{aligned}
$$

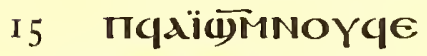

$$
\begin{aligned}
& \text { N̄AГג-O.wC AYW } \\
& \text { aYNor NিPAde } \\
& \text { (1) }
\end{aligned}
$$

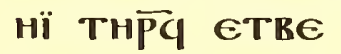

$$
\begin{aligned}
& 20 \text { П"т21бо ÑTAq } \\
& \text { Ф)пе їп(1)не } \\
& \text { ФнM ЕITALH } \\
& \text { NE OYMONOTENHC }
\end{aligned}
$$

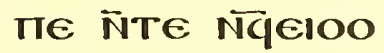

$$
\begin{aligned}
& 25 \text { TE AYU OYON }
\end{aligned}
$$

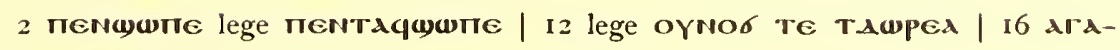

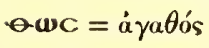


p. 45 , col. $i$

$$
\begin{aligned}
& \text { NIM N̄TAYCW } \\
& \text { TM АҮР̈(пнне } \\
& \text { emate aYteo } \\
& \text { oY M̈TNoYTE } \\
& 5 \text { М̈пархАгте } \\
& \text { 入OC ETOYAAB } \\
& \text { ГАвРIHА АYल } \\
& \text { А ПCOCIT } \Pi \omega_{2} \\
& \text { (1) OYON NIM } \\
& \text { Iо ज̈ாविрдсте } \lambda \in \\
& \text { ENE TKYPIAKн } \\
& \text { TE A прМмE } \\
& \text { MOYTE Еतеप } \\
& \text { (1) А Аपтї } \\
& \text { I } 5 \text { NAq ज̈ாсоOY } \\
& \text { N̄2OגOKOTINNOC } \\
& \text { MTN ZENKEAW } \\
& \text { PON ETTAHY } \\
& \text { AपTNNNOOYG } E \\
& 20 \text { ТполIC 2Ршмн } \\
& \text { етётадץ } 6 \\
& \text { Птопос мпגР } \\
& \text { xגтזеגOC } \epsilon \\
& \text { TOYAגB ГגBPi } \\
& 25 \text { нл прос ө. }
\end{aligned}
$$


p. 45 , col. ii

$$
\begin{aligned}
& \text { 1द } \\
& \text { N̄TAq€P[HT } \\
& \text { MOC пथि[(1)PE] } \\
& \lambda \in \lambda q X I T[O Y] \\
& \overline{2 N} \text { OYPA(1)E } A[q] \\
& 5 \text { TAגq ETq2ïrH] }
\end{aligned}
$$

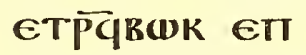

$$
\begin{aligned}
& \text { TOIIOC MitTapxar } \\
& \text { reNoc etoYAdR } \\
& \text { ГАврIHА АYल N } \\
& \text { Io Tер̆м⿴囗十) } \\
& \text { MAYAAq IIAN } \\
& \text { TGLWN EROYN } \\
& \text { ETTTIONIC GAN } \\
& \text { TE WOMNT } \bar{M} \\
& \text { I5 MixION AqEI } \\
& \text { EX্XN 2ENNOE } \bar{N} \\
& \text { 2YAH ÑXEPCOC } \\
& \text { EYMOKू MMO } \\
& \text { O(1) } \mathrm{N} 2 \mathrm{HTOY} \\
& 20 \text { AYW ÑTEYNOY } \\
& \text { EIC OYMOŸ̈ } \bar{N} \\
& \text { ATrPION AqEi } \\
& \text { EROA } \overline{2 N} \bar{N} 2 Y A H \\
& \text { ETXAXU ETM̃ } \\
& 25 \text { MAY EqEATM } \\
& \text { EZOYN EП(1)нРE }
\end{aligned}
$$

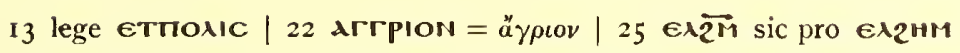


M之

p. 46 , col. $\mathrm{i}$

[山HM] Астшт

[6200]YN EPOq

[Aq] ME2 PUq E

пестाр М̈пшн

5 ре Фнн Аq

сшк M̄MOG $\epsilon$

2OYN $\overline{2 N}$ OY

तH Eq(1) EOY

омव пथне

Iо $А \in$ ФнM АqОY

(19) EROA $\overline{2 N}$

oYNo6 N̈ANAT

Гн EqXu $\bar{M}$

MOC $x \in \pi \lambda \overline{X C}$

I 5 ПлРХАГгелос

ГАвРIHA вO

[H]-1ג EPOÏ $\overline{2 N}$

TI Noб ÑANAT

ГH KCOOYN

20 ГАP пा $\overline{X C} x \in$

N̄Td TIAEIWT

TNNIOLOYT $\epsilon$

ПЕКTOTOC ET

TPdT [N] KOYI

25 N̄AGPON

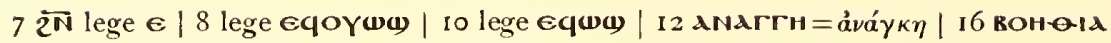
$=\beta o \eta \theta \epsilon i v \mid$ I9 $\mathrm{cf}$ I2 
p. 46, col. ii

\begin{tabular}{|c|c|}
\hline & G2OYN єாєK \\
\hline & MAPTYYPION \\
\hline & eTOYAAR $\bar{N}$ \\
\hline & ТАКтої єाА \\
\hline 5 & Hï a \\
\hline & २HHTE TINA \\
\hline & MOY ĪTOOT̄ \\
\hline & Mாте-YYPION \\
\hline & N̄TEYNOY $\Lambda \epsilon \in$ \\
\hline IO & TM̈MAY GIC IIAP \\
\hline & ХАГтелос $€$ \\
\hline & TOYAAB ГAB \\
\hline & 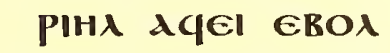 \\
\hline & $\overline{2 N}$ TIE EपథO \\
\hline 15 & peï ÑoYCXH \\
\hline & MA N̄OYOEIN \\
\hline & Аqqї ज̈пшнре \\
\hline & ФHM EROX $\overline{2^{N}}$ \\
\hline & P@q ज̈пाMоYІ \\
\hline 20 & АपсфРАГІZе \\
\hline & М̈пчспाр Аq \\
\hline & 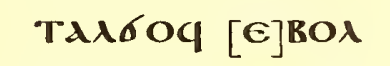 \\
\hline & 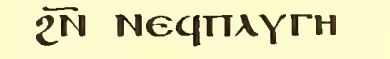 \\
\hline & $\bar{N} T d$ TIMOYI TA \\
\hline & Aq NAq $\overline{2 N} \overline{N q}$ \\
\hline
\end{tabular}


p. 47, col. i

$$
\begin{aligned}
& \text { NAXe AYW ITE } \\
& \text { XגG NAq } x \in \text { AK } \\
& \text { COYWNT } \overline{\mathbf{w}} \text { пाшн } \\
& \text { PE Фнм АपФ(1)ह } \\
& 5 \text { xє ज̈пдї пा } \overline{X C} \\
& \text { IEXAG NAq Xe } \\
& \text { ANOK пе ГARPї } \\
& \text { нג пАрхАГГе }
\end{aligned}
$$$$
\text { АОС петеквнк }
$$$$
\text { IO ETTqTOTIOC } \boldsymbol{A}
$$$$
\text { NOK ПENTAÏє }
$$$$
\text { (1)גPOK AїTAג }
$$$$
\text { бок } \overline{2 M} \text { ПЕк(1) }
$$$$
\text { NE ANOK ON ITN }
$$

I5 TAINO2 M̂K ET тАтिо ज̈пєеY

PION AYW $\bar{N}$ TE TqXe Naï גq АMA2TE N̄TqбוX

20 АqтАлоq $E \overline{X M}$ ITCOÏ ज̄TIMOYi Аүш Mாе пMOYï

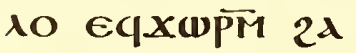

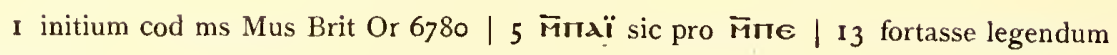
EBON $\overrightarrow{2^{M}}$ 
$\overline{M Z}$ (sic !)

p. 47, col. ii

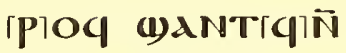

TQ ГEI2OYN EII

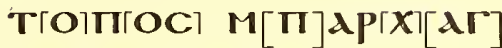

ГEA ГOC $[$ E]TOYA

5 AR ГАврIнА 6

PE $\Pi[\mathbf{M}]$ ннше $\mathbf{T}[\mathrm{H}]$

$\overrightarrow{P Q} \cdot O[G(W] P G I M$

MOQ EYР̈(I)

PE MMOG $\bar{N} T E$

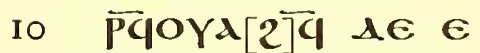

песн[T 2]

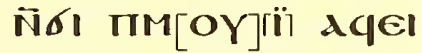

EROX [2]ГN] TMH[H]

те їтмннше

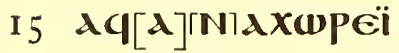

N[dC] ETTMMA $E$

PE OYON NIM $\sigma \omega$

(1)T ज̄CWG

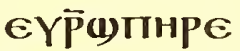

20 MTN ÑCA Nai

А ПтннФе Тн $\overrightarrow{P G}$

XNE ПШНРE ФнМ

XE OY IIENTAG 
p. 48 , col. i

$$
\begin{aligned}
& \text { MH } \\
& \text { Ф)ПЕ М̈MOK } \\
& \text { ÑTOKEI TWN } \\
& \text { єкдАнY етеї } \\
& \text { MOYï N̄TOC } \lambda \epsilon
\end{aligned}
$$

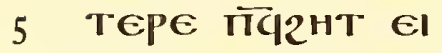

$$
\begin{aligned}
& \text { EPOC } 21[\bar{X}]^{N} \text { O. } \\
& \text { OTE N「TAC]W(W } \\
& \text { IIE MM[OC] } \overline{\mathbf{N}} \\
& \text { тере плגос тн } \\
& \text { IO } \overline{P G} \overline{M N} \text { TCYГतH } \\
& \text { TOC EIT'[C]WOY2 } \\
& \text { E2OYN [EIT]TרOIIOC }
\end{aligned}
$$

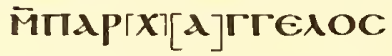

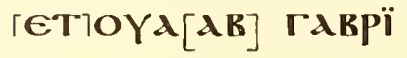

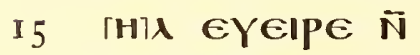

$$
\begin{aligned}
& \text { TCYNAそC N } \\
& \text { TepoYCw[TM] } \\
& \text { eNdï aүu(1) } E \\
& \text { BOג EYXw } \\
& 20 \\
& \text { MOC } X E \text { OY } \\
& \text { d IIE IINOY } \\
& \text { Tе M̈пархגГ }
\end{aligned}
$$

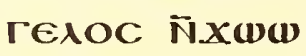

$$
\begin{aligned}
& \text { ре гаврінд OY }
\end{aligned}
$$

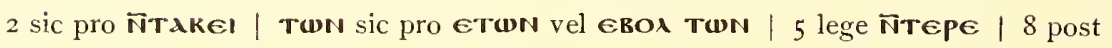

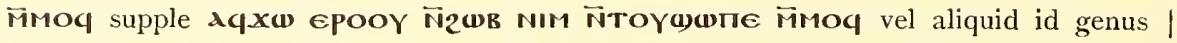
20-2I membrana jam ante hac lacerata litterae nullae hoc loco omissae sunt 
p. 48 , col. ii

$$
\begin{aligned}
& \text { No6 TE TER } \\
& \text { (i) TIApxarrenoc } \\
& \text { GTOYAגR ГגBPI }
\end{aligned}
$$

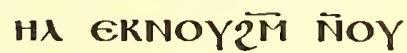

$$
\begin{aligned}
& 5 \text { ON NIM ET2EA } \\
& \text { пI>е EPOK пा)н }
\end{aligned}
$$

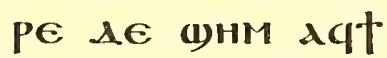

$$
\begin{aligned}
& \text { ÑÑAMPON ETÑTO } \\
& \text { OTA EROYN ET }
\end{aligned}
$$

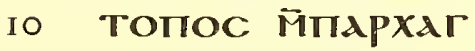

$$
\begin{aligned}
& \text { rEAOC GTOYAdR } \\
& \text { ГАврIнд MT̃ NंCOC } \\
& \text { ज̈TवOYW2 ЕTOO } \\
& \text { Tव धкTOQ Еाт } \\
& \text { I Hii dald aqo(i) } \\
& \text { 2M ПтопоC } \bar{M} \\
& \text { пाрХАГГеגOC } \\
& \text { ГАврина єч(у)на } \\
& \text { AYW EqNHCTEYE } \\
& 20 \text { AYW ÑTEPE NEq } \\
& \text { EIOOTE EI GגPOC } \\
& \text { AqXa EPOOY N̄2(1) } \\
& \text { NIM N̄TAY(1) } \\
& \text { пе MMOQ AYPA } \\
& 25 \text { WE EMATE }
\end{aligned}
$$


p. 49 , col. i

\author{
AYW aYteooY \\ M̈ாNOYTE ர̄TOG \\ $\lambda \in \lambda q \sigma a 2^{M}$ Птто \\ поC EqTIATW \\ 5 Nel ud nezOOY \\ MाTqMOY EqEi \\ pe ÑzeN Nor M \\ полYTï AYW \\ Аqฒ)ात N̄oY \\ Io PEME N̄CWTTா

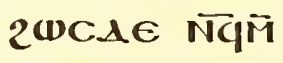 \\ II()A ÑNAY E2A2

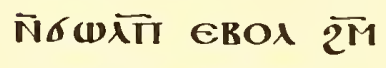 \\ птопоО ज̈пाР \\ I5 ХАГГСАОС ЕTOY \\ AAR ГАвРIHА \\ AYU NGMHN E \\ BOA $\overline{2 N}$ COICT

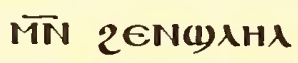 \\ 20 ENAWWOY \\ ATETTNNAY $\bar{\omega}$ \\ NAMEPATE \\ xe eүб̆̈бOM \\ Ñda) Ñ2E N \\ 25 б1 MNTT(1)גNE2
}

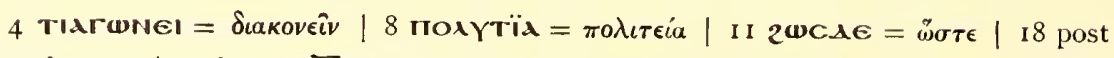

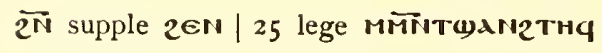


M०O.

\author{
THq M̈TNOYTE \\ MN ज̄COात̄ जे \\ пдрхАГГСлос $€$ \\ TOYAAB ГABP!

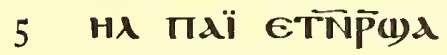 \\ NAq ज̈ா[O]OY A \\ NON $\lambda \in$ zWWN

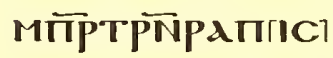 \\ TOC ENIMAEIN \\ Iо MTN Ne(1)THPE $\bar{N}$ \\ TАYल(1) $\overline{2 M}$ \\ Птवтопос ETOY \\ AdR $x \in R d C \bar{N}$ \\ NENXI N̈OYNO6 \\ I5 N̈KPIMA Kaï ГגP \\ 2N OYME AYOE

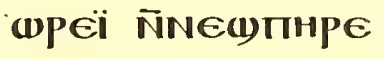 \\ THPOY $\overline{2 N}$ Na \\ BAA AYU A 2 A2 \\ 20 N̄2HTTHYTTN \\ NAY EPOOY $\lambda$ IÖ̈ \\ TON MAPNTIC \\ TEYE N̄6OM MN

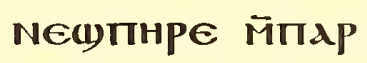 \\ 25 ХАггелос ГАврIнג
}

23 lege єก̃бом 
p. 5o, col. i

$$
\begin{aligned}
& \bar{N} \\
& \text { TM TIEN2HT TH } \\
& \text { PQ XEKaC N̄NA } \\
& \text { xï N̄OYNOK } \bar{N} \\
& \text { AMPEA EBOX } 2 \\
& 5 \text { TM INOYTE IIHN } \\
& \text { CWTM EPOI } \bar{\omega} \\
& \text { NAMEPATE N̄TA } \\
& \text { Xw EPWTN } \bar{N} \\
& \text { TiNoб N̄ฒтнре } \\
& \text { IO MITAPAגO之ON } \\
& \text { EIOYWÑ2 EROX } \\
& \text { जัTMัTNTAHT } \bar{M} \\
& \text { пגPXAГTEגOC } \\
& \text { гАврIн入 MTN } \\
& \text { I } 5 \text { TMNTOYA2ї } \\
& \text { 2HT М̈TALАBO } \\
& \text { IOC MTN NEqAגi } \\
& \text { MUN XE CEGO } \\
& \text { рб Eтрфме } \bar{M} \\
& 20 \text { ПЕЕООY MN TËY } \\
& \text { (1) EYOYW(1) } \\
& \text { ерाIIET-OOY } \\
& \text { NAq NEYTN } \\
& \text { OYPAME } \lambda \in
\end{aligned}
$$


p. $5 \mathrm{O}$, col. ii

ON N̄P̄TATHC

OYH2 2їTOYल4

M̈TाTOTIOC M̈ாג[P]

XAГTEAOG ГA[R]

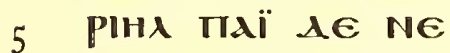

OүАMPH пе $\overline{2 N}$

OYТTTHXHN

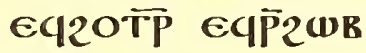

$2 \lambda$ пеqівеке еq

Io ตANTwOYN $\Lambda E$

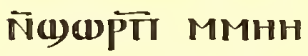

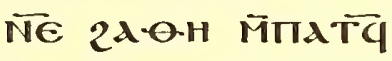

RWK ETTपMA

N̄P2WR Ф)גपाWK

I5 E2OYN EITOIIOC M̈пाрхагтелос

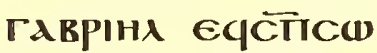

ரтव Eqxw M̄MOC

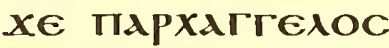

20 ETOYAגB ERE

COOYTT ÑNA

2lOOYE AYW $\bar{N}$

TEï 2E шגपTגY

O N̄ฒOMNT $N$

7 оүт̈ттин lege теqтехnн 
p. 5 I, col. i

$$
\begin{aligned}
& \text { соп ज̄ฒ1нג } \overline{\mathrm{Nq}} \\
& \text { СФРАГІZе } \bar{M} \\
& \text { MOQ NEqRWK }
\end{aligned}
$$

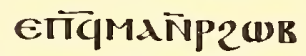

$$
\begin{aligned}
& 5 \text { ENE MNAdAY } \bar{M} \\
& \text { пETNANOYG } \overline{2 M} \\
& \text { Tावвї тн } \overline{P q} \\
& \text { OYAE NHCTIA } \\
& \text { OYde MNTHA } \\
& \text { Io N̄CA пגï M̈MATE }
\end{aligned}
$$

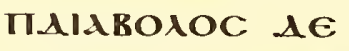

$$
\begin{aligned}
& \text { пMOCTE пет } \\
& \text { NANOYG NIM } \\
& \text { मq2ICE Eqбo } \\
& \text { I5 } \overline{\mathrm{PO}} \text { epoq eqoY } \\
& \text { (w) EeIPE Naq } \\
& \text { Nं zENTETOO } \\
& \text { OY М̈ாеqеш } \\
& \text { бOM EPOQ ET } \\
& 20 \text { ве пथОМัNT } \bar{N}
\end{aligned}
$$

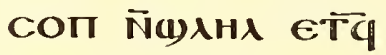

$$
\begin{aligned}
& \text { EIPE M̈MOOY } \\
& \text { MN TROHOEIA } \\
& \text { M̈INOYTE MN }
\end{aligned}
$$

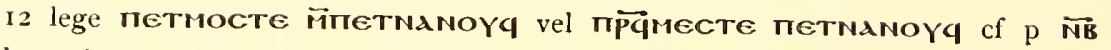
col i 15 vel mmactmetnanoya $\mid 17$ post litteram primam litura in qua î̀ 
p. 5 I, col. ii

$$
\begin{aligned}
& \text { пархагтелос } 6 \\
& \text { TоYגАB Г̈גврї } \\
& \text { HA ECRETIZE M̀ } \\
& \text { MOQ АCWUாE } \\
& 5 \text { Ає M̄MOQ N̄oY2O } \\
& \text { OY AqW } \overline{C K} \text { EपशI } \\
& \text { NHB A TाPH G) M }
\end{aligned}
$$

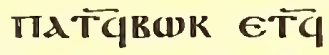

$$
\begin{aligned}
& \text { PTAcïl aүa пе } \\
& \text { Io xגq eqpaï Ñ2HTq } \\
& x \in A \ddot{A} \overline{C K} \text { TWNOY } \\
& \text { MTOOY ETPAвШK } \\
& \text { CIIAMAN̄̄̄TE } \\
& \text { NTTEi 2E бe a TAME } \\
& \text { I5 AIA MTN ITPOOY() } \\
& \text { ET(1)OYEIT CWK }
\end{aligned}
$$

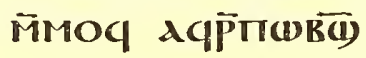

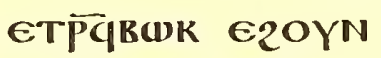

$$
\begin{aligned}
& \text { єாтопос ज̈ாगР } \\
& 20 \text { XATГEAOC ETOY } \\
& \text { AdB ГАRPIHA KA } \\
& \text { Td TACYNHO-IA } \\
& \text { MัMHнNE АqREK }
\end{aligned}
$$

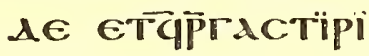


p. 52 , col. i

$$
\begin{aligned}
& \overline{\mathrm{NB}} \\
& \text { ON AqTAגO } \lambda \in E \\
& \text { TETPIP ETPिMO }
\end{aligned}
$$

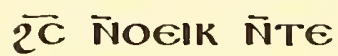

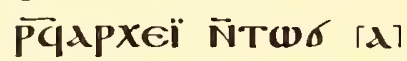

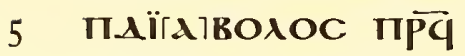

$$
\begin{aligned}
& \text { MECTE ПETNA } \\
& \text { NOYG NIM Aq } \\
& \text { AMA2TE N̈TC̄OY } \\
& \text { РнHTE 2IITA2OY } \\
& \text { Io AqXaNA M̄MOq } E \\
& \text { песнт ЕтЕтрIP }
\end{aligned}
$$

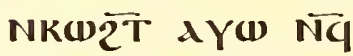

$$
\begin{aligned}
& x \omega \text { M̈MOC } x \in \overline{\mathbf{w}} \\
& \text { пАTАKтоC גї }
\end{aligned}
$$

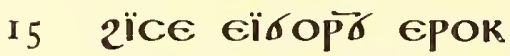

$$
\begin{aligned}
& \text { EIC ()OMTE N̈POM } \\
& \text { пе тїертла) } є
\end{aligned}
$$

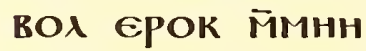

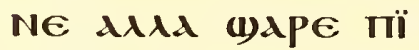

$$
\begin{aligned}
& 20 \text { घOMNT N̄COT } \bar{N} \\
& \text { Ф人нд єтекеіре } \\
& \text { MMOY M̈MHнNE } \\
& \text { २M Птопос } \bar{M} \\
& \text { ПАРХАГГелОС }
\end{aligned}
$$

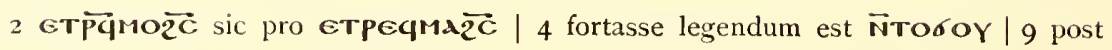
оүрннте supple cente 


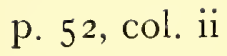

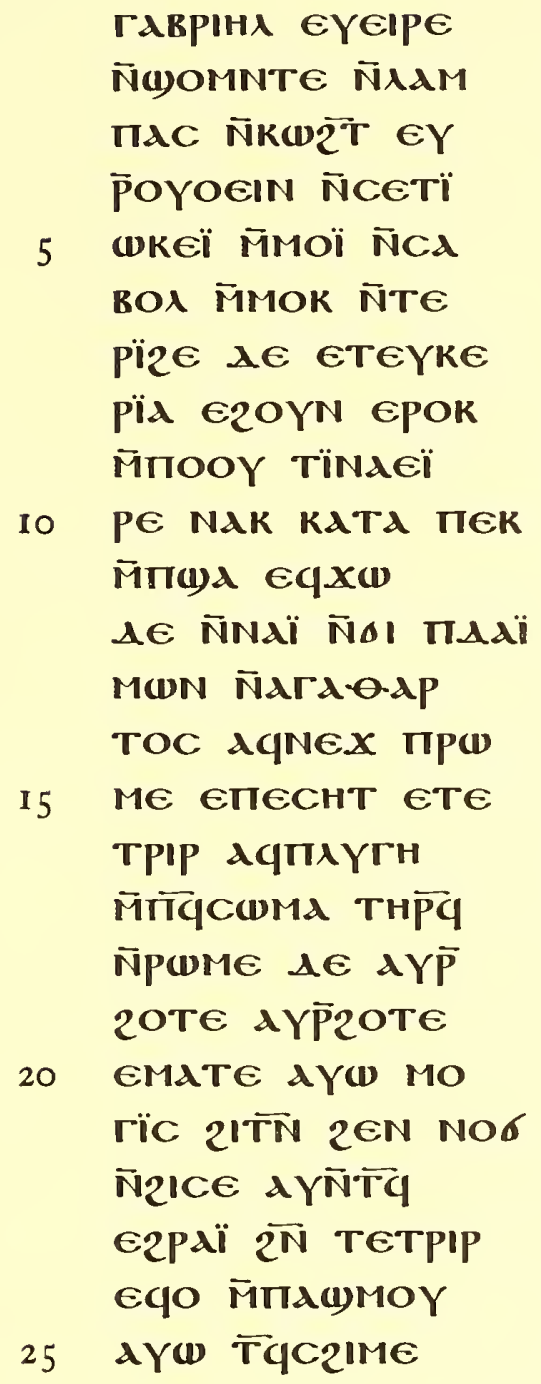


p. 53, col. i

$$
\begin{aligned}
& \text { MN NeqщнPE AY } \\
& \text { пE(1) NEY2OÏTE } \\
& \text { AYA()KAK EROA } \\
& \text { AYPIME } \overline{2 N} \text { OY } \\
& 5 \text { No6 N̄Cläe dY } \\
& \text { TAlOG EX } \overline{X N} \text { OY } \\
& \text { MAN̄N̄KOTTK } \\
& \text { AYXITG E2OYN } \\
& \text { हाтопос } \bar{M} \\
& \text { Iо ПАРХАГГеגОС } \\
& \text { ГАвРІнА Еүпрос } \\
& \text { AOKI } x \in \text { EqNa } \\
& \text { MOY M̈IATE прH } \\
& \text { 2थTत AYW AY } \\
& \text { I } 5 \text { кште ероч Тн }
\end{aligned}
$$

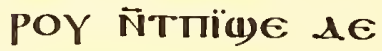

$$
\begin{aligned}
& \text { N̄TEY(H EIC } \\
& \text { пАРХАГГЕАОС } \\
& \text { ГАвРІнА АपOY } \\
& 20 \text { ON2 EBOA } \bar{M} \\
& \text { IтPMME } \overline{2 N} \text { OY } \\
& \text { 2OPOMA Eqo } \\
& \text { M̈IECMOT } \bar{N} \\
& \text { OYCTPATYAג } \\
& 25 \text { THс }
\end{aligned}
$$


$\overline{N T}$

p. 53, col. ii ЄqфOPEi ÑOYCXH

MA ÑOYOEIN TIE

Xגq NAq $x \in$ גKCOY

WNT \& TPQME

5 А

Me пеXגq $x \in M$

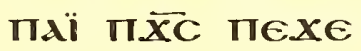

IтAPXAгтелOC

Naq $x \in$ edi) $x \in \bar{M}$

IO IIE ANOK ITETAR

PIHA TIAPXATTE

IOC ПETKNHY M

MHнNe E2OYN E

गापTOாОС धКСОा

I5 $\overline{C T}$ जIMOQ ETPप्NA2

MEK EROA $\overline{2 N}$ NEK

o-dïtic THPOY a

NOK TITNOY $\overline{2 M}$

M̈MOK N̈бOPб̄C

20 MाTALABOAOC $\bar{N}$

$2 \lambda 2$ NTCOTT ANA

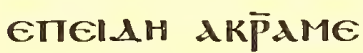

入HC ÑCAq MITIEK

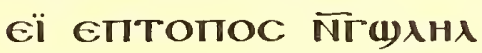

25 eтве плї а пАIдволос

EINE EXWK N̈TINO6

6 мпגі sic pro мпе | 7 п $\overrightarrow{x C}$ correctio $\Pi \lambda$ (xocıc) in margine | I9 fortasse legendum ймок євол $\overline{2 \mathbb{N}} \overline{\mathbf{N}}$ борб $\overline{\mathrm{C}}$ sed cf $\mathrm{p} \overline{\mathrm{MZ}}$ col i 19 
p. 54 , col. i

$$
\begin{aligned}
& \text { M̈IAYГH ANOK } \Lambda E \\
& \text { M̈ாIकв(1) ЕРОК } \epsilon
\end{aligned}
$$

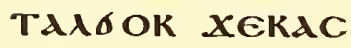

$$
\begin{aligned}
& \text { EPE OYON NIM क̄ } \\
& 5 \text { Nर AYW N̄CEEI } \\
& \text { ME ETMNTбWR } \\
& \text { M̄TAIABOAOC AY } \\
& \text { (1) } x \in \text { TвOH-Eї } \\
& \text { a M̈INNOYTE MN } \\
& \text { Io NEqATTEAOG ET } \\
& \text { CKEாגZE N̄TMNT } \\
& \text { PUME TH } \\
& \text { AYW } \bar{N} T E \overline{P G} X E \\
& \text { Naï АqCфрAгї } \\
& \text { I5 Ze M̄IĆ̂ma TH }
\end{aligned}
$$

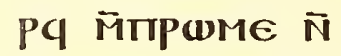

$$
\begin{aligned}
& \text { TАपРОКू } \overline{2 M} \text { пाкш } \\
& \text { 2T } \overline{2 M} \text { TMAEIN } \\
& \text { M̈ாЕGTAYPOC } \\
& 20 \text { Аүल пехגq NAq } \\
& \text { xe EIC2HнTE AI } \\
& \text { тАлбок мітр } \\
& \mathrm{OYW}_{2} \text { ETOOTK } \\
& \text { ерамелнс е }
\end{aligned}
$$

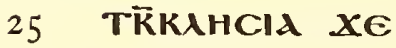


p. 54 , col. ii

$$
\begin{aligned}
& \text { ÑNE TIETOOOY } \\
& \text { eтral wwTE } \bar{M} \\
& \text { MOK AYW } \bar{N} \\
& \text { TEPप̄Xe Naï NAq } \\
& 5 \text { Aq1O EqNaY EPOq }
\end{aligned}
$$

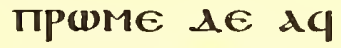

$$
\begin{aligned}
& \text { qоб̆ E2pגï eq } \\
& \text { CTWT } 2 \lambda \text { O.OTE } \\
& \text { AqRe EPOY EA NE } \\
& \text { Iо пАYГн тнроY Ет } \\
& 2 \bar{M} \text { ПपСсшм } \lambda O \\
& \text { AqP̈2RA Aqda) } \\
& \text { KAK EROA } \overline{2 N} \text { OY } \\
& \text { No6 N̈CMH } X E \\
& \text { I5 OYA חIE חNOYTE } \\
& \text { M̈тархגгеєАоC }
\end{aligned}
$$

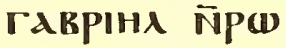

$$
\begin{aligned}
& \text { ME } \lambda \in \text { ThPOY ET } \\
& \text { cWOY2 E2OYN E } \\
& 20 \text { ПтTOाIOC MாגP } \\
& \text { хגгтелос ГАв } \\
& \text { РінА АүாПт } \\
& \text { e2pdi exuq } \\
& \text { EYOYW(1) ENAY }
\end{aligned}
$$


p. $55, \mathrm{col}$. i

\author{
ETNOo N̄ฒTHPE \\ N̄TАCฒ凹TE \\ AYW AYNAY E \\ петоYMEєYє \\ 5 POq $x \in$ eqNa \\ MOY МПגТЕ прH \\ 2WTTI EqOYOX \\ EMTN OYாАYГH \\ N̄OYWT 2M ПЕQ \\ IO CWMA AYP2O \\ TE GMATE AY \\ (1) EYX(1) M̄MOC \\ XE TICMOY $E$ \\ POK IINOYTE $\bar{M}$ \\ I 5 ПגРXAгтелоC \\ eTOYAAB ГAB \\ PIHA MTN $\bar{N}$ \\ CA NAI AYXNE \\ TिФME $X \in \overline{\text { iv }}$ \\ 20 TENTAqWWTE \\ M̄MOK N̄CAC \\ АК2Е ЄПЕСН' \\ єтетрір Акрш \\ К2 тнТ̈ Аүш \\ $25 x \in \bar{N} T$ Trגo $\bar{N}$ \\ (1) $\bar{N} 2 E 2 N$ Tे
}

I9 $\overline{\boldsymbol{\omega}}$ sic pro or 
p. 55 , col. ii

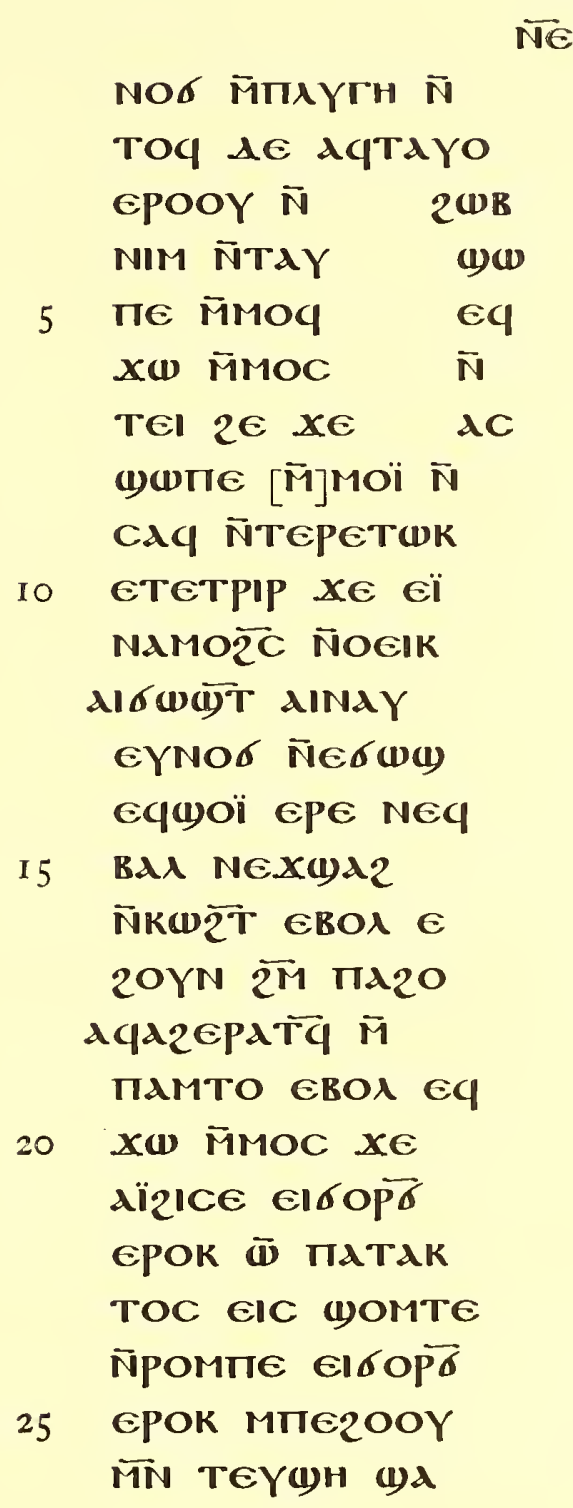

3-7 membrana jam antehac lacerata nullae litterae hoc loco omissae sunt $\mid 9$ lege

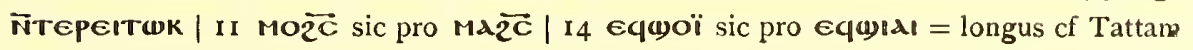

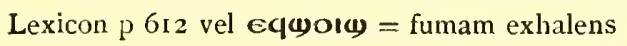


p. 56 , col. i

$$
\begin{aligned}
& \text { N5 } \\
& \text { PE } \Pi \text { IEI }
\end{aligned}
$$

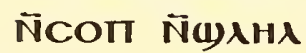

$$
\begin{aligned}
& \text { e TekeIpe } \\
& \text { M MOOY } \overline{2 M} \\
& 5 \\
& \text { ПTOПTOC } \\
& \text { MiтAрхגГ } \\
& \text { ГEAOC ГAR }
\end{aligned}
$$

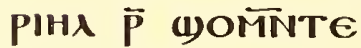

$$
\begin{aligned}
& \text { N̄AAMIIAC N̄OY } \\
& \text { IO OEIN N̄KW2T } \\
& \text { N̄ceTEIOKE M̀ } \\
& \text { MOÏ ÑCA BOA } \bar{M} \\
& \text { MOK స̄TEPÏ }
\end{aligned}
$$

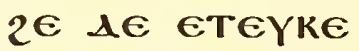

$$
\begin{aligned}
& \text { I5 PÏג ЕРОК Мाто } \\
& \text { OY AIEI NAK } E \\
& \text { TPdeIPE NAK KA } \\
& \text { Tג пекज̈пाय } \\
& \text { АYU ÑTE } \overline{P G} X E \\
& 20 \text { NAÏ АqAMג2TE } \\
& \text { ज̄TАОҮРннте } \\
& \text { CENTE AqNOY } \\
& x \in \text { M̈Mö̈ єाє } \\
& \text { СHT ETETPIP }
\end{aligned}
$$

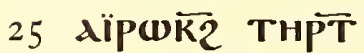

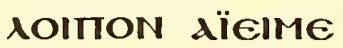

$$
\begin{aligned}
& x \in \text { AYNTT EחIMA }
\end{aligned}
$$

3-7 membrana antehac lacerata litterae nullae hoc loco omissae sunt 8 घoMT̃Te

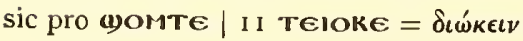


p. 56, col. ii

INTICOOY[N AN] XE

a)diOYOÑ $[H] x \in$

(1)AIMOY IEIBOA $X E$

גї2WN EROYN $E$

5 ПMOY AYW $\overline{2 N}$

TïOYwH dïnaY

emapxartedoc

eTOYAdB ГABPI

н入 дqєї сарої

Io גqсфрגг̈̈zе ї

мої аqталбої

Аqвек є2рдї $\epsilon$

TाE $\overline{2 N}$ OYCXH

Md ÑNOYOEIN

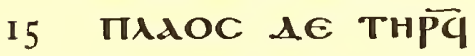

ÑTE TिNAY ENAI"

АүР̈шпнре $\epsilon$

MATE AYW AY

MOY2 N̄ZOTE $A$

20

TETNNAY (i)

NAMEPATE $x \in$

EYढ̆MбOM Nंגa)

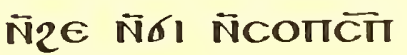

М̈ாגрXАГГЕАОС

25 ETOYAגB ГАВ

рінд aүल $x \in$

PUME NIM

2 oүo $\overrightarrow{N 2}$ sic pro $\omega \overline{N 2}$ 
p. 57, col. i

\author{
ЄТвнк ЕТЕКкАн \\ cId MMHнNe \\ MEPE AAAY $\bar{N}$ \\ AגIMUNION $\in(1)$ \\ 5 ZWN E2OYN E \\ POQ EITHPप \\ ANAג TROHOEIA \\ MINNOYTE NA

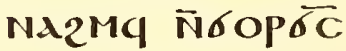 \\ io MiाmCATANAC \\ пє(1)лнд пете \\ (1) גqNOYXE $E$

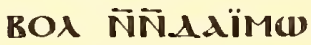 \\ NION TIE(1)גHג \\ I 5 ПЕTNO $\overline{\text { NM }}$ \\ NET $\overline{2 N}$ TIKYN \\ TINOC MTN $\in X \ddot{i}$ \\ MWN MTN N̈TA

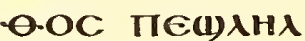 \\ 20 петсол $\overline{\mathrm{C \lambda}} \overline{\mathrm{N}}$ \\ NET2HE TEK \\ кגнсїд пе пाג

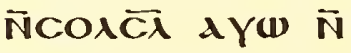 \\ cWOY2 N̄N̄Ar \\ 25 ГЕАОС ТЕККАн
}

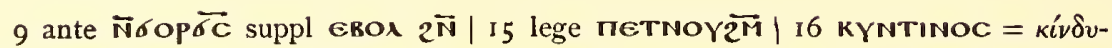

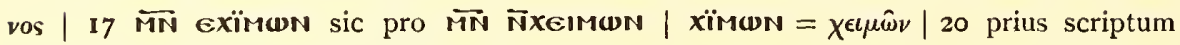

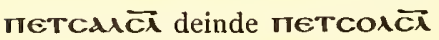


p. 57, col. ii

\author{
$\overline{N Z}$

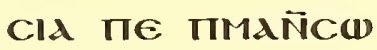 \\ OY2 ÑNEIXEIPOY \\ BEIN M̄N ÑCEPAфEIN

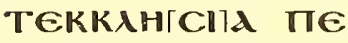 \\ 5 TIMAN̄MTTON $\bar{M}$ \\ †YXH NIM ANON \\ Le zUN MAPNME

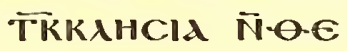

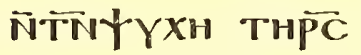 \\ IO пето ÑAMENIC \\ ЄТТкКннІА Eq \\ NOYXE N̈-OTE

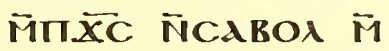 \\ MOG AYW TIETNA

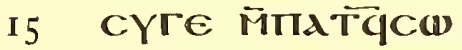 \\ TM ENANATNGCIC \\ THPOY EqCYNATE \\ ज̈m(1) М̈ாє⿻с⿰ \\ NATE ETXHK $€$ \\ 20 ROג IIETNAEï $\epsilon$ \\ BOג $\overline{2 N}$ TKKNHCIA

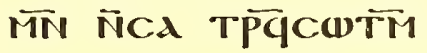 \\ eNANATNUCIC TH \\ POY $\overline{N G 2 M O O C ~} 21 \overline{P N}$

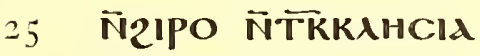 \\ птаї N̄Tï MINE OY
}

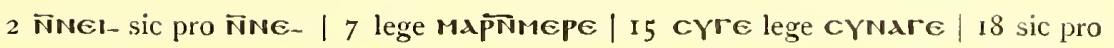

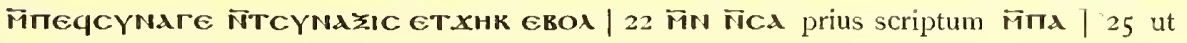
videtur litterae 21 deletae et legendum त̄Po 


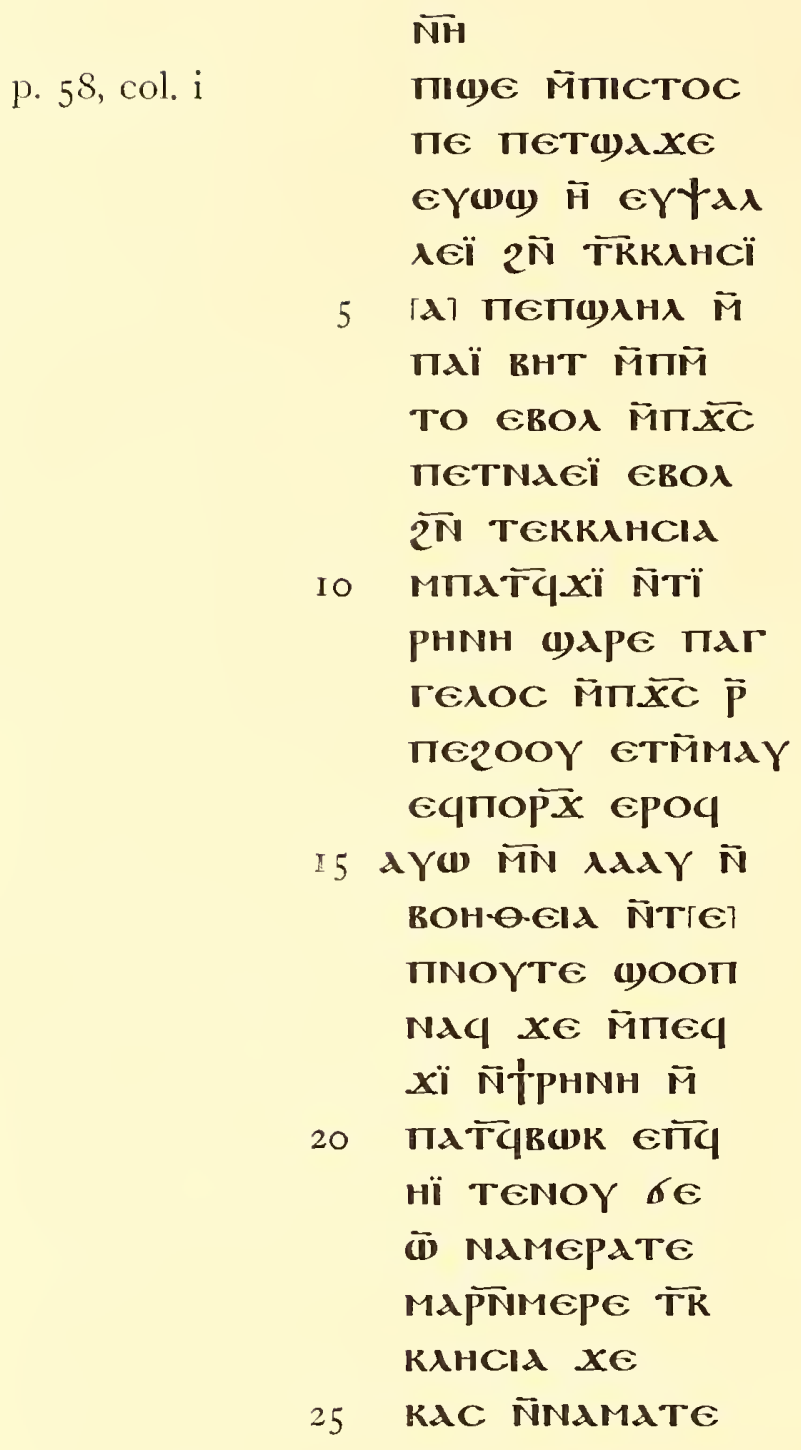

5 пєпшлнх lege пєшлнх | 16 ante $\mathbf{N}$ erat $\boldsymbol{\omega}$ vel $\boldsymbol{\omega}$ ut videtur deletum 
p. 58 , col. ii

\author{
ӤпKW EROA N̄ \\ NENNORE [M]A \\ PN̄̄ed M̈TIOOY \\ M̈ாАРХАГГЕАОС \\ 5 ГАврІнА ПтАवाँ \\ NOYGE ÑNIIIIWN \\ ज̈ாOYTOEIIN $\overline{2 N}$ OY \\ (1) MIITINEYMA† \\ KON 2WC NTCOOYN \\ Io $x \in \bar{N} T O 4$ пE TIA \\ гелос M̈трдае

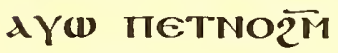 \\ ÑOYON NIM $e$ \\ TOYXII M̄MOOY \\ 15 N̄ \\ TTN TAIABOAOC \\ MAT̄PNCCNTE \\ NัTOOT̄N $\bar{N} \bar{N}$ \\ 2нке тPГOाC TTN \\ 20 бом поYА поY \\ d MMON XeKAC \\ epe пархатте \\ АOC ГАВPIHA \\ NAOYWN NAN \\ 25 N̄NA2WwP $\bar{N}$
}

I2 lege петмoy 
p. 59, col. i.

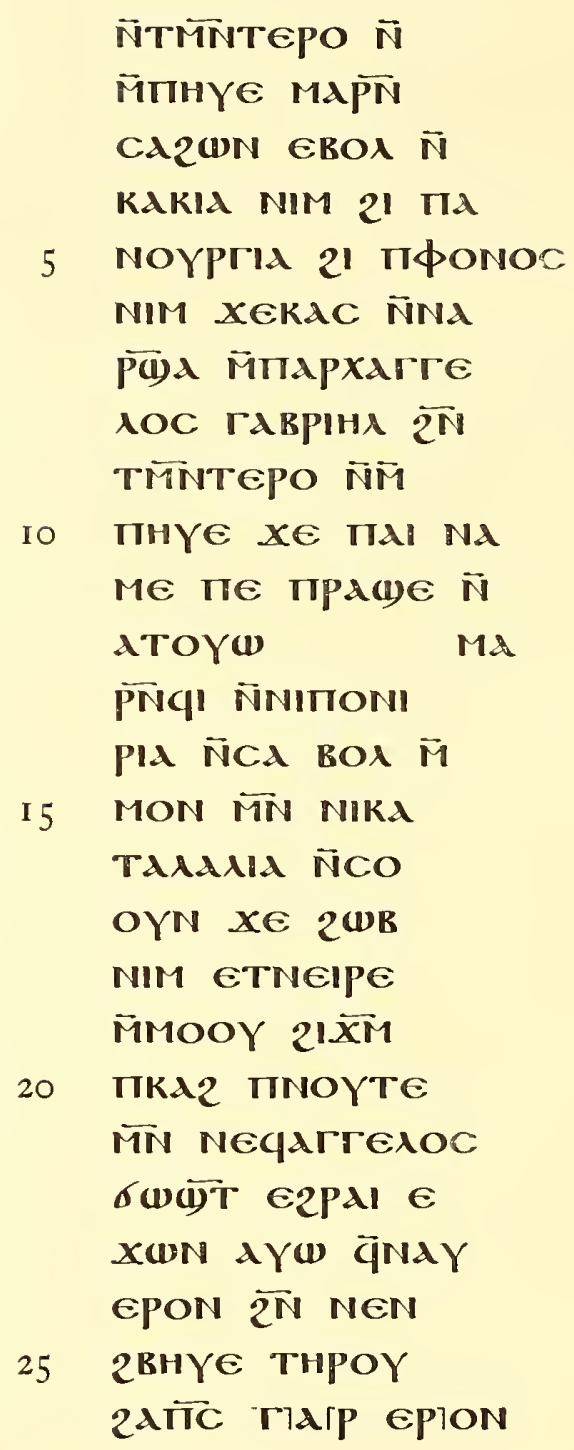

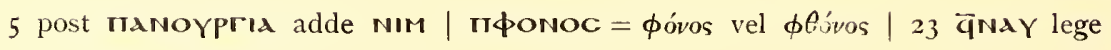
eynay vel fortasse îcenay 
NOO

p. 59 , col. ii

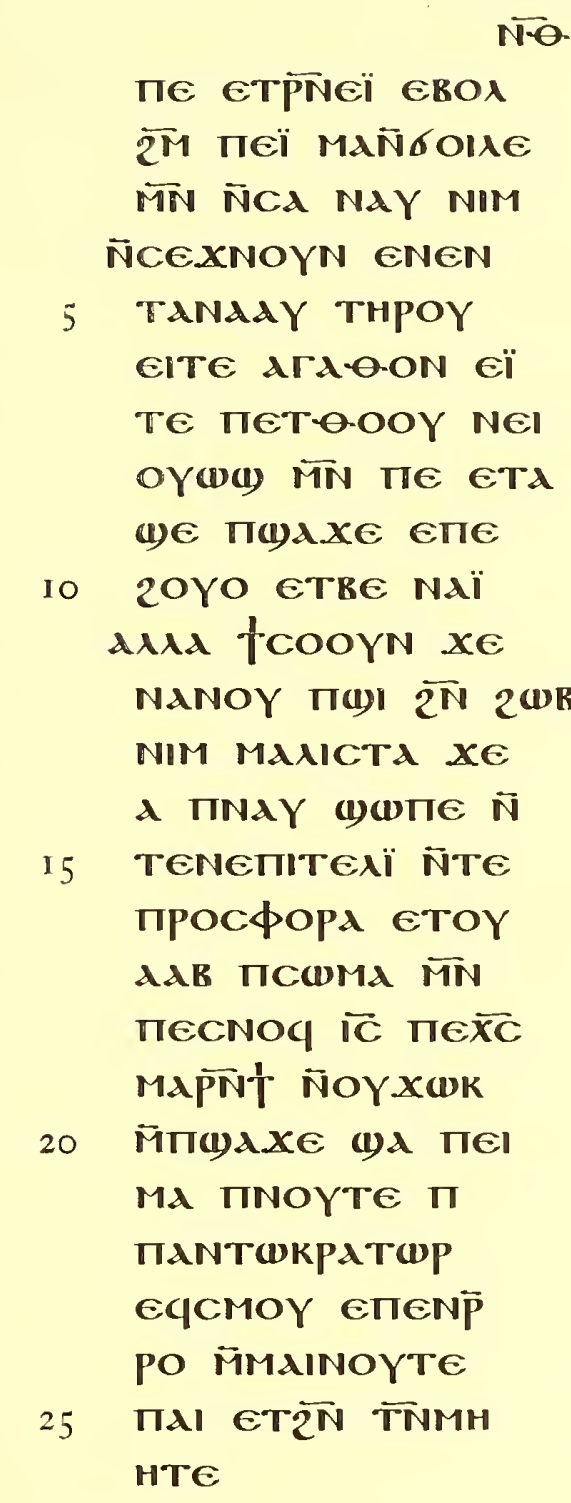

$5 \mathrm{~N}$ prius scriptum d $\mid 8$ M̄N $=\mu \epsilon^{\prime} \mid 23$ lege eqecrior 
p. 60, col. i

产

ज̈ாоOY AYW $\overline{N q}$

POEIC EPOC N̄-E

ÑAdA M̄N COAO

MUN MTN IECEKï

5 גC $\overline{M N}$ ÏwcïdC

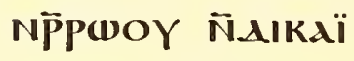

oC AYw eqe2d

$\overline{P 2}$ ETCYNKNY

TOC MN TKYPOY

Io cId THPC ÑNE2P

MAÏOC MTN TAY

MOC TH $\overline{P Q} \bar{N} T \pi O$

NIC EITE 2OOYT

EITE C2IME EITE

I5 KOYï eITE NOr $\overline{N q}$

$\bar{P}$ OYNA ÑTNYYXH

M̈ாє2OOY ETNNA

AחגNTA EPOY $2 i$

$\overline{T N} \bar{N} C O \Pi \overline{C T} \overline{M N}$

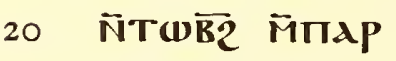

XАГГEגOC ETOY

AגB ГАврIH入 тגї

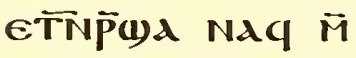

поOY $\overline{2^{N}}$ тाா $d Y$

25 (1) $21 \overline{X M} \pi \mathrm{KA} 2$

2ITN TEXAPIC

9 кYPOYCld $=\gamma \epsilon \rho o v \sigma i \alpha$ 
p. 60 , col. ii

\author{
MN TMTNTMAÏ \\ PQMe M̈ITEN \\ xoeIC $\overline{I C} \pi \epsilon \overline{X C}$ \\ пеNCWTHP \\ 5 TIÄ EBO人 2ITOO \\ Tq MN TाबEWT \\ NंArA O.WC $\overline{M N}$ \\ TINA ETOYAAR \\ TENOY AYW \\ IO OYOEIA NIM \\ (1) ENE2 ÑE \\ NE2 2АMHN प्.
}

6 post 21Toot $\vec{q}$ adde neooY NAq of Brit Mus Ms Orient numero 7597 p $\overline{\mathbf{z}}$ apud Budge Miscellaneous Coptic Texts in the Dialect of Upper Egypt 1915 p $155 \mid 8$ lege

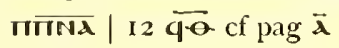



PLA'TE X.

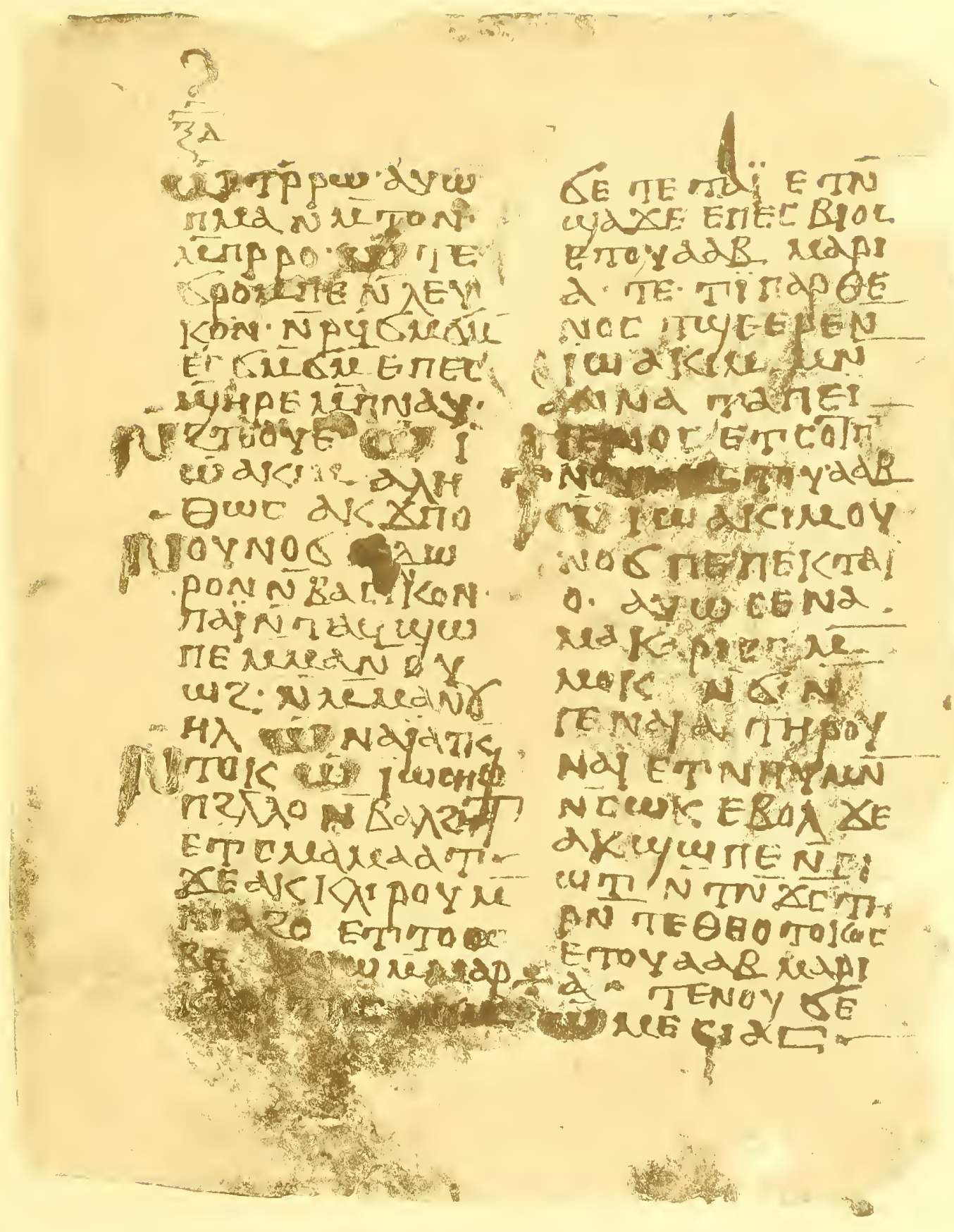

COPTIC HOMILY ON THE VIRGIN

Washington Coptic Manuscript No. 2 [p. 64] 

A HOMILY ON THE VIRGIN BY THEOPHILUS,
ARCHBISHOP OF ALEXANDRIA

p. 60 , col. ii

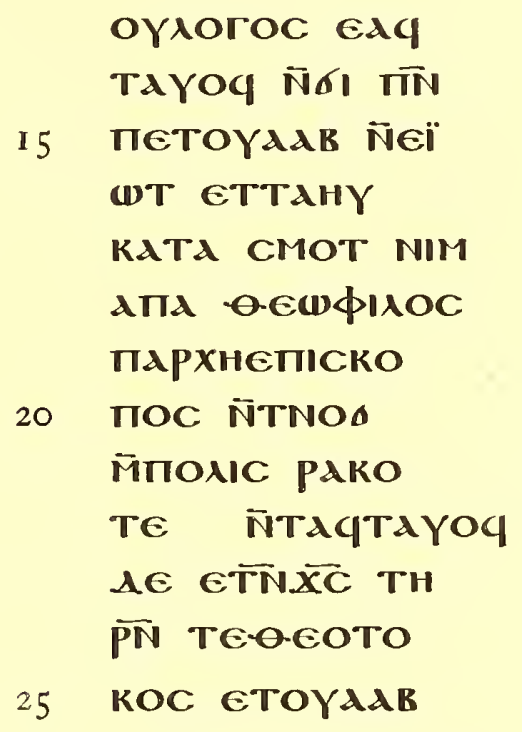

22 ก̄TAqTAYoq prius scriptum N̈TAYTAYOq 
p. $6 \mathrm{r}, \mathrm{col} . \mathrm{i}$

$$
\begin{aligned}
& \text { MA[PIA] TPपXतE } \\
& \text { п[NOYTE } \overline{2 N} \text { OY } \\
& \text { M[E N̄TAqT]גY }
\end{aligned}
$$

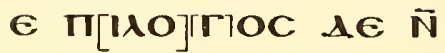

$$
\begin{aligned}
& 5 \text { CoYM̄NTACE M̀ } \\
& \text { MECOYPE ETE } \\
& \text { candaIMTIC } E \\
& \text { TOYAAB N̄TAY } \\
& \text { xाTC EzPaï EM } \\
& \text { Iо пнYе ज̄2HTि } \\
& \text { AqUגXe } 1 E \text { ON } \\
& \text { eтвE OYPWME } \bar{N} \\
& \text { ¿̈вPAIOC ETOYH } \\
& \text { H2 } \overline{2 N} \text { TTIONIC } \\
& \text { I } 5 \text { Pגкоте Аqпाс } \\
& \text { TEYE EாE } \overline{X C} \text { ET } \\
& \text { RE }- \text { GIKWN } \bar{N} T \bar{T} \\
& \overline{X C} \text { THPि TOATI } \\
& \text { d MApla ECCH2 }
\end{aligned}
$$

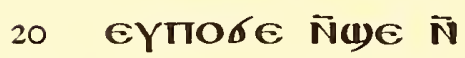

$$
\begin{aligned}
& \text { 2OYN EYATIO-Y } \\
& \text { кн АqФдxe } \lambda \epsilon \\
& \text { ON ETBE TNOб } \\
& \text { MMNTEBÏHN M } \\
& 25 \text { TтршME 2N OYE } \\
& \text { PHNH ÑTE TNOY }
\end{aligned}
$$

I4 $\overline{2 \pi}$ prius scriptum $\mathrm{CT}$ vel $\mathrm{cm} \mid 20$ prius scriptum поке deinde побе| EY-lege T̃zoYN ÑOY-vel T̈2OYN $2 \bar{N}$ OY- 
$\overline{\mathrm{K} \lambda}$

p. 6I, col. ii

[TE] 2AMHN qब्̈.

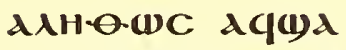

NAN M̈TIOOY Ñ

5 бा пірптеєүе

Tєеєотокос $€$

TOYAdR MAPIA

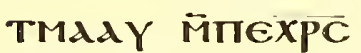

†сWTM rap eात

Iо профнтнс 6

TOYAAB $\overline{A \lambda A} \in q$

(wi) EBOA $\overline{2^{N}} \mathrm{NE}$

talMOC eqXa $\bar{M}$

MOC XE AYXW

I 5 Ñ2ENTAIO ET

внHте тाIONIC

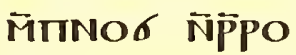

(i) Namepate

MH TNXXOeIC

$20 \quad \lambda N$ TE T.e-drïd

MAPId Tגї ÑTגC

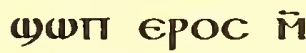

пNOYTE ПגOГOC

$\Pi \in \overline{X P C} \overline{I C} \Pi \in N \overline{X C}$

25 d(1) TE THOAIC $\bar{M}$

חINO6 N̄PPO 
p. 62, col. i

$$
\begin{aligned}
& \mathrm{2k} \\
& \text { TeIMeEYe } x \in \\
& \text { TKdגd2E TE Md } \\
& \text { pïa тїпарөє } \\
& \text { Noc Taï ÑTAC } \\
& 5 \text { Ф)ШாЕ MিMAN̄ } \\
& \text { (1)шпе ज̈пגогос } \\
& \text { M̈IEIWT EAq } \\
& \text { eï גqoYar } \overline{2 N} \\
& \text { TECKAגA2H } \bar{M} \\
& \text { IO †ї N̄EROT AC } \\
& \text { хпоव } E \overline{X M} \text { пкג2 } \\
& \text { АपशाாОMїN } \overline{\mathrm{N}} \\
& 2 \omega B \text { NIM NTTN } \\
& 2 € \text { MANTT TNO } \\
& 15 \text { ве М̈L̈वрNoве } \\
& \text { OYAe MாOYze } \\
& \text { EKPOQ } \overline{2 N} \overline{T Q T A} \\
& \text { про дqвшк } E \\
& \text { 2Pגї єйпнүЕ Єגq } \\
& 20 \text { 2MOOC 2ï TOYNAM } \\
& \text { MIT|CIaT } \overline{2 N} \\
& \text { XOCE AYW ON }
\end{aligned}
$$

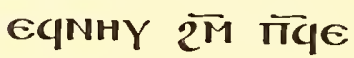

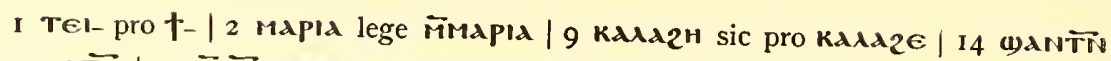
sic pro 
p. 62, col. ii

$$
\begin{aligned}
& \text { OOY NGIKP[INE] } \bar{N} \\
& \text { NETON2 [MN N] [TT] } \\
& \text { MOOYT } A[Y(w] \text { NG } \\
& \text { тї їпоYа пाоYа } \\
& 5 \text { КАТА ज̆व2внүЕ } \\
& \text { EITIE] ATAQON [EI] } \\
& \text { TE петөОOY TE } \\
& \text { NOY } 6 \in \text { ii NAME } \\
& \text { PАTE MAPNKK } \\
& \text { IO 2ENCMOT NंTII } \\
& \text { ПIP.QGNOC } \overline{2 N} \\
& \text { 2WB NIM } X E A C
\end{aligned}
$$

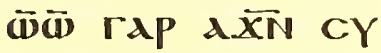

$$
\begin{aligned}
& \text { NOYCIA Ñ2OOYT } \\
& \text { I5 ACMICE AXXN Tï } \\
& \text { TKAC AC2AOOAE } \\
& \text { ज̈п̆сунне пєС } \\
& \text { (ब) } \\
& \text { бOOIG N̄2ENTO } \\
& 20 \text { EIC ACWTOQ } 2 \overline{\mathrm{N}} \\
& \text { OYOM̆G ज̄T̈RNH } \\
& \text { ÑTOQ TE TENNOY } \\
& \text { TE } 2 \bar{N} \text { OYME AYU } \\
& \text { กัтоq пе пाнне }
\end{aligned}
$$

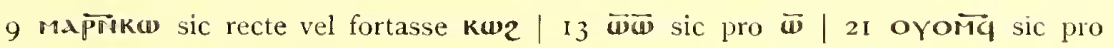
oyoyomव 
p. $6_{3}$, col. i

$$
\begin{aligned}
& \text { M̄TNOYTE ET } \\
& \text { CMAMAdT GA } \\
& \text { ENE2 } \overline{\mathbf{\omega}} \text { TIIAP } \\
& \text {-ENOC ETOYAdR }
\end{aligned}
$$

5 MAPIA MATA

Гїд тнр्ष тї

IO MEEYE TAP ANOK

xє пнї єтрџळооп

Nं2HTि TENOY

TE ब.IAHM $\overline{\mathrm{N}}$

тाЕ 2АरTN поY

I5 Фнне РПард

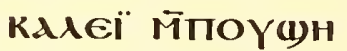

PE N̄oYoeIC

NIM 2APON A

NON NIIPÄPNO

20 BE (I)ANTqबN

2THC 2APON

xe OYZIKANOC

ГАР Пе єсCоп

$\overline{C \pi} 2 \lambda$ ПГENOC

8 өpıcriid $=\theta_{p \eta \sigma \kappa \epsilon i a} \mid$ I I eTp-sic pro єTepe- $\mid$ I5 $\overline{\mathbf{p}}$ - sic pro epe- 22 lege OYZIKANOC . . п пе 
p. 63 , col. ii

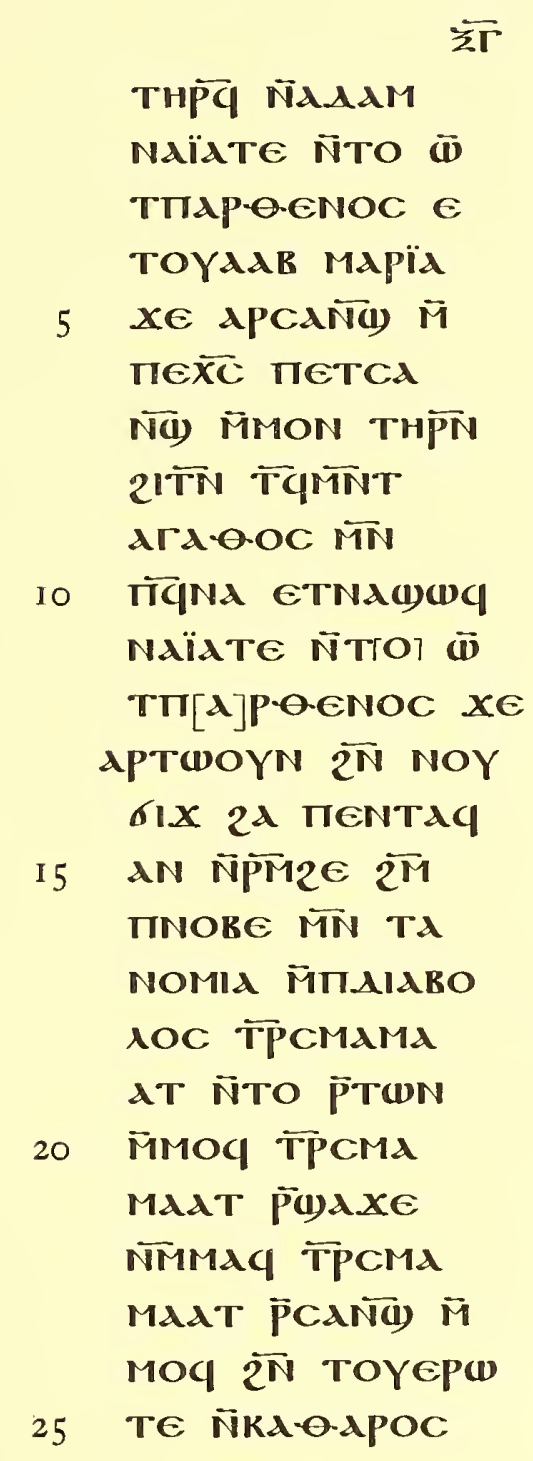

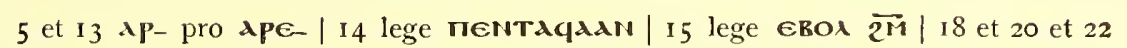

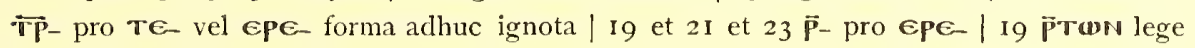
PTWOYN 
p. 64 , col. i

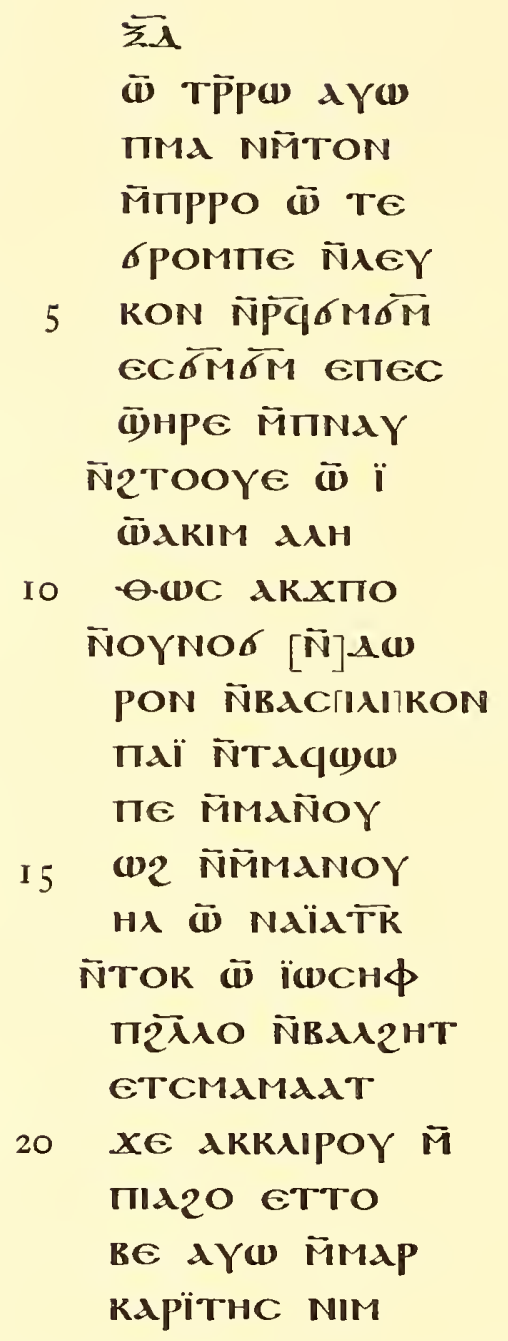

究

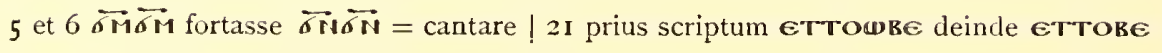


p. 64 , col. ii

\author{
бE TE Taï ETT̄ \\ (1)גXe EIECBIOC \\ ETOYAdB MAPI

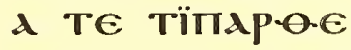 \\ 5 NOC TUEEPE $\bar{N}$ \\ ïגКाM Мल \\ ANNA TA TEi \\ ГENOC ETCOTTI \\ PNOYNE ECTOYAdB \\ IO $\overrightarrow{\mathrm{D}}$ ÏWARIM OY \\ Nor ne neRTdï \\ O AYU CeNd \\ MAKגPIZE M̀ \\ MOK N̄́l Nं \\ I 5 ГENAIA THPOY \\ NAÏ GTNHY MTN \\ N̄CWK EROA $x \in$

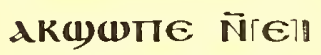 \\ 1T $\overline{\mathrm{N} T} \overline{\mathrm{N}} \overline{\mathrm{XC}}$ Тн \\ 20 PN TEOEOTOKOC \\ eTOYAגB MAPI \\ a TeNOY $6 E$ \\ (i) Mecide
}

9 ectoydak lege eтоүAגв 
p. 65, col. i

INOYTE MME XAPIZE NAN

2WOK $\bar{N}$ AwPE

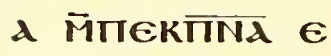

5 TOYAגB $\bar{N} \overline{T N}$

XE zENkOYï $\bar{N}$

eПIENOC EாTГגї

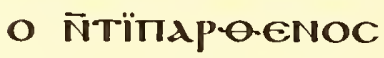

ANON $A \in 2 \omega W N$

IO $\overrightarrow{\mathrm{w}}$ NCNHY MA

PN $2 A \overline{P 2}$ EPON $N$

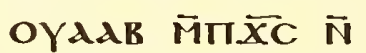

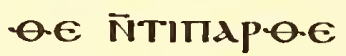

ETOYAAR MAPï

I5 A Ñ2OYO $\lambda \in M \lambda$

PN T̈вBO M̈TाEN

CWMA MN TIEN

2HT ज̈TNCA

2WWN EROA $\bar{N}$

20 POOYG NIM $\bar{N}$

TE IIBIOC $\overline{\mathbf{N}}$

COOYN Mitraï $x \in$

ज̄внк E2pגI N̄бוx

M̈ாINYYE ETO

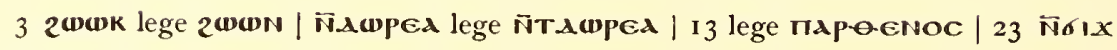

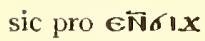


产

p. 65 , col. ii

N2 a Yu E6Ix

N̈PaMe AN $\overline{\mathbf{w}}$

TM̃NTIAP.O.E

NOC TRA.O-APIOIC

5 MாTCWMA MN TE

†YXH MTN пETाNA

21 OYCOा пеXA

PICMA MITETINA

eTOYAAB $\overline{\mathrm{D}}$

IO TMINTIIPAENOC

теткג-APIZE $\bar{M}$

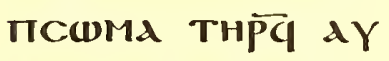

(1) ETEIPE N̄TE

†YXH N̈Kג-APOC

I5 EBOג 2M пЕ2топ

M̈TAIABOAOC MN

NEqTIA) EO-OOY

(b) XE TMNT

TIAP-ENOC $\ddot{\mathrm{P}}$

202 2WB EOYHP

АРETH NIM IIENTAC

TAגTE IIOC E

NE2 AqXIMITE

TM̃NTIAP-OENOC

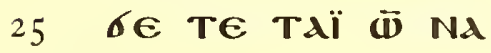

4 lege тит̄ткג-aגpoc 
p. $66, \mathrm{col} . \mathrm{i}$

$$
\begin{aligned}
& \text { گद } \\
& \text { MepdTе eтpe } \\
& \text { TOYA TIOYA } 2 A \\
& \text { P2 ETिMMAN̄̄N }
\end{aligned}
$$

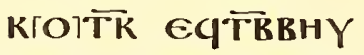

$$
\begin{aligned}
& 5 \text { २M ПCABBATWN } \\
& \text { MN TKYPIAKH } \\
& \text { MN 2OOY NIM } \bar{N} \\
& \text { (1) } \\
& \text { NIM N̄CYNATE } \\
& \text { IO KגTA N̄KANON } \\
& \text { ÑNENEIOOTE } E \\
& \text { TOYAdB N̄AITOC }
\end{aligned}
$$

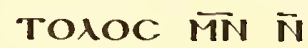

$$
\begin{aligned}
& \text { COC חe2ME Ñ } \\
& \text { I } 5 \text { 2OOY ETOYAגB } \\
& \text { MN TNICTIA } \\
& \text { CENTE MN } \bar{N} \\
& \text { (1) TнPOY } \bar{M} \\
& \Pi \overline{X C} \text { גï̈ тї } \\
& 20 \text { Cоात̄ ज̈MW } \\
& \overline{T N} \overline{\mathbf{\omega}} \text { NACNHY } \\
& \text { MAT̄ } \overline{P N} \text { POPEI } \bar{M} \\
& \text { ITPAN N̄TMTT }
\end{aligned}
$$

I3 
p. 66 , col. ii

\author{
TIPAENOC "Nं" \\ ()еере шнм ГТ) \\ TOYAגK M̈ாגP \\ बENOC M̈IATE \\ 5 TEN2TER $X E$ \\ NA NTTOOTTN \\ N̈CGKAdN ÑCd \\ BOג MITPO M̈TIMA

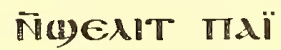 \\ Io eте \\ NAKOT"q AN EY \\ АYTIH ПNE2 \\ бE ET 2T ÑNAM \\ TIAC TIE TINA $E$ \\ I 5 2OYN $\bar{N} 2 H$ KE \\ MÑ NETṔ́PO2 \\ MN NOPQANOC \\ KA"TA MIIXXE \\ MiTCA2 їккивос \\ 20 XOOQ $\overline{2 N}$ NंKA-OO \\ 入ïkoN $x \in$ пापM \\ (1) $⿱ \epsilon \in$ GTYYAB \\ AYW $\bar{N} A T T \omega \bar{M}$
}

2 ante $\varepsilon$ vestigia $\mathbf{T l}$ aut $\dagger$ lege

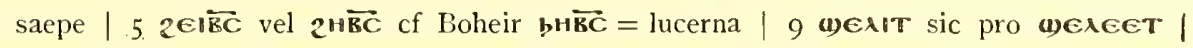

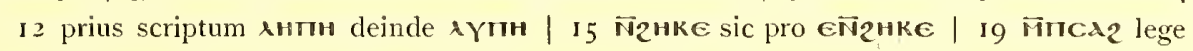

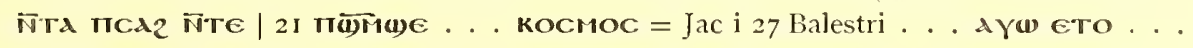

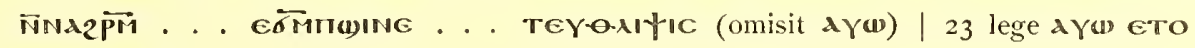
जิATTWत̄T 
p. 67, col. i

$$
\begin{aligned}
& \text { ÑNA2 T̄N INOY } \\
& \text { TE пеIवт пе }
\end{aligned}
$$

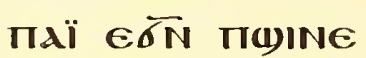

$$
\begin{aligned}
& \text { ÑNOPФANOC } \overline{M N} \\
& 5 \text { Nexнрд e2pגï }
\end{aligned}
$$

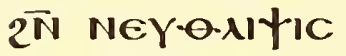

$$
\begin{aligned}
& \text { AYW ETPप12APZ } \\
& \text { epoq EтWत्रा } \\
& \text { 2M тाкOCMOC тाе } \\
& \text { Iо XAq ON } x \in \text { пр(1) } \\
& \text { ME ETO N̄2HT } \\
& \text { CNAY EqUT⿱卄一 } \\
& \text { TwP } \overline{2 N} \overline{N q 2 I O} \\
& \text { OYE THPOY А(1) } \\
& \text { I5 бE пе пештортр } \\
& \text { eTNAGWTE M }
\end{aligned}
$$

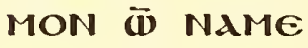

$$
\begin{aligned}
& \text { PATE TïMeY } \\
& \text { e гаp } x \in \text { пाештор } \\
& 20 \text { TP ETNג山шпе } \\
& \text { M̈MON TIE INAY } \\
& \text { етере пршмє } \\
& \text { NAÑKOTK ET̃प } \\
& \text { WWNE NqUTOP }
\end{aligned}
$$

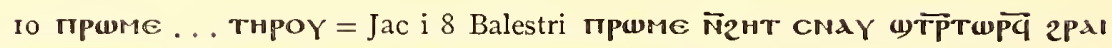

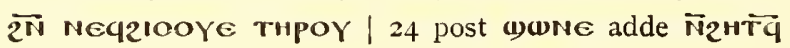


p. 67, col. ii

$$
\begin{aligned}
& \overline{2} \\
& \text { TP eqmeeYe e } \\
& \text { BOA ENENTAY } \\
& \text { (1)חЕ N̄2HTOY } \\
& \text { THPOY MTN } \bar{N} \text { пET } \\
& 5 \text { OOOY ÑTAqAגY } \\
& \text { Eк(1)ТРTळP } x \in \\
& \text { NETNHY N̄CWK }
\end{aligned}
$$

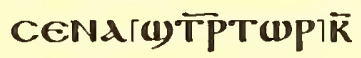

$$
\begin{aligned}
& \text { 2N NeqIOOYE } \\
& \text { IO (1)ANTKOYW(1)T } \\
& \text { ज̈пекрітнс ї } \\
& \text { ME } \overrightarrow{I C} \pi \in \overline{X C} \text { EK } \\
& \text { (1)ТРTwP } x \in \overline{M T} \\
& \text { ÑCd keкOYï (1)גK } \\
& \text { I } 5 \text { X(1) їாєк(1)dxe }
\end{aligned}
$$

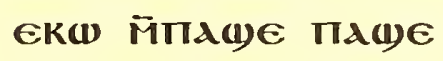

$$
\begin{aligned}
& \text { MTN N̄Cd KEKOYï } \\
& \text { TE2MOM NAlOG } \\
& \text { त̄ ज̈пексшмд } \\
& 20 \text { Тнस् EROX IITN } \\
& \text { TANAKTH ETNA } \\
& \text { TA2OK EK(1) } \overline{T P} \\
& \text { TwP } x \in \overline{M N} \bar{N} \\
& \text { cג oүATिमTE }
\end{aligned}
$$

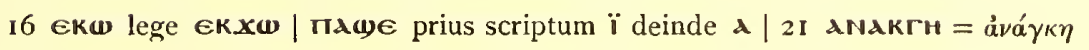


p. 68, col. i

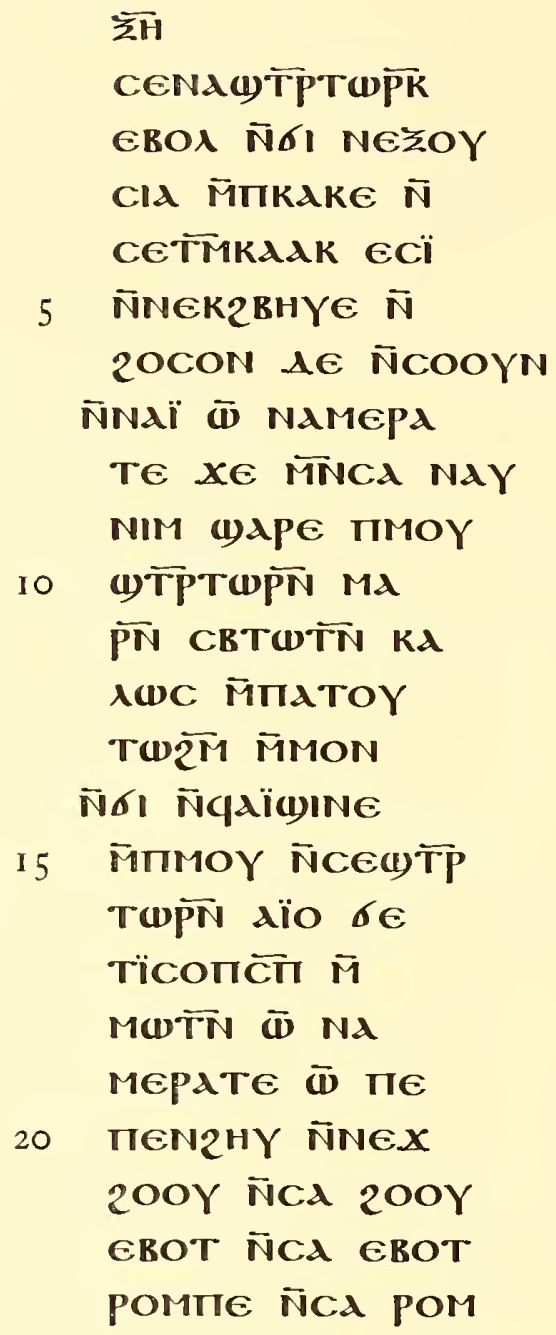

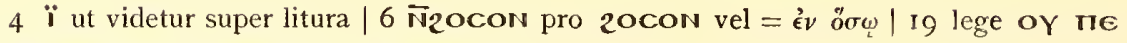
lege $\mathbf{\omega} \boldsymbol{\lambda}(\boldsymbol{y}) \Pi \in \mid 20$ Ninex pro enNex 
p. 68 , col. ii

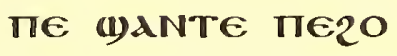 \\ oy $\bar{M} \pi \overline{x c}$ ei $\in x \omega N$ \\ N̈.Q૯ N̄OYTIAU $\in q$ \\ NHY TAP EX̄N NET \\ 52 MOOC EX̄ \\ MTIKA2 THPप MA \\ PNC̈ $\overline{C B} T \overline{T N}$ KA \\ AWC MITATOY

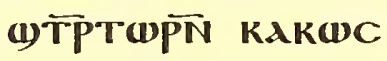 \\ IO AYW NंTTNWWIE \\ 2N OY2BA MN \\ OYNO6 MIMNT \\ ERBIHN $\overline{2 M}$ TTE \\ 2OOY N̄TNNANAK \\ I5 TH TIEXAq rap \\ $\bar{N} \sigma \| \Pi \overline{X C} \overline{2 M} \pi \in Y$ \\ AГTEAION XE \\ P(1) IтPAME Ti \\ 2нY М̈ாкосмос \\ 20 THPप $\overline{\mathrm{Nq}}$ TíOCE \\ ก̃Tव \\ петере ттршме \\ NATAdq N̄ERBI \\ O N̄T्भYXH
}

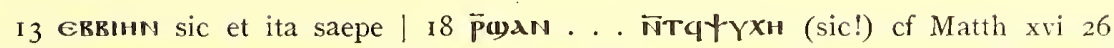

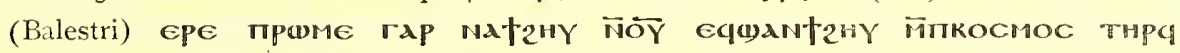
Nqtoce גe NTeq†Yरн 
p. 69, col. i

$$
\begin{aligned}
& \text { сшTM бE EPOÏ } \bar{N} \\
& \text { TAXw ePwTN } \\
& \text { M̄TITIAPAגITMA } \\
& \text { N̄ฒпнре OYMO } \\
& 5 \text { NON EqO M̄ME } \\
& \text { ANAY NAK EYPW } \\
& \text { ME EquANOY } \\
& \text { w2 } \overline{2 N} \text { OYHï KaN } \\
& \text { EYMITTE M̈MOY } \\
& \text { Iо М̈пє(1) }
\end{aligned}
$$

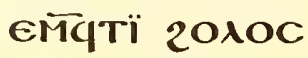

$$
\begin{aligned}
& \text { ПАНN ФАС̆ ОY } \\
& \text { МнHФE } \overline{2} \text { OOY } \\
& \text { ЄсОҮн2 } \overline{2 N} \text { пнї }
\end{aligned}
$$

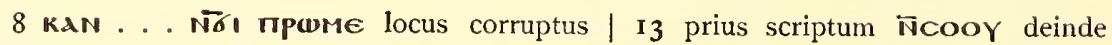
N̄ं2ooY | I6 त̄ delendum 
p. 69, col. ii

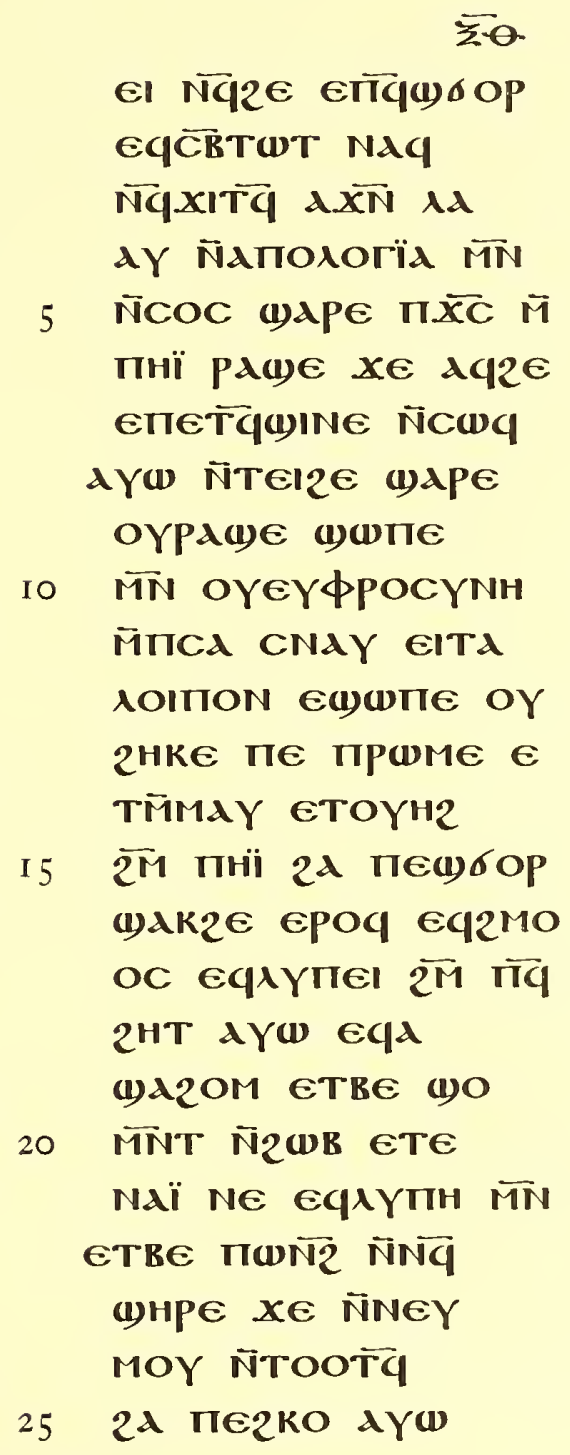

坴O 
p. 70 , col. i

$$
\begin{aligned}
& \text { ō } \\
& \text { ON ETRE пе(I) OP }
\end{aligned}
$$

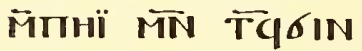

$$
\begin{aligned}
& \text { MOY ETO Ñ2HKE } \\
& \text { MN TQMTNTERRIHN } \\
& 5 \text { Nä̈ Ne mMeeYe Ñ } \\
& \text { 2HT М̈ாршME ர̄ } \\
& \text { 2HKE EIAd MOITION } \\
& \text { P(1) пе(1)бOP } \bar{M} \\
& \text { пнї MOY2 ERON (y) } \\
& \text { Iо PE п } \overline{X C} \text { M̈пні ФINe } \\
& \text { ÑCd пете пюq пе }
\end{aligned}
$$

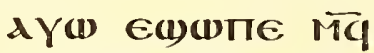

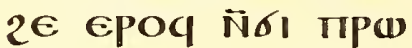

$$
\begin{aligned}
& \text { ME (I) }
\end{aligned}
$$

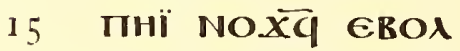

$$
\begin{aligned}
& \text { ¿N OYбWNT MN } \\
& \text { OүАүПн АYल (1) } \\
& \text { PE пршме шलте } \\
& \text { ¿N OY(1)TOPTP } \in \\
& 20 \text { NAY ENqCKEYE EY } \\
& \text { NOYXE MMOOY } E \\
& \text { RO人 } \overline{2 M} \text { Пто мпнї } \\
& \text { ANAY Ф̈ NCNHY } E \\
& \text { ПЕ(1)TOPTP ETNA }
\end{aligned}
$$


PLATE XI.

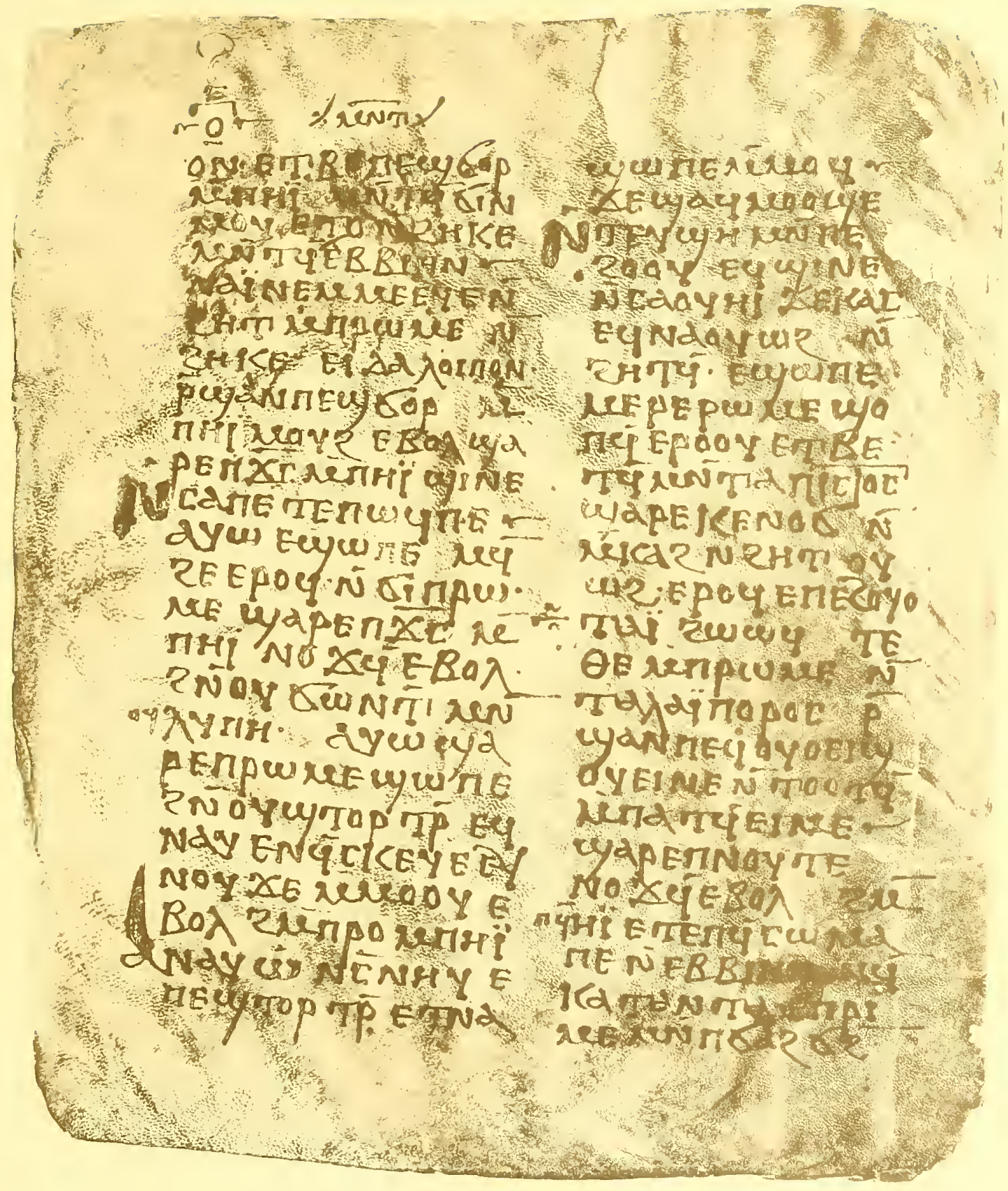

COPTIC HOMILY ON THE VIRGIN

Washington Coptic Manuscript No. 2 [p. 70] 

p. 7o, col. ii

\author{
(1)ШாЕ М̈MOप \\ $x \in$ ()גqMOоฒе


p. 7 I, col. i

$$
\begin{aligned}
& \text { N̄N̄OR2€ ЄயMाE } \\
& \text { equań́w eqNix } \\
& \text { 2OOY N̄CA } 200 Y \\
& \text { OY2OOY EqTOPNEY } \\
& 5 \text { E MTN OYC2ÏME } \\
& \text { NंTOG AN TE } \\
& \text { oyzooy eqxḯYe } \\
& \text { еqqї М̈пете Mे }
\end{aligned}
$$

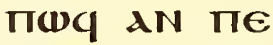

$$
\begin{aligned}
& \text { IO OYZDOY EqMOCTE } \\
& \text { MitIeqCoN } \\
& \text { OY20OY EqKdTd }
\end{aligned}
$$

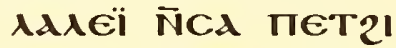

$$
\begin{aligned}
& \text { TOYNA } x \in \text { OYTN } \\
& \text { I } 5 \text { бOM ETï OY } \overline{M N} \\
& \text { OY OYzOOY Eq } \\
& \text { EாI-ӨYMï } \epsilon_{2} \in N \\
& \text { N̄KA N̄ NOQ AN NE } \\
& \text { OY2OOY EqXe ad } \\
& 20 \text { XE ÑAPTON EBOA } \\
& \overline{2 N} \text { PलG OY2OOY } \\
& \text { eqtame male } \\
& \text { N̄KГPlOG OY2OOY } \\
& \text { єqqiі М̈пвєекє }
\end{aligned}
$$


p. 7 I, col. ii

\author{
$\overline{\mathrm{OA}}$ \\ NิNP̄ГАTHC ก̃TO \\ OTOY OYZOOY \\ EqIIMOOC Eq4O \\ ÑAPTON OYZOOY \\ 5 Eqб(1)T NंCA NE \\ 2lOOME KAKWC \\ 2N OYETEI-YYMII \\ GQOOY MTN OYBAd

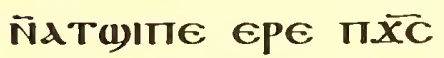 \\ Io $x(1)$ जिMOC $x \in p(1)$ \\ ME NIM ETIIOOI \\ $\widehat{2 N}$ OYCOOG $\overline{M N}$ NE \\ TEPE IIMOOY aOYO \\ M̄MOOY ETETNAIIO \\ I5 PXOY NAÏ GROג $\epsilon$ \\ TCYNATWГH AY \\ (w) ANOK TÏNdEï \\ N̄TAM)IE $\overline{2 N}$ TE \\ TNMHнTE пе \\ $20 x \in \pi \overline{x C} \in(1) x \in$ \\ дстардгтеплеї \\ ÑGI INOYTE ET \\ вє Naï Єïє посш \\ MAlAON ANON Nï

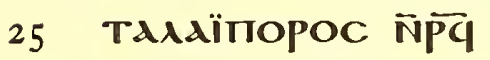

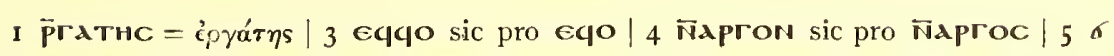
prius scriptum к 6 2 Zech ii to 
p. 72, col. i

\author{
$\widehat{\mathrm{OB}}$

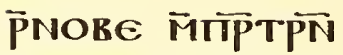 \\ ш)ताE бE N̄OYÑ \\ AMEAIC $\overrightarrow{\mathrm{w}}$ NAME

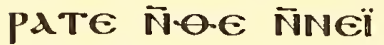

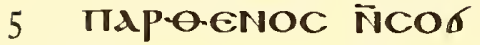 \\ ÑNEX 2OOY ÑCA 20 \\ OY घגNTE пE2O \\ OY M̄ா $\overline{X C}$ ETM̄MAY \\ EI EXWN $\overline{2 N}$ oYбE \\ Iо Пн qNHY пеXגq \\ E2PAï EX̄XN NET \\ 2 MOOC $21 \overline{X M} \pi 20$ \\ MாTKג2 NTTCOOYN

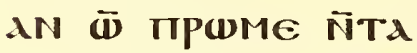 \\ I 5 גïrmopoc $x \in \overline{2 M}$ \\ TE2OOY N̈TA IINOY \\ те плגСсе М̈MOK

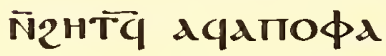 \\ NE EXWK ЄqXW \\ 20 M̄MOC $x \in \bar{N} \overline{T K}$ OY \\ KA2 AYW EKNAK \\ TOK AN EாKג2 $\bar{N}$ \\ TTK2E $\lambda$ OITION \\ P(ג) Tннте $\bar{N}$ \\ 25 NEK2OOY XWK
}

2 NoYN- lege oYN $\bar{M}-$ vel eNo $\bar{N}-\mid 22$ AN lege on 
p. 72, col. ii

\author{
GBOA М̈ITATKME \\ TANÖ̈ CENAETTP

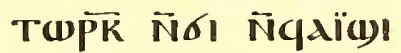 \\ Ne $\overline{2 M}$ TTEKCA N2OYN \\ 5 N̄TE ITEฒMON wOYO \\ EROA $\overline{2 N}$ TKTATPO

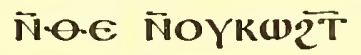 \\ స̄TE пексшмА \\ Тнप्रq $6 \omega \overline{\lambda 2} \bar{N}$ \\ IO TE TTER2O MIBE \\ ÑTE NERRAA NAY $E$ \\ NeTNAEI N̄CaK \\ 2N OY(1)TOPTP $\overline{M N}$ \\ OY2RA ÑTE NEK \\ I 5 6IX KA бOM EROA \\ N̄TE NEKTHнBE ФI \\ ве NEKџ)П $\overline{2 N}$ \\ OY2BA MIN OY2O \\ TE N̄TE NETNAY \\ 20 EPOK тнроY $\overline{\boldsymbol{\omega}}$ \\ птАлАITOPOC A \\ NOK ITPUME NIM \\ пеTNA2MNT $E$ \\ BOA $\overline{2 M}$ ICWMA $\bar{M}$ \\ 25 пाMOY пाЕ२МОТ \\ גe M̈IINOYTE घнत
}

5 a)mon fortasse sic pro 2MOM $\mid 96 \mathbf{\omega} \overline{\lambda 2}$ pro $* 60 \overline{\lambda 2}=$ inquietum, commotum,

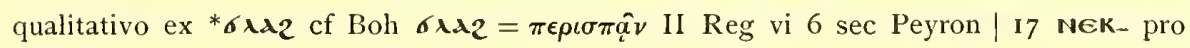

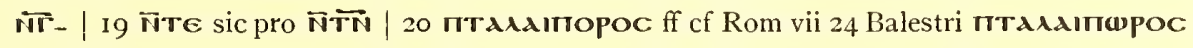

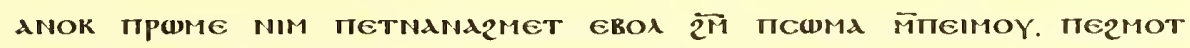

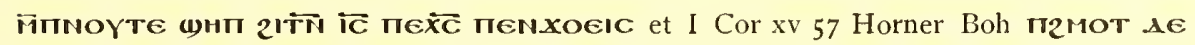

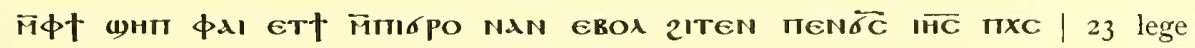
петNandz"TT 
p. 73 , col. i

$$
\begin{aligned}
& \text { TAÏ ETNA† NAN } \\
& \text { ज̈ாЕхPO 21Tस TC } \\
& \text { TE } \overline{X C} \text { TENIOIY } \\
& \text { бE a Nameiplate } \\
& 5 \text { MAPN[KAdN] ENCE } \\
& \text { TWT [NOTYOEIC } \\
& \text { NIM їாגTOYฒї } \\
& \text { NE N̈CWN N̄TT } \\
& \text { COOYN AN } \bar{N} \\
& \text { IO TNC2OYP nE2OOY } \\
& \text { N̄TAYXTON ज̄ंH } \\
& \text { Tq EाTाKOCMOC } \\
& \text { ETETNCOOYN } x E \\
& \text { Ñ()ANMOY MÑqï } \\
& 15 \text { AdAY NIMMAN EI }
\end{aligned}
$$

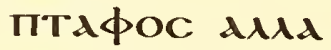

$$
\begin{aligned}
& \text { KAN OYTITETNA } \\
& \text { NOYG TENTANAAG } \\
& \text { KAAN OYTETOOOY } \\
& 20 \text { ПЕ (I)AN2E EPOOY } \\
& \text { THPOY EY } 21-0 . H \\
& \text { M̈MON KATA O.E } \\
& \text { етсH2 } \overline{2 N} \text { NeTPd } \\
& \text { фн пехגq гар } \bar{N}
\end{aligned}
$$

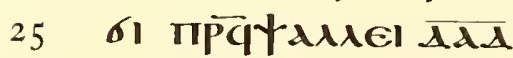

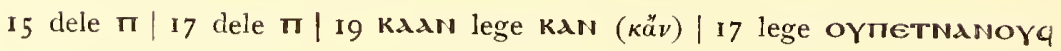


p. 73, col. ii $\quad x \in$ ÑTOK пеTNA

ô

Tï Pinoyd поYd

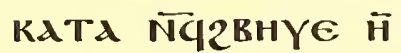

NTCOOYN AN $\overline{\mathbf{D}}$ ПTW

5 ME ก̃dT

(1)OMNT MिMA2E IN

кА2 пе пекнї (1)ג

ENE2 EKP OY TiNi

к(1) THPOY MTN Ni

Iо Hï eTXOOCE MTN Nï

Nor ÑZwгрAфId

NAÏ E(1)גKR(1)K NT

KAdY ÑzenKOOYE

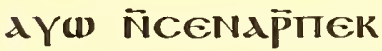

I5 MeEYe AN N̈́r NT

Оүнн2

(1)ג TNAY EKCWOY2

G2OYN ÑZENKOOYE

AYE TINTOC NEK

20 XגXE NETNAKAH

PONOMEI TIMOOY

(1)A ENE2 NTCO

OYN AN XE ÑTOKEï

E2OYN EாїкосMOC

25 ЄKKH KA2HY $\bar{N}$

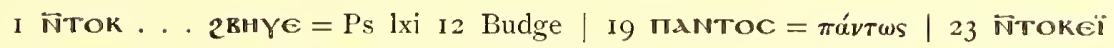

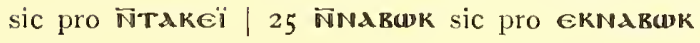


p. 74 , col. i

$$
\begin{aligned}
& \overline{O A} \\
& \text { NARWK ON N̄KH KA } \\
& \text { 2HY N̄Tокєї EBON } \\
& \overline{2 N} \text { 2HTC ÑTKMAAY } \\
& \text { єкPіме єкNАвШK } \\
& 5 \text { ON ERPÏ⿴\zh11 Аүш } \in Y \\
& \text { TOEIT EPOK MAY } \\
& \text { АגK EIC2HHTE }
\end{aligned}
$$

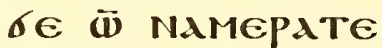

$$
\begin{aligned}
& \text { етве пе2Оүо } \bar{N} \\
& \text { Io Nanobe aiтi M } \\
& \text { пртாтеєүе ÑNAï } \\
& \text { NHTN } \bar{M} T O O Y \overline{2 M} \\
& \text { пा) } \\
& \text { (w) TMAdY M̈LEN } \\
& \text { I } 5 \text { OYलत̃र THPT } \\
& \Pi \in N \overline{X C} \text { IC } \Pi \epsilon \overline{X C} \\
& \text { пגї N̄TגqCoOY } \\
& \text { टN E2OYN M̈ாOOY }
\end{aligned}
$$

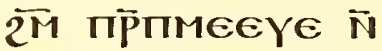

$$
\begin{aligned}
& 20 \text { ТqMadY ज̈пגрөє } \\
& \text { NOC MAPNEAPE }
\end{aligned}
$$

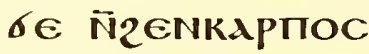

$$
\begin{aligned}
& \text { ENANOOY } \bar{N} \overline{T N T}
\end{aligned}
$$

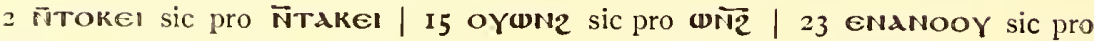
enanoYOY 
p. 74, col. ii

$$
\begin{aligned}
& \text { MTTON MIINOY } \\
& \text { TE Ñ2HTOY MTN } \\
& \text { TqMAdY FitTaP } \\
& \text { o-ENOC EPE пा } \overline{X C} \\
& 5 \text { TAP NAMAKAPI } \\
& \text { ZE TIMON } x \in \\
& \text { ANEÏ G2OYN E } \\
& \text { пITTOIIOC ETOYA } \\
& \text { AR MTIOOY } \bar{N} \\
& \text { IO घOOT } \overline{2 N} \text { NINO6 } \\
& \text { NiNOBE TIOYA } \\
& \text { TOYA KATA Tq } \\
& \text { MiNe AY(1) TOY } \\
& \text { EI TOYEI KATA }
\end{aligned}
$$

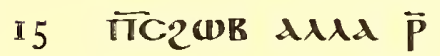

$$
\begin{aligned}
& \text { (1)AN TIIIAP-OE } \\
& \text { NOC e-ewPEI MT } \\
& \text { ПKAPाIOC MITNA } \\
& \text { eTOYAdR } E \\
& 20 \text { AqAY⿱ANE } \overline{2 N} \\
& \text { NENMEAOC ETE } \\
& \text { Tגї Пढ тAГגTH } \\
& \text { MTN TINA MTN ITPA } \\
& \text { (1)E TIPHNH }
\end{aligned}
$$

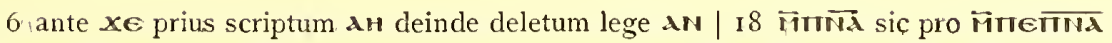

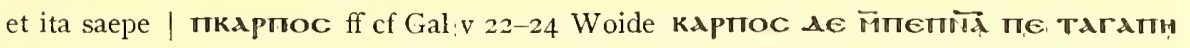

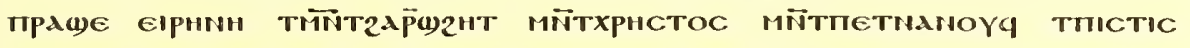

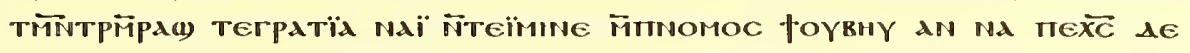

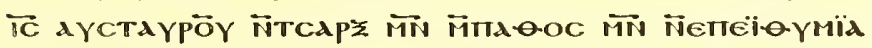


p. 75 , col. i

$$
\begin{aligned}
& \text { TMNT2А Рष्) } \\
& \text { TMT̃TXPICTOC } \\
& \text { IETNANOYG } \\
& \text { THICTIC TMNT }
\end{aligned}
$$

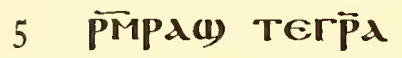

$$
\begin{aligned}
& \text { Aïd Naï rap } \\
& \text { N̄TIMINN пе } \\
& \text { Xגq N̄́I INOMOC } \\
& \text { XE TI OYBHY AN } \\
& \text { Io ENATE } \overline{X C} \perp E \overline{I C} \\
& \text { AYCTAYPOY } \bar{N} \\
& \text { TCגP竞 MTN ÑTA } \\
& \text { O.OC MTN Nerii } \\
& \text { OYMï пEXAq }
\end{aligned}
$$

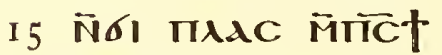

$$
\begin{aligned}
& \text { NOYqE חIYYOC } \\
& \text { MATNGï ÑNEN } \\
& \text { blx erpal e } \\
& \text { TIE ÑTNCOT } \\
& 20 \text { CT NTTNMAXY } \\
& \text { MitapegNOC } \\
& \text { TodrId MAPId } \\
& \text { TNт̈есвеYTHC } \\
& \text { oYzIKANOC rap TE }
\end{aligned}
$$

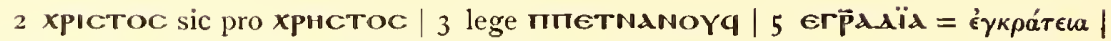

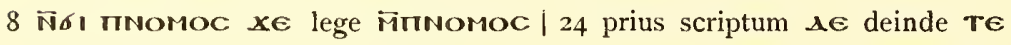


p. $75, \mathrm{col}$. ii

$$
\begin{aligned}
& \overline{O E} \\
& \text { AY(W CNATAPA }
\end{aligned}
$$

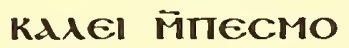

$$
\begin{aligned}
& \text { NOTENHC } \overline{\mathbf{N}}(1) \\
& \text { PE } \overline{\mathrm{IC}} \Pi \boldsymbol{\overline { X C C }} \Theta \\
& 52 \text { PAI EXWN THPN } \\
& \text { XE ÑNE } 2 Y B W \\
& \text { WN OYAE NOIMOC } \\
& \text { (1)шाE E2Pגї } \bar{N} \\
& 2 \text { HTM AYW NC } \\
& \text { I० } 2 \lambda \overline{P 2} \text { EPON EாTाO } \\
& \text { NYPOC חAÏ ET } \\
& \text { חגANג N̄TIKOY } \\
& \text { MENH THPC AY } \\
& \text { (1) } \bar{N} C \lambda \bar{N} K O T \overline{T C} \in \\
& \text { I } 5 \text { T-O.OOY } \overline{\mathbf{d}} \text { TN } \\
& \overline{X C} \text { M̄MdAY MA } \\
& \text { Pld Tерpo aYo }
\end{aligned}
$$

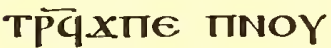

$$
\begin{aligned}
& \text { TE } \overline{2 N} \text { OYME Ñ } \\
& 20 \text { COOYN TAP XE } \\
& \text { TP2EN E2OYN } \\
& \text { EாNOYTE ПIOY } \\
& \text { шнре їMеріт } \\
& \text { TAPA NETOYAdB }
\end{aligned}
$$

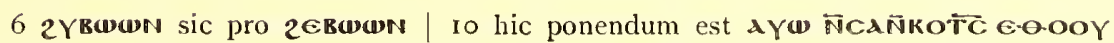

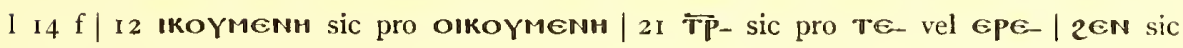
pro 2 HN 
p. 76, col. i

$$
\begin{aligned}
& \overline{05} \\
& \text { THPOY } \overline{\mathrm{P}} \boldsymbol{\text { TIN }}
\end{aligned}
$$

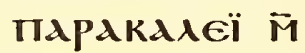

$$
\begin{aligned}
& \text { MOQ EXUN OY } \\
& \text { AïkdïOC пाE } \overline{2 N} \\
& 5 \text { 2UR NIM AYW } \\
& \text { EqOYAdB AYU } \\
& \text { EqNAKW NAN E } \\
& \text { BOA ÑNEN⿴囗WT } \\
& \text { THPOY MTN NEN } \\
& \text { io TANAMEAeI E } \\
& \text { POOY AYU NEq } \\
& \text { TI OE NAN N̄TN }
\end{aligned}
$$

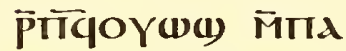

$$
\begin{aligned}
& \text { TquINE N̄CUN } \\
& \text { I5 GROA } \overline{2 M} \text { TIIMA } \\
& \text { N̄ฒшாє ЄIC Naï } \\
& \text { MEN ANXOOY } \\
& \text { EாTגїO N̄T.A } \\
& \text { rïd MAPId TMd } \\
& 20 \text { AY M̈INOYTE } \overline{2 N} \\
& \text { OYME AOITION MA } \\
& \text { PNIRTON EX̄N TIE } \\
& \text { IIPOKYMENON ET } \\
& \text { кH NaN eqpaï Te } \\
& 25 \text { NOY } \bar{N} T \overline{T N} X \\
& \text { 2ENROYi N̄ETï }
\end{aligned}
$$


p. 76, col. ii

\author{
NOC ETBE TTAI \\ O T̄TN $\overline{X C}$ THPN \\ TI-єОтокос $E$ \\ TOYAdR MAPIA \\ 5 пPOC O.E ÑTNMTNT \\ 2HкE АCW( \\ $\pi \in \lambda \epsilon \overline{2^{M}} \Pi(1) \lambda$ \\ $\overline{\mathrm{N} T N} \overline{\mathrm{XC}}$ AYC

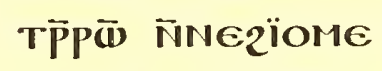 \\ Iо тhPOY Tө.drïd \\ Mapla EாGIAH \\ ṄeIPE MTtTC(y) \\ ÑOYCOח АЕРОM \\ пाе $\overline{2 M}$ пе2OOY \\ 15 NTTCANAAYMYIC \\ eTOYAdR eTE \\ пגї пе COYMT \\ TACE MTMECOY \\ PH KATd OE Ñ \\ 20 Td NENEIOOTE \\ TMMAKAPIOC TA \\ AC ETOOTN KA \\ T'A II)גXX iT \\ фіеро фалтнс \\ 25 AYW TENEIWT \\ AdA EqXW $\bar{M}$
}

I $3 \Delta \in$ sic pro Te 
p. 77, col. i

$$
\begin{aligned}
& \text { MOC XE NEN } \\
& \text { TANCOTMOY } \\
& \text { ANEIME EPOOY } \\
& \text { NENTA NENEI } \\
& 5 \text { OOTE XOOY } 6 \\
& \text { PON ज̄TIOYरшा } \\
& \text { ENEYшнPE } € \\
& \text { RE ХWM EYXWM } \\
& \text { ज़MOY ज̈ாI } \overline{X C} \\
& \text { I0 } \overline{M N} \overline{N G} \text { бOM } \overline{M N} \\
& \text { Neqштнре N̄TAq } \\
& \text { AAY ACWUTE }
\end{aligned}
$$

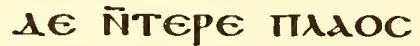

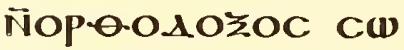

$$
\begin{aligned}
& \text { I } 5 \text { Oү2 єТккинсї } \\
& \text { d M̄TINAY N̄TCY } \\
& {[N] d そ I C \quad x \in \text { N̄NA }} \\
& \text { [X] EBOA } \overline{2 N} \bar{M} \\
& \text { MICTIPION } \bar{M} \\
& 20 \text { TIE } \overline{X C} \text { ETEi OYN } \\
& \text { EPE TCYNAそIC } \\
& \text { ПIOP(i) EBOA GIC } \\
& \text { OYPWME N̄2BPAї } \\
& \text { oC aqEi NaN } E \\
& 25 \text { ZOYN } 2 \bar{N} \text { TCYNA } \\
& \text { そІ } \widehat{2 N} \text { TMHн }
\end{aligned}
$$

I NeNTANCOTMOY . . N̈TAqAaY = Ps lxxvii 3-4 Budge ex quo post NenTANCOTMOY addendum aYw et eYXwM legendum eYXw $\bar{N}-$ 
p. 77 , col. ii

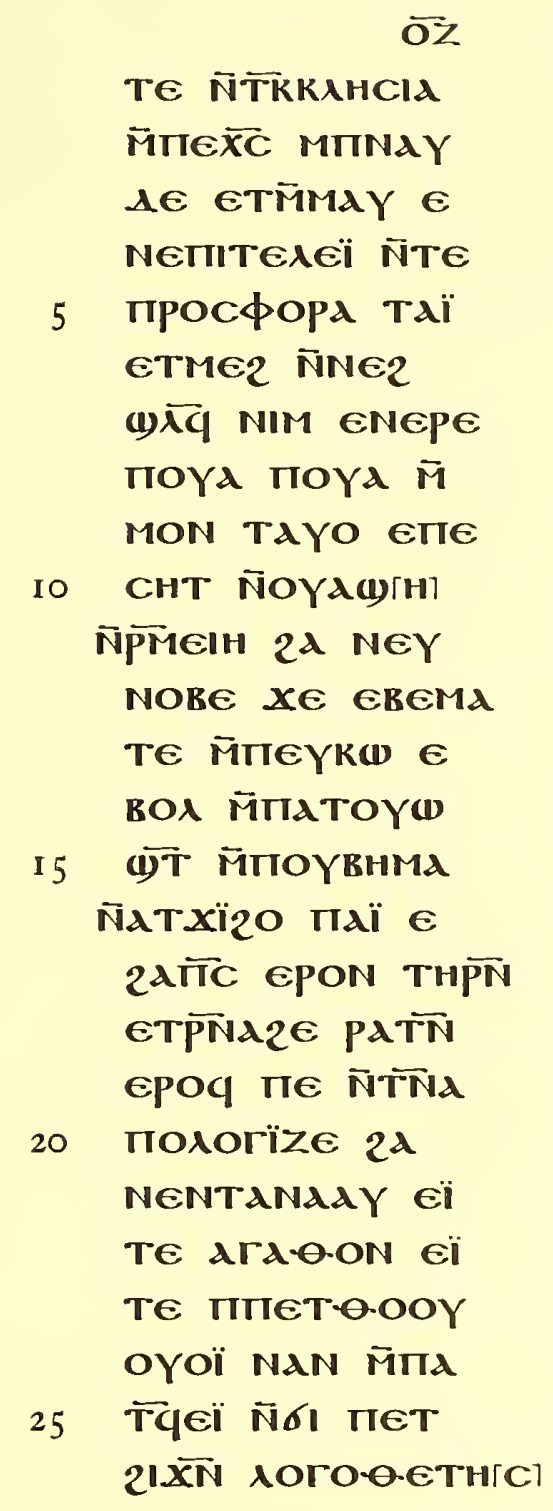

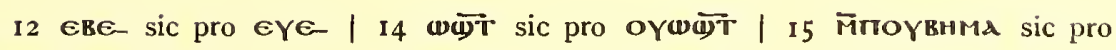
Mтtвнma 


$$
\begin{aligned}
& \overline{\mathrm{OH}} \\
& \text { NIM пEर्XC NG }
\end{aligned}
$$

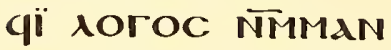

$$
\begin{aligned}
& \text { AY(1) (1)ג2Pגï EY } \\
& \text { (1)גXE ÑOYUT } \\
& 5 \text { ÑAPгळN GANXo } \\
& \text { OY TNNATINO } \\
& \text { rOC 2APOG } \lambda \text { ï̈ } \\
& \text { TION A прФME } \\
& \text { Ñ2BPdïOC } \\
& \text { IO TM̈MAY АCKW } \\
& \text { пе N̄oүMннше } \\
& \text { N̈PAME } \overline{2 N} \text { NE } \\
& \overline{T N} 20 Y N \text { 2N TK }
\end{aligned}
$$

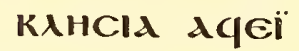

$$
\begin{aligned}
& \text { I NE MMOOY ad } \\
& \text { TIAYTOYCTANI } \\
& \text { ос етреY } \\
& \text { NAq N̈XINXH } \\
& \text { ТкКАнсІа де }
\end{aligned}
$$

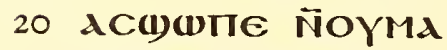

$$
\begin{aligned}
& \text { N̄xגїн MitrNaY } \\
& \text { ETM̈MAY ANOK } \\
& \lambda \in \text { O.EOфYAOC }
\end{aligned}
$$

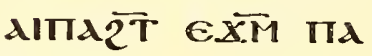

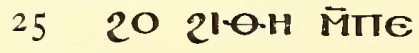

p. 78, col. i 
p. 78 , col. ii

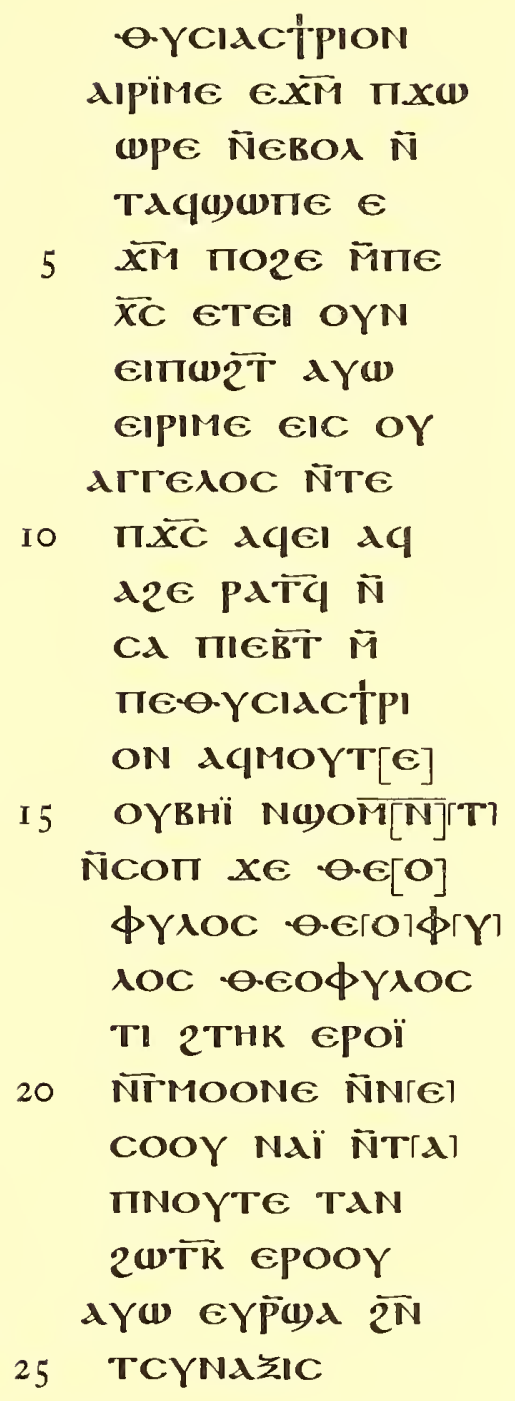

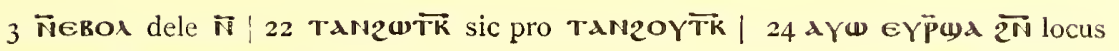
corruptus lege a $\boldsymbol{y} \boldsymbol{\omega} \overrightarrow{\boldsymbol{P}}(\boldsymbol{y}) \boldsymbol{N}$ 
p. 79, col. i

\author{
XWK EBOA ANA \\ Xupei \\ NAK E \\ TEחIC \\ 5 KOTIION] \\ Naï $\lambda \epsilon$ \\ స̄TEрqхо \\ OY EPOI AINAY \\ EINAY EPOG a \\ IO NOK $\Lambda \in$ dïEIPE

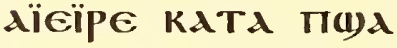

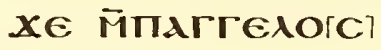

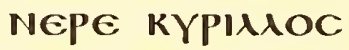 \\ 2N TEIICKOпї \\ I 5 ON MITINAY E \\ TM̈MAY EqOY \\ (1) $\overline{2 N}$ NeTPA \\ фH ETOYAגB \\ $\overline{2 N}$ NCBOOYE $\bar{N}$ \\ 20 NENEIOOTE $\bar{M}$ \\ IM1AKAPÏOC Nä̈ \\ N̄TAYฒ)ाE 2A \\ Tतिभ NE Пए⿴囗十 \\ pe rap пाE N̄TA
}

I-7 membrana jam antehac lacerata litterae nullae hoc loco omissae sunt 4 et $\mathbf{1} 4$

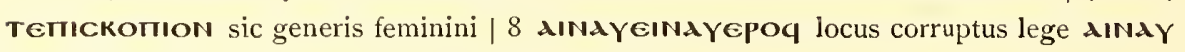

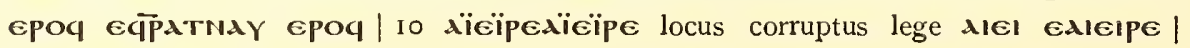

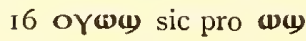


p. 79, col. ii

\author{
$\overline{0.0}$ \\ CONE KATA III \\ KOCMOC dïNTA \\ E2PAï $\overline{2 N} \overline{M N T}$ \\ EYCERHC NIM \\ 5 EqMOOWE $\overline{2 N}$ \\ NENTOXH THPOY

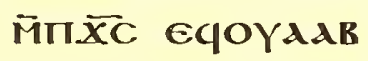 \\ N̄TEPIX(1) $\lambda \in$ Naq \\ जाா(1)גxe $\lambda q[\overline{\mathbf{P}}]$ \\ Iо $)[\Pi H] P \in$ M̈ாENTAq \\ Г(d) \\ TIEII 2E AINT ANGO \\ ÑITIÏ ElOlOY M̈InNOY \\ те петеїре N̄Ne \\ I5 ()ாПн1PE MAYAdq \\ 2Pdï $\lambda \epsilon \overline{2 N}$ NE2O \\ OY ETM̃MAY NE \\ OYTN OYATO-Y.YKH \\ N̄CAPHC N̄TTIOAIC \\ 20 N̄OYKOYï TAl ג€ \\ NE OYTN PUME $\lambda \in$ \\ ÑXPICTIANOC \\ ОүнН2
}

I2 AN lege on

2 I lege oYpume 
p. So, col. i

$$
\begin{aligned}
& \text { iิ } \\
& \text { тршме } \Lambda \epsilon \overline{\mathbf{N}} \overline{2 B} \\
& \text { pä̈oc IIAİ ÑTAN } \\
& \text { (1)גxe ePOQ } 2 \lambda \\
& \text { O.H ÑoYkoYï aq } \\
& 5 \text { TWOYN АपREK } \\
& \text { Аqтале (1)бор є } \\
& \text { IIPAME } \bar{N} x P I C \\
& \text { TIIANOC AGNO } \\
& \overline{X q} \text { EROA } \overline{2 N} \text { TA } \\
& \text { Iо поӨүкн пехрIс } \\
& \text { TIANOC } \lambda \in \text { ÑTE }
\end{aligned}
$$

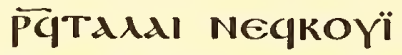

$$
\begin{aligned}
& \text { N̄(1)не } \overline{2 N} \text { OYलTOPTP } \\
& \text { MN NGCKHYE Аq }
\end{aligned}
$$

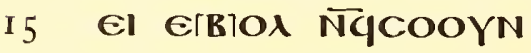

$$
\begin{aligned}
& \text { an } x \in \text { eqNamo } \\
& \text { Ode ETWN AY } \\
& \text { (1) 2їТм пе2вд } \\
& \text { етशїшप N̄б। } \\
& 20 \text { птршME ÑXPIC } \\
& \text { TIANOC } \overline{M N} \overline{N q} \\
& \text { кOYï ÑCKнYe גq }
\end{aligned}
$$

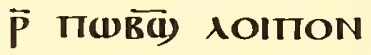

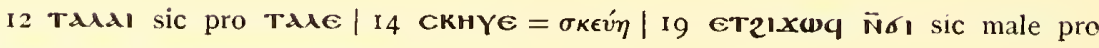

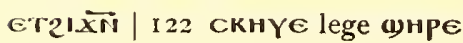


p. 8o, col. ii

$$
\begin{aligned}
& \text { ÑOYKOYi MTOKE } \\
& \text { N̄ue } \epsilon \text {. } \\
& \text { тАнY } \\
& \text { eMdTE } \\
& 5 \\
& \text { ePE T2I } \\
& \text { KWN } \bar{N} \\
& \text { TÑ } \overline{X C} \text { M̈ாגPaE } \\
& \text { NOC } \mathrm{CH} 2 \text { EPOC } \\
& \text { EстגАн EP2Pגï } \\
& \text { Io E.XXN OYXOE ÑCd } \\
& \text { 2OYN N̄TAITO-OY } \\
& \text { кн пехрIстіА } \\
& \text { NOC } \lambda \in \lambda q \bar{P} \text { пEC } \\
& \text { OY(WB̈) AGBWK } \\
& \text { I5 АCाкגАC Пгврגї } \\
& \text { OC } \lambda \in \bar{N} \lambda T N O Y \\
& \text { TE גQANE } 2 E N \\
& \text { Г̈кАтнс етреY } \\
& \text { Kd-AdPIZE NTd } \\
& 20 \text { ПО्УҮКн ज̈ता } \\
& \text { T्वOYW2 } \\
& \text { ITPOC ПMEEYE M̈ }
\end{aligned}
$$

I поке sic pro побе | 2-6 vide paginam superiorem i 2-7 | 3 єстАнY lege

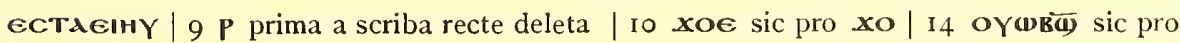
(w) $\overline{\text { Bii }}$ 
p. $8 \mathrm{I}$, col. i

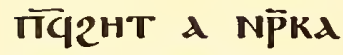

THC бш(1) $A Y$

NAY ETTOKE ÑuE

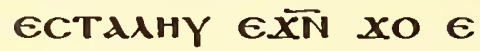

5 PE T२їKWN ÑTtIAP

O-ENOC CH2 EPOC

N̄TOOY $\lambda \in$ AYXITC

AүגCாגZE MMOC

AYOYATM E2OYN

10 EPOC AYOYW(T)

$\epsilon \overline{X N} \overline{N C} \sigma \mid x$ MN

NCOYPHHTE AY

бш EYגCחגZe M

MOC ÑOYNo6 $\bar{N}$

15 NaY EYTwW6e

M̈MOC ETEYMC

OHT $\overline{2 N}$ OYNOE

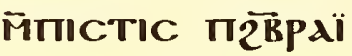

OC $\lambda \in$ NTTEPGNAY

20 epooY eYdcாגZe

M̄MOC AqбWNT

AqMOOWE E2OYN

EPOOY $\overline{2 N}$ OY NOK

N̈OYMOC MIN OY

25 АпүАн пеХАव

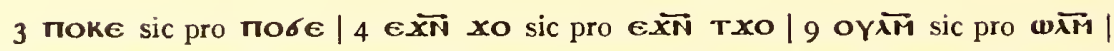
I2 оүрннте sic pro oyephte et ita constanter | 15 TwW6e sic pro тw6e |

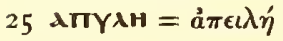


$\overline{\pi \lambda}$

p. $8 \mathrm{I}$, col. ii

NAY XE OY TE

Tdï ETETÑOY

WलिT EXWC AY

(1) ETETT̃ATI

5 ZE MMOC AYOY

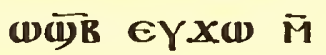

MOC NAq Xe TAi

TE O.̈KWN $\bar{N}-\boldsymbol{d}$

rï MAPIA TMd

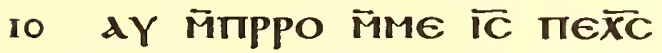

IEN $\overline{X C}$ пाi $\bar{N}$

TAYCPOY ज̈MOQ

2APON AYW AC

TWOYN EBOA $\overline{2 N}$

I 5 NETMOOYT $\overline{2 M}$

ПME2UOMNT $\bar{N}$

2OOY KATA NE

ГPАфH MTN ÑCOC

ON ACOYWN2

20 ЕNप̆MAнTHC

2ï.XM птоOY $\bar{N}$

N̄XOEIT EqCW

TM $\Lambda \in$ ENdï $\bar{N} \sigma \mid$

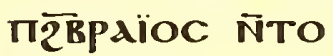

25 OTOY N̄NP̄KATHC

Iо ज̈прp० in margine suppletum 
p. 82 , col. i

$$
\begin{aligned}
& \overline{T B} \\
& \text { AYW AqNAYe } \\
& \text { ӨIKWN N̄TTIAP } \\
& \text { OENOC ETOYAdB } \\
& \text { MaPï2 EC ÑTOTOY } \\
& 5 \text { ЄүПРОскүNH } \\
& \text { NdC Аपढ WNT }
\end{aligned}
$$

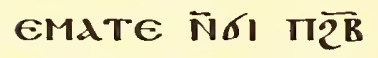

$$
\begin{aligned}
& \text { PAÏOC N̄ATNOY } \\
& \text { TE AqAMA2TE } \\
& \text { Iо N̈тпобе N̄(1) } \mathrm{EC} \\
& \text { N̄TOOTOY AqOY } \\
& \text { w6TाC AqAdC } \bar{N}
\end{aligned}
$$

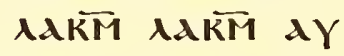

$$
\begin{aligned}
& \text { w AqNOYXE } \bar{M} \\
& \text { I } 5 \text { MOC E2pגï eүвнр } \\
& \text { EqMe2 } \bar{N} \overline{\mathrm{P}} \overline{M C} \\
& \text { AqTPE OYA } \overline{2 N} \mathrm{NP} \\
& \text { кATHC TWOYN } \\
& 2 \lambda \text { пाвї єqхu } \\
& 20 \text { M̄MOC NAq } x \in \\
& \text { TwOYN 2d пגї } 6 \\
& \text { pe тїтобе N̄(1) } \\
& \text { М̈гா]ЕСНT ज̈MOQ } \\
& \text { epe O.IK@N }
\end{aligned}
$$

I AqNaY sic pro EqNaY of Levy Syntax d. kopt. Apophth. Patr. Aeg. \$ 69 et

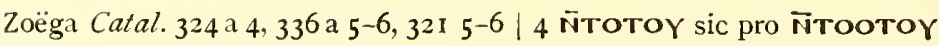


p. 82, col. ii

$$
\begin{aligned}
& \text { TMAAY M̈TINA } \\
& \text { ZMPAIOC CH2 } \\
& \text { EPOC } \overline{N T} X I \overrightarrow{11} \\
& \text { MOOY NTIIO2TOY } \\
& 5 \text { ЕПЕСнт ЕாМОоY } \\
& \text { пістеүе naï } \overline{\mathbf{w}} \\
& \text { NCNHY XE AYP } \\
& \text { MTTTPE Nä̈ } \overline{\mathrm{N}} \\
& \text { бї zeNPUME M } \\
& \text { Iо ПICTOC AY(N } \bar{N} \\
& \text { P्22OTE 2HTA } \\
& \text { M̈пNOYTE } \\
& \text { eMATE XE } \\
& \text { Ñтере пршмE } \overline{\mathrm{N}} \\
& \text { I5 PKATHC TEOYN } \\
& 2 \lambda \text { Пाв ज̈пє пеС } \\
& \text { NOG } 10 \text { equOYO } \\
& \text { EROA 2M TRIP TAI } \\
& \text { етере өїкшN } \bar{N} \\
& 20 \text { TTAP-eENOC N̄2H } \\
& \text { Tq TAї NTTA ח } \overline{2 B} \\
& \text { PAIOC OYलOTC }
\end{aligned}
$$

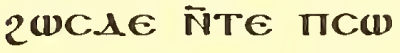

$$
\begin{aligned}
& \text { Md M̈пршме Ñ } \\
& 25 \text { ргдтнс 20ர़ा }
\end{aligned}
$$

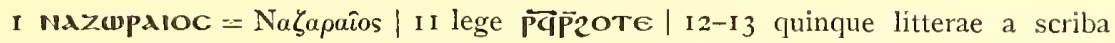
deletae ut videtur дите | 22 оүшб $(\mathscr{\omega} \sigma \tau \epsilon)$ et ita saepe 
p. 83 , col. i

\author{
ÑCNOY EBOA $2 \bar{M}$ \\ TECNOG $\bar{N} \cdot$ OIKWN \\ N̄TIAPO-ENOC \\ ETOYAגB MAPI \\ 5 А ЄC N̈ாЕСHT $\bar{M}$ \\ TाBIP 2WCAE $\bar{N}$ \\ OYON NIM N̄TAY \\ NAY EPOC Pिता \\ Ре їпгшв Аүш

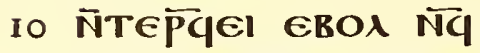

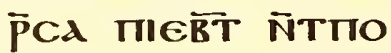 \\ AIC ÑOYKOYï AY \\ TWMNT EPOQ $\bar{N}$ \\ GI zeNAPXWN \\ I 5 M̈ாICTOC N̄TE \\ TIOAC AYW $\bar{N}$ \\ TEPOYNAY $E$ \\ пвIP Eq()OYE \\ CNOQ EПECHT \\ 20 EாKג2 AYAMA2 \\ те M̄MOq EYW( \\ ETi NAG N̄2EN \\ BACANOC EYXW \\ MMOC NAq XE
}

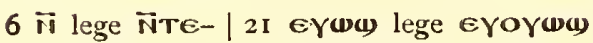


p. 83 , col. ii

\author{
ताก

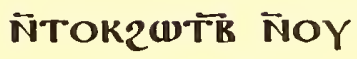 \\ PWME АKqा $\overline{\mathbf{N}}$ \\ NGXPHMA TAPE \\ OYMTNT PMMAO \\ 5 шलाT NAK AYW

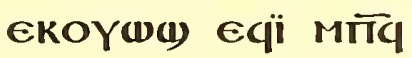 \\ CWMA NTNO $\overline{X q}$ \\ EாMOOY пршME \\ גe N̄P̈гАTHC גq \\ Io Pïe EqXW $\bar{M}$ \\ MOC XE OYOÏ Naï \\ 2AMOÏ PW ENE OY \\ PWME TE N̄TN2H \\ N̄TAÏMOOYT̄G $d Y$ \\ I 5 (v) TINATI M̈II \\ CNOG $2 \lambda$ TIWG dA \\ ld IECNOG maï 6 \\ TETTNNAY EPOY \\ IIA TCorpdфid \\ 20 пе $\overline{\mathbf{N}} \overline{T N} \overline{X C}$ TH \\ PN TE-COTO \\ KOC GTOYAAB \\ Mapid TE T*di $\bar{N}$

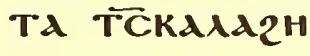

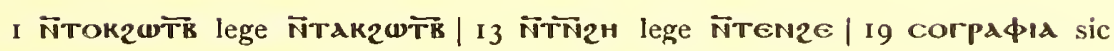
pro zwrpaфid et ita saepe 
p. 84, col. i

Tाג

TWOYN 2A TET

CAN(1) M̄MON TH

$\overline{\mathrm{PN}} \overline{2 M}$ Tप्YOW(

AYW N̄TOG IIEN

5 TAqMIE проме

MTN ÑKA NIM ET

WOOII NETNNAY

EPOOY MN NETT

NAY EPOOY AN EIC

IO 2HHTE $\lambda O ̈$ TION

CC N̄TECHT M̈TIRIP

ETETNNAY EPOY

TENOY EITWOYN

ज̈MOQ N̄TA OY PW

I5 ME N̄ $\overline{2 B} P d i O C$ OY

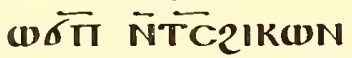

АqтגАOC EPOї єq $x(1)$ Mimoc $x \in x i$

TC $\overline{N T N O} \overline{X C} \in$

20 TIMOOY $\overline{M N}$ NiK̄

MC ÑAPXCN AE

ETTגїнY N̄TePOY

CWTM ENAI ÑTOO

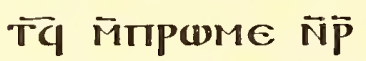

25 ГАтнс етере пाвІ

NंTOOTั EqฒOYE

4 пеNтגqme lege пеNTAqTame

I 3 post Terroy supple $x \in$ 
p. 84 , col. ii

$$
\begin{aligned}
& \text { CNOY EROA ETECHT } \\
& \text { AYAMA2TE M̈MOY } \\
& \text { AYXïTq ETETIC } \\
& \text { KOTION EA TAMTT } \\
& 5 \text { EגАXICTOC AYW } \\
& \text { NEPE 2ENKEETIC } \\
& \text { KOTIOC } 2 \text { MOOC } 2 A \\
& \text { 2THї M̈ாINAY } E \\
& \text { TM̈MAY ÑOYCW } \\
& \text { Io Eeipe M̃Itad } \bar{N} T \overline{T N} \\
& \overline{X C} \text { THPN TPिPW } \\
& \text { AYQ TMAdY MTTP } \\
& \text { PO M̄TGÑ MN } \\
& \text { N̄CA Nä̈ ÑTEPOY } \\
& \text { I5 Ei (1)גpoï dïEINE } \\
& \text { eqpaï } \overline{2 N} \text { TाBIP } \bar{N} \\
& \text { тாобе ÑuE ETE } \\
& \text { pe TcorpdфIA Ñ } \\
& \text { TIIAPQENOC CH2 } \\
& 20 \text { EPOC THCTEYE } \\
& \text { NAï (i) NACNHY }
\end{aligned}
$$

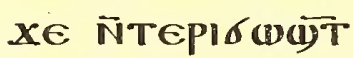

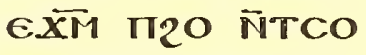

$$
\begin{aligned}
& \text { IPAфIA ANOK } \\
& 25 \text { MTN NeTICKOTIOC }
\end{aligned}
$$


p. 85, col. i

ETNMMAII ANNAY

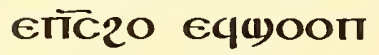
2N OYOKM 2WC xe ECPIME ECTA

5 BE CNOY EBOA $\bar{N}$ TET̃NAY $\lambda E \in$ POC ÑTEIRE AN TWOYN M̈MOC AN XITC E2OYN ETEK

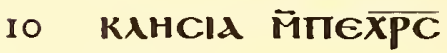

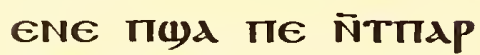
oENOC EPE OYNOK М̈мннГшle CWOY? E2OYN EITCTOTIOC

I 5 EToYגar N̄eIpe

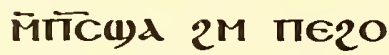
oY м̈пр̈пMєеүе N̈TCANANIMtIC $E$ TOYAAB EPE NAI

20 OYN wOOT N̄T2E ANXI N̄OYMOOY ETCOTT MTN OY Ne2 N̄CTINOYGE ANEIAAC EROA $\bar{N}$

25 2HTOY ANEINE 4 тAвE sic pro тAYe 
p. 85 , col. ii

$$
\begin{aligned}
& \pi \overline{T E} \\
& \text { NCE) EROYN ENEY } \\
& \text { EPHY ANTALO M } \\
& \text { MOC E2PAï ExO } \bar{N} \\
& \text { тетрдпIze aүш } \\
& 5 \text { mICTEYE NAÏ ON } \\
& \text { xe EC(y)OT wd } \\
& \text { TENOY AYW ON } \\
& \text { WA ENE2 N̄ENE2 } \\
& \text { AYW ON PEME NIM } \\
& \text { Io ET(1)OOI } \overline{2 N} 2 \mathrm{EN} \\
& \text { GINAINNE EYUO } \\
& \text { BE MN NETO } \bar{N} \\
& \text { AdIMMUNION } \overline{M N} \\
& \text { NETRITTE 2ATIAZ } \\
& \text { I5 } 2 A \Pi \lambda \omega C \text { OYON NIM } \\
& \text { ET(1)ОOா } \overline{2^{N}} \text { जा) } \\
& \text { NE NIM EYUIN }
\end{aligned}
$$

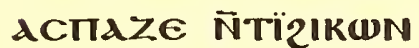

$$
\begin{aligned}
& \text { N̄TIIAPQENOC } \\
& 20 \quad \overline{{ }^{N}} \text { OYMICTIC WAY } \\
& \text { MAdTE M̄TIOYXגï }
\end{aligned}
$$

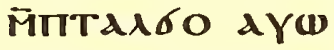

$$
\begin{aligned}
& \text { N̄TEÏ2E ANeIPE }
\end{aligned}
$$

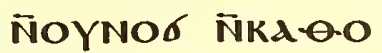

$$
\begin{aligned}
& 25 \text { АIKH CYNA元C }
\end{aligned}
$$

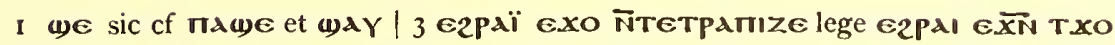

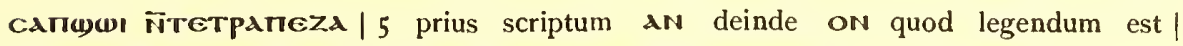

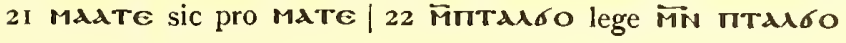


p. 86, col. i

\author{
Tा5 \\ N̄Tï હOOY M̈TEN \\ $\overrightarrow{X C}$ IC $\Pi E \overline{X C} \quad \overline{M N}$ \\ T्MMAY M̈тtAPOE \\ NOC ANOK de $\lambda \ddot{i}$ \\ 5 TNNNOOY AIEINE

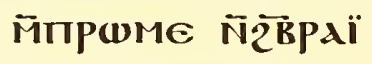 \\ OC ETM̈MAY ETK

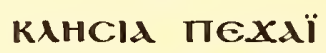 \\ NAq XE тлфнне

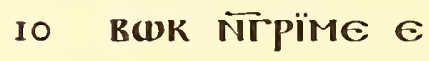 \\ POK MAYAAK EROIA] \\ XE OYNOF TE TK \\ KOגАCIC M̈ாTि \\ TO EBOA M̈TINOY \\ I 5 TE के ПАT-OHT \\ 2M пе2OOY ETप \\ NAGINE ÑCWK \\ eIE NTГि20TE AN \\ N̄TOK 2HTि $\bar{M}$ \\ 20 пNOYTE паї \\ Tepe TाкNICE \\ रेल Tिбוx $\bar{N}$ \\ TАКтолMА АK

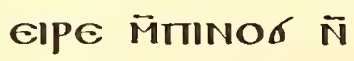

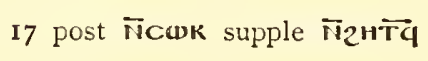


p. 86 , col. ii

$$
\begin{aligned}
& \text { N̄2WB N̄ROTE AK }
\end{aligned}
$$

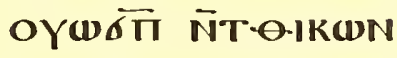

$$
\begin{aligned}
& \text { ÑTIAP-ENOC } € \\
& \text { TOYAdR Mapla } \\
& 5 \text { TMdAY ज̈ாTENA } \\
& \text { AYOINOC ÑNOY } \\
& \text { TE IC } \Pi \in \overline{X C} \text { AYW } \\
& \text { NัTePÍ́(1) El(1)d } \\
& x \in \text { NTMMAq } \overline{2 M}
\end{aligned}
$$

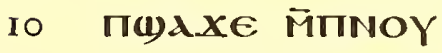

$$
\begin{aligned}
& \text { TE AqTï MeTd } \\
& \text { Noïd Naï aYu dï } \\
& \text { Kג-O-Irel MimOG } \\
& \text { AITI NAq ज̈пिாM } \\
& 15 \text { EY€ ก̃N̄KOגАCIC } \\
& \text { ElXW M̄MOC NAq } \\
& \text { xe дpї пмєєүє } \bar{N} \\
& \text { NEKNORE ПגАH } \\
& \text { PE MTN ÑCA OYROYï } \\
& 20 \text { ERNAEI EROA TTN }
\end{aligned}
$$

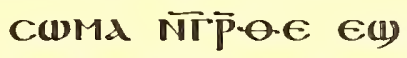

$$
\begin{aligned}
& \text { XE ПоҮХПок єाI }
\end{aligned}
$$

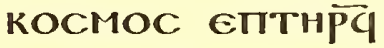

$$
\begin{aligned}
& \text { AY(W EK(I)AN ME }
\end{aligned}
$$

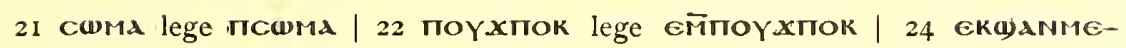
TANOI lege eKwaNTTMETANOI 
p. 87, col. i

$$
\begin{aligned}
& \text { TANÖ̈ M̄TATK } \\
& \text { MOY ПTКKMAN̄ฒW }
\end{aligned}
$$

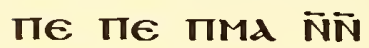

$$
\begin{aligned}
& \text { PGPNORE TTHPOIY }
\end{aligned}
$$

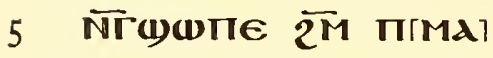

$$
\begin{aligned}
& \text { ETE M̄ IIAENE? } \\
& \text { бINE AN M̈Iq⿴囗̈ } \\
& \text { NE KAN N̄TK OY }
\end{aligned}
$$

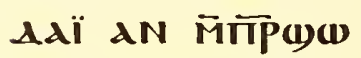

$$
\begin{aligned}
& \text { Io IIE ÑATNOYTE } \\
& \text { AYU OГYIUAN2TГHIG } \\
& \text { IIE INOYTE q[NA] } \\
& \text { KW NAK EBOA } \epsilon \\
& \text { TEIAH OYEBOA } \overline{2 N} \\
& \text { I } 5 \text { NIOYAdi TE TIIAP } \\
& \text { बENOC ETOYAAB } \\
& \text { Mapid aYw E } \\
& \text { PE IINOYTE бw } \\
& \text { घT ETKMETA } \\
& 20 \text { NoÏd ENE M̄MON } \\
& \text { AqEINE N̄TTGOP } \\
& \text { гн ЄXฒK XI1 } \\
& \text { N̄ TEYNOY ÑITA7K }
\end{aligned}
$$

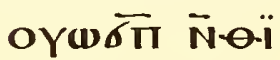


p. 87, col. ii

\author{
$\overline{\pi z}$ \\ KWN ÑTGMAdY \\ M̈TAP-QENOC \\ IINOMOC rap $\mathrm{xw}$ \\ M̈MOC NีTEI2E \\ 5 XE OYOPГH MN \\ OYбWNT MTN OY

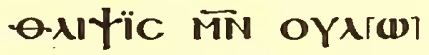 \\ $\overline{x 2} 2 \ddot{I} \overline{X M}$ hYXin] \\ NIM N̈PWME ETP \\ IO 2山B EாढTөOOY \\ Td mIOYAdï ÑळO \\ PTा MT TOYEEININ \\ Ndï $\lambda \in$ ÑTEPIXO \\ oY NAG A TITNOYC \\ I5 XI OYOEIN nEXגG \\ Naï $x \in \Pi \pi \overline{X C}$ Ñeï

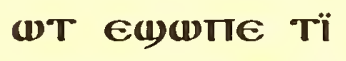 \\ M̈T(1) MAPE TाK \\ Nd TA20ï NTd \\ 20 AT NXPICTIA \\ NOC MN TAC2I \\ Me MTN NaqHPE \\ 2APHY ĪTE IINOY \\ TE KW NAl EBOA
}

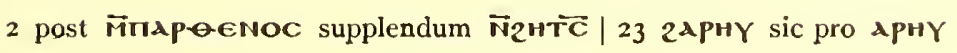


p. 88 , col. i

\author{
กับ \\ M̈TEINITAïdq d \\ NOK 「Ale [ா]EXAl NAq \\ xE (1)גсте ткYPї \\ АКн $2 \bar{M}$ поҮГШ]⿻ \\ 5 ज̈ாNOYTГEI AMOY \\ (1)גPON ЄTKКגH \\ CIA AYW TINA \\ RATtZE M̈MOK \\ МN текнї тнроү

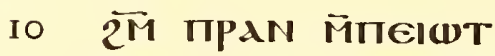 \\ MN пाнне $\overline{M N}$ пात्र \\ ETOYAAB 2TOOY \\ E $\lambda \in$ N̄TKYPIдкн \\ дїTшOYN АIвळK \\ I5 GTKклнсіА $\mathrm{E}$ \\ TpdeIPE N̄TKג-O-O \\ 入їкн CYNAそlC Nं \\ TEPIRWK $\lambda \in \Theta$ \\ 2OYN EITMA ETOY \\ 20 AגR גїтफि(i) $N$ \\ NÁIX EROA 6 \\ 2Pגï ЄПNAHT $\bar{N}$ \\ NOYTE IC

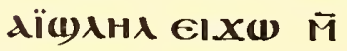 \\ 25 MOC $X \in$ INOYTE
}

3 шдсте lege pגcte vel шג пєсрдсте 
p. 88 , col. ii

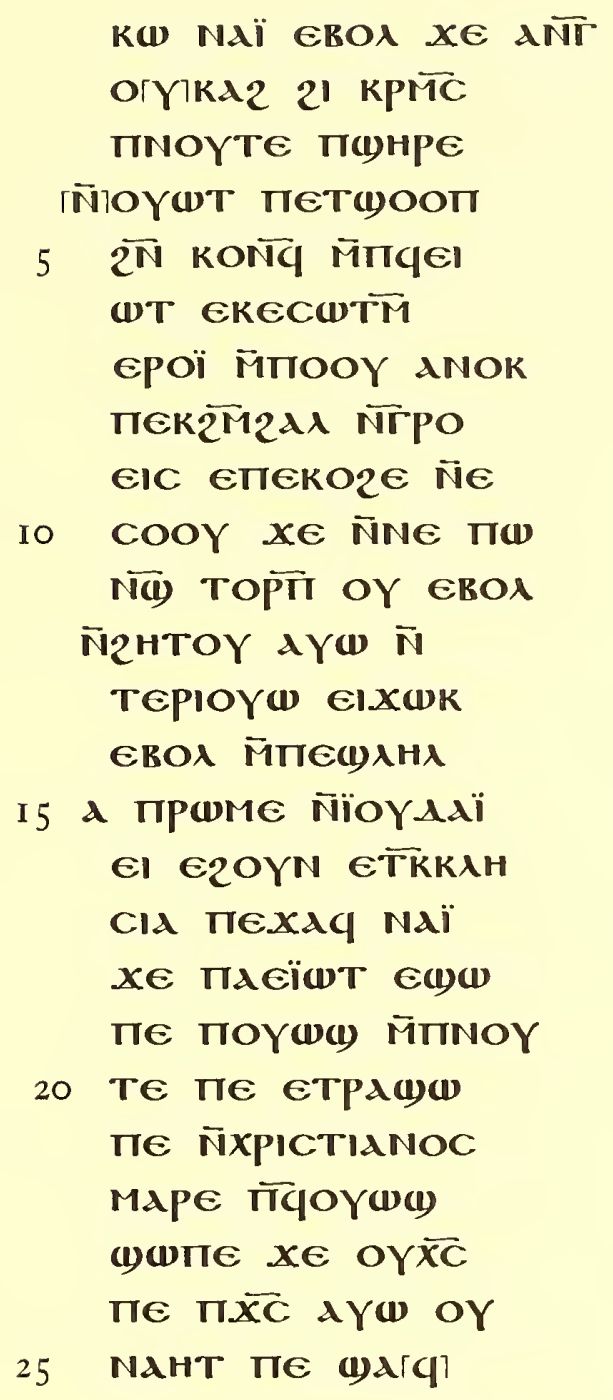

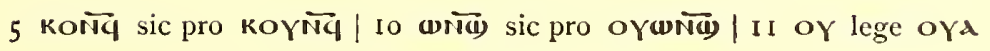


p. 89, col. i

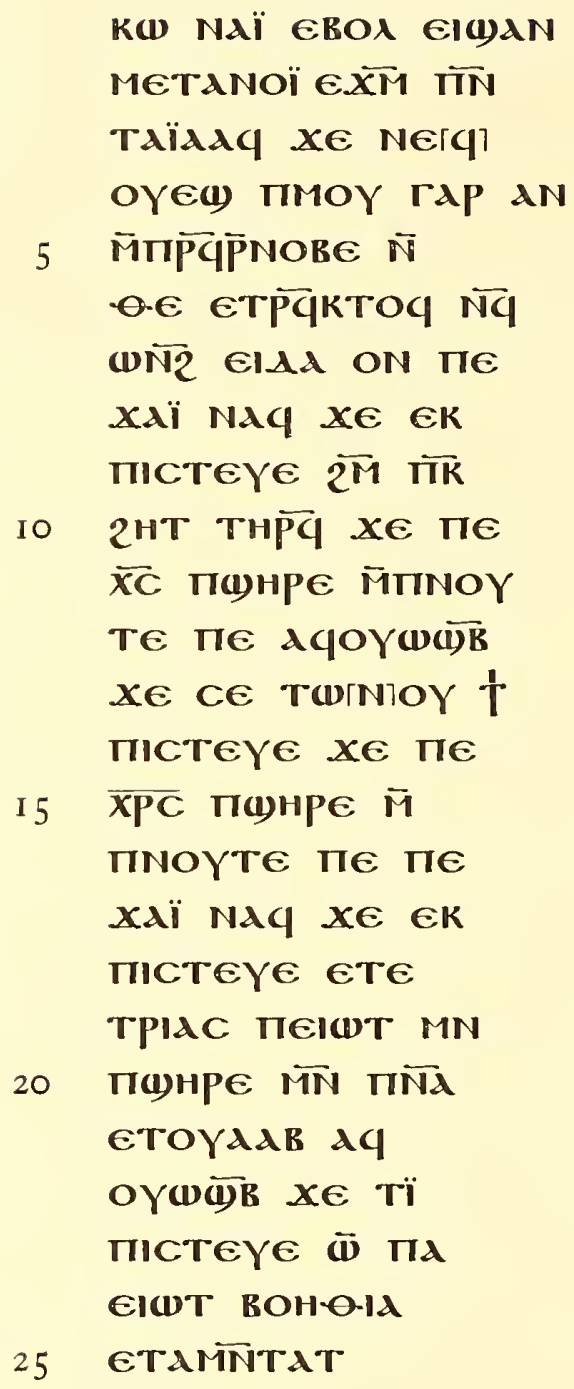

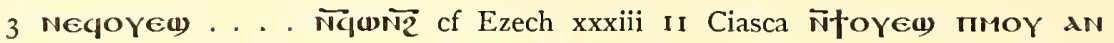

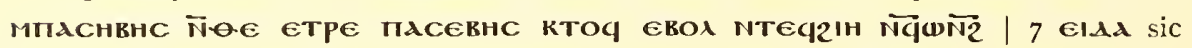

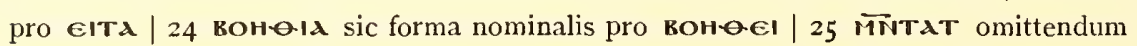


p. 89 , col. ii

ПT्ध.

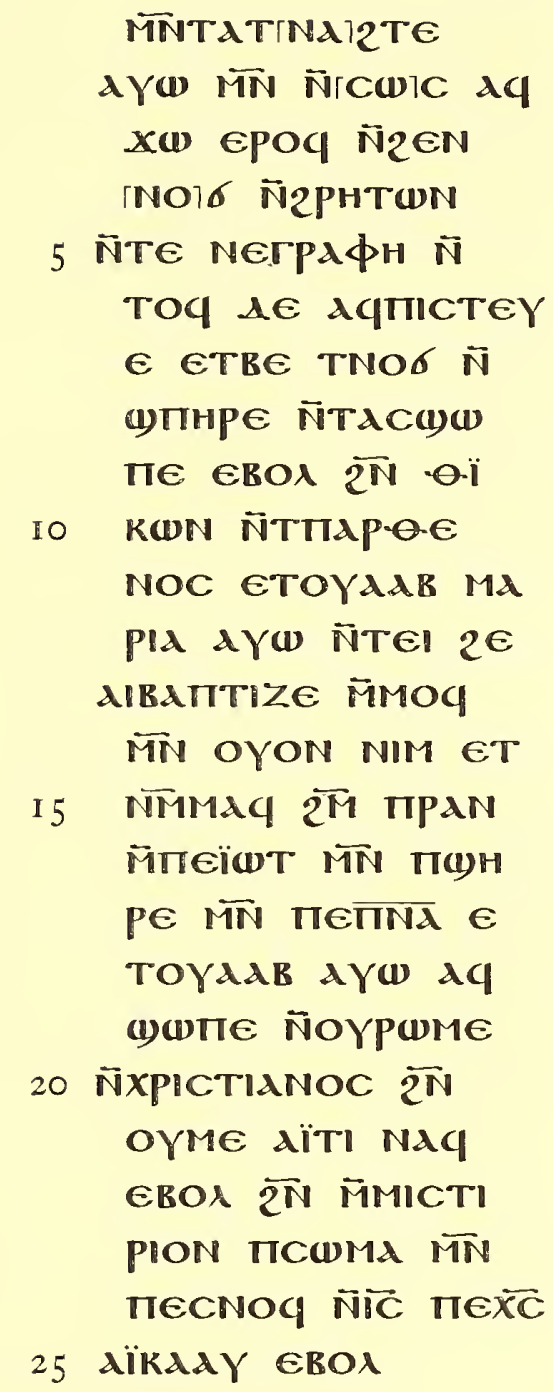

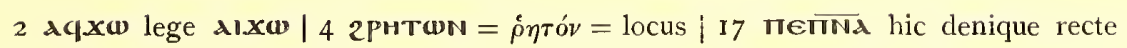

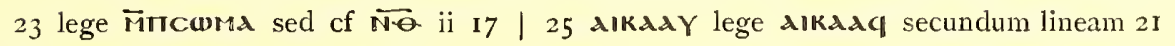
aut legendum NaY et dIKadY 


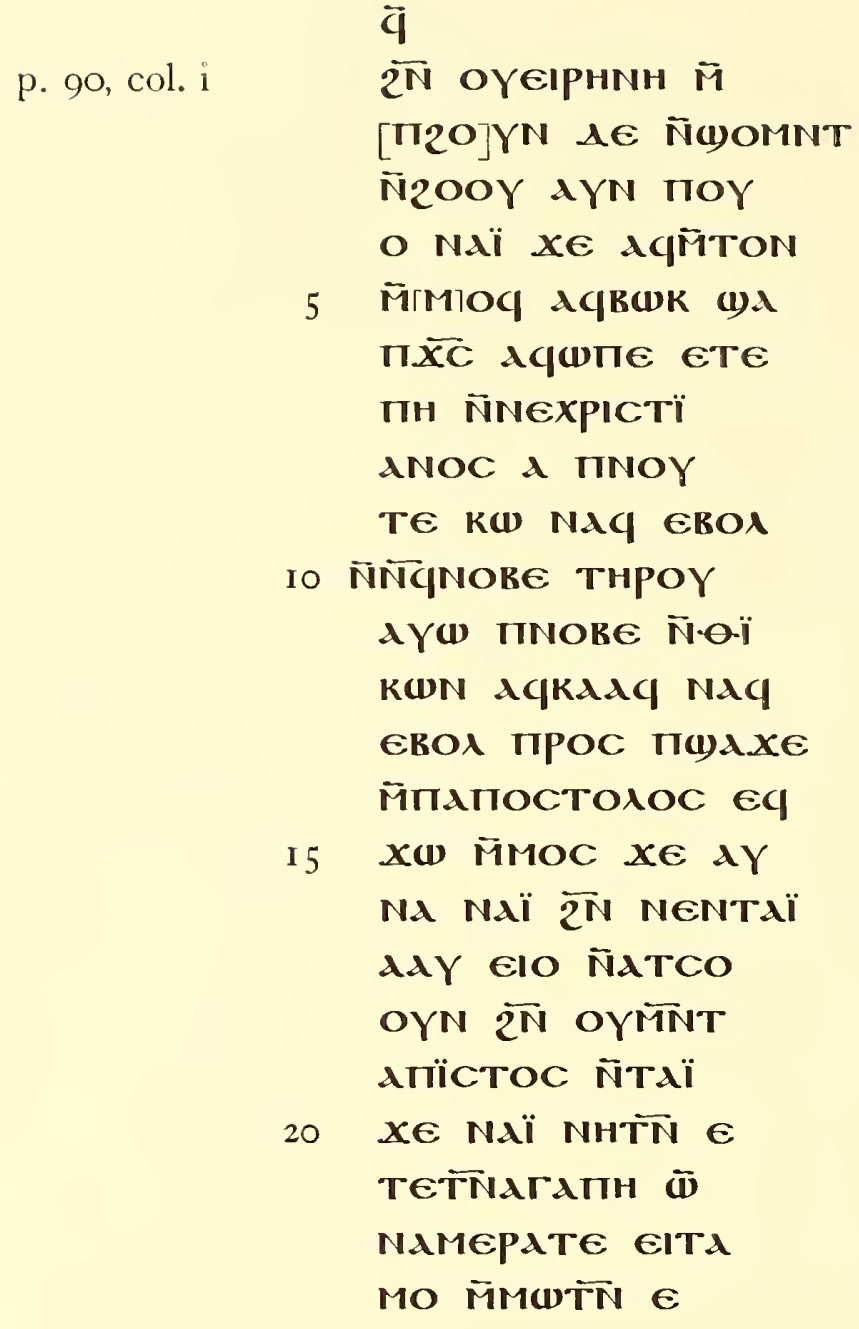

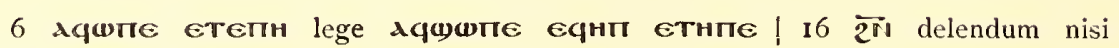

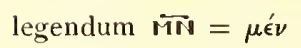


p. 90, col. ii

maÏ xe MaPNPOO

EIC EPON EBOA $\overline{2 N}$

NoRE NIM E(I)XE

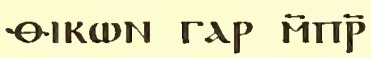

5 РО П̈пїкосмос

EY(1)גNZ(1ГPd

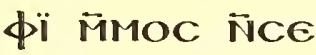

TA2OC EPATC

2N TMHнTE ÑTA

IO ГUPd E(1)גC(1)(1)

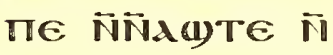

TтолIC THPC AY

(1) EY(1)ANXI OY

a Ñroñ NqGi

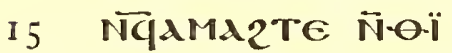

KWN M̈ாїPO MTN

lddY Nd(b)Tï OY

вHq Kגї ГAP OY

ршме пе прро

20 E(I)גqMOY AY(1)

cexï MিMOq eYMs

ก̈TÏ2AT ANON $\lambda \epsilon$

zOMN (1) NAME

PATE MAPिTTї

25 COOY T̃T2IKUN 
p. 9 I, col. $i$

\author{
$\bar{N} \overline{T N} \cdot \overline{X C}$ TнसN

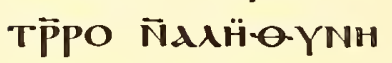 \\ TEOEOTOKOC 6 \\ TOYdAB Mäpla \\ 5 TMAdY M̈ாENNOY \\ TE TC $\Pi \overline{T C} \overrightarrow{X C}$ AYW \\ ÑTNMEPE T̄KRH \\ cId пнї MாINOYTE

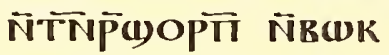 \\ Io EPOC Ñ2TOOYE MN \\ POYZE M̆MHN EROA \\ КАTג OЕ ЄTСH2 \\ xe тїламоY Eрок \\ MMmNe M̈MnNe \\ I 5 TNגсMоY епекрдN \\ (1) ENE2 AYW \\ ENE2 N̄ENE2 $x \in$ \\ KגC XEKdC ÑNA \\ 2E EYNA M̈Tिप̆ \\ 20 TO EROA $\overrightarrow{2 M}$ пе2O \\ OY N̄TT̃NANKTH \\ 2OMAÏOC $\lambda \in$ ON TA \\ ПО⿴囗Кн N̈TAY2E

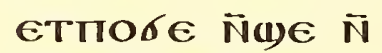 \\ 25 2HTC ETEPE O.IKWN \\ N̄TTAPOENOC $\mathrm{CH} 2 \in$

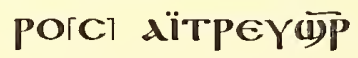

I8 dele $x \in \kappa \lambda \mid 22$ 20mגïoc $=$ ó $\mu$ oíws 
p. 9 I, col. ii

$$
\begin{aligned}
& \overline{q \lambda} \\
& \text { (1) } \overline{\mathrm{PC}} \text { dïddC } \overline{\mathrm{N}}[\mathrm{O}] \mathrm{Y}] \\
& \text { No6 їாגANTTOIXI } \\
& \text { ON AY(D) Aq(1) }
\end{aligned}
$$

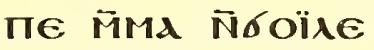

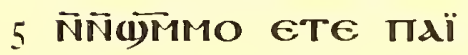

$$
\begin{aligned}
& \text { пE TENOY ETAIO } \\
& \text { оा Гॉ̃र TKecdpld } \\
& \text { NTTIOAIC PAKOTE } \\
& \text { AYW QMHN GBOA } \\
& \text { IO (I) ZOYN ETOOY Nं } \\
& \text { 2 ГOOY AYC PEME } \\
& \text { NIM ET()OOП } \overline{2 N} \\
& \text { GING)(WNE NIM } \\
& \text { EY(I)AXIITA GIAN } \\
& \text { I5 AOXION ETMTMAY }
\end{aligned}
$$

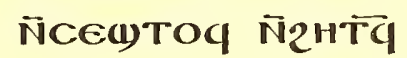

$$
\begin{aligned}
& \text { 2A2TT TMA ETEPE } \\
& \text { T2IKWN N̄TாAP } \\
& \text { OENOC Ñ2HTq } \\
& 20 \text { (1)дqMAте ज̈ாтגА } \\
& \text { 6O ÑTEYNOY } 21 \\
& \text { TN NE(I)AHA NTTAP } \\
& \text { aeNOC MAPIA TMA } \\
& \text { dY MTINT } \overline{X C} \overrightarrow{I C} \text { пढ } \\
& 25 \overline{X C} \text { TENOY } 6 \in \overrightarrow{\mathbf{w}} \\
& \text { NAMEPATE MITP }
\end{aligned}
$$

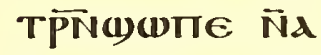

$$
\begin{aligned}
& \text { MENIC ENICIA }
\end{aligned}
$$

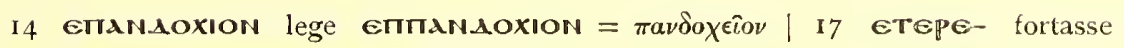

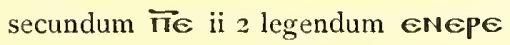




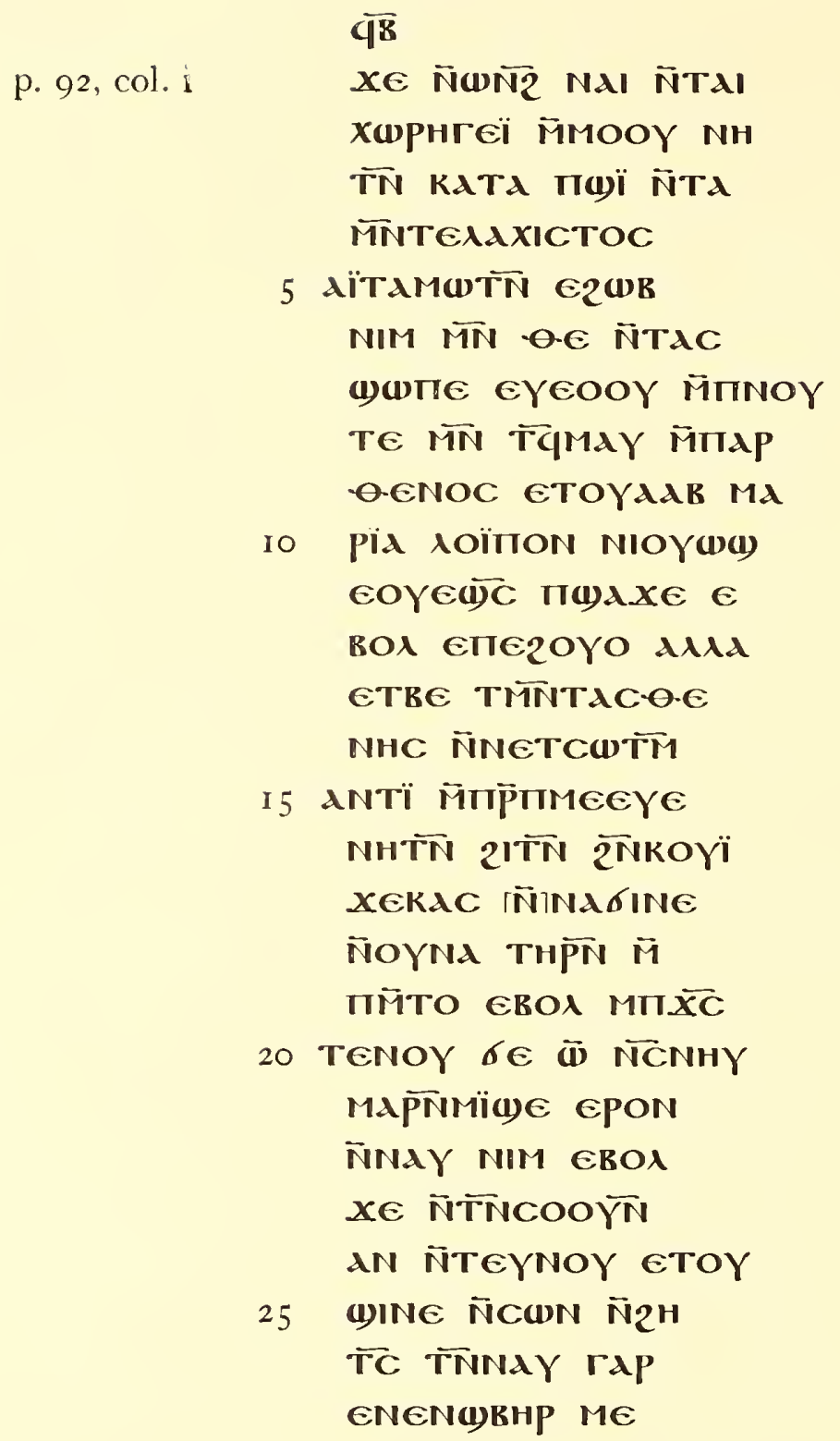

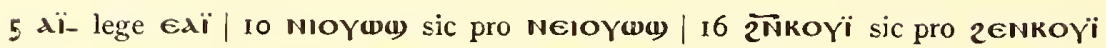


p. 92, col. ii

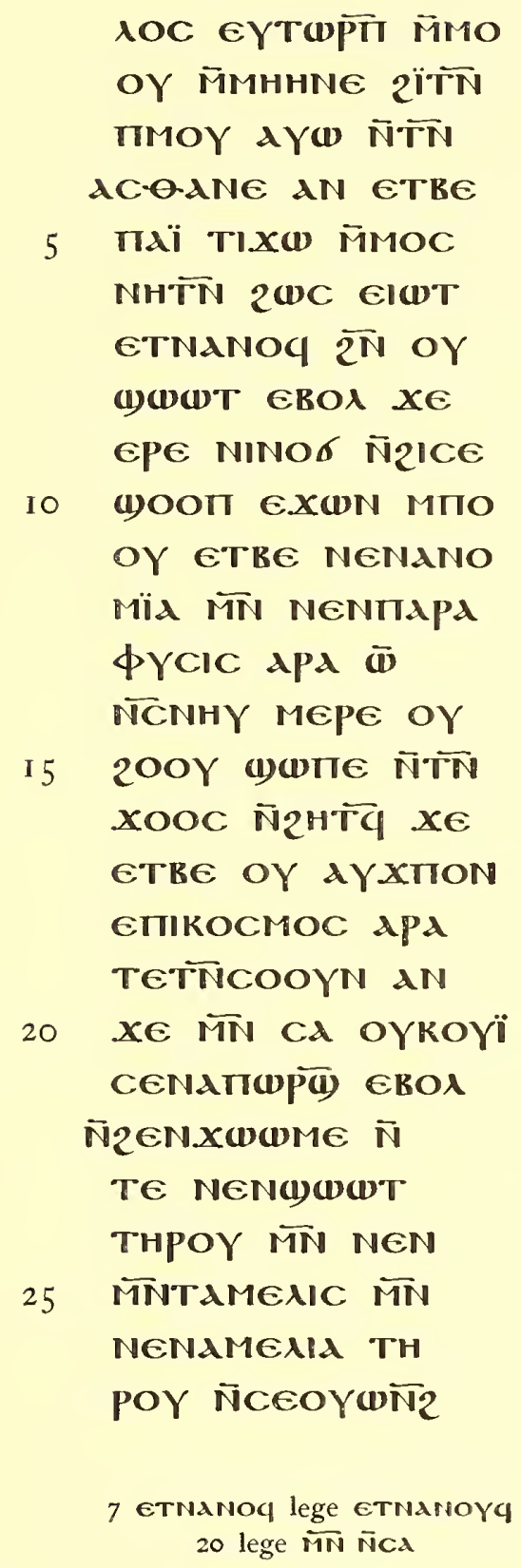


p. 93, col. i

EBOA NA TIE2OOY MÑ Na теүलH Apï IIME

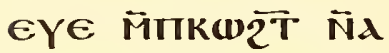

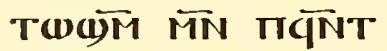

5 N̄גTN̄кOT̄K MN TPM ЄIH ETNHY EROA $\overline{2 N}$ NENBAג N̄TNYYXH тגï $\epsilon$ MN 2AE OYн2 N̈COC MTN OYPÏME Ñ

Io ATW $\overline{X N}$ APA EK NAqї OY ЄKBHK ERO» 2M ПIKOCMOC $\overline{\mathbf{\omega}}$ NCNHY M̈MEPIT Ñ Cd NEKNORE MNN $\overline{N K}$

I 5 ANOMiA NTCOOYN

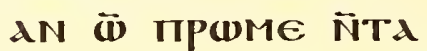
AגIIIOPOC $X E$ WOMTN M̄MA2є N̄KA2 Tढ пекнї шג ENE2 20 yOOT $\lambda \in$ OYN $\bar{N}^{2}$ $2 \in \overline{\mathbf{\omega}} \overline{\mathrm{NCNHYE}} \overline{2 M}$ Tाі

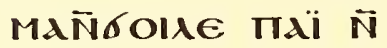
(1)ANNAY EYPWME E IIUN TIE EdqMOY

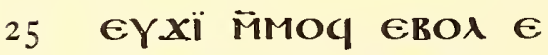
ПTגфOC KAN EWW пе OYलMMO TIE घAN 
p. 93 , col. ii

$$
\overline{q \Gamma}
$$

xooc ñtre Xe eIC

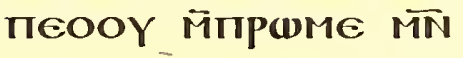

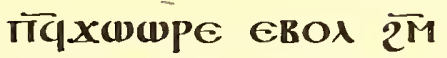
ПтАфос (1)גPE $2 \in \mathrm{N}$

5 KOOYE XOOC $\overline{2 N} \bar{N}$ COфOC XE 2OITC $E$

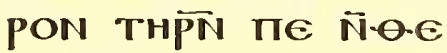

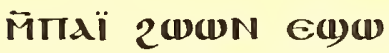
пе $\overline{M N}$ גqр orkoyï

Io M̈ாIIETNANOYG (1)גq2€ ePOG $2^{i}$ өн ज̄пाекрітнс

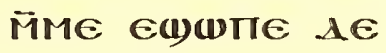
zWWG OYATNA

I5 ()ACINAY ENET2O OY TнPOY EI(1) XE eNEzOYCIA $\bar{M}$ ПKגKE EYO ÑAAГ MA AגTMA EYMO

20 O(1)E NMMAq (1)AN

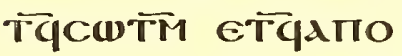
中ACIC EROA 2ITM INOYTE TENOY бE (i) Namepate

25 KAN OYIIETNANOYA пе KaN OYTIICT-O-O oY IIE Cenamooile 


\section{p. 94 , col. i}

$$
\begin{aligned}
& \overline{\varphi \lambda} \\
& \text { 2I-H MMMON THPN } \\
& \text { (1) ANTNOYW(1)T M } \\
& \text { пекрітнс М̈Mе пе }
\end{aligned}
$$

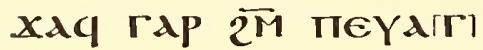

$$
\begin{aligned}
& 5 \text { TENION N̄KATA Ïฒ } \\
& \text { 2ANNHC XE NEN } \\
& \text { TAYEipe N̄zenteT } \\
& \text { NANOOY EYANAC } \\
& \text { TACIC N̈WN2 NEN } \\
& \text { Io TAYEIPE N̄zeNTITET } \\
& \text { OOOY EYANACTA } \\
& \text { GIC N̄kpïcïc }
\end{aligned}
$$

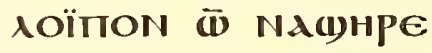

$$
\begin{aligned}
& \text { MMEPIT MA广̃N.XI } \\
& \text { I } 5 \text { CвW М̈ாАTE CRE } \\
& \text { NIM } \boldsymbol{\omega} \overline{X N} \text { ÑTOO } \\
& \text { TN MAPNE } \overline{C B T W}
\end{aligned}
$$

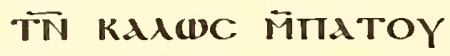

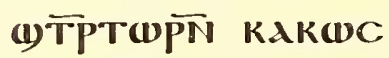

$$
\begin{aligned}
& 20 \text { MAPN(1)גнА ETINOY } \\
& \text { TE N̄OYOEI() NIM } \\
& \text { 2N 2ENCOTITT } \overline{M N} \\
& \text { 2ENPMEIOOYE AYW } \\
& \text { ECINACWTM ETTN }
\end{aligned}
$$

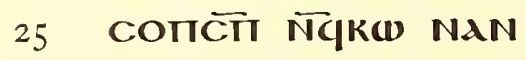

$$
\begin{aligned}
& \text { EBOA тाр̈PO гAP M̄тіi }
\end{aligned}
$$

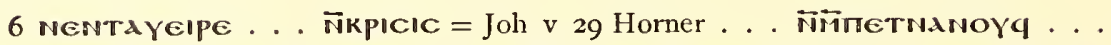

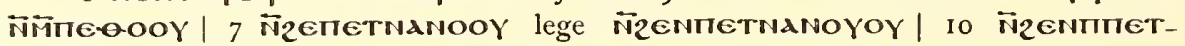
booy sic pro ñzenteroooy 
p. 94, col. ii

KOCMOC Eq(uAN"

АCERHC ÑOYPPO AN

tTe XIN MitIINAY dA

IA OYAYPANNOC TIE

5 AYW PलME NIM Eव wOOI $\overline{2 N}$ NAÏ ГTEINOY

eTINAXOOY ÑOY

XPICTIANOC AN TE

$\overline{2 N}$ OYME didd OY

Io PAN ÑNOYX пTET̃

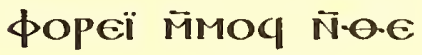
ÑANANIAC MN CAM MEdC $\overline{2 M}$ TICPAHA

AYW ON TENOY Ñ.eE

I 5 N̄ZGAGKIAC MT $\lambda$ XIAC N̄Td TTिPPO Ñ TBABYACN 2OTBOY кATג Tा(1)גXe Ñï PHMIAC пепिO

20 фнтHC TENOY $6 \mathrm{E}$ MAPNPOEIC EPON eтміраtтістос $\epsilon$ NE(IITHE M̄INNOY TE MN $\overline{\text { Nqq }}$

25 AdB N̄2OYO $\lambda \epsilon$ TqMAdY जि̈Iय e-ENOC MAPIA 
p. 95 , col. i

Tגï ETNCWOY2 $\epsilon$ 2OYN EITCTOIOC $E$ TOYAגR M̄TIOOY ज̄

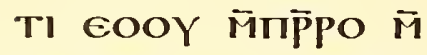

5 ME $\overrightarrow{I C} \Pi \in \overrightarrow{X C}$ IIN $\overline{X C}$ пגї N̄TAqua NAN GBOA $\bar{N}_{2}[\mathrm{H}] \overline{\mathrm{TC}} \overline{\mathrm{N}} \boldsymbol{T}$ IIAPOENOC TAl $\mathrm{E}$

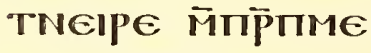

Io EYe N̈TCANAגIM fïc eTOYAAR $\bar{M}$ חOOY M̄N O.E NंTA INOYTE TI MTON NAC $\overline{2 N}$ 2कB NIM $\bar{N}$

I 5 AГגOON EIIAH Nï OY(W(1) MN

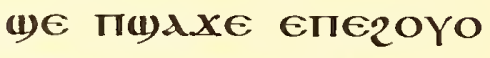
ЄтвE а пNAY про коптї MA

20 lO $6 \mathrm{E}$ E2PAI N̈2EN OYCIA N̄CMOY 21 NA $2 і$ АГАПн 2 МाNT PMÑ̃HT NIM AY (1) Mัтртре NAdY $\bar{M}$

25 TIOPNOC $2 i$ NOEIK oYAE MANAKOC 
p. 95, col. ii

\author{
[ब्षब]

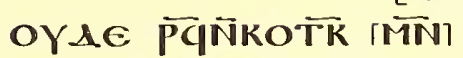 \\ ÑN2OOYT OYAE $\overline{P Q}$ \\ 2 WTE OY[AE MAI]TTOI \\ e Ñ2oYo oyAe $\overline{\mathrm{Pq}}$

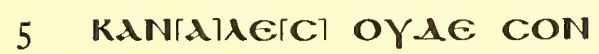 \\ 21 ()внр IีNOYX OY \\ de MAIEOOY ET(I)YY \\ eIT MiTptpe OOYA \\ 2N NENTdixOOY \\ Io TाTqoYoï eTKoino \\ NId ETOYdAB $\mathbb{N}$ \\ ÑMYCTIPION $\bar{M}$ \\ пє $\overline{X C}$ еІмннте

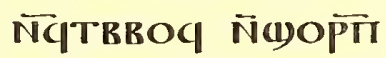

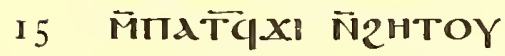

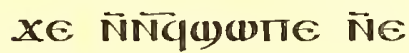 \\ NOXOC EITCWMA \\ MTN TICNOQ MITIXC

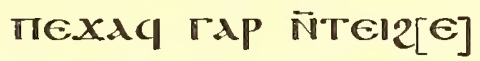

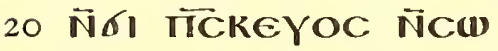 \\ TाI maYAOC $x \in E$ \\ TRE TAÏ OYTN 2 A2 \\ (1)WNE İ2HTTHY \\ TN AYW Cectó \\ 25 lor AYU CeÑKo \\ TK \\ AYU ON XE NENMO
}

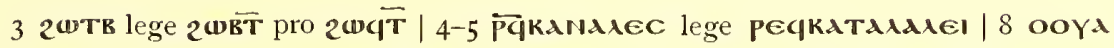

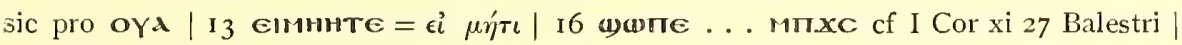

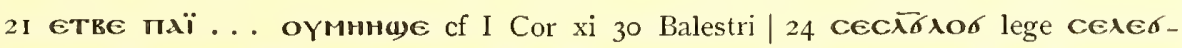

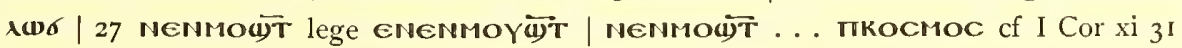
Balestri 


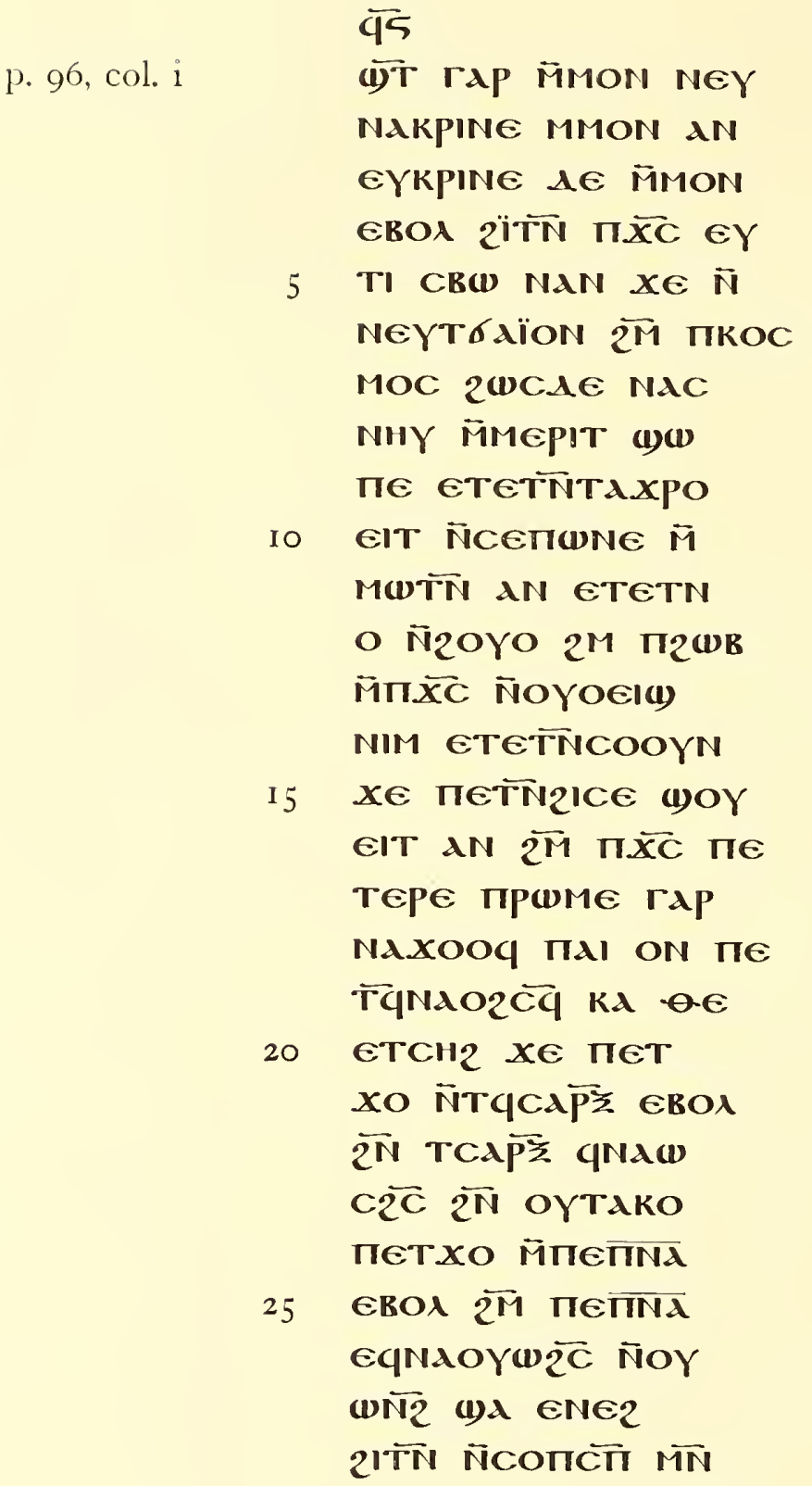

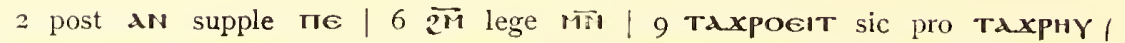

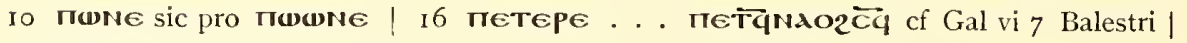
I9 кג lege кגтג| 20 петхо ... eNe2 cf Gal vi 8 Balestri| 22 qnaduc $\overrightarrow{2 C}$ lege

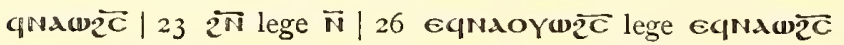




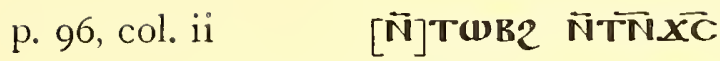

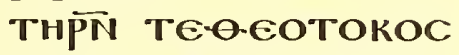

eTOYAdB MAPIA $2 \|$

TN TExapIC MT

5 TMNTMAIP⿴囗十

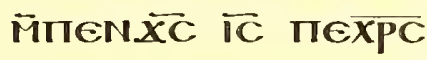

паї євол 2ІтоОТ̆

neOOY NAq MTN

EIWT NiATA-O.WC

Io MT пеताNA ETOYd

AR NিPCTAN2O AY

(1) त̄2OMOOYCIOY

TENOY AYU ÑOYO

GII) NIM (I)d ENE2

15 N̈ENE2 2АMHN

GTW IWANNOY GAd

прСвүтерОY YІе коג

גO-O-OC TOY ArIOY MEP

KOYPÏOY KAl OїкшNOMOY

20 TOY APXATIEAOY ГAB

рннд опос прєсвієс

KAl EYXAIC EгPAфH MENH

IAPTIZOT A NAIK

AIONOC [B] АПO АIOKAH

$25 \overline{x q}$ ETOYC TミГ

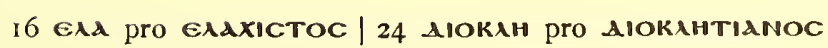


2ITÑ TC̄ாOAH MÑ TMNTqAїрOOY(i) MாENMAINOYTE ÑCON CICINNIOC ПШ)нE

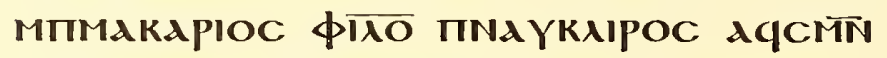

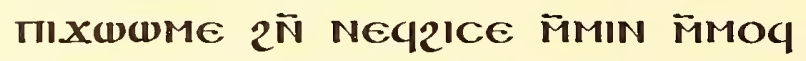

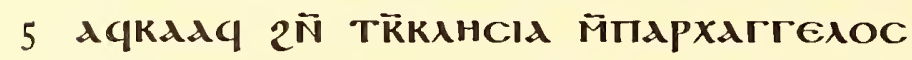

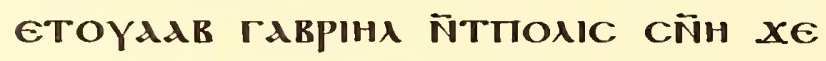
KAC EPE ПNOYTE MПAPXAГTEAOC ГАR

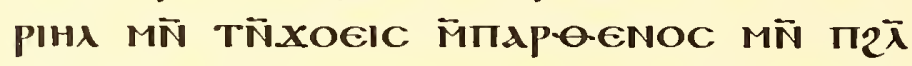

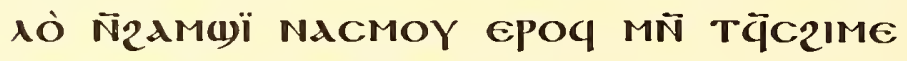

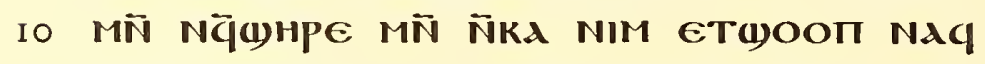
Ñ̈e ÑTAqCMOY EeYAPONIOC MN

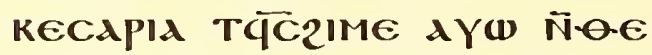
N̄TAqCMOY ENENEIOOTE MIIA TPIAPXHC AвPAגM MÑ CAPA ICAK MÑ

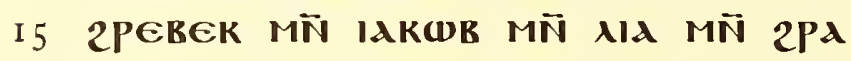

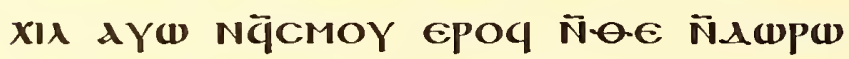

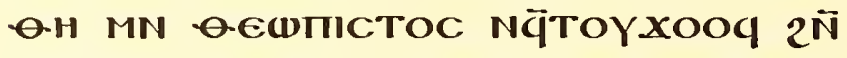

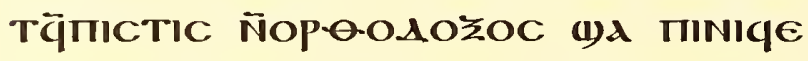

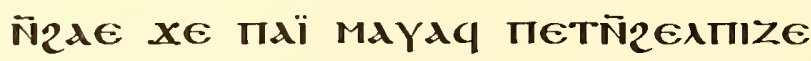

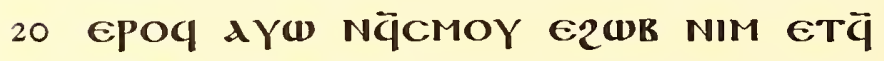

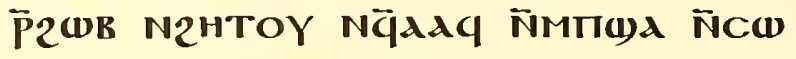

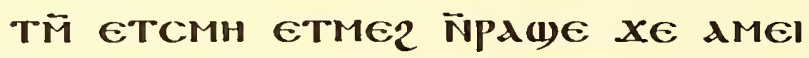
TÑ ()גPOї NeTCMdMAdT ÑTE חגєIOT ÑTETÑ KAIPONOMEI ÑTMNTEPO ÑTAY 25 CRTWTC̈ NHTÑ XIN TKגTגBOגH MПKOC

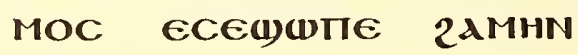




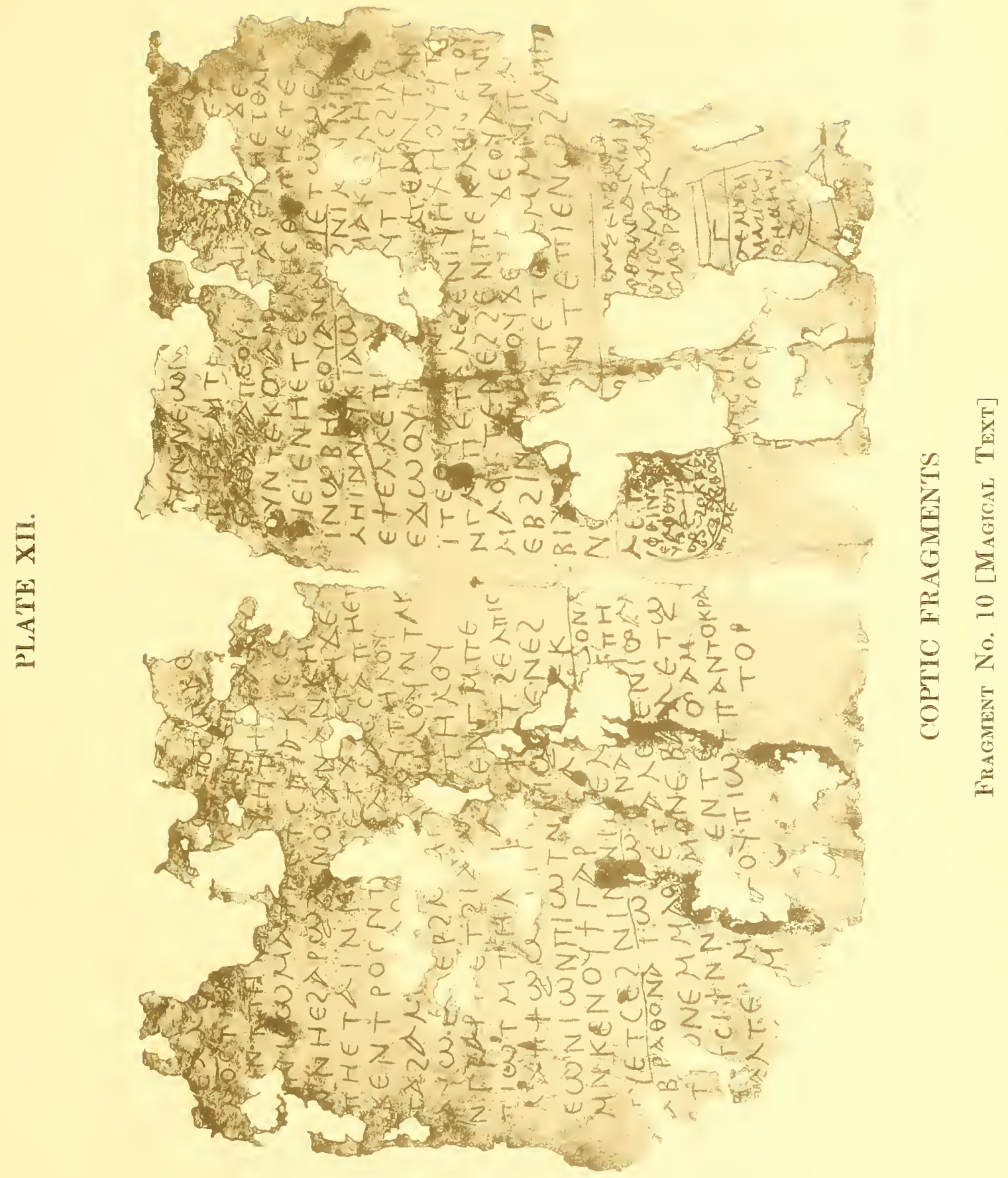





\section{A MAGICAL TEXT}

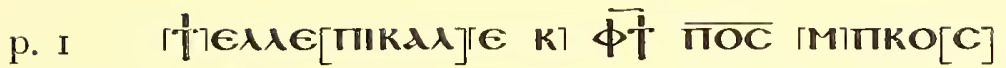
MOC THג[Q NEM T]KE2I тाH] ET[CA2[P]

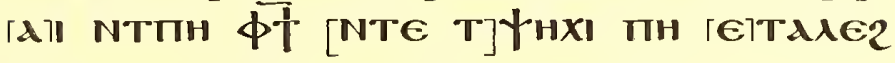
[G]NICWMA ПTH ETCTIPIAKICCIG]

5 ГNINH E2AP(I) 2EN OYANANKH

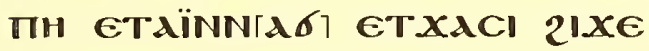

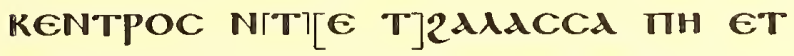

TA2A M[M] גOY [EA]

AYU E[T]EP2MMI ГM]MAOY NTAK

IO

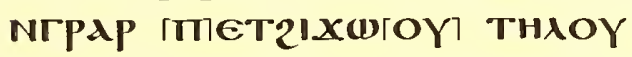

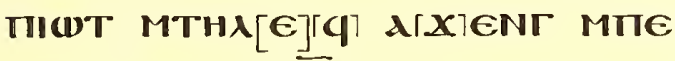

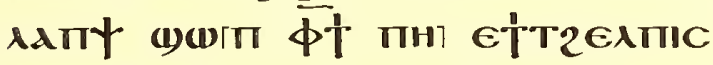

EWNICN IIICT NNICIATENE?

MN KE NOYT ГAP [N]CA[MAY]ГTK

I 5 TY ETCE2NI [NNIIMTEA[I] [ATAONA

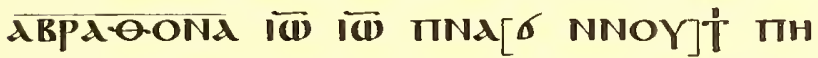

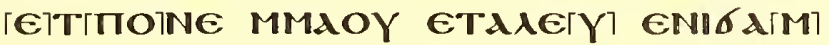

[IT]H ETרCIT NNIIT][AIMON GRAג ET(y)

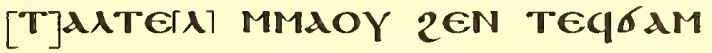

20

..... M[M]AOY IIWT IIANTORPA

TOP

I. Teлмепікдле supplied from ii, 8. Perhaps the intention is to write teıent-

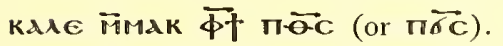

2. There is not room for the expected Tнй $\pi \kappa \epsilon_{21}$, while grammatical, is not satisfactory.

3. The letters $x_{I}$ are by a second hand, in darker ink. †нхו is for Түхн.

4. спрдкіссе is probably for сфрдгіze, "to seal" with the sign of the Cross, "to bless," "to heal" (cf. the homily manuscript p. ज̈z), "to choose," "to keep."

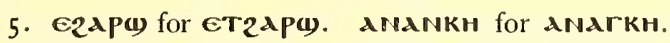

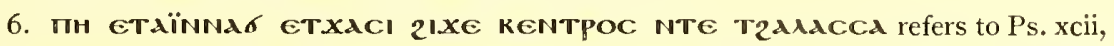

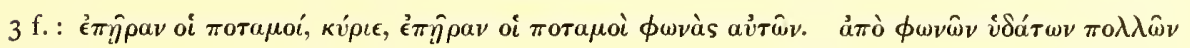

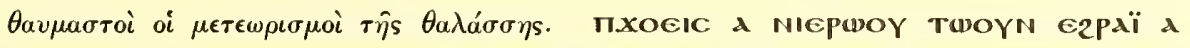

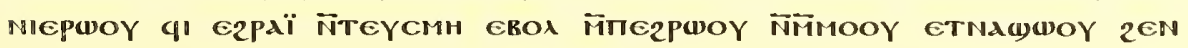


штнре Ne T̃xice T̃exldccA (Sahidic, there being no Fayúmic extant); cf. Ps.

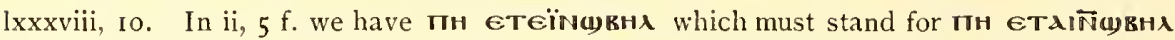
(= Sahidic пето п̄щвнр). Accordingly we read пн етдїнидб (=Sahidic пето

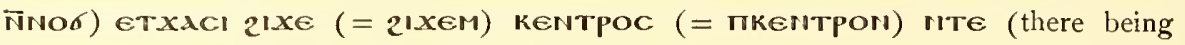
too much room for $\overline{\mathrm{N}}$ ) T2גudcc.

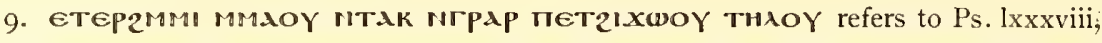

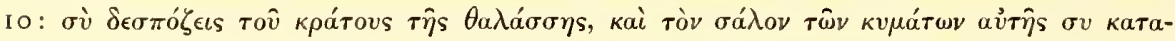

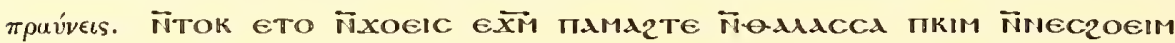
їток еттреqбњ; cf. Ps. lxiv, 8 ; cvi, 29. Perhaps the scribe intended етергmm

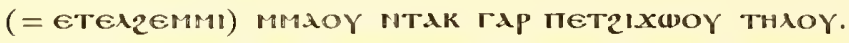

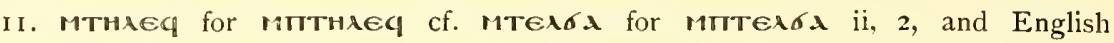

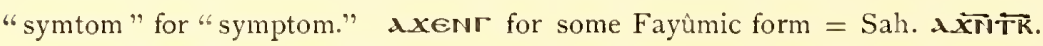

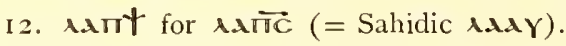

I4. NGAMAYak.

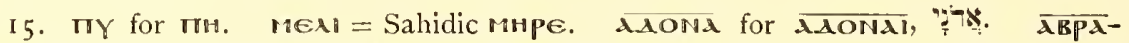
$\overline{\text { QONd }}$ is also connected with are common enough in Christian and Moslem magic; cf. Deissmann, Bible Studies, I909, pp. 32 I ff. ; Deissmann, Licht vom Osten, I909. p. 308 ; Hastings' Encyclopedia of Religion and Ethics, loc. cit. p. 40I ; Zeitschrift fïr Assyriologie, xx, 412; xxi, 244; xxix, I I6.

I7. ג৯eY perhaps for גie.

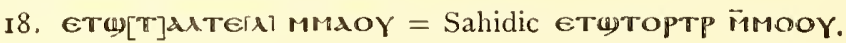




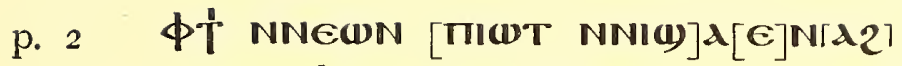

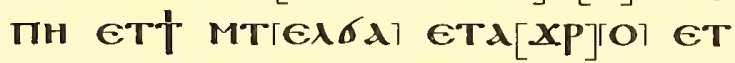

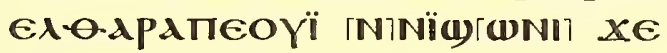

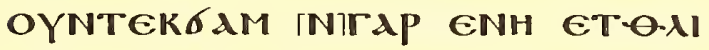

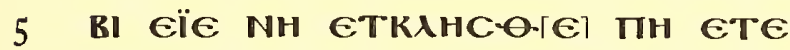

INNARHA GOYAN NIRI GTAW $\epsilon_{2}$

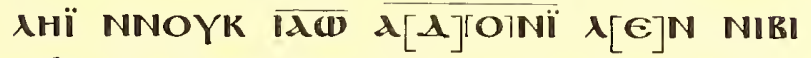

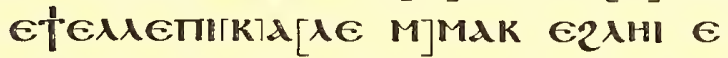

EXWOY ï[T] [E] [2גO]YT їTе C2IMI

Io ITTE OYAN NIIB][1] ГEYרAITE MO NTAK

NIIP TETAגe2 ENIYHXHOY ITE

MAOY ITTE NE2 ZEN TIERAEN ETOY

EB zINA NCEOYXEi $x \in$ OYAN NII

BI $x \in[T][\boldsymbol{W}] \mathrm{T}$ TE Tб「גרM MN TIAГOY

I.5 NIG][A ENE]2 NTE TIENTEI2 2AMH[N]

I. Cf. eNd2 with eNe2, i, I3 and perhaps also ii, I5, and with TAre2, i, 3 .

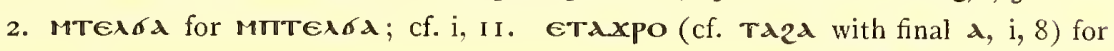

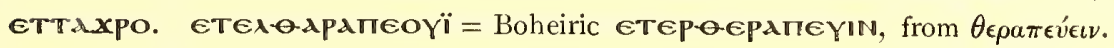

4. There was a letter after $6 \mathbf{\lambda} \mathbf{M}$, perhaps $\mathbf{N}$, cf. i, Io; ii, I I.

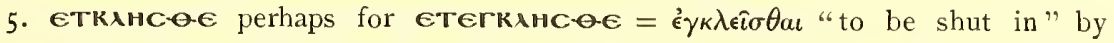
sickness or the law. пн етёімавнд (cf. i, 6, пн етגїnмar) perhaps for пн етАाп̃ฒвня (= Sahidic петойळвнр).

7. NNOYK apparently "thy (name)." $\overline{\mathbf{I A g}} \overline{\text { AlONï, }} \mathrm{cf}$. i, I $5 \mathrm{f}$.

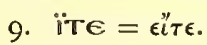

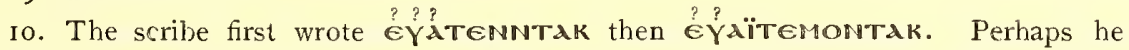

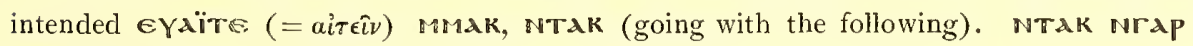
for NTAK rAP.

I . тетдле2 = Sahidic петгдре2. †нхноу plural of trхн.

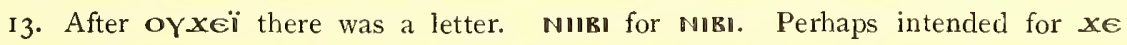

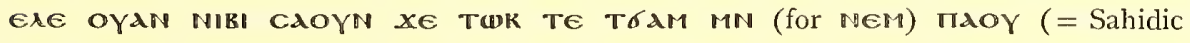
пеOOY).

I5. The second eNe2 may be eNd2, cf. ii, I. The letters and other characters which follow, like the strings of signs in Jewish, Greek and Abyssinian amulets, are intended to have magical efficacy and cannot be translated. 



\title{
A HOMILY ON THE ARCHANGEL GABRIEL BY CELESTINUS, ARCHBISHOP OF ROME ${ }^{1}$
}

\author{
ALPHA OMEGA JESUS THE CHRIST CONQUERETH \\ HELPETH $99^{2}$
}

A discourse of the honored patriarch who beCame the Dwelling place of the Holy Spirit, Apa Celestinus, ArchBishop OF THE GREAT CITy OF ROME, WHICH HE DELIVERED CONCERNING THE GREAT HONOR OF THE MESSENGER OF GOOD TIDINGS of the eons of the light, the Archangel Gabriel, on the DAY OF HIS HOLY FEAST WHICH IS THE TWENTY-FIRST OF THE month of Choiahk. ${ }^{3}$ And he spake concerning the mighty WORKS AND WONDERS WHICH HAD TAKEN PLACE IN HIS HOLY SHRINE WHICH WAS BUILT TO HIM IN THE CITy of Rome; AND ALSO CONCERNING THE WORD Which IS WRITTEN IN THE LAw: That Which ThOU WOUldest NOT THAT IT HAPPEN UNTO THEE

1 The homily alludes to Rome (pp. $\overline{\mathbf{\lambda}} \overline{\mathbf{M S}}$ ) and the Imperial Court ( $\overline{\mathbf{M}} \cdot \bar{\Sigma})$; but the local color is sometimes Egyptian, as e.g. the rising of the Nile ( $\overrightarrow{\boldsymbol{I}})$; and the material is often incompatible with either country ( $\overline{\mathbf{M}}$ ), as indeed might be expected in the case of legends which, like the last ( $\overline{\mathbf{M S}}$ ), also occur, without any local color, in the Synaxarium. What is possibly an arabism occurs on p. $\overline{k Z}$. Pope Celestine I (Bardenhewer, Patrology, p. 5I4), A.D. 422-432, left behind him sixteen epistles.

2 The words are talismanic, as often in Coptic epitaphs. The numeral 99 is a cryptogram for AmHr, being the sum of $\mathbf{I}+40+8+50$, according to Springer, Die Zahl $\overline{\mathbf{q}} \overrightarrow{\boldsymbol{\theta}}$.

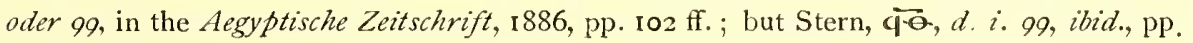
$73 \mathrm{ff}$., holds to the explanation of the famous A. Bsciai, that in writing 99 for one's self or another, one regards that person as the lost sheep (cf. Mt. xviii, I2; Lk. xiv, 4) and begs the intercession of the other ninety-nine. The numerical correspondence could hardly be accidental.

${ }^{3}$ According to the Synaxarim Alexandrinum, ed. Forget, 1905 (Corp. Script. Chr. Oriental.), p. 167, the $22 \mathrm{~d}$ of Choiak, Dec. I8. Cf. Le Synaxaire Arabe-Jacobite, Rédaction Copte, ed. Basset (Patr. Oriental.), p. 506. The Abyssinians celebrated this feast on June 20 and in addition noted June 7 as the day of St. Gabriel according to the Egyptians. Cf. Le Synaxaire Ethiopien, ed. Guidi (Patr. Oriental.), p. 606. 
DO IT NOT UNTO ANY ONE; ${ }^{4}$ AND ALSO THAT LYING IS A GREAT SIN, ESPECIALLY WITH THEM THAT CONFIRM THE LIE WITH A FALSE OATH; ${ }^{5}$ AND ALsO THAT PRAYER HELPETH A MAN AND SAVETH HIM FROM THE SNARES OF SATAN.

With the peace of God. Bless us. Amen. $99^{2}$

Thanks be unto God! His grace aboundeth; for He hath protected us and guided us with his invisible hand and hath brought us into the cycle of this year of our lives. He hath gathered us together in the shrine of the messenger of good-tidings of the aeons of the light, the holy archangel Gabriel.

Therefore I shall take unto myself the voice of David, the holy singer of hymns and collector of sweet songs and the just king, and say with my poor tongue: This is the day which the Lord hath made; let us assemble and rejoice and be glad in it. $^{6}$ He saith moreover: Judah, make thy feasts and perform thy vows.

Therefore blessed is God that $\mathrm{He}$ hath made us worthy and gathered us together in the shrine of His holy steward and the messenger of good-tidings of the aeons of the light, the holy archangel [3] Gabriel. But let us assemble in purity of heart and purity of body, and celebrate him whom God and His angels celebrate. Let us put away from us every uncleanness and every hypocrisy and celebrate the feast of the archangel Gabriel, crying out and saying with the psalmist David: Bless the Lord all ye angels of $\mathrm{His}$, strong in their might, that perform His word. ${ }^{7}$

Verily, thou holy archangel Gabriel, greater is the honor that God hath given thee than of all the incorporeal angel host that is in the height of the heavens! O thou Archangel, who wert called of God from the beginning with this name: Gabriel, ${ }^{8}$ that

${ }^{4}$ Cf. Mt. vii, I2; Lk. vi, 31 ; Tob. iv, I5 (Vulg.). ${ }^{5}$ Cf. Exod. xx, I6; Zech. viii, 17.

- Ps. cxvii, 24. T T. cii, $20 . \quad{ }^{8}$ I.e. ל. "Man of God." 
thou mightest continue to serve the generation of God, the Word, [4] since the interpretation of "Gabriel" is "God-andman," ${ }^{9}$ according to the type of Our Lord who came in humility and put on a body of flesh for our sakes, being God-and-man, His divinity not being separated from His humanity for the twinkling of an eye-God forbid! - but being God-and-man in perfection at one time in the womb. of His mother. She brought Him forth as God-and-man; He was crucified as God-and-man; He made the flesh one and the same with divinity in a single nature, indivisible, immutable, inseparable the one from the other. ${ }^{10}$

Come now, Nestorius, thou truly godless man, with that mouth which should be stopped, and surfeit thyself with shame and confound thyself! Come now and see the King, the Christ - He is both the one and the other ${ }^{11}$ - being God-and-man kings and their retinues coming and bowing down to Him and

${ }^{9}$ Lit. : "God intimately associated with Man"; cf. the use of the preposition 21 in

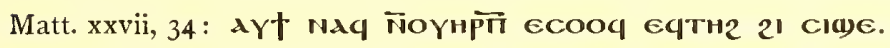

10 "He made the flesh one and the same with divinity in a single nature." $\mathbf{A q \mathbb { P }}$

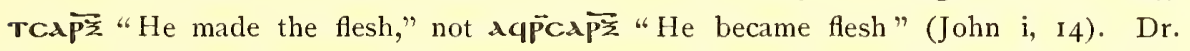
Vaschalde calls my attention to the following passage in the Anaphora of St. Basil, to be found in the Boheiric-Arabic liturgy and, in its Greek form, in Renaudot's Liturgiarum Orientalium Collectio, I, 79 (= p. 83, old ed.), which explains the expression. O-Al Te

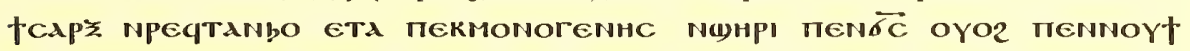

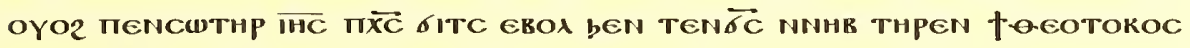

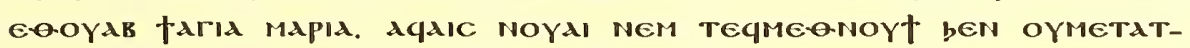
MOYXT NEM OYMETATEWb NEM OYMETATMIRt

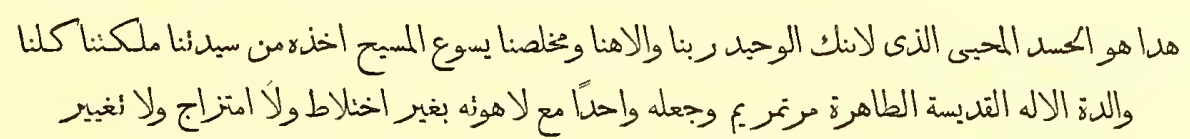

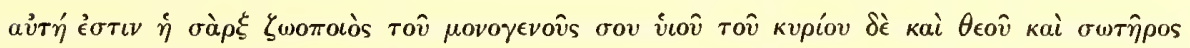

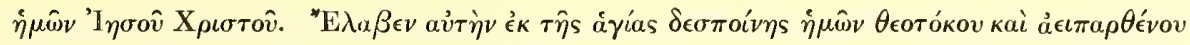

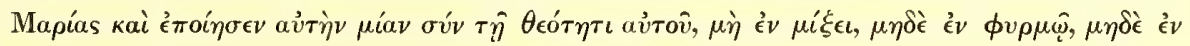
$\dot{\alpha} \lambda \lambda \circ \iota \dot{\omega} \sigma \epsilon$.

The Monophysite writer does not wish to say aqP्PCAP罙 "He became flesh," because if he says that God became something he denies that the divine nature remained $a \dot{\tau} \rho \dot{\epsilon} \pi \tau \omega s$.

But cf. the homily (?) on the Conception (Lk. i, 39 ff.) in Crum, Catalogue of the Coptic Manuscripts in the Rylands Library at Manchester, no. 73 TCגp TגI ETO NOYA NOYUT MN TMNTNOYTE.

11 Lit. "He is the one, the one." 
the whole world partaking of [5] His body and His blood, crying out with the voice of that blessed one: Remember me, Lord, when thou comest unto thy kingdom! ${ }^{12}$

But thou, impious one, didst change thine honorable estate. And thou shalt die in exile because of thy blasphemy and thy poisonous tongue. ${ }^{13}$

But we had nearly forgotten thine honor and thy glory, O thou great Archangel Gabriel. Nevertheless grant us forgiveness; for I am very deficient in the things which I do, and my feeble mouth is unable to tell of thine honor, which God hath given thee, $\mathrm{O}$ Gabriel, Angel of Joy! What tongue of flesh or mouth of man shall be able to tell of thy glory, O holy Minister of God the great King! O thou of the joy illumined face! O Gabriel, Messenger of Glad Tidings of the angel hosts! [6] $\mathrm{O}$ thou true Ministrant that didst minister unto the birth of thy Lord! O thou Proclaimer of the truth, thou Herald of the Kingdom of Heaven, I desire that I may see thee, holy Archangel Gabriel, speaking with the Virgin! $O$ thou Stranger from the far country of God, the Almighty Ruler of mankind, I desire that I may see thee speaking with the Queen of Women! I desire that I may see thy joyful countenance whilst speaking with her that excelleth all the women of heaven and earth, saying: Hail to thee that hast found grace, the Lord is with thee! ${ }^{14} \mathrm{O}$ thou Commander of hosts of the King of Kings, my poor tongue is unable to tell of thy glory; but I shall only say: Blessed is the merciful God that hath said with His divine mouth: The place in which two

${ }^{12}$ The use of this quotation from Lk. xxiii, 42 in the Eucharist is not otherwise recorded.

${ }^{13}$ The Ecclesiastical History of Evagrius (ed. Bidez and Parmentier, 1898), ch. iv, describes the deposing of Nestorius by the third synod of Ephesus, and ch. vii his wanderings and miserable end in Upper Egypt, his tongue at last being eaten out by worms .

${ }^{14}$ Lk. i, 28. 
[7] or three are gathered together in my name, I am with them in their midst. ${ }^{15}$ If God is present with two or three, then who shall be able to take account to-day of His joy with His good Father and the Holy Ghost, seeing this great multitude of listeners assembled and giving honor to His great and holy archangel, Gabriel the archangel of Joy!

But let us for our part put aside all violence and all slander and all blasphemy and all hatred and all lying and every evil deed, the doers of which things are under the curse. For every man that lieth is like unto the Devil who hath never stood in the truth. Hearest thou not what is written: The Lord shall destroy [8] everyone that uttereth a lie; ${ }^{16}$ and moreover: Everyone that uttereth a lie is not of the truth but of Satan. ${ }^{17}$ Wherefore he that shall lie or swear falsely touching worldly goods, and goeth and layeth them up, is like unto Judas Iscariot that betrayed his Lord for money. Hast thou not heard what is written: Cursed is he that shall utter the name of the Lord over a vain thing. ${ }^{18}$

Now I would speak with you to the profit of your souls; but it is the praise of the archangel Gabriel, whom to-day we celebrate, that compelleth me. For all the angels whom God sent in the Old Testament were to serve mortal men. But thee, $\mathrm{O}$ holy Archangel, the Father sent in the New Testament unto the holy Virgin Mary, to announce to her the birth according to the flesh ${ }^{19}$ of His only-begotten Son.

O thou Archangel Gabriel, to whom honor is due! O thou wise Herald! O thou holy Innocent, whose [9] wings bear sweet odor! O Steward of the House, that preparest and makest ready the house of $\mathrm{Him}$ that laid the foundations of the whole world, God looked abroad in the whole of creation and

\footnotetext{
I5 Mt. xviii, 20.

${ }^{16}$ Ps. v, 7 ; lxiii ; Prov. xix, 9.

${ }_{17}$ Cf. I John, ii, 2I, 22 ; iii, 8.
}

${ }^{18}$ Cf. Deut. v, II. Exod. $x x, 7$.

\footnotetext{
19 I.e. as the son of David.
} 
found no one like unto Mary among the whole race of women; and He was pleased to dwell within her according to a dispensation, to the end that He might redeem our race. He looked abroad among all the angel host and He found no one like unto thee in the prophetic fitness of thy holy name. Wherefore He sent thee to His virgin mother to bear her glad tidings. He sent the incorporeal being to the holy, faithful woman. He sent the bearer of good tidings of life to the queen of the race of women. He sent Gabriel bearing ${ }^{20}$ the cloud of life, in which was the life of every man, to cause him to dwell nine months in the womb of her that was more excellent than all women of heaven and earth. And when the angel appeared unto her he said unto her: Hail to thee that hast found grace, the Lord is with thee! ${ }^{21}$ Behold thou shalt conceive and bring forth [10] a son and shalt call his name Jesus.. ${ }^{22}$ And at the moment when he thus spake unto her she conceived through the hearing of her ears and the salutation of the archangel Gabriel; and the Son of God went down into her womb, no man having knowledge of her. ${ }^{23}$

I beseech thee, holy Archangel Gabriel, Messenger of the good-tidings of life, when thou comest into our midst this day in this great feast which is celebrated ${ }^{24}$ this day, to bless this great multitude assembled in thy holy name. For behoid thy Lord, and the Lord of all of us, and all His holy angels, and His virgin mother are with us celebrating in thine honor,

${ }^{20}$ I.e. "borne upon his shoulders."

${ }^{21}$ Lk. i, 28.

${ }^{22}$ Lk. i, 3 I.

${ }^{23} \overline{2 N}$ oymintatime epoc literally: "in ignorance of her." On this theory of the conception "through the hearing of her ears" and of the miraculous descent of the perfect child into the womb of the Virgin, see Hartland, Primitive Paternity, I9og, i, $20 \mathrm{ff}$,, A. Maury, Croyances et Légendes du Moyen Age, I896, p. 265, n. 2, and sources cited by Maury.

${ }^{24}$ Or, "extended." 
and all the incorporeal angel host, rejoicing with thee on the day of the revelation of thy holy name.

O Gabriel, pride of the angel host, boast of the ranks of heaven, great is the honor which God hath given thee [11] in heaven and earth. For in heaven thou art called Messenger of Good Tidings of the Eons of Light, and on earth thou art called the Angel of Joy, because of the great and marvellous plan which was intrusted to thee: Gabriel, thou truly faithful ministrant!

For when the officers rose against Daniel the Prophet and cast him into the den of lions, he was in sore distress by reason of hunger and thirst. But the holy archangel Gabriel took pity upon him by the command of his Lord; he seized Habakuk by the hair of the head, together with the repast which he was carrying, and by the fervor of the Spirit transported him suspended from Judaea unto Babylon, a journey of forty-six stages, and brought him instantly to the den of lions and gave the repast unto Daniel. And Daniel ate and praised God saying: Thou hast [12] remembered me, O Lord; thou hast not forsaken them that love thee. ${ }^{25}$

And it was also he that closed the mouths of the lions so that they should not molest him for seven days while he was down in the den of lions. ${ }^{26}$ It was Gabriel the Archangel that appeared unto Daniel and taught him concerning the vision, even as it is written: Gabriel, Teach this man the vision; and: Behold the man Gabriel came unto me and made me strong even as before. ${ }^{27}$

It was Gabriel the Archangel that appeared unto Zachariah in the Temple and announced unto him the birth of John, and, when he disbelieved, condemned him to dumbness. ${ }^{28}$ And

${ }^{25}$ Cf. Bel and the Dragon, 3I-38 (Vulgate, Daniel xiv, 32-37)

${ }^{26} \mathrm{Cf}$. Bel and the Dragon, $3 \mathbf{I}$.

${ }^{27}$ Cf. Daniel viii, I6; ix, 2 I.

${ }^{28}$ Cf. Lk. i, I9. 
moreover it was Gabriel that appeared unto the shepherds, announcing unto them the great tidings of joy, which is to say the birth of God the Word from the truly spotless Lamb, ${ }^{29}$ Mary Theotokos. ${ }^{30}$

It was Gabriel the Archangel that brought the Hebrews out of captivity and delivered the people from the land of their servitude. [13] And he saved them in the desert and wrought these signs by the hand of Moses the chief of prophets. And moreover it is Gabriel the message-bearer who is faithful amidst the angelic host, and he is the bearer of good tidings among the angels. And Gabriel is the land-measurer of the Almighty, and the steward of the Kingdom of Heaven. ${ }^{31} \mathrm{O}$ Gabriel, faithful messenger of good tidings, who shall be able to tell of thy great glory; what tongue of flesh of man living upon earth shall be able to tell of thy great glory! O thou Archangel Gabriel that standest ever in the presence of God, even as thou didst testify unto Zachariah in the Temple, saying: I am Gabriel that stand in the presence of God, ${ }^{32}$ what human heart, though it be wiser than any that have been upon earth, shall be able to declare unto us thine honor, thou Archangel Gabriel, thou of the countenance beaming with gladness and $[14]$ joy!

There is no honor like unto thine honor, for thou standest ever in the presence of God, supplicating him in behalf of the whole race of Adam. And when all the orders of angels and cherubim and seraphim cast themselves down before God and ascribe glory and honor and might unto God, the King of heaven and earth, crying out and saying continually: ${ }^{33}$ Holy, holy, holy, Lord Sabaoth: ${ }^{34}$ Heaven and earth are full of thy glory! ${ }^{35}$ the great com-

${ }^{29}$ I.e. agna. $\quad{ }^{30}$ Cf. Lk. ii, 8 ff. $\quad{ }^{31}$ Cf. Ezek. xl. $\quad{ }^{32}$ Lk. i, I9. $\quad{ }^{33}$ Cf. Rev. iv, I I.

${ }^{34} \mathrm{LXX}$ Kúpes $\sigma \alpha \beta a \omega \theta$, Vulg.: Dominus Deus exercituum. In the Coptic version $\Sigma_{\alpha} \beta a \omega \theta$ is a proper name just as it is in magical literature among Christians and Muslims, and so it also seems to be in LXX.

35 Is. vi, 3 . 
mander in chief, the messenger of good tidings of life, Gabriel, and the great commander in chief, ${ }^{36}$ Michael, the governor of the heavens, cast themselves down before God, who sitteth upon His throne, saying: [15] Lord God Almighty, have pity upon thy people; have mercy upon thy likeness and thine image; redeem the work of thy hands; accept not the accusation of the Enemy ${ }^{37}$ against them, for he hateth thy servants; strengthen these men whom thou hast created according to thy likeness and image; bless the work of their hands; increase the fruit of their land; send them the dew and rain at all times; make the waters of the River to rise for them, according to thy will; forgive them their negligences; reckon not with them over their iniquities; for thou knowest that it is that hard-hearted Devil who leadeth astray their hearts from thee. And the great archangel Gabriel and the great archangel Michael have ceased not to cast themselves down upon their faces, thus supplicating Him day and night, that $\mathrm{He}$ might [16] forgive His likeness and His image, and that His compassion be vouchsafed them all through the supplications of the archangels.

But I beseech thee, holy Archangel, forgive me; for I have made bold to speak of thine honor, a thing of which I am not worthy and to which I am not equal. And while the apology of my halting tongue hath not sufficed to compass even a small part ${ }^{38}$ of thine honor, the great King of truth Jesus Christ sufficeth to honor thee according to thy merit.

I shall now return to my theme and declare unto you some few of the miracles and wonders of the great archangel Gabriel which took place in the holy shrine in which we are gathered together

36 Sic!

${ }^{37}$ As in Job j, $6 \mathrm{ff}$.

${ }^{38}$ Mєגoc if intended for $\mu$ é $\lambda$ os would require some such rendering as "praise, eulogy,"

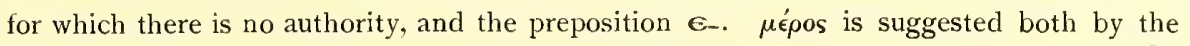
context and the following preposition $\overline{2 M}$. Cf. p. $\overline{M_{\lambda}}$. 
to the glory of God and of His great and holy archangel whom to-day we celebrate.

There was a certain rich man in this city whose name was Philip; and this [17] man was very rich in gold and silver and cattle; and, according to what was said of him, he possessed more than fifty hundredweight of gold, being a great merchant that trafficked in many lands, many men being employed by him in foreign countries who brought him a great trade in all the lands. And he was greatly praised for his beneficence; and he was beloved, devoted to the poor, and good. And he gave many large alms to the poor and the orphaned; and he gave large alms to the shrine of the holy archangel Gabriel, for he lived hard by the shrine of the holy archangel Gabriel.

And there was a poor man living near him [18] whose name was Stephen; and this man used to beg his bread day by day; and Philip the rich man used in his goodness to provide the poor man with all that he needed for the love of God, ${ }^{39}$ since he lived near him as I said before.

Now Stephen the poor man knew well how to write, but the rich man was unlettered and knew not how to write; and oft times he let Stephen the poor man arrange for him his accounts with his agents and the men that trafficked under him, and he trusted him with all his accounts, he keeping account of all his property. And many times the men that bought and sold with the rich man gave large sums to the poor [19] man secretly to the end that they might take for themselves that which they owed ${ }^{40}$ without its being noticed by the rich man in his accounts.

One day the poor man found that he had little by little become rich. But riches which are gathered in unrighteous-

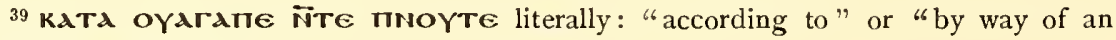
alms" or "love of God."

${ }^{40}$ The exact sense of the passage is somewhat uncertain.
} 
ness shall be scattered suddenly, even as we shall disclose the matter to you, according to the saying of the poet David: Trust not in wrongdoing neither set thy heart to snatch at riches; if they come, set not thy heart upon them. ${ }^{41}$ And as the poor man profited ${ }^{42}$ little by little he found bread and ate it. And he betook himself to the rich man one day and said unto him: I wish for thee to do me a favor and give me also ${ }^{43}$ a little gold, and I will engage in traffic therewith, and I with my children will make known concerning it; ${ }^{44}$ and $[20]$ when thou shalt require thy gold I will give it thee together with thy portion of that which I shall have gained thereby, being grateful unto thee.

The rich man said unto him: Go thou and prepare the bond for me for whatever thou hast need of and I will give it thee. And the poor man sat down and prepared the bond for the rich man for seven hundred denarii, ${ }^{45}$ swearing by God the Almighty that he would give them to him at the end of a year together with the half of that which he should have gained by them. And the rich man took the bond and gave the seven hundred denarii to the poor man. And he went and trafficked with them for three years and gained great sums of

${ }^{41}$ Ps. 1xi, I I.

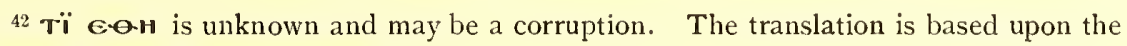
emendation of the note to the text.

43 I.e. as he had given the agents.

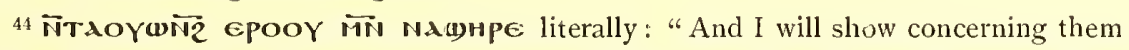
with my children." The expression is obscure. Cf. Crum, Coptic Ostraca, 1902, nos. 78 and II3, where (oY)onzor $\mathbf{E B O}$ is used of spreading abroad a ban of excommunication and of appearing openly after the danger of prosecution had passed. The writer intends to say that the fact of the transaction shall be established by noising it abroad or perhaps by giving a bond, and that no secret shall be made of it. But p. $\overline{\boldsymbol{\lambda}}$ we have a reference to the poor man's offer to sell his house and children to satisfy his creditor, which suggests that the phrase in question may refer to giving his children as security. Otherwise, read $\overline{\mathbf{N}} \mathbf{T} \boldsymbol{\lambda} \boldsymbol{\omega} \overline{\mathrm{N}} \mathbf{2}$ and translate: "And I shall live thereby with my children."

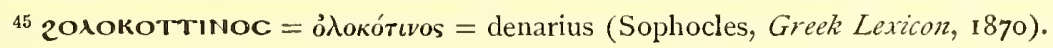


money; but he sought not to give anything to the rich man, either principal or interest. ${ }^{46}$

Then, after many days, [21] the rich man took the poor man into his house to let him arrange his accounts for him. And when he opened the repository in which were the documents and books, wishing to find the records of which he was in search, and when Stephen, the man of whom we are speaking, found his bond, he put it under his tunic without the rich man's knowing - in his innocence and guilelessness - and departed without the man's knowing.

One day the poor man found that he had little by little become rich. But, after all, riches which are gathered in unrighteousness shall perish and be scattered suddenly, as I have said before. ${ }^{47}$ And when Stephen had entered his house he tore the bond and rent it in pieces; and he said in his heart - through his evil thoughts which Satan had put into his heart - Verily [22] I shall pay nothing to this man at any time of these seven hundred denarii which I owe him. And if he shall say unto me: Give them unto me, I shall say unto him: I owe thee nothing. Produce the bond and I will pay it thee in full ${ }^{48}$ if at all I owe thee anything; nor indeed had I any need of money. And I shall take them and become rich thereby; and they shall suffice me for a long time, eating and drinking and making good cheer with them - remembering not even that which is written: Thou fool, thy soul shall be taken from thee! The things which thou hast prepared, whose shall they be? ${ }^{49}$

46 raparii-ald occurs here and twice on p. $\vec{k}$ with the same spelling and in the same sense, and evidently is $\pi \alpha \rho \alpha \mu v \theta i a$; but $I$ find no record of such a use of the word elsewhere.

47 Cf. p. $\overrightarrow{1 \vec{\theta}}$.

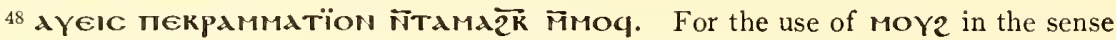
of "paying in full," see the many examples in Crum, Coptic Ostraca, index; p. $\overline{\mathrm{k} \lambda} 11, \mathrm{f}$.; Stern, Koptische Grammatik, $\$$ 509, end.

${ }^{49}$ Cf. Lk. xii, 20. 
Then - not to delay the narrative - after four years the rich man perceived that the poor man had given him nothing, [23] neither principal or interest. He made haste and sent his servants after him and said unto him, when he had come unto him: Give me the seven hundred denarii which thou owest me, for I have need of them. But that godless man began to make denial to that pious rich man, saying: I owe thee nought, neither hast thou ever given me anything. The rich man said to him in anger: O thou truly wicked man! Behold, have I not the bond which thou didst prepare with me for seven hundred denarii! Behold, it is five years since I gave them to thee. It is not only that thou hast paid me no interest on them, but see now, thou desirest to take what belongeth to another.

The poor man answered [24] and said: If the bond is in thy possession, bring it unto me that I may see it and go and sell my house and my children and pay thee in full seven hundred denarii. And the rich man searched through all the bonds and documents without finding it, and knew at once that he had stolen it secretly. And he said unto him: Of a truth I knew that it was thou that didst take it secretly; nevertheless, if thou art confident in thine heart that I did not give thee the seven hundred denarii, come let us go to the shrine of the holy archangel Gabriel which is in our quarter, and swear to me, and satisfy me, and I will let thee go. ${ }^{50}$

But that fool thrust aside from him the fear of the Lord and the multitude of benefits which he had received at the hands [25] of that pious rich man, having forgotten that which is written: The Lord shall destroy everyone that speaketh a

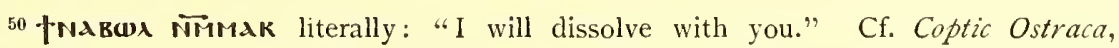
no. 285 , where $\mathbf{в} \boldsymbol{\alpha} \boldsymbol{\lambda}$ is used possibly of releasing someone from an oath. 
lie $;^{51}$ and he said unto the rich man: Come let us go, and I will swear unto thee. And straightway they went with one another until they entered the shrine of the archangel Gabriel. And a great crowd had gathered unto them, marvelling at what was happening.

And the rich man said unto the poor man: Give me the seven hundred denarii which I gave unto thee. Swear not falsely lest evil betide thee. And that fool said: I owe thee nothing. The rich man said unto him: If thou owest me nothing, swear unto me and I will depart. And that godless man despised the miracles of the holy archangel Gabriel [26] for the sake of perishable riches; and he made bold in his folly to place his hand upon the doors of the sanctuary, and to swear to him saying: By the great miracles of the archangel Gabriel, I owe thee nothing; neither hast thou ever given me anything of these seven hundred denarii for which thou dost bring action against me!

And straightway, while yet the words were in his mouth a Power smote him, and he fell down upon his face, and he became blind in both his eyes, and his face was twisted backwards, and his tongue swelled in his mouth, and he fell down upon the pavement of the shrine, and he bit at his own tongue and lips in great agony, and he emitted foam from his mouth like them that are possessed of a devil..$^{52}$

And when the multitude beheld that which had befallen [27] him, they marvelled and were greatly troubled and cried out with a loud voice saying: One is the God of the archangel Gabriel!

${ }^{51}$ Cf Ps. v, 7 ; lxiii ; Prov. xix, 9.

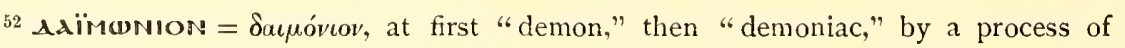
identification well known in the lore of magic; cf. $=i s$ in the Old Testament; Jastrow, Religion Babyloniens und Assyriens, i. 309; Stern, Wanderings among the Falashas in Abyssinia, ch. xi (1862); Parkyns, Life in Abyssinia, ch. xxxiii (1853); Basset, Les Apocryphes Éthiopiens, iv, 25; especially Zeitschrift fiir Assyriologie, xxiii, 181, xxiv, 67, xxix. 87. This usage is not found in the Greek New Testament. 
And after a long time, suffering torture the while, he cried out in a loud voice: Forgive me, my Lord, thou Archangel Gabriel, that I dared to swear a false oath in thy name! This rich man did give me seven hundred denarii five years ago, and I trafficked with them; but I gave him nothing for them. And when I found opportunity I took the bond by stealth; and I deceived him, wishing to take them and to become rich thereby. Woe is me! What shall I do? For I have sworn falsely!

And straightway he sent to his house in haste. And his wife brought the seven hundred denarii, and gave them to the rich man. But that man [28] continued to suffer torture the whole of the day as if he were being pierced with a lance. And he yielded up his spirit more miserably than ever a man did. He swore falsely and he gained nothing but the destruction of his soul and his body.

Ye have seen, my beloved, how powerful are the miracles of the archangel Gabriel, whom to-day we celebrate; and that he who shall falsely swear in his name shall surely die in misery. Let us divorce ourselves completely from the evil vice of lying, especially the vice of them that confirm the lie with a false oath. Do ye not hear the Lord crying out unto every man: Let your word be, the yea yea and the nay nay, that ye be not judged..$^{53}$ And let us, for our part, keep our mouth from these [29] fearful oaths which we swear, especially concerning a vain matter, lest we receive a great condemnation and punishment everlasting in the place which the Everlasting visiteth not.

But I remember that I promised to tell you some few of the miracles and wonders of the great archangel Gabriel, whom to-day we celebrate, to the glory of God and of His great archangel Gabriel, the messenger of good tidings. 
Now there was also a certain man in this city, blind in both eyes from his youth. And when he heard of the miracles and the wonders of the archangel Gabriel he begged his people to bring him into the shrine of the archangel Gabriel, saying: Surely his mercy will be vouchsafed unto me also, and he will graciously grant me the light of mine eyes. And they took him to the shrine [30] of the archangel Gabriel, he saying the while: Surely his mercy will be vouchsafed unto me.

And when he had entered he cast himself down upon his face in front of the holy sanctuary and wept saying: $O$ my lord the holy Archangel Gabriel, have mercy upon my wretched state and graciously grant me the light of mine eyes, for I suffer greatly. And while he was yet weeping and saying these things he perceived a human hand touching his eyes. . . . ${ }^{54}$ And all the people that were assembled at the shrine of the archangel Gabriel.... And when they beheld the blind man seeing and crying out for joy and exclaiming: One is the God of the holy archangel Gabriel, for his mercy hath been vouchsafed unto me [31] and he hath graciously granted me the light of mine eyes. . . .55 And the whole multitude rushed upon him and questioned him, saying: Tell us what hath befallen thee; in what manner hast thou regained thy sight! And he related to them everything that had befallen him saying: It befell me, after I had entered the holy shrine, that I cast myself down before the sanctuary and besought the holy archangel Gabriel. And straightway I perceived a human hand come down upon my face and make the sign of the cross over my eyes, and forthwith I was able to see. And I heard a voice saying unto me: Behold I have granted thee the light of thine

${ }^{54}$ A passage describing the result of the touch, as on the following page, may be presumed to have been omitted at this point. The following clause seems to be part of another fragmentary sentence.

${ }_{55}^{5}$ The principal verb of the sentence has been omitted. 
eyes even as thou didst beseech me. And I said: Who art thou, my lord? And he said unto me: [32, (33)] I am the archangel Gabriel. And that is what I heard, but I saw not him that spake with me. And the people, when they heard these things, cried out with a loud voice, saying: One is the God of the archangel Gabriel!

And the man that had regained his sight remained in the shrine of the archangel Gabriel, ministering until the day of his death.

And there was also a man, rich in gold and silver, who was dumb. After a time he chanced to take a disease, the same which is called gout, ${ }^{56}$ in that a violent pain gnawed at his feet day and night, he having spent a considerable amount ${ }^{57}$ on the physicians without benefiting at all thereby but rather growing worse. And when he heard of the miracles and wonders which had come to pass in the shrine of the holy archangel [33, (34)] Gabriel, he caused his servants to place him upon a beast and bring him unto his holy shrine. He lay down in that shrine in great pain, and he cried out ${ }^{58}$ saying: My lord the holy Archangel Gabriel, have mercy upon me and graciously grant me to be healed, for I suffer greatly.

Moreover a man who in like manner was palsied ${ }^{59}$ in his legs, dragging himself along the ground in the manner of little children was also in the shrine, lying down, beseeching the archangel graciously to grant him healing. And the gouty man ${ }^{60}$ was lying near him in the shrine, suffering pain in his feet; and they, side by side, were together seeking health at the hand of the holy archangel Gabriel. Then at that time the archangel had compassion upon them and was graciously

\footnotetext{
56 The identification is based partly upon symptoms.

57 Literally: "the sum of expense."

58 I.e. within himself, for he was dumb.

59-60 The one was gouty and dumb, the other palsied.
} 
pleased $[34,(35)]$ to grant them health, the two together at one time.

And so he revealed himself to the palsied $\operatorname{man}{ }^{61}$ in a vision, in the form of a man of light whose countenance shed rays of light. And he said to the palsied man: If thou earnestly desirest to be healed and restored to health and to walk upon thy feet as any man, watch thou till all the men that are in the shrine have lain down and gone to sleep. When thou art sure that they are all asleep, arise thou and drag thyself upon thy hands and feet and go unto the couch of the rich man that suffereth pain in his feet, and begin to take the bed covering which is upon him, and thou shalt obtain health and shalt walk upon thy feet forthwith and be restored and go unto thy house as one that never [35, (36)] suffered illness.

And when the man awoke from his vision he marvelled and said within himself: Verily I have been deceived by this dream. If $I$ do this thing and go to take the bed-covering of the rich man, it will come to pass that he will awaken and his servants will seize me, since I am helpless, being palsied, and they will deliver me up unto the eparch of the city and he will put me to death, and that I shall have gone seeking the health of my body and forfeit my life in consequence of the torture which will be visited upon me. No, I will never do this thing!

And in the ensuing night the archangel Gabriel came to him, clad in royal majesty and shining more brightly than the sun. He spake with the palsied man, while a great sweetness [36, (37)] exhaled from his mouth, and he said to him: Why hast thou neglected to do that which I commanded thee? Be sure of this, that if thou hearken not unto me thou shalt not cease from thy disease until thou diest. But if thou wilt do what I commanded thee thou shalt recover forthwith. And 
as the archangel spake these things unto him he departed from him. Immediately he awoke in perturbation and fear. And he strengthened his heart well, saying: Of a truth this was the archangel Gabriel which spake with me. And now, even if it come about that I be seized and put to death, I shall not this time be disobedient. The will of the Lord be done! In this manner did the man lay up the saying in his heart, in that he told no man of that which had befallen him.

And when it had become even $[3 \mathbf{3 7},(\mathbf{3 8})]$ the palsied man waited until all the men that lay in the shrine were asleep. And he arose, and crawled on his hands and his feet, and went to the man that was dumb and that suffered pain in his feet, and he seized the clothing that covered him, and began to drag it away. But the man that suffered pain in his feet and that was dumb awakened from sleep in terror, and the God of the archangel Gabriel had compassion upon him, and straightway the bond of his tongue was loosed, and he spake - although he had never before spoken at all - and he cried out in a loud voice unto his servants, saying: Arise in haste and seize this thief who hath come and taken my clothing which covered me! And the palsied man was afraid lest the servants of the rich man should seize him; and straightway [38, (39)] his hands and feet were set right and he leapt up and ran upon his feet like a runner, until he came unto his couch. Likewise he whose feet were stiff with pain ${ }^{62}$ was set right, and he sprang down from his couch like one that had never suffered illness and pursued with his servants the palsied man, trying to catch him.

But after a little, when they perceived the grace that had been vouchsafed them both, namely, the rich man who had ceased to suffer pain and had ceased to be dumb, and the palsied man whose hands and feet ${ }^{63}$ had been set right, they came into the

$$
62 \text { I.e. the rich man who was gouty and dumb. } \quad{ }^{63} \text { According to the emendation. }
$$


midst together and confessed the healing that had come to them together, and cried out with one voice: [39, (40)] One is the God of the holy archangel Gabriel. And when all the multitude that were gathered together in the shrine of the holy archangel Gabriel had seen this great wonder, they were filled with great fear, and trembling seized them. Then they asked the palsied man, saying: What hath befallen thee that thou doest this? And he recounted to them all that had befallen him, saying: Behold it befell me thus and so; and: It was the archangel Gabriel who revealed himself to me twice, wearing a garment of light, so that I have done this. And all the people that were assembled in the shrine of the holy archangel Gabriel cried out in a loud voice, saying: One is the God of the mighty archangel! [40, (41)] Great are the miracles of the messenger of good tidings of life, Gabriel whose name is worthy to be honored! And the men that had obtained healing remained in the shrine of the archangel Gabriel, praying and fasting therein, unto the day of their death.

What shall I say and what shall I leave unsaid among the wonders which thou hast wrought, thou great proclaimer of God the Word, thou holy Archangel Gabriel! Verily if I should spend my whole time attempting to exhaust thy wonders I should not be able to tell any great part thereof. But I shall tell some few of the mighty deeds which thou hast wrought, to the glory of Christ our Savior and His great and holy archangel Gabriel whom to-day we celebrate.

Now ${ }^{64}$ there was a man moreover living in a village which was distant about six or eight miles from the city. And this man had an only son who had $[4 \mathbf{1},(\mathbf{4 2})]$ reached the age of man-

${ }^{64}$ See note to p. $\overline{\mathbf{x}}$ and Basset, Le Synaxaire Arabe-Jacobite, Rédaction Copte, pp. $506 \mathrm{f}$., of which the translation follows. The Arabic text of this passage is of no interest; its orthography is bad in places, after the manner of such texts; and it is not inaccessible to those who might require to use it. The Copts, unlike the Syrians, never cared for the 
hood. After a time he fell sick of a disease, suffering pain in his hands and his feet both by day and by night, and he was unable to lift up his head at all, in that he had passed six years lying upon a bed, not being able to arise from the place in which he lay. And his parents and his kinsfolk often despaired of him saying: Verily death were a relief to him rather than the suffering which afflicteth him. And his parents spent

Arabic which replaced their older language; and to this day Christian Egyptians are sadly to speak, as they are sadly to write, in the language of the Prophet. This is all the worse, since they have no knowledge of and no interest in Coptic either. As the meaning of the passage is clear, however, it has not been thought necessary to reproduce it. I have not been able to determine the relative ages of this passage and of the homily.

"There was a man in a distant city who had lived there a long time, sick; and no relief was found for him. And he heard about the wonders and signs which were in the church of the angel Gabriel. And it chanced to be the night of his feast; and behold he vowed, saying: O Gabriel, mention me in the presence of God that He may grant me healing and that I may find repose. And after it had come to be midnight he broke into a sweat, and was delivered, and God granted him healing. And he arose early with rejoicing, and gave unto his son twenty-five denarii of gold, and sent him unto the church. But whilst he was journeying in the way, behold a lion came forth from the thicket and rent him. And he cried out, saying: O thou Angel Gabriel, mention me in the presence of God that he may deliver me : And straightway Gabriel descended from heaven and took the youth away from the lion, and made him to ride upon the back of the lion and brought him unto the church - and he urged him on as though he were riding upon a beast of burden - until he reached the church. And when the multitude beheld him they marvelled. And with rejoicing he presented the vow which he brought. And as for the lion, he set him down at the door of the church, and went his way, whilst all the people marvelled and gazed at the wondrous thing which had come to pass."

The Arabic version is evidently translated from a different, shorter Coptic text of the same story.

Marvellous stories of the ability of saints to tame wild beasts, particularly lions, are very common in legend. They are due no doubt in part to speculation - as the Muslim idea that lions are the "dogs of Allah" (Goldziher, Muhammedanische Studien, I8go, p. 293; Jacob, Beiträge zur Kenntnis des Derwischordens der Baktaschis, I9o8, p. 56, note 3 ; Jacob. Die Bakteschïa in ihrem Verhältuis zu verwandten Erscheinungen, Sitzungsber. Bayr. Akad. I909, p. 7) - he who fears Allah need fear none of His creatures (alDamîri, Kitâb Hajat al-Hajawân.sub. voc. (w) - but also without doubt to actual experiences of lonely dwellers among wild creatures which in varying degrees become tame when unmolested. Cf. I Kings xiii, 24 ff. (Vulgate III Regum xiii, 24 ff.), Daniel vi, 22 (idem), Bel and the Dragon 3I-38 (Daniel xiv, 32-37). A number of Christian and Jewish references have been collected by Günther, Die Christliche Legende des Abendlandes, I 9 IO, p. 8I. 
great sums of money on physicians without his getting relief, but he, on the contrary, grew worse.

And when his father heard of the works of power and the wonders which occurred in the shrine of the holy archangel Gabriel which is in the city of Rome, he made a vow saying: If the God of the archangel Gabriel hear my supplication and graciously grant health to my son, I will give six $[42,(43)]$ denarii to thy shrine yearly unto the day of my death; for I know, $\mathrm{O}$ my lord the Archangel, that thou art able to do all things. I cannot bring my son to thy shrine for that he is unable to walk or to ride upon a beast of burden by reason of the great pain that he suffereth; but I believe that thy holy power filleth every place. May thy mercy be vouchsafed me that thou graciously grant the healing of my little son; and I shall confess thy miracles unto the day of my death.

And straightway the archangel Gabriel heard the supplication of the man, and was pleased to grant the healing of his son, for he is merciful. And he brought a brief slumber upon the young man, and he slept against his wont, — not having been accustomed indeed to sleep at all, by night or by day, but crying out $[43,(44)]$ because of the great pain that devoured him.

And when he had fallen asleep the holy archangel came to the young man in a vision, clad in great and unspeakable glory, enveloped in a robe that shone with light; and said to him: Behold I have taken away the pain from thee; and no suffering shall lay hold of thee from this hour henceforth. And the youth said: My lord, who art thou, thus encompassed with so great glory? He said to him: I am the archangel Gabriel. And straightway he vanished from before him.

And when he awoke on the morrow he found his body covered with sweat, ${ }^{65}$ and perceived that healing had come to

${ }_{65}$ The clinical significance of perspiration in acute disorders, such as fevers, and the therapeutic value of the means of inducing perspiration were both anciently and widely 
him, and that there was no pain in his body, and he leapt upon his couch $[\mathbf{4 4},(\mathbf{4 5})]$ and stood up and walked about, leaping and praising God; and he was as one that had never been ill at all. And straightway he cried out saying: I will glorify thee, thou great and holy Archangel Gabriel, that thou hast remembered me and graciously granted healing unto me.

And his father and his mother, when they saw the healing that had suddenly come upon their son, wept sorely. ${ }^{66}$ And they asked their son: What hath befallen thee, $O$ beloved son? And he related to them the vision which he had seen. And straightway they cried out saying: One is the God of the holy archangel Gabriel! Great is the benefaction which thou hast wrought with us, $\mathrm{O}$ thou good bearer of glad tidings! And there was great rejoicing in all their house because of the healing that had come to the young man, since he was an only son of his parents. And everyone $[\mathbf{4 5},(\mathbf{4 6})]$ that heard marvelled exceedingly and glorified the Grod of the holy archangel Grabriel. And the report reached everyone.

And on the morrow, it being the Lord's Day, the man called to his son and gave him the six denarii and other valuable presents and sent him to the city of Rome to have him present them to the shrine of the holy archangel Gabriel, even as he had vowed. And the young man took them with joy and set about his journey ${ }^{67}$ to go to the shrine of the holy archangel Gabriel.

And as he journeyed thus alone until he approached to within three miles of the city, he came upon great desolate forests through which it was difficult to travel. And behold straightknown. But the case in question is chronic and not acute. Von Hovorka and Kronfeld in a remarkable medico-anthropological collection, V'ergleichende Volksmedizin, Stuttgart, 1909, have brought together (i. pp. 46 ff.; ii, pp. 63, I 59, 293) an extraordinary array of facts.

${ }^{66}$ Or, "rejoiced exceedingly."

${ }^{67}$ Aqтגaq Crqұ̈̈н literally "he gave himself to his way." 
way a fierce lion came out of those inhospitable forests and roared at the young man $[46,(47)]$ and sprang upon him and seized ${ }^{68}$ the side of the young man with his mouth and dragged him into the forest, wishing to devour him. And the young man cried out in great distress, saying: $O$ my lord the Archangel Gabriel, help me in this great distress, for thou knowest that my father hath sent me to thy shrine to present these few gifts to thy holy martyrium ${ }^{69}$ and to return to my house, but behold I shall die at the hand of the wild beast. And at that instant behold the holy archangel Gabriel came out of heaven, clad in a robe of light, and took the young man out of the mouth of the lion, and made the sign of the cross upon his side, and healed him of his wounds which the lion had given him with his ${ }^{70}[47]$ teeth..$^{71}$ And he said unto him: Dost thou know me, O young man? He answered him: Nay, my lord! He said unto him: I am the archangel Gabriel unto whose shrine thou goest; I am he that came to thee and healed thee of thy disease; and I am also he that saved thee out of the mouth of the wild beast. And as he said these things he took hold of his hand and set him upon the back of the lion. And the lion ceased not to run, bearing him upon his back, until he had brought him into the shrine of the holy archangel Gabriel, while all the throng gazed at him marvelling at him. And when the lion had set him down from off his back he went out from the midst of the throng and returned to his place while every man gazed after him marvelling.

After these things all the throng questioned the young man saying: What is it that hath [48] befallen thee? Whence comest thou, mounted upon this lion? But he, when he had

\footnotetext{
${ }^{68}$ AqMe2 Pwq literally "he filled his mouth."

${ }^{69}$ MxpTYPION $=\mu \alpha \rho$ róptov, at first the shrine of a martyr, then any shrine.

${ }^{70}$ The end of BMMsOr 7028 .

${ }^{71}$ Beginning of BMMsOr 6780 .
} 
recovered from his fear ... ${ }^{72}$ When all the people and the Senate, which were gathered together at the shrine of the holy archangel Gabriel attending the synaxis ${ }^{73}$ heard these things, they cried out, saying: One is the God of the mighty archangel Gabriel! Great is thy power, O holy Archangel Gabriel! Thou deliverest everyone that putteth his trust in thee!

And the young man presented the gifts which he had to the shrine of the holy archangel Gabriel. Thereafter he did not again return to his house, but remained in the shrine of the archangel Gabriel, praying and fasting. And when his parents came unto him he related unto them all that had befallen him, and they greatly rejoiced, [49] and they praised God. And he remained in the shrine, ministering unto the day of his death, performing many pious works; and he became a man of election such that he was found worthy to behold many visions in the shrine of the holy archangel Gabriel; and he persevered in many supplications and prayers.

Ye have seen, $\mathrm{O}$ my beloved, how potent are the mercies of God and the supplications of the holy archangel Gabriel, whose festival we celebrate this day. But let us not show ourselves unbelieving toward the signs and wonders which have occurred in his holy shrine lest we suffer a great condemnation; for in very truth all the wonders were seen with mine own eyes, and many of you also have seen them. Nay rather let us believe the works of power and the wonders of the archangel Gabriel [50] with all our hearts, that we may receive a great favor from the hand of God.

But give ear to me and I will relate to you, my beloved, this great and marvellous miracle, making manifest the mercy of the archangel Gabriel and the insolence of the Devil and

72 Evidently something has been omitted here, having the general sense: "told them all that had befallen him."

${ }^{73}$ The term "synaxis" has been used throughout for the church service meant. 
his demons; for they lie in wait for man by day and by night, wishing to do him evil.

There was a certain workman living near the shrine of the archangel Gabriel; and this man was a baker by trade, being an hireling and working for his wage. And when he arose each morning he was wont, before he had gone to his place of work, to enter the shrine of the archangel Gabriel and to pray to him saying: O holy Archangel, guide thou my ways! And thus he was wont to utter thrice [51] an invocation, and he made the sign of the cross, and departed unto his place of work, there being nothing good in all his life whatsoever except this, neither fasting nor works of compassion. But the Devil, hater of all good, took pains to ensnare him, wishing to inflict evil upon him, yet he could not prevail over him, because of the thrice repeated invocation which he performed, and because of the help of the God of the holy archangel Gabriel protecting him.

And it befell him one day that he overslept. The sun had arisen and he was not yet gone to his work. And he said to himself: I am late indeed to-day in going to my place of work. And thus carelessness and vain anxiety seduced him. He forgot to enter the shrine of the holy archangel Gabriel according to his daily custom; and he went to his shop [52] and mounted the oven to fill it with bread.

When he began to bake, the Devil, hater of all good, seized his feet from behind and let him down into the fiery oven, and said: O thou rebellious one! These three years have I striven to ensnare thee, I have set a trap for thee daily; but the thrice repeated invocation which thou dost perform daily in the shrine of the archangel Gabriel hath become three fiery shining lamps, driving me away from thee. But since I have found opportu- 
nity to approach thee to-day I shall do to thee according as thou deservest.

And the foul fiend, as he spake these words, cast the man down into the oven, and his whole body was wounded. And the men were sore afraid, and scarcely did they with great effort pull him half dead out of the oven. And his wife [53] and his children rent their garments and cried out and wept with great bitterness. They placed him upon a bed and brought him into the shrine of the archangel Gabriel, thinking that he would die before sunset; and they were all standing round about him.

But at midnight behold the archangel Gabriel appeared to the man in a vision, having the form of a royal general, wearing a robe of light. He said to him: Dost thou know me, O man? The man answered and said: Nay, my lord. Said the archangel to him: If not, then know thou that I am Gabriel the archangel into whose shrine thou hast daily come, beseeching me to deliver thee out of all thy tribulations. I am he that hath delivered thee many times from the snares of the Devil. But since thou wert negligent yesterday, not coming to the shrine to pray, for this cause hath the Devil brought upon thee this great [54] affliction. But I have not forgotten thee, to restore thee to health, that every man may live and know the feebleness of the Devil and that it is the help of God and his angels which protecteth all mankind. And as he spake these words he made the sign of the cross upon the whole body of the man who had been burned with the fire; and he said unto him: Behold I have healed thee. Be not again careless as touching the church lest a worse thing than this befall thee. And as he spake these things to him he ceased to see him. And the man leaped up, trembling with fear, and found himself with all the wounds which were in his body vanished. And 
he was perturbed, and cried out in a loud voice: One is the God of the archangel Gabriel!

But all the people that were gathered together in the shrine of the archangel Gabriel rushed upon him wishing to see [55] the great miracle which had taken place. And they beheld him, of whom they had thought that he would die before sunset, sound, there being not one wound upon his body. They were sore afraid and cried out saying: I bless thee, thou God of the holy archangel Gabriel!

After these things they asked the man: What hath befallen thee! Yesterday thou didst fall into the oven, and didst burn thy whole body. How then didst thou cease to have this great affliction? And he related to them everything that had befallen him, saying in this wise: It happened to me yesterday that, after I had heated the furnace, in order to fill it with bread, I looked and beheld a huge Nubian, ${ }^{74}$ tall, his eyes emitting

${ }^{74}$ There may be nothing strange, on the part of fairer-skinned races, in picturing the Devil and evil spirits generally as negroes. It appears to have been an opinion generally held by both negroes and whites of the southern states of the Union that God was white and the Devil black (Booker T. Washington, The Story of the Negro, i, p. 23). Among the coffee-colored Hamito-Semites of Abyssinia and the yellow Copts of Egypt proper the black man is a figure, not only comic and grotesque, but also strange and uncanny.

In Abyssinian magical texts the lycanthropous wizards are called "black," and are associated with the negro tribes of the country; and demons generally are pictured in the illustrations as of darker hue than the saints and angels (Worrell, Studien zum abessinischen Zauberwesen, Zeitschrift für Assyriologie. xxiii, p. 152 and note). In Greece the Devil is called $\mu a \hat{v} \rho$ s "black" (Pradel, Griechische und süditalienische Gebete, Beschwörungen und Rezepte des Mittelalters, p. 95, cf. English "Moor"). In Syria and Armenia, as I am assured by Fransîs Zaitûn of Nazareth and Professor M. H. Ananikian of Aintab, the Devil is thought of as black. In early Greek-Egyptian Christian papyri he is called $\delta$

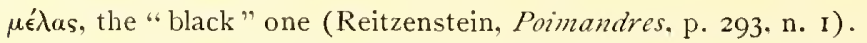

Muslim Arab tradition makes Upper Egypt the home of the gùl (al-Mas ûdi, Murîg

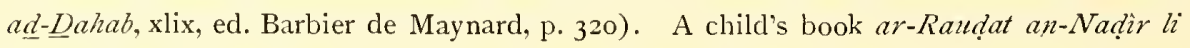
Bahgat kull Walad Șagîr, by the Rev. Henry Jessup. Bairût 1882 (cf. Goldziher in an article in Zeitschrift der deutschen morgenländischen Gesellschaft, xxxiii, $608 \mathrm{ff.}$, Jugendund Strassenpoesie in Kairo, on Jessup's The Women of the Arabs. with a Chapter for Children, which contains translations of the rhymes published in the first-named work) has on p. I 87: Hal turîdi an al-gabâbera ja"tu min bilâd al-Barâbera, "Do you want the Ogres to come, from the land of the Nubians?" Apparently, the Orientals thought of the Devil, 
fiery flames in my face. And he stood before me saying: I have labored to ensnare thee, thou lawless one, these three years, lying in wait for thee by day and by night. [56] This thrice repeated invocation which thou performedst in the shrine of the arch-

or devils, as not merely black because to be so was strange or fearful, but because of some connection of thought with the country of the blacks, far up the Nile. That the idea is a very old one we see from Tobit, viii, 3 , where the exorcised demon flees "into the uttermost parts of Egypt."

It is a very curious fact, if nothing more, that the god Bes, identified by the Greeks with the evil giant Typhon (Wiedemann, Religion of the Egyptians, p. 164) and held by Egyptians of later dynasties to be the frightful, avenging god of the underworld, is of Nubian origin; and that he was in the later period represented as a large man or even a giant (Wiedemann, p. 167 f.), as in the present homily (cf. Spitta, Grammatik des arabischen Vulgärdialektes von Aegypten, p. X: nòš, nùs, Ausdruck für etwas sehr Grosses [kebyr zê en nûš] = Nor "Grösse"). Sa'ad "Abd al-Masì h, a Copt of Asyût, tells me that this phrase: "big as the Nûš" means "as tall as the Devil," and that nôš means any "ifrît tawill, "long devil," but with the article, "the Devil."

This tall and terrible god of Hades seems to have been Bes as the Nubians themselves conceived him to be (Wiedemann, p. I67). The squat, bow-legged, megalocephalic, dwarfish figure, clad in a leopard skin and crowned with feathers, protector of childbed and infancy, by which Bes is represented in earlier Egyptian remains, must accordingly have been only a foreigner's symbolism for the Up-River god $\kappa \alpha \tau^{\prime} \boldsymbol{\epsilon}^{\prime} \xi \boldsymbol{\xi} \chi \chi^{\prime} \nu$, for it was from the Up-River country that Egypt procured the grotesque little Sudanese dancers called Denga (= Dinkas of the Sudan? see Petrie, Religion of Ancient Egypt, p. 63) as early as the fifth dynasty.

Sahidic $€ \sigma \boldsymbol{\omega} \boldsymbol{\omega}$ (older form $€ \sigma \mathbf{\sigma o w})$, as has long been recognized, corresponds phonetically to Egyptian ek'ôš and Hebrew ש̇ (Cheyne, Encyclopedia Biblica, sub. voc. Ethiopia). The strange Boheiric form ea-wa, which does not follow the established principles of sound change, might be explained as an attempt of delta Egyptians to represent in their orthography the late palatalized pronunciation of Sahidic $\sigma$, which had changed from [k] to [c]. Similarly the Greek ai $\theta_{i} \omega \psi$ (even in Homer, Odyssey, i, 22, where they are merely the remotest and least known of men) is probably only another attempt to represent the palatal [c] in terms of another orthography. The meaningless ending -ôs was made into -òps and the word understood to mean "swarthy of face" $(\alpha \ddot{\prime \prime} \omega+\ddot{\omega} \psi)$.

Not only in origin but in meaning also are eroow and ai $\theta$ i $\omega \psi$ the same (cf. Acts viii, 27 in the versions) in Coptic times. By both terms are meant the people at the extreme southern boundary of Egypt (cf. Zoëga, Catalogus, p. 379: Newdxe A€ 2woY Mாє-

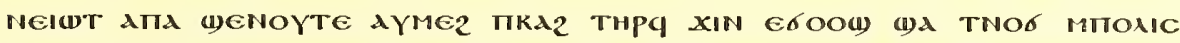
рдкоте, "And the sayings also of our father Aba Shenute have filled the whole land, from $€ 6$ oo( ) even unto Alexandria the Metropolis").

It must however be admitted that the Western idea, that the Devil is red --derived perhaps from the prevailing tint of his heated surroundings - is also found in Eastern lands. The red devils which are occasionally mentioned in Abyssinian magical texts (Worrell, op. cit. ZDMG, xxiv, 62, 75, 95 ; xxix, 94-95,96, 105, 132; and Hastings' Encyclopedia of 
angel Gabriel was like unto three glowing, fiery lamps, and it drove me away from thee. But when I found opportunity to-day to attack thee I came to thee to do unto thee according as thou deservest. And as he spake these words he seized my two feet and threw me down into the oven; and I was all burned. For the rest, I was conscious that I was brought to this place, but I did not know whether I was alive or dead, for I had entered into death. And in that night I beheld the holy archangel Gabriel. He came to me and signed me with the sign of the cross, and restored me to health, and departed into the heavens in a garment of light.

And when all the people had seen these things they marvelled exceedingly and were filled with fear.

Ye see, $\mathrm{O}$ my beloved, how potent are the petitions of the holy archangel Gabriel, and that [57] no demon can ever approach any man that goeth to the church daily; but the help of God shall keep him from the snares of Satan.

It is prayer that casteth out the demons.

It is prayer that keepeth them that are in danger and distress, and suffering.

It is prayer that solaceth them that are distressed.

The church is the place of consolation and of the assembling of the angels.

Religion and Ethics, sub. voc. Charms and Amulets - Abyssinian, p. 401) seem to arise from speculations regarding lighter and darker tribes of the same name, Bâryâ. Nevertheless red devils are mentioned in Arabic texts where no such explanation is possible, unless a connection with the foregoing is assumed. In an article, Ink, Oil, and Mirror gazing Ceremonies in Modern Egypt, Journal of the American Oriental Society, xxxvi, I9r6, pp. 49, 50, 52 and note, the present writer calls attention to the magical words Tarš or Tâaraš — better Târiš — and al-Aḥmar, both evidently names of demons, occurring in certain Arabic receipts for performing the scrying ceremonies known as darb al-mandal. Both these names occur also in the well-known charm of ch. xii, Lane, Manners and Customs of the Modern Egyptians. That the Arabic al-Ahmar, "the Red One" is only a translation of Ṭ̂riš (= Coptic e.(1) (1), Tpowp(1), "red") was suggested to me by Mr. W. E. Crum. The emendation eqøolø would mean "smoke breathing." 
The church is the place of assembling of the cherubim and seraphim.

The church is the resting place of every soul.

But let us love the church like our very souls. He that is negligent of the church casteth away from himself the fear of the Lord. And he that shall attend the synaxis without having first heard all the lections, attendeth only by half, not completely. He that shall depart from the church after hearing all the lections and shall sit at the door of the church, a man of this sort [58] is only a half believer. He that speaketh while they are reading or singing in the church, the prayer of that man is abominable in the sight of the Lord. He that departeth from the church without first having received the peace ${ }^{75}$ the angel of the Lord shall spend that day far from him; and no help from God is his, because he received not the peace before departing to his house. ${ }^{76}$

But now, O my beloved, let us love the church that we may obtain the forgiveness of our sins. Let us celebrate to-day the feast of the archangel Gabriel, the messenger of good tidings of the eons of the light, with a spiritual feast, knowing that he is the archangel of joy and the succorer of all who suffer violence at the hands of the Devil. Let us succor the poor as we are able each one of us, in order that the archangel Gabriel may open unto us the treasuries of [59] the Kingdom of Heaven. Let us keep ourselves from all naughtiness and all knavery and all bloodshed in order that we may celebrate the feast of the archangel Gabriel in the Kingdom of Heaven,

${ }_{75} \dot{\alpha} \sigma \pi \alpha \sigma \mu o ́ s$, the kiss of peace.

76 The faults here enumerated are strikingly common in Coptic village churches of today, as every traveller knows. The seeming indifference to what is going on is, however, apparently an ancient fault and not due entirely to the complete unintelligibility of the Coptic and all but complete unintelligibility of the classical Arabic in which prayers are said. But village Copts will listen any number of hours to discourses which are simple in language and content. 
for this verily is joy without end. Let us put away from us these base and slanderous deeds; for we know that God and His angels look down upon us and see us in all our works, all things whatsoever we do upon earth. For sooner or later we must depart from this place of sojourn, and we shall be questioned concerning all that we have wrought, whether good or evil.

Now I desired to prolong discourse upon these matters, but I know that moderation in all things is good; ${ }^{77}$ and, especially as the time has come for us to perform the holy oblation of the body and blood of our Lord Jesus Christ, let us finish the discourse at this place.

May God Almighty bless our god-loving Emperor who is this day in our midst, [60] and watch over him even as over the just kings David and Solomon and Hezekiah and Josiah; and may He keep the Senate and all the Council of Elders ${ }^{78}$ of the Romans, and all the people of the city, both male and female, young and old; and may He have mercy upon our souls on the day when we shall meet Him, through the supplications and petitions of the holy archangel Gabriel whom to-day we celebrate in heaven and upon earth; by the grace and philanthropy of our Lord Jesus Christ, our Savior, through whom glory is due: unto himself and His good Father and the Holy Ghost, now and forever, world without end, Amen. $99^{79}$

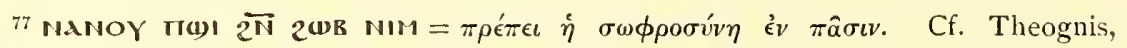

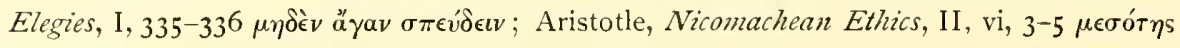
$\dot{\epsilon} \sigma \tau i \nu \dot{\eta} \dot{\alpha} \rho \epsilon \tau \dot{\eta}$.

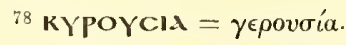

${ }^{79} \mathrm{On}$ the meaning of this cryptogram cf. the note to p. $\overrightarrow{\boldsymbol{A}}$. 


\section{A HOMILY ON THE VIRGIN BY THEOPHILUS, ARCHBISHOP OF ALEXANDRIA}

[60] A discourse which our holy and all-honorable father, Apa Theophilus, Archishop of the metropolis of AlexanDria, ${ }^{1}$ Delivered Concerning our Lady the holy Theotokos, [61] Mary, very mother of God, on the sixteenth of Mesore, ${ }^{2}$ THE FEAST OF IIER HOLY ASSUMPTION, WHEN SHE WAS TAKEN UP into Heaven. And he also spake concerning a Hebrew, biving in the city of Alexandria, who believed on the Christ because of the eikon of our Lady St. Mary, drawn upon a tablet of Wood within a storehouse. And he also SPAKE CONCERning the GREAT Misery of man. ${ }^{3}$ With the PEACE OF God! Amen! $99^{4}$

Of a truth the commemoration of the holy Theotokos Mary, Mother of Christ, hath dawned upon us this day! For I hear the holy prophet David crying out in the Psalms, and saying: ${ }^{5}$ Honorable things of thee are spoken, thou city of the great

1 Tradition makes Theophilus, archbishop of Alexandria, the author of many discourses. Cf. Evett's edition of the History of the Coptic Church of Alexandria, p. 426. A homily similar to the present one is found in Budge's Coptic Homilies in the Dialect of Upper Egypt. I9I0, no. iv, pp 66-79, especially fol. 95 b, col. 2, fol. 96 a, coll. I and 2, fol. 86 b, col. 2; and both may belong to the same "Theophilus." Against the acceptance of the present homily as a genuine work of Theophilus of Alexandria may be urged the legendary character of material ( $\mathrm{p} . \overline{\mathrm{o} z}$ note) which the homilist represents to be of the nature of personal experience, the topographical inconsistencies regarding the city of Alexandria (pp. $\vec{\pi} \mathbb{8}, \overrightarrow{\mathrm{q} \lambda}$ notes), and the very ordinary character of both substance and expression.

${ }^{2}$ Cf. Synaxarium Alexandrinum, ed. Forget, I9I2, tom. ii, p. 28o; Le Synaxaire Ethiopien, ed. Guidi, iii, pp. 335-340.

3 Apparently the great misery of people of his own time is what is meant by the homilist's editor. P. $\overline{\mathbf{q}} \mathbf{B}$ seems to refer to persecution or to plague. At the time of Theophilus of Alexandria the disorders were within the church. It is impossible to say to what the title or the text refers.

${ }^{4}$ For the meaning of this cryptogram see the note to $\mathrm{p} . \overline{\mathrm{\lambda}}$

5 Cf. Ps. Ixxxvi, 3 ; xlvii, 2. 
King! O my beloved, is it not our Lady the holy Mary who received unto herself God the Word, Christ Jesus, our Lord? What is the city of the great King? [62] I think that it is the womb of the Virgin Mary, which became the dwelling place of the Word of the Father, when He came and dwelt in her womb nine months, and she brought Him forth upon earth. ${ }^{6}$ He was subject unto all things such as we ourselves, save only sin; for He sinned not, neither was guile found in $\mathrm{His}$ mouth. ${ }^{7}$ He ascended into heaven, and sat upon the right hand of His Father on high. And $\mathrm{He}$ cometh in His glory and judgeth the quick and the dead, ${ }^{8}$ and giveth unto each according to his works, whether good or evil. ${ }^{9}$

But now, my beloved, let us thoroughly consider ${ }^{10}$ certain qualities of this Virgin. She conceived without associating with any man. ${ }^{11}$ She gave birth without suffering pain, for she merely brought forth ${ }^{12}$ her first-born Son. She wrapped Him in swaddling clothes; she laid Him in a manger of beasts: ${ }^{13} \mathrm{Him}$ our very God, Him the Son [63] of ${ }^{13 a}$ God forever blessed.

" His being brought forth upon earth is contrasted with His having been "begotten before all worlds," having had no mother in heaven and no father upon earth.

7 Cf. I Pet. ii, 22.

$8 \mathrm{Cf}$. the creeds.

${ }^{9} \mathrm{Cf}$. Rom. ii, 6.

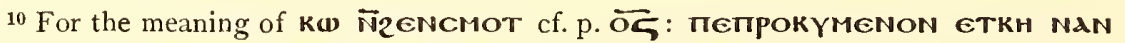
e2pגi. If $\mathbf{K u}_{2}$ is read, it is translated "emulate."

11 cYNoYcid $=$ coiltus.

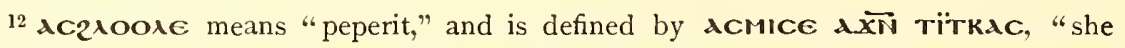
gave birth without suffering pain." Peperit sed non parturivit. Cf. Harris, Odes of Solomon, no. xix, $6 \mathrm{ff}$ : " (The Spirit) opened the womb of the Virgin and she received conception and brought forth; and the Virgin became a mother with many mercies; and she travailed and brought forth a Son without incurring pain; and because she was not sufficiently prepared and she had not sought a midwife (for He brought her to bear), she brought forth, as if she were a man, of her own will." Cf. Harris, Ephrem on the Gospel, p. 31: "For there are no pangs in the case of a virgin that man has not known . . ." and Bar Salibi's imitation (quoted from Harris) : "If thou requirest proof or illustration, hear how He came forth of the Sepulchre unopened, and entered into the Parlour being shut up; so He came forth of the womb not prejudicing virginity." Cf. note 15; Ezek. $\mathrm{xliv}, 2$.

${ }^{13}$ Cf. Luke ii, 7 .

13a Beginning of Washington Ms 2. 
O holy Virgin Mary, tell me of thy kinship, and of the house of thy father, and of all thy religious observances: for I think that the house in which thou now art is the heavenly Jerusalem, with thy Son, beseeching Him continually in behalf of us sinners that He may have mercy upon us: for she is sufficient to make intercession for the whole race of Adam.

Blessed art thou, holy Virgin Mary, for thou didst nourish the Christ who nourisheth us all ${ }^{14}$ in His kindness and abundant mercy! Blessed art thou, O Virgin, for thou didst carry in thy hands Him that made us free from the sin and iniquity of the Devil! Blessed art thou, taking Him up! Blessed art thou, speaking to Him! Blessed art thou, nourishing Him with thy chaste milk! [64] O Queen and resting place of the King! O cooing white dove, cooing to thy Son in the morning!

O Joakim, truly didst thou beget a great and royal gift which became the dwelling place of Immanuel! Fortunate art thou, Joseph, blessed innocent old man, for thou wast allotted this Treasury, sealed and of Pearls. ${ }^{15}$

But who is she of whose life we speak? It is Mary, this virgin, the daughter of Joakim and Anna, of this chosen line, this holy root. O Joakim, great is thine honor; and all the gen-

${ }^{14} \mathrm{Cf}$. Odes of Solomon, no. viii : "My own breasts I prepared for them that they might drink my holy milk and live thereby"; and no. xix: "The Son is the cup, and He who was milked is the Father: and the Holy Spirit milked Him."

${ }^{15}$ It is the doctrine of the perpetua virginitas that is here alluded to. Cf. Ezek. xliv, 2. There is also some allusion to that strange Talmudic legend of Joseph "the Sabbathkeeper" which will be found in Wünsche, Der Babylonische Talmud, 1886, vol. I, p. I63, or Tractate Shabbath, fol. I 9 a. Cf. Gïnther, Die Christliche Legende des Abendlandes, I9Io, p. 83, where, however, it is misunderstood. According to this legend a certain gentile sells all his worldly goods and buys with the proceeds thereof a pearl of great price (cf. Matt. xiii, 46). He does this fearing that his Jewish neighbor, Joseph the Sabbathkeeper, will inherit all his property. The fear is justified by events; for the gentile loses the pearl, it is swallowed by a fish, and Joseph obtains it by happening to buy the latter. Perhaps in the phrase "royal gift" there is a similar allusion to the Egyptian tribute of Jehoiakim mentioned in II Kings xxiii, 35 . 
erations that come after thee shall call thee blessed; for thou didst become father of our Lady, the holy Theotokos Mary.

And now, O Messiah, [65] God of truth, do thou graciously grant us thy Holy Spirit that we may utter a few words of praise to the honor of this virgin.

But let us, O my brethren, keep ourselves holy unto the Lord like this holy virgin Mary. And above all let us cleanse our body and our heart and free ourselves from every care of this life, knowing that we are going up into the hands of the living God and into no human hand. ${ }^{16}$

Oh the virginity, purity, at once of body, soul, and spirit, the gift of the Holy Spirit! Oh the virginity which cleanseth the whole body and purifieth it from the destruction of the Devil and his evil snares! Oh how many virtues doth virginity produce! Who, having put thee on, hath ever been confounded?

But virginity ${ }^{17}$ is this, my [66] beloved: that each one keep his couch undefiled upon the Sabbath and the Lord's Day ${ }^{18}$ and every feast-day and every day of the synaxis, according to the canons of our holy apostolic fathers ${ }^{19}$ in addition, during the Quadragesima and the two fasts ${ }^{20}$ and all the feasts of the Lord. ${ }^{21}$ Oh I beseech you, my brethren, let us put on the name of the virginity of this maiden, this holy virgin, lest ${ }^{22}$ our lamp be extinguished in our hands and we be put outside the door of that bridal place whose joy shall not turn to grief! ${ }^{23}$

${ }^{16}$ Cf. Hebr. $\mathrm{x}, 3 \mathrm{I}$.

${ }^{17}$ Here, at least, chastity, not virginity is meant.

18 Sabbath and the Lord's Day of course were never confused in Egypt until the advent of Scotch Presbyterian influences. Even the Arabic language bears witness to the true Christian tradition: jòm es-sabt is the seventh day, and jôm el-ahạad the first day, of the week.

${ }^{19} \mathrm{I}$ am unable to identify this reference.

${ }^{20}$ I.e. the paschal fast, at that time separate and beginning on the Friday before Palm Sunday, and the fast of Jonas, or of Nineveh, occurring one week before Lent.

${ }^{21}$ I.e. all the feasts commemorating events in the life of our Lord.

22 їпдте_ "lest," as often in the homily.

${ }^{23}$ Cf. Matt. xxv, 8. 
But the oil which is in the lamps is mercy toward the poor and needy and the orphans, according to the word which the scripture of James saith in the Catholic Epistles: But pure service and undefiled [67] before God the Father is this: to visit the fatherless and the widows in their afflictions and keep himself unspotted from the world. ${ }^{24}$

And he saith further: The double-minded man is unstable in all his ways. ${ }^{25}$ Then how great is the trouble which shall be ours, my beloved! For I think that the distraction which shall be ours is at the time when the man lieth down for his last illness, and is distraught, thinking of all the things in which he hath been engaged and the evils which he hath done. Thou art distraught because the things ${ }^{26}$ which pursue thee are about to torment thee in the ways, ${ }^{27}$ till thou come to adore the Judge of truth, Jesus the Christ. Thou art distraught because, after another brief space, thou sayest thy say in faltering accents. ${ }^{28}$ Again after a little the fever consumeth thy whole body by reason of the distress which is about to encounter thee. Thou art distraught because, after a space, [68] the Powers of Darkness shall hurry thee out and shall not let thee enjoy thy labors. Now inasmuch as we know these things, $O$ my beloved, that after a time however long death troubleth us, let us prepare ourselves well, ere the messengers of death summon us and trouble us.

But I beseech you, beloved! What is our profit, wasting day after day, month after month, year after year, until the Day of the Lord cometh upon us like a snare! For it surely cometh upon the dwellers over the face of the whole earth. Let us prepare ourselves well lest we be sorely troubled and come to grief

\footnotetext{
24 Jas. i, 27.

25 Jas. i, 8.

${ }^{26}$ Diabolical creatures which beset the road on which the soul must travel. Cf. p. $\overline{q \pi}$.

${ }^{27}$ The word "ways" is thus literally understood.

${ }^{28}$ Referring to the gasping utterance of the dying.
} 
and great wretchedness in the day of our distress. For the Lord saith in the gospel: If the man gain the whole world and lose his soul! Or what shall the man give in exchange for his soul ? 29

[69] But give ear to me and I will relate to you this example, wonderful besides being true.

Behold a man, if he dwelleth in a house, whether, being asked for the rent, he payeth, or payeth nothing at all, nevertheless he hath dwelt many days in the house. ${ }^{30}$ If that man inhabiting the rented house be wealthy, you find him sitting in the house, his mind at ease. For, after a certain time, the landlord cometh and findeth his rent ready for him and taketh it without hearing any excuse. Then the landlord rejoiceth that he hath found what he sought; and thus there is joy and gladness on both sides.

But if that man inhabiting the rented house is poor, you find him sitting grieved in his mind and sighing because of three things which are these: grieving, on the one hand, for the life of his children, lest they die of hunger and leave him; and, [70] moreover, because of the rent of the house; and his dying in poverty, and his wretchedness. These are the reflections of the poor man.

And then, if the rent of the house is due, the landlord seeketh that which is his; and, if the man findeth it not, the landlord casteth him forth in anger and distress; and the man is troubled, seeing his goods cast out of the door of the house. Behold, brethren, the trouble that cometh to him: for he walketh about by night and by day, seeking a house to live in. If no man receive them because of his untrustworthiness, yet another great trouble of mind settleth upon him.

${ }^{29}$ Cf. Matt. xvi, 26.

${ }^{30}$ This sentence is unclear or possibly corrupt. From the context it would appear that the reference is to paying rent in advance or otherwise. 
This verily is the manner of the wretched man, when his time slippeth from him without his knowing it: God casteth him out of his house, which is his wretched body; and he encountereth weeping and gnashing [71] of teeth, if he continueth wasting day after day: one day committing adultery, with a woman not his own; one day stealing, taking what is not his own; one day hating his brother; one day slandering his neighbor, saying that he might give so-and-so much to the church; ${ }^{31}$ one day coveting things which are not his own; one day speaking vain words out of his mouth; one day making balances of deceit; one day taking away the hire of the laborer from him; one day sitting idly; one day gazing wickedly after the women, with lustfulness and a shameless eye; while the Lord saith: Every man that is in uncleanness and those from whom the issue runneth, ye shall put out from the congregation, and I shall come and be in your midst, saith the Lord. ${ }^{32}$ If God hath given charge concerning these persons, then the more we wretched sin- [72] ners ought not to be heedless, $O$ my beloved, like those foolish virgins, wasting day after day until that Day of the Lord cometh upon us suddenly. He cometh, saith He, upon those that dwell upon the face of the earth. Knowest thou not, $O$ wretched man, that, upon the day in which God formed thee, He gave sentence against thee, saying: Dust thou art and thou shalt return unto the dust according to thy manner. ${ }^{33}$

But if the number of thy days be fulfilled before thou repent, the messengers shall trouble thee in thine inward parts, and the ... ${ }^{34}$ flow from thy mouth like fire, and thy whole body be agitated ${ }^{35}$ and thy countenance be changed, and thine

\footnotetext{
31 oY MN OY, "what and what," "so and so much." Cf. Zoëga, Catalogus, 527.

${ }^{32}$ Cf. Levit. xv, $\mathrm{I}$ f. $\quad{ }^{34}$ cmon perhaps for 2 mom, heat.

${ }^{33} \mathrm{Gen}$. iii, I9. $\quad{ }^{35} \mathbf{6} \mathbf{6} \mathbf{1 2}$ is of uncertain meaning in this connection.
} 
eyes see them that are about to pursue thee with torment and troubling, and thy hands become weak, and thy fingers become changed, and thou becomest troubled and terrified at all them that shall see ${ }^{36}$ thee. Oh wretched man that I am! Who shall deliver me out of the body of this death! But thanks be to God [73] who shall give us the victory through Jesus Christ. $^{37}$

But now, O my beloved, let us keep ourselves ready at all times lest we be required when we know it not and curse the day in which we were born into this world. ${ }^{38}$ Ye know that when we die we take nothing with us to the tomb. But whether it be a good thing which we have wrought or whether it be an evil thing, we find all of them all in front of us, even as it is written in the scriptures. For the psalmist David saith: Thou art He who shall give each one according to his deeds. ${ }^{39}$ Or knowest thou not, $\mathrm{O}$ foolish man, that three cubits of earth are thy house forever! What doest thou with all these buildings and these lofty houses and these great pictures, which thou, departing, leavest to others? And they that dwell therein shall not think of thee. How long dost thou gather for others? And they are thine enemies who are to inherit them forever. Dost thou not know that thou camest into this world naked, and [74] that naked thou shalt go hence? Thou camest out of the womb of thy mother, weeping; and thou shalt depart, weeping and thyself bewept.

Behold then, my beloved; because of the multitude of our sins I have mentioned these things to you to-day at the feast of the Queen and Mother of our Life, our Lord Jesus Christ, who hath gathered us together to-day at the commemoration of His virgin mother. Let us then bring good fruits and

\footnotetext{
${ }^{36}$ Or else, reading îTe: a cause of troubling and terror to "all them that shall see thee." ${ }^{37}$ Cf. Rom. vii, 24 ; I Cor. xv, 57.

${ }^{38}$ Cf. Job iii, I.

${ }^{39}$ Cf. Ps. 1x1. I 2.
} 
satisfy God therewith and His virgin Mother. For the Lord will not bless us for having come into this holy place to-day, being in these great sins, each man according to his manner and each woman according to her deed; but if the Virgin beholdeth the fruit of the Holy Spirit to have increased in our members - which is this: love and mercy and joy, peace, [75] long-suffering, kindness, goodness, faith, temperance, continence - for these of this sort, he saith, the law is not against them, they that are of Christ Jesus having crucified the flesh, and the passions and the desires: saith the tongue of Paul, ${ }^{40}$ the fragrance.

Let us raise our hands to the heaven and beseech our virgin mother, St. Mary, our ambassadress; for she is powerful; and she will exhort her only-begotten Son, Jesus Christ, in behalf of all of us, that no famine nor pestilence descend among us; and she will keep us from the Evil One, the crafty wicked one, who leadeth astray the whole world.

O our Lady-Mother Mary, Queen and Mother of God in truth, we know indeed that thou art nearer to God, thy beloved Son, than all the saints. [76] When thou dost supplicate Him in our behalf $\mathrm{He}$ is just in everything and holy; and $\mathrm{He}$ will forgive us all our faults and our negligences, and enable us to do His will, ere He requires us out of this dwelling-place.

Behold now these things we have spoken to the honor of St. Mary, the very mother of God. But let us return to the subject which now lies before us, and say a few words of praise to the honor of Our Lady, the holy Theotokos, Mary, according to the measure of our poor ability.

It befell at the feast of Our Lady, the Queen of all women, St. Mary - since we celebrate her feast once a year, on the day of her holy assumption, which is the sixteenth of Mesore, in 
the manner which our fathers of blessed memory have handed down to us, according to the word of the psalmist, our father David: [77] The things which we have heard and have known, the things which our fathers have said unto us, have not been concealed from their children, from another generation: telling the praises of the Lord, and His mighty deeds and His wonders which he hath wrought. ${ }^{41}$

It befell, as the people of the true faith, assembled in the church at the time of the synaxis, in order that we might partake of the mysteries of the Christ, at the most solemn moment of the synaxis: behold a Hebrew ${ }^{42}$ came to us during the synaxis, into the midst of the church of the Christ, we being in the act of performing the awful oblation, each of us having shed a multitude of tears for his sins, that he might obtain the forgiveness thereof before he should come to adore the Tribunal which is no respecter of persons - that Tribunal before which all of us must stand and render account for the things which we have done, whether good or evil - Woe to us if we repent ${ }^{43}$ not ere $\mathrm{He}$ come, the Christ, who is the strictest

${ }^{41}$ Cf. Ps. Ixxvii, 3-4.

${ }^{42}$ Cf. note I. Günter, Die Christliche Legende des Abendlandes, 1910, cites a number of interesting parallels to our story. (a) A Jew becomes Christian upon seeing the host upon the altar to be in fact real flesh and blood (p. 159, from Schönbach in the Wiener Sitzungsberichte, 1908, I 56, pp. 50-70). (b) Certain Jews in the city of Toledo once abused and marred a figure of the Christ. One day, on the feast of the Assumption or the blessed Virgin, a cry of pain led to an investigation and to the discovery of the crime (p. 4I, from Willame [?] Adgar of the 12th cent., in Förster's Altfranzosische Bibliothek, r886, no. 9, ed. Karl Neuhaus, p. ir). (c) At Lidda, near Diospolis, certain Jews had wished to dispossess Christians from a building which they had sold them and which they were unwilling for them to use as a church. The miraculous appearance of an image or picture of the Virgin frightened the Jews away (p. 40 f., from Boto von Prifening, ed. B. Pez, I73I, p. 20). (d) A picture of Christ at Constantinople is thrown by a Jew into a deserted pit; and as punishment his bowels gush forth; but the picture is found to be unsoiled (p. 41. from Adgar, 37). These stories of the desecration of images by Jews are supposed to have reached the West through the Crusades. On stories of bleeding images see Sébillot, Le Folk-Lore de France, 1907, vol. iv, p. 389.

${ }^{43}$ Or else, "woe unto us lest $\mathrm{He}$, the Christ, come . . ." 
of accountants, [78] and make reckoning of our accounts; and we shall give account even as to a single idle word which we have uttered.

But that Hebrew man took a crowd from among those who were within the church, and took them away to the augustalius ${ }^{44}$ to make them work for him without pay; and the church then became deserted.

But I, Theophilus, prostrated myself upon my face before the altar, and wept at the scattering which had overtaken the flock of the Christ. While yet prostrated and weeping, behold an angel of the Lord came and stood at the east of the altar, and called out to me thrice: Theophilus, Theophilus, Theophilus! Attend to me, and pasture the sheep over which God hath given thee charge! And when the synaxis ${ }^{4 a_{a}}$ [79] hath come to an end, betake thee to the episcopal residence.

And as he spake these words to me I saw him vanish from sight; and I went, doing in accordance with the saying of the angel.

At that moment Cyrillus was in the episcopal residence, reading in the holy scriptures, in the teachings of our fathers of blessed memory, who were before us. For he was the son of my sister, after the manner of this world. I had reared him in every piety, walking as a saint in all the commands of the Lord. Now when I told him of the matter he marvelled at what had happened. And thus we continued to give praise unto God who alone performeth wonders.

And in those days there was a storehouse, a little south of the city, and a Christian used to live therein. [80] And that Hebrew, of whom we spoke a little time before, arose and went and offered a higher rent than ${ }^{45}$ the Christian and thrust him out of the storehouse. And the Christian, when he had loaded up 
his little children in haste and his goods, departed, not knowing whither he was to go. And, because of the perturbation which was upon the Christian and his little children, he forgot somehow a little tablet of wood, very precious, upon which the eikon of our virgin Lady was drawn, set up on top of a wall within the storehouse. So the Christian forgot it and departed and left it behind.

But the godless Hebrew brought in some workmen to have them clean the storehouse before he should dwell therein according to the wish of his [81] heart.

The workmen looked and saw the tablet of wood, set up on top of the wall, the eikon of the Virgin being drawn thereon. And they took it, and saluted it, and embraced it, and kissed its hands and feet ${ }^{46}$ and continued to salute it a long time, pressing it to their bosoms in great faith.

But when the Hebrew saw them saluting it he became angry, and he came in unto them in great wrath and with threatening. And he said to them: What is this to which ye are paying homage and which ye are kissing?

They answered, saying to him: This is the eikon of St. Mary, the mother of the King of truth, Jesus Christ our Lord, who was crucified for us, and rose from the dead on the third day according to the scriptures, and afterward appeared to His disciples upon the Mount of Olives.

And the Hebrew, hearing these things from the workmen, [82] and seeing the eikon of the holy Virgin Mary in their hands, they bowing down thereto, he, the godless Hebrew, became very wroth, and seized the tablet of wood in their hands, and shattered it, and brake it into small pieces, and cast it into a basket filled with ashes, ${ }^{47}$ and made one of the workmen

\footnotetext{
${ }^{46}$ In token of reverence; cf. Peyron Lex. and Zoega Catal. I22.

${ }^{47} \mathrm{Cf}$. the baskets still used in Egypt by workmen in excavating ruins.
} 
take up the basket, saying to him: Take up this in the bottom of which is this tablet of wood, on which the mother of this Nazarene is drawn, and take and empty them into the water. ${ }^{48}$

Believe me, brethren, for believing and very god-fearing men testify unto me. When the workman took up the basket, blood flowed continually from the basket in which was the eikon of the Virgin, which the Hebrew had destroyed, so that the body of the workman [83] was soaked with blood from the blood of the eikon of the holy Virgin Mary in the bottom of the basket, so that everyone that saw him marvelled at the matter.

As he went out and passed a little eastward of the city, there met him certain believing rulers of the city; and, when they saw the basket dripping blood down upon the ground, they seized him, wishing to torture him, and saying to him: Thou hast slain a man and hast taken his money in order that thou mightest become rich; and thou wishest to take his body and throw it into the water.

But the workman wept, saying: Woe is me! Would that it had only been a man of our kind that I had slain, and I would give my blood for his! But the blood which ye see is that of the eikon of our Lady, the Holy Theotokos, Mary, whose womb [84] did carry Him who nourisheth us all with his love, Him who hath made man and everything which is, visible and invisible. But behold she is at the bottom of the basket which ye now see me carrying. A Hebrew shattered her eikon and gave it me to carry, saying: Take it and cast it into the water with these ashes.

But the honorable rulers, when they heard these things

48 I.e. most naturally into the Flavizs Nozus, or else Lake Mareotis near which the store-house lay (p. $\overline{\mathbf{0}-\boldsymbol{\theta}}$ ); but vid. p. $\overline{\boldsymbol{\pi}}$, where the workman is made to pass to the east of the city, and p. $\overline{\mathbf{q}}$, where it is said to be in the Caesareum at the north of the city. 
from the workman who had the basket dripping with blood, seized him and took him to the episcopal residence, to my unworthy self; and there were other bishops staying at that time with me, we wishing to keep the feast of our Lady the queen and mother of the King of life. Thereupon, when they had come to me I brought up, out of the basket the tablet of wood upon which the picture of the Virgin was drawn. Betieve me, my brethren: As I gazed at the face of the picture - I and the bishops [85] which were with me - we saw its countenance to be sad, as if weeping tears of blood.

And when we saw it thus we picked it up and took it in to the Church of the Christ, it being the feast of the Virgin, and a great multitude being assembled at her holy-place, since we were keeping her feast on the day of the commemoration of her holy assumption. The circumstances being thus, we took a portion of excellent water and of fragrant oil and washed it with some of these, and put the fragments together, and set it up upon the wall above the table. And believe this, moreover: It remaineth even unto now, and it shall remain world without end. And every man that is in divers diseases and they that are possessed of devils, and they that writhe - in a word: anyone that hath any disease - if they salute this eikon of the Virgin in faith, obtain good health and restoration.

And thus we held a great general synaxis ${ }^{49}$ [86] in honor of our Lord Jesus Christ and His virgin Mother.

But I sent and brought that Hebrew unto the church, and I said unto him: My Son, go and weep for thyself, for thy chastisement is great before God, O foolish one, on the day when He shall require thee! Fearest thou not God in whose hand is thy soul? Hast thou dared to commit this great abomination, to shatter the eikon of the holy Virgin Mary, the mother of our true 
God, Jesus Christ? And as I continued to speak with him by the word of God he repented before me and I instructed him, and I reminded him of the chastisements, saying to him: Bethink thee of thy sins, my son. After a little thou shalt depart from the body and shalt be as though thou hadst not been born into this world at all. And if thou repent not [87] before thou diest thy dwelling place shall be the abode of all the sinners, and thou shalt come into the place which the Eternal never visiteth. And even if thou art a Jew, be not godless. And $I$ also said: God is merciful; $\mathrm{He}$ will forgive thee, since the holy Virgin Mary was from among the Jews. And I also said: Surely God expecteth thy repentance; otherwise he would have brought His wrath down upon thee from the hour when thou didst shatter the eikon of His virgin mother. For thus saith the law: Anger, and wrath, and tribulation, and anguish upon every soul of man that worketh evil, that of the Jew first, and the Greek also. ${ }^{50}$

While I was saying these things to him, his mind received illumination; and he spake thus to me: My lord and father, if I am worthy let thy mercy be vouchsafed me, and do thou make me a Christian, together with my wife and my children; and perchance God forgiveth me [88] that which I have done.

But I said unto him: By ${ }^{51}$ to-morrow, the Lord's day, come to me, God willing, unto the church, and I will baptize thee and thy whole household, in the name of the Father, and of the Son, and of the Holy Ghost.

And upon the morning of the Lord's day I arose and went to the church to hold the general synaxis. And as I entered into the holy place I extended my hands toward the merciful God, Jesus Christ, and prayed, saying: O God, forgive men for I am a thing of dust and ashes. O God, the only-begotten 
Son, who art in the bosom of thy Father, mayest thou hear me this day, even me, thy servant, and watch over thy flock of sheep, that the wolf seize not any from among them.

And as I ceased, having finished the prayer, the Jew came into the church and said unto me: My father, if it be the will of God that I become a Christian, let His will be done; for the Lord is kind and merciful, and forgiveth [89] me if I repent concerning what I have done; for He desireth not the death of the sinner, so that he may turn about and live. ${ }^{52}$

Then I said to him: Dost thou believe with all thy heart that the Christ is the Son of God? He answered: Yea, verily, I believe that the Christ is the Son of God. I said to him: Believest thou on the Trinity, the Father, and the Son, and the Holy Ghost? He answered: I believe, my father. Help thou mine unbelief!

And afterward I recited to him many passages of the Scriptures. But he believed rather because of the great wonder which had proceeded from the eikon of the holy Virgin Mary.

And thus I baptized him and everyone with him, in the name of the Father, and the Son, and the Holy Ghost; and he became in very truth a Christian man; and I gave him of the mysteries of the body and the blood of Jesus Christ; and I let him depart [90] in peace.

But within the space of three days report was brought to me that he had found rest and gone to the Lord; and was reckoned to the number of the Christians. God forgave him all his sins, and $\mathrm{He}$ forgave him even the sin of the breaking of the eikon; according to the word of the Apostle, saying: I obtained mercy in the things which I did, being ignorant, in unbelief. ${ }^{5}$; 
I have related these things to you, ${ }^{54}$ my beloved, to show you this thing: Let us guard ourselves from every sin. For if the image of the Emperor of this world, when painted and set up in the midst of the marketplace, becoming a protection to the whole city, and if violence is committed against any one, and he goeth and taketh hold of the image of the Emperor: then no man will be able to oppose him, ${ }^{54 a}$ even though the Emperor is naught but a mortal man; and he is taken to a court of law. Let us, therefore, my beloved, honor the eikon [91] of our Lady the veritable Queen, the holy Theotokos Mary, the mother of our God, Jesus Christ, and love the church, the house of God, and visit it promptly morning and evening, with perseverance, even as it is written: I will praise thee daily, I will praise thy name forever and forever, ${ }^{55}$ that we may obtain mercy in His presence in the day of our sore distress.

And so I caused to be torn down the storehouse, in which was found the tablet of wood upon which was drawn the eikon of the Virgin, and I made it into a great caravansary; and it became a hospice for strangers. This is now the one in the Caesareum ${ }^{56}$ of the city of Alexandria; and it remaineth unto the present day. And if any man that hath any disease be brought to that caravansary and be made to lie down therein at the place where is ${ }^{57}$ the eikon of the Virgin, he findeth health forthwith through the prayers of the Virgin Mary, the mother of our Lord Jesus Christ.

But now, O my beloved, let us not be heedless of the words [92] of life which I have supplied to you, according to the measure

\footnotetext{
${ }^{54}$ Literally, "to your charity." $\quad 5{ }^{5} a$ Cf. Tacitus, Annales, III, $3^{6 .}$

55 Cf. Ps. cxliv, 2.

${ }^{56}$ This building lay in the northern part of the middle portion of the city, far from the place of the storehouse as given on p. $\overline{\mathbf{0}}$.

57 But we are told on $p$. $\overline{\Pi \epsilon}$ that the image remained in the church above the table.
} 
of my littleness having told you everything, and the manner in which it occurred, to the glory of God and His holy Virgin mother, Mary.

I had wished to speak yet more; but, because of the weakness of the hearers, we have mevely reminded you with a few words, in order that we may all obtain mercy in the presence of the Lord.

But now, brethren, let us combat ourselves ever; for we know not the hour when we are required. Yea, we see our fellow-members snatched away daily by death; yet we perceive not. Therefore I say unto you, as a good father, bluntly, that these great troubles are to-day upon us because of our transgressions and our unnatural practices. ${ }^{58}$ Doth a day never dawn, brethren, on which we say: Why were we born into this world? Do ye not know that, after a little, books shall be spread out, and all our shortcomings and our negligences, and all our sins of carelessness shall be revealed: [93] those of the day and those of the night? ${ }^{58}$ Bethink ye of the fire that is

${ }^{58}$ пגрАфүCıс, "unnaturalness," which our homilist gives as the cause of divine visitation in the shape of persecution or pestilence, can hardly be taken here in a general

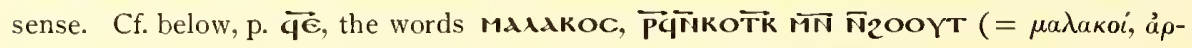
бєขокоітац, cf. I Cor. vi, 9). Cf. Crum, Catalogue of the Coptic Manuscripts in the British Museum, no. I98, BMMsOr no. 358 I A (26) fol. I :

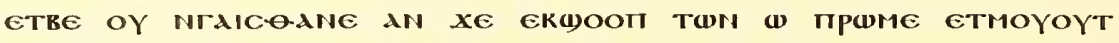

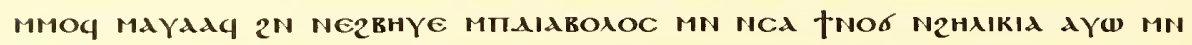

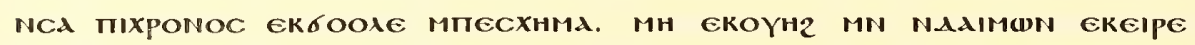

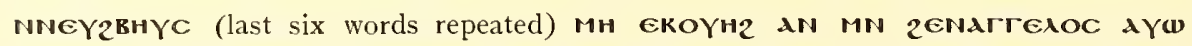

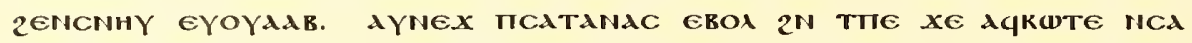

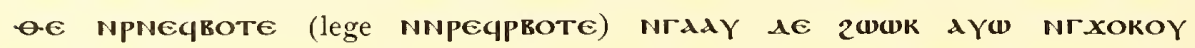

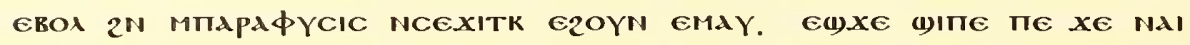

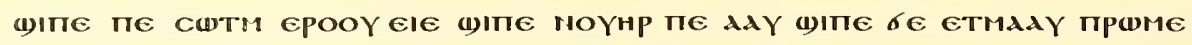
NAKA-AגPTOC ETMEEYE ENANAגMON NHAY NIM AYW ETMEeYe AN eNANATREAOC NOYOYNOY N[OY]WT.

"Why perceivest thou not where thou art, $O$ man that slayest thyself with the things of the Devil, at such an advanced age and after wearing so long the habit of a monk? Dost thou dwell perchance with the devils that thou doest their works? Dost thou not dwell with angels and holy brethren? Satan was cast out of heaven because he inclined 
not quenched, and of the worm that sleepeth not, and of the tears that well from the eyes of our souls, which are to have no end, and ceaseless weeping.

What wilt thou take when thou goest out of this world, O beloved brethren, except thy sins and thy transgressions? Knowest thou not, $\mathrm{O}$ wretched man, that three cubits of earth are thy house for eternity?

But thus are we, $\mathrm{O}$ brethren, in this place of sojourn, that when we see a man from among our relatives dead and being carried out to the tomb - or even if he be a stranger - we are wont to speak thus: Behold the glory of man and its dissolution in the tomb! Others, of the wise ones, say: To all of us it needs must be in the manner of this one.

If, on the one hand, he hath wrought a little of good, he findeth it in the presence of the Judge of truth. But if he be a man without compassion he seeth all the evil things - I mean the powers of darkness - rank on rank, marching along with him until he heareth his sentence at the hands of God.

But now, $\mathrm{O}$ my beloved, whether it be a good deed or whether it be an evil deed, they shall march [94] before us all until we come to adore the Judge of truth. For $\mathrm{He}$ saith in the Gospel according to John: They that have done good deeds unto a resurrection of life, they that have done evil deeds unto a resurrection of judgment. ${ }^{59}$

to the way of workers of abomination. And shalt thou do them thyself and accomplish them in unnatural ways and be admitted there? If it is a shame to say these things, a shame to hear them, then how great a shame is it to do them! Be then thou ashamed so as not to do them, $\mathrm{O}$ unclean man, that thinkest of the things of devils ever, and thinkest not of things of angels for even a single hour!"

Cf. a homily by a disciple of Shenute, Crum, Coptic Manuscripts in the John Rylands Library, no. 70. Cf. Milman's note no. 200 to Gibbon, ch. xliv. Cf. M. H. E. Meier in Ersch and Griber's Encyclopädie, 1837, iii, 9. Cf. Burton, Thousand Nights and a Night, vol. $x$, pp. $224 \mathrm{ff}$.

${ }^{59}$ Cf. John v, 29. 
But, my beloved children, let us receive instruction ere all instruction hath ceased among us. Let us prepare ourselves well lest we be sorely troubled. Let us ever pray to God with supplications and tears, and He will hear our petitions and forgive us. For the king of this world, if he becometh impious, is not a king but a tyrant from that time on; and every man that liveth in these things which I am now about to name is not a Christian in truth but it is a false name that he beareth, like Ananias and Sammeas ${ }^{60}$ in Israel or again like Zedekias and Achias whom the king of Babylon slew, according to the word of Jeremiah the prophet. ${ }^{61}$

But now let us guard ourselves from unbelief toward the miracles of God and His saints, especially those of His virgin mother, Mary, [95] in whose holy-place we are this day assembled to give glory to the King of truth, Jesus Christ our Lord, who was born to us out of the womb of this virgin, the com. memoration of whose holy assumption we make to-day, and the manner in which God did satisfy her with every good thing.

Now I wished to prolong my discourse concerning these things, but, since the time is far spent, let us send up offerings of praise, and mercy, and love, and all wisdom. And let no fornicator, nor adulterer, nor effeminate, ${ }^{62}$ nor bed-fellow of men, nor thief, ${ }^{63}$ nor covetous person, nor slanderer, nor false brother and friend, nor lover of vainglory ${ }^{64}$ - let not one among them that I have named resort to the holy communion of the mysteries of the Christ unless he purify himself before he partake of them, that he may not become guilty of the body and the blood of the Lord. ${ }^{65}$ For thus saith the chosen vessel Paul:

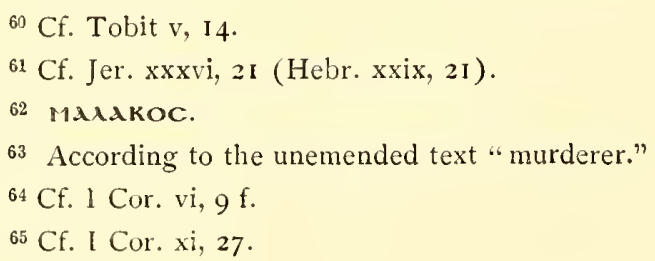


For this cause many among you are sick and weak, and many do sleep. ${ }^{66}$ And furthermore: For if we examined [96] ourselves we should not be judged. But being judged of the Lord, we are chastened that we may not be condemned with the world. ${ }^{67}$ Wherefore, my beloved brethren, be ye steadfast and unmoved, always abounding in the work of the Lord, forasmuch as ye know that your labor is not vain in the Lord. ${ }^{68}$ For whatsoever a man shall sow, that it is which he shall also reap; ${ }^{69}$ even as it is written: For he that soweth of his flesh, shall of the flesh reap corruption; but he that soweth of the spirit, shall of the spirit reap eternal life. ${ }^{69}$

Through the supplications and the prayers of our Lady, the holy Theotokos, Mary, through the grace and the benevolence of our Lord Jesus Christ, from whom is glory to himself with His good Father and the revivifying, consubstantial Holy Spirit; now and forever, world without end! Amen! $99^{70}$

I am John, the humble priest, the son of Kollothos, of [the Monastery of] St. Merkourios, and steward of [the Church of] the archangel Gabriel. [Oh may there be] intercessions and prayers [for me]!

It was written on the fourth of the month of Paremhot [in the] second [year] of the [current] indiction [in the year] six hundred and ninety after Diocletian [and] in the year [of the Saracens] three hundred and sixty-three. ${ }^{71}$

${ }^{66}$ Cf. I Cor. xi, 30.

${ }^{69}$ Cf. Gal. vi, $7,8$.

${ }^{67}$ Cf. I Cor. $x i, 31$ f. $\quad{ }^{68}$ Cf. I Cor. $x v, 5^{8 .}$

${ }^{70}$ See the note to p. $\overline{\mathbf{\lambda}}$.

${ }^{71}$ Though Greek words are used, the order is Coptic; and the Greek inflectional endings must be disregarded. 


\section{[SECOND COLOPHON]}

Through the zeal and solicitude of our God-loving brother Sisinnios, son of the blessed Philotheos, the ship-owner, he hath himself borne the expenses of this book by his own labors. He hath deposited it in the Church of the Holy Archangel Gabriel, ${ }^{1}$ of the city of Esneh, in order that the God of the Archangel Gabriel and of our Virgin Lady and of the Venerable Carpenter may bless him and his wife and his children and everything that is his: in the manner in which he blessed Eudronios and Kesaria, his wife; and as he blessed our patriarchal fathers, Abraham and Sarah, Isaac and Rebecca, and Jacob and Leah and Rachel; and bless him like Dorothe and Theopistos; ${ }^{2}$ and preserve him in his orthodox faith till the last breath - for this alone it is for which we hope-and bless every undertaking in which he is engaged; and make him worthy to hear the joyful voice: Come unto me, ye blessed of my Father, and inherit the kingdom which hath been prepared for you since the foundation of the world! ${ }^{3}$ So be it. Amen.

1 The Church of the Holy Archangel Gabriel of the city of Esneh is not found among the twenty-two churches of Gabriel in Egypt recorded by Abu Șâlị (Evetts and Butler, Churches and Monasteries of Egypt, 1895, p. 362), who, however, wrote over two hundred years later than our manuscript. According to the two colophons this church of St. Gabriel belonged to the Monastery of St. Merkurios in Esneh.

${ }^{2}$ Dorothe and Theopistos are doubtless intended for Dorotheos and Theopiste, concerning whose pious devotion of their wealth to charity, as followers of the Holy Archangel Michael, mention is often made. The British Museum Ms. Or. 702I, pp. 47-79 (Budge, Miscellaneous Coptic Texts in the Dialect of Upper Egypt, pp. 365-403, 918-938. Cf. Budge, Coptic Martyrdoms in the Dialect of Upper Egypt, pp. 47, 929; Budge, St. Michael the Archangel, p. xviii and indices; Wistenfeld. Synaxarizm, p. II2, where we find the names: Theodorus and Theopista) affords a detailed and lengthy account of their deeds, in the course of an encomium of Theodosius, Archbishop of Alexandria, on St. Michael, the Archangel.

${ }^{3}$ Cf. Matt. xxv, 34. 


\section{[A MAGICAL TEXT] ${ }^{4}$}

I invoke thee, God, Lord ${ }^{1}$ of the whole universe and of the world,

Which is beneath the heaven,

God of the soul,

That keepeth the bodies,

That blesseth ${ }^{2}$ them that labor in distress,

That is great,

That is exalted upon the midst of the sea,

That establisheth them ${ }^{3}$ all,

And that ruleth them.

For it is thou that art above them all,

Father of the universe,

Without thee nothing is,

God, that giveth hope,

Eternal,

Father of the everlasting,

1 Or, "God, Theos."

" I.e., by "sealing" with the sign of the cross.

${ }^{3}$ I.e., the seas.

${ }^{4}$ Coptic amulets are comparatively rare, considering the magical tendencies of the Egyptian people and the abundance of Abyssinian amulets, many of which show Coptic influence. Crum, Catalogue of the Coptic Manuscripts in the British Museum, 1905, Nos. 316, 317, 369, 370,371, 372,373,524,525,526; Crum, Catalogue of the Coptic Manuscripts in the Rylands Library at Manchester, I909, Nos. I00, I0I, I02, 103, I04, I05, III, 467; Aegyptische Urkunden aus den Königlichen Museen zu Berlin, I904, I, Nos. I, 2, 3, 4, 5, 6, 7, 8, 9, I0, I I, I2, I3, I4, I 5, 16, I 7, 18, 22, 23, 24, 25; Krall, Koptische Amilete (Mitteilungen aus der Sammlung der Papyrus Erzherzog Rainer, V), 1892, Nos. 65, 78, 2434, 2435. 2436, 17354; Crum, Coptic Ostraka, 1902, No. 490: are the only examples which I have seen. For Abyssinian amulets see the present writer's articles in Zeitschrift fiir Assyriologie, xxiii, xxiv, xxix, and in Hastings' Encyclopeclia of Religion and Ethics, sub voc. Charms. The "ring-signs" and other elements are Hebrew, cf. Journal of the American Oriental Society, xxxi, part 3. They are found in a Greek papyrus of the 4 th-5th cent.: Kenyon, Greek Papyri in the British Museum, I, I893, No. 125. The Arabic charm, Rylands Library No. 467, resembles very closely certain Ethiopic charms. The Coptic transmission of at least some of the parts of the Abyssinian amulets is seen in the Ethiopic form of the so-called Sator Formula. The Latin form of the palindrom is SATOR. AREPO. TENET. OTERA. ROTAS; and the 
For there is none other god beside thee,

That dispensest the floods.

Adonai! Abrathona! Jaw! Jaw!

The Great God,

That poureth them out,

That ridest upon the powers,

That casteth forth the demons,

That terrifieth them in thy might,

The Father Almighty!

God of the ages,

Father of the everlasting,

Coptic form (Crum, British Museum No. 524, of A.D. 600-750) CATap - ApeTw - TENET. wTepd. portac, with the $p$ changed to $t$ under the influence of the other four $t \mathrm{~s}$. In the

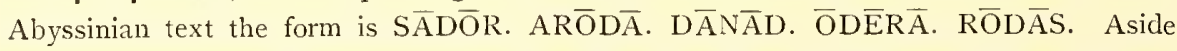
from the corruption of the second word, which was not discovered because it was not used in a palindrome, the changes are due to the characteristic Coptic confusion between $t$ and $d$. Coptic origin and Arabic transmission are seen in Ethiopic Ba'ūnāt, with its variant Bawwāna, which are derived from Coptic Boore, invida, the Woman with the Evil Eye, through

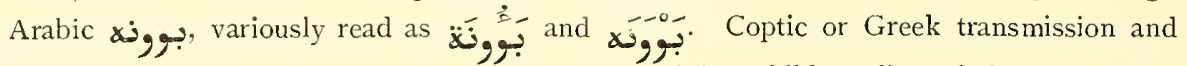
Hebrew origin are seen in the name of the Abyssinian child-stealing witch Werzelyā, in Coptic верzналд (Crum, British Museum No. 524), where bis a kind of $w$, and in Hebrew the theoretical feminine adjective 3 ב כ the Woman of Iron. Is it possible that the fury of Shenute and the tradition of it drove out and kept out of Egypt most of this sort of superstition? Nowadays Copts do not, to my knowledge, manufacture or wear amulets in either Coptic or Arabic; whereas Abyssinia is full of them. Shenute (died 45I), opposing not merely pagan survivals but paganism itself, says in a fragment in the Borgia Collection (Zoëga, Catalogus Codicum Copticorum, i8ıo, p. 459):

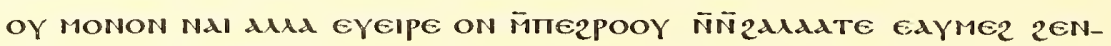

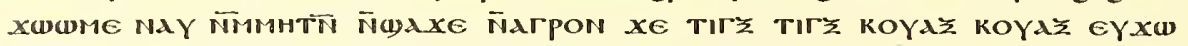

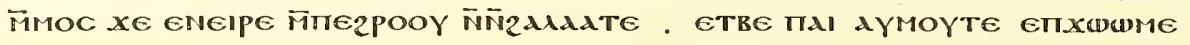
$x \in$ opNi-a-cc

"Not only these things! They also make the sound of birds, having filled books, for themselves and you, with vain words: Tinks! Tinks! Kwaks! Kwaks! saying: We are making the sound of birds. Hence they call the book Ornithes."

The incorrigibly sarcastic Shenute treats his opponents with the unfairness of a caricaturist. The title, "Ornithes," birds or auguries, of some real or imagined book, gave him opportunity to laugh at the chirping Tinks! Tinks! and the quacking Kwaks! Kwaks!, words which, by the way, resemble Abraxas, Abrasax, and many other magical "names." Shenute's ridicule is probably designed for the ears of men less learned in these matters than himself. But it was needed, and seems to have been effective. 
That giveth healing,

That strengtheneth,

That healeth diseases,

For thou hast power in respect to them that are oppressed,

Those who are imprisoned,

That art friendly to every one that calleth upon thy names:

Jaw! Adonai!

Every name by which I invoke thee,

Be it male, be it female,

Be it any one asking thee,

For thou guardest the souls,

Be it by water or oil,

By thy holy name,

That they may be well,

To the end that every man may know that

Thine is the power, and the glory,

World without end,

Amen! 



\section{INDEX OF COPTIC WORDS AND FORMS}
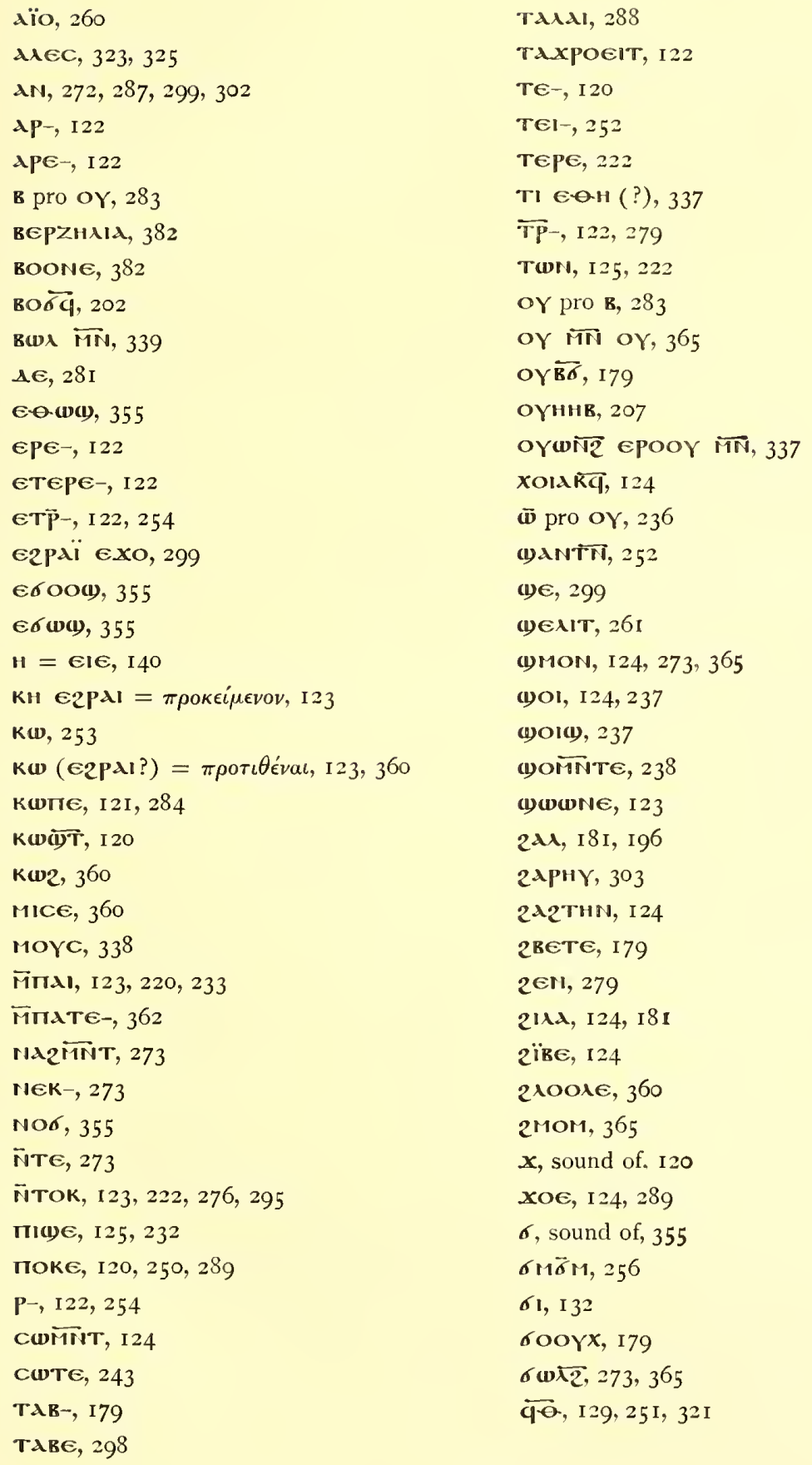


\section{INDEX OF GREEK WORDS}

$$
\text { A, } 129
$$

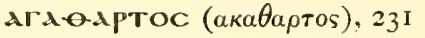

ayatos (Arגaon, -oc, -wC), I 40, ct passim

$\alpha \gamma \alpha \pi \eta, \mathrm{I} 6 \mathrm{I}$, et passim

a $а \pi \eta \pi T o s, ~ I 6 I$

a $\gamma \boldsymbol{\epsilon} \iota \iota \kappa \eta$, I 33, et passim

$\alpha \gamma \gamma \epsilon \lambda$ os, passim

a yı (o-drid), I45, ct passim

a ylos, $32 \mathrm{I}$

aypeov (ArrPION), 2 I 7

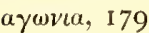

$\alpha \gamma \omega \rho \alpha, 309$

aıvos, I 43

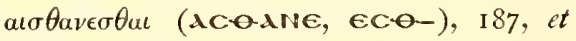

$$
\text { passim }
$$

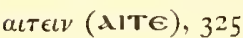

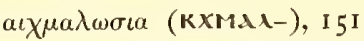

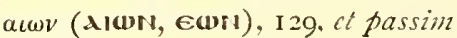

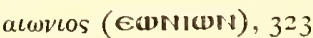

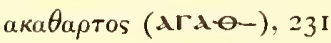

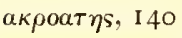

akтls (АKTIN), I94, 2 I 2

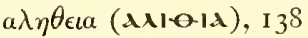

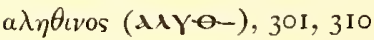

$\alpha \lambda \eta \theta \omega s, \mathrm{I} 33$, et passim

$\alpha \lambda \lambda \alpha$, I36, et passim

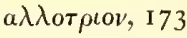

$\alpha \mu \epsilon \lambda \epsilon \iota \nu, 198,280$

$\alpha \mu \epsilon \lambda \eta s$ (-HC, -IC), 233, 24I, 272, $3 \mathrm{I} 3$

$\alpha \mu \epsilon \lambda \epsilon \iota \alpha, 3$ I 3

$\alpha \mu \eta v$ (2גмнг), 247, et passim

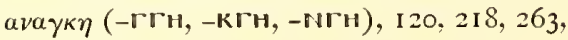
323

$\alpha \nu \alpha \gamma \nu \omega \sigma \iota s, 24 \mathrm{I}$

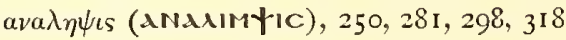
$\alpha \nu \alpha \lambda \omega \mu \alpha$ (גN2OגOMd), I9I

$\alpha \nu \alpha \sigma \tau \alpha \sigma \iota s, 3 \mathrm{I} 6$

$\alpha \nu \alpha \chi \omega \rho \epsilon \iota \nu, 22 \mathrm{I}, 286$

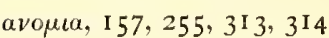

Anzoגor1d $(\alpha \nu \alpha \lambda \omega \mu \alpha)$, I9I avoolos (AN2OCIOC), I36

$\alpha \xi \cos$, I 3 I

$\alpha \xi \omega \omega \mu \alpha$, I 97

иоратоv (АzOpגToN), I 30

$\alpha \pi \alpha \nu \tau \alpha \nu, 246$

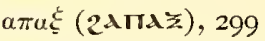

$\alpha \pi \epsilon \iota \lambda \eta$ (АПҮАн ), 290

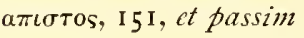

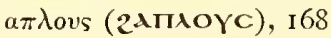

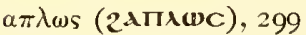

$a \pi 0,32 \mathrm{I}$

АпотגкрІх ( $\pi \circ \delta \alpha \gamma \rho \alpha)$, I9I

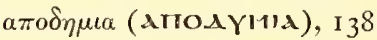

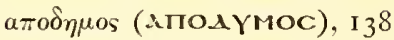

$\alpha \pi \circ \theta \eta \kappa \eta$ (Апо-оүкн), 250 , et passim

$\alpha \pi 0 \lambda$ oу $а$, I 58,267

$\alpha \pi \circ \lambda \circ \gamma \iota \zeta \epsilon \sigma \theta \alpha, 283$

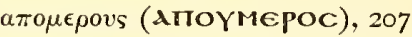

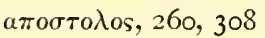

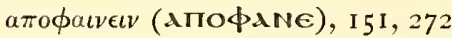

$\alpha \pi \circ \phi \alpha \sigma \iota s, 3$ I 5

$\alpha \rho a, 3 \mathrm{I} 3$

apyos (-ON, -(WN), 270, 27I, 284

$\alpha \rho \epsilon \tau \eta, 259$

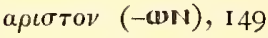

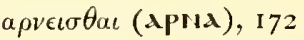

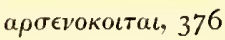

$\alpha \rho \chi \alpha \gamma \gamma \in \lambda o s, 129$, et passim

$\alpha \rho \chi \epsilon \iota \nu, 195,200,230$

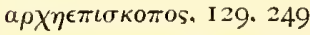

$\alpha \rho \chi \eta \pi \rho \circ \phi \eta \tau \eta s$, I 52

$\alpha \rho \chi \eta \sigma \tau \rho \alpha \tau \eta \gamma o s, \mathrm{I} 55$

$\alpha \rho \chi \omega \nu$, I 55, 294, 296

$\alpha \sigma \epsilon \beta \eta \varsigma, 3$ I 7

$\alpha \sigma \theta \epsilon v \eta s, \mathrm{I} 37,3 \mathbf{I} 2$

$\alpha \sigma \pi \alpha \zeta \epsilon \sigma \theta \alpha \iota, 290,29 \mathrm{I}, 299$

$\alpha \sigma \pi \alpha \sigma \mu \rho \varsigma, 146,357$

$\alpha \sigma \chi \eta \mu o v \epsilon \iota \nu$ (АCXYMONH), I 35

$\alpha \sigma \omega \mu \alpha \tau 05$, I 33, I 45, I 47

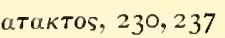

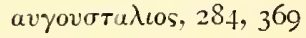


${ }_{a} v \xi \alpha \nu \epsilon t, \mathrm{I}_{5} 6,277$

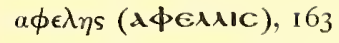

$\beta a \pi \tau \iota \zeta \epsilon l \nu$ (вАпヤZє), 304, 307

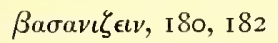

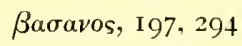

$\beta \alpha \sigma \iota \lambda \iota к о \nu, ~ 197,256$

$\beta \eta \mu \alpha, 283$

$\beta \iota 0$, 228, 257, 258

во-O $(\beta$ o $\theta \epsilon \iota v)$, I 29

$\beta o \eta \theta \epsilon \iota, 122$, et passim

$\beta o \eta \theta \epsilon \iota \nu, 129,2$ I 8

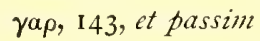

$\gamma \in \nu \epsilon a$ (геIIdId), 257

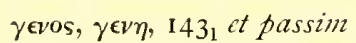

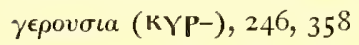

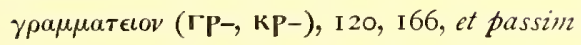

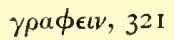

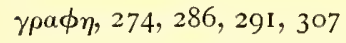

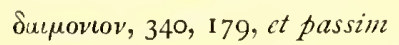

$\delta a \mu \mu \omega \nu$ (Aגmwr, Tdimorv), 226, 231, 323

$\delta \epsilon, \mathrm{I} 48$, et passim

$\delta \eta \mu o s$ (АүMOC), 246

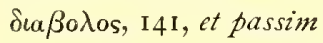

$\delta \iota \theta \eta \kappa \eta$ (Аाג-о-ҮКн), I43

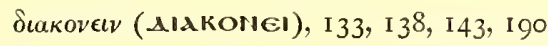

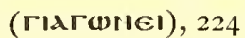

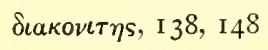

Sıкацоь, I 3I, 246, 280

$\delta \iota \omega \kappa \iota v$ (тішкєІ, ТєІокє), 23I, 238

$\delta \rho 0 \mu \epsilon \mathrm{s}\left(\mathrm{\Gamma P}^{-}\right), 202$

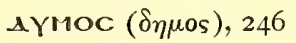

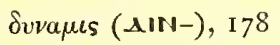

AYPANNOC (Tvpavvos), 317

$\delta \omega \rho \epsilon \alpha, 215,226,258$

$\delta \omega \rho o v, 216,218,223,256$

$\epsilon \gamma \kappa \lambda \epsilon \iota \theta \theta$ a (кинс-є), 325

$\epsilon \gamma \kappa \rho \alpha \tau \epsilon \iota \alpha$ (єгрגАІа), 278

$\epsilon \gamma \omega, 32$ I

є $\mu \eta \tau \epsilon$ (єІмннте), 3 I9

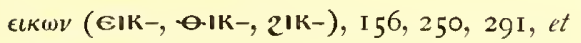

$$
\text { passim }
$$

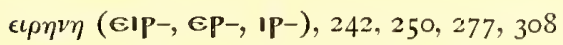

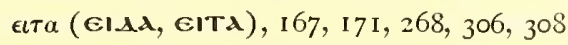

єITE (ITE), 325

$\epsilon \kappa \kappa \lambda \eta \sigma \iota \alpha, 234$, et passim

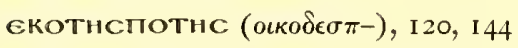

$\epsilon \lambda \alpha \chi^{\iota \sigma \tau o s, ~ 297, ~ 312, ~} 32$ I $\epsilon \lambda \pi \iota \epsilon \nu(2 \Theta \lambda \pi \mid Z \mathcal{C}), 223,322$

$\epsilon \mu \phi \alpha \nu \epsilon \sigma \theta \alpha \iota$ (МфАMHс-о-д), I 53

$\epsilon \nu$ o $\sigma \omega$ (N2OCON), I 23,264

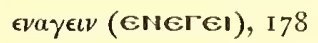

$\epsilon \nu \circ$, I 43

$\epsilon \nu \tau o \lambda \eta, 287$

$\epsilon \nu \omega \chi \lambda \epsilon \iota, \mathrm{I} 50$

$\epsilon \xi \circ \mu \nu \lambda \circ \gamma \epsilon \iota \sigma \theta \alpha \iota, 2$ I I

$\epsilon \xi \circ \mu \mathrm{i} \sigma 0 \gamma \eta \sigma \iota \mathrm{s}, \mathrm{I} 54$

$\epsilon \xi o p \iota \sigma \tau \iota \alpha$ (K₹OPIC†ג), I36

$\epsilon \xi o v \sigma \iota \alpha, 264,3$ I 5

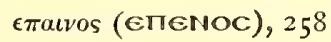

$\epsilon \pi \alpha \rho \chi^{\circ S}$, I 97

$\left.\epsilon \pi \epsilon \delta \delta_{\eta}(\epsilon \Pi\lrcorner \Lambda н\right)$, I20, I23, 318, et passin

$\epsilon \pi \epsilon \tau \alpha$, I 20, I69

$\epsilon \pi \imath \theta v \mu \epsilon \iota v, 270$

$\epsilon \pi \iota \theta v \mu \iota a, 27 \mathbf{I}, 278$

$\epsilon \pi \iota k \alpha \lambda \epsilon \iota \nu, 323,325$

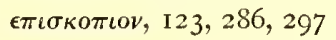

$\epsilon \pi \iota \sigma \kappa о \pi \circ \%, 297$

$\epsilon \pi \iota \sigma \tau o \lambda \circ \phi o \rho \circ$, I 52

$\epsilon \pi \iota \tau \epsilon \lambda \epsilon \iota v, 245,283$

$\epsilon \pi \iota \tau \rho \circ \pi \circ$ s, 163

$\epsilon \rho \gamma a \sigma \iota \alpha, 229$

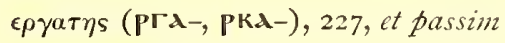

$\epsilon \rho \eta \mu o s, ~ I 52$

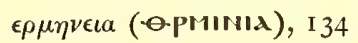

EC-OANE ( $\alpha \sigma \theta \alpha \nu \epsilon \sigma \theta \alpha \iota)$, I 89

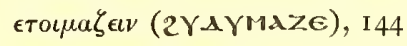

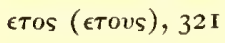

$\epsilon v a \gamma \gamma \epsilon \lambda \iota 0 \nu, 265,316$

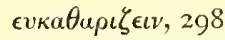

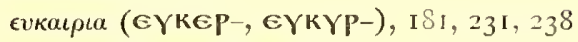

$\epsilon v \sigma \epsilon \beta \eta s$, I 72, I 76, 287

$\epsilon v \phi \rho o \sigma v v \eta, 267$

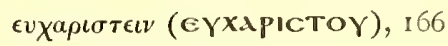

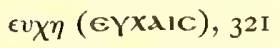

EWN $(\alpha \iota \omega \nu), 325$

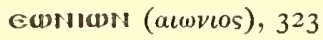

$\zeta \omega \gamma \rho \alpha \phi \epsilon \iota \nu$ (ZаргАф1), 309

$\zeta \omega \gamma \rho \alpha \phi \iota \alpha$ (Zwr-, cor-), 275, 295, 297

$\eta$, passim

$\eta \lambda \iota \kappa \iota \alpha$ (-Оүхігіג), 208

Odrid (aju), I45, et passim

Ouppєuv (-Aגpeı), I 75

$\theta \cos$ (गОC), 323

Өєотокоs, 249, et passim 


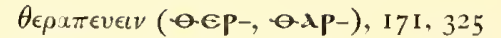

$\theta \epsilon \omega \rho \epsilon \iota, 138,22 \mathrm{I}, 225,277$

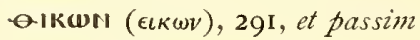

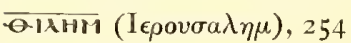

$\theta \lambda \iota \beta \epsilon \iota v$, I 48, 325

$\theta \lambda \iota \psi \iota s, 232,262,303$

o.opacic (opaбıs), I 50

өормн $(о \rho \mu \eta)$, I 49

$\theta \rho \eta \sigma \kappa \epsilon l a ~($ өрістіа), 254

opMINId ( $\epsilon \rho \mu \eta v \epsilon \epsilon)$, I 34

$\theta$ ovoos, I 55

өүлн $(v \lambda \eta), 2$ I 8

$\theta v \mu o s, 290$

$\theta v \rho \iota 0 v, 2$ I 9, 220

$\theta v \sigma \iota \alpha, 3 \mathrm{I} 8$

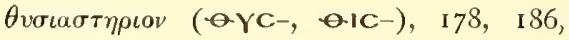
I 88,285

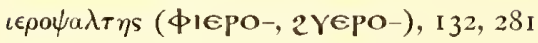

ıкаvos (2ıкגNoc), 254, 278

iva (2INA), 325

$\iota \nu \delta \iota \tau \iota \omega \nu$ (NAIKAIONOC), 32I

Iте ( $\epsilon$ T $\epsilon), 325$

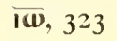

$\kappa \alpha \theta \alpha \rho \iota \zeta \epsilon \iota \nu, 259$

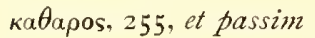

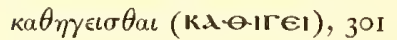

$\kappa a \theta$ oגєкоs (-H, -ON), 26I, 299, 304

каı, $32 \mathrm{I}$

$\kappa \alpha \iota \gamma \alpha \rho, \mathrm{I} 4 \mathrm{I}$, et passim

$\kappa a \iota v \eta, \mathrm{I} 43$

$\kappa \alpha \kappa \iota, 244$

$\kappa a \kappa \omega s, 265,27 \mathrm{I}, 3 \mathrm{I} 6$

$\kappa a \lambda \omega s$, I 99, 264, 265, 3 I 6

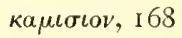

$\kappa \alpha \nu, 274$

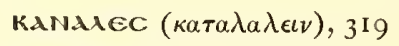

$\kappa a v \omega v, 260$

$\kappa \alpha \rho \pi \circ$ s, I 56, 276, 277

$\kappa a \tau \alpha$, I 34, et passim

$\kappa \alpha \tau \alpha \beta o \lambda \eta, 322$

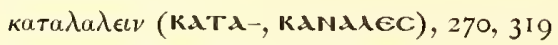

$\kappa \alpha \tau \alpha \lambda \alpha \lambda \iota \alpha$, I 4 I, 244

кат $\alpha \lambda \alpha \mu \beta \alpha \nu \epsilon \iota$ (КАTג入גвגNE), I 58

катаvтаv, 269

катафроvєє (КАтגфронн), I 77

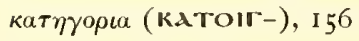

кгрАМмגтіоN ( $\gamma \rho \alpha \mu \mu \alpha \tau \epsilon \iota \nu)$, I 20 $\kappa \epsilon \lambda \epsilon v \sigma \iota s, \mathbf{I} 49$

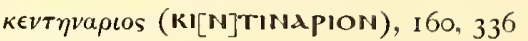

$\kappa \epsilon \nu \tau \rho o s, 323$

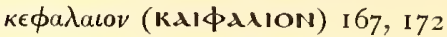

$\kappa \eta \rho v \xi$ (КҮPIร), I 38, I 43

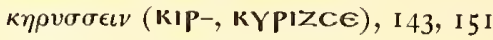

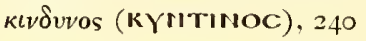

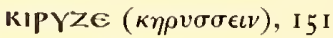

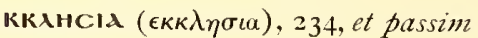

$\kappa \lambda \eta \rho о \nu о \mu \epsilon \nu$ (К入нр-, Кхір-), 275, 322

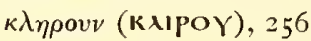

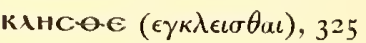

кรорістג ( $\left.\epsilon \xi_{\text {op } \iota \sigma \tau \iota \alpha)}\right)$ I 36

кoเvovıa, 3 I9

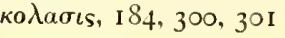

ко $\lambda \lambda \circ \theta_{\circ}, 32 \mathrm{I}$

коб $\mu \circ$ s, I 42

кPAMMATON ( $\rho \rho \mu \mu \alpha \tau \epsilon$ I $)$, I 20

$\kappa \rho \iota \mu \alpha, ~ I 84,225$

$\kappa \rho \iota v \epsilon \iota v$, I 83, 319

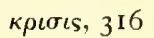

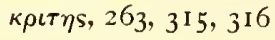

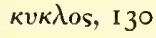

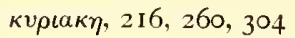

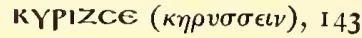

күPI之 $(\kappa \eta \rho v \xi)$ I 38, I 43

кYPOYGId ( $\gamma \epsilon \rho o v \sigma \iota \alpha), 246$

KXMAגOCIA ( $\alpha \iota \chi \mu \alpha \lambda \omega \sigma \iota \alpha)$, I 5 I

$\lambda \alpha \mu \pi \alpha$ s, 23 I, 238, 26I

$\lambda a \nu \theta a v \epsilon \iota, \mathrm{I} 64$

$\lambda$ aos, I 5 I, et passim

$\lambda \epsilon \iota \tau o v \rho \gamma o s$ (入IT-), I 37

$\lambda \epsilon$ ко , 256

хнпн $(\lambda v \pi \eta), 26$ I

$\lambda_{\eta} \sigma \tau \eta \mathrm{s}$ (入ICTHC), $20 \mathrm{I}$

$\lambda \circ \gamma_{o} \theta \epsilon \tau \eta s, 283$

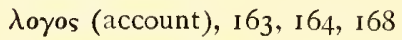

(discourse), 249

(Christ), I 29, et passim

$\lambda \circ \gamma \times \eta, \mathbf{I} 82$

$\lambda o \iota \mu o s, 279$

入остоv, 225, et passzm

$\lambda v \pi \eta$ (Антн, Аүтн, АүтеІ), 26I, 267, 268

$\mu \alpha \theta \eta \tau \eta \mathrm{s}, 29 \mathrm{I}$

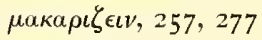

накарьоs, I 36, 28I, 286, 322

малакоь, 318, 376, 378 
$\mu \alpha \lambda \iota \sigma \tau \alpha$, I 29, I83, I 84, 245

$\mu \alpha \rho \gamma \alpha \rho \iota \tau \eta \mathrm{s}, 256$

$\mu \alpha \rho \tau$ ขtov, I 59, 2 I 9, 350

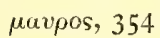

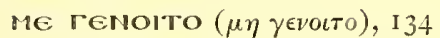

$\mu \epsilon \lambda \alpha s, 354$

$\mu \in \lambda \iota 0 \gamma \rho \alpha \phi o s,{ }_{1} 6_{4}$

$\mu \epsilon \lambda$ os, I I 7, I 23, 277, 335

$\mu \in \nu$ ( $\overline{M M}), ~ I 2 I, 245,308$

MeNH ( $\mu \eta \eta \nu), 32 \mathrm{I}$

$\mu \epsilon \rho 0$, I I 7 , et passin

$\mu \epsilon \sigma o \tau \eta \bar{s}, 5,8$

$\mu \in \tau \alpha \nu \circ \epsilon \nu, 273,306$

$\mu \in \tau \alpha \nu \circ \iota, 3 \mathrm{OI}, 302$

$\mu \in \tau \rho \circ \nu, \mathrm{I}_{3} 8$

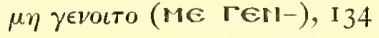

$\mu \eta \nu, 321$

$\mu \eta \tau \alpha \tau \omega \rho$ (MITdTop), I 52

$\mu \iota \lambda \iota 0 \nu, 207,2$ I 7

MN $(\mu \in \nu), 245,308$

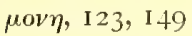

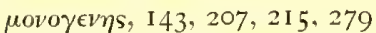

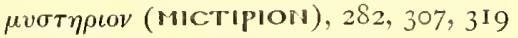

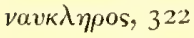

MAIKAIOHOC (

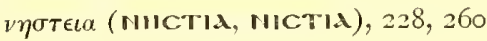

$\nu \eta \tilde{\sigma \epsilon} \epsilon \epsilon \nu, 206,223$

vเкаv, I 29

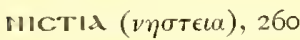

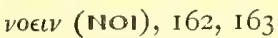

vouos, I 29, I 73

vovs, 303

N2OCON $(\epsilon \nu$ o $\sigma \omega), 123,264$

o ( (ov), 32 I, et passim

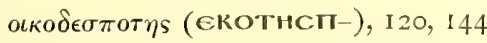

оєкоуонеа, I 44, I48

оєкоуоноs, I 3 I, I 52, 32 I

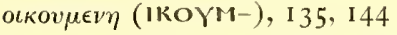

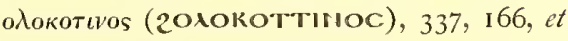
passin

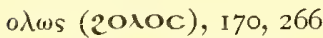

opows (2OMdioc), I92, 202, 310

о

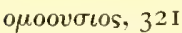

o $\pi \omega_{s}$ (omoc), 32 I

орана (2орома), I94, et passim

oparis (-o.opdcic), I 50 орүฑ, 302, 303

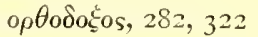

орнү (-оOрмн), 149

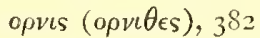

горомх $(о \rho \alpha \mu \alpha), 194$

opфavos, I61, 261, 262

ov $\epsilon, \mathrm{I} 6 \overline{5}$

ov $\mu$ ovov, 173

$\pi \alpha \theta \epsilon \iota \nu, 175$

$\pi \alpha \theta 0 s, 240,278$

$\pi \alpha \lambda \alpha \omega, \mathbf{I} 43$

$\pi \alpha \nu \delta \sigma \chi \epsilon i O V$ (ПАNTOXION), 3 I I

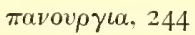

$\pi \alpha \nu \tau о к \rho \alpha \tau \omega \rho, \mathrm{I} 38$, et passim

$\pi \alpha v \tau \omega$ s (ПАптос), I 85, I 86, 275

$\pi \alpha \rho a$, I 33, ct passin

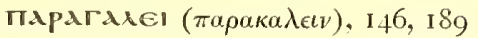

$\pi \alpha \rho \alpha \gamma \gamma \epsilon \lambda \lambda \epsilon \iota \nu$, I 88, 204, 27 I

$\pi \alpha \rho \alpha \delta \iota \gamma \mu \alpha, 266$

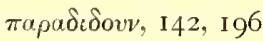

$\pi \alpha \rho \alpha \delta \circ \xi o v, 226$

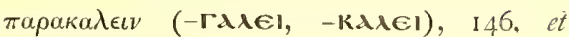
passim

$\pi \alpha \rho \alpha \mu v \theta \iota a(-\mathbf{M 1 \cdot 0 - 1 \lambda}=$ interest $), 123,167$, 172,338

$\pi \alpha \rho a \phi v \sigma \iota s, 376$

$\pi \alpha \rho \theta \epsilon v o s, \mathbf{I} 38$, et passim

$\pi \alpha \tau \rho \iota \rho \chi \eta \uparrow$, I 29, 322

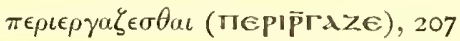

$\pi \epsilon \rho \iota \sigma \pi \alpha \nu, 273$

$\pi \iota \sigma \tau \epsilon v \epsilon \iota \nu, 2 \mathrm{I} \mathrm{0}$, et passim

$\pi \iota \sigma \tau \iota \mathrm{S}, 278,290,299.322$

$\pi \iota \sigma \tau o s, 145,242,293,294$

$\pi \lambda \alpha v \alpha \nu, 279$

$\pi \lambda \alpha \xi, 179$

$\pi \lambda \alpha \sigma \sigma \epsilon \iota, 272$

$\pi \lambda \eta \gamma \eta$ (п入үгн) 2I 9, et passin

$\pi \lambda \eta \nu, 175,207,226,266$

$\pi \lambda \eta \rho \circ \phi о \rho \epsilon \iota$, I 75

$\pi v \epsilon v \mu \alpha$ (Tतर), I 29, et passim

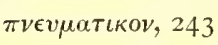

$\pi$ по $\alpha \gamma \rho \alpha$ (Апотגкріג), I9I

$\pi \circ \lambda \iota s, 129,159,185$

$\pi \circ \lambda \iota \tau \epsilon \iota \alpha$ (по入үтіג), 224

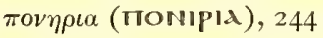

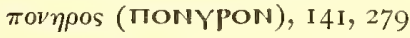

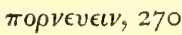


$\overline{\operatorname{IOC}}(\theta \epsilon O \varsigma), 323$

$\pi \circ \sigma \omega \mu \alpha \lambda \lambda o \nu, 27 \mathrm{I}$

$\pi \rho а \gamma \mu а \tau \epsilon \iota \alpha, \mathrm{I} 60$

$\pi \rho \alpha \gamma \mu \alpha \tau \epsilon v \epsilon \sigma \theta \alpha, 163,165,167$

$\pi \rho \alpha \gamma \mu \alpha \tau \epsilon v \tau \eta \mathrm{s}, \mathrm{I} 60$

$\pi \rho a \xi \iota s$, I 37

$\pi \rho \epsilon \sigma \beta \epsilon i \alpha$ (прєсвієс), 32 I

$\pi \rho \epsilon \sigma \beta \epsilon v \tau \eta \mathbf{s}, 278$

$\pi \rho \epsilon \sigma \beta v \tau \epsilon \rho \circ$, $32 \mathrm{I}$

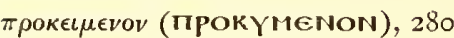

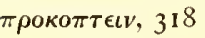

$\pi \rho 05,189$

$\pi \rho о \sigma \delta о к \epsilon \iota \nu, 232$

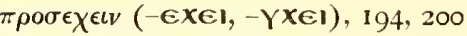

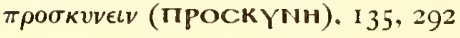

просүхє। ( $\pi \rho \circ \sigma \epsilon € \iota \nu), 200$

$\pi \rho \circ \sigma \phi о \rho а, 245,283$

$\pi \rho \circ \phi \eta \tau \eta \mathrm{s}, 148,251,317$

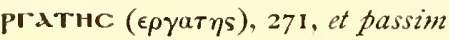

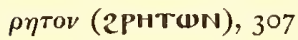

$\rho \eta \tau \omega \rho$ (грнтшр), I39

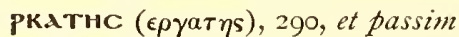

$\sigma \alpha \beta a \omega \theta$, I 55, 334

$\sigma \alpha \beta \beta a \tau o v, 260$

$\sigma \alpha \rho \xi$, I 34, et passim

$\sigma \epsilon \rho \alpha \phi \epsilon \iota, 154,241$

Clᄉd ( $\sigma v \rho \epsilon \iota), 195$

$\sigma \kappa \epsilon \pi \alpha \zeta \epsilon \iota \nu, 129,234$

бкєи (скєүє, скнүє), І 42, 268, 288

$\sigma \kappa \in v o s, 319$

согрגф1ג ( $\left.\omega_{\omega \gamma \rho \alpha \phi(\alpha)}\right), 295,297$

бoфos, 153,315

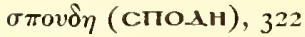

стрдкіссе ( $\left.\sigma \phi \rho \alpha \gamma \iota \epsilon_{\iota \nu}\right), 323$

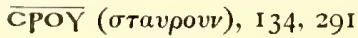

бтаvроs, 234

бтavpovv, I 34, 278, 291

$\sigma \tau o \lambda \eta, 212$

$\sigma \tau \rho \alpha \tau \epsilon v \mu \alpha$, I 35

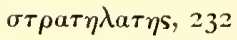

$\sigma \tau \rho \alpha \tau 0 \pi \epsilon \delta \alpha \rho \chi \eta s, \mathrm{I} 39$

$\sigma v \gamma \gamma \epsilon v \eta s$ (CYNr-), I20, 208

$\sigma v \gamma \gamma \nu \omega \mu \eta, \mathrm{I} 37$

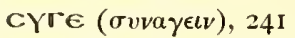

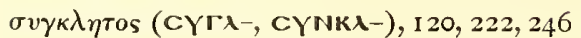

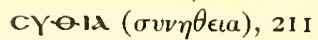

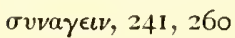

$\sigma v v a \gamma \omega \gamma \eta, 271$

$\sigma v \nu \alpha \xi \iota s, 222,35 \mathrm{I}$, et alibi

$\sigma v \nu \eta \theta \epsilon \iota a, 21$ I

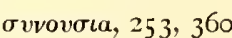

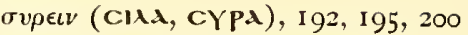

$\sigma \phi \rho \alpha \gamma \iota \zeta \epsilon \iota \nu, 189$, et passim

$\sigma \chi \eta \mu \alpha, 205$, et passim

$\sigma \omega \mu a, 132$, et passim

$\sigma \omega \tau \eta \rho, 207,247$

$\sigma \omega \phi \rho о \sigma v v \eta, 358$

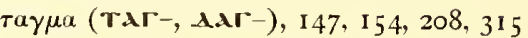

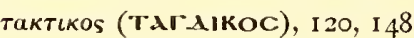

Tגimon $(\delta a \mu \mu \omega), 323$

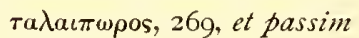

тафоs, 274, 314, 315

$\tau \epsilon, 278$

теוоке $(\delta \iota \omega \kappa \epsilon \nu), 238$

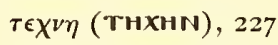

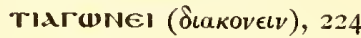

$\tau \iota \mu \eta$, I 47

тішкє। ( $\delta \iota \omega \kappa \epsilon \nu), 23$ I

$\tau o \lambda \mu \alpha$, I 58, I $78,180,300$

тотоs, 129, et passim

$\tau \rho \alpha \pi \epsilon \zeta \alpha, 299$

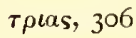

тромєүс ( $\delta \rho \mu \epsilon \epsilon \varsigma), 202$

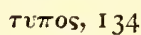

vios, $32 \mathrm{I}$

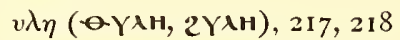

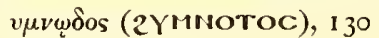

vлокрเбเs, 132

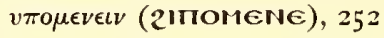

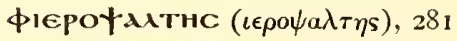

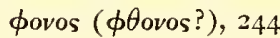

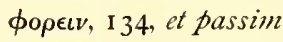

$\phi v \sigma \iota s, I 35$

$\phi \omega v \eta, 136$

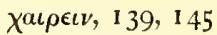

$\chi^{a \lambda a v,}, 230$

$\chi \alpha \rho \iota \zeta \epsilon \sigma \theta \alpha \iota$, I 85, et passim

$\chi \alpha \rho \iota s, 129,246,321$

$\chi \alpha \rho \tau \eta \mathrm{s}, 168,174$

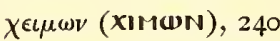

$\chi \epsilon \iota \rho 0 v \beta \epsilon \iota \nu$, I 54, 241

$\chi \in \rho \sigma o s, 217$

$\chi \eta \rho \alpha, 262$

$\chi \rho \epsilon \iota \alpha$ (Хр1ג), І62, I66, I70, I72 


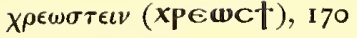

$\chi \rho \eta \mu \alpha$, I $_{22}$, et passim

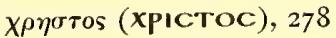

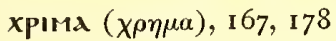

$\chi \omega \rho a, \mathbf{1} 60$

$\chi^{\omega \rho} \eta \gamma \epsilon \iota \nu, \mathbf{1 6 2}, 312$

$\psi \alpha \lambda \lambda \epsilon \iota v, 242,274$

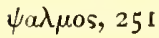

$\psi v \times \eta$ (†Үхн, †нх1), I7I, et passim

$\omega$, interjection, I29, I35, et passim

$\omega \phi \epsilon \lambda \epsilon \iota \nu$, I 9 I ws (20C), 243, 3I 3

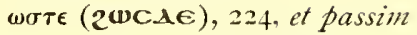

2Амнм ( $\alpha \mu \eta v), 247$, et passim

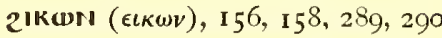

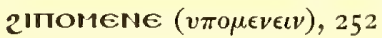

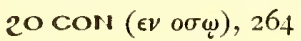

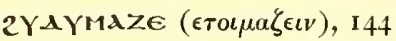

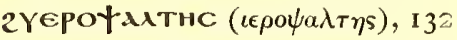

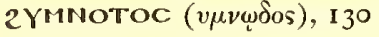

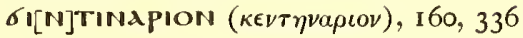




\section{INDEX OF BIBLICAL PASSAGES}

Gen. iii, I9. 365

Exod. xx, 7, $33 \mathrm{I}$

Exod. xx, 16, 328

Levit. $x v$, I I f., 365

Deut. v, I I, 33 I

I Kings xiii, $24 \mathrm{ff}$, 347

II Kings xxiii, $35,36 \mathbf{I}$

Job i, 6 ff., 335

Job iii, I, 366

Ps. v, 7, $33 \mathrm{I}, 340$

Ps. xlvii, 2, 359

Ps. lxi, I2, 366

Ps. Ixiii, 33 I, 340

Ps. lxxvii. $3^{-4}, 368$

Ps. Ixxxvi, 3, 359

Ps. cii, 20, 328

Ps. cxvii, 24. 328

Ps. cxliv, 2, 375

Prov. xix, 9. 33 I. 340

Is. vi, $3 \cdot 334$

Jer. xxxvi, 2 I, 378

Ezek. xxxiii. I I, 374

Ezek. xl. 334

Ezek. xliv, 2, 360, 36I

Dan. vi, 22, 347

Dan. viii, I6, 333

Dan. ix, 2I, 333

Bel and the Dragon, $3 \mathbf{I}-38,333,347$

Nahum i, I 5, I3 I

Tob. iv, I 5.328

Tob. v. 14,378

Tob. viii, 3, 355

Zech. viii, I 7. 328

Matt. v. 37. 34I

Matt. vii, I2, 328
Matt. xiii, 46, 36 I

Matt. xvi, 26, 364

Matt. xviii, I2, 327

Matt. xviii, 20, 33 I

Matt. xxy, 8, 362

Matt. xxv, 34, 380

Luke i, I9, 333. 334

Luke i, 28, 330. 332

Luke i, 3 I, 332

Luke ii. 7, 360

Luke ii, 8 ff.. 334

Luke vi, 3I, 328

Luke xiv, 4, 327

Luke xxiii, 42. 330

John v, 29, 377

Rom. ii, 6. 360

Rom. ii, 8-9. 373

Rom. vii. 24, 366

I Cor. vi, 9 f., 366,376

I Cor. xi, 27, 378

I Cor. xi, 30,379

I Cor. xi, 3 I f., 379

I Cor. xv, 57, 366

I Cor. $\mathrm{xv}, 58,379$

Gal. v, 22-24, 367

Gal. vi, 7-8, 379

I Tim. i, I3, 374

Hebr. x, 3I, 362

Hebr. xxix, 21,378

Jas i, 8, 363

Jas. i, 27, 363

Jas. v, 12 b, 34 I

I Pet. ii, 22, 360

I John ii, 2 I f., 33 I

I John iii, 8. 33 I

Rev. iv. I I, 334 


\section{INDEX OF NAMES AND SUBJECTS}

Abraham, 322

Abrasax, 382

Abrathona, 323, 382

Abraxas, 382

Abū-Ṣālih, 380

Abyssinian amulets, I28, 38 I

Abyssinian magic, 355

accounts, 336

Achias, 317, 378

Adam, 1 54, 255

agents, 356

Akhmīm, $x$

al-Ahmar, 356

Albright, Wm. F., v

Alexandria, 355, 359, 375

'Alì al 'Arabī, x

allegorical interpretation, 125

Alpha-Omega, 327

amulets, $128,38 \mathrm{I}$

Ananias, 317,378

angels, 126,369

Anna, 257, 36 I

Annunciation, 332

Apostolic Canons, 362

Aristotle, 358

Assumption. 359

" augustalius," 284, 369

authorship of homily, 359

Barnett, L. D., v

Bar Salībì, 368

Bāryā, 356

Bes, 355

birth from a man, 360

black (devil), 354

bleeding images, 368

Boak, A. E. R., I 26

bond, 337

Budge, Sir E. A. W., v, I I 5, I I6

Burton, Sir Richard F., 377

Caesareum, 31 I, 322, 371, 375

Celestinus, 125, 126, 327
Christ, 129, ct passim

Christian, 287, et passim

Christology, 329

church, behavior at, 357

Church of the Archangel Gabriel, I I 5, 379,

380

circumstantial negative, "lest," 123

City of the Great King (womb of the Virgin), 360

Cloud of Life, 332

colophons, I I 5, I I 7, I I 8, I 26, 379, 380

conception by hearing. 332

conversions of Jews, $368,373,374$

Coptic amulets, $38 \mathrm{I}$

Council of Elders, 358

cross, 356,380

Crum, W. E., v, I I 5. 356

cure by shock, 345

cursive, I 18

Cyrillus, nephew of Theophilus, I24. 369

Daniel, I49, 333

dating of MS., xv ff., I I 8, I 25

David, 25I, 274, 28I, 358

de Revières, Arthur, xxvi

de Rustafjaell, xi

death, 377

demons, $340,356,363,3^{82}$

Denga. 355

desecration by Jews, 368

Devil. 335, 35 I, 357

devil, red, 355

devil, tall, 355

Dinkas, 355

Diocletian, 321, 379

dogs of Allah, 347

Dorothe, 322, 388

Dorotheos, 380

dove (Mary), $36 \mathrm{I}$

dumbness. 343

Edfü, xi, I I 5

edition, plan of, xxiii f. 
eikon, 359

eikon restores health, 372

Emmanuel, 256

Emperor, 358, 375

Enemy (Satan), 355

eons of light, $327,328,333$

Ephrem, 360

Eshmunēn, $x$

Esnē, I I 5, 380

Ethiopian, 355

Eucharist, 330

Eudronius, 322, 380

Evagrius, $33^{\circ}$

evil-eye, 382

Exodus, 334

Fayùm, x, xi, I I 5, et passion

Flavius Novus, $37 \mathrm{I}$

Freer, Charles L., v, x

Freer Collection, ix

future tense, 123

Gabriel, 32 I, 327. et passim meaning, 328, 332

Messenger, 327, et passim shrine of, 336, et passim

ghūl, 354

Gibbon, 377

gout, 345

"graft," $33^{6}$

Greek language, I I 8, 379

Habakkuk, 333

Hebrew, 250, et passim, 359, 368

Hezekiah, 358

Hyvernat, Henri, v, xvi, I I 5, I 8

illiteracy, 336

image of Emperor, 375

intercession, I 26, 358, 36 I

interest, $33^{8}$

inundation, 335

"invida," 382

Isaac, 322

Israel, 317

Jacob, 322

James, 26I

Jeremiah, 3 I 7

Jesus, I29, I 58, et passim

Jews, 302, 305, 368, 37.3

Joakim, 256, 257, 36I
Job fragment, I Io

John, 3 I6, 32 I

Jonas, fast of, $3^{62}$

Joseph, 256, 36r, 380

Josiah, $35^{8}$

Judas Iscariot, I I 7, 33 I

Kennard Papyri, xxvi

Kenyon, Sir Frederic G., v

Kesaria, 380

king, title of emperor, I 26

(vs. tyrant), 378

kissing of eikon, 370

Kollothos, 379

lamb (name of the Virgin), 334

land-measurer (name of Gabriel), 334

Lane, E. W., 356

Leah, 322

lions, $333 \cdot 347,35^{\circ}$

and saints, 333

loans, 337

Logos (the Word), I 29, et passim

Mareotis, 37 I

Mary, 278, 359 ff., et passim

Mercurius, St., I I 5, 32 I, 379

Messiah, 257

Mesore, 359

"metator," I 52

Michael, 355

Middle Egyptian, I 16, I 28

minute volumes, $\mathrm{xi}$ ff.

miracles, seen by homilist, 35 I

moderation, 358

Monastery of St. Mercurius, I I 5

Monastery of the Vinedresser, $x$

Morgan Library, vi

Moses, 334

names, in magic, $3^{8} 3$

Nazarene, 293

negro, 354

Nestorius, 125, 329, 350

Nile, 335

ninety-nine, $327,35^{8}, 359$

Nineveh, fast of, 362

Nubian, 354

oath, at shrine, 340

obelus, I I 8, I I9

Odes of Solomon, 360,361 
Odyssey, 355

oil or water, magic, 383

ornament, I I 7

one God, 354

palsy, 343

painless birth, 360

patriarchs, 380

Paul, 278, 319

penetration of matter, 360

perjury, 328, 33I

Perry, A. M., v

persecution (?), 359

Philip, the rich man, 336

Philotheos, 322, 380

plague (?), 359

"praefectus augustalis," 369

prayer, 328

provenance of MSS., $x$, I 5

psalter mss., xxiii

punctuation, xviii, I 8

questioning of soul, $35^{8}$

Rachel, 322

Rebecca, 322

repository, $33^{8}$

riches, 337

ring-signs, I 28, 38I

River Nile, 335

Romans, 246, 358

Rome, 327, 348, 349, 358

"round " letters, xv

Sabaoth, 334

sabbath and Lord's Day, 362

saints, I 26

and lions, 333

Sammeas, 3I 7, 378

Sanclers, H. A., v, x

Saracens, 379

Sarาh, 322

Satan, I69, 240

sātōr-formula, $38 \mathbf{I}, 382$

scrying, 356

Senate, 358

Shenute, 355, 377, 382

ship-owner, 380 shrine, 35 I

sign of cross, 353

sins, 365,378

Sisinnios, 322,380

Solomon, $35^{8}$

spelling, xviii f., I I 9

Stephen, the poor man, 356

storehouse, 359, 369, 375

sweating, 348

syllable division, I 19

synaxarium, $327,347,359$

synaxis, 351,372

tablet of wood, 359,370

Tacitus, 375

tall, Devil, 355

Talmud, 36I

text of psalter, xxiii

Theognis, 358

Theophilus, I 25, 249, 284, 359, 369

Theopiste, 380

Theopistos, 322. 380

theotokos, 334, 359

doctrine of, 125

torment of the dying, 363, 365

Treasury of Pearls (Mary), 36r

Typhon, 355

unbelief, 378

ungrateful steward, 336

" unnaturalness," 376, 378

Vaschalde, A. A., v

vellum, $x v$

Virgin, $359 \mathrm{ff}$.

virginity, $360 \mathrm{ff}$.

vow, 348

water or oil, magic, 383

Werzēlyā, 382

White Monastery, $\mathrm{x}$

wild beasts, 347

Winstedt, E. O., v

worm-holes, $x$, i 6

writing, $\mathrm{xv}$ ff., I I 7, I I 8, I 28

Zachariah, 333, 334

Zedekias, 3 I 7, 378 


\section{INDEX OF WORDS IN OTHER LANGUAGES}

บบำ, $324,382,383$

จิหม, 340

dee Lol, 123

xigg; $3^{82}$

Ba' ūnāt, $3^{82}$

Bawwāna, 382

הילוา 382

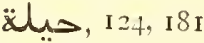

กทศฺ", 324

Jaw, $3^{82}, 3^{8} 3$
ํำ 355

กอ๊ร้, 355

ghūl, 354

centenarius, I60, 336

centrum, 323

charta, I 68, I 74

indictio, $32 \mathrm{I}$

milium, 207, 217.

praefectus augustalis, 284, 369 


\section{University of Michigan Studies}

HUMANISTIC SERIES

General Editors. FRANCIS W. KELSEY and HENRY A. SANDERS

Size, $22.7 \times 15.2 \mathrm{~cm}$. $8^{\circ}$. Bound in cloth

Vol. I. Roman Historical Sources and Institutions. Edited by Henry A. Sanders, University of Michigan. Pp. vii +402 . $\$ 2.50$ net.

\section{CONTENTS}

I. The Myth about Tarpeia: Henry A. Sanders.

2. The Movements of the Chorus Chanting the Carmen SaeCUlARE: Walter Dennison.

3. Studies in the Lives of Roman Empresses, Julia Mamaea: Mary Gilmore Williams, Mt. Holyoke College.

4. The Attitude of Dio Cassius toward Epigraphic Sources: Duane Reed Stuart, Princeton University.

5. The Lost Epitome of Livy: Henry A. Sanders.

6. The Principales of the Early Empire: Joseph H. Drake, University of Michigan.

7. Centurions as Substitute Commanders of Auxiliary Corps: George H. Allen.

Vol. II. Word Formation in Provençal. By Edward L. Adams, University of Michigan. Pp. xvii $+607 . \$ 4.00$ net.

Vol. III. Latin Philology. Edited by Clarence Linton Meader, University of Michigan. Pp. vii +290 . \$2.00 net.

Parts Sold Separately in Paper Covers:

Part I. The Use of idem, ipse, and Words of Related MeanING. By Clarence L. Meader. Pp. I-II2. \$0.75.

Part II. A Study in Latin Abstract Substantives. By Manson A. Stewart, Yankton College. Pp. II3-78. \$o.40.

Part III. The Use of the Adjective as a Substantive in the De Rerum Natura of Lucretius. By Frederick T. Swan. Pp. I79-2I4. \$0.40.

Part IV. Autobiographic Elements in Latin Inscriptions. By Henry H. Armstrong, Beloit College. Pp. 2I 5-86. \$o.40.

\section{THE MACMILLA N COM PA N Y \\ Publishers \\ 64-66 Fifth Avenue \\ New York}




\section{University of Michigan Studies_Continued}

Vol. IV. Roman History and Mythology. Edited by Henry A. Sanders. Pp. viii $+427 . \$ 2.50$ net.

Parts Sold Separately in Paper Covers:

Part I. Studies in the Life of Heliogabalus. By Orma Fitch Butler, University of Michigan. Pp. I-I69. \$I.25 net.

Part II. The Myth of Hercules at Rome. By John G. Winter, University of Michigan. Pp. I $7 \mathrm{I}-273$. \$0.50 net.

Part III. Roman Law Studies in Livy. By Alvin E. Evans. Pp. 275-354. \$0.40 net.

Part IV. Reminiscences of Eninius in Silius Italicus. By Loura B. Woodruff. Pp. 355-424. \$o.40 net.

Vol. V. Sources of the Synoptic Gospels. By Rev. Dr. Carl S. Patton. Pp. xïi +263 . \$1.30 net.

Size, $28 \times 18.5 \mathrm{~cm}$. $4^{\text {to. }}$

Vol. VI. Athenian Lekythoi with Outline Drawing in Glaze Varnish on a White Ground. By Arthur Fairbanks, Director of the Museum of Fine Arts, Boston. With 5 plates, and 57 illustrations in the text. Pp. viii +37 r. Bound in cloth. $\$ 4.00$ net.

Vol. VII. Athenian Lekythoi With OUtline Drawing in Matt Color on a White Ground, and an Appendix: Additional Lekythoi With Outline Drawing in Glaze Varnish on a White Ground. By Arthur Fairbanks. With 4x plates. Pp. $x+275$. Bound in cloth. $\$ 3.50$ net.

Vol. VIII. The Old Testament Manuscripts in the Freer Collection. By Henry A. Sanders, University of Michigan. With 9 plates showing pages of the Manuscripts in facsimile. Pp. viii +357 . Bound in cloth. $\$ 3.50$ net.

Parts Sold Separately in Paper Covers:

Part I. The Washington Manuscript of Deuteronomy and Joshua. With 3 folding plates. Pp. vi + ro4. \$1.25 net.

Part II. The Wasmington Manuscript of the Psalms. With i single plate and 5 folding plates. Pp. viii + 105-357. \$2.00 net.

THE MACMILLAN COMPAN Y

Publishers

64-66 Fifth Avenue

New York 


\section{University of Michigan Studies_-Continued}

Vol. IX. The New Testament Manuscripts in the Freer Collection. By Henry A. Sanders, University of Michigan. With 8 plates showing pages of the Manuscripts in facsimile. Pp. $x+323$. Bound in cloth. $\$ 3.50$ net.

Parts Sold Separately in Paper Covers:

Part I. The Washington Manuscript of the Four Gospels. With 5 plates. Pp. vii + 247. \$2.00 net.

Part II. The Washington Manuscript of the Epistles of Paul. With 3 plates. Pp. vii, 249-3I5. \$1.25 net.

Vol. X. The Coptic Manuscripts in the Freer Collection. By William H. Worrell, Hartford Seminary Foundation. With I 2 plates. Pp. xxvi +396 . Bound in cloth. $\$ 4.75$ net.

Parts Sold Separately in Paper Covers:

Part I. The Coptic Psalter. The Coptic text in the Sahidic Dialect, with an Introduction, and with 6 plates showing pages of the Manuscript and Fragments in Facsimile. Pp. xxvi + II 2. \$2.00 net.

Part II. A Homily on the Archangel Gabriel by Celestinus, Archishop of Rome, and A Homily on the Virgin by TheopHillus, Archbishop of Alexandria, from MANuscript Fragments in the Freer Collection and the British Museum. The Coptic Text, with an Introduction and Translation, and with 6 plates showing pages of the Manuscripts in facsimile. Pp. I $13-396$. \$2.5० net.

Vol. XI. Contributions to the History of Science. (Parts $I$ and $I I$ ready.)

Part I. Robert of Chester's Latin Translation of the Algebra of Al-Khowarizmi. With an Introduction, Critical Notes, and an English Version. By Louis C. Karpinski, University of Michigan. With 4 plates showing pages of manuscripts in facsimile, and 25 diagrams in the text. P p. vii $+\mathrm{I}_{4}$. Paper covers. \$2.00 net.

Part II. The Prodromus of Nicolaus Steno's Latin Dissertation on a Solid Body Enclosed by Process of Nature within A Solid. Translated into English by John G. Winter, University of Michigan, with a Foreword by William H. Hobbs. With 7 plates. Pp. vii + r69-283. Paper covers. \$r.3o net.

Part III. Vesuvius in Antioumity. Passages of Ancient Authors, with a Translation and Elucidations. By Francis W. Kelsey. Illustrated. (In preparation).

\section{THE MACMILLAN COMPANY \\ Publishers \\ 64-66 Fifth Avenue \\ New York}




\section{University of Michigan Studies_Continued}

Vol. XII. Studies in East Christian and Roman Art. By Charles R. Morey, Princeton University, and Walter Dennison. With 67 plates (Io colored) and 91 illustrations in the text. Pp. xiii $+\mathrm{I} 75$. Bound in cloth. $\$ 4.75$ net.

Parts Sold Separately:

Part I. East Christian Paintings in the Freer Collection. By Charles R. Morey. With I3 plates (ro colored) and 34 illustrations in the text. Pp. xiii +86 . Bound in cloth. \$2.50 net.

Part II. A Gold Treasure of the Late Rohan Period from EgYPT. By Walter Dennison. With 54 plates and 57 illustrations in the text. Pp. 89-175. Bound in cloth. \$2.50 net.

Vol. XiII. Documents from the Cairo Genizah in the Freer Collection. Text, with Translation and an Introduction by Richard Gottheil, Columbia University. (In preparation.)

Vol. XiV. Aspects of Roman Law and Admintstration.

Part I. The Master of Offices in the Later Roman and Byzantine EMpires. By Arthur E. R. Boak, University of Michigan. Pp. x + I6o. Paper covers. \$I.00 net.

Part II. The Office of the Grand Chamberlain in the Later Roman and Byzantine Empires. By James E. Dunlap, Indiana University. (In press.)

Vol. XV. Greek Themes in Modern Musical Settings. By Albert A. Stanley, University of Michigan. (In press.)

Part I. Incidental Music to Percy Mackaye's Drama of Sappho and Phaon.

Part II. Music to the Alcestis of Euripides with English Text.

Part III. Music to the Iphigenta among the Taurians by Euripides, with Greek Text.

Part IV. Two Fragments of Ancient Greek Music.

Part V. Music to Cantica of the Menaechum of Plautus.

Part VI. Attis: A Symphonic Poem.

Vol. XVI. Nicomachus of Gerasa: Introduction to ArithMETIC. Translated into English by Martin Luther D'Ooge, with Studies in Greek Arithmetic by Frank Egleston Robbins and Louis C. Karpinski. (In preparation.)

Vols. XVII, XVIII. Correspondence of the Assyrian Kings: Sargon Period. Translated into English, with a transliteration of the Text and a Commentary. By Leroy Waterman, University of Michigan. (In press.)

THE MA CMILLAN COMPAN Y

Publishers

64-66 Fifth Avenue

New York 


\title{
FACSIMILES OF MANUSCRIPTS
}

\author{
Size, $40.5 \times 35 \mathrm{~cm}$.
}

Facsimile of the Washington Manuscript of Deuteronomy ANd Joshua in the Freer Collection. With an Introduction by Henry A. Sanders. Pp. x; 2or heliotype plates. The University of Michigan. Ann Arbor, Michigan, rg го.

Limited edition, distributed only to Libraries, under certain conditions. A list of Libraries containing this Facsimile is printed in University of Michigan Studies, Humanistic Series, Volume VIII, pp. $35^{1-353}$.

Size, $34 \times 26 \mathrm{~cm}$.

Facsimile of the Washington Manuscript of the Four Gospels in the Freer Collection. With an Introduction by Henry A. Sanders. Pp. x; 372 heliotype plates and 2 colored plates. The University of Michigan. Ann Arbor, Michigan, IgI 2.

Limited edition, distributed only to Libraries, under certain conditions. A list of Libraries containing this Facsimile is printed in University of Michigan Studies, Humanistic Series, Volume IX, pp. 3I 7-320.

\section{SCIENCE SERIES}

Size, $28 \times 18.5 \mathrm{~cm} .4^{\circ}$. Bound in cloth

Vol. I. The Circulation and Sleep. By John F. Shepard, University of Michigan. Pp. ix +83 , with an Atlas of $6_{3}$ plates, bound separately. Text and Atlas, $\$ 2.50$ net.

Vol. II. Studies on Divergent Series and Summability. By Walter B. Ford, University of Michigan. Pp. xi + 194. \$2.50.

\section{University of Michigan Publications}

General Editor: EUGENE S. McCARTNEY.

Size, $22.7 \times 15.2 \mathrm{~cm}$. $8^{\circ}$. Bound in cloth

The Life and Works of George Sylvester Morris: A Chapter in the History of American Thought in the Nineteenth Century. By Robert M. Wenley, University of Michigan. Pp. $\mathrm{xv}+332$. $\$ \mathrm{r} .50$ net.

Latin and Greek in American Education, with Symposia on the Value of Humanistic Studies. Edited by Francis W. Kelsey. Pp. $\mathrm{x}+396$. \$1.50. (Out of print; new edition in preparation.)

The Present Position of Latin and Greek, tie Value of Latin and Greek as Educational Instruments, the Nature of Culture Studies.

THE M A C M L L A N COM PA N Y

Publishers 64-66 Fifth Avenue New York 


\section{University of Michigan Publications - Continued}

Symposia on the Value of Humanistic, particularly Classical, Studies as a Preparation for the Study of Medicine, Engineering, LaW and TheOLOgY.

A Symposium on the Value of Humanistic, particularly Classical, Studies as a Training for Men of Affairs.

A Symposium on the Classics and the New Education.

A Symposium on the Doctrine of Formal Discipline in the Ligit of Contemporary Psychology.

The Senate and Treaties, i789-I8I7: The Development of the Treaty-Making Functions of the United States Senate during Their Formative Period. By Ralston Hayden, University of Michigan. Pp. xvi +237 . Cloth \$I.5० net.

Size, $23.5 \times 15.5 \mathrm{~cm}$. $8^{\circ}$. Bound in cloth

William Plumer's Memorandum of Proceedings in the United States Senate, I803-I807. Edited by Everett Somerville Brown, University of Michigan. Pp. xi +673 . Cloth. $\$ 3.50$.

Papers of the Michigan Academy of Science, Arts and LetTERS. Volume I, containing Papers submitted at the Annual Meeting in I92I. Edited by Paul S. Welch and Eugene S. McCartney. With 38 plates and 5 maps. Pp. xi +424 . \$2.00 net.

Size, $\mathrm{I} 8 \times \mathrm{I} 2 \mathrm{~cm}$.

The Menaechim of Plautus. The Latin Text, with a Translation by Joseph H. Drake, University of Michigan. Pp. xi + I 29. \$0.60 net.

This edition of the Menaechmi was prepared, with stage directions, as a libretto for the presentation of the play at the University of Michigan in 1890. It was revised and republished in 1916.

THE MACMILLA N C OMPA N Y

Publishers

64-66 Fifth Avenue

New York 


\title{
HANDBOOKS OF ARCHAEOLOGY AND ANTIQUITIES
}

\author{
EDITED BY PERCY GIRDNER AND FRANCIS W. KELSEY
}

\section{THE PRINCIPLES OF GREEK ART}

By Percy Gardner, Litt. D., Lincoln and Merton Professor of Classical Archaeology in the University of Oxford.

Makes clear the artistic and psychological principles underlying Greek art, especially sculpture, which is treated as a characteristic manifestation of the Greek spirit, a development parallel to that of Greek literature and religion. While there are many handbooks of Greek archaeology, this volume holds a unique place.

New Edition. Illustrated. Cloth $\$ 3.25$

\section{HANDBOOK OF GREEK SCULPTURE}

By Ernest A. Gardner, M. A., Professor of Archacology in University College, London.

A comprehensive outline of our present knowledge of Greek sculpture, distinguishing the different schools and periods, and showing the development of each. This volume, fully illustrated, fills an important gap and is widely used as a text-book.

Illustrated. Cloth $\$ 4.25$

\section{ATHENS AND ITS MONUMENTS}

By Charles Heald Weller, of the University of Iowa.

This book embodies the results of many years of study and of direct observation during different periods of residence in Athens. It presents in concise and readable form a description of the ancient city in the light of the most recent investigations. Profusely illustrated with Half-tones and Line Engravings.

Illustrated. Cloth $\$ 4.00$

\section{ROMAN FESTIVALS}

By W. Warde Fowler, M. A., Fellow and Sub-Rector of Lincoln College, Oxford.

This book covers in a concise form almost all phases of the public worship of the Roman state, as well as certain ceremonies which, strictly speaking, lay outside that public worship. It will be found very useful to students of Roman literature and history as well as to students of anthropology and the history of religion.

Cloth $\$ 2.50$

On sale wherever books are sold

\section{THE MACMILLAN COMPA N Y}

Publishers 


\section{HELLENIC HISTORY}

\section{By George Willis Botsford}

A survey of Greek life from its primitive beginnings to the year 30 B.C., with an account of the political, social, economic, artistic, intellectual, and religious development. The book is abundantly illustrated.

\section{TABLE OF CONTENTS}

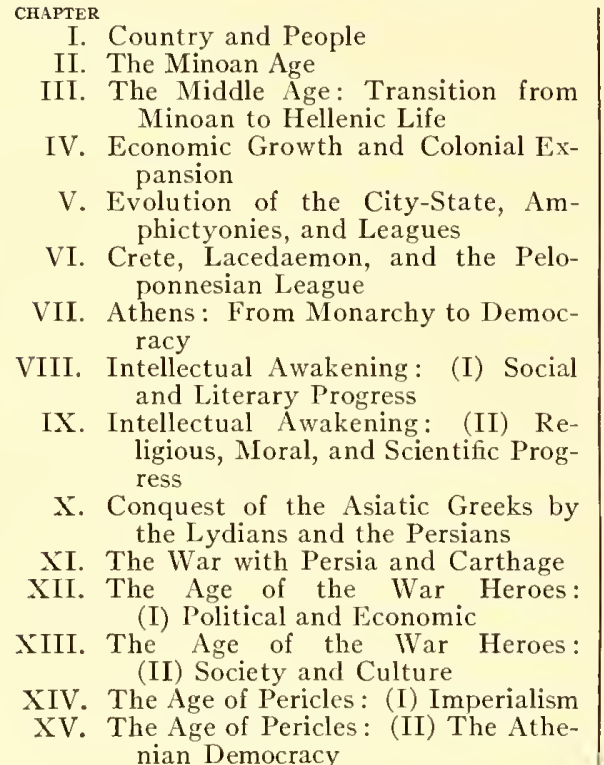

CHAPTER

XVI. The Age of Pericles: (III) Society and Public Works

XVII. The Age of Pericles:(IV) Thought, Culture, and Character

XVIII. The Peloponnesian War to the Beginning of the Sicilian Expedition

XIX. The Sicilian Expedition and the Last Years of the War

XX. A Cultural Revolution

XXI. The Lacedaemonian Empire and the Ascendancy of Thebes

XXII. Sicily and Magna Graecia

XXIII. The Rise of Macedon to 337

XXIV. Economy and Society

XXV. Social Aspects of the State

XXVI. Art and Intelligence in the Fourth Century

XXVII. Alexander's Empire and the Hellenistic Kingdoms

XXVIII. The Organization and Administration of the Hellenistic States

XXIX. Hellenistic Culture: (I) City Construction and Art

XXX. Hellenistic Culture: (II) Philosophy, Science, and Literature

Price $\$ 4.00$

\section{A HISTORY OF ROME TO 565 A.D.}

By Arthur E. R. BoAk, Professor of Ancient History in the University of Michigan

A well-proportioned and accurately written history of Rome from the beginning of civilization in Italy to $565 \mathrm{~A} . \mathrm{D}$.

\section{TABLE OF CONTENTS}

INTRODUCTION

The Sources for the Study of Early Roman History

PART I

The Forerunners of Rome in Italy

PART II

The Early Monarchy and the Republic, from Prehistoric Times to 27 B.C. PART III

The Principate or Early Empire: 27 B.C. -285 A.D. PART IV

The Autocracy or Late Empire: 285-565 A.D.

Epilogue Chronological Table Bibliographical Note Index

$$
\text { Price } \$ 3.25
$$

On sale wherever books are sold

THE MACMILLA N COMPANY

Publishers 64-66 Fifth Avenue New York 



INIMUTSONIAN INSTTUTION LIBRARIES

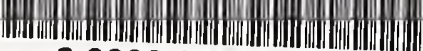
39088016743544 